\title{
Untersuchungen zur Synthese und Struktur von ein- und mehrkernigen Komplexen der Münzmetalle mit Chelatliganden
}

\author{
Dissertation \\ zur Erlangung des Doktorgrades \\ der Mathematisch-Naturwissenschaftlichen Fakultäten \\ der Georg-August-Universität zu Göttingen
}

vorgelegt von

Ruth Dinger

aus Renchen

Göttingen 1999 
D7

Referent: Prof. Dr. J. Magull

Korreferent: Prof. G.M. Sheldrick, PhD

Tag der mündlichen Prüfung: 3. November 1999 


\section{Inhaltsverzeichnis}

1 Einleitung 1

$\begin{array}{llr}2 & \text { Aufgabenstellung } & 7\end{array}$

3 Gang der Untersuchung $\quad 9$

3.1 Umsetzungen der Methanderivate $\operatorname{dppmE}_{2} \mathrm{H}(\mathrm{E}=\mathrm{O}, \mathrm{S}, \mathrm{Se}) \ldots \ldots$

3.1.1 Die Synthese und Struktur von $\left[\mathrm{Cu}\left(\mathrm{dppmO}_{2} \mathrm{H}\right)_{3}\right]\left[\mathrm{CuCl}_{4}\right] \underline{1} \ldots$. . . 9

3.1.2 Die Synthese und Struktur von $\left[\mathrm{Cu}_{2}\left(\mathrm{dppmS}_{2} \mathrm{H}\right)_{2} \mathrm{Cl}\right]\left[\mathrm{CuCl}_{2}\right] \underline{2}$. . . . 11

3.1.3 Die Synthese und Struktur von $\left[\mathrm{Cu}_{2}\left(\mathrm{dppmSe}_{3}\right)_{2}\right]$ 3 . . . . . . . . . 16

3.1.4 Die Synthese und Struktur von $\left[\mathrm{Au}_{2}\left(\mathrm{dppmS}_{2}\right)\left(\mathrm{PPh}_{3}\right)_{2}\right] \underline{4} \ldots \ldots$

3.2 Umsetzungen von $\mathrm{CuCl}$ und $\mathrm{CuCl}_{2}$ mit dem $\mathrm{Amin}_{\mathrm{dppaO}} \mathrm{H}$. . . . . . . . 24

3.2.1 Die Synthese und Struktur von $\left[\mathrm{Cu}\left(\mathrm{dppaO}_{2}\right)_{2}(\mathrm{MeOH})\right] \underline{5}$. . . . . . 24

3.2.2 Die Synthese und Struktur von $\left[\mathrm{Cu}_{2}\left(\mathrm{dppaO}_{2}\right)_{2} \mathrm{Cl}_{2}(\mathrm{thf})_{2}\right] \underline{6} \ldots \ldots 27$

3.2.3 Die Synthese und Struktur von $\left[\mathrm{Cu}_{2}\left(\mathrm{dppaO}_{2}\right)_{2}(\mathrm{OMe})_{2}\right] \underline{7} \ldots \ldots$

3.2.4 Die Synthesen und Strukturen von

$\left[\mathrm{Cu}_{3}\left(\mathrm{dppaO}_{2}\right)_{2}\left(\left(\mathrm{OSiMe}_{2}\right)_{2} \mathrm{O}\right)_{2}(\mathrm{thf})_{2}\right] \underline{8}$ und $\left[\mathrm{Cu}_{4}(\mathrm{O})\left(\mathrm{dppaO}_{2}\right)_{4} \mathrm{Cl}_{2}\right] \underline{\underline{9}} \quad 32$

3.3 Umsetzungen von $\mathrm{CuCl}$ und $\mathrm{CuCl}_{2}$ mit den Aminen dppaOEH ( $\left.\mathrm{E}=\mathrm{S}, \mathrm{Se}\right) \quad 39$

3.3.1 Die Synthese von $\left[\mathrm{Cu}_{3}(\mathrm{dppaOS})_{3}\right] 10 \ldots 39$

3.3.2 Die Synthese und Struktur von $\left[\mathrm{Cu}\left(\mathrm{dppaO}_{2}\right)_{2}\left(\mathrm{NH}_{3}\right)_{3}\right]_{\infty} \underline{11} \ldots \ldots$

3.3.3 Die Synthese und Struktur von $\left[\mathrm{H}\left((\mathrm{O}) \mathrm{PPh}_{2} \mathrm{NPPh}_{2}\right)_{2} \mathrm{O}\right]\left[\mathrm{CuCl}_{2}\right] \underline{12}$. 42

3.4 Umsetzungen von $\mathrm{CuCl}$ und $\mathrm{AgCl}$ mit den Aminen dppaE $\mathrm{E}_{2} \mathrm{H}(\mathrm{E}=\mathrm{S}, \mathrm{Se})$. 45

3.4.1 Die Synthesen und Strukturen von $\left[\mathrm{NaCu}_{2}\left(\mathrm{dppaS}_{2}\right)_{3}(\mathrm{thf})_{2}\right] \underline{13}$ und $\left[\mathrm{NaCu}_{2}\left(\mathrm{dppaSe}_{2}\right)_{3}(\mathrm{thf})_{2}\right] 14 \ldots \ldots \ldots$. . . . . . . . . . . 45

3.4.2 Die Synthesen und Strukturen von $\left[\mathrm{Cu}_{3}\left(\mathrm{dppaE}_{2}\right)_{3}\right]$

$\left(\mathrm{E}=\mathrm{S}\right.$ und $\mathrm{O}: \underline{10} ; \mathrm{S}: \underline{15}$; Se: 16), $\left[\mathrm{Ag}_{3}\left(\mathrm{dppaS}_{2}\right)_{3}\right] \underline{17}$ und $\left[\mathrm{Na}_{2} \mathrm{Ag}_{2}\left(\mathrm{dppaS}_{2}\right)_{4}(\mathrm{thf})_{4}\right]_{18}^{18} \ldots \ldots \ldots \ldots$

3.4.3 Die Synthesen und Strukturen von $\left[\mathrm{Cu}_{4}\left(\mathrm{dppaS}_{2}\right)_{3}\right]_{2}\left[\mathrm{CuCl}_{2}\right][\mathrm{OH}] \underline{19}$ und $\left[\mathrm{Cu}_{4}\left(\mathrm{dppaSe}_{2}\right)_{3}\right]\left[\mathrm{CuCl}_{2}\right] \underline{20} \ldots \ldots \ldots \ldots \ldots$

3.4.4 Die Synthese und Struktur von $\left[\mathrm{Cu}\left(\mathrm{dppaS}_{2}\right)(\mathrm{th})\right] \underline{21} \ldots \ldots . . . .57$

3.4.5 NMR-spektroskopische Untersuchungen . . . . . . . . . . . . . . . . 59

3.5 Umsetzungen von $\mathrm{CuCl}$ und $\mathrm{CuCl}_{2}$ mit dem Amin dmpaS $\mathrm{S}_{2} \mathrm{H}$. . . . . . . . 62

3.5.1 Die Synthese und Struktur von $\left[\mathrm{Cu}_{2}\left(\mathrm{dmpaS}_{2} \mathrm{H}\right)_{2} \mathrm{Cl}_{2}\right] \underline{22} \ldots$. . . . . 62

3.5.2 Die Synthesen und Strukturen von $\left[\mathrm{Cu}_{5}\left(\mathrm{dmpaS}_{2}\right)_{5}\right] \underline{23}$,

$\left[\mathrm{Cu}_{8}\left(\mathrm{dmpaS}_{2}\right)_{6} \mathrm{Cl}_{2}\right] \underline{24}$ und $\left[\mathrm{Cu}_{10}\left(\mathrm{dmpaS}_{2}\right)_{6} \mathrm{Cl}_{4}\right] \underline{25} \ldots \ldots . . .64$ 
3.5.3 Die Synthese und Struktur von $\left[\mathrm{KCu}\left(\mathrm{dmpaS}_{2}\right)_{2}(\mathrm{thf})\right]_{\infty} \underline{26} \ldots \ldots$

3.5.4 NMR-spektroskopische Untersuchungen . . . . . . . . . . . . . 75

3.6 Orientierende Versuche . . . . . . . . . . . . . . . . . . . . . . . 79

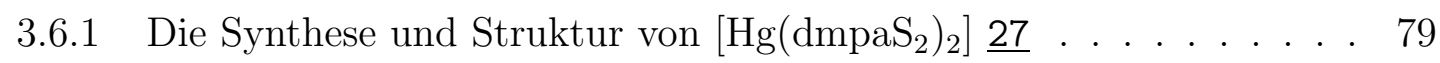

4 Experimenteller Teil $\quad 81$

4.1 Allgemeines . . . . . . . . . . . . . . . . . . . 81

4.2 Darstellung der Ausgangsverbindungen . . . . . . . . . . . . . . . 82

4.2.1 Darstellung von Bis(diphenylphosphoryl)amin $\left(\right.$ dppaO $\left._{2} \mathrm{H}\right) \quad \ldots \quad$. . . 82

4.2.2 Darstellung von Diphenylphosphino(diphenylphosphoryl)amin

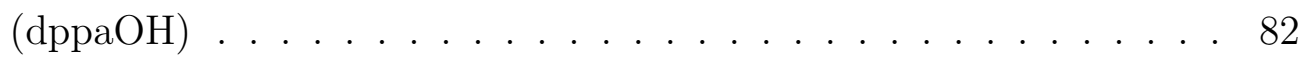

4.2.3 Darstellung von Diphenylphosphoryl(diphenylthiophosphoryl)amin (dppaOSH) und Diphenylphosphoryl(diphenylselenophosphoryl)-

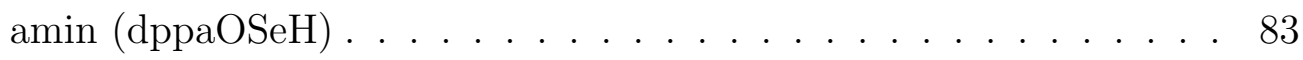

4.2.4 Darstellung von Bis(diphenylphosphoryl)methan $\left(\mathrm{dppmO}_{2} \mathrm{H}\right)$. . . . 83

4.2.5 Darstellung von Bis(diphenylselenophosphoryl)methan (dppmSe $\left.\mathrm{e}_{2} \mathrm{H}\right) \quad 84$

4.2.6 Darstellung von $\mathrm{CuO}^{\mathrm{t}} \mathrm{Bu}$. . . . . . . . . . . . . . . . . 84

4.3 Umsetzungen von $\mathrm{CuCl}, \mathrm{CuCl}_{2}$ und $\mathrm{AuCl}\left(\mathrm{PPh}_{3}\right)$ mit dppmE $\mathrm{H}_{2} \mathrm{H}$ $(\mathrm{E}=\mathrm{O}, \mathrm{S}, \mathrm{Se}) \ldots \ldots \ldots \ldots \ldots$

4.3.1 Darstellung von $\left[\mathrm{Cu}\left(\mathrm{dppmO}_{2} \mathrm{H}\right)_{3}\right]\left[\mathrm{CuCl}_{4}\right]$ 1. . . . . . . . . . 84

4.3.2 Darstellung von $\left[\mathrm{Cu}_{2}\left(\mathrm{dppmS}_{2} \mathrm{H}\right)_{2} \mathrm{Cl}\right]\left[\mathrm{CuCl}_{2}\right] \underline{2} \ldots \ldots . . . . . . \quad 85$

4.3.3 Darstellung von $\left[\mathrm{Cu}_{2}\left(\mathrm{dppmSe}_{3}\right)_{2}\right]$ 3. . . . . . . . . . . . 85

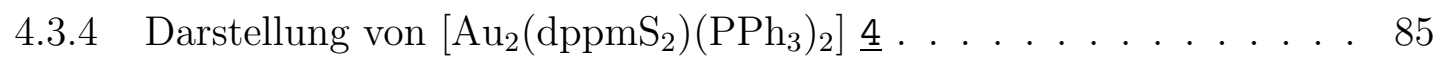

4.4 Umsetzungen von $\mathrm{CuCl}$ und $\mathrm{CuCl}_{2}$ mit dppaO $\mathrm{O}_{2} \mathrm{H} \ldots \ldots$. . . . . . . 86

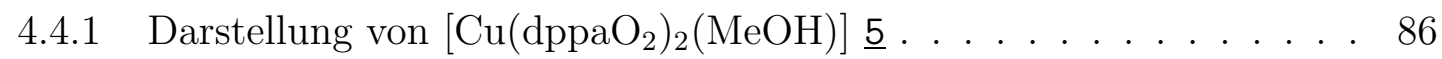

4.4.2 Darstellung von $\left[\mathrm{Cu}_{2}\left(\mathrm{dppaO}_{2}\right)_{2} \mathrm{Cl}_{2}(\mathrm{thf})_{2}\right] \underline{6} \ldots \ldots \ldots$. . . . . 86

4.4.3 Darstellung von $\left[\mathrm{Cu}_{2}\left(\mathrm{dppaO}_{2}\right)_{2}(\mathrm{OMe})_{2}\right] \underline{7} \ldots \ldots \ldots$. . . . . 86

4.4.4 Darstellung von $\left[\mathrm{Cu}_{3}\left(\mathrm{dppaO}_{2}\right)_{2}\left(\left(\mathrm{OSiMe}_{2}\right)_{2} \mathrm{O}\right)_{2}(\mathrm{thf})_{2}\right] \underline{8}$ und $\left[\mathrm{Cu}_{4}(\mathrm{O})\left(\mathrm{dppaO}_{2}\right)_{4} \mathrm{Cl}_{2}\right] \underline{9} \ldots \ldots \ldots . \ldots . \ldots . \ldots . \ldots 7$

4.5 Umsetzungen von $\mathrm{CuCl}$ und $\mathrm{CuCl}_{2}$ mit dppaOSH . . . . . . . . . . 87

4.5.1 Darstellung von $\left[\mathrm{Cu}_{3}(\mathrm{dppaOS})_{3}\right] \underline{10}$ und $\left[\mathrm{Cu}(\mathrm{dppaOS})_{2}\right] \ldots . . .87$

4.6 Umsetzungen von $\mathrm{CuCl}$ mit dppaOSeH . . . . . . . . . . . . . . . . . 87

4.6.1 Darstellung von $\left[\mathrm{Cu}\left(\mathrm{dppaO}_{2}\right)_{2}\left(\mathrm{NH}_{3}\right)_{3}\right]_{\infty} \underline{11} \ldots \ldots \ldots$. . . . . . 87

4.6.2 Darstellung von $\left[\mathrm{H}\left((\mathrm{O}) \mathrm{PPh}_{2} \mathrm{NPPh}_{2}\right)_{2} \mathrm{O}\right]\left[\mathrm{CuCl}_{2}\right] \underline{12} \ldots \ldots . . . . \quad . \quad 88$

4.7 Umsetzungen von $\mathrm{CuCl}$ und $\mathrm{AgCl}$ mit dppaE $\mathrm{E}_{2} \mathrm{H}(\mathrm{E}=\mathrm{S}, \mathrm{Se}) \ldots . . . \quad 88$ 
4.7.1 Darstellung von $\left[\mathrm{NaCu}_{2}\left(\mathrm{dppaE}_{2}\right)_{3}(\mathrm{thf})_{2}\right](\mathrm{E}=\mathrm{S}: \underline{13} ;$ Se: $\underline{14}) \ldots 88$

4.7.2 Darstellung von $\left[\mathrm{Cu}_{3}\left(\mathrm{dppaS}_{2}\right)_{3}\right] \underline{15} \ldots \ldots \ldots$. . . . . . . . 88

4.7.3 Darstellung von $\left[\mathrm{Cu}_{3}\left(\mathrm{dppaSe}_{2}\right)_{3}\right] \underline{16} \ldots \ldots$. . . . . . . . . . . . 89

4.7.4 Darstellung von $\left[\mathrm{Cu}_{4}\left(\mathrm{dppaS}_{2}\right)_{3}\right]_{2}\left[\mathrm{CuCl}_{2}\right][\mathrm{OH}] \underline{19} \ldots \ldots . . . . . \quad . \quad 89$

4.7.5 Darstellung von $\left[\mathrm{Cu}_{4}\left(\mathrm{dppaSe}_{2}\right)_{3}\right]\left[\mathrm{CuCl}_{2}\right] 20 \ldots \ldots$. . . . . . . 89

4.7.6 Darstellung von $\left[\mathrm{Cu}\left(\mathrm{dppaS}_{2}\right)(\mathrm{th})\right] \underline{21} \ldots \ldots \ldots$

4.7.7 Darstellung von $\left[\mathrm{Ag}_{3}\left(\mathrm{dppaS}_{2}\right)_{3}\right] \underline{17} \ldots \ldots$. . . . . . . . . . . . 90

4.7.8 Darstellung von $\left[\mathrm{Na}_{2} \mathrm{Ag}_{2}\left(\mathrm{dppaS}_{2}\right)_{4}(\mathrm{thf})_{4}\right] \underline{18} \ldots \ldots$. . . . . . . . 90

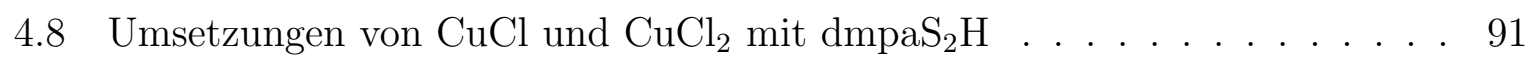

4.8.1 Darstellung von $\left[\mathrm{Cu}_{2}\left(\mathrm{dmpaS}_{2} \mathrm{H}\right)_{2} \mathrm{Cl}_{2}\right] 22 \ldots \ldots$. . . . . . . . . 91

4.8.2 Darstellung von $\left[\mathrm{Cu}_{5}\left(\mathrm{dmpaS}_{2}\right)_{5}\right] \underline{23} \ldots \ldots \ldots$. . . . . . . . 91

4.8.3 Darstellung von $\left[\mathrm{Cu}_{8}\left(\mathrm{dmpaS}_{2}\right)_{6} \mathrm{Cl}_{2}\right] \underline{24} \ldots \ldots . \ldots . . \ldots 91$

4.8.4 Darstellung von $\left[\mathrm{Cu}_{10}\left(\mathrm{dmpaS}_{2}\right)_{6} \mathrm{Cl}_{4}\right] \underline{25} \ldots \ldots \ldots . \ldots . \ldots . \ldots 92$

4.8.5 Darstellung von $\left[\mathrm{KCu}\left(\mathrm{dmpaS}_{2}\right)_{2}(\mathrm{thf})\right]_{\infty} \underline{26} \ldots \ldots . \ldots . . \ldots 92$

4.9 Darstellung von $\left[\mathrm{Hg}\left(\mathrm{dmpaS}_{2}\right)_{2}\right] 27 \ldots \ldots \ldots 2 \ldots \ldots \ldots$

5 Kristallstrukturuntersuchungen $\quad 94$

5.1 Daten zur Kristallstrukturanalyse von $\left[\mathrm{Cu}\left(\mathrm{dppmO}_{2} \mathrm{H}\right)_{3}\right]\left[\mathrm{CuCl}_{4}\right]_{1} \ldots \ldots 6$

5.2 Daten zur Kristallstrukturanalyse von $\left[\mathrm{Cu}_{2}\left(\mathrm{dppmS}_{2} \mathrm{H}\right)_{2} \mathrm{Cl}\right]\left[\mathrm{CuCl}_{2}\right] \underline{2}$. . . . 102

5.3 Daten zur Kristallstrukturanalyse von $\left[\mathrm{Cu}_{2}\left(\mathrm{dppmSe}_{3}\right)_{2}\right] \underline{3} \ldots \ldots$. . . . . . 105

5.4 Daten zur Kristallstrukturanalyse von $\left[\mathrm{Au}_{2}\left(\mathrm{dppmS}_{2}\right)\left(\mathrm{PPh}_{3}\right)_{2}\right] \underline{4}$. . . . . 108

5.5 Daten zur Kristallstrukturanalyse von $\left[\mathrm{Cu}\left(\mathrm{dppaO}_{2}\right)_{2}(\mathrm{MeOH})\right] \underline{5} \ldots . . .113$

5.6 Daten zur Kristallstrukturanalyse von $\left[\mathrm{Cu}_{2}\left(\mathrm{dppaO}_{2}\right)_{2} \mathrm{Cl}_{2}(\text { thf })_{2}\right] \underline{6} \ldots \ldots$

5.7 Daten zur Kristallstrukturanalyse von $\left[\mathrm{Cu}_{2}\left(\mathrm{dppaO}_{2}\right)_{2}(\mathrm{OMe})_{2}\right] \underline{7}$. . . . . 120

5.8 Daten zur Kristallstrukturanalyse von $\left[\mathrm{Cu}_{3}\left(\mathrm{dppaO}_{2}\right)_{2}\left(\left(\mathrm{OSiMe}_{2}\right)_{2} \mathrm{O}\right)_{2}(\text { thf })_{2}\right] \underline{8} 123$

5.9 Daten zur Kristallstrukturanalyse von $\left[\mathrm{Cu}_{4}(\mathrm{O})\left(\mathrm{dppaO}_{2}\right)_{4} \mathrm{Cl}_{2}\right] \underline{9}$. . . . . . 127

5.10 Daten zur Kristallstrukturanalyse von $\left[\mathrm{Cu}_{3}(\mathrm{dppaOS})_{3}\right] \underline{10}$. . . . . . . 131

5.11 Daten zur Kristallstrukturanalyse von $\left[\mathrm{Cu}\left(\mathrm{dppaO}_{2}\right)_{2}\left(\mathrm{NH}_{3}\right)_{3}\right]_{\infty} \underline{11} \ldots \ldots$

5.12 Daten zur Kristallstrukturanalyse von $\left[\mathrm{H}\left((\mathrm{O}) \mathrm{PPh}_{2} \mathrm{NPPh}_{2}\right)_{2} \mathrm{O}\right]\left[\mathrm{CuCl}_{2}\right] \underline{12} \quad 140$

5.13 Daten zur Kristallstrukturanalyse von $\left[\mathrm{NaCu}_{2}\left(\mathrm{dppaS}_{2}\right)_{3}(\text { thf })_{2}\right] \underline{13} \ldots \ldots 4$

5.14 Daten zur Kristallstrukturanalyse von $\left[\mathrm{NaCu}_{2}\left(\mathrm{dppaS}_{2}\right)_{3}(\text { thf })_{2}\right]_{13}^{13} \ldots \ldots$

5.15 Daten zur Kristallstrukturanalyse von $\left[\mathrm{NaCu}_{2}\left(\mathrm{dppaSe}_{2}\right)_{3}(\mathrm{thf})_{2}\right]_{14}^{14} \ldots . .154$

5.16 Daten zur Kristallstrukturanalyse von $\left[\mathrm{Cu}_{3}\left(\mathrm{dppaS}_{2}\right)_{3}\right] \underline{15} \ldots \ldots$

5.17 Daten zur Kristallstrukturanalyse von $\left[\mathrm{Cu}_{3}\left(\mathrm{dppaSe}_{2}\right)_{3}\right] \underline{16} \ldots \ldots 5$

5.18 Daten zur Kristallstrukturanalyse von $\left[\mathrm{Ag}_{3}\left(\mathrm{dppaS}_{2}\right)_{3}\right] \underline{17} \ldots \ldots$. . . . 170 
5.19 Daten zur Kristallstrukturanalyse von $\left[\mathrm{Na}_{2} \mathrm{Ag}_{2}\left(\mathrm{dppaS}_{2}\right)_{4}(\mathrm{thf})_{4}\right] \underline{18}$. . . . 175

5.20 Daten zur Kristallstrukturanalyse von $\left[\mathrm{Cu}_{4}\left(\mathrm{dppaS}_{2}\right)_{3}\right]_{2}\left[\mathrm{CuCl}_{2}\right][\mathrm{OH}] \underline{19} \ldots 180$

5.21 Daten zur Kristallstrukturanalyse von $\left[\mathrm{Cu}_{4}\left(\mathrm{dppaSe}_{2}\right)_{3}\right]\left[\mathrm{CuCl}_{2}\right] \underline{20} \ldots 183$

5.22 Daten zur Kristallstrukturanalyse von $\left[\mathrm{Cu}\left(\mathrm{dppaS}_{2}\right)(\mathrm{th})\right] \underline{21}$. . . . . . . 189

5.23 Daten zur Kristallstrukturanalyse von $\left[\mathrm{Cu}_{2}\left(\mathrm{dmpaS}_{2} \mathrm{H}\right)_{2} \mathrm{Cl}_{2}\right] \underline{22} \ldots$. . . . . 194

5.24 Daten zur Kristallstrukturanalyse von $\left[\mathrm{Cu}_{5}\left(\mathrm{dmpaS}_{2}\right)_{5}\right] \underline{23}$. . . . . . . . 196

5.25 Daten zur Kristallstrukturanalyse von $\left[\mathrm{Cu}_{8}\left(\mathrm{dmpaS}_{2}\right)_{6} \mathrm{Cl}_{2}\right] \underline{24}$. . . . . . 199

5.26 Daten zur Kristallstrukturanalyse von $\left[\mathrm{Cu}_{10}\left(\mathrm{dmpaS}_{2}\right)_{6} \mathrm{Cl}_{4}\right] \underline{25} \ldots 203$

5.27 Daten zur Kristallstrukturanalyse von $\left[\mathrm{KCu}\left(\mathrm{dmpaS}_{2}\right)_{2}(\mathrm{thf})\right]_{\infty} \underline{26} \ldots . . .210$

5.28 Daten zur Kristallstrukturanalyse von $\left[\mathrm{Hg}\left(\mathrm{dmpaS}_{2}\right)_{2}\right] \underline{27} \ldots . . . . . .213$

$\begin{array}{lll}6 & \text { Zusammenfassung } & 215\end{array}$

7 Tabellarische Zusammenfassung der durchgeführten Reaktionen 219

8 Verzeichnis der verwendeten Abkürzungen $\quad 224$

8.1 Moleküle und organische Gruppen . . . . . . . . . . . . . . . . . . 224

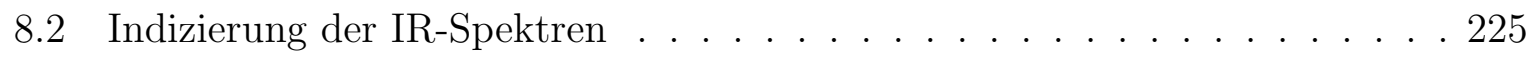

8.3 Sonstiges . . . . . . . . . . . . . . . . . . . 225

$9 \begin{array}{ll}2 & \text { Verzeichnis der Abbildungen }\end{array}$

$\begin{array}{llr}10 & \text { Literaturverzeichnis } & 229\end{array}$

11 Danksagung und Lebenslauf $\quad 238$ 


\section{Einleitung}

Die Komplexchemie der Münzmetalle Kupfer, Silber und Gold ist geprägt von Verbindungen, in denen die Metallionen in den Oxidationsstufen I bis III vorliegen. Im Falle des $\mathrm{Cu}^{\mathrm{I}}$-Kations wird meist eine tetraedrische Koodinationsgeometrie beobachtet, jedoch sind auch trigonal-planare und lineare Koordinationen möglich. Die häufigsten Koordinationszahlen für $\mathrm{Cu}^{\mathrm{II}}$ sind vier, fünf und sechs, doch werden nur selten regelmäßige, geometrische Anordnungen angetroffen, so daß die Unterscheidung zwischen quadratisch-planarer und Jahn-Tellerverzerrter oktaedrischer Koordinationsumgebung nicht leicht fällt. In $\mathrm{Ag}^{\mathrm{I}}$ - und $\mathrm{Au}^{\mathrm{I}}$ Komplexverbindungen werden meist linear und trigonal koordinierte Kationen vorgefunden, während eine tetraedrische Koordinationssphäre seltener beobachtet wird. Für Komplexe des $\mathrm{Au}^{\mathrm{III}}$-Ions sind Verbindungen mit quadratisch-planarer Geometrie typisch $[1-3]$.

Komplexe der Münzmetalle mit Chalkogenoliganden, insbesondere sauerstoff- und schwefelhaltige Verbindungen, sind in der Literatur häufig beschrieben [4,5]. Unterstützt durch die Brückenfunktion von Chelatliganden, konnten mehrkernige Verbindungen, wie z.B. der vierkernige 1,1-Dithiophosphato-Komplex $\left[\mathrm{Cu}_{4}\left(\mathrm{~S}_{2} \mathrm{P}\left(\mathrm{O}^{\mathrm{i}} \mathrm{Pr}\right)_{2}\right)_{4}\right]$ [6], dessen Grundkörper aus einem $\mathrm{Cu}_{4}$-Tetraeder besteht, dargestellt werden.

Im Vergleich zu den 1,1-Dithiophosphaten weisen Phosphorylamide des Typs $\left[\mathrm{R}_{2} \mathrm{P}(\mathrm{E}) \mathrm{NP}(\mathrm{E}) \mathrm{R}_{2}\right]^{\ominus}(\mathrm{E}=$ Chalkogen $)$ einen deutlich größeren Abstand der Donoratome ("Biß") auf, was eine ungewöhnlich hohe Flexibilität bezüglich des EPNPE-Gerüstes zur Folge hat und eine Vielfalt an Koordinationsarten an Metallatome ermöglicht. Gleichfalls gestatten die entsprechenden Phosphorylmethanide $\left[\mathrm{R}_{2} \mathrm{P}(\mathrm{E}) \mathrm{CHP}(\mathrm{E}) \mathrm{R}_{2}\right]^{\ominus}$ als chelatisierendes, meist zweizähniges Ligandensystem einen einfachen Weg zur Synthese von Komplexverbindungen.

Die Gruppe der Bis(diorganochalkogenophosphoryl)amine $\mathrm{R}_{2} \mathrm{P}(\mathrm{E}) \mathrm{NHP}(\mathrm{E}) \mathrm{R}_{2}$ und -methane $\mathrm{R}_{2} \mathrm{P}(\mathrm{E}) \mathrm{CH}_{2} \mathrm{P}(\mathrm{E}) \mathrm{R}_{2}(\mathrm{E}=\mathrm{O}$, S, Se) (Abb. 1) sind leicht in mittleren bis hohen Ausbeuten darstellbar und bieten die Möglichkeit, sowohl die Donorsubstituenten $\mathrm{E}$ als auch die organischen Reste $\mathrm{R}$ zu variieren, so daß ihr unterschiedliches Koordinationsverhalten in Komplexierungsreaktionen untersucht werden kann.

Die Synthesen und Kristallstrukturen der Liganden Bis(diphenylchalkogenophosphoryl)methan, im folgenden abgekürzt mit $\operatorname{dppmE}_{2} \mathrm{H}(\mathrm{E}=\mathrm{O}, \mathrm{S}, \mathrm{Se})$, sind gut untersucht [7-12]. Sie können sowohl in ihrer neutralen Form als auch als Methanide und Methandiide auf unterschiedliche Weisen an Metallionen koordiniert sein. Abb. 2 stellt die Koor- 
<smiles>[R][PH]([R])(F)N[PH]([R])([R])F</smiles>

(a)<smiles>[R][PH]([R])(F)C[PH]([R])([R])F</smiles>

(b)

Abb. 1: Das Ligandensystem Bis(diorganochalkogenophosphoryl)amin (a) und -methan (b)

dinationsmöglichkeiten des Methanderivates dppmE $\mathrm{E}_{2} \mathrm{H}$ und der mono- und dianionischen Liganden $\operatorname{dppmE}_{2}{ }^{\ominus}$ und $\operatorname{dppmE}_{2}{ }^{2 \ominus}$ dar.

Das Phosphorylmethan kann nicht nur chelatisierend wirken (Abb. 2 (a)), was die meist beschriebene Koordinationsform darstellt, oder einzähnig an ein Metallzentrum gebunden sein (b), sondern es besitzt auch die Fähigkeit, bimetallische Einheiten zu verbrücken $((\mathrm{c})$ und $(\mathrm{d}))$. Das Methanid $\mathrm{dppmE}_{2}{ }^{\ominus}$ zeigt mehrere, in Abb. 2 (e) bis (l) dargestellte, Bindungsmodi an ein, zwei oder drei Metallatome zu binden. Im Falle von dppmE ${ }_{2}^{2 \ominus}$ ist es zudem möglich, bis zu vier Metallzentren durch die Koordination eines Liganden miteinander zu verbinden (Abb. $2(\mathrm{~m})$ bis $(\mathrm{p})$ ).

Auch Bis(diorganochalkogenophosphoryl)amine des Typs $\mathrm{R}_{2} \mathrm{P}(\mathrm{E}) \mathrm{NHP}(\mathrm{E}) \mathrm{R}_{2}$, abgekürzt mit $\operatorname{dppaE}_{2} \mathrm{H}(\mathrm{R}=\mathrm{Ph})$ und $\operatorname{dmpaS}_{2} \mathrm{H}(\mathrm{R}=\mathrm{Me})$, sind vielseitig einsetzbare Liganden [10,20-25], die die Bildung von Metallchelatringsystemen ermöglichen, in denen der Ligand üblicherweise über die Chalkogenatome in einer symmetrischen Koordinationsweise auftritt [26-28].

Der Ligand kann aufgrund des sauren Charakters des Aminprotons leicht mit Basen wie $\mathrm{KO}^{\mathrm{t}} \mathrm{Bu},{ }^{\mathrm{n}} \mathrm{BuLi}$ und $\mathrm{NaN}\left(\mathrm{SiMe}_{3}\right)_{2}$ deprotoniert werden, was zur Bildung der anionischen Spezies $\left[\mathrm{R}_{2} \mathrm{P}(\mathrm{E}) \mathrm{NP}(\mathrm{E}) \mathrm{R}_{2}\right]^{\ominus}$ führt. In Komplexverbindungen des Phosphorylamids werden P-E- bzw. P-N-Bindungslängen gefunden, die zwischen einer Einfach- und einer Doppelbindung liegen. Das Stickstoffatom trägt formal eine negative Ladung, die über die Chalkogenatome, die an positiv geladene Metallionen gebunden sind, delokalisiert ist. Dies äußert sich, verglichen mit dem neutralen Amin, in einer P-E-Bindungsverlängerung und einer P-N-Bindungsverkürzung. Aus diesem Grund kann der Ligand mit seinen kanonischen Grenzstrukturformeln (a) und (b) (Abb. 3) oder in seiner delokalisierten Darstellungsform (c) beschrieben werden. 
$\mathrm{dppmE}_{2} \mathrm{H}$ :<smiles>[Y1]1PCCCCP1</smiles>

(a)<smiles>[M]CCCCF</smiles>

(b)<smiles>[Y1]CP[I+]CCC[IH+]</smiles>

(c)<smiles>[M]C1=[V]PCCCCP1</smiles>

(d)

$\operatorname{dppmE}_{2} \odot$ :<smiles>[Y1]1[CH]CC[Te]CC1</smiles>

(e)<smiles>[M]C1CC[Te]CC1[O-]</smiles>

(i)<smiles>[M]C(CF)[O+]CF</smiles>

(f)

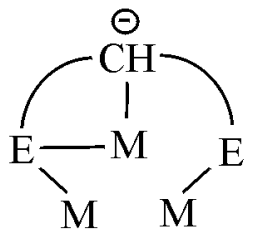

(j)<smiles>FC[Al]1[M][Te]CC1</smiles>

(g)

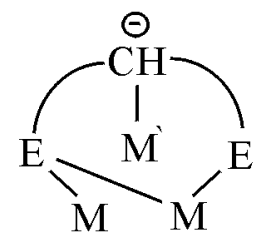

(k)

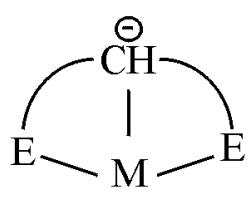

(h)

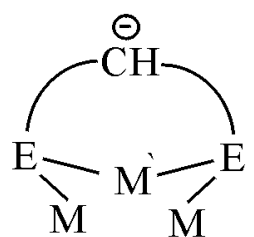

(1)

$\operatorname{dppmE}_{2}^{2 \Theta}$<smiles>[X][M]C1([O])CC[Y14](F)CC1</smiles>

(m)<smiles>[M]C([M])(CF)CF</smiles>

(n)<smiles>[M]CC[C@]1([O-])CC[Y14]1[M]</smiles>

(o)

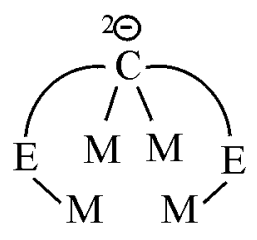

(p)

Abb. 2: Koordinationsformen der Liganden $\operatorname{dppmE}_{2} \mathrm{H}, \operatorname{dppmE}_{2}{ }^{\ominus}$ und $\operatorname{dppmE}_{2}{ }^{2 \ominus}[13-19]$ 
<smiles>[R]P([R])(=[V])N=P([R])([R])[Si]</smiles>

(a)<smiles>[R]P([R])(=[V])N=P([R])([R])[Na]</smiles>

(b)<smiles>[R][R]([R])(F)N=[P+]([R])([R])F</smiles>

(c)

Abb. 3: Grenzstrukturformeln der Bis(diorganochalkogenophosphoryl)amide

Die koordinationschemische Anpassungsfähigkeit der phenylsubstituierten Amine und Amide ist in Abb. 4 verdeutlicht, in der die Koordinationsmöglichkeiten der Liganden $\mathrm{dppaE}_{2} \mathrm{H}$ und $\mathrm{dppaE}_{2}{ }^{\ominus}$ dargestellt sind. Unter den zahlreichen Übergangsmetallkomplexen ist die Bildung von sechsgliedrigen kohlenstofffreien Cyclen, in denen der Ligand das Metallatom zweizähnig chelatisiert, vorherrschend (Abb. 4 (c)). Auch ein- und dreizähnige Koordinationen werden beobachtet (Abb. 4 (d), (f) und (j)). Mittels der Chalkogenatome und des Amido-Stickstoffatoms können bis zu drei Metallionen durch diese Liganden miteinander verknüpft werden. Im Gegensatz zum Amid $\operatorname{dppaE}_{2}{ }^{\ominus}$ sind nur wenige Beispiele von Komplexverbindungen des neutralen Amins dppaE ${ }_{2} \mathrm{H}$ bekannt.

Die bisher einzigen in der Literatur beschriebenen Komplexverbindungen des methylsubstituierten Liganden $\operatorname{dmpaS}_{2} \mathrm{H}$ sind homoleptische, einkernige Verbindungen des Typs $\left[\mathrm{M}\left(\mathrm{dmpaS}_{2}\right)_{2}\right](\mathrm{M}=\mathrm{Fe}, \mathrm{Co}, \mathrm{Ni}[26,27,34])$, in denen der Ligand immer als Anion zweizähnig an das Metallion gebunden ist. Es existieren jedoch einige weitere Koordinationsarten, auch des Neutralliganden, die in Abb. 5 dargestellt sind. Das Amid kann nicht nur ein Metallion chelatisieren (Abb. 5 (b)), sondern ermöglicht durch mehrfache Koordination der Chalkogenatome die Bindung an bis zu fünf Metallionen (Abb. $5(\mathrm{j}))$. 


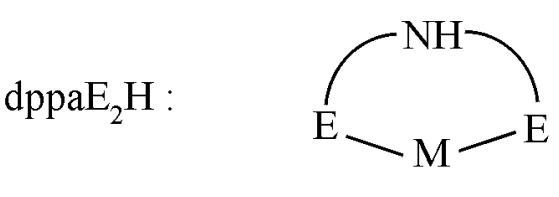

(a)

$\operatorname{dppaE}_{2}^{\Theta}$

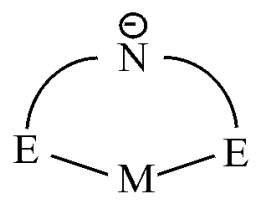

(c)

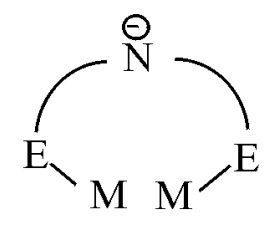

$(\mathrm{g})$

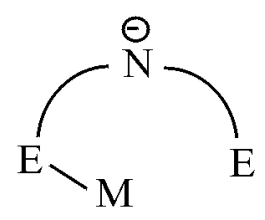

(d)

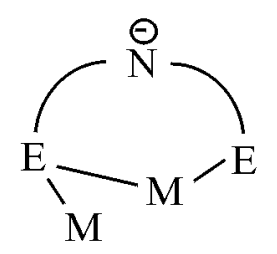

(h)

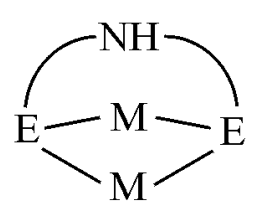

(b)

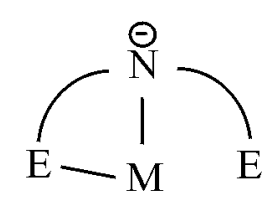

(e)

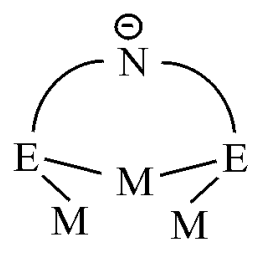

(i)

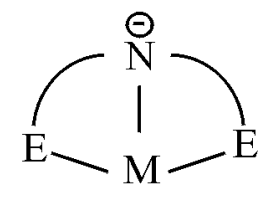

(f)

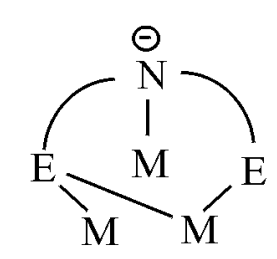

(j)

Abb. 4: Koordinationsformen der Liganden $\operatorname{dppaE}_{2} \mathrm{H}$ und $\operatorname{dppaE}_{2}{ }^{\ominus}$ [29-33] 
$\operatorname{dmpaS}_{2} \mathrm{H}: \quad \quad \operatorname{dmpaS}_{2}^{\ominus}$ :

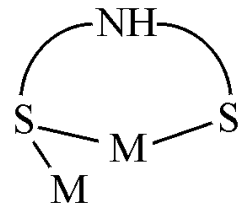

(a)

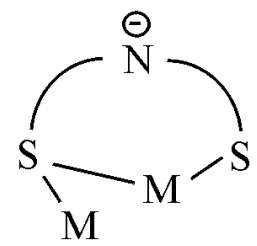

(d)

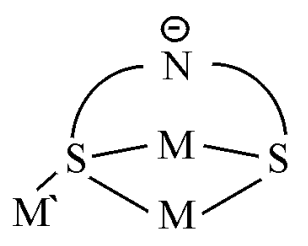

(h)

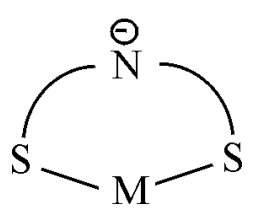

(b)

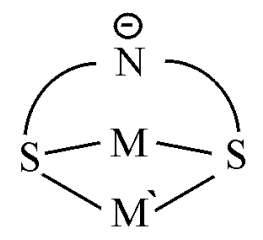

(e)

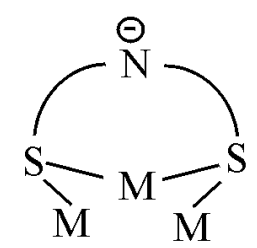

(f)<smiles></smiles>

(i)

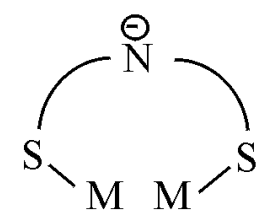

(c)<smiles>[M][Si]1([Y14])[M]SC[N]NC1</smiles>

(g)

Abb. 5: Koordinationsformen der Liganden $\operatorname{dmpaS}_{2} \mathrm{H}$ und $\operatorname{dmpaS}_{2}{ }^{\ominus}[26,27,34]$ 


\section{Aufgabenstellung}

Im Rahmen der vorliegenden Arbeit sollen systematische Untersuchungen zeigen, ob es möglich ist, unterstützt durch den Einsatz chelatisierender Liganden, gezielt mehrkernige Metallkomplexe aufzubauen.

Interessant ist in diesem Zusammenhang vor allem die Fragestellung, was geschieht, wenn ein zweizähniger, anionischer Ligand L mit z.B. $\mathrm{CuCl}$ umgesetzt wird und sich die hypothetische Einheit " $\mathrm{Cu}(\mathrm{L})$ " bildet, in der $\mathrm{Cu}^{\mathrm{I}}$ eine Koordinationszahl von zwei besitzt (Abb. 6). Es soll geklärt werden, wie sich diese Einheit verhält, wenn sie sich selbst überlassen wird oder zusätzlich andere Liganden angeboten werden.

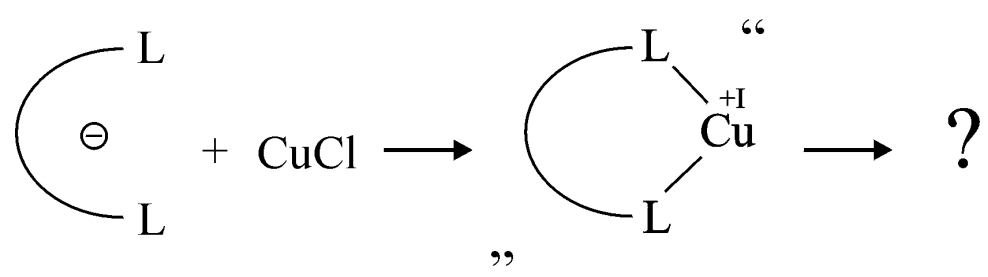

Abb. 6: Reaktion zur hypothetischen Spezies " $\mathrm{Cu}(\mathrm{L})$ " mit Koordinationszahl $=2$

Die Untersuchungen konzentrieren sich hierbei auf die Ligandensysteme der Bis(chalkogenophosphoryl)methane $\mathrm{Ph}_{2} \mathrm{P}(\mathrm{E}) \mathrm{CH}_{2} \mathrm{P}(\mathrm{E}) \mathrm{Ph}_{2} \quad(\mathrm{Abb} . \quad 7 \quad$ (a)) und -amine $\mathrm{R}_{2} \mathrm{P}(\mathrm{E}) \mathrm{NHP}(\mathrm{E}) \mathrm{R}_{2}$ (Abb. 7 (b)). Die vorliegende Arbeit soll am Beispiel der Münzmetalle zeigen, wie ein Zugang zu ein- und mehrkernigen Verbindungen durch Variation des Liganden und des Metalls ermöglicht wird. Es sollen die Halogenide und Alkoholate der Metallionen $\mathrm{Cu}^{\mathrm{I}}, \mathrm{Cu}^{\mathrm{II}}, \mathrm{Ag}^{\mathrm{I}}$ und $\mathrm{Au}^{\mathrm{I}}$ eingesetzt werden und mit den Chelatliganden Bis(diphenylchalkogenophosphoryl)methan (dppmE $\mathrm{E}_{2} \mathrm{H}$ mit $\left.\mathrm{E}=\mathrm{O}, \mathrm{S}, \mathrm{Se}\right)$, Bis(diphenylchalkogenophosphoryl)amin (dppaE $\mathrm{E}_{2} \mathrm{H}$ mit $\mathrm{E}=\mathrm{O}, \mathrm{S}, \mathrm{Se}$ ) und Bis(dimethylthiophosphoryl)amin $\left(\mathrm{dmpaS}_{2} \mathrm{H}\right)$ bzw. deren Alkalisalzen unter verschiedenen Reaktionsbedingungen umgesetzt werden.

Untersuchungen sollen zeigen, wie die Komplexbildungstendenz der Münzmetalle und die Koordinationseigenschaften der Liganden beeinflußt werden, wenn durch eine Variation der Chalkogensubstituenten $(\mathrm{E}=\mathrm{O}, \mathrm{S}, \mathrm{Se})$ die Donoreigenschaften der Liganden verändert werden. In diesem Zusammenhang sind die unterschiedlichen Bindungsmodi der Liganden an Metallionen von besonderer Bedeutung.

Weiterhin soll der sterische Einfluß organischer Reste $(\mathrm{R}=\mathrm{Ph}, \mathrm{Me})$ auf die Bildung von mehrkernigen Komplexverbindungen durch eine unterschiedliche Substitution der 
<smiles>[R][R]([R])(F)C[PH]([R])([R])F</smiles>

(a)
Bis(diphenylchalkogenophosphoryl)methan :

\section{$\operatorname{dppmE}_{2} \mathrm{H}$}

$$
\mathrm{E}=\mathrm{O}, \mathrm{S}, \mathrm{Se}
$$

$$
\mathrm{R}=\mathrm{Ph}
$$<smiles>[R][PH]([R])(F)N[PH]([R])([R])F</smiles>

(b)
Bis(diphenylchalkogenophosphoryl)amin :

$$
\begin{array}{r}
\operatorname{dppaE}_{\mathbf{2}} \mathbf{H}=\mathrm{O}, \mathrm{S}, \mathrm{Se} \\
\mathrm{R}=\mathrm{Ph}
\end{array}
$$

Bis(dimethylthiophosphoryl)amin :

\section{$\operatorname{dmpaS}_{2} H$}

$$
\mathrm{R}=\mathrm{Me}
$$

Abb. 7: Die verwendeten Ligandensysteme

Phosphoryle aufgeklärt werden.

In orientierenden Untersuchungen zum Komplexbildungsverhalten der Chelatliganden sollen Reaktionen mit weiteren Übergangsmetallen durchgeführt werden.

Im Vordergrund der Arbeiten steht die Synthese neuer Verbindungen und deren röntgenstrukturanalytische Strukturaufklärung. ${ }^{31}$ P-NMR-spektroskopische Messungen sollen zusätzliche Informationen über die Lösungseigenschaften einiger dargestellter Verbindungen liefern. 


\section{Gang der Untersuchung}

Im folgenden sollen die systematischen Untersuchungen des Komplexverhaltens von Bis(diorganodichalkogenophosphoryl)aminen und -methanen gegenüber den Münzmetallen $\mathrm{Cu}^{\mathrm{I}}, \mathrm{Cu}^{\mathrm{II}}, \mathrm{Ag}^{\mathrm{I}}$ und $\mathrm{Au}^{\mathrm{I}}$ beschrieben werden.

In den synthetisierten Verbindungen werden zum Teil recht kurze $\mathrm{Cu}-\mathrm{Cu}$-Abstände in einem Bereich von 263 bis 308 pm (im Mittel: 270 bis 280 pm) und Au-Au-Abstände von 288 pm beobachtet, was immer zur Annahme bindender Metall-Metall-Wechselwirkungen geführt hat [35]. Aus sehr geringen $\mathrm{Cu}-\mathrm{Cu}$-Abständen, wie sie z.B. in der Verbindung $\left[\mathrm{Cu}_{2}((\mathrm{p} \text {-tol }) \mathrm{NCHN}(\mathrm{p} \text {-tol }))_{2}\right]$ mit einem Cu-Cu-Abstand von $250 \mathrm{pm}$ auftreten [36], kann jedoch nicht gefolgert werden, daß eine $\mathrm{Cu}-\mathrm{Cu}$-Bindung vorliegt. Sehr kurze $\mathrm{Cu}-\mathrm{Cu}-$ Abstände sind lediglich auf die $\mathrm{Cu}$-Chalkogen-Bindungen, die sich aufgrund der Brückenfunktion der Chalkogenatome ausbilden können, und den Abstand der Donoratome eines Liganden ("Biß") zurückzuführen. Auch Molekülorbitalberechnungen [36,37] von Verbindungen mit $\mathrm{d}^{10}-\mathrm{d}^{10}$-Elektronenkonfiguration wie $\left[\mathrm{M}_{2}(\mathrm{RNCHNR})_{2}\right](\mathrm{M}=\mathrm{Cu}, \mathrm{Ag})$ zeigen, daß die formale M-M-Bindungsordnung bei diesen Verbindungen tatsächlich null beträgt. Nach neuesten Dichtefunktionaltheorie-Rechnungen geben nicht einmal $\mathrm{Cu}^{\mathrm{I}}-\mathrm{Cu}^{\mathrm{I}}$ Abstände von 238 pm Anlaß zur Annahme von Cu-Cu-Bindungen [38].

\subsection{Umsetzungen der Methanderivate $\operatorname{dppmE}_{2} \mathrm{H}(\mathrm{E}=\mathrm{O}, \mathrm{S}, \mathrm{Se})$}

Komplexverbindungen der Liganden $\mathrm{dppmE}_{2} \mathrm{H}$ sind in der Literatur gut untersucht, jedoch finden sich überwiegend nur einkernige Verbindungen, in denen die Liganden $\eta^{2}$-artig an ein Metallatom binden (vgl. Abb. 2 (a) und (e)).

\subsubsection{Die Synthese und Struktur von $\left[\mathrm{Cu}\left(\operatorname{dppmO}_{2} \mathrm{H}\right)_{3}\right]\left[\mathrm{CuCl}_{4}\right] \underline{1}$}

Von Komplexen des Liganden $\mathrm{dppmO}_{2} \mathrm{H}$ sind hauptsächlich die der Hauptgruppenelemente beschrieben, in denen der Ligand in neutraler Form vorliegt [39,40]. Übergangsmetallkomplexe sind bisher nur von den Metallen Eisen, Nickel und Kupfer strukturell charakterisiert $[26,41,42]$.

Es konnten mehrere $\mathrm{Cu}^{\mathrm{II}}$-Komplexe von $\mathrm{dppmO}_{2} \mathrm{H}$ und $\mathrm{dppmO}_{2}{ }^{\ominus}$ dargestellt werden. Beispielsweise wurde die Verbindung $\left[\mathrm{Cu}\left(\operatorname{dppmO}_{2} \mathrm{H}\right)_{3}\right]\left[\mathrm{CuCl}_{4}\right] \underline{1}$ erhalten, die sowohl aus der Reaktion mit dem neutralen als auch mit dem anionischen Liganden isoliert werden 
kann (Gl. 1 und 2).

$$
\begin{aligned}
& 2 \mathrm{CuCl}_{2}+3 \mathrm{dppmO}_{2} \mathrm{H} \stackrel{\mathrm{THF}}{\longrightarrow}\left[\mathrm{Cu}\left(\mathrm{dppmO}_{2} \mathrm{H}\right)_{3}\right]\left[\mathrm{CuCl}_{4}\right] \underline{1} \\
& 2 \mathrm{CuCl}_{2}+3\left[\mathrm{~K}\left(\mathrm{dppmO}_{2}\right)\right] \stackrel{\mathrm{THF}}{\longrightarrow}\left[\mathrm{Cu}\left(\mathrm{dppmO}_{2} \mathrm{H}\right)_{3}\right]\left[\mathrm{CuCl}_{4}\right] \underline{1}
\end{aligned}
$$

Die Struktur dieser Komplexverbindung entspricht dem Aufbau der von K. B. Yatsimirskii et al. [42] synthetisierten Verbindung $\left[\mathrm{Cu}\left(\mathrm{dppmO}_{2} \mathrm{H}\right)_{3}\right]\left[\mathrm{Cu}_{2} \mathrm{Cl}_{6}\right] \cdot \mathrm{OCMe}_{2}$ [43], die bei der Reaktion von $\mathrm{CuCl}_{2}$ mit dppmO $\mathrm{m}_{2} \mathrm{H}$ in Aceton entstand.

\section{Die Struktur von $\left[\mathrm{Cu}\left(\operatorname{dppmO}_{2} \mathrm{H}\right)_{3}\right]\left[\mathrm{CuCl}_{4}\right] \underline{1}$ :}

Verbindung 1 kristallisiert monoklin in der Raumgruppe $P 2_{1} / n$ (Abb. 8). Das Kation besteht aus einem $\mathrm{Cu}^{\mathrm{II}}$-Ion, an das drei Liganden gemäß Abb. 2 (a) binden.

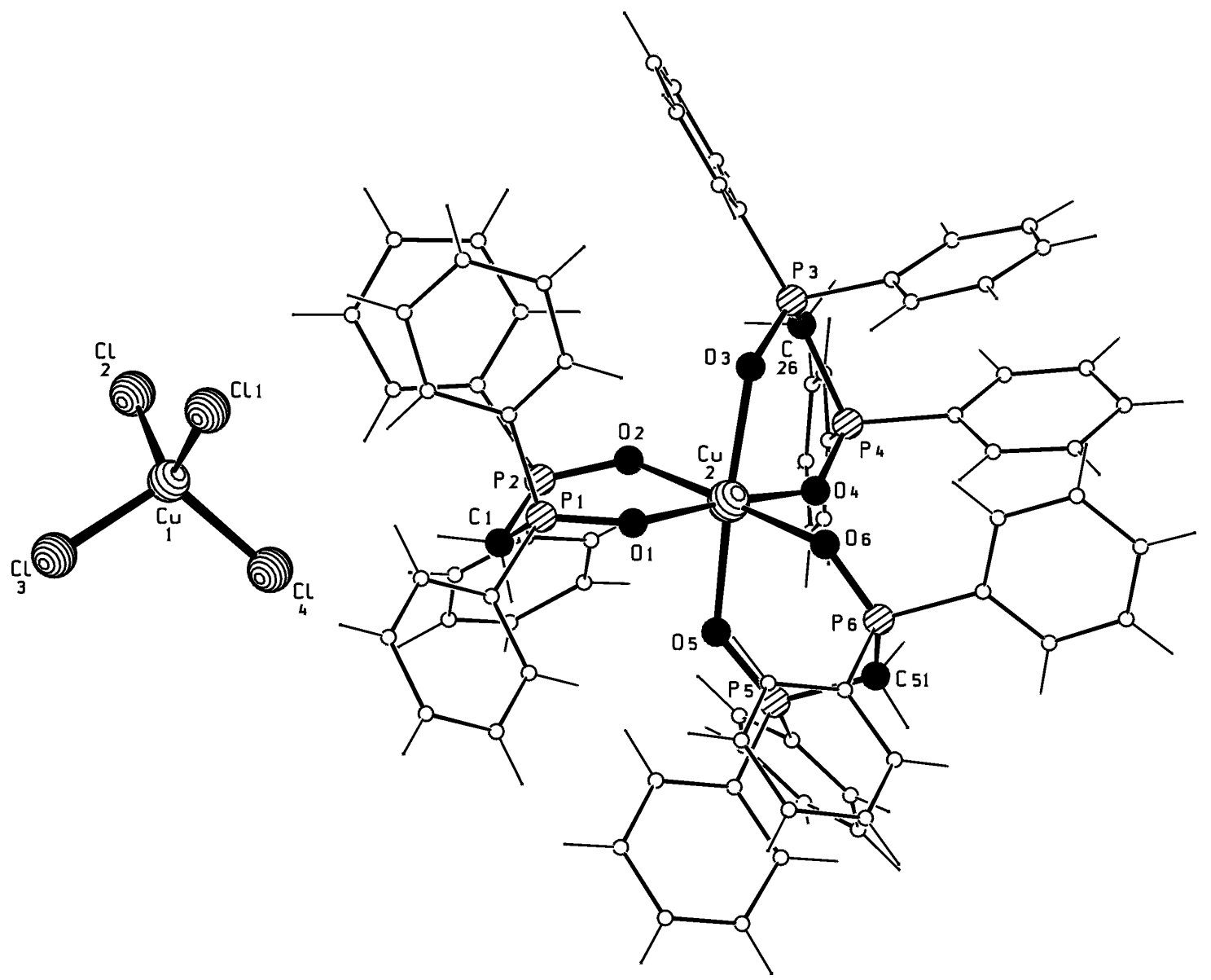

Abb. 8: Die Struktur von $\left[\mathrm{Cu}\left(\mathrm{dppmO}_{2} \mathrm{H}\right)_{3}\right]\left[\mathrm{CuCl}_{4}\right] \underline{1}$ im Kristall 
An $\mathrm{Cu}(2)$ sind sechs Sauerstoffatome koordiniert, so daß sich ein Jahn-Teller-verzerrter, gestreckter Oktaeder als Koordinationspolyeder ergibt.

In 1 muß zwischen zwei Cu-O-Abständen unterschieden werden. Die vier O-Atome mit den kürzeren Abständen zu $\mathrm{Cu}(2)$ von 219 pm liegen auf den äquatorialen Positionen des $\mathrm{CuO}_{6}$-Oktaeders, während $\mathrm{O}(3)$ und $\mathrm{O}(5)$ einen Abstand von $225 \mathrm{pm} \mathrm{zu} \mathrm{Cu}(2)$ aufweisen. Sie besetzen somit die zwei axialen Positionen. Diese deutliche Abstufung der Bindungslängen ist in der Verbindung $\left[\mathrm{Cu}\left(\mathrm{dppmO}_{2} \mathrm{H}\right)_{3}\right]\left[\mathrm{Cu}_{2} \mathrm{Cl}_{6}\right] \cdot \mathrm{OCMe}_{2}$ [43] nicht zu erkennen. Hier variieren die $\mathrm{Cu}-\mathrm{O}$-Bindungslängen von 200 bis $225 \mathrm{pm}$.

Der Vergleich der P-O- und P-C-Bindungslängen in 1 mit denen von Verbindung $\left[\mathrm{Cu}\left(\mathrm{dppmO} \mathrm{H}_{2} \mathrm{H}\right)_{3}\right]\left[\mathrm{Cu}_{2} \mathrm{Cl}_{6}\right] \cdot \mathrm{OCMe}_{2}$ zeigt eine gute Übereinstimmung, denn in beiden Verbindungen betragen die P-O- und die P-C-Bindungslängen durchschnittlich 149 pm und $181 \mathrm{pm}$.

\subsubsection{Die Synthese und Struktur von $\left[\mathrm{Cu}_{2}\left(\operatorname{dppmS}{ }_{2} \mathrm{H}\right)_{2} \mathrm{Cl}\right]\left[\mathrm{CuCl}_{2}\right] \underline{2}$}

Unter den zahlreich synthetisierten Verbindungen des dithioanalogen Liganden $\operatorname{dppmS}_{2} \mathrm{H}$ werden meist einkernige Komplexverbindungen der Übergangsmetalle gefunden, in denen der Ligand ebenfalls ungeladen als Phosphorylmethan an das Metall gebunden ist $[44,45]$. Komplexe der Münzmetalle, in denen der Ligand entweder neutral oder dianionisch an das Metall bindet, konnten ebenfalls synthetisiert werden [13,16,46,47]. Allerdings sind mehrkernige Komplexverbindungen, vor allem des Kupfers, bisher nur wenig zu finden. Durch die röntgenstrukturanalytische Charakterisierung der Verbindung $\left[\mathrm{Cu}_{2}\left(\mathrm{dppmS}_{2} \mathrm{H}\right)_{2} \mathrm{Cl}\right]\left[\mathrm{CuCl}_{2}\right] \underline{2}$ konnte die bisher schon bekannte Reihe der Komplexe mit der Zusammensetzung $\mathrm{Cu}_{\mathrm{x}}\left(\mathrm{dppmS}_{2} \mathrm{H}\right)_{\mathrm{y}} \mathrm{Cl}_{\mathrm{x}}(\mathrm{x}=1,2)[13,16]$ um ein Glied erweitert wer$\operatorname{den}(\mathrm{x}=3)$.

Werden $\mathrm{CuCl}$ und $\operatorname{dppmS}_{2} \mathrm{H}$ im Molverhältnis 1:1 zusammengeschmolzen und anschließend in $\mathrm{CH}_{2} \mathrm{Cl}_{2}$ aufgenommen, so bildet sich nach Überschichten mit Heptan Verbindung $\underline{2}$ (Gl. 3).

$$
\mathrm{CuCl}+\mathrm{dppmS}_{2} \mathrm{H} \underset{\text { 2) } \mathrm{CH}_{2} \mathrm{Cl}_{2}}{\longrightarrow}\left[\mathrm{Cu}_{2}\left(\mathrm{dppmS}_{2} \mathrm{H}\right)_{2} \mathrm{Cl}\right]\left[\mathrm{CuCl}_{2}\right] \underline{2}
$$

\section{Die Struktur von $\left[\mathrm{Cu}_{2}\left(\operatorname{dppmS}_{2} \mathrm{H}\right)_{2} \mathrm{Cl}\right]\left[\mathrm{CuCl}_{2}\right] \underline{2}$ :}

Abb. 9 zeigt den molekularen Aufbau von Verbindung $\underline{2}$, die orthorhombisch in der Raumgruppe Pccn kristallisiert. Die Kupferionen sind trigonal-planar von einem Chloratom und je zwei Schwefelatomen eines Chelatliganden koordiniert, wodurch sich zwei sechsgliedrige 


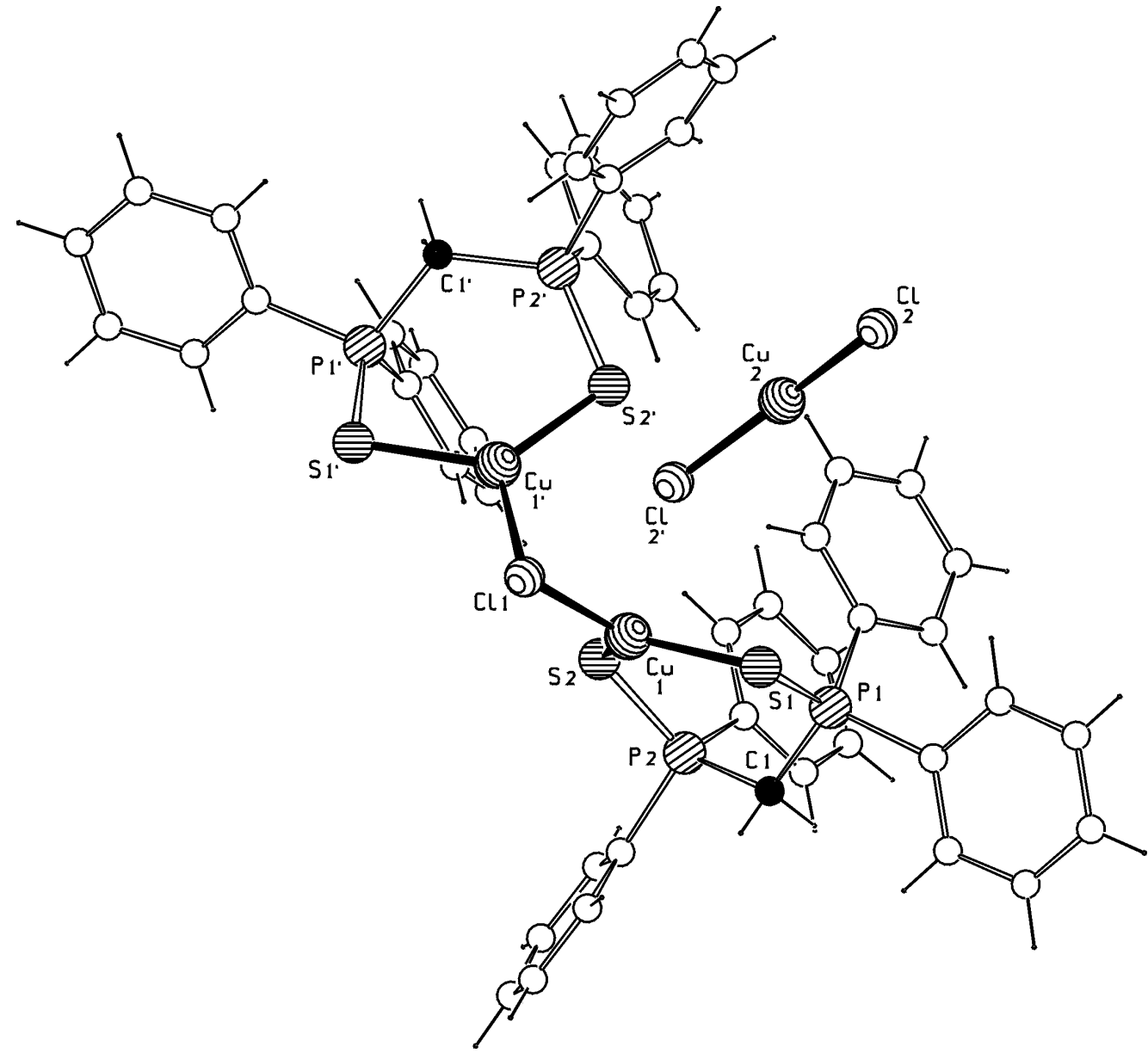

Abb. 9: Die Struktur von $\left[\mathrm{Cu}_{2}\left(\operatorname{dppmS}_{2} \mathrm{H}\right)_{2} \mathrm{Cl}\right]\left[\mathrm{CuCl}_{2}\right] \underline{2}$ im Kristall 
Cyclen ausbilden, die durch das Chloratom so miteinander verbunden sind, daß sich für den Winkel $\mathrm{Cu}(1)-\mathrm{Cl}(1)-\mathrm{Cu}\left(1^{\prime}\right)$ fast ein rechter Winkel ergibt $\left(\angle 91^{\circ}\right)$.

Die Bindungslängen der P-S-, P-C(1)- und Cu-Cl-Bindungen betragen 197 pm, 180 pm und $221 \mathrm{pm}$ und unterscheiden sich nur wenig von denen in der monomeren Verbindung $\left[\mathrm{Cu}\left(\mathrm{dppmS}_{2} \mathrm{H}\right) \mathrm{Cl}\right]$, in der das $\mathrm{Cu}$-Atom ebenfalls trigonal koordiniert ist (P-S: $198 \mathrm{pm}$, P-C: 180 pm, P-Cl: 218 pm [16]). In 2 sind die Cu-S-Abstände mit 220 pm zwar um 4 pm kürzer als in der einkernigen Verbindung, dies kann jedoch durch die Ladung des Kations $\left[\mathrm{Cu}_{2}\left(\mathrm{dppmS} \mathrm{S}_{2} \mathrm{H}\right)_{2} \mathrm{Cl}\right]^{\oplus}$, die die Donoratome stärker zum Zentralion zieht, erklärt werden.

Von den mit dppmS $\mathrm{H}_{2} \mathrm{H}(\mathrm{n}=1)$ eng verwandten Ligandensystemen $\mathrm{R}{ }_{2} \mathrm{P}(\mathrm{S})\left(\mathrm{CH}_{2}\right)_{\mathrm{n}} \mathrm{P}(\mathrm{S}) \mathrm{R}_{2}$ $(\mathrm{n}=0,2)$ sind mehrere zweikernige $\mathrm{Cu}^{\mathrm{I}}$-Komplexe bekannt, die verschiedene Koordinationsmodi der Liganden aufgrund der unterschiedlichen Ringgröße der Metall-ChelatCyclen und der damit verbundenen verschiedenartigen sterischen Gegebenheiten im Ring aufweisen.

Wird der Abstand der Donoratome des Liganden verkleinert $(\mathrm{n}=0)$, so kann der dimere Komplex $\left[\mathrm{Cu}_{2}\left(\mathrm{Me}_{4} \mathrm{P}_{2} \mathrm{~S}_{2}\right)_{2} \mathrm{Cl}_{2}\right]$ isoliert werden [48]. Da der Abstand der Schwefelatome des Liganden zu klein ist, um mit den Kupferionen einen trigonal-planaren Komplex zu bilden, entsteht ein Komplex mit tetrahedral umgebenen Cu-Zentren. Das Molekül besteht aus zwei $\mathrm{Cu}(\mathrm{L}) \mathrm{Cl}-H a ̈ l f t e n$, die miteinander über zwei verbrückende Schwefelatome verbunden sind, so daß sich ein viergliedriger, planarer $\mathrm{Cu}_{2} \mathrm{~S}_{2}$-Ring ausbildet. Analog aufgebaut ist die von E.W. Ainscough et al. $[13,16]$ synthetisierte Verbindung $\left[\mathrm{Cu}_{2}\left(\mathrm{dppmS}_{2} \mathrm{H}\right)_{2} \mathrm{Cl}_{2}\right]$ $(\mathrm{n}=1)$. Durch die gezielte Wahl des Lösungsmittels kann auch das entsprechende Monomer $\left[\mathrm{Cu}\left(\mathrm{dppmS}_{2} \mathrm{H}\right) \mathrm{Cl}\right]$ aus $\mathrm{CuCl}_{2}$ und $\mathrm{dppmS}_{2} \mathrm{H}$ synthetisiert werden.

Die kupferhaltige Verbindung des Ethanderivates $\mathrm{Ph}_{2} \mathrm{P}(\mathrm{S})\left(\mathrm{CH}_{2}\right)_{2} \mathrm{P}(\mathrm{S}) \mathrm{Ph}_{2}$ ( $\mathrm{n}=2$ ) [49], in dem der "Biß" des Liganden um eine weitere $\mathrm{CH}_{2}$-Einheit vergrößert ist, ist ebenfalls dimer. Allerdings koordinieren hier die Chloratome nicht terminal, wie in den zwei eben beschriebenen Komplexen, sondern verbrücken zwischen den zwei Cu-Atomen. Die Schwefelatome des Liganden binden nur terminal an die Kupferionen (Abb. 10).

Die Verbindung $\left[\mathrm{Cu}\left(\operatorname{dppmS}_{2} \mathrm{H}\right) \mathrm{Cl}\right]$ und das Dimer $\left[\mathrm{Cu}_{2}\left(\mathrm{dppmS}_{2} \mathrm{H}\right)_{2} \mathrm{Cl}_{2}\right]$ zeigen im ${ }^{31} \mathrm{P}$ NMR-Spektrum in $\mathrm{CDCl}_{3}$ eine mit $\underline{2}$ fast identische chemische Verschiebung von 32,8 ppm. Für 2 wird in $\mathrm{CDCl}_{3}$ ebenfalls ein Singulett bei $\delta=33,2$ ppm gefunden, was darauf hindeutet, daß in allen drei Fällen in Lösung die gleiche Spezies vorliegt, in der alle Phosphoratome chemisch äquivalent sind.

Wird nun das Eduktverhältnis $\mathrm{CuCl}$ : Ligand variiert, indem $\mathrm{CuCl}$ statt mit einem Äqui- 


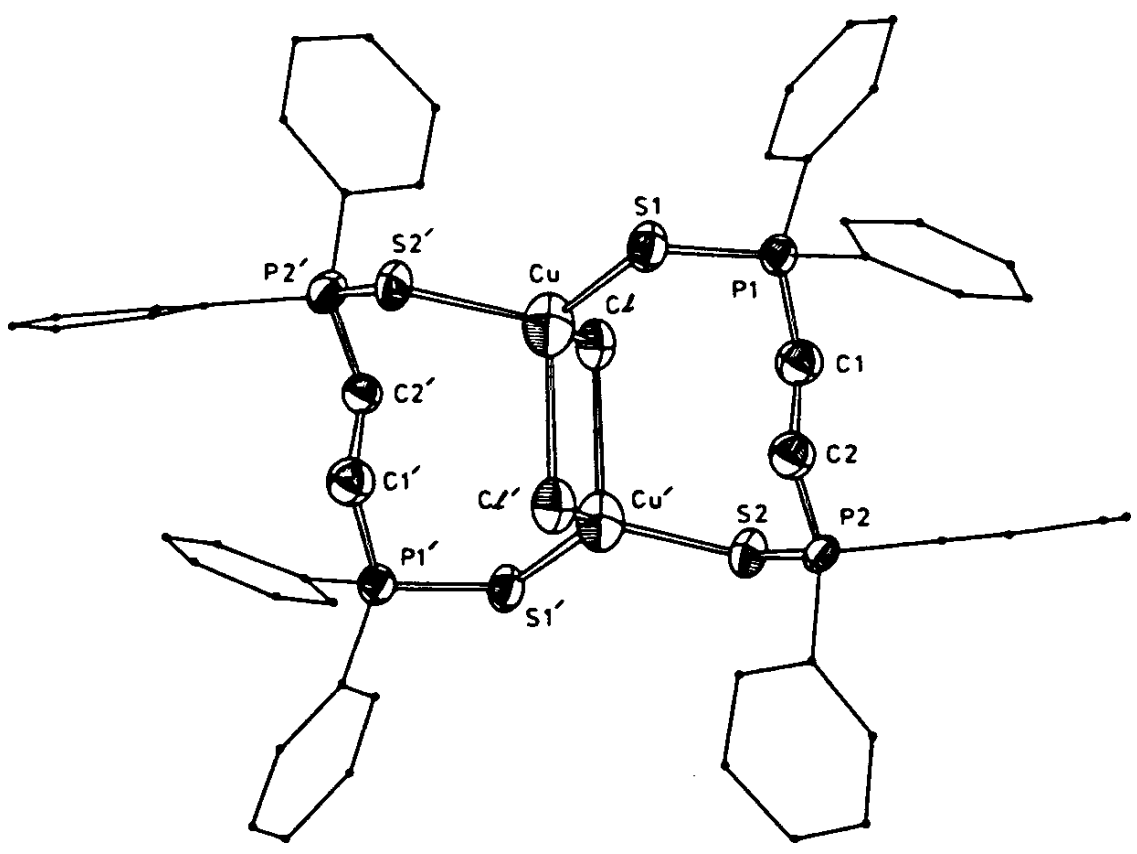

Abb. 10: Die Struktur von $\left[\mathrm{Cu}_{2}\left(\mathrm{dppeS}_{2} \mathrm{H}\right)_{2} \mathrm{Cl}_{2}\right]$ im Kristall [49]

valent mit zwei Äquivalenten $\mathrm{dppmS}_{2} \mathrm{H}$ und $\mathrm{KO}^{\mathrm{t}} \mathrm{Bu}$ umgesetzt wird, so erhält man den vierkernigen Komplex $\left[\mathrm{K}_{2} \mathrm{Cu}_{2}\left(\mathrm{dppmS}_{2}\right)_{4}(\text { thf })_{2}\right]$ (Gl. 4, Abb. 11).

$$
2 \mathrm{CuCl}+4\left[\mathrm{~K}\left(\mathrm{dppmS}_{2}\right)\right] \stackrel{\text { THF } / \text { Heptan }}{\longrightarrow}\left[\mathrm{K}_{2} \mathrm{Cu}_{2}\left(\mathrm{dppmS}_{2}\right)_{4}(\mathrm{thf})_{2}\right]+2 \mathrm{KCl}
$$

In dieser Verbindung sind vier anionische Liganden und zwei THF-Moleküle an die vier Metallatome koordiniert. Der anionische Ligand bindet in diesem Komplex auf zwei verschiedene Weisen: Zum einem kann das Ligandensystem an insgesamt drei Metallatome so gebunden sein, daß die beiden Schwefelatome $\mu_{2}$-artig zwischen je zwei Metallatomen verbrücken (vgl. Abb. 2 (l)). Zum anderen kann der Ligand gemäß Abb. 2 (k) zusätzlich durch das Methanid-Kohlenstoffatom an ein weiteres Metallion koordiniert sein, wodurch sich in der Verbindung $\left[\mathrm{K}_{2} \mathrm{Cu}_{2}\left(\mathrm{dppmS}_{2}\right)_{4}(\mathrm{thf})_{2}\right]$ ein planarer $\mathrm{K}_{2} \mathrm{~S}_{2}$-Ring ausbildet. 


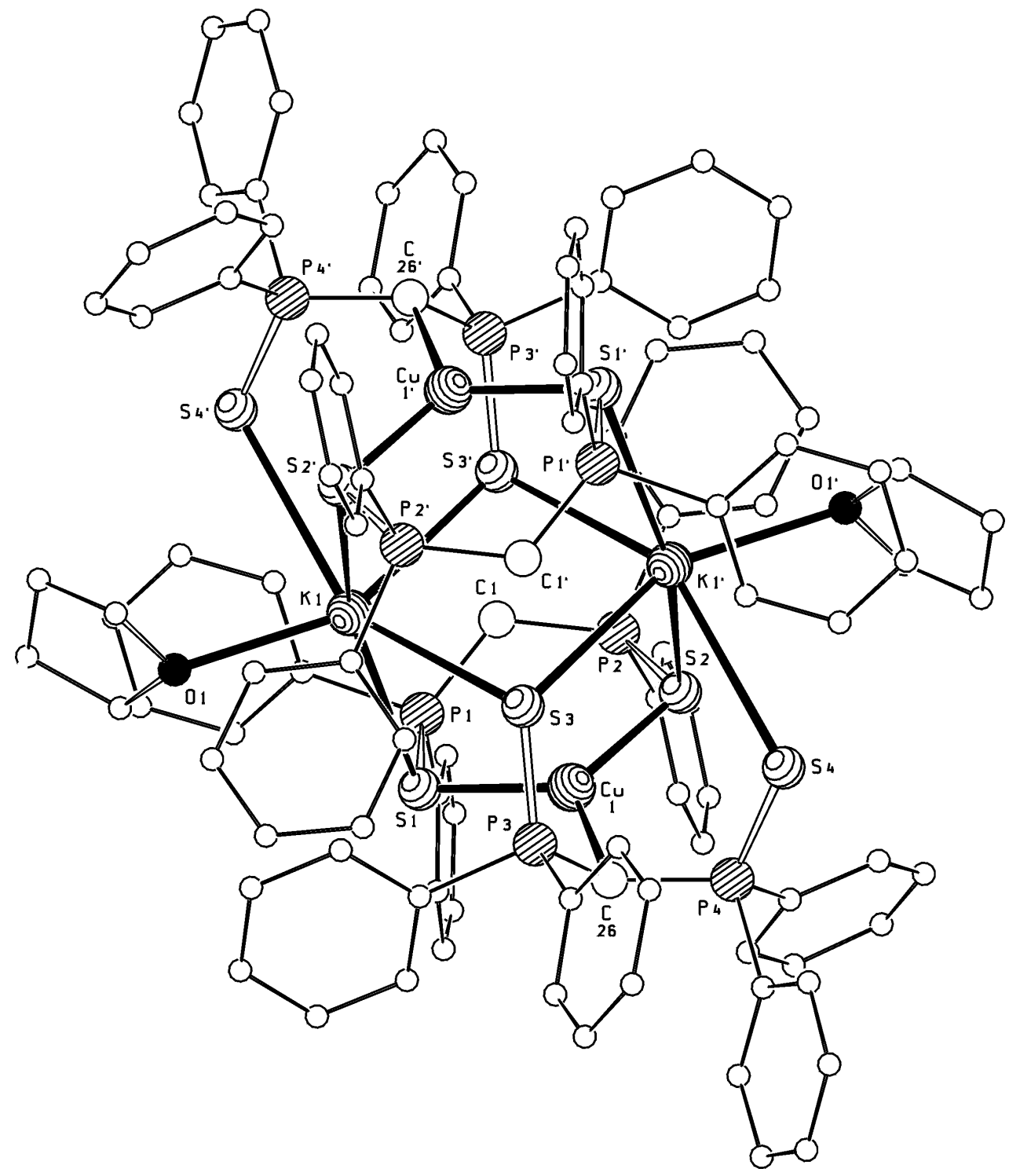

Abb. 11: Die Struktur von $\left[\mathrm{K}_{2} \mathrm{Cu}_{2}\left(\mathrm{dppmS}_{2}\right)_{4}(\text { thf })_{2}\right]$ im Kristall 


\subsubsection{Die Synthese und Struktur von $\left[\mathrm{Cu}_{2}(\operatorname{dppmSe})_{2}\right] \underline{3}$}

Die Umsetzung des selenanalogen Liganden dppmSe ${ }_{2} \mathrm{H}$ mit $\mathrm{CuCl}$ ergab ein unerwartetes Ergebnis: Bei der Reaktion von $\mathrm{CuCl}$, dppmSe $\mathrm{H}_{2}$ und $\mathrm{NaN}\left(\mathrm{SiMe}_{3}\right)_{2}$ in THF bilden sich violette Kristalle der Verbindung $\left[\mathrm{Cu}_{2}\left(\mathrm{dppmSe}_{3}\right)_{2}\right] \underline{3}$ (Gl. 5).

$$
\mathrm{CuCl}+\left[\mathrm{Na}\left(\mathrm{dppmSe}_{2}\right)\right] \stackrel{\mathrm{THF}}{\longrightarrow}\left[\mathrm{Cu}_{2}\left(\mathrm{dppmSe}_{3}\right)_{2}\right] \underline{3}
$$

\section{Die Struktur von $\left[\mathrm{Cu}_{2}(\mathrm{dppmSe})_{2}\right] \underline{3}$ :}

In Abb. 12 ist die Struktur der Verbindung $\underline{3}$ (Kristallsystem triklin, Raumgruppe $P \overline{1}$ ) dargestellt, in der zwei dppmSe ${ }_{3}^{\ominus}$-Liganden mit jeweils drei Selenatomen an die beiden Kupferionen gebunden sind. Während die vier Selenatome, die an die Phosphoratome binden, terminal an die zwei Kupferionen koordiniert sind, verbrücken die beiden Se-Atome, die an $\mathrm{C}(1)$ und $\mathrm{C}\left(1^{\prime}\right)$ binden, $\mu_{2}$-artig zwischen den Metallatomen und verknüpfen somit beide Molekülhälften miteinander. Dadurch ist jedes Metallatom verzerrt tetraedrisch von vier Selenatomen umgeben. Die beiden $\mathrm{CuSe}_{4}$-Tetraeder sind durch die gemeinsame Se-Se-Kante miteinander verbunden, woraus sich ein planarer $\mathrm{Cu}_{2} \mathrm{Se}_{2}$-Vierring ergibt.

Nach F.H. Allen et al. [50] beträgt die Bindungslänge einer Se-Se-Einfachbindung 234 pm. Im Vergleich dazu ist in $\underline{3}$ der Se(3)-Se(3')-Abstand mit 265 pm um 31 pm größer. Folglich handelt es sich in $\underline{3}$ zwar um keine Se-Se-Einfachbindung, jedoch um einen schwachen SeSe-Kontakt, da der Se-Se-Abstand sehr viel geringer als die Summe der Van-der-WaalsRadien (400 pm) ist.

Damit zusammenhängend wird in $\underline{3}$ eine C-Se-Bindungslänge von 186 pm gefunden, die kleiner ist als die in der Literatur für eine C-Se-Einfachbindung mit $\mathrm{sp}^{2}$-hybridisiertem Kohlenstoffatom angegebene von 189 pm [50]. Sie ist jedoch nur geringfügig größer als die C-Se-Doppelbindunglänge in der Verbindung $\left[\mathrm{Cu}^{\mathrm{II}}\left(\mathrm{Cu}^{\mathrm{I}} \mathrm{Cl}_{2}\left(\mathrm{Se}=\mathrm{C}\left(\mathrm{Ph}_{2} \mathrm{PO}\right)_{2}\right)\right)_{2}\right] \cdot 2 \mathrm{CH}_{2} \mathrm{Cl}_{2}$ von 185 pm [51]. Somit kann angenommen werden, daß in $\underline{3}$ eine C-Se-Bindung mit einer Bindungsordnung von annähernd zwei vorliegt. Die P-Se-Bindungen in $\underline{3}$ sind mit 217 pm um ca. 7 pm länger als in dppmSe ${ }_{2} \mathrm{H}$ [10], was, wie erwartet, eine Verringerung der Bindungsordnung der P-Se-Bindung im Vergleich zum neutralen Liganden bedeutet und im Bereich von bisher beobachteten Bindungslängen von $\eta^{2}$-artig koordiniertem dppmSe ${ }_{2}^{\ominus}$ von 216 pm liegt [52].

Die bimetallische Verbindung $\underline{3}$ enthält zwei monoanionische, dreizähnige Liganden $\operatorname{dppmSe}_{3}{ }^{\ominus}$, die durch intermolekularen Selentransfer von einer P-Se-Gruppierung an ein Methanid-Kohlenstoffatom entstanden sind. In der Literatur sind ähnli- 


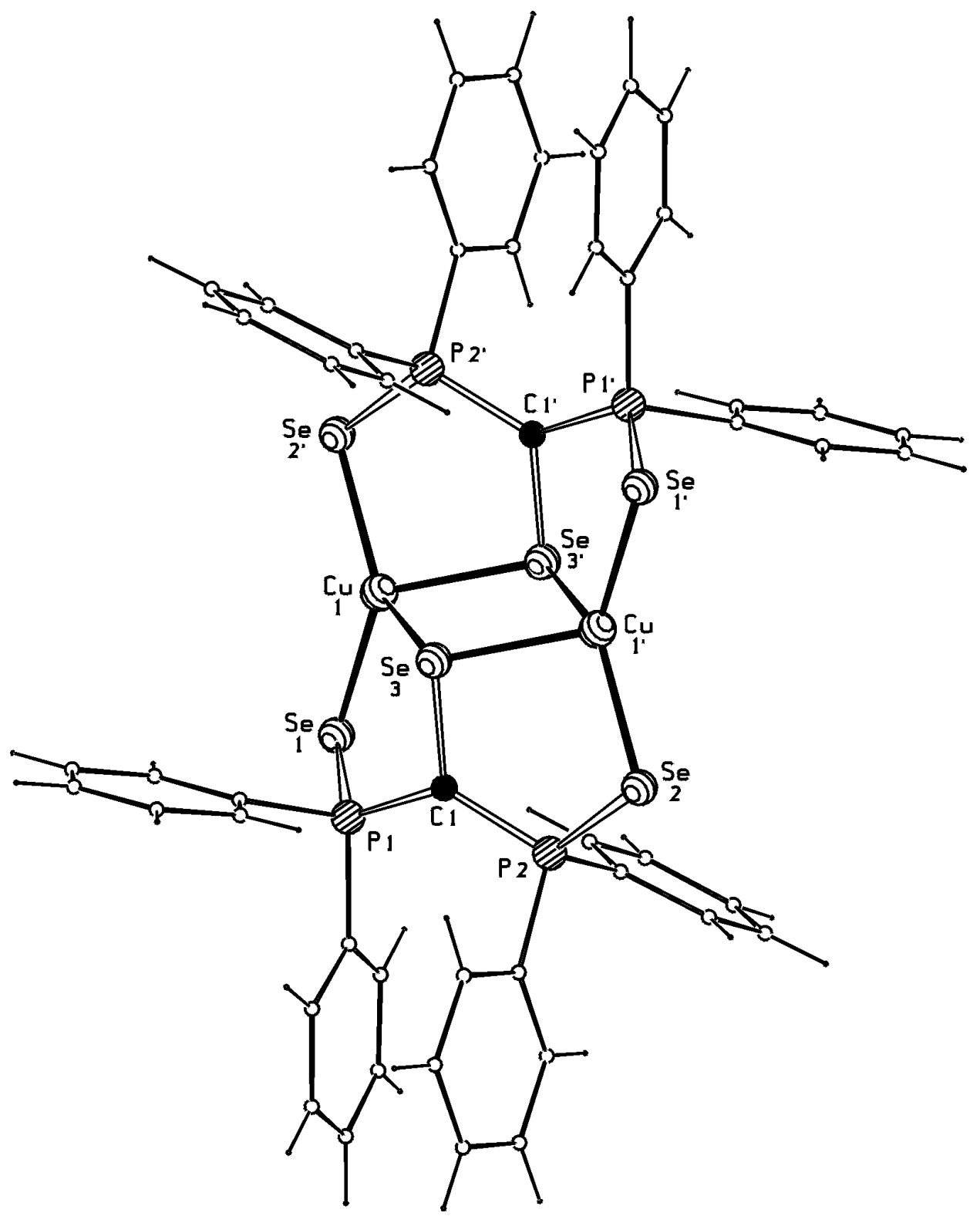

Abb. 12: Die Struktur von $\left[\mathrm{Cu}_{2}\left(\mathrm{dppmSe}_{3}\right)_{2}\right] \underline{3} \mathrm{im} \mathrm{Kristall}$ 
che Selentransferreaktionen schon beschrieben [51]. Beispielsweise bildet sich aus der Umsetzung von dppmSe $\mathrm{H}_{2}$ mit $\mathrm{CuCl}_{2}$ der oben erwähnte dreikernige Komplex $\left[\mathrm{Cu}^{\mathrm{II}}\left(\mathrm{Cu}^{\mathrm{I}} \mathrm{Cl}_{2}\left(\mathrm{Se}=\mathrm{C}\left(\mathrm{Ph}_{2} \mathrm{PO}\right)_{2}\right)\right)_{2}\right]$, der einen Selenonliganden enthält, in dem ebenfalls ein Selenatom intramolekular auf das Methanid-Kohlenstoffatom übertragen wurde. Die anschließende Oxidation an Luft ergab den gemischt-valenten Komplex.

Als weiteres Beispiel eines Komplexes, der ebenfalls einen transformierten Liganden enthält, sei die von L.C. Göldner [52] synthetisierte Verbindung $\left[\mathrm{Cu}\left(\mathrm{dppmSe}_{2}\right)(\mathrm{dppmSeH})\right]$ (Abb. 13) genannt, die aus der Umsetzung von $\mathrm{CuCl}$ mit zwei Äquivalenten $\left[\mathrm{K}\left(\mathrm{dppmSe} \mathrm{e}_{2}\right)\right]$ entstand (Gl. 6).

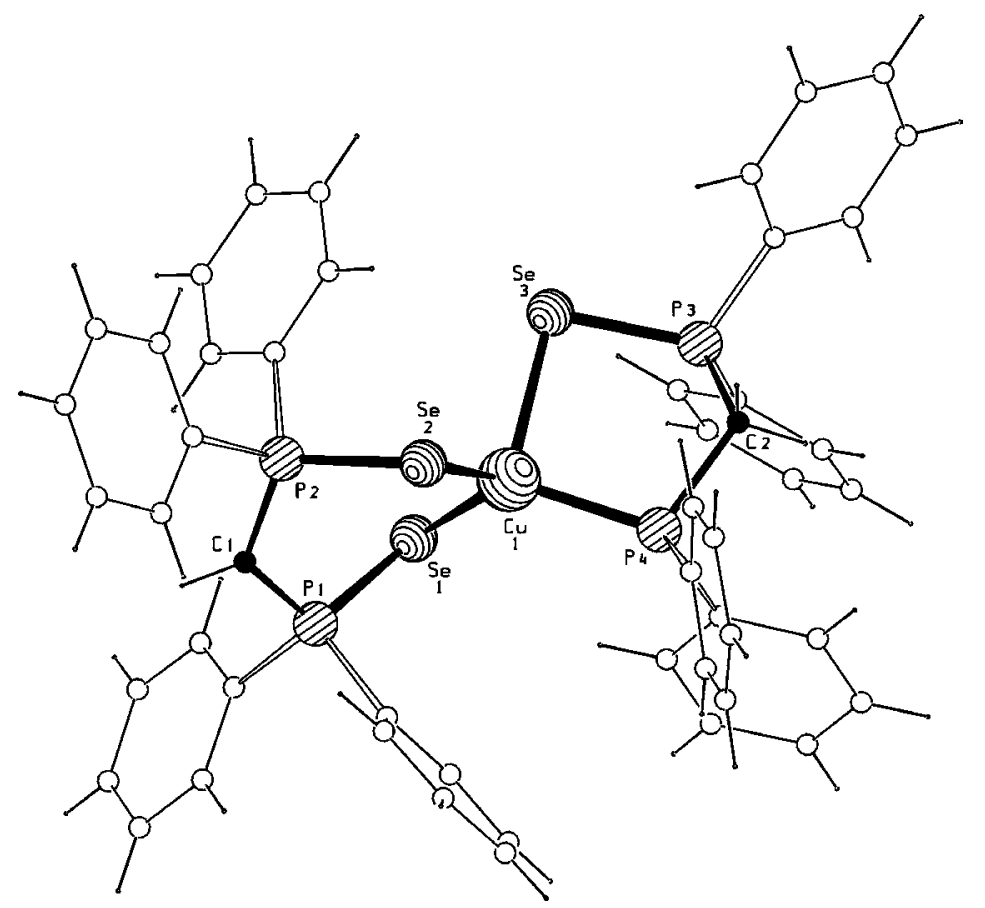

Abb. 13: Die Struktur von $\left[\mathrm{Cu}\left(\mathrm{dppmSe}_{2}\right)(\mathrm{dppmSeH})\right]$ im Kristall

$$
\mathrm{CuCl}+2\left[\mathrm{~K}\left(\mathrm{dppmSe}_{2}\right)\right] \stackrel{\text { THF } / \text { Heptan }}{\longrightarrow}\left[\mathrm{Cu}\left(\mathrm{dppmSe}_{2}\right)(\mathrm{dppmSeH})\right]
$$

Überraschenderweise liegen in dieser Verbindung zwei verschiedene Liganden vor: Der neutrale Ligand dppmSeH ist $\eta^{2}$-artig über das $\mathrm{P}^{\mathrm{III}}$ - und das Selenatom an das Metallion gebunden; auch der anionische Ligand $\mathrm{dppmSe}_{2}{ }^{\ominus}$ chelatisiert über die zwei Selenatome das Cu-Ion, so daß sich für das Kupferatom eine verzerrt tetraedrische Koordinationssphäre ergibt. 
Derartige Reduktionsreaktionen, wie z.B. die Reduktion von $\operatorname{dppaSe}_{2}{ }^{\ominus} \mathrm{zu} \mathrm{dppaSe}^{\ominus}$, sind ebenfalls von P. Bhattacharyya et al. [53] beobachtet worden. Sie erhielten den Komplex $\left[\mathrm{Pd}\left(\mathrm{dppaSe}_{2}\right)(\mathrm{dppaSe})\right]$ als Nebenprodukt aus der Reaktion von $\left[\mathrm{PdCl}_{2}\left(\mathrm{PMe}_{2} \mathrm{Ph}\right)_{2}\right]$ mit $\left[\mathrm{K}\left(\mathrm{dppaSe}_{2}\right)\right]$. Der Mechanismus der Reaktion zum gemischten, quadratisch-planaren Komplex konnte bisher noch nicht aufgeklärt werden. Jedoch wurde für die Reaktion von $\left[\mathrm{Pt}\left(\mathrm{dppmSe} \mathrm{H}_{2}\right)_{2}\right]\left[\mathrm{O}_{3} \mathrm{SCF}_{3}\right]_{2} \mathrm{zu}\left[\mathrm{Pt}(\mathrm{dppmSeH})_{2}\right]\left[\mathrm{O}_{3} \mathrm{SCF}_{3}\right]_{2}$ und Selen von P. Peringer und J. Schwald ein Reaktionsmechanismus vorgeschlagen, der in Abb. 14 dargestellt ist [54].
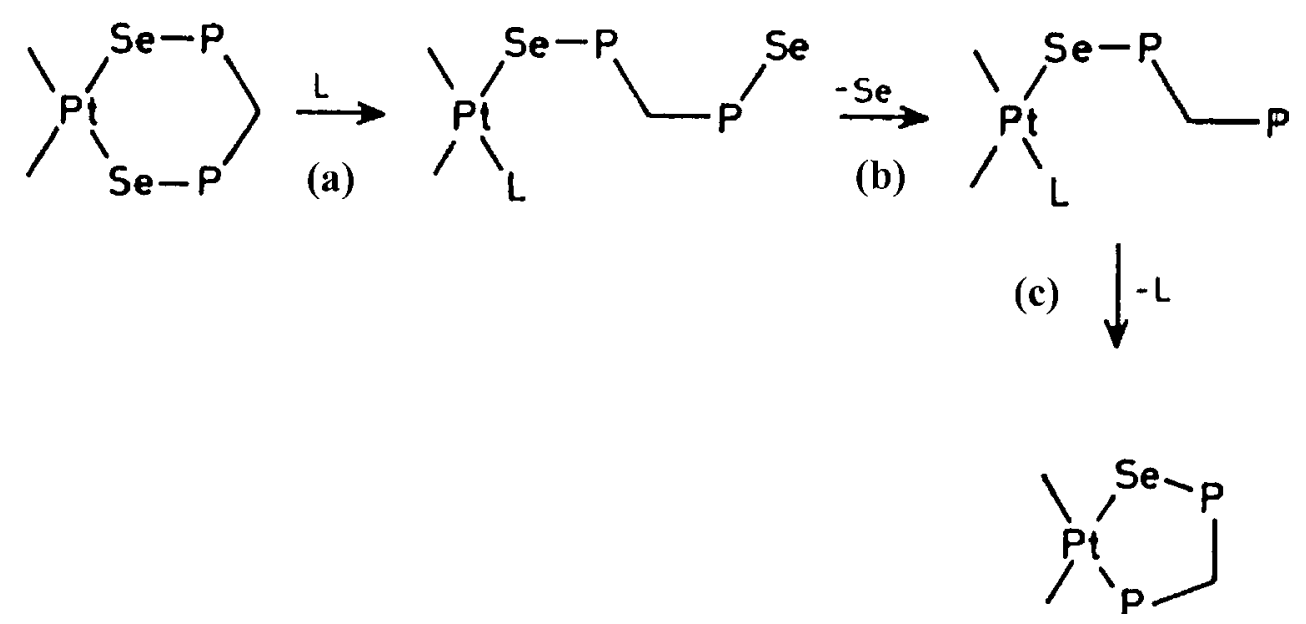

Abb. 14: Vorgeschlagener Reaktionsmechanismus der Reduktion von $\operatorname{dppmSe}_{2} \mathrm{H}$ zu dppmSeH [54]

$$
\mathrm{EPR}_{3} \rightleftharpoons \mathrm{E}+\mathrm{PR}_{3}(\mathrm{E}=\mathrm{Se}, \mathrm{Te})
$$

Demnach zersetzt sich im Verlauf der Reaktion das Diphosphoryldiselenid zu Phosphinophosphorylselenid und Selen, wobei $\mathrm{P}^{\mathrm{V}}$ zu P $\mathrm{P}^{\mathrm{III}}$ reduziert und $\mathrm{Se}^{-\mathrm{II}} \mathrm{zu} \mathrm{Se}^{ \pm 0}$ oxidiert wird. In einem ersten Schritt (a) (Abb. 14) wird die Pt-Se-Bindung eines vierfach koordinierten Pt-Intermediates gebrochen. Das Dissoziationsgleichgewicht nach Gl. 7, wie es sehr gut von Phosphortelluriden bekannt ist, führt dann im zweiten Schritt zum Bruch der P-SeBindung. Es bilden sich Selen und das Phosphan (Schritt (b)), welches anschließend in einem dritten Schritt an das Pt-Atom koordinieren kann (Schritt (c)). 


\subsubsection{Die Synthese und Struktur von $\left[\mathrm{Au}_{2}\left(\operatorname{dppmS} S_{2}\right)\left(\mathrm{PPh}_{3}\right)_{2}\right] \underline{4}$}

Komplexverbindungen des Liganden $\operatorname{dppmS}_{2} \mathrm{H}$ und der Münzmetalle Silber und Gold wurden vielfach beschrieben $[19,46]$.

So gelang auch die Synthese der zweikernigen Verbindung $\left[\mathrm{Au}_{2}\left(\mathrm{dppmS}_{2}\right)\left(\mathrm{PPh}_{3}\right)_{2}\right] \underline{4}$ durch die Umsetzung von $\left[\mathrm{AuCl}\left(\mathrm{PPh}_{3}\right)\right] \operatorname{mit}\left[\mathrm{K}\left(\mathrm{dppmS}_{2}\right)\right]$ in äquimolarem Mengenverhältnis. Jedoch entsteht bei der Reaktion Verbindung $\underline{4}$ nur in geringen Mengen neben viel $\mathrm{dppmS}_{2} \mathrm{H}$ (Gl. 8).

$$
\begin{aligned}
{\left[\mathrm{AuCl}\left(\mathrm{PPh}_{3}\right)\right]+\left[\mathrm{K}\left(\mathrm{dppmS}_{2}\right)\right] \stackrel{\mathrm{THF} / \mathrm{Heptan}}{\longrightarrow} } & {\left[\mathrm{Au}_{2}\left(\mathrm{dppmS}_{2}\right)\left(\mathrm{PPh}_{3}\right)_{2}\right] \underline{4} } \\
& +\mathrm{dppmS}_{2} \mathrm{H}
\end{aligned}
$$

A. Laguna et al. [19] konnten die $\mathrm{zu} \underline{4}$ verwandten Verbindungen der analytischen Zusammensetzungen $\left[\mathrm{Au}\left(\mathrm{dppmS}_{2} \mathrm{H}\right) \mathrm{PPh}_{3}\right]$ und $\left[\mathrm{Au}_{2}\left(\mathrm{dppmS}_{2}\right)\left(\mathrm{PPh}_{3}\right)_{2}\right]$ darstellen und untersuchten diese NMR-spektroskopisch. Der Aufbau des Komplexes $\left[(\mathrm{S}) \mathrm{PPh}_{2} \mathrm{C}\left(\mathrm{AuPPh}_{3}\right)_{2} \mathrm{PPh}_{2} \mathrm{CH}\left(\mathrm{AuPPh}_{3}\right) \mathrm{COOMe} \mathrm{ClO}_{4}\right.$ konnte röntgenstrukturanalytisch aufgeklärt werden und zeigt das gleiche Strukturelement $\left(\mathrm{Ph}_{3} \mathrm{P}\right) \mathrm{AuCAu}\left(\mathrm{PPh}_{3}\right)$, das ebenfalls in $\underline{4}$ auftritt.

\section{Die Struktur von $\left[\mathrm{Au}_{2}\left(\mathrm{dppmS}_{2}\right)\left(\mathrm{PPh}_{3}\right)_{2}\right] \underline{4}$ :}

Die zweikernige Verbindung $\underline{4}$ kristallisiert monoklin in der Raumgruppe $C 2 / c$ mit einem Komplexmolekül und 2,5 THF-Molekülen in der asymmetrischen Einheit [55]. 4 ist aus einer trigonalen $\mathrm{Au}_{2} \mathrm{C}$-Einheit aufgebaut. An die zwei Goldatome sind in einer annähernd linearen Anordnung jeweils ein Phosphoratom eines Triphenylphosphanmoleküls und das Methandiid-Kohlenstoffatom eines $\mathrm{dppmS}_{2}{ }^{2}{ }_{-}$-Liganden (Abb. 15) gebunden.

Der Au-Au-Abstand beträgt 288 pm, liegt also im Bereich von bisher bekannten GoldClusterverbindungen (260 bis $320 \mathrm{pm}$ ) [56].

Aufgrund des kleinen Au-C-Au-Winkels ( $\angle 85^{\circ}$ in $\underline{4}$ ), den im Vergleich zu dppmS ${ }_{2} \mathrm{H}[8]$ um 5 pm verkürzten $\mathrm{P}-\mathrm{C}(1)$-Bindungen und dem recht großen $\mathrm{P}-\mathrm{C}-\mathrm{P}-$ Winkel $\left(\angle 122^{\circ}\right.$ in 4), wurde für Komplexe des Typs $\left(\mathrm{Ph}_{3} \mathrm{P}\right) \mathrm{AuCAu}\left(\mathrm{PPh}_{3}\right)$ von B.F.G. Johnson et al. [57] und J. Vincente et al. [58] das Modell einer geschlossenen Drei-Zentren-Zwei-ElektronenBindung innerhalb der $\mathrm{Au}_{2} \mathrm{C}$-Einheit vorgeschlagen, die durch die in Abb. 16 dargestellten Grenzstrukturen beschrieben werden kann [55].

Werden die P-S-Bindungslängen in Betracht gezogen, so stellt man fest, daß sie in $\underline{4}$ mit 197 pm um 3 pm größer sind als im neutralen Liganden [8], was eine Verringerung der 


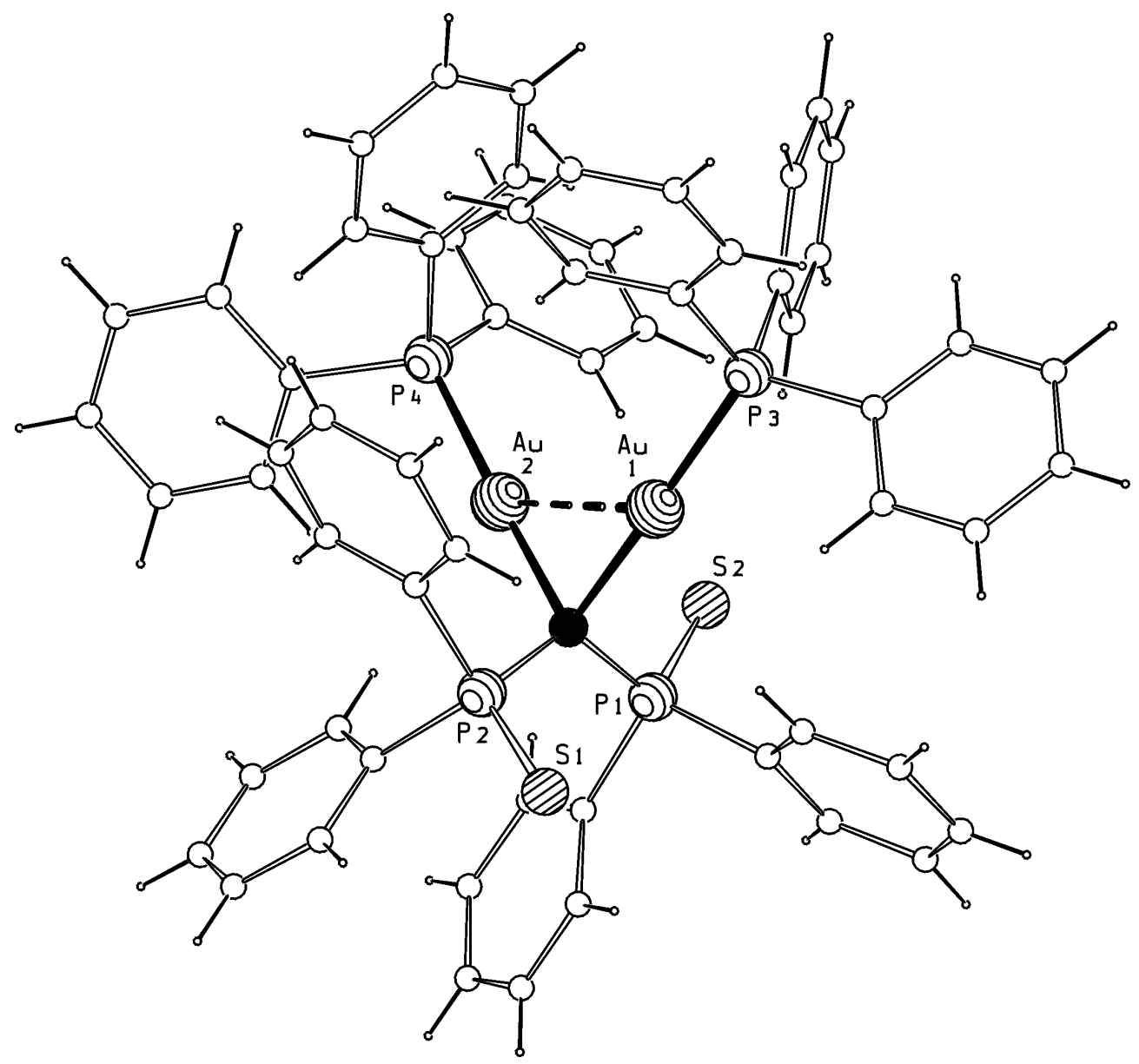

Abb. 15: Die Struktur von $\left[\mathrm{Au}_{2}\left(\mathrm{dppmS} \mathrm{S}_{2}\right)\left(\mathrm{PPh}_{3}\right)_{2}\right] \underline{4}$ im Kristall

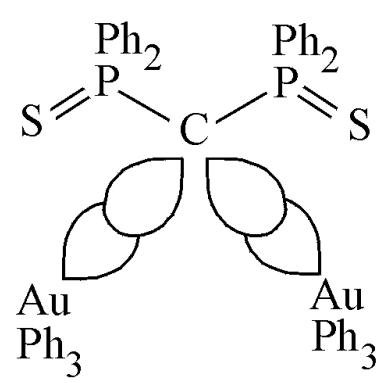

(a)

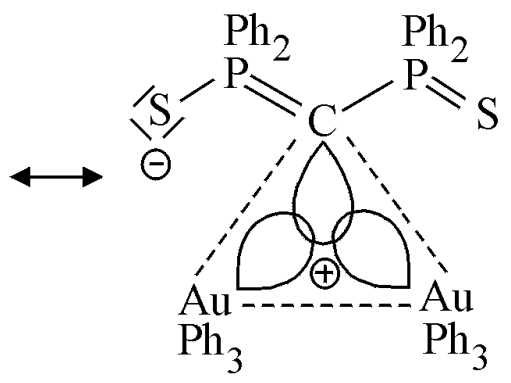

(b)

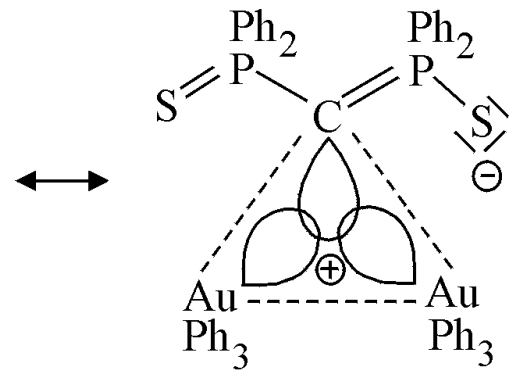

(c)

Abb. 16: Modell einer Drei-Zentren-zwei-Elektronen-Bindung [55] 
P-S-Bindungsordnung zur Folge hat und mit den in Abb. 16 formulierten Grenzstrukturen (b) und (c) gut vereinbar ist.

Die Au-C-Abstände in $\underline{4}$ mit 213 pm sind vergleichbar mit denen, die bereits in ähnlichen Methanid- und Ylidkomplexen bestimmt wurden (217 pm [59], 208 pm und 214 pm [60]). Auch ist die Koordination eines $\mathrm{Au}\left(\mathrm{PPh}_{3}\right)$-Fragmentes an ein Kohlenstoffatom nicht überraschend, da Gold-Methanid-Komplexe besonders stabil sind, und viele Schwefel-DonorLiganden in Goldverbindungen leicht durch andere Liganden ersetzt werden können [61]. Eine Verbindung, die ebenfalls das Strukturelement $\left(\mathrm{PPh}_{3}\right) \mathrm{AuCAu}\left(\mathrm{PPh}_{3}\right)$ enthält, konnte von J. Vincente et al. [60] röntgenstrukturanalytisch untersucht werden. Es handelt sich um den ylidischen Komplex $\left[\left(\mathrm{AuPPh}_{3}\right)_{2}\left(\mathrm{C}\left(\mathrm{PPh}_{3}\right) \mathrm{C}(\mathrm{O}) \mathrm{NMe}_{2}\right)\right] \mathrm{ClO}_{4}$. Auch wurde von A. Laguna et al. [19] eine Röntgenstrukturanalyse des Komplexes $\left[\mathrm{Au}_{5}\left(\mathrm{C}_{6} \mathrm{~F}_{5}\right)\left(\mathrm{dppmS}_{2}\right)_{2}\left(\mathrm{PPh}_{3}\right)\right]$ durchgeführt, in dem der zweifach anionische Ligand $\operatorname{dppmS}_{2}{ }^{2 \ominus}$ sowohl mit dem Methandiid-Kohlenstoffatom als auch mit den zwei Schwefelatomen an insgesamt vier Goldatome koordiniert ist (vgl. Abb. 2 (p)). Die Bildung des einkernigen Komplexes $\left[\mathrm{Au}\left(\mathrm{PPh}_{3}\right)\left(\mathrm{dppmS} \mathrm{S}_{2}\right)\right]$, wie sie A. Laguna [19] beschreibt, in dem der Ligand nur einfach deprotoniert vorliegt, konnte in Umsetzungen mit dem Liganden jedoch nicht beobachtet werden.

Im Verlauf der Untersuchungen von Silberkomplexverbindungen von $\mathrm{dppmS}_{2} \mathrm{H}$ wurde ein $\mathrm{Au}$-analoges Verhalten nicht festgestellt, denn bei der Umsetzung von Silberacetat und Triphenylphosphan mit einem Äquivalent $\mathrm{dppmS}_{2} \mathrm{H}$ und zwei Äquivalenten Deprotonierungsmittel entsteht der Komplex $\left[\mathrm{Ag}\left(\mathrm{dppmS}_{2}\right)\left(\mathrm{PPh}_{3}\right)\right]$ [52], in dem der Ligand nur einfach deprotoniert ist (Gl. 9).

$$
\begin{gathered}
2 \mathrm{Ag}(\mathrm{Ac})+2 \mathrm{PPh}_{3}+\mathrm{dppmS}_{2} \mathrm{H}+2{ }^{\mathrm{n}} \mathrm{BuLi} \stackrel{\text { THF } / \text { Heptan }}{\longrightarrow} \\
2\left[\mathrm{Ag}\left(\mathrm{dppmS}_{2}\right)\left(\mathrm{PPh}_{3}\right)\right]+2{ }^{\mathrm{n}} \mathrm{BuH}+2 \mathrm{LiAc}
\end{gathered}
$$

Auch koordiniert hier nicht das Methanid-Kohlenstoffatom, sondern der Ligand chelatisiert mit den im Sinne des HSAB-Konzeptes weicheren Schwefelatomen das weiche Ag $\mathrm{I}_{-}$ Ion. Die trigonal-planare Koordinationssphäre des Silberions wird durch das Phosphoratom des $\mathrm{PPh}_{3}$-Moleküls vervollständigt (Abb. 17). 


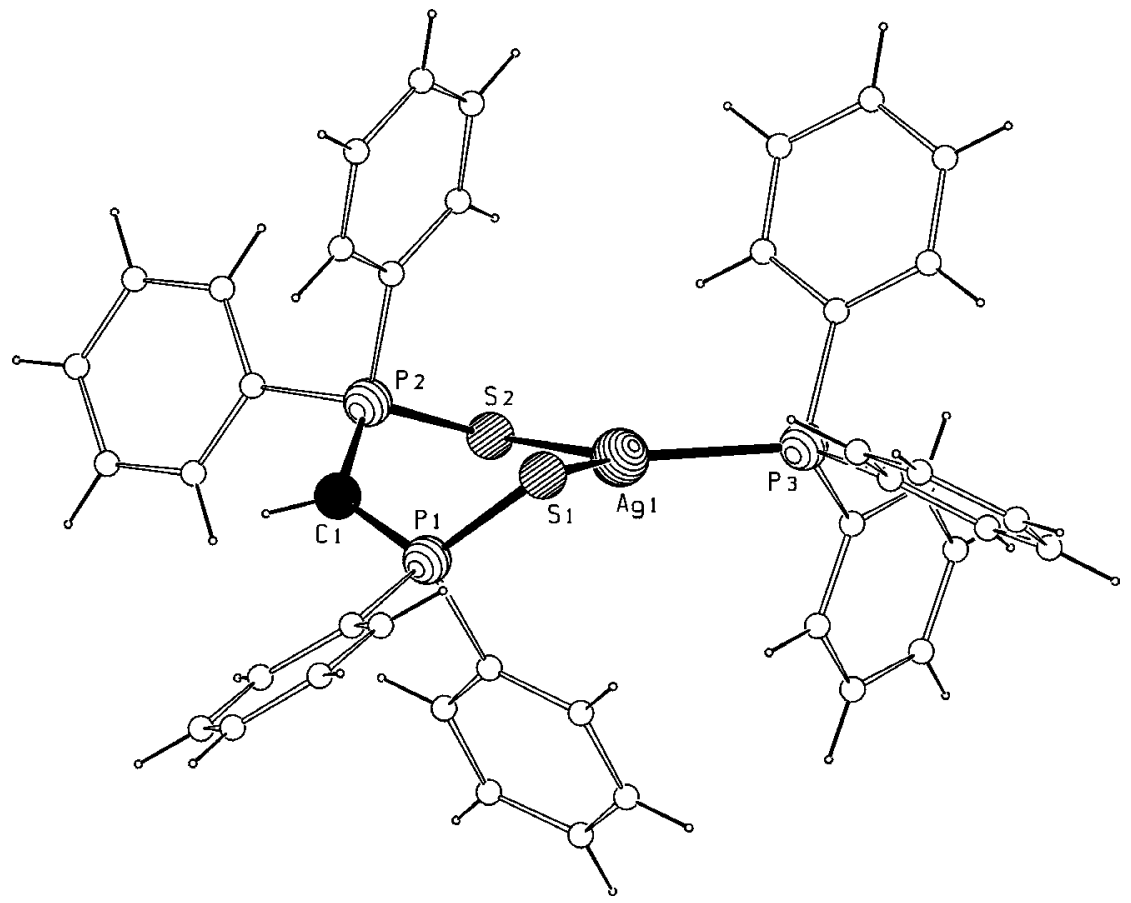

Abb. 17: Die Struktur von $\left[\operatorname{Ag}\left(\operatorname{dppmS}_{2}\right)\left(\mathrm{PPh}_{3}\right)\right]$ im Kristall 


\subsection{Umsetzungen von $\mathrm{CuCl}$ und $\mathrm{CuCl}_{2}$ mit dem $\mathrm{Amin}$ dppaO ${ }_{2} \mathrm{H}$}

In der Literatur ist schon von zahlreichen Verbindungen des Amid-Liganden $\mathrm{dppaO}_{2}{ }^{\ominus}$, sowohl von Hauptgruppen- als auch von Nebengruppenelementen und Lanthanoiden, berichtet worden. So konnten beispielsweise der Komplex $\left[\mathrm{Sn}\left(\mathrm{dppaO}_{2}\right)_{2}\right]$ [62] und die Verbindungen $\left[\mathrm{Fe}\left(\mathrm{dppaO}_{2}\right)_{3}\right][63]$ und $\left[\operatorname{Pr}\left(\mathrm{dppaO}_{2}\right)_{3}\right]$ [64] synthetisiert werden.

Unter den Komplexen der Münzmetalle konnte die Struktur der vierkernigen Verbindung $\left[\mathrm{Ag}_{4}\left(\mathrm{dppaO}_{2}\right)_{4}(\mathrm{EtOH})_{2}\right]$ [33] röntgenstrukturanalytisch aufgeklärt werden. Diese Verbindung stellt zudem noch das erste Beispiel eines Übergangsmetallkomplexes dar, in dem der Ligand dppaO ${ }_{2}{ }^{\ominus}$ sowohl mit den Sauerstoffatomen als auch mit dem Amido-Stickstoffatom an insgesamt drei Metallatome koordiniert ist (vgl. Abb. 4 (j)). Bisher war diese Art der Koordination nur in Metallkomplexen der Seltenen Erden bekannt.

Da bislang jedoch keine weiteren mehrkernigen Verbindungen des Liganden, außer einige zweikernige Verbindungen des Praseodyms [65], strukturell charakterisiert sind, wurden Umsetzungen von $\mathrm{CuCl}$ und $\mathrm{CuCl}_{2}$ mit verschiedenen Salzen von $\mathrm{dppaO}_{2} \mathrm{H}$ in unterschiedlichen Mengenverhältnissen und Lösungsmitteln durchgeführt.

\subsubsection{Die Synthese und Struktur von $\left[\mathrm{Cu}\left(\mathrm{dppaO}_{2}\right)_{2}(\mathrm{MeOH})\right] \underline{5}$}

Wird $\mathrm{CuCl}_{2}$ mit zwei Äquivalenten $\left[\mathrm{K}\left(\mathrm{dppaO}_{2}\right)\right]$ umgesetzt, so können blaue Kristalle der einkernigen Verbindung $\left[\mathrm{Cu}\left(\mathrm{dppaO}_{2}\right)_{2}(\mathrm{MeOH})\right] \underline{5}$ isoliert werden (Gl. 10). Da die Synthese zunächst in THF erfolgte und keine für eine Röntgenstrukturanalyse geeigneten Kristalle erhalten werden konnten, wurde die Reaktion in wässrig-methanolischer Lösung durchgeführt.

$$
\mathrm{CuCl}_{2}+2\left[\mathrm{~K}\left(\mathrm{dppaO}_{2}\right)\right] \underset{\mathrm{H}_{2} \mathrm{O}}{\stackrel{\mathrm{MeOH}}{\longrightarrow}}\left[\mathrm{Cu}\left(\mathrm{dppaO}_{2}\right)_{2}(\mathrm{MeOH})\right] \underline{5}
$$

\section{Die Struktur von $\left[\mathrm{Cu}\left(\mathrm{dppaO}_{2}\right)_{2}(\mathrm{MeOH})\right] \underline{\mathbf{5}}$ :}

Verbindung $\underline{5}$ kristallisiert triklin in der Raumgruppe $P \overline{1}$ (Abb. 18) [66]. Das Kupferatom ist annähernd quadratisch-planar von vier Sauerstoffatomen der zwei Ligandmoleküle umgeben. Weiterhin bindet ein Methanolmolekül mittels zweier Wasserstoffbrückenbindungen an ein Sauerstoffatom je eines Liganden.

Die ähnlich $\underline{5}$ aufgebaute Verbindung $\left[\mathrm{Cu}\left((\mathrm{PhO})_{2} \mathrm{P}(\mathrm{O}) \mathrm{NP}(\mathrm{O})(\mathrm{OPh})_{2}\right)_{2}\right]$ (Abb.19) wurde von H. Richter et al. [67] in einer analogen Transmetallierungsreaktion synthetisiert, indem $\mathrm{CuCl}_{2}$ mit $\left[\mathrm{Ba}\left((\mathrm{PhO})_{2} \mathrm{P}(\mathrm{O}) \mathrm{NP}(\mathrm{O})(\mathrm{OPh})_{2}\right)_{2}\right]$ umgesetzt wurde. Auch in dieser Verbindung besitzt das $\mathrm{Cu}$-Ion eine annähernd quadratisch-planare Koordinationssphäre. 


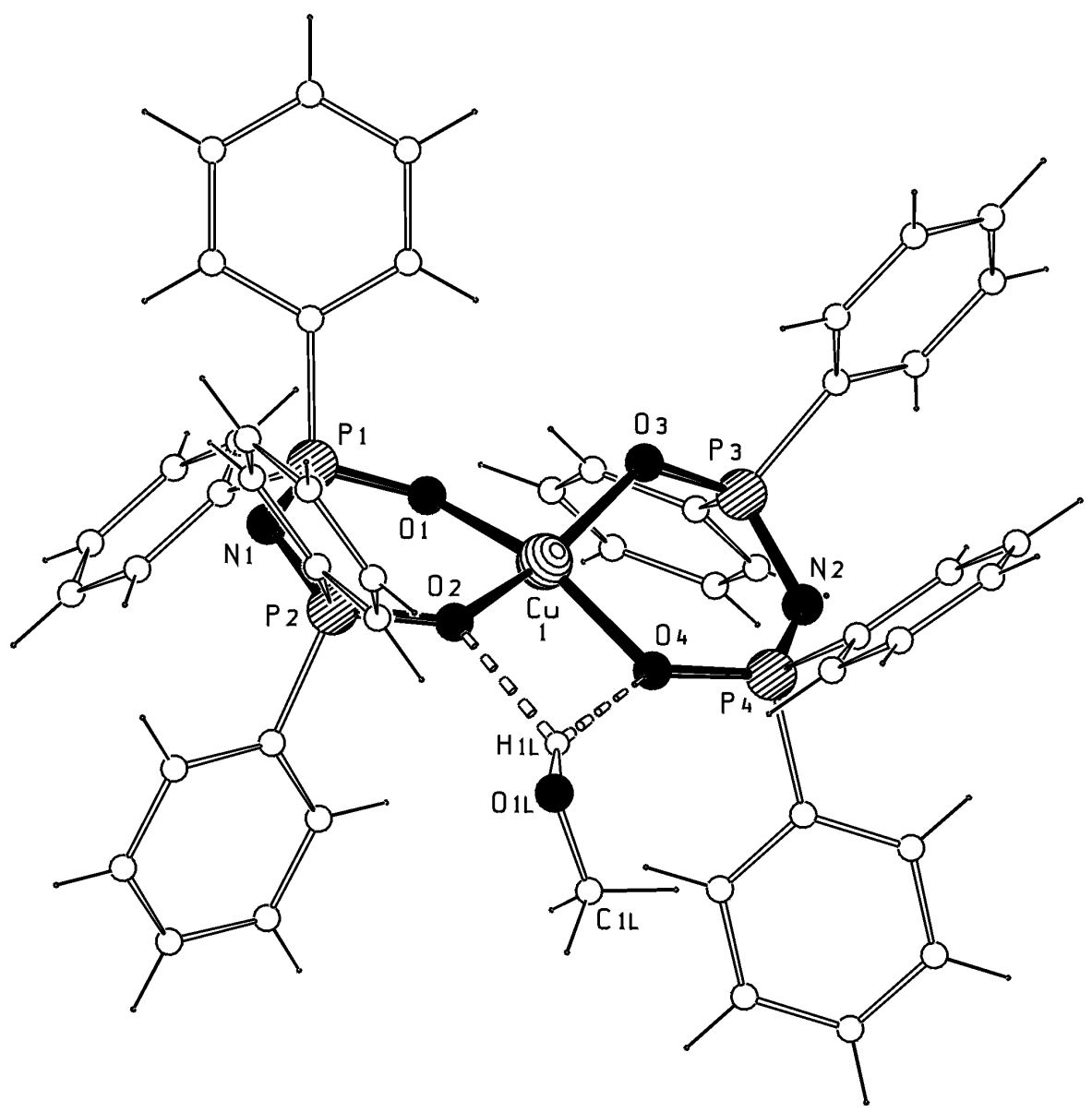

Abb. 18: Die Struktur von $\left[\mathrm{Cu}\left(\mathrm{dppaO}_{2}\right)_{2}(\mathrm{MeOH})\right] \underline{5}$ im Kristall 


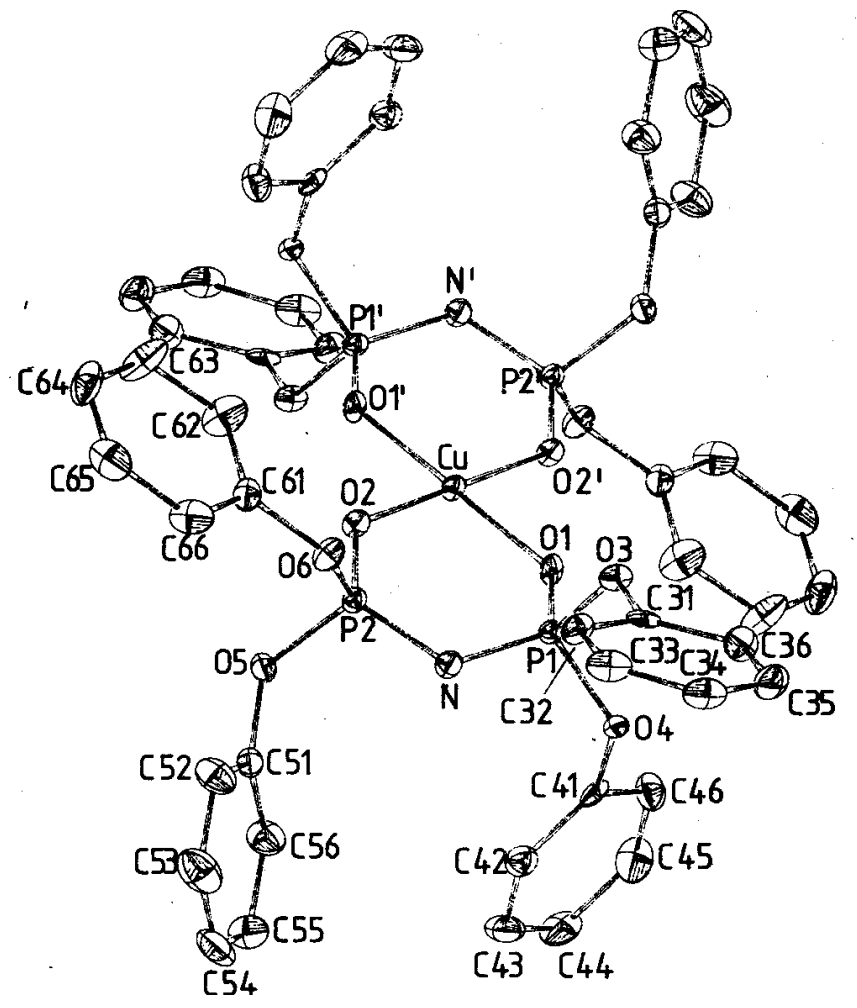

Abb. 19: Die Molekülstruktur von $\left[\mathrm{Cu}\left((\mathrm{PhO})_{2} \mathrm{P}(\mathrm{O}) \mathrm{NP}(\mathrm{O})(\mathrm{OPh})_{2}\right)_{2}\right][67]$ 
Die P-N-Bindungslängen betragen in $\underline{5}$ durchschnittlich 159 pm. Sie sind charakteristisch für Phosphazene mit hohem Doppelbindungsanteil [50] und entsprechen den Abständen, die in anderen Komplexen für das an ein Metallatom bindende $\mathrm{dppaO}_{2}{ }^{\ominus}$-Molekül gefunden werden $[62,68]$. Ebenso weisen die P-O-Abstände mit durchschnittlich 152 pm auf das Vorliegen von P-O-Doppelbindungsanteilen hin, denn für P-O-Einfachbindungslängen werden Werte von 161 pm und für P-O-Doppelbindungslängen Werte von 149 pm [50] gefunden. Folglich kann von einer $\pi$-Elektronendelokalisierung innerhalb der OPNPO-Systeme der Liganden ausgegangen werden.

Die Cu-O-Bindungen sind ca. 192 pm lang und mit anderen Cu-O-Abständen des vierfach koordinierten $\mathrm{Cu}^{\mathrm{II}}$-Atoms von ca. 195 pm vergleichbar [69]. Die zwei unsymmetrischen Wasserstoffbrückenbindungen, die H(1L) bildet, sind 212 pm und 233 pm lang. Im Gegensatz dazu weist der neutrale Ligand $\mathrm{dppaO}_{2} \mathrm{H}$ zwei symmetrische Wasserstoffbrückenbindungen auf: das Phosphoryl liegt im festen Zustand nicht in der Amin- sondern in der Säureform $\mathrm{HOPPh}_{2} \mathrm{NP}(\mathrm{O}) \mathrm{Ph}_{2}$ vor und bildet somit ein lineares PNP-System aus [70]. Die O-H-Abstände betragen 220 pm, liegen also genau zwischen den Werten von Verbindung 32 .

\subsubsection{Die Synthese und Struktur von $\left[\mathrm{Cu}_{2}\left(\operatorname{dppaO}_{2}\right)_{2} \mathrm{Cl}_{2}(\operatorname{thf})_{2}\right] \underline{6}$}

Wird nun die Reaktion von einem Äquivalent $\mathrm{CuCl}_{2}$ mit einem Äquivalent $\left[\mathrm{K}\left(\operatorname{dppaO} \mathrm{O}_{2}\right)\right]$ in THF durchgeführt, so entstehen die zweikernige Verbindung $\left[\mathrm{Cu}_{2}\left(\mathrm{dppaO}_{2}\right)_{2} \mathrm{Cl}_{2}(\mathrm{thf})_{2}\right]$ $\underline{6}$ und der Komplex $\left[\mathrm{Cu}\left(\mathrm{dppaO}_{2}\right)_{2}\right]$ (Gl. 11).

$$
\left.\mathrm{CuCl}_{2}+\left[\mathrm{K}(\mathrm{dppaO})_{2}\right)\right] \stackrel{\text { THF }}{\longrightarrow}\left[\mathrm{Cu}_{2}\left(\mathrm{dppaO}_{2}\right)_{2} \mathrm{Cl}_{2}(\mathrm{thf})_{2}\right] \underline{6}+\left[\mathrm{Cu}(\mathrm{dppaO})_{2}\right](11
$$

\section{Die Struktur von $\left[\mathrm{Cu}_{2}\left(\mathrm{dppaO}_{2}\right)_{2} \mathrm{Cl}_{2}(\text { thf })_{2}\right] \underline{6}$ :}

Die triklin in der Raumgruppe $P \overline{1}$ kristallisierende Verbindung $\underline{6}$ ist in Abb. 20 dargestellt. An die zwei Kupferatome bindet $\eta^{2}$-artig jeweils ein Ligand (vgl. Abb. 4 (c)); zwei Chloroliganden wirken zusätzlich zwischen den zwei Metallionen verbrückend. Die Ligandensphäre der Metallionen wird durch je ein THF-Molekül, das mit dem Sauerstoffatom an ein Kupferion bindet, ergänzt, so daß sich eine quadratisch-pyramidale Koordinationsumgebung für beide Kupferionen ergibt. $\mathrm{O}(1), \mathrm{O}(2), \mathrm{Cl}(1)$ und $\mathrm{Cl}\left(1^{\prime}\right)$ bilden hierbei die Grundfläche des Polyeders, über der sich das Cu-Ion in einem Abstand von 22 pm befindet. Unter den $\mathrm{Cu}-\mathrm{O}-$ Bindungen muß zwischen zwei kürzeren und zwei längeren Bindungen unterschieden werden. Die Cu-O-Abstände zu den Sauerstoffatomen der Liganden 


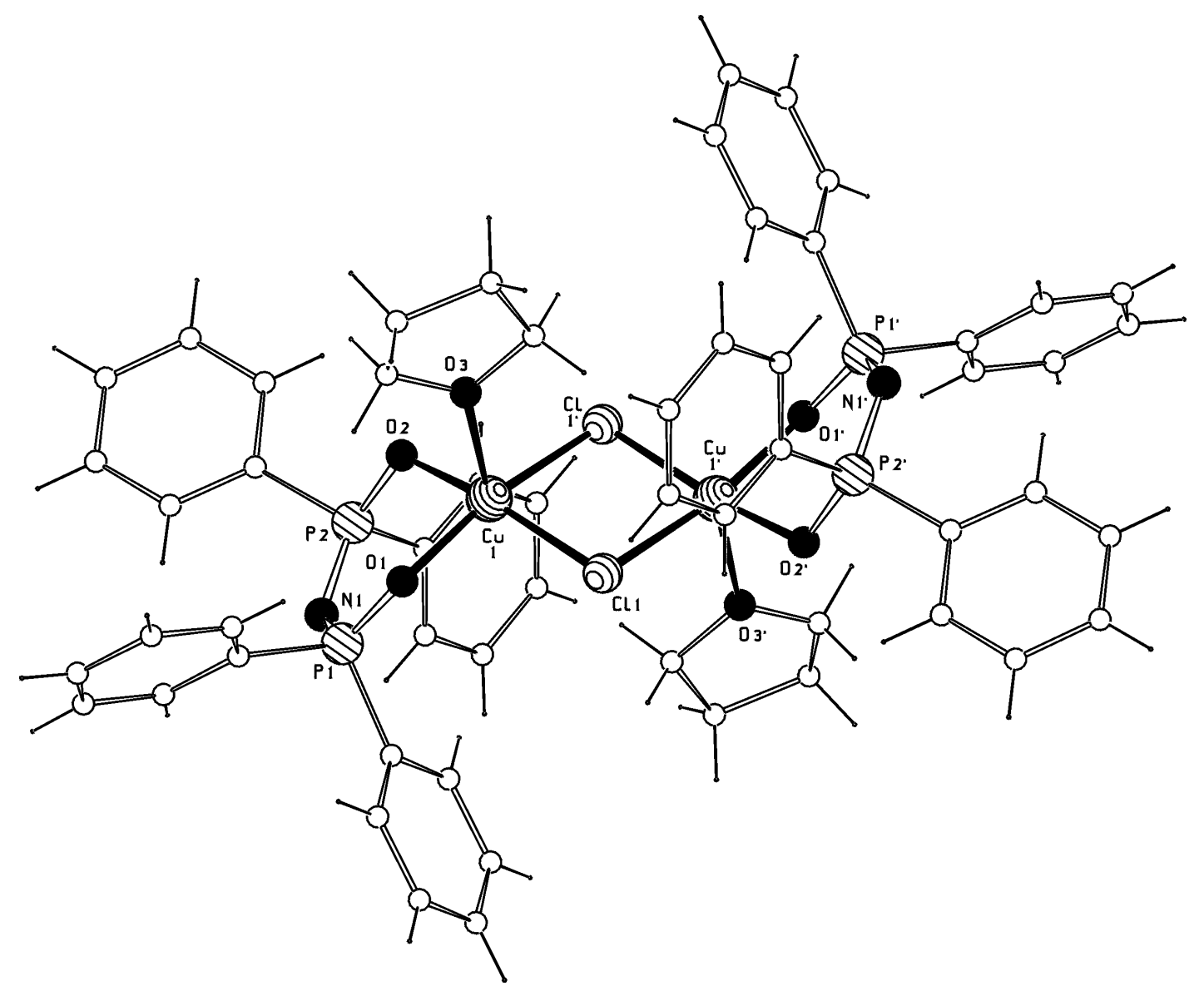

Abb. 20: Die Struktur von $\left[\mathrm{Cu}_{2}\left(\operatorname{dppaO}_{2}\right)_{2} \mathrm{Cl}_{2}(\text { thf })_{2}\right] \underline{6}$ im Kristall 
betragen durchschnittlich 195 pm, während die Cu-O-Abstände zu den O-Atomen der THF-Moleküle mit $224 \mathrm{pm}$ deutlich größer sind. Letztere werden jedoch ebenfalls in $\mathrm{Cu}-$ THF-Komplexverbindungen gefunden, wie z.B. in der Verbindung $\left[\mathrm{Cu}_{2}\left(\mathrm{O}_{2} \mathrm{CR}\right)_{4}(\mathrm{thf})_{2}\right]$ $\left.\left(\mathrm{R}=\left(\mathrm{C}_{5} \mathrm{H}_{4}\right) \mathrm{FeCp}\right)\right)[71]$, in der die $\mathrm{Cu}^{\mathrm{II}}$-Ionen von fünf Sauerstoffatomen koordiniert sind und die $\mathrm{Cu}-\mathrm{O}$ (thf)-Abstände zu 223 pm bestimmt wurden. Mit 230 pm sind die Cu-ClBindungen etwas kürzer als in der Literatur für $\mu_{2}$-bindende Chloroliganden, die an ein fünffach koordiniertes $\mathrm{Cu}^{\mathrm{II}}$-Ion binden, angegeben werden (237 pm) [69]. In Chlorocupraten werden jedoch die gleichen $\mathrm{Cu}$-Cl-Bindungslängen für $\mu_{2}$-verbrückende Cl-Liganden, wie beispielsweise im Anion $\left[\mathrm{Cu}_{2} \mathrm{Cl}_{6}\right]^{2 \ominus}$, vorgefunden [43].

\subsubsection{Die Synthese und Struktur von $\left[\mathrm{Cu}_{2}\left(\mathrm{dppaO}_{2}\right)_{2}(\mathrm{OMe})_{2}\right] \underline{7}$}

Wird dppaO ${ }_{2} \mathrm{H}$ an Stelle von $\mathrm{KO}^{t} \mathrm{Bu}$ mit $\mathrm{NEt}_{3}$ im Überschuß deprotoniert und mit äquimolaren Mengen von $\mathrm{CuCl}_{2}$ in Methanol umgesetzt, so kann die Verbindung $\left[\mathrm{Cu}_{2}(\mathrm{dppaO})_{2}(\mathrm{OMe})_{2}\right] \underline{7}$ isoliert werden (Gl. 12). Diese weist einen zu $\underline{6}$ analogen Aufbau auf, da die in $\underline{6}$ verbrückenden Chloroliganden formal durch die Alkoholatliganden substituiert sind.

Den gleichen Aufbau zeigt Verbindung $\left[\mathrm{Cu}_{2}\left(\mathrm{dppaO}_{2}\right)_{2}\left(\mathrm{O}^{\mathrm{t}} \mathrm{Bu}\right)_{2}\right]$, in der die MethanolatGruppen durch Tertiärbutanolat-Liganden ersetzt sind. Sie kann synthetisiert werden, wenn statt $\mathrm{CuCl}_{2}$ das Alkoholat $\mathrm{Cu}\left(\mathrm{O}^{\mathrm{t}} \mathrm{Bu}\right)_{2}$ mit dppaO ${ }_{2} \mathrm{H}$ in THF umgesetzt wird (Gl. 13, Abb. $21[52])$.

$$
\begin{aligned}
& \mathrm{CuCl}_{2}+\mathrm{dppaO}_{2} \mathrm{H} \underset{3,5-\mathrm{DTBC}}{\stackrel{\mathrm{MeOH} / \mathrm{NEt}_{3}}{\longrightarrow}}\left[\mathrm{Cu}_{2}\left(\mathrm{dppaO}_{2}\right)_{2}(\mathrm{OMe})_{2}\right] \underline{7} \\
& \mathrm{Cu}\left(\mathrm{O}^{\mathrm{t}} \mathrm{Bu}\right)_{2}+\mathrm{dppaO}_{2} \mathrm{H} \underset{\mathrm{THF}}{\stackrel{\Delta}{\longrightarrow}}\left[\mathrm{Cu}_{2}\left(\mathrm{dppaO}_{2}\right)_{2}\left(\mathrm{O}^{\mathrm{t}} \mathrm{Bu}\right)_{2}\right]
\end{aligned}
$$




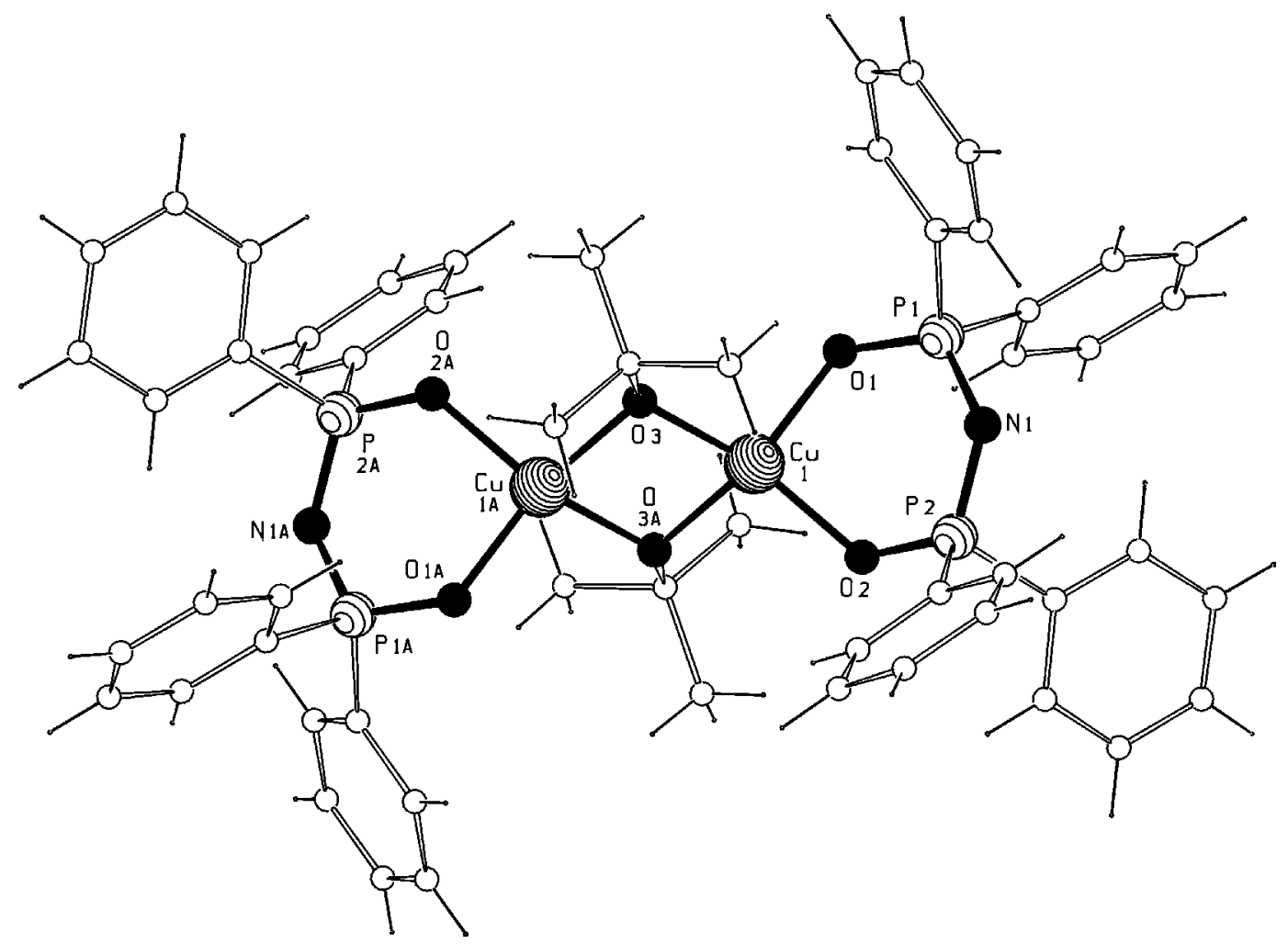

Abb. 21: Die Struktur von $\left[\mathrm{Cu}_{2}\left(\mathrm{dppaO}_{2}\right)_{2}\left(\mathrm{O}^{\mathrm{t}} \mathrm{Bu}\right)_{2}\right]$ im Kristall 


\section{Die Struktur von $\left[\mathrm{Cu}_{2}\left(\mathrm{dppaO}_{2}\right)_{2}(\mathrm{OMe})_{2}\right]$ 7:}

Verbindung 7 kristallisiert triklin in der Raumgruppe $P \overline{1}$. Die Molekülstruktur setzt sich zusammen aus zwei Cu-Ligand-Einheiten, in denen der Ligand ein Kupferatom zweizähnig chelatisiert (vgl. Abb. 4 (c)), und zwei Methanolationen, die $\mu_{2}$-artig über ihre Sauerstoffatome an die Kupferionen binden, so daß sie die beiden Cu-Ligand-Einheiten zum dimeren Komplex verbinden. Die Koordinationsgeometrie der Cu-Ionen ist annähernd quadratischplanar (Abb. 22).

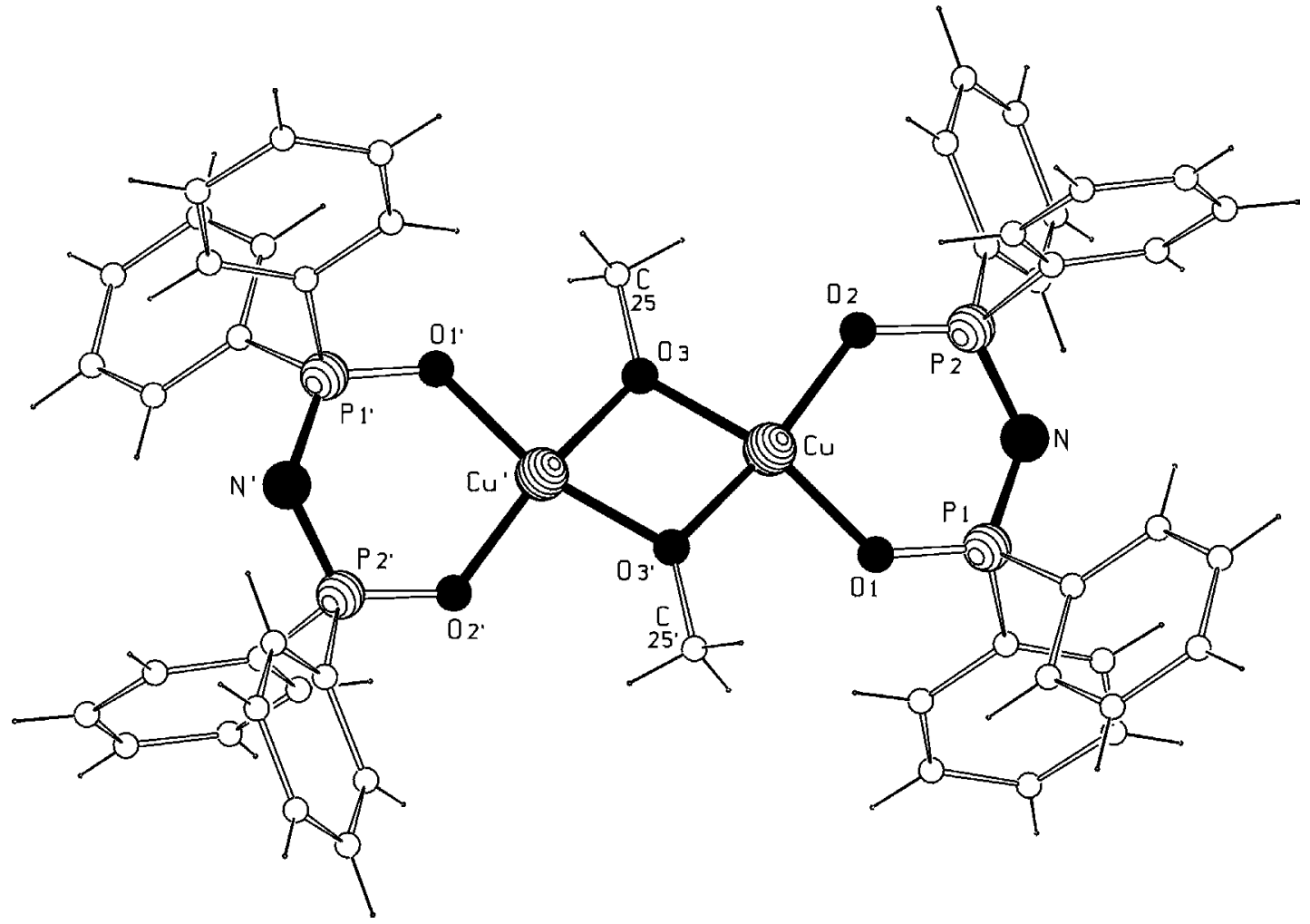

Abb. 22: Die Struktur von $\left[\mathrm{Cu}_{2}\left(\mathrm{dppaO}_{2}\right)_{2}(\mathrm{OMe})_{2}\right] \underline{7}$ im Kristall

Die Cu-O(3)- bzw. Cu-O(3')-Abstände betragen ca. 191 pm, während die C(25)-O(3)Bindung 141 pm lang ist, was damit der in Methanol auftretenden C-O-Bindungslänge entspricht [50]. Auch in vergleichbaren Methanolatkomplexen, in denen die $\mathrm{Cu}^{\mathrm{II}}$-Ionen ebenfalls quadratisch-planar von vier Sauerstoffatomen umgeben sind, wie z.B. in der Verbindung $[\mathrm{Cu}(\mathrm{Ac})(\mathrm{OMe})]_{4}[72]$, treten fast identische Bindungslängen auf. Der Komplex besteht aus zwei $\mathrm{Cu}_{2}(\mathrm{Ac})_{2}$-Einheiten, die durch vier $\mu_{2}$-koordinierte Methanolat-Liganden verbrückt sind. Die C-O-Bindungslängen der Methanolationen betragen hier 140 pm, die entsprechenden Cu-O-Abstände 192 pm. 


\subsubsection{Die Synthesen und Strukturen von}

$$
\left[\mathrm{Cu}_{3}\left(\mathrm{dppaO}_{2}\right)_{2}\left(\left(\mathrm{OSiMe}_{2}\right)_{2} \mathrm{O}\right)_{2}(\mathrm{thf})_{2}\right] \underline{8} \text { und }\left[\mathrm{Cu}_{4}(\mathrm{O})\left(\mathrm{dppaO}_{2}\right)_{4} \mathrm{Cl}_{2}\right] \underline{\mathbf{9}}
$$

Überraschenderweise konnte die dreikernige, in blauen Kristallen erhaltene Verbindung $\left[\mathrm{Cu}_{3}\left(\mathrm{dppaO}_{2}\right)_{2}\left(\left(\mathrm{OSiMe}_{2}\right)_{2} \mathrm{O}\right)_{2}(\mathrm{thf})_{2}\right] \underline{8}$ aus der Reaktion von $\mathrm{CuCl}, \mathrm{dppaO}_{2} \mathrm{H}$ und $\mathrm{KO}^{\mathrm{t}} \mathrm{Bu}$ im Molverhältnis 1:0,5:1 nach mehreren Wochen Reaktionszeit isoliert werden (Gl. 14). Im Verlauf der Reaktion wird $\mathrm{Cu}^{\mathrm{I}} \mathrm{zu} \mathrm{Cu}^{\mathrm{II}}$ oxidiert, das überschüssige $\mathrm{KO}^{\mathrm{t}} \mathrm{Bu}$ reagiert mit dem im Kolben befindlichen Schliffett Baysilone $\left(\left[\mathrm{OSiMe}_{2}\right]_{\infty}\right)$ zu Tetramethyldisiloxandiolat, das nun als dianionischer Ligand an die Kupferionen binden kann.

$$
\begin{aligned}
\mathrm{CuCl}+0,5 \mathrm{dppaO}_{2} \mathrm{H}+\mathrm{KO}^{\mathrm{t}} \mathrm{Bu} \stackrel{\mathrm{THF}}{\longrightarrow} \\
{\left[\mathrm{Cu}_{3}\left(\mathrm{dppaO}_{2}\right)_{2}\left(\left(\mathrm{OSiMe}_{2}\right)_{2} \mathrm{O}\right)_{2}(\text { thf })_{2}\right] \underline{8} }
\end{aligned}
$$

8 kann ebenso direkt durch die Reaktion von $\mathrm{CuCl}_{2}$ mit Schliffett bei einer Reaktionszeit von einer Woche synthetisiert werden. Diese Umsetzung führt zusätzlich zu einem braunen kristallinen Nebenprodukt, der Verbindung $\left[\mathrm{Cu}_{4}(\mathrm{O})\left(\mathrm{dppaO}_{2}\right)_{4} \mathrm{Cl}_{2}\right] \underline{9}(\mathrm{Gl} .15)$.

$$
\begin{array}{r}
3 \mathrm{CuCl}+2 \mathrm{dppaO}_{2} \mathrm{H}+4\left[\mathrm{OSiMe}_{2}\right]+6 \mathrm{KO}^{\mathrm{t}} \mathrm{Bu} \stackrel{\mathrm{THF}}{\longrightarrow} \\
{\left[\mathrm{Cu}_{3}\left(\mathrm{dppaO}_{2}\right)_{2}\left(\left(\mathrm{OSiMe}_{2}\right)_{2} \mathrm{O}\right)_{2}(\mathrm{thf})_{2}\right] \underline{8}+\left[\mathrm{Cu}_{4}(\mathrm{O})\left(\mathrm{dppaO}_{2}\right)_{4} \mathrm{Cl}_{2}\right] \underline{9}}
\end{array}
$$

\section{Die Struktur von $\left[\mathrm{Cu}_{3}\left(\mathrm{dppaO}_{2}\right)_{2}\left(\left(\mathrm{OSiMe}_{2}\right)_{2} \mathrm{O}\right)_{2}(\mathrm{thf})_{2}\right] \underline{8}$ :}

Die Struktur der Verbindung $\underline{8}$, die monoklin in der Raumgruppe $P 2_{1} / c$ kristallisiert [55], ist in Abb. 23 gezeigt.

Das Molekül ist aus drei linear angeordneten $\mathrm{Cu}^{\mathrm{II}}$-Ionen aufgebaut. An die beiden äußeren Metallatome ist zweizähnig jeweils ein dppaO ${ }_{2}{ }^{\ominus}$-Ligand koordiniert (vgl. Abb. 4 (c)), während an das mittlere Kupferion zwei Schliffettfragmente $\left[\left(\mathrm{OSiMe}_{2}\right)_{2} \mathrm{O}\right]^{2 \ominus}$ in der Weise gebunden sind, daß sie das in der Mitte befindliche $\mathrm{Cu}$-Atom über die SiloxandiolatSauerstoffatome chelatisieren. Weiterhin verbrücken diese Sauerstoffatome $\mu_{2}$-artig zwischen dem mittleren und je einem äußeren Cu-Atom, was zu einer dreikernigen Struktur führt. Da zusätzlich zwei THF-Moleküle an das mittlere Cu-Atom koordiniert sind, ergibt sich für $\mathrm{Cu}(2)$ ein gestreckter, Jahn-Teller-verzerrter Oktaeder als Koordinationspolyeder. Die beiden anderen Kupferionen sind annähernd quadratisch-planar von vier Sauerstoffatomen umgeben.

Ähnlich aufgebaut ist die Verbindung $\left[\mathrm{Cu}\left(\left(\mathrm{OSiPh}_{2}\right)_{2} \mathrm{O}\right)_{2}\left(\mathrm{Li}(\mathrm{py})_{2}\right)_{2}\right]$ [73], die aus einer linearen Li-Cu-Li-Kette besteht und in Abb. 24 dargestellt ist. Das Cu-Atom wird von 


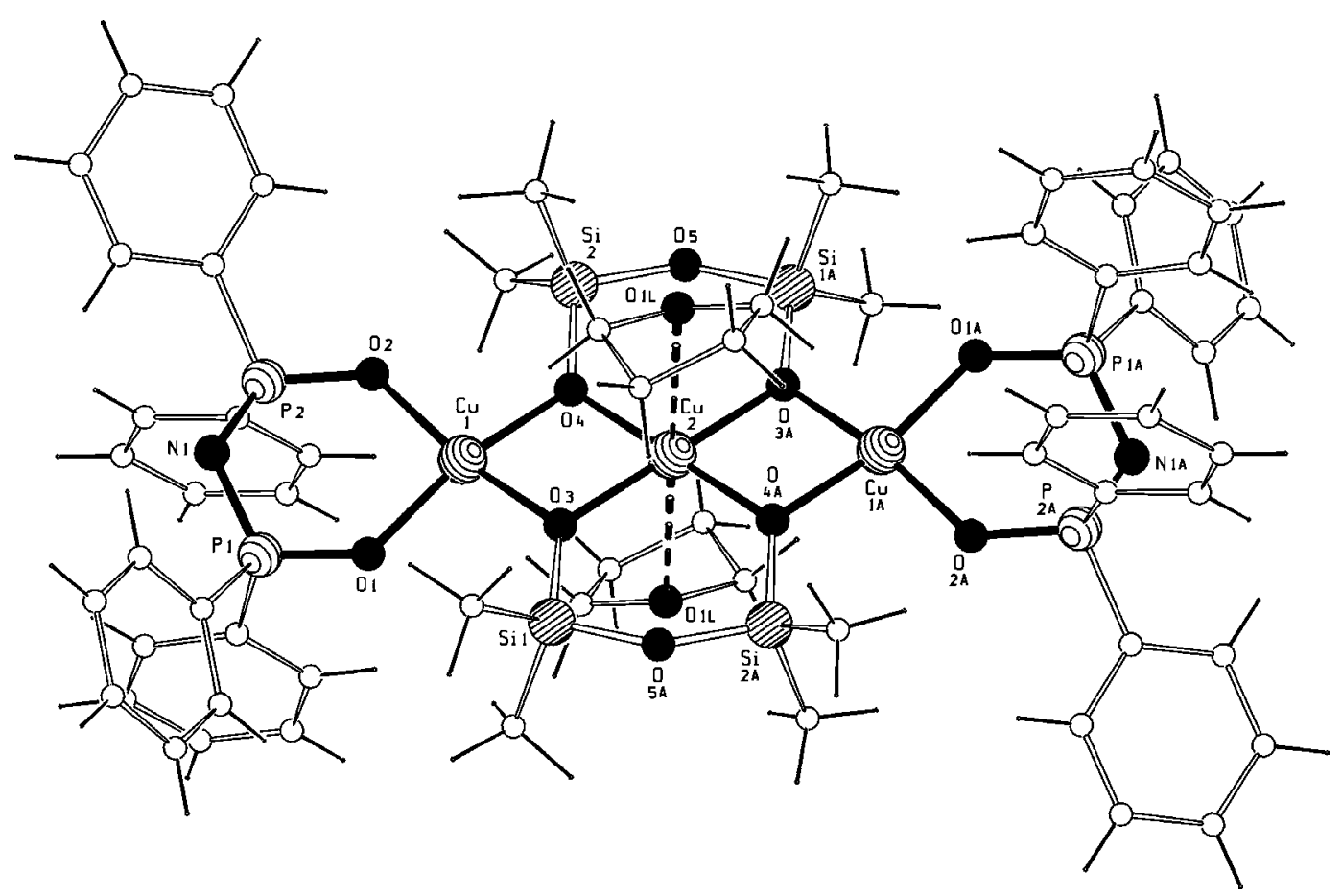

Abb. 23: Die Struktur von $\left[\mathrm{Cu}_{3}\left(\mathrm{dppaO}_{2}\right)_{2}\left(\left(\mathrm{OSiMe}_{2}\right)_{2} \mathrm{O}\right)_{2}(\text { thf })_{2}\right] \underline{8}$ im Kristall

den zwei Siloxandiolat-Liganden chelatisiert, an die weiterhin die zwei Li(py) $)_{2}$-Einheiten über die $\mu_{2}$-verbückenden Sauerstoffatome gebunden sind, wodurch sich der trimetallische Komplex ausbilden kann.

Die Si-O-Bindungslängen in $\underline{8}$ liegen mit 162 und 164 pm im Bereich von Si-O-Einfachbindungen, die z.B. in Tetramethyldisiloxandiol gefunden werden (162 und 163 pm) [74]. Die Abstände von $\mathrm{Cu}(2)$ zu den Sauerstoffatomen der THF-Moleküle sind mit 293 pm vergleichsweise groß, denn man findet in der Literatur meist kleinere $\mathrm{Cu}-\mathrm{O}$ (thf)-Abstände. So beträgt der Abstand des $\mathrm{Cu}^{\mathrm{II}}$-Ions zum Sauerstoffatom eines koordinierten THF-Liganden in der polymeren Verbindung $\left[\mathrm{Cu}_{2} \mathrm{Cl}_{4}\left(\mathrm{C}_{6} \mathrm{H}_{10}(\mathrm{OH})_{2}\right)(\text { thf })_{2}\right]_{\infty} 272 \mathrm{pm}$ [75]. Folglich sind die THF-Moleküle in 8 nur sehr schwach an $\mathrm{Cu}(2)$ gebunden.

W.J. Evans et al. konnten ebenfalls einen Komplex isolieren, in dem der Tetramethyldisiloxandiolat-Ligand aus einer Reaktion mit Schliffett vorliegt [76]. Es handelt sich um den zweikernigen Komplex $\left[\mathrm{SmCp}_{2}{ }_{2}\left(\left(\mathrm{OSiMe}_{2}\right)_{2} \mathrm{O}\right)(\text { thf })_{2}\right]$. Jedoch ist es bisher noch nicht gelungen, diesen Komplex aus der direkten Umsetzung mit Schliffett zu isolieren. Lediglich durch die Umsetzung mit Hexamethylcyclotrisiloxan konnte diese Verbindung synthetisiert werden.

Weiterhin konnte bei der Reaktion von überschüssigem Thalliumethanolat mit Schliff- 


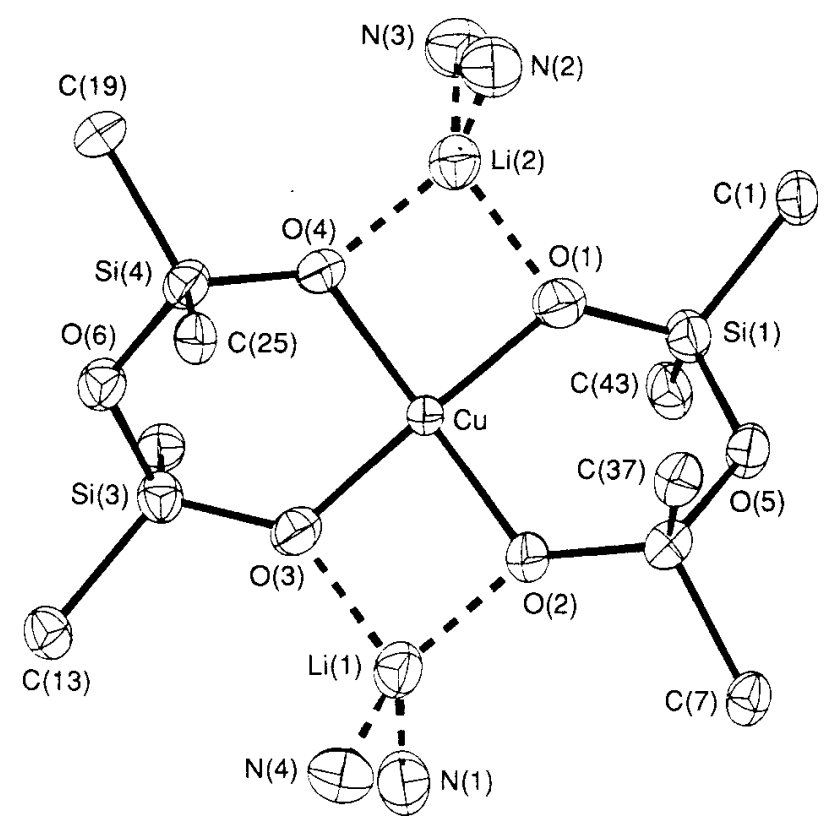

Abb. 24: Der molekulare Aufbau von $\left[\mathrm{Cu}\left(\left(\mathrm{OSiPh}_{2}\right)_{2} \mathrm{O}\right)_{2}\left(\mathrm{Li}(\mathrm{py})_{2}\right)_{2}\right][73]$

fett die Struktur der polymeren Verbindung $\left[\mathrm{Tl}_{2}\left(\left(\mathrm{OSiMe}_{2}\right)_{2} \mathrm{O}\right)_{2}\right]_{\infty}$ aufgeklärt werden, die ebenfalls den Siloxandiolat-Liganden enthält. Von S. Harvey et al. wurde ein zugrundeliegender Reaktionsmechanismus vorgeschlagen, in dem die Degradierung von Schliffett, eines Polysiloxans (Abb. 25 (a)), zum Siloxandiolat (b) auf einen nucleophilen Angriff des Alkoholates zurückzuführen ist [77].

Ein Beispiel eines Münzmetallkomplexes von Tetramethyldisiloxandiolat stellt die Verbindung $\left[\mathrm{Ag}_{4}\left(\left(\mathrm{OSiMe}_{2}\right)_{2} \mathrm{O}\right)_{2}\left(\mathrm{PPh}_{3}\right)_{4}\right]$ dar [78]. Aus Schliffett, $\mathrm{PPh}_{3}$ und dem metallorganischen Komplex $\left[\mathrm{Ag}_{4}\left(\mathrm{C}_{6} \mathrm{H}_{4} \mathrm{CH}_{2} \mathrm{NMe}_{2}\right)_{4}\right]$, der möglicherweise in der Reaktion als Base diente, konnte in Toluol die vierkernige Verbindung nach einer Reaktionszeit von mehreren Wochen kristallisiert werden.

Bei Reaktionen von polymerem Schliffett mit Nucleophilen können sich auch andere Zersetzungsprodukte bilden, wie folgende Beispiele zeigen: $\left[\mathrm{OSiMe}_{2} \mathrm{C}_{3} \mathrm{~N}_{2} \mathrm{HMe}_{2}\right]^{\ominus}$, das aus einem unsubstituierten Pyrazolanion $\left[\mathrm{C}_{3} \mathrm{~N}_{2} \mathrm{HMe}_{2}\right]^{\ominus}$ gebildet wird [79], $\left(\mathrm{OSiMe}_{2}\right)_{7}$, ein cyclisches Heptasiloxan, das kronenetherartig ein Kaliumion umgibt [80], [OSiMe $\left.{ }_{2} \mathrm{NPh}\right]^{2 \ominus}$ und $\left[\mathrm{OSiMe}_{2} \mathrm{NPhSiMe}_{2} \mathrm{O}\right]^{2 \ominus}$, die aus Natriumanilid entstehen [81], [OSiMe $\mathrm{Naph}^{\ominus}$, das sich aus Naphthyllithium bildet [82].

Auch konnte von K. Dehnicke et al. aus der Reaktion von Siliconfett mit ${ }^{n}$ Butyllithium das Fragment $\left[\mathrm{OSiMe}_{2}{ }^{\mathrm{n}} \mathrm{Bu}\right]^{\ominus}$ nachgewiesen werden [83]. 

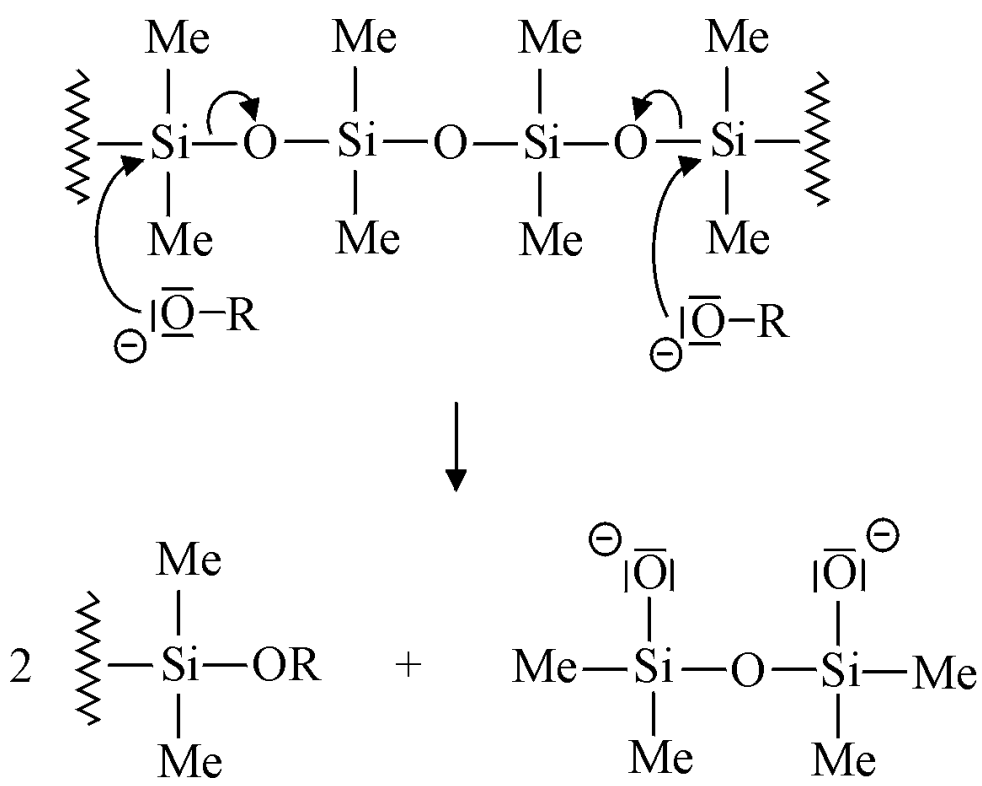

Abb. 25: Von S. Harvey et al. vorgeschlagener Mechanismus der Bildung von $\left[\left(\mathrm{OSiMe}_{2}\right)_{2} \mathrm{O}\right]^{2 \ominus}[77]$

Bei der Überprüfung, ob Verbindung $\underline{8}$ aus einer Reaktion mit Schliffett hervorgeht, konnte bei der direkten Synthese neben dem Hauptprodukt $\underline{8}$, das durch einen Vergleich der Gitterkonstanten identifiziert wurde, die Bildung des vierkernigen Komplexes $\left.\left[\mathrm{Cu}_{4}(\mathrm{O})(\mathrm{dppaO})_{2}\right)_{4} \mathrm{Cl}_{2}\right] \underline{9}$ beobachtet werden (vgl. Gl. 15).

\section{Die Struktur von $\left[\mathrm{Cu}_{4}(\mathrm{O})\left(\mathrm{dppaO}_{2}\right)_{4} \mathrm{Cl}_{2}\right] \underline{9}$ :}

Die vierkernige Verbindung 9 kristallisiert monoklin in der Raumgruppe $C 2 / c$, deren zentrale Baueinheit aus einem $\mathrm{Cu}_{4}$-Tetraeder mit eingelagertem Oxidion besteht (Abb. 26). Um den $\mathrm{Cu}_{4}$-Tetraeder wird durch zwei $\mu_{2}$-verbrückende Chloroliganden und vier ebenfalls $\mu_{2}$-bindende Sauerstoffatome von vier Liganden ein Oktaeder aufgebaut. Werden nur die $\mathrm{Cu}$-Atome, die verbrückenden Sauerstoffatome und die Chloroliganden betrachtet, so ergibt sich eine adamantanähnliche Grundstruktur. 


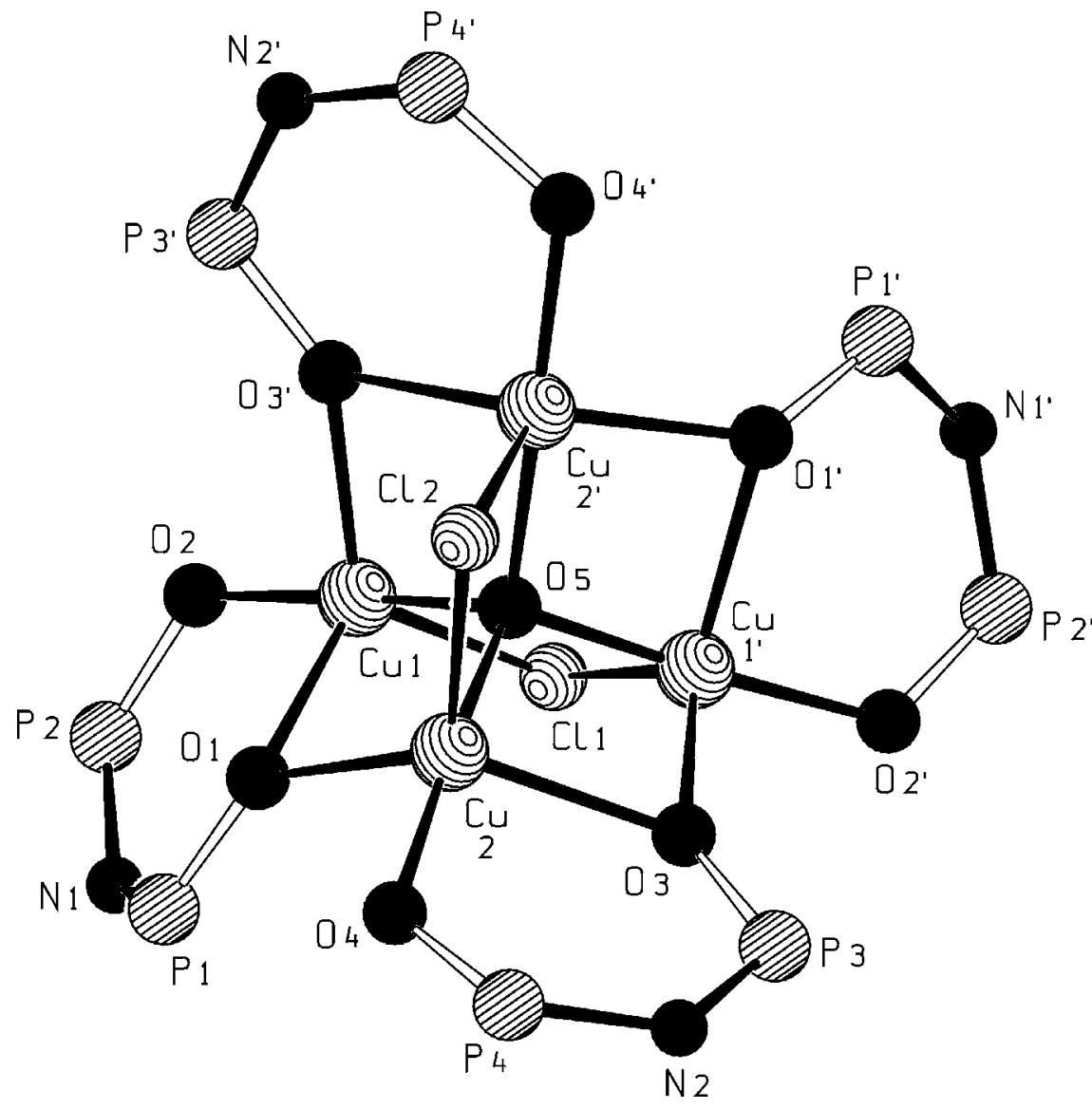

Abb. 26: Die Struktur von $\left[\mathrm{Cu}_{4}(\mathrm{O})\left(\mathrm{dppaO}_{2}\right)_{4} \mathrm{Cl}_{2}\right] \underline{9}$ im Kristall (zur besseren Veranschaulichung sind die Phenylgruppen nicht mit eingezeichnet) 
Die Liganden chelatisieren je ein Kupferatom, so daß jeweils ein Sauerstoffatom terminal an ein $\mathrm{Cu}$-Ion bindet und das andere O-Atom zusätzlich $\mu_{2}$-artig zu einem zweiten $\mathrm{Cu}$ Atom verbrückt (vgl. Abb. 4 (h)).

Die Cu-Ionen besitzen eine trigonal-bipyramidale Koordinationssphäre, wobei je ein terminal bindendes O-Atom und das Oxidion $\mathrm{O}(5)$ die axialen Positionen besetzen. Die äquatorialen Positionen werden von einem verbrückenden Chloroliganden und zwei verbrückenden Sauerstoffatomen mit einem längeren $\mathrm{Cu}-\mathrm{O}-\mathrm{Abstand}$ von $221 \mathrm{pm}$ und einem kürzeren $\mathrm{Cu}-\mathrm{O}-\mathrm{Abstand}$ von 213 pm eingenommen. Diese Cu-O-Abstände sind im Vergleich zu anderen Abständen in Kupferkomplexen des gleichen Liganden, die normalerweise um 190 bis 195 pm liegen, recht groß. Es sind jedoch auch Verbindungen mit der $\mathrm{Cu}_{4} \mathrm{O}$-Einheit beschrieben, die gleichfalls $\mu_{2}$-koordinierte Sauerstoffatome aufweisen und in denen ebenfalls ein längerer und ein kürzerer Cu-O-Abstand von ca. 235 und 195 pm beobachtet wird [84]. Die Abstände der Cu-Atome zum eingeschlossenen O(5)-Oxidion betragen 188 pm und sind, wie die Cu-Cl-Abstände mit 237 pm, etwas kürzer als die Abstände, die für das Anion $\left[\mathrm{Cu}_{4}(\mathrm{O}) \mathrm{Cl}_{10}\right]^{4 \ominus}$ gefunden werden $\left(\mathrm{Cu}-\mu_{4}-\mathrm{O}\right.$ : $\left.192 \mathrm{pm}, \mathrm{Cu}-\mu_{2}-\mathrm{Cl}: 241 \mathrm{pm}\right)$ [85]. Dies kann jedoch auf die vierfach negative Ladung des Cuprats zurückgeführt werden.

Kupferverbindungen mit dem tetraedrischen $\mathrm{Cu}_{4} \mathrm{O}$-Bauelement sind schon seit längerer Zeit und auch in großer Zahl bekannt. Das $\mathrm{Cu}_{4} \mathrm{O}$-Gerüst repräsentiert ein Strukturelement, welches auch in vielen Komplexen mit der Einheit $\left[\mathrm{Cu}_{4}(\mathrm{O}) \mathrm{Cl}_{6} \mathrm{~L}_{4}\right]$ vorliegt, in denen alle Chloroliganden über die sechs Kanten des $\mathrm{Cu}_{4}$-Tetraeders verbrücken, während die Liganden L, die leicht ausgetauscht werden können, terminal binden. So konnten mehrere Komplexe dieses Aufbaus mit unterschiedlichen Liganden L (L = $\mathrm{OPPh}_{3}$ [86], Cl [85,87], py [88]) isoliert werden.

Über die Herkunft des Oxidions in $\underline{9}$ kann keine sichere Aussage getroffen werden. Beispielsweise wurden für die Bildung von Komplexverbindungen der Lanthanoide mit interstitiellem Oxidion Wasserspuren als Oxidquelle verantwortlich gemacht [89].

R. Belfort et al. [85] schlugen einen Mechanismus der Bildung des $\mathrm{Cu}_{4} \mathrm{O}$-Strukturelementes vor, in dem zwei $\left[\mathrm{Cu}(\mathrm{OH}) \mathrm{L}_{\mathrm{n}}\right]_{2}$-Einheiten durch eine Kondensationsreaktion zum Clusterkomplex $\left[\mathrm{Cu}_{4}(\mathrm{O}) \mathrm{L}_{\mathrm{m}}\right]$ reagieren (Abb. 27).

Möglicherweise stammt das Oxidanion aus dem eingesetzten Schliffett, denn es konnte ebenfalls ein vierkerniger Lanthanoidkomplex mit eingelagertem Oxidion und zusätzlich koordiniertem Schliffettfragment isoliert werden [81]. 


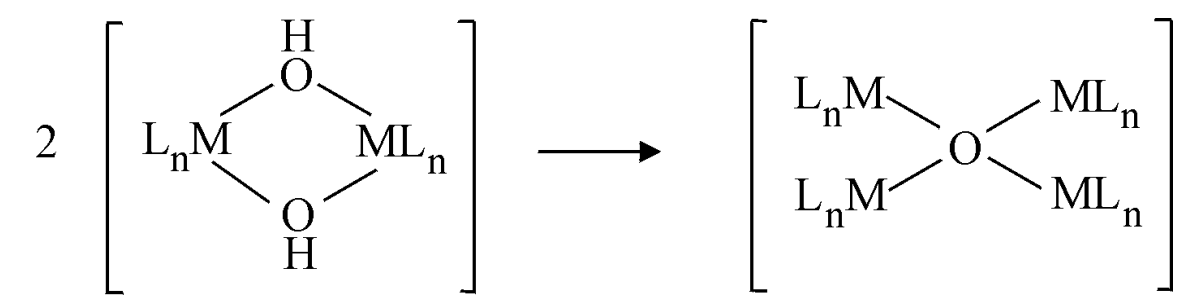

Abb. 27: Von R. Belfort et al. vorgeschlagener Mechanismus der Oxidioneneinlagerung $[85]$ 


\subsection{Umsetzungen von $\mathrm{CuCl}$ und $\mathrm{CuCl}_{2}$ mit den Aminen dppaOEH $(\mathrm{E}=\mathrm{S}, \mathrm{Se})$}

Obgleich die Synthesen der Liganden $\mathrm{R}_{2} \mathrm{P}(\mathrm{E}) \mathrm{NHP}(\mathrm{E}) \mathrm{R}_{2}$ mit unterschiedlichen Chalkogenen bzw. organischen Resten schon lange beschrieben sind [23,90] und später O. Siiman et al. von den spektroskopischen Eigenschaften von $\mathrm{Ni}^{\mathrm{II}}$ - und $\mathrm{Cu}^{\mathrm{II}}-\mathrm{Komplexen}$ mit dppaOS $^{\ominus}$ berichteten $[91,92]$, stieg erst in letzter Zeit das Interesse an Verbindungen der gemischten Liganden dppaOSH und dppaOSeH [20,93-97].

Die strukturellen Untersuchungen von Komplexverbindungen dieser Liganden beschränken sich jedoch überwiegend auf Hauptgruppenelemente wie $\mathrm{Na}$ [98], $\mathrm{Sn}^{\mathrm{IV}}$ [93], $\mathrm{Pb}^{\mathrm{II}}$ [94], $\mathrm{Sb}^{\mathrm{V}}$ [95] und $\mathrm{Te}^{\mathrm{IV}}$ [99] neben einigen wenigen Übergangsmetallen wie $\mathrm{Mo}^{\mathrm{V}}$ [100], $\mathrm{Pd}^{\mathrm{II}}, \mathrm{Pt}^{\mathrm{II}}[20]$ und $\mathrm{Au}^{\mathrm{I}}[97]$.

In Umsetzungen der Liganden mit $\mathrm{CuCl}_{2}$ und $\mathrm{CuCl}$ gelang es, sowohl Komplexe mit $\mathrm{Cu}^{\mathrm{II}}$ als auch mit $\mathrm{Cu}^{\mathrm{I}}$ zu synthetisieren, in denen der Ligand entweder in seiner ursprünglichen Form vorliegt oder ein Chalkogenatom substituiert wurde. Auch konnte ein Kopplungsprodukt zweier Liganden isoliert werden.

\subsubsection{Die Synthese von $\left[\mathrm{Cu}_{3}(\operatorname{dppaOS})_{3}\right] \underline{10}$}

In wasserfreiem Lösungsmittel (THF) führen Umsetzungen von äquivalenten Mengen $\mathrm{CuCl}_{2}$ und $\mathrm{CuCl}$ mit zwei Äquivalenten $[\mathrm{Na}(\mathrm{dppaOS})]$ zur Bildung der in grünen Kristallen erhaltenen Verbindung $\left[\mathrm{Cu}(\text { dppaOS })_{2}\right]$ und des dreikernigen $\mathrm{Cu}^{\mathrm{I}}-\mathrm{Komplexes}$ $\left[\mathrm{Cu}_{3}(\mathrm{dppaOS})_{3}\right] \underline{10}$, dessen Festkörperstruktur durch eine Röntgenstrukturanalyse aufgeklärt werden konnte (Gl. 16).

$$
\begin{aligned}
\mathrm{CuCl}_{2}+\mathrm{CuCl}+2[\mathrm{Na}(\text { dppaOS })] \stackrel{\mathrm{THF}}{\longrightarrow} & {\left[\mathrm{Cu}(\text { dppaOS })_{2}\right] } \\
& +\left[\mathrm{Cu}_{3}(\operatorname{dppaOS})_{3}\right] \underline{10}
\end{aligned}
$$

Von O. Siiman und J. Vetuskey wurden die Synthese und die spektroskopischen Eigenschaften einer Verbindung der Zusammensetzung $\left[\mathrm{Cu}(\mathrm{dppaOS})_{2}\right]$, die jedoch als braunes Reaktionsprodukt aus der Reaktion von $[\mathrm{Na}(\mathrm{dppaOS})]$ und $\mathrm{CuCl}_{2}$ in Wasser entstand, schon beschrieben [91].

Auf den molekularen Aufbau der Verbindung 10 wird gemeinsam mit den isostrukturell kristallisierenden Verbindungen $\left[\mathrm{Cu}_{3}\left(\mathrm{dppaE}_{2}\right)_{3}\right](\mathrm{E}=\mathrm{S}: \underline{15}$, Se: $\underline{16})$ in Kapitel 3.4.2 näher eingegangen. 
Einige weitere, unerwartete Resultate ergaben Umsetzungen von $\mathrm{CuCl}_{2}$ und $\mathrm{CuCl}$ mit dem unsymmetrischen selensubstituierten Amid dppaOSe ${ }^{\ominus}$.

\subsubsection{Die Synthese und Struktur von $\left[\mathrm{Cu}\left(\operatorname{dppaO}_{2}\right)_{2}\left(\mathrm{NH}_{3}\right)_{3}\right]_{\infty} \underline{11}$}

Wird $[\mathrm{Na}($ dppaOSe $)]$ mit $\mathrm{CuCl}$ in THF umgesetzt, so kann nach einigen Wochen Reaktionszeit die Verbindung $\left[\mathrm{Cu}\left(\mathrm{dppaO}_{2}\right)_{2}\left(\mathrm{NH}_{3}\right)_{3}\right]_{\infty} \underline{11}$ aus der Reaktionslösung kristallisiert werden (Gl. 17).

$$
\mathrm{CuCl}+[\mathrm{Na}(\text { dppaOSe })] \stackrel{\mathrm{THF}}{\longrightarrow}\left[\mathrm{Cu}\left(\mathrm{dppaO}_{2}\right)_{2}\left(\mathrm{NH}_{3}\right)_{3}\right]_{\infty} \underline{11}+\mathrm{Se}
$$

Die Struktur von $\left[\mathrm{Cu}\left(\mathrm{dppaO}_{2}\right)_{2}\left(\mathrm{NH}_{3}\right)_{3}\right]_{\infty} \underline{11}$ :

Verbindung 11, die triklin in der Raumgruppe $P \overline{1}$ kristallisiert, ist aufgebaut aus $\left[\mathrm{Cu}\left(\mathrm{dppaO}_{2}\right)_{2}\left(\mathrm{NH}_{3}\right)_{3}\right]$-Einheiten, welche durch Wasserstoffbrückenbindungen der $\mathrm{H}$ Atome der Ammoniakmoleküle zu den Sauerstoffatomen der Liganden zu einer polymeren Kette verknüpft werden (Abb. 28).

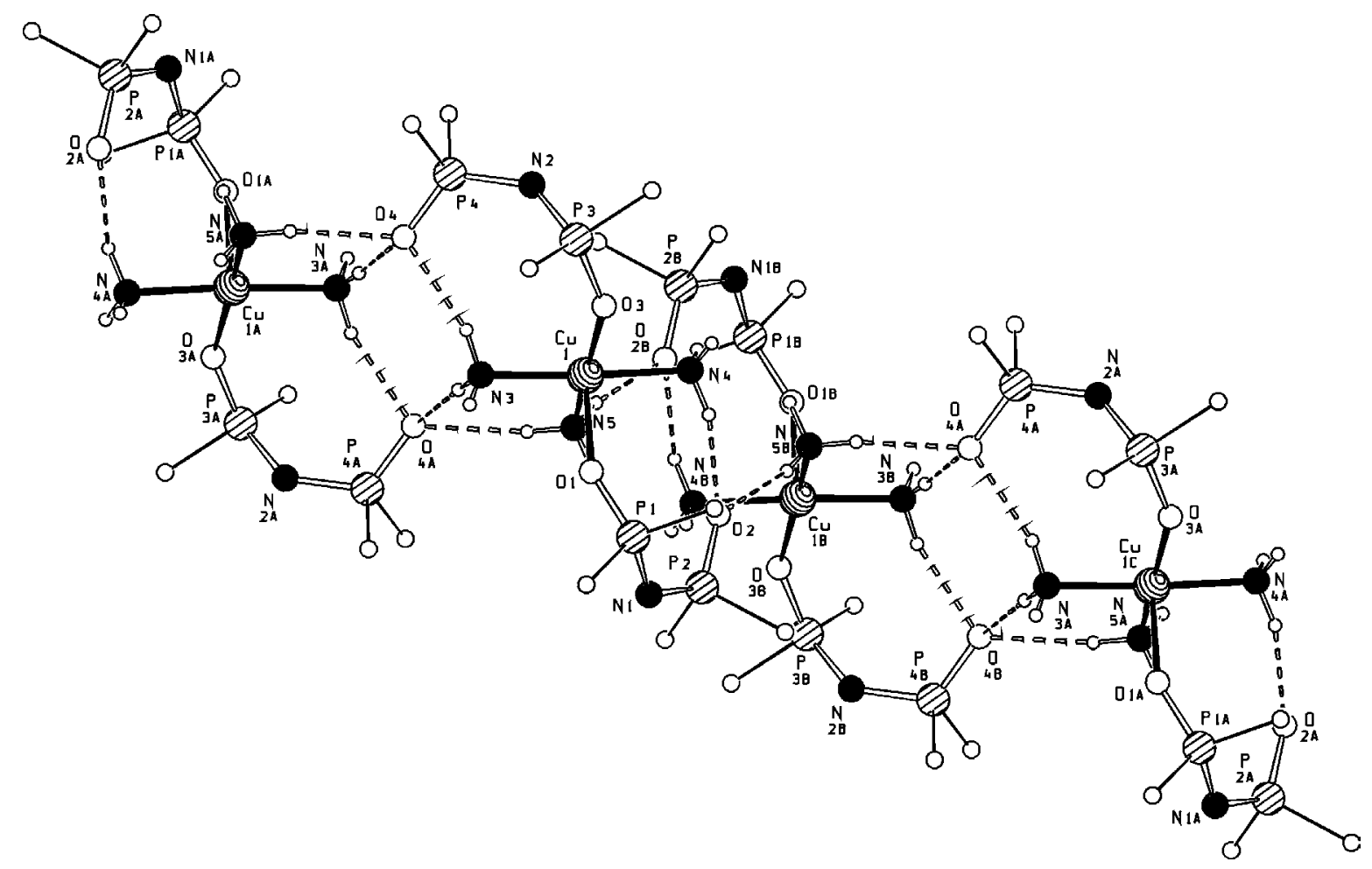

Abb. 28: Ausschnitt aus der Struktur von $\left[\mathrm{Cu}\left(\mathrm{dppaO}_{2}\right)_{2}\left(\mathrm{NH}_{3}\right)_{3}\right]_{\infty} \underline{11}$ im Kristall 
11 stellt das einzige Beispiel dar, in dem der Ligand $\mathrm{dppaO}_{2}{ }^{\ominus}$ das Metallion nicht chelatsiert, sondern nur mit einem der Sauerstoffatome terminal an ein Cu-Ion gebunden ist (vgl. Abb. 4 (d)). Im Komplex binden pro Formeleinheit zwei derartig koordinierte Liganden mit drei weiteren Ammoniakmolekülen an jeweils ein $\mathrm{Cu}$-Ion, so daß sich eine verzerrt quadratisch-pyramidale Koordinationssphäre für jedes Cu-Atom ergibt. N(3), N(4), N(5) und $\mathrm{O}(3)$ bilden die Grundfläche des $\mathrm{N}_{3} \mathrm{O}_{2}$-Koordinationspolyeders, während $\mathrm{O}(1)$ in der Pyramidenspitze positioniert ist.

Die Cu-N-Abstände liegen in einem Bereich von 200 bis 201 pm, der auch in den vergleichbaren Cu-Amin-Komplexen $\left[\mathrm{Cu}\left(\mathrm{NH}_{3}\right)_{4}\left(\mathrm{O}_{2} \mathrm{NC}(\mathrm{Cl}) \mathrm{C}(\mathrm{O}) \mathrm{NH}_{2}\right)_{2}\right]$ (199 bis 202 pm) [101] und $\left[\mathrm{Cu}\left(\mathrm{NH}_{3}\right)_{4}\left(\mathrm{H}_{2} \mathrm{O}\right)\right][18-\mathrm{K}-6]\left[\mathrm{PF}_{6}\right]$ (203 bis $\left.204 \mathrm{pm}\right)$ [102] gefunden wird.

Ob das Sauerstoffatom an Kupfer bindet oder stattdessen Wasserstoffbrückenbindungen zu zwei bzw. drei Amin-H-Atomen eingeht, hat keine feststellbaren Auswirkungen auf die P-O-Bindungslängen. Sie betragen einheitlich 151 pm.

Werden die Atomabstände mit den bisher besprochenen Verbindungen $\underline{6}$ oder $\underline{8}$ verglichen, so stellt man fest, daß die $\mathrm{Cu}-\mathrm{O}$-Bindungen in $\underline{11}$ deutlich länger sind, was zur Folge hat, daß die P-O-Bindungen entsprechend kürzer sind. Wiederum kann im koordinierten Liganden eine $\pi$-Elektronen-Delokalisierung angenommen werden, da die P-N- und P-OAbstände zwischen Einfach- und Doppelbindungslängen liegen.

Die Länge der Wasserstoffbrückenbindungen zu den Ligand-O-Atomen betragen 199 bis 217 pm. Im Festkörper liegt der Ligand dppaOSH ebenfalls als polymere Kette vor, die durch relativ schwache Amin-H-Brückenbindungen zu den Sauerstoffatomen gebildet wird. Diese Abstände liegen in einem Bereich von 203 bis 215 pm [98], wie sie annähernd in $\underline{11}$ bestimmt wurden. Folglich handelt es sich in $\underline{11}$ ebenfalls nur um geringe H. . O-Wechselwirkungen, die jedoch zu der polymeren Struktur führen.

Wie es zu der Bildung von Ammoniak kam, der nicht nur in Spuren in der Reaktionslösung vorhanden sein muß, ist ungeklärt. Denkbar wäre, daß der Ligand selbst mit Ammoniak verunreinigt war. Es ist möglich, daß während der Ligandsynthese bei der Umsetzung von Diphenylchlorphosphan mit Hexamethyltrisilazan Wasser in den Reaktionskolben gelangte, welches mit dem Disilazan zu Ammoniak und Trimethylsilanol reagierte. Da nur ein ${ }^{31}$ P-NMR-Spektrum des synthetisierten Liganden angefertigt wurde, kann die Anwesenheit von Ammoniak nicht ausgeschlossen werden. Auch ist nicht bekannt, auf welche Weise der Ligand $\mathrm{dppaO}_{2}{ }^{\ominus}$ gebildet werden konnte. Es wurde lediglich nach einigen Wochen die Bildung von Selen beobachtet.

Einen möglichen Anhaltspunkt einer mechanistischen Erklärung des Reaktionsablaufes 
bietet die Reaktion von $\mathrm{CuCl}_{2}$ und $\mathrm{dppmSe}_{2} \mathrm{H}$ in Ethanol und Toluol unter Stickstoffatmosphäre, bei der ein Rohprodukt, von G. Predieri et al. [51] als $\mathrm{Cu}^{\mathrm{I}}-\mathrm{Komplex}$ des dppmSeH-Liganden postuliert, und elementares Selen entstehen.

Bei der Reaktion zu 11 könnte also $\mathrm{Cu}^{2 \oplus}$, das wohl im Edukt vorhanden gewesen sein muß, $\mathrm{zu} \mathrm{Cu}{ }^{\oplus}$ reduziert worden sein, worauf die anfängliche Grünfärbung der Reaktionslösung mit anschließender Gelbfärbung hinweist. Selenid wurde zu Selen oxidiert, wobei sich dppaOH bildete. Die anschließende Oxidation des Phosphino-Phosphoryl-Liganden durch $\mathrm{Cu}^{\mathrm{II}}$ führt dann zur reinen Phosphoryl-Verbindung dppaO ${ }_{2} \mathrm{H}$.

\subsubsection{Die Synthese und Struktur von $\left[\mathrm{H}\left((\mathrm{O}) \mathrm{PPh}_{2} \mathrm{NPPh}_{2}\right)_{2} \mathrm{O}\right]\left[\mathrm{CuCl}_{2}\right] \underline{\mathbf{1 2}}$}

Ein weiteres, überraschendes Ergebnis zeigte die Umsetzung von $\mathrm{CuCl}_{2}$ und $\mathrm{CuCl}$ mit $[\mathrm{K}(\mathrm{dppaOSe})]$ in THF. Es wurde versucht, den bei der Reaktion gebildeten roten Niederschlag in gängigen Lösungsmitteln zu lösen, was jedoch nicht gelang. In $\mathrm{CH}_{2} \mathrm{Cl}_{2}$ konnte nur eine farblose Produktkomponente gelöst werden, die nach einer Röntgenstrukturanalyse als Verbindung $\left[\mathrm{H}\left((\mathrm{O}) \mathrm{PPh}_{2} \mathrm{NPPh}_{2}\right)_{2} \mathrm{O}\right]\left[\mathrm{CuCl}_{2}\right] \underline{12}$ identifiziert wurde (Gl. 18).

$$
\begin{aligned}
\mathrm{CuCl}_{2}+\mathrm{CuCl}+2[\mathrm{~K}(\text { dppaOSe })] \frac{1) \mathrm{THF}}{2) \mathrm{CH}_{2} \mathrm{Cl}_{2}} \\
{\left[\mathrm{H}\left((\mathrm{O}) \mathrm{PPh}_{2} \mathrm{NPPh}_{2}\right)_{2} \mathrm{O}\right]\left[\mathrm{CuCl}_{2}\right] \underline{12} }
\end{aligned}
$$

\section{Die Struktur von $\left[\mathrm{H}\left((\mathrm{O}) \mathrm{PPh}_{2} \mathrm{NPPh}_{2}\right)_{2} \mathrm{O}\right]\left[\mathrm{CuCl}_{2}\right] \underline{12}$ :}

In Abb. 29 ist der molekulare Aufbau der Verbindung 12 dargestellt, die monoklin in der Raumgruppe $P 2_{1} / n$ kristallisiert. Die monokationische Einheit $\left[\mathrm{H}\left((\mathrm{O}) \mathrm{PPh}_{2} \mathrm{NPPh}_{2}\right)_{2} \mathrm{O}\right]^{\oplus}$ kann als Kopplungsprodukt zweier Liganden betrachtet werden und besteht aus einem zehngliedrigen Cyclus, der durch zwei unsymmetrische Wasserstoffbrückenbindungen von $\mathrm{O}(1)$ und $\mathrm{O}(3)$ zu H(49) gebildet wird. Der Mittelwert der O . H-Abstände in 12 von $122 \mathrm{pm}$ ist vergleichbar mit dem Abstand der symmetrischen H-Brückenbindungen von $120 \mathrm{pm}$, die im Liganden dppa $\mathrm{O}_{2} \mathrm{H}$ vorliegen [70].

Der $\mathrm{P}(1)-\mathrm{O}(1)$-Abstand ist mit 153 pm geringfügig größer als der $\mathrm{P}(4)-\mathrm{O}(3)$-Abstand (152 pm), was damit erklärt werden kann, daß $\mathrm{O}(1)$ die stärkere H-Brückenbindung eingeht und somit entsprechend auch die P-O-Bindung etwas schwächer sein muß. Beide Bindungslängen liegen jedoch in einem Abstandbereich, der annähernd einer P-ODoppelbindung entspricht ( $\mathrm{P}=\mathrm{O}: 149$ pm [50], P-O: $162 \mathrm{pm}$ in Triphenylphosphat [103]). Demnach muß es sich bei den P-O-Bindungen zu O(2) mit einer Länge von 162 pm um P-O-Einfachbindungen handeln. 


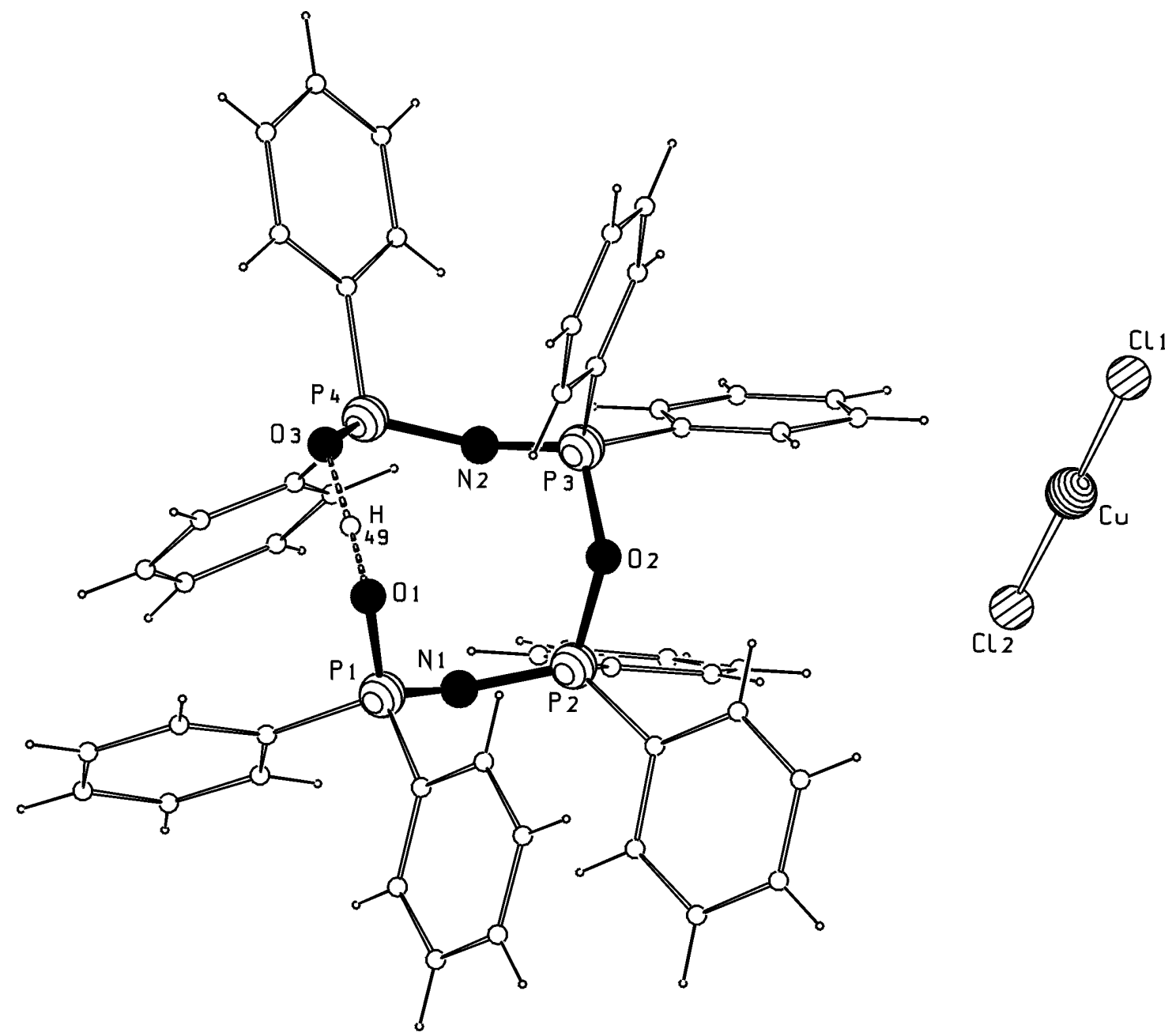

Abb. 29: Die Struktur von $\left[\mathrm{H}\left((\mathrm{O}) \mathrm{PPh}_{2} \mathrm{NPPh}_{2}\right)_{2} \mathrm{O}\right]\left[\mathrm{CuCl}_{2}\right] \underline{12}$ im Kristall 
Dagegen kann man den P-N-Bindungen in 12 mit Längen von 155 und 160 pm nicht eindeutig eine Bindungsordnung zuweisen, da diese, verglichen mit den auftretenden P-NBindungslängen der Verbindung $(\mathrm{S}) \mathrm{PPh}_{2} \mathrm{NP}(\mathrm{SMe}) \mathrm{Ph}_{2}$ [98] von 157 und 161 pm, zwischen einer Einfach- und einer Dopppelbindung liegen.

Das analoge und ebenfalls sauerstoffverbrückte Kopplungsprodukt zweier Liganden [((Se) $\left.\left.\mathrm{PPh}_{2} \mathrm{NPPh}_{2}\right)_{2} \mathrm{O}\right]$ konnte aus der Umsetzung von $\left.\left[\mathrm{K}(\mathrm{dppaSe})_{2}\right)\right]$ mit Wasser isoliert werden [104], was für die Bildung von 12 bedeutet, daß wahrscheinlich das eingesetzte Lösungsmittel nicht wasserfrei war. Jedoch wurden bisher keine Vorschläge für einen Mechanismus der Ligandkopplung gemacht.

Derartige Kopplungsreaktionen könnten zu einer neuen Klasse von makrocyclischen Ligandensystemen führen, die in Komplexsynthesen interessante Strukturen ausbilden. 


\subsection{Umsetzungen von $\mathrm{CuCl}$ und $\mathrm{AgCl}$ mit den Aminen $\operatorname{dppaE}_{2} \mathrm{H}(\mathrm{E}=\mathrm{S}, \mathrm{Se})$}

Unter den Komplexen des Ligandensystems dppaE ${ }_{2} \mathrm{H}(\mathrm{E}=\mathrm{S}, \mathrm{Se})$ sind die Verbindungen des Dithiophosphoryls am besten untersucht. Von einkernigen bis hin zu polymeren Verbindungen konnten die Strukturen einiger Komplexe aufgeklärt werden.

Besonders Verbindungen der Nebengruppenelemente spielen hierbei eine große Rolle [28,105-107]. Es wurde fast ausschließlich von Komplexverbindungen berichtet, in denen der Ligand als Amid und dabei in verschiedenen Koordinationsmodi vorliegt (vgl. Abb. 4).

Das Reaktionsverhalten von $\mathrm{CuCl}$ und $\mathrm{AgCl}$ gegenüber den Alkaliamiden der oben genannten Ligandensysteme wurde nun systematisch untersucht, denn über $\mathrm{Cu}^{\mathrm{I}}$ - oder $\mathrm{Ag}^{\mathrm{I}}$ Verbindungen im Zusammenhang mit Chalkogenophosphorylamiden war bis zu Beginn der vorliegenden Arbeit nur wenig bekannt [32,108].

In Reaktionen der Amine dppaE ${ }_{2} \mathrm{H}(\mathrm{E}=\mathrm{O}, \mathrm{S}, \mathrm{Se})$ können, abhängig vom Mengenverhältnis der Edukte, des Deprotonierungsmittels oder der Temperatur, gezielt ein- bis vierkernige $\mathrm{Cu}^{\mathrm{I}}$ - und $\mathrm{Ag}^{\mathrm{I}}$-Verbindungen isoliert und charakterisiert werden. Die Deprotonierung der Liganden erfolgt entweder durch eine $\mathrm{NaN}\left(\mathrm{SiMe}_{3}\right)_{2}$-Lösung in $\mathrm{THF}, \mathrm{KO}^{\mathrm{t}} \mathrm{Bu}$ oder eine Lösung von ${ }^{\mathrm{n}}$ Butyllithium in Hexan. Nach der Deprotonierung des Amins in THF wird dieses zu einer Suspension von $\mathrm{CuCl}$ bzw. $\mathrm{AgCl}$ in THF gegeben. Nach einem Tag wird der gebildete Niederschlag von der Lösung abfiltriert und aus dem Filtrat können durch Überschichten mit Heptan Kristalle der im folgenden besprochenen Verbindungen erhalten werden.

\subsubsection{Die Synthesen und Strukturen von $\left[\mathrm{NaCu}_{2}\left(\operatorname{dppaS}_{2}\right)_{3}(\text { thf })_{2}\right] \underline{13}$ und $\left[\mathrm{NaCu}_{2}\left(\operatorname{dppaSe}_{2}\right)_{3}(\text { thf })_{2}\right] \underline{14}$}

Durch die Reaktion von einem Äquivalent $\mathrm{CuCl}$ mit 1,5 Äquivalenten $\left[\mathrm{Na}\left(\mathrm{dppaE}_{2}\right)\right]$ in THF können die Verbindungen $\left[\mathrm{NaCu}_{2}\left(\mathrm{dppaS}_{2}\right)_{3}(\text { thf })_{2}\right] \underline{13}$ und $\left[\mathrm{NaCu}_{2}\left(\operatorname{dppaSe}_{2}\right)_{3}(\text { thf })_{2}\right]$ 14 erhalten werden (Gl. 19).

$$
\begin{aligned}
2 \mathrm{CuCl}+3\left[\mathrm{Na}\left(\mathrm{dppaE}_{2}\right)\right] \stackrel{\mathrm{THF}}{\longrightarrow} & {\left[\mathrm{NaCu}_{2}(\operatorname{thf})_{2}\left(\mathrm{dppaE}_{2}\right)_{3}\right]+2 \mathrm{NaCl} } \\
& (\mathrm{E}=\mathrm{S}: \underline{13}, \mathrm{Se}: \underline{14})
\end{aligned}
$$


Da beide Verbindungen nahezu isostrukturell sind, werden diese hier gemeinsam besprochen.

\section{Die Strukturen von $\left[\mathrm{NaCu}_{2}\left(\operatorname{dppaS}_{2}\right)_{3}(\mathrm{thf})_{2}\right] \underline{13}$ und}

$\left[\mathrm{NaCu}_{2}\left(\operatorname{dppaSe}_{2}\right)_{3}(\text { thf })_{2}\right] \underline{14}$ :

Die Einkristallröntgenstrukturanalyse zeigt, daß $\underline{13}$ in zwei verschiedenen Kristallsystemen, in den Raumgruppen $P 1$ bzw. $P 2_{1} / c$, kristallisiert. Die Verbindung 14 , die in Abb. 30 dargestellt ist, kristallisiert ebenfalls in der Raumgruppe $P 2_{1} / c$. Es handelt sich in beiden Fällen um dreikernige Verbindungen, in denen zwei $\left[\left(\mathrm{Cu}\left(\mathrm{dppaE}_{2}\right)\right]\right.$-Fragmente durch eine $\left[\mathrm{Na}\left(\mathrm{dppaE}_{2}\right)(\text { thf })_{2}\right]$-Einheit verknüpft werden.

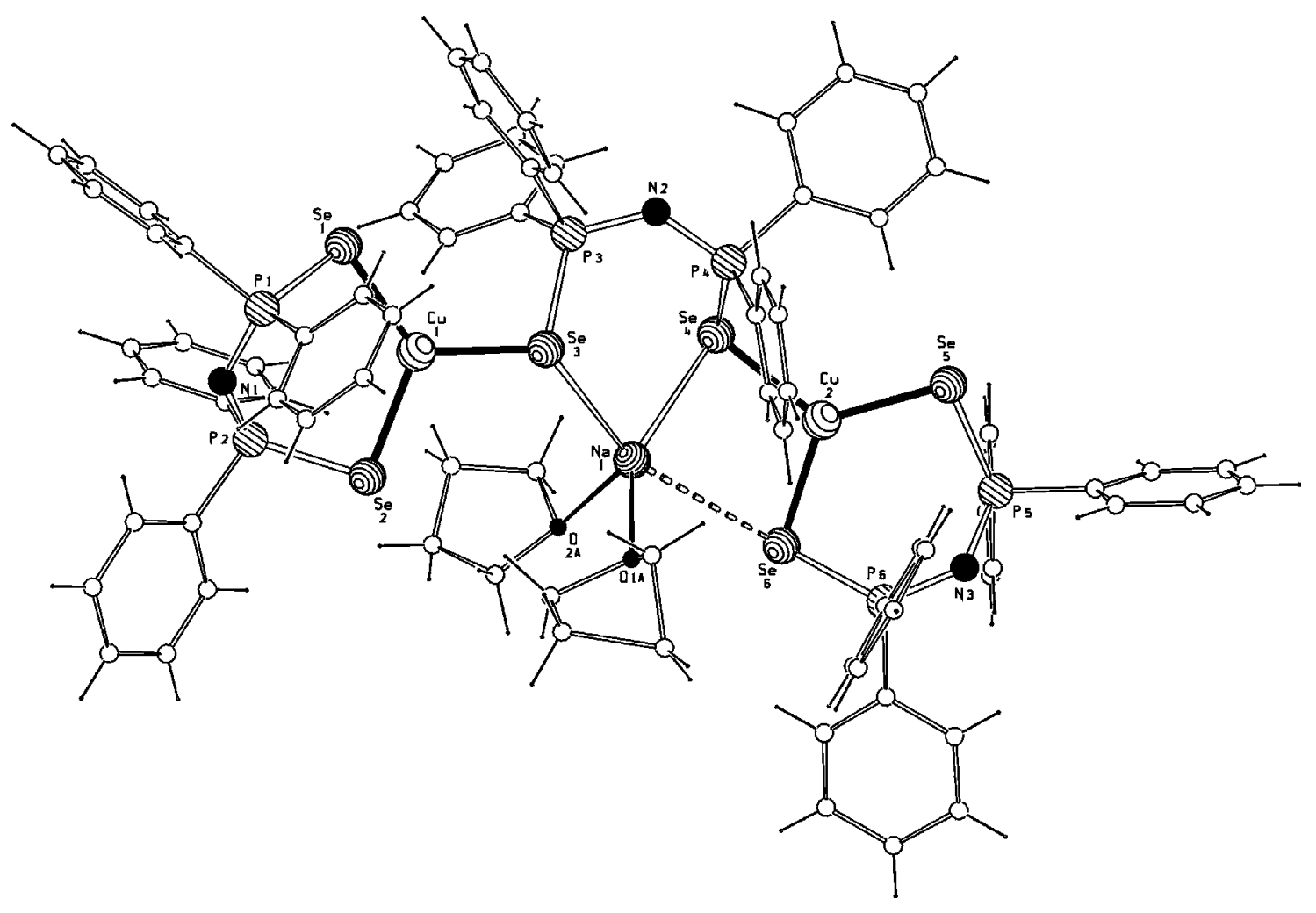

Abb. 30: Die Struktur von $\left[\mathrm{NaCu}_{2}\left(\mathrm{dppaSe}_{2}\right)_{3}(\text { thf })_{2}\right] \underline{14}$ im Kristall

Beide $\mathrm{Cu}^{\mathrm{I}}$-Atome werden von je einem $\mathrm{dppaE}_{2}{ }^{\ominus}$-Liganden chelatisiert, wodurch ein sechsgliedriger Cyclus gemäß Abb. 4 (c) entsteht. Die $\mathrm{Cu}^{\mathrm{I}}$-ionen sind annähernd trigonal-planar von je drei Chalkogenatomen umgeben, während der verzerrt tetraedrische Koordinationspolyeder des Natrium-Ions durch zwei Chalkogenatome eines dritten dppaE ${ }_{2}{ }^{\ominus}$-Liganden 
und die Sauerstoffatome zweier THF-Moleküle gebildet wird. Die Chalkogendonoratome dieses Liganden bilden zwei zusätzliche Bindungen zu den $\left[\mathrm{Cu}\left(\mathrm{dppaE}_{2}\right)\right]$-Fragmenten aus, so daß eine Verknüpfung von drei Metallzentren gemäß Abb. 4 (i) formuliert werden kann. Somit liegen in $\underline{13}$ und $\underline{14}$ zwei verschiedene Koordinationsarten der gleichen Ligandensorte vor.

Im Unterschied zu 13 existiert in 14 noch eine weitere schwache Wechselwirkung zwischen den Atomen $\mathrm{Na}(1)$ und $\mathrm{Se}(6)$, die sich in einem Abstand von 344 pm voneinander entfernt befinden (Abb. 30). Verglichen mit ähnlichen Na-Se-Wechselwirkungen, wie sie z.B. in der Verbindung $\left[\mathrm{Na}_{2}\left(\mathrm{Se}_{2} \mathrm{PPh}_{2}\right)(\text { thf })\left(\mathrm{H}_{2} \mathrm{O}\right)_{5}\right]_{\infty}\left[\mathrm{Se}_{2} \mathrm{PPh}_{2}\right]_{\infty}[109]$ mit einem Na-Se-Abstand von 298 pm auftreten, handelt es sich in 14 nur um einen schwachen Na-Se-Kontakt. Deshalb kann die Koordination des Na-Ions als eine [4+1]-Koordination formuliert werden.

Der zweizähnig koordinierte Dithio- bzw. Diseleno-Ligand bildet jeweils einen $\mathrm{CuE}_{2} \mathrm{P}_{2} \mathrm{~N}$ Ring aus, in dem die Cu-S-Abstände ca. 224 pm, die Cu-Se- 235 pm, die P-S- 200 pm, die P-Se- 217 pm und die P-N-Abstände 159 pm betragen. Die Bindungslängen von Verbindung 13 stimmen sehr gut überein mit den Atomabständen der von I. Haiduc et al. [108] dargestellten einkernigen Verbindung $\left[\mathrm{Cu}\left(\mathrm{dppaS}_{2}\right)\left(\mathrm{PPh}_{3}\right)\right]$, in der der dppaS ${ }_{2}{ }^{\ominus}$-Ligand ebenfalls zweizähnig an ein $\mathrm{Cu}$-Atom, an das ein weiterer $\mathrm{PPh}_{3}$-Ligand bindet, koordiniert ist. Die Bindungslängen des Cu-S-, P-S- und P-N-Bindungen betragen in dieser Verbindung 224 pm, 201 pm und 159 pm.

Auch entsprechen die Atomabstände im dppaSe ${ }_{2}$-Liganden in $\underline{14}$ denen in vergleichbaren Verbindungen, wie z.B. im homoleptischen Komplex [Cd(dppaSe $)_{2}$ ] [31]. Da sich diese P-S- bzw. P-Se- und P-N-Bindungslängen nur geringfügig von denen in Verbindung $\underline{13}$ und 14 unterscheiden, liegt die Annahme einer $\pi$-Elektronen-Delokalisierung über das gesamte EPNPE-Fragment auch in diesen Fällen nahe.

Die Bindungslängen im zweifach $\mu_{2}$-verbrückenden dritten Liganden unterscheiden sich nicht wesentlich von denen im zweizähnigen, die $\mathrm{Cu}$-Atome chelatisierenden Liganden. Durch die $\mu_{2}$-Koordination der Schwefelatome sind die entsprechenden P-S-Bindungen in 13 mit 202 pm um 2 pm länger im Vergleich zu den P-S-Bindungslängen mit terminal koordinierten S-Atomen. Die zusätzliche Koordination von $\mathrm{Se}(6)$ an $\mathrm{Na}(1)$ in 14 bewirkt nur eine schwache P-Se-Bindungsverlängerung, denn der P(6)-Se(6)-Abstand ist nur um 2 pm länger als der Durchschnitt der anderen P-Se-Abstände. Gleichermaßen besteht kein signifikanter Unterschied zwischen den Cu-S- bzw. Cu-Se-Abständen, wenn die Schwefelbzw. Selenatome terminal oder $\mu_{2}$-artig gebunden sind.

Um die Reihe der Verbindungen des Typs $\left[\mathrm{NaCu}_{2}\left(\mathrm{dppaE}_{2}\right)_{3}(\mathrm{thf})_{2}\right]($ mit $\mathrm{E}=\mathrm{O}, \mathrm{S}, \mathrm{Se})$ 
zu vervollständigen, wurde versucht, den analogen Komplex mit dppaOS ${ }^{\ominus}$ zu synthetisieren. Bislang konnten jedoch nur komplexe Produktgemische erhalten werden, deren Zusammensetzung noch nicht bestimmt wurde.

\subsubsection{Die Synthesen und Strukturen von $\left[\mathrm{Cu}_{3}\left(\mathrm{dppaE}_{2}\right)_{3}\right](\mathrm{E}=\mathrm{O}$ und $\mathrm{S}$ : 10; S: 15; Se: 16), $\left[\mathrm{Ag}_{3}\left(\operatorname{dppaS}_{2}\right)_{3}\right] \underline{17}$ und $\left[\mathrm{Na}_{2} \mathrm{Ag}_{2}\left(\operatorname{dppaS}_{2}\right)_{4}(\operatorname{thf})_{4}\right] \underline{18}$}

Wird das Reaktandenverhältnis $\mathrm{CuCl}$ : Amid nicht 1:1,5 sondern 1:1 eingestellt, so können mehrere dreikernige, homoleptische Verbindungen des Typs $\left[\mathrm{Cu}_{3}\left(\mathrm{dppaE}_{2}\right)_{3}\right]$ isoliert werden (Gl. 20). Wählt man ein $\mathrm{CuCl}$ : Amid-Verhältnis, das kleiner ist, so entstehen anteilig nebeneinander die trimere Verbindung $\underline{15}$ bzw. 16 und die Verbindung $\underline{13}$ bzw. 14 .

$$
\begin{aligned}
3 \mathrm{CuCl}+3\left[\mathrm{~K}\left(\mathrm{dppaE}_{2}\right)\right] \stackrel{\mathrm{THF}}{\longrightarrow} & {\left[\mathrm{Cu}_{3}\left(\mathrm{dppaE}_{2}\right)_{3}\right]+3 \mathrm{KCl} } \\
& (\mathrm{E}=\mathrm{S}: \underline{15}, \mathrm{Se}: \underline{16})
\end{aligned}
$$

Verbindung $\underline{15}$ wurde erstmals von O. Siiman [32,91,92] synthetisiert. Sie entstand als Disproportionierungsprodukt beim Zerfall der gemischtvalenten Verbindung der Zusammensetzung $\left[\mathrm{Cu}_{3}\left(\mathrm{dppaS}_{2}\right)_{4}\right]$, die sich in wässriger Lösung aus der Reaktion von $\left[\mathrm{Na}\left(\mathrm{dppaS}_{2}\right)\right]$ mit $\mathrm{CuCl}_{2}$ bildete. Allerdings konnte der Aufbau dieser Verbindung nicht bestimmt werden. Ebenfalls wurden die zu $\underline{15}$ und $\underline{16}$ isostrukturelle Verbindung $\left[\mathrm{Cu}_{3}\left(\mathrm{~d}^{\mathrm{i}} \mathrm{PrpaS}_{2}\right)_{3}\right]$, ausgehend von $\mathrm{CuBr}_{2}$ und $\mathrm{d}^{\mathrm{i}} \mathrm{PrpaS}_{2}{ }^{\ominus}[110]$, und der Komplex $\left[\mathrm{Cu}_{3}\left((\mathrm{PhO})_{2} \mathrm{P}(\mathrm{S}) \mathrm{NC}(\mathrm{S}) \mathrm{NEt}_{2}\right)_{3}\right]$ [111] dargestellt. Im Gegensatz zu den Synthesemethoden dieser dreikernigen Verbindungen konnte die Verbindungsreihe $\left[\mathrm{M}_{3}\left(\mathrm{dppaE}_{2}\right)_{3}\right]\left(\mathrm{E}=\mathrm{S}\right.$, Se), ausgehend von $\mathrm{Cu}^{\mathrm{I}}$-Chlorid und $\mathrm{dppaE}_{2}{ }^{\ominus}$, in wasserfreiem Lösungsmittel dargestellt werden.

Welche Komplexverbindungen im Falle von $\mathrm{Ag}^{\mathrm{I}}$ gebildet werden, ist vor allem davon abhängig, welches Deprotonierungsmittel eingesetzt wird. Die Mengenverhältnisse von $\mathrm{Ag}^{\mathrm{I}}$ $\mathrm{zu}$ Ligand sind weniger von Bedeutung, denn werden $\mathrm{AgCl}$ und $\mathrm{dppaS}_{2}{ }^{\ominus}$ im Verhältnis 1:1 eingesetzt, so bildet sich der Komplex 17, wenn die Deprotonierung des Amins mit ${ }^{\mathrm{n}} \mathrm{BuLi}$ erfolgt (Gl. 21), während Verbindung 18 entsteht, wenn das Amin mit $\mathrm{NaN}\left(\mathrm{SiMe}_{3}\right)_{2}$ deprotoniert wird (Gl. 22).

$$
\begin{aligned}
& 3 \mathrm{AgCl}+3\left[\mathrm{Li}\left(\mathrm{dppaS}_{2}\right)\right] \stackrel{\mathrm{THF}}{\longrightarrow}\left[\mathrm{Ag}_{3}\left(\mathrm{dppaS}_{2}\right)_{3}\right] \underline{17}+3 \mathrm{LiCl} \\
& \mathrm{AgCl}+\left[\mathrm{Na}\left(\mathrm{dppaS}_{2}\right)\right] \stackrel{\mathrm{THF}}{\longrightarrow}\left[\mathrm{Na}_{2} \mathrm{Ag}_{2}\left(\mathrm{dppaS}_{2}\right)_{4}(\mathrm{thf})_{4}\right] \underline{18}
\end{aligned}
$$


Die Strukturen von $\left[\mathrm{Cu}_{3}\left(\operatorname{dppaE}_{2}\right)_{3}\right](\mathrm{E}=\mathrm{O}$ und S: 10; S: 15; Se: 16 $)$ und $\left[\operatorname{Ag}_{3}\left(\operatorname{dppaS}_{2}\right)_{3}\right]$ 17:

Die in Abb. 31 dargestellte trimere Verbindung 10 kristallisiert monoklin in der Raumgruppe $C 2 / c, \underline{15}$ und $\underline{16}$ kristallisieren monoklin in der Raumgruppe $P 2_{1} / n$ und Verbindung 17 (Abb. 33) triklin in der Raumgruppe $P \overline{1}$.

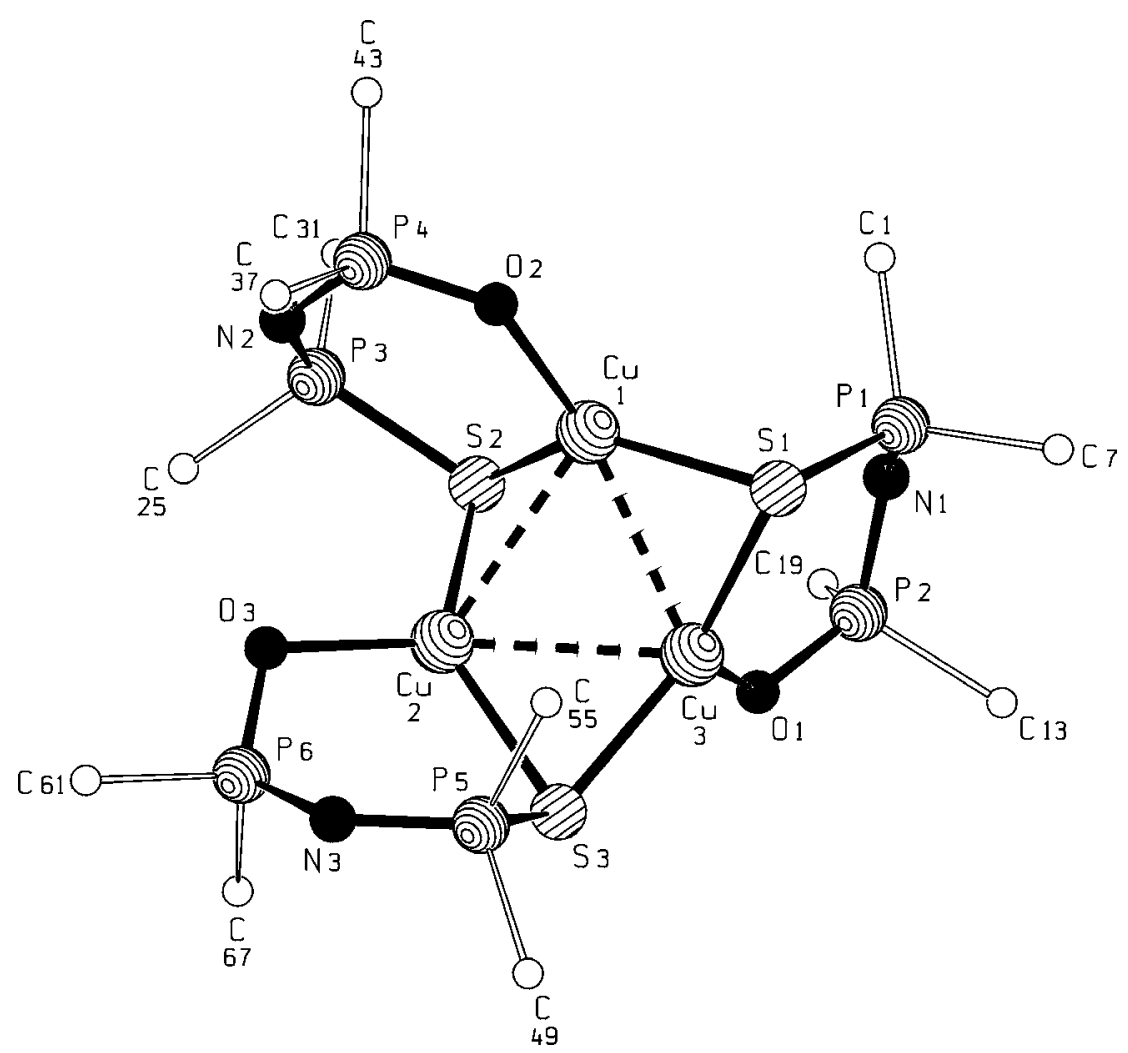

Abb. 31: Die Struktur von $\left[\mathrm{Cu}_{3}(\mathrm{dppaOS})_{3}\right] 10$ im Kristall (zur vereinfachten Darstellung sind von den Phenylgruppen nur die ipso-Atome eingezeichnet)

Allen vier Verbindungen ist gemeinsam, daß sie aus einem trigonalen $\mathrm{M}_{3}$-Grundgerüst aufgebaut sind, wobei die $\mathrm{Cu}-\mathrm{Cu}$-Kontakte in Verbindung 10 bei ca. $269 \mathrm{pm}$ liegen. Sie sind damit kürzer als in Verbindung $\underline{15}$ und $\underline{16}$, in denen die $\mathrm{Cu}-\mathrm{Cu}$-Abstände 285 pm bzw. 287 pm betragen, was mit dem elektronegativeren Sauerstoff-Donoratom in $\underline{10}$ erklärbar ist. Im Vergleich dazu sind die $\mathrm{Cu}-\mathrm{Cu}$-Abstände der vierkernigen Verbindung $\left[\mathrm{Cu}_{4}\left(\mathrm{dppaS}_{2}\right)_{3}\right]\left[\mathrm{CuCl}_{2}\right] \cdot \mathrm{CCl}_{4}[32,91,92]$ zu ca. $279 \mathrm{pm}$ bestimmt worden, liegen also zwischen den entsprechenden Werten in $\underline{10}$ bzw. 15 und 16. Die Ag-Ag-Abstände in 17 sind, verglichen mit anderen Ag-S-Komplexen, sehr lang und variieren von 412 bis 435 pm. In schwefelverbrückten Silber-Dithiophosphato-Komplexen, beispielsweise in der Verbin- 
dung $\left[\mathrm{Ag}_{6}\left(\mathrm{~S}_{2} \mathrm{P}\left(\mathrm{O}^{\mathrm{i}} \mathrm{Pr}\right)_{2}\right)_{6}\right][112]$, werden $\mathrm{Ag}$-Ag-Kontakte in einem Bereich von 315 bis 326 pm gefunden, so daß bezüglich 17 von keinem Ag-Ag-Kontakt ausgegangen werden kann.

In $\underline{10}$ und $\underline{15}$ bis $\underline{17}$ sind die drei Kupfer- bzw. Silberatome so mit den Chelatliganden verbunden, daß jeder der drei Liganden mit einem Chalkogenatom endständig an je ein $\mathrm{Cu}$ bzw. Ag-Atom gebunden ist und das jeweils andere Chalkogenatom $\mu_{2}$-artig verbrückend zwischen zwei Metallatomen wirkt (vgl. Abb. $4(\mathrm{~h})$ ), woraus sich die trimere Molekülstruktur mit einer trigonal-planaren Koordinationssphäre für die $\mathrm{Cu}$ - und Ag-Ionen ergibt. In 10 sind alle drei O-Atome terminal koordiniert, wobei sich zwei oberhalb und eines unterhalb einer gedachten $\mathrm{Cu}_{3}$-Ebene befinden (vgl. Abb. 31). Die drei S-Atome sind $\mu_{2}$-artig an je zwei $\mathrm{Cu}$-Atome gebunden. Abweichend davon liegen in den Verbindungen $\underline{15}$ und $\underline{16}$ alle terminal koordinierten Chalkogenatome auf der gleichen Seite der gedachten $\mathrm{Cu}_{3}$-Ebene, was ebenfalls in der analog aufgebauten Verbindung $\left[\mathrm{Cu}_{3}\left((\mathrm{PhO})_{2} \mathrm{P}(\mathrm{S}) \mathrm{NC}(\mathrm{S}) \mathrm{NEt}_{2}\right)_{3}\right][111]$ beobachtet werden kann (Abb. 32).

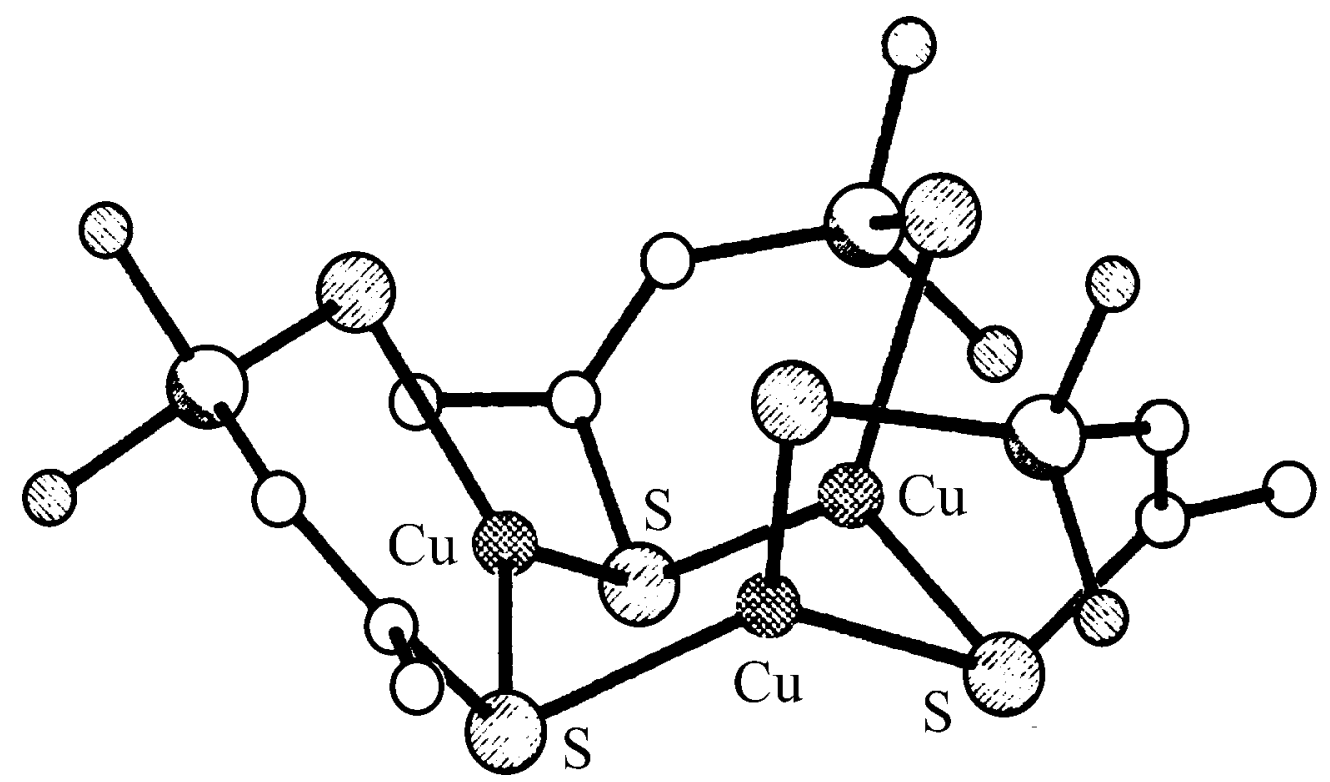

Abb. 32: Vereinfachte Darstellung des molekularen Aufbaus von $\left[\mathrm{Cu}_{3}\left((\mathrm{PhO})_{2} \mathrm{P}(\mathrm{S}) \mathrm{NC}(\mathrm{S}) \mathrm{NEt}_{2}\right)_{3}\right][111]$

Im Unterschied zu den $\left[\mathrm{Cu}_{3}\left(\mathrm{dppaE}_{2}\right)_{3}\right]$-Verbindungen $\underline{10}, \underline{15}$ und $\underline{16}$ weisen in $\underline{17}$ nicht alle drei Liganden vom $\mathrm{M}_{3} \mathrm{~S}_{3}$-Cyclus weg, sondern ein Ligand ist zu einer Seite hin gekippt, denn die Ebene eines der drei sechgliedrigen $\mathrm{AgS}_{2} \mathrm{P}_{2} \mathrm{~N}$-Ringe steht in einem Winkel von $67^{\circ} \mathrm{zu}$ der $\mathrm{Ag}_{3} \mathrm{~S}_{3}$-Ebene, während die beiden anderen nur in Winkeln von $5^{\circ}$ und $12^{\circ}$ dazu 
geneigt sind (Abb. 33).

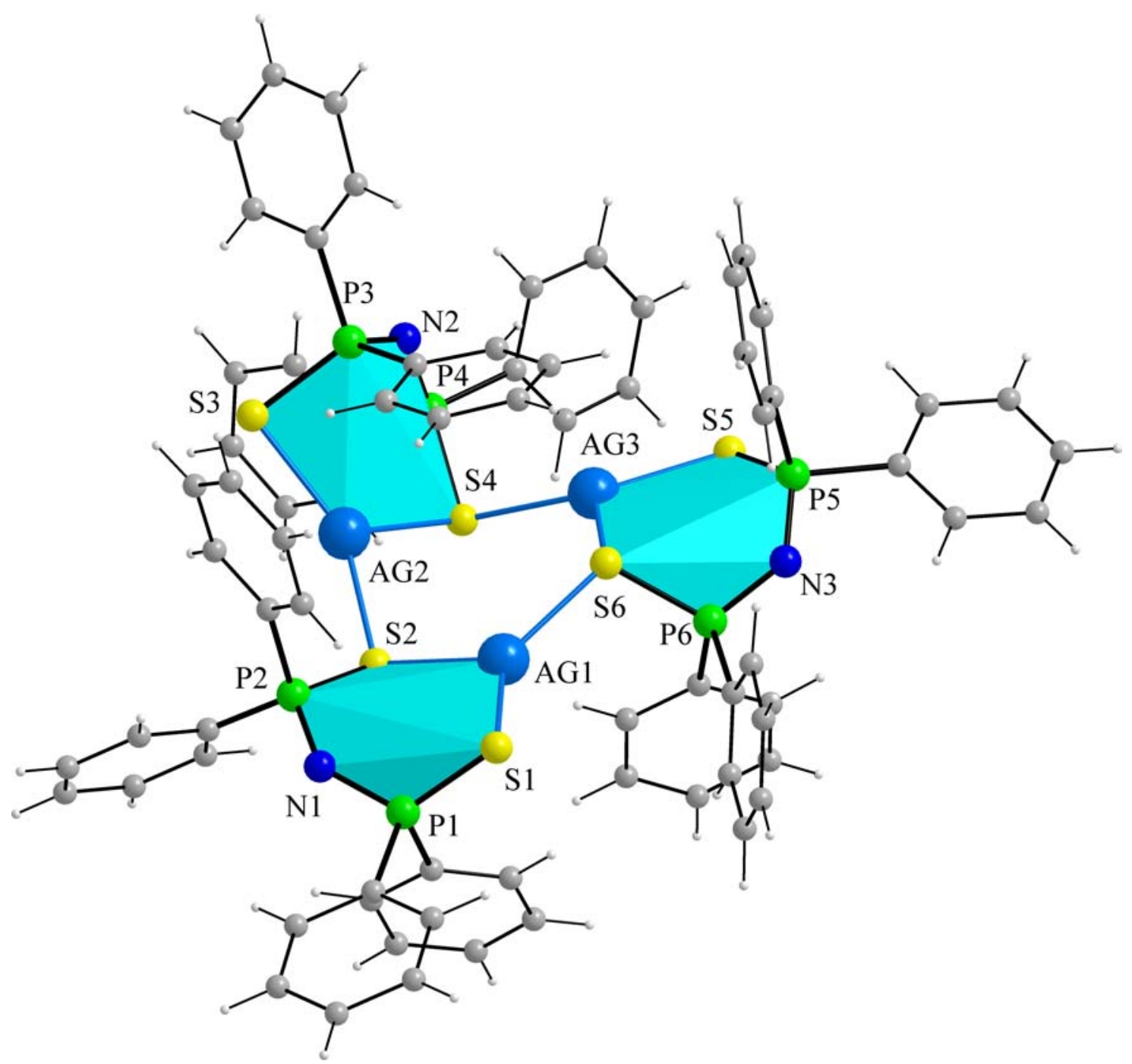

Abb. 33: Die Struktur von $\left[\mathrm{Ag}_{3}\left(\mathrm{dppaS}_{2}\right)_{3}\right] \underline{17}$ im Kristall

Abhängig von der Koordinationsart des Chalkogenatoms können zwei unterschiedliche CuChalkogenbindungslängen unterschieden werden. Die Cu-S- bzw. Cu-Se-Bindunglängen für $\mu_{1}$-koordinierte S- und Se-Atome betragen 223 und $233 \mathrm{pm}$. Bindet das Chalkogenatom $\mu_{2}$-artig, so werden $\mathrm{Cu}-\mathrm{S}-\mathrm{Abstände}$ von $227 \mathrm{pm}$ in $\underline{10}$ und $228 \mathrm{pm}$ in 15 und Cu-SeAbstände von 238 pm in 16 gefunden.

In Verbindung 17 ist eine derartige Unterscheidung nicht möglich, denn die Ag- $\mu_{1}$-SAbstände betragen 244 pm und die Ag- $\mu_{2}$-S-Abstände 244 und 255 pm. Beide Werte liegen jedoch in einem Bereich, der auch beispielsweise in Silber-Dithiocarbamato-Komplexen 
gefunden wird, in denen Ag- $\mu_{1}$-S-Abstände von 243 bis 248 pm und Ag- $\mu_{2}$-S-Abstände von 247 bis 256 pm beobachtet werden [113].

Im Gegensatz zu den trimeren Komplexen des Kupfers 10, $\underline{15}$ und $\underline{16}$, in denen sich die Metallatome mittels der Liganden annähern, wird in 17 innerhalb des $\mathrm{Ag}_{3} \mathrm{~S}_{3}$-Sechsringes eine deutliche Alternanz der Ag-S-Bindungslängen beobachtet. Der Unterschied zwischen langen und kurzen Ag-S-Abständen beträgt im Durchschnitt 11 pm.

Im Vergleich zum ungebundenen Phosphorylamin dppaOSH [98] sind in 10 durch die Deprotonierung und Bindung an die Cu-Ionen die P-S-Bindungslängen mit 207 pm um ca. 14 pm und die P-O-Bindungslängen mit 152 pm nur um ca. 2 pm größer. Dagegen findet man, daß die P-N-Abstände mit 159 pm um ca. 9 pm erwartungsgemäß kürzer sind. Diese Befunde unterstützen die Annahme, daß die negative Ladung über das gesamte SPNPO-System delokalisiert ist.

Bei den P-S- und P-Se-Bindungen kann deutlich zwischen zwei Gruppen unterschieden werden: Die P-E-Abstände der $\mu_{2}$-verbrückenden Chalkogenatome in $\underline{15}$ und $\underline{16}$ sind mit 206 pm und 222 pm durchschnittlich um 6 pm und in 17 mit 203 pm um 3 pm länger als die entsprechenden P-E-Bindungen der terminal bindenden Chalkogenatome. Geht man davon aus, daß die P-S-Einfachbindungslänge 208 pm und die P-S-Doppelbindungslänge 195 pm beträgt, wie z.B. in $\mathrm{Ph}_{2} \mathrm{P}(\mathrm{S}) \mathrm{SH}$ [114], dann kann den $\mathrm{P}-\mu_{2}$-S-Bindungen eine Bindungsordnung von annähernd eins und den $\mathrm{P}-\mu_{1}$-S-Bindungen eine Bindungsordnung von über 1,5 zugeordnet werden. Einen analogen Gang beobachtet man für die P-Se-Bindungen in 16. Wird eine P-Se-Doppelbindungslänge von 210 pm und eine P-SeEinfachbindungslänge von $227 \mathrm{pm}$ angenommen, wie sie z.B. in $\left[{ }^{\mathrm{t}} \mathrm{BuP}(\mathrm{Se}) \mathrm{Se}\right]_{2}[115]$ vorgefunden werden, so liegen die $\mathrm{P}-\mu_{1}$-Se-Bindungen in $\underline{16}$ genau zwischen einer Einfachund einer Doppelbindung. Die P- $\mu_{2}$-Se-Bindungen jedoch besitzen überwiegend Einfachbindungscharakter.

Setzt man nun diesen Befund in Beziehung mit den auftretenden P-N-Bindungslängen, so ist erkennbar, daß die P-N-Bindung eines Phosphoratoms, das an ein $\mu_{2}$-koordiniertes Chalkogenatom gebunden ist, mit einer Länge von 158 pm leicht kürzer ist als die P-NBindung eines Phosphoratoms, das an ein terminal koordiniertes Chalkogenatom gebunden ist. Sie sind in 10, 15 und $16160 \mathrm{pm}$ und in $\underline{17} 159 \mathrm{pm}$ lang. Diese Alternanz in den Bindungslängen kann jedoch nicht beobachtet werden, wenn beide Chalkogenatome gleichartig, also alle terminal oder alle $\mu_{2}$-verbrückend, koordiniert sind, wie beispielsweise in $\underline{13}$ und $\underline{14}$ beobachtet wird. 
Die Struktur von $\left[\mathrm{Na}_{2} \mathrm{Ag}_{2}\left(\mathrm{dppaS}_{2}\right)_{4}(\mathrm{thf})_{4}\right] \underline{18}$ :

Die in Abb. 34 gezeigte Verbindung 18 kristallisiert triklin in der Raumgruppe $P \overline{1}$, deren achtgliedriger $\mathrm{Na}_{2} \mathrm{Ag}_{2} \mathrm{~S}_{4}$-Ring den zentralen Baustein des Komplexes darstellt.

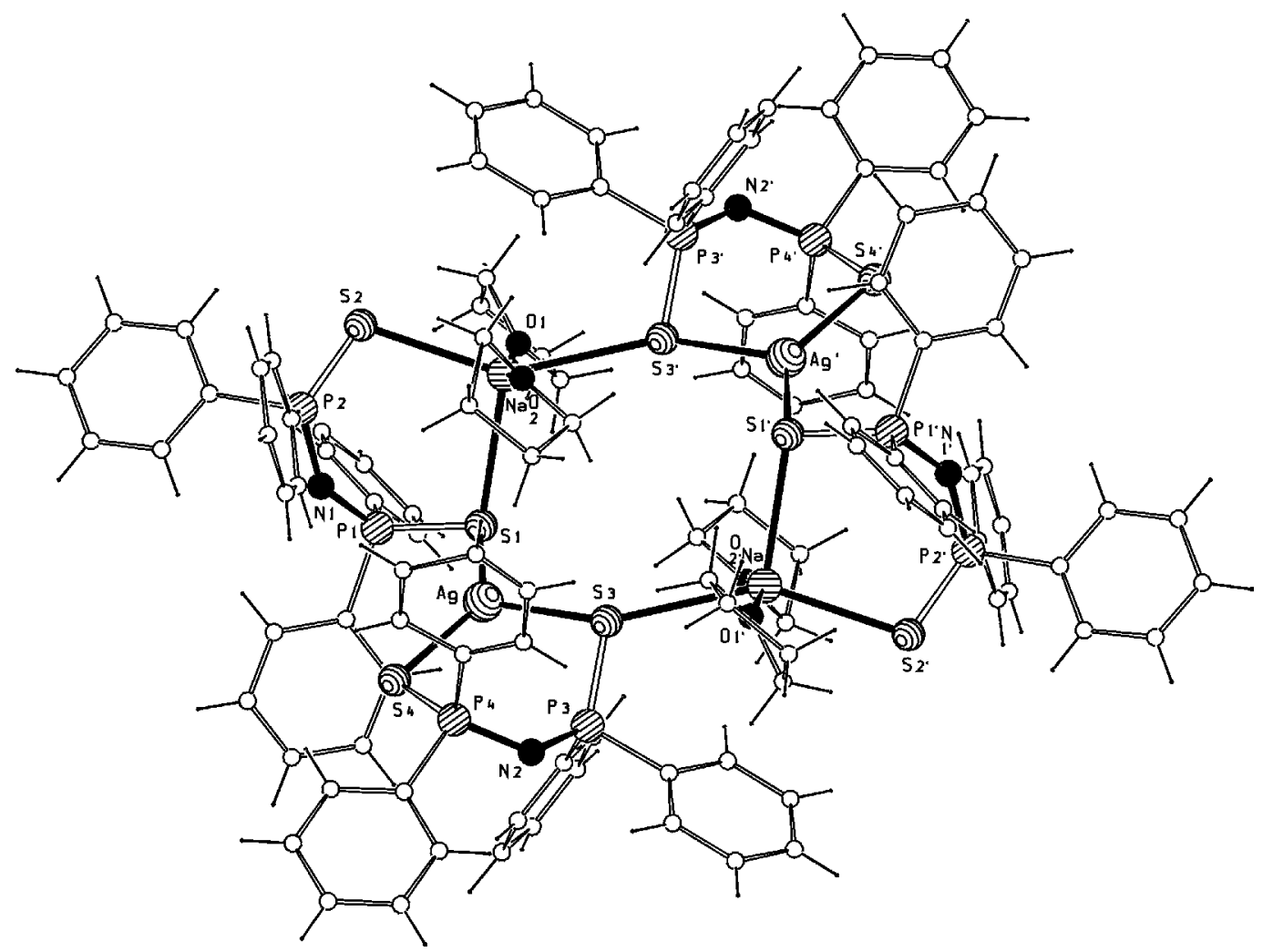

Abb. 34: Die Struktur von $\left[\mathrm{Na}_{2} \mathrm{Ag}_{2}\left(\operatorname{dppaS}_{2}\right)_{4}(\text { thf })_{4}\right] 18$ im Kristall

Die vier Liganden sind gemäß Abb. 4 (h) an jeweils zwei Metallatome koordiniert, so daß jeder Ligand ein Metallion chelatisiert und zusätzlich über ein S-Atom zu einem zweiten Metallatom verbrückt. Die Ag-Ionen sind trigonal-planar von je drei Schwefelatomen umgeben, während sich für die Na-Ionen eine trigonal-bipyramidale Koordinationsumgebung ergibt, da jedes Na-Atom von drei Schwefel- und den Sauerstoffatomen zweier THF-Liganden, die die apicalen Positionen des Na-Koordinationspolyeders besetzen, koordiniert ist.

Die P-S-Bindungslängen mit 198 bis 200 pm befinden sich im Bereich zwischen einer Einfach- und einer Doppelbindung, ebenso wie die P-N-Bindungslängen mit 159 bis 160 pm. Lediglich die $\mathrm{P}(1)-\mathrm{N}(1)$-Bindung ist mit einer Länge von $156 \mathrm{pm}$ recht kurz, was die im Vergleich zu den anderen P-S-Bindunglängen leicht größere $\mathrm{P}(1)-\mathrm{S}(1)$ Bindungslänge von 202 pm erkärt. 
Da der $\mathrm{P}(1)-\mathrm{N}(1)-\mathrm{P}(2)-$ Winkel mit $143^{\circ}$ ungewöhnlich aufgeweitet $(\angle \mathrm{P}(3)-\mathrm{N}(2)-\mathrm{P}(4)$ : $128^{\circ}$ ) und damit zusammenhängend der "Biß" dieses Liganden mit 440 pm um 27 pm größer ist als der des anderen Liganden, ist der $\mathrm{AgS}_{2} \mathrm{P}_{2} \mathrm{~N}$-Cyclus sehr stark verdrillt. Entsprechend beobachtet man eine starke Stauchung des S(1)-Na-S(2)-Winkels (100 statt $120^{\circ}$ ). Aufgrund des dadurch erhöhten sterischen Anspruchs der vier Phenylgruppen durch die kürzere P-N-Bindung ist auch der Winkel S(1)-Ag-S(4) von $134^{\circ}$ gegenüber dem idealen Winkel von $120^{\circ}$ aufgeweitet. 


\subsubsection{Die Synthesen und Strukturen von $\left[\mathrm{Cu}_{4}\left(\mathrm{dppaS}_{2}\right)_{3}\right]_{2}\left[\mathrm{CuCl}_{2}\right][\mathrm{OH}] \underline{19}$ und $\left[\mathrm{Cu}_{4}\left(\mathrm{dppaSe}_{2}\right)_{3}\right]\left[\mathrm{CuCl}_{2}\right] \underline{20}$}

Um auch Reaktionen von $\mathrm{CuCl}$ mit den Aminen bei höheren Temperaturen untersuchen zu können, werden diese in der Schmelze des Liganden durchgeführt, in denen die Deprotonierung der Liganden durch die Bildung von $\mathrm{HCl}$, das aus dem Reaktionssystem gasförmig entweichen kann, stattfinden kann. So gelingt z.B. die Synthese der vierkernigen Verbindungen $\left[\mathrm{Cu}_{4}\left(\mathrm{dppaS}_{2}\right)_{3}\right]_{2}\left[\mathrm{CuCl}_{2}\right][\mathrm{OH}] \underline{19}$ und $\left[\mathrm{Cu}_{4}\left(\mathrm{dppaSe}_{2}\right)_{3}\right]\left[\mathrm{CuCl}_{2}\right] \underline{20}$.

Das Phosphorylamin und $\mathrm{CuCl}$ werden hierbei im Molverhältnis 1:1 unter Stickstoffatmosphäre vermischt und am Schmelzpunkt des Amins (Smp. $220^{\circ} \mathrm{C}$ ) für 5 min. erhitzt. Aus dem zurückbleibenden Reaktionsgemisch können die Verbindungen $\underline{19}$ und 20 aus $\mathrm{CH}_{2} \mathrm{Cl}_{2}$ kristallisiert werden (Gln. 23 und 24).

$$
\begin{array}{cl}
\mathrm{CuCl}+\operatorname{dppaS}_{2} \mathrm{H} \underset{2) \mathrm{CH}_{2} \mathrm{Cl}_{2}}{\stackrel{1) \Delta}{\longrightarrow}}\left[\mathrm{Cu}_{4}\left(\mathrm{dppaS}_{2}\right)_{3}\right]_{2}\left[\mathrm{CuCl}_{2}\right][\mathrm{OH}] \underline{19} \\
\mathrm{CuCl}+\operatorname{dppaSe}_{2} \mathrm{H} \underset{2{ }_{2 \mathrm{CH}_{2} \mathrm{Cl}_{2}}}{\stackrel{1) \Delta}{\longrightarrow}}\left[\mathrm{Cu}_{4}\left(\mathrm{dppaSe}_{2}\right)_{3}\right]\left[\mathrm{CuCl}_{2}\right] \underline{20}
\end{array}
$$

Im Unterschied zur Synthesemethode in der Schmelze konnte von O. Siiman die zu $\underline{19}$ analog aufgebaute Verbindung $\left[\mathrm{Cu}_{4}\left(\mathrm{dppaS}_{2}\right)_{3}\right]\left[\mathrm{CuCl}_{2}\right] \cdot \mathrm{CCl}_{4}$ durch die Reaktion von $\mathrm{CuCl}_{2}$ mit dppaS ${ }_{2}{ }^{\ominus}$ in wässriger Lösung isoliert werden $[32,91,92]$.

Da 19 und 20 annähernd isostrukturell zueinander sind, werden sie gemeinsam besprochen.

\section{Die Strukturen von $\left[\mathrm{Cu}_{4}\left(\mathrm{dppaS}_{2}\right)_{3}\right]_{2}\left[\mathrm{CuCl}_{2}\right][\mathrm{OH}] \underline{19}$ und $\left[\mathrm{Cu}_{4}\left(\mathrm{dppaSe}_{2}\right)_{3}\right]\left[\mathrm{CuCl}_{2}\right] \underline{20}$ :}

Die in orangefarbenen Kristallen erhaltene Verbindung 19 kristallisiert rhomboedrisch in der Raumgruppe $R \overline{3}$, während der Komplex $\underline{20}$, der triklin in der Raumgruppe $P \overline{1}$ kristallisiert, in Form von farblosen Kristallen isoliert werden kann. In Abb. 35 ist die Struktur der einfach positiv geladenen $\left[\mathrm{Cu}_{4}\left(\mathrm{dppaSe}_{2}\right)_{3}\right]^{\oplus}$-Einheit dargestellt.

Sie besteht aus einem verzerrten $\mathrm{Cu}_{4}$-Tetraeder, an das drei anionische Liganden binden. Jedes Chalkogenatom verbrückt $\mu_{2}$-artig zwischen je zwei $\mathrm{Cu}$-Ionen, so daß jedes $\mathrm{Cu}$-Atom annähernd trigonal-planar von drei Chalkogenatomen umgeben ist. Die Koordinationsart der Liganden (vgl. Abb. 4 (i)) führt somit zum adamantanartigen $\mathrm{Cu}_{4} \mathrm{E}_{6}$-Grundkörper, der ebenfalls als Oktaeder, dessen Ecken von sechs Chalkogenatomen besetzt sind, angesehen werden kann. Dann befinden sich die Kupferatome in der Mitte von vier alternierenden Oktaederflächen. 


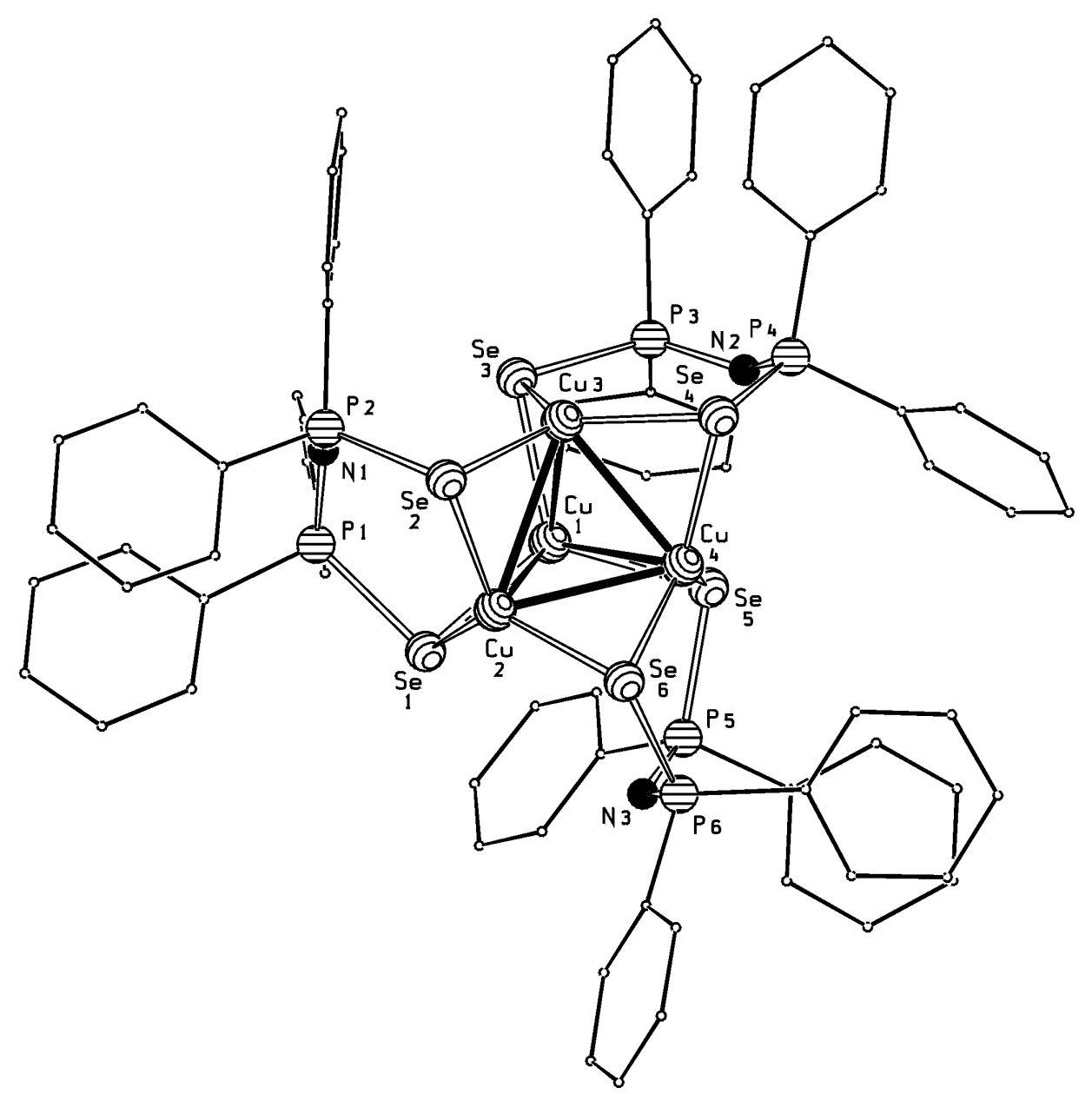

Abb. 35: Die Struktur des Kations $\left[\mathrm{Cu}_{4}\left(\mathrm{dppaSe}_{2}\right)_{3}\right]^{\oplus}$ von $\underline{20}$ im Kristall

Die mittleren Abstände der Cu-Cu-Kontakte und der Cu-S-, P-S- und P-N-Bindungen der analog aufgebauten Verbindung $\left[\mathrm{Cu}_{4}\left(\mathrm{dppaS}_{2}\right)_{3}\right]\left[\mathrm{CuCl}_{2}\right] \cdot \mathrm{CCl}_{4}[32,92]$ sind vergleichbar mit denen, die in 19 gefunden werden. Sie weichen maximal um 1 pm von einander ab (in $\underline{19}$ : Cu-Cu: 279 pm, Cu-S: 226 pm, P-S: 206 pm, P-N: 159 pm). Auch in 20 werden ähnliche $\mathrm{Cu}-\mathrm{Cu}$-Kontakte von $278 \mathrm{pm}$ beobachtet. Die Längen der Cu-Se-Bindungen in $\underline{20}$ sind mit $238 \mathrm{pm}$ typisch für $\mu_{2}$-verbrückende Se-Atome, denn auch in anderen Verbindungen mit dem Bauelement $\mathrm{Cu}_{4} \mathrm{Se}_{6}$ findet man vergleichbare $\mathrm{Cu}-\mathrm{Cu}$-Abstände von ca. $277 \mathrm{pm}$ und Cu-Se-Abstände von ca. 238 pm [116]. 


\subsubsection{Die Synthese und Struktur von $\left[\mathrm{Cu}\left(\operatorname{dppaS}_{2}\right)(\operatorname{th})\right] \underline{21}$}

Es wurden auch Reaktionen untersucht, in denen dem System aus $\mathrm{CuCl}$ und $\operatorname{dppaE}_{2}{ }^{\ominus}$ zusätzliche Liganden wie Thioharnstoff, N,N-Diphenylthioharnstoff und $\mathrm{PPh}_{3}$ angeboten wurden.

So reagieren $\mathrm{CuCl},\left[\mathrm{Na}\left(\mathrm{dppaS}_{2}\right)\right]$ und Thioharnstoff zu der einkernigen Verbindung $\left[\mathrm{Cu}\left(\mathrm{dppaS}_{2}\right)(\mathrm{th})\right] \underline{21}$. Gleichzeitig wird auch die Bildung der oben beschriebenen, trimeren Verbindung 15 beobachtet (Gl. 25). Außerdem können mit thPh $\mathrm{h}_{2}$ oder $\mathrm{PPh}_{3}$ die analogen Komplexe $\left[\mathrm{Cu}\left(\mathrm{dppaS}_{2}\right)\left(\mathrm{thPh}_{2}\right)\right](\mathrm{Gl} .26$ [52]) und die von I. Haiduc et al. synthetisierte Verbindung $\left[\mathrm{Cu}\left(\mathrm{dppaS}_{2}\right)\left(\mathrm{PPh}_{3}\right)\right]$ dargestellt werden (Gl. 27 [108]).

$$
\begin{aligned}
& \mathrm{CuCl}+\left[\mathrm{Na}\left(\text { dppaS }_{2}\right)\right]+\text { th } \underset{2) \mathrm{THF}}{\stackrel{1) \mathrm{EtOH}}{\longrightarrow}}\left[\mathrm{Cu}\left(\text { dppaS }_{2}\right)(\text { th })\right] \underline{21} \\
& +\left[\mathrm{Cu}_{3}\left(\mathrm{dppaS}_{2}\right)_{3}\right] \underline{15} \\
& \mathrm{CuCl}+\left[\mathrm{Na}\left(\text { dppaS }_{2}\right)\right]+\operatorname{thPh}_{2} \stackrel{\text { THF }}{\longrightarrow}\left[\mathrm{Cu}\left(\text { dppaS }_{2}\right)\left(\operatorname{thPh}_{2}\right)\right] \\
& \mathrm{CuCl}+\left[\mathrm{Na}\left(\mathrm{dppaS}_{2}\right)\right]+\mathrm{PPh}_{3} \stackrel{\mathrm{THF}}{\longrightarrow}\left[\mathrm{Cu}\left(\mathrm{dppaS}_{2}\right)\left(\mathrm{PPh}_{3}\right)\right]
\end{aligned}
$$

\section{Die Struktur von $\left[\mathrm{Cu}\left(\operatorname{dppaS}_{2}\right)(\mathrm{th})\right] \underline{21}$ :}

Die Röntgenstrukturanalyse von Verbindung 21 ergibt, daß sie monoklin in der Raumgruppe $P 2_{1} / c$ kristallisiert mit zwei Komplexmolekülen und drei THF in der asymmetrischen Einheit. Die Molekülstruktur ist in Abb. 36 dargestellt. Die einkernige Verbindung ist aus einem trigonal-planar umgebenen $\mathrm{Cu}^{\mathrm{I}}$-Ion aufgebaut, das von einem Liganden $\eta^{2}$-artig koordiniert ist (vgl. Abb. 4 (c)). Die dritte Koordinationsstelle des Cu-Ions besetzt das Schwefelatom eines Thioharnstoff-Liganden.

In 21 fällt auf, daß ein größerer Cu-S-Abstand eine etwas längere P-S-Bindung und damit eine leicht kürzere P-N-Bindung bedingt und umgekehrt. Denn beträgt der Cu-SAbstand 228 pm, wird eine entsprechende P-S- und P-N-Bindungslänge von 202 pm und 160 pm beobachtet, während bei einem Cu-S-Abstand von 225 pm die entsprechenden P-S- und P-N-Bindungen 200 bis 201 pm und 161 bis 162 pm lang sind. Da P-S- und P-N-Bindungslängen gefunden werden, die einer Bindungsordnung zwischen eins und zwei entsprechen, kann davon ausgegangen werden, daß die einfach negative Ladung des Liganden über das SPNPS-System delokalisiert ist.

Der Neutralligand Thioharnstoff bindet terminal durch das S-Atom an das Kupferion mit Cu-S-Abständen von 224 pm, S-C-Bindungslängen von 169 und 175 pm und CN-Abständen von 129 bis 138 pm. Ähnliche Werte werden in anderen Thioharnstoffkomplexen gefunden, wie z.B. in der polymeren Verbindung $\left[\left[\mathrm{Cu}_{2} \mathrm{Cl}(\mathrm{th})_{4}\right] \mathrm{Cl}\right]_{\infty}[117]$, in der 


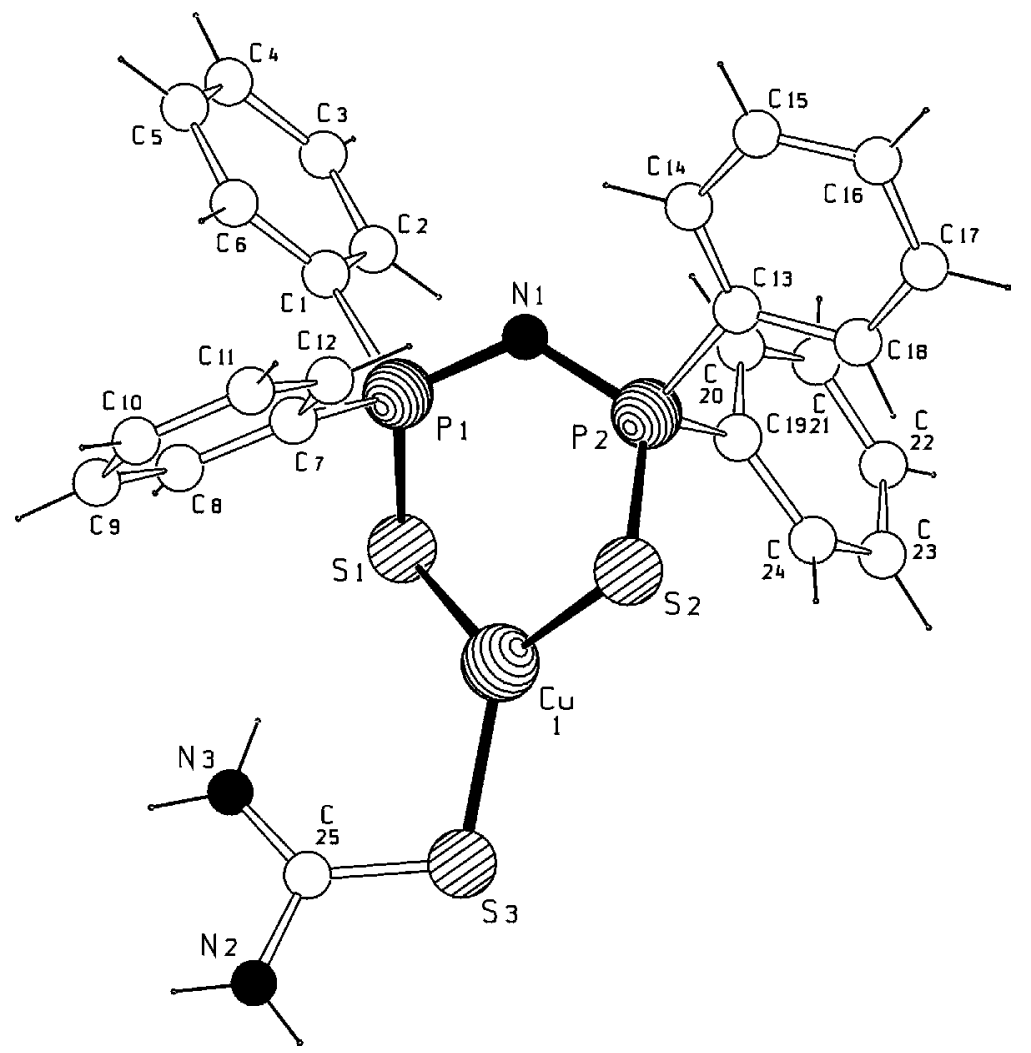

Abb. 36: Die Struktur von $\left[\mathrm{Cu}\left(\mathrm{dppaS}_{2}\right)(\right.$ th) $) \underline{21}$ im Kristall

die $\mathrm{Cu}-\mu_{1}$-S-Abstände 223 bis 225 pm, die S-C- 164 bis 174 pm und C-N-Bindungslängen 129 bis 135 pm betragen. 


\subsubsection{NMR-spektroskopische Untersuchungen}

Zur weiteren Charakterisierung der isolierten Komplexe und zur Untersuchung ihres Lösungsverhaltens wurden ${ }^{31} \mathrm{P}-\mathrm{NMR}$-Spektren von einigen ausgewählten Verbindungen aufgenommen.

Das ${ }^{31} \mathrm{P}-\mathrm{NMR}-\mathrm{Spektrum}$ von $\left[\mathrm{Cu}_{3}\left(\mathrm{dppaS}_{2}\right)_{3}\right] \underline{15}$ in THF $\left(\mathrm{C}_{6} \mathrm{D}_{6}\right)$ zeigt bei Raumtemperatur ein Singulett bei einer chemischen Verschiebung von 31,82 ppm, das chemisch äquivalenten Phosphoratomen des Liganden zugeschrieben werden kann. Wird die Meßtemperatur von $+50{ }^{\circ} \mathrm{C}$ auf $-10{ }^{\circ} \mathrm{C}$ verringert, so führt dies zu einer anfänglichen Signalverbreiterung, an die sich bei weiterer Verringerung der Temperatur auf $-70{ }^{\circ} \mathrm{C}$ eine Abnahme der Signalbreite anschließt. Zudem wird über den ganzen Temperaturbereich eine Tieffeldverschiebung um $\Delta \delta=0,4 \mathrm{ppm}$ beobachtet (31,7 ppm bei $+50{ }^{\circ} \mathrm{C}$ gegen $32,1 \mathrm{ppm}$ bei $-70{ }^{\circ} \mathrm{C}$ ). J.D. Woollins et al. [110] untersuchten die dreikernige Verbindung $\left[\mathrm{Cu}_{3}\left(\mathrm{~d}^{\mathrm{i}} \mathrm{PrpaS}_{2}\right)_{3}\right]$ NMR-spektroskopisch sowohl im Festkörper als auch in Lösung und stellten ebenfalls fest, daß in Lösung nur ein Singulett im ${ }^{31} \mathrm{P}$ NMR-Spektrum auftritt. Im Festkörper jedoch konnten fünf Signale detektiert werden, wobei zwei chemische Verschiebungen im ${ }^{31} \mathrm{P}-\mathrm{NMR}$-Spektrum zusammmentreffen. Da über den gesamten Temperaturbereich im ${ }^{31}$ P-NMR-Spektrum von 15 keine Signalauftrennung erkennbar ist, liegt die Vermutung nahe, daß nur eine einzige Spezies, möglicherweise $\left[\mathrm{Cu}\left(\mathrm{dppaS}_{2}\right)(\right.$ thf $\left.)\right]$ oder $\left[\mathrm{Cu}\left(\mathrm{dppaS}_{2}\right)(\text { thf })_{2}\right]$, in Lösung vorhanden ist. In diesen Komplexen wäre die von $\mathrm{Cu}^{\mathrm{I}}$ bevorzugte Koordinationszahl von drei bzw. vier gegeben.

Um diese Annahme zu unterstützen, wurden die Verbindungen 21, $\left[\mathrm{Cu}\left(\mathrm{dppaS}_{2}\right)\left(\mathrm{thPh}_{2}\right)\right]$ und $\left[\mathrm{Cu}\left(\mathrm{dppaS}_{2}\right)\left(\mathrm{PPh}_{3}\right)\right]$ zu weiteren spektroskopischen Untersuchungen herangezogen. Die Synthesewege dieser Verbindungen nach den Gln. 25, 26, 27 legen die Annnahme nahe, daß in Lösung das koordinierte Lösungsmittel in der vermuteten Spezies durch andere, ungeladene Donorliganden leicht verdrängt werden kann.

So zeigen die Phosphoratome von Lösungen dieser Komplexe im ${ }^{31}$ P-NMR-Spektrum unterschiedliche Resonanzsignale von scharfen Singuletts. Verbindungen, in denen im Festkörper das Cu-Ion von drei Schwefelatomen umgeben ist, wie es bei den Verbindungen 21 und $\left[\mathrm{Cu}\left(\mathrm{dppaS}_{2}\right)\left(\mathrm{thPh}_{2}\right)\right]$ der Fall ist, beobachtet man in THF und $\mathrm{CDCl}_{3}$ im ${ }^{31} \mathrm{P}$-Spektrum Resonanzsignale bei chemischen Verschiebungen von $\delta=33,47 \mathrm{ppm}$ und $\delta=33,31 \mathrm{ppm}$, während die Verbindung $\left[\mathrm{Cu}\left(\mathrm{dppaS}_{2}\right)\left(\mathrm{PPh}_{3}\right)\right]$, in der das $\mathrm{Cu}-\mathrm{Ion}$ im Festkörper nur von zwei S-Atomen umgeben ist, eine höhere chemische Verschiebung von $\delta=34,54$ ppm für das Phosphorylatom zeigt. Da eine Abhängigkeit der chemischen Ver- 
schiebung der Phosphoratome von der Koordinationsart des in der Lösung befindlichen Metallions besteht, kann demnach angenommen werden, daß in diesen Fällen das koordinierte Lösungsmittel der vermuteten Spezies durch die Thio- und Phosphanliganden verdrängt wurde.

Wählt man statt eines Cu-Ligand-Verhältnisses von 1:1 ein geringeres Verhältnis, so gelangt man zu neuen Verbindungen: Wird beispielsweise ein ${ }^{31} \mathrm{P}-\mathrm{NMR}-$ Spektrum der in THF und $\mathrm{C}_{6} \mathrm{D}_{6}$ gelösten Verbindung 13 bei Raumtemperatur aufgenommen, so beobachtet man ein Signal eines ungewöhnlich breiten Singuletts bei $\delta=34,0$ ppm mit einer Signalhalbwertsbreite von $\nu_{1 / 2}=469,9 \mathrm{~Hz}$. Da sehr breite Signale oft auf in Lösung stattfindende, schnelle Austauschprozesse hinweisen, wurden von 13 mehrere ${ }^{31} \mathrm{P}-\mathrm{NMR}$ Spektren bei Temperaturen von $+50{ }^{\circ} \mathrm{C}$ bis $-70{ }^{\circ} \mathrm{C}$ aufgenommen. In Abb. 37 ist das ${ }^{31}$ P-NMR-Spektrum von $\underline{13}$ in Abhängigkeit von der Temperatur dargestellt.

Bei einer Meßtemperatur von $+50{ }^{\circ} \mathrm{C}$ tritt im ${ }^{31} \mathrm{P}-\mathrm{NMR}$-Spektrum nur ein Singulett mit $\delta=33,5 \mathrm{ppm}$ auf, das sich bei einer Temperaturverringerung erst verbreitert und dann in zwei ebenfalls breitere Signale mit einem Intensitätsverhältnis von ca. 2:1 aufspaltet $\left(-10{ }^{\circ} \mathrm{C}: \delta=34,5 \mathrm{ppm} ; 31,2 \mathrm{ppm}\right)$. Wird die Temperatur weiter herabgesetzt, kann erst eine erneute Aufspaltung in drei und dann in vier Signale mit unterschiedlichen Intensitäten $\left(-55^{\circ} \mathrm{C}: \delta=34,7 \mathrm{ppm} ; 34,0 \mathrm{ppm} ; 32,7 \mathrm{ppm} ; 31,5 \mathrm{ppm}\right)$ beobachtet werden. Schlußendlich werden bei $-70{ }^{\circ} \mathrm{C}$ nur noch zwei Signale mit einem Intensitätsverhältnis von $1: 2$ ( $\delta=33,8 \mathrm{ppm}, 32,5 \mathrm{ppm})$ unterschieden.

Aufgrund unterschiedlicher ${ }^{31} \mathrm{P}-\mathrm{NMR}$-Signale liegen in Lösung in Abhängigkeit von der Temperatur verschiedene, chemisch nicht äquivalente Phosphoratome vor, die unterschiedlich gebundenen Ligandmolekülen entsprechen. Aus diesen Beobachtungen kann also gefolgert werden, daß in Lösungen, in denen das Cu-Ligand-Verhältnis nicht 1:1 beträgt, mehrere Spezies vorliegen, die bei Raumtemperatur durch schnelle Austauschprozesse nur ein breites ${ }^{31} \mathrm{P}-\mathrm{NMR}$-Signal zeigen. Bei tieferen Temperaturen jedoch werden die Austauschreaktionen soweit verlangsamt, daß die chemischen Verschiebungen der vorhandenen Spezies NMR-spektroskopisch unterscheidbar werden. 


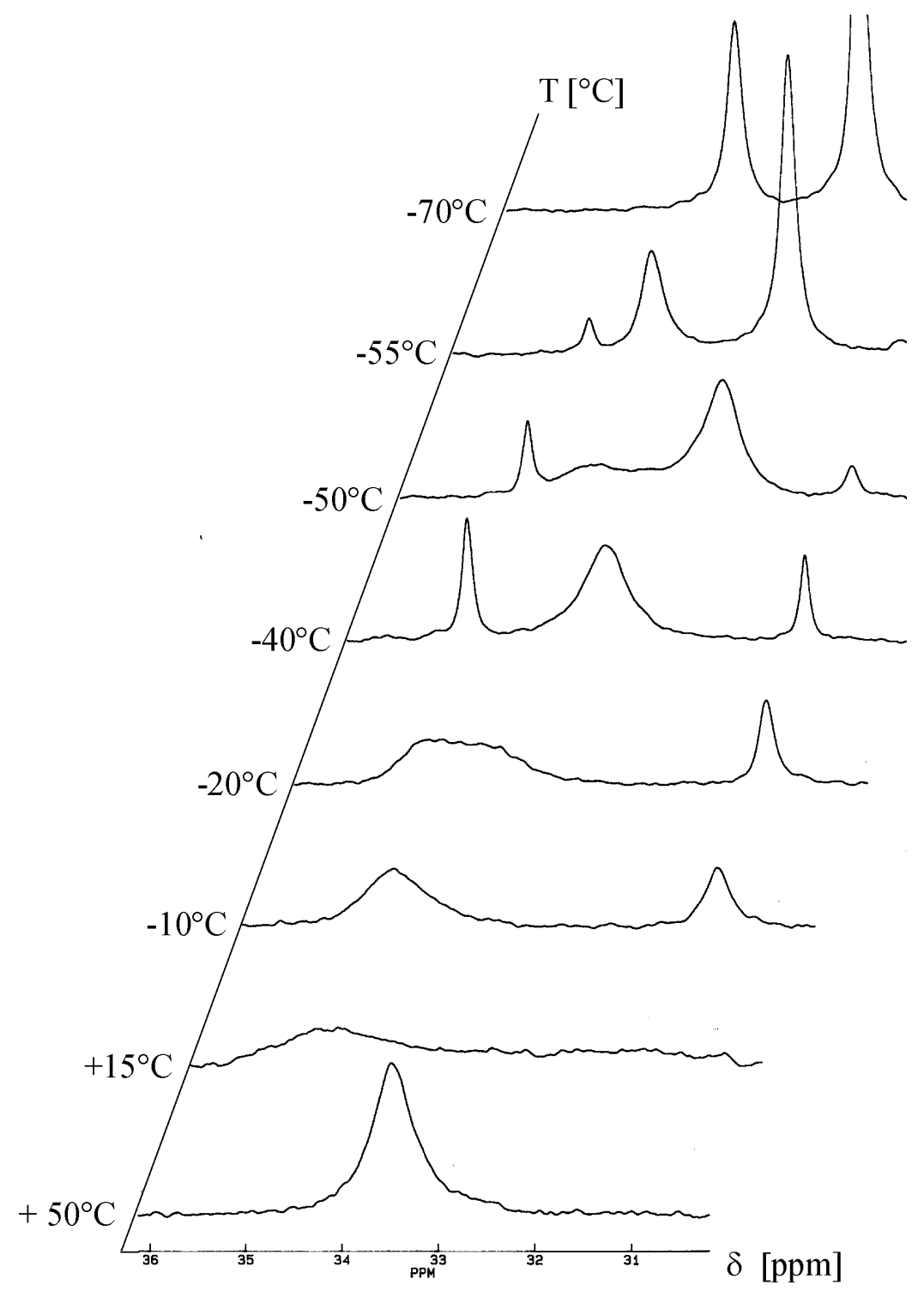

Abb. 37: ${ }^{31} \mathrm{P}-\mathrm{NMR}-\mathrm{Spektrum}$ von $\left[\mathrm{NaCu}_{2}\left(\mathrm{dppaS}_{2}\right)_{3}(\mathrm{thf})_{2}\right] \underline{13}$ in THF und $\mathrm{C}_{6} \mathrm{D}_{6}$ in Abhängigkeit von der Temperatur 


\subsection{Umsetzungen von $\mathrm{CuCl}$ und $\mathrm{CuCl}_{2}$ mit dem $\mathrm{Amin}$ dmpaS $\mathrm{S}_{2} \mathrm{H}$}

Es sind bislang schon mehrere Synthesen von Metallkomplexen des methylsubstituierten Liganden dmpaS $_{2}{ }^{\ominus}$ beschrieben worden. A. Davison und E.S. Switkes berichteten von der Synthese der homoleptischen Verbindungen $\left[\mathrm{M}\left(\mathrm{dppmS}_{2}\right)_{2}\right](\mathrm{M}=\mathrm{Fe}, \mathrm{Co}, \mathrm{Ni}, \mathrm{Pd}, \mathrm{Pt}$, Zn) [118]. Jedoch wurden bisher lediglich die drei Verbindungen für $M=F e$, Co und $\mathrm{Ni}$ röntgenstrukturanalytisch charakterisiert, in denen die Metallatome eine tetrahedrale Koordinationsumgebung aufweisen $[26,27,34]$.

Komplexverbindungen des neutralen Liganden $\mathrm{dmpaS}_{2} \mathrm{H}$ oder des anionischen Liganden $\mathrm{dmpaS}_{2}{ }^{\ominus}$ mit Kupfer sind bisher nicht bekannt. Dennoch gelang es, ein- und mehrkernige $\mathrm{Cu}^{\mathrm{I}}$-Verbindungen zu synthetisierten, die sowohl den neutralen als auch den anionischen Liganden enthalten.

\subsubsection{Die Synthese und Struktur von $\left[\mathrm{Cu}_{2}\left(\operatorname{dmpaS}_{2} \mathrm{H}\right)_{2} \mathrm{Cl}_{2}\right] \underline{22}$}

Wird $\mathrm{CuCl}_{2}$ mit einem Äquivalent dmpaS ${ }_{2} \mathrm{H}$ in Ethanol umgesetzt, so entsteht eine gelbe Lösung, die sich sofort nach Zugabe von NaOMe entfärbt, was auf eine Reduktion von $\mathrm{Cu}^{\mathrm{II}}$ $\mathrm{zu} \mathrm{Cu}{ }^{\mathrm{I}}$ hindeutet. Aus der Reaktionslösung können durch Überschichten mit $\mathrm{Et}_{2} \mathrm{O}$ farblose Kristalle der $\mathrm{Cu}^{\mathrm{I}}$-Verbindung $\left[\mathrm{Cu}_{2}\left(\mathrm{dmpaS}_{2} \mathrm{H}\right)_{2} \mathrm{Cl}_{2}\right] \underline{22}$ erhalten werden (Gl. 28). Sie ist isostrukturell mit den Verbindungen $\left[\mathrm{Cu}_{2}\left(\mathrm{dppmS}_{2} \mathrm{H}\right)_{2} \mathrm{Cl}_{2}\right][16]$ und $\left[\mathrm{Cu}_{2}\left(\mathrm{Me}_{4} \mathrm{P}_{2} \mathrm{~S}_{2}\right)_{2} \mathrm{Cl}_{2}\right]$ [48], die in Kapitel 3.1.2 besprochen wurden.

$$
\mathrm{CuCl}_{2}+\mathrm{dmpaS}_{2} \mathrm{H} \underset{\mathrm{EtOH}}{\stackrel{\mathrm{NaOMe}}{\longrightarrow}}\left[\mathrm{Cu}_{2}\left(\operatorname{dmpaS}_{2} \mathrm{H}\right)_{2} \mathrm{Cl}_{2}\right] \underline{22}
$$

\section{Die Struktur von $\left[\mathrm{Cu}_{2}\left(\operatorname{dmpaS}_{2} \mathrm{H}\right)_{2} \mathrm{Cl}_{2}\right] \underline{22}$ :}

Abb. 38 zeigt den molekularen Aufbau von Verbindung 22, die orthorhombisch in der Raumgruppe Pbca kristallisiert. In $\underline{22}$ sind zwei $\left[\mathrm{Cu}\left(\mathrm{dmpaS}_{2} \mathrm{H}\right) \mathrm{Cl}\right]$-Einheiten durch ein Symmetriezentrum zu einem dimeren Komplex verknüpft. Gemäß Abb. 5 (a) chelatisiert der Ligand ein $\mathrm{Cu}$-Ion und bindet zusätzlich über ein Schwefelatom an ein zweites $\mathrm{Cu}$-Atom. Die verzerrt tetraedrische Koordinationssphäre der Kupferatome wird durch ein Schwefel- und ein Chloratom, die beide terminal koordiniert sind, und zwei weitere Schwefelatome, die die beiden $\mathrm{Cu}$-Atome $\mu_{2}$-artig verbrücken, gebildet, so daß ein planarer $\mathrm{Cu}_{2} \mathrm{~S}_{2}$-Ring entsteht.

In 22 sind die Längen der Cu-S-Bindungen mit 231 und 243 pm, der P-S-Bindungen mit 198 und 199 pm und der Cu-Cl-Bindungen mit 321 pm nahezu identisch mit denen, die in der Verbindung $\left[\mathrm{Cu}_{2}\left(\mathrm{dppmS}_{2} \mathrm{H}\right)_{2} \mathrm{Cl}_{2}\right][16]$ gefunden werden. In beiden Strukturen 


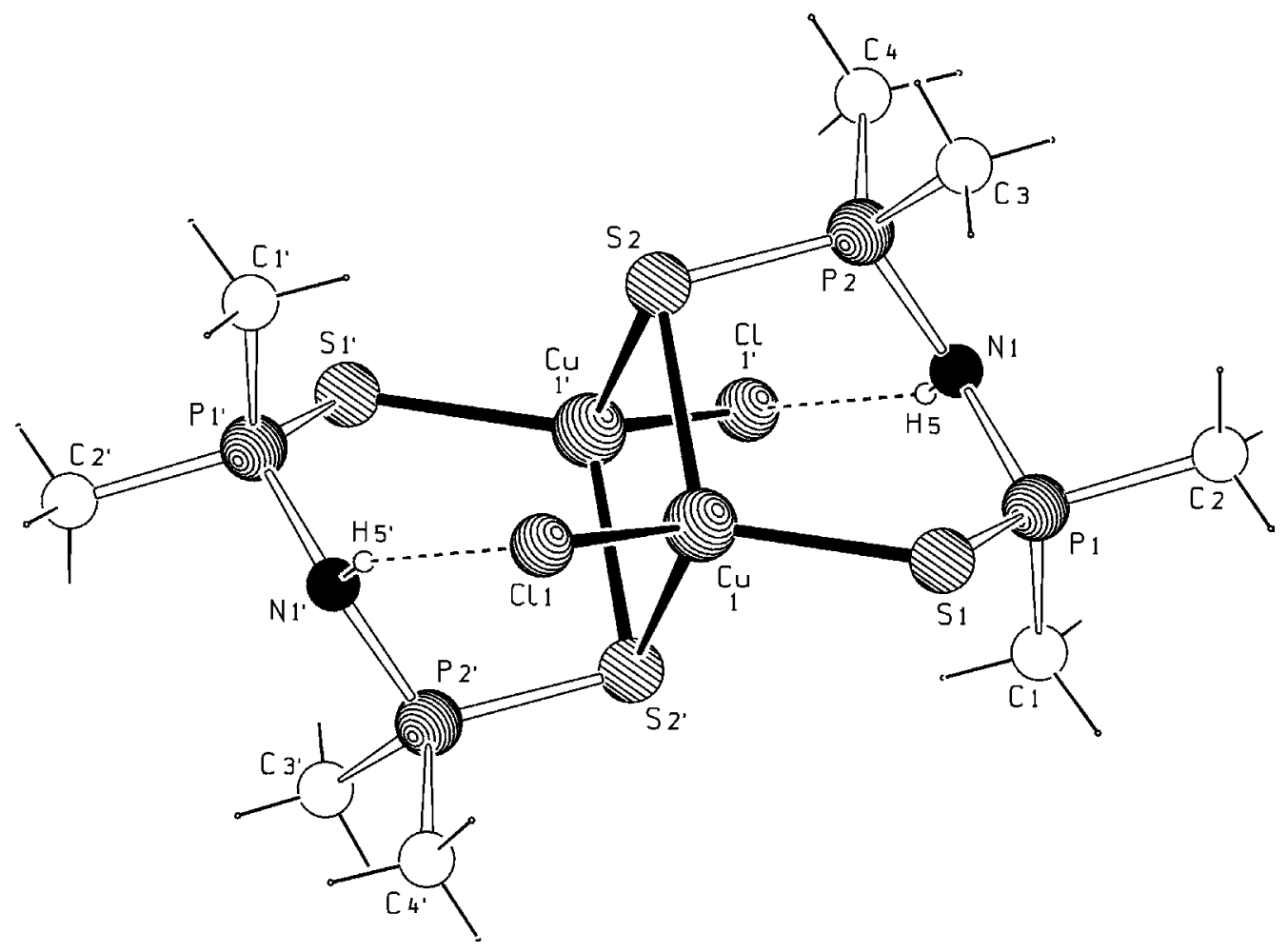

Abb. 38: Die Struktur von $\left[\mathrm{Cu}_{2}\left(\mathrm{dmpaS}_{2} \mathrm{H}\right)_{2} \mathrm{Cl}_{2}\right] \underline{22}$ im Kristall

hat die Art der Bindung des Schwefelatoms an das Metallion einen Einfluß auf die CuS-Bindungslänge, denn die Abstände der Cu-Ionen zu $\mu_{2}$-koordinierten Schwefelatomen sind um 12 bzw. 11 pm größer als zu den terminal koordinierten S-Atomen.

Die P-N-Abstände in $\underline{22}$ von 168 pm entsprechen den Längen von P-N-Einfachbindungen im ungebundenen Liganden [26], der im Festkörper über S ․H-Brücken zu einem Polymer verknüpft ist. Auch die beiden Chloroliganden in 22 bilden zusätzlich noch je eine Wasserstoffbrückenbindung zu den Amin-Wasserstoffatomen aus. Der Abstand des Chlordonoratoms zum Amin-Wasserstoffatom beträgt $255 \mathrm{pm}$ und ist im Vergleich zu anderen H-Brückenbindungen zwischen Chloroliganden und Aminwasserstoffatomen, wie sie z.B. im quadratisch-planaren Komplex $\left[\mathrm{Pd}\left(\mathrm{d}^{\mathrm{i}} \mathrm{PrpaS}_{2}\right)\left(\mathrm{d}^{\mathrm{i}} \mathrm{PrpaS}_{2} \mathrm{H}\right)\right] \mathrm{Cl}$ [119] mit Längen von ca. $224 \mathrm{pm}$ auftreten, recht lang. Es handelt sich folglich nur um eine sehr schwache H-Brückenbindung. 


\subsubsection{Die Synthesen und Strukturen von $\left[\mathrm{Cu}_{5}\left(\mathrm{dmpaS}_{2}\right)_{5}\right] \underline{23}$, $\left[\mathrm{Cu}_{8}\left(\operatorname{dmpaS}_{2}\right)_{6} \mathrm{Cl}_{2}\right] \underline{24}$ und $\left[\mathrm{Cu}_{10}\left(\operatorname{dmpaS}_{2}\right)_{6} \mathrm{Cl}_{4}\right] \underline{25}$}

Komplexe des Kupfers mit dem anionischen Liganden konnten in einer großen Variationsbreite synthetisiert werden. So gelang es, abhängig von den eingesetzten Mengenverhältnissen der Edukte $\mathrm{CuCl}$ und $\mathrm{dmpaS}_{2}{ }^{\ominus}$, die mehrkernigen Komplexe $\left[\mathrm{Cu}_{5}\left(\mathrm{dmpaS}_{2}\right)_{5}\right] \underline{23}$, $\left[\mathrm{Cu}_{8}\left(\mathrm{dmpaS}_{2}\right)_{6} \mathrm{Cl}_{2}\right] \underline{24}$ und $\left[\mathrm{Cu}_{10}\left(\mathrm{dmpaS}_{2}\right)_{6} \mathrm{Cl}_{4}\right] \underline{25}$ strukturell zu charakterisieren (Gln. 29, 30 und 31).

$$
\begin{aligned}
\mathrm{CuCl}+\left[\mathrm{Na}\left(\mathrm{dmpaS}_{2}\right)\right] \stackrel{\mathrm{THF}}{\longrightarrow}\left[\mathrm{Cu}_{5}\left(\mathrm{dmpaS}_{2}\right)_{5}\right] \underline{23} \\
6 / 5\left[\mathrm{Cu}_{5}\left(\mathrm{dmpaS}_{2}\right)_{5}\right] \underline{23}+2 \mathrm{CuCl} \stackrel{\mathrm{THF}}{\longrightarrow}\left[\mathrm{Cu}_{8}\left(\mathrm{dmpaS}_{2}\right)_{6} \mathrm{Cl}_{2}\right] \underline{24} \\
2 \mathrm{CuCl}+\left[\mathrm{Na}\left(\mathrm{dmpaS}_{2}\right)\right] \stackrel{\mathrm{THF}}{\longrightarrow}\left[\mathrm{Cu}_{10}\left(\mathrm{dmpaS}_{2}\right)_{6} \mathrm{Cl}_{4}\right] \underline{25}
\end{aligned}
$$

In Umsetzungen von $\mathrm{CuCl}$ mit $\mathrm{dmpaS}_{2}{ }^{\ominus}$ wurde jedoch niemals die Bildung einer trimeren Verbindung $\left[\mathrm{Cu}_{3}(\mathrm{~L})_{3}\right]$, die in analogen Reaktionen mit dem phenylsubstituierten Derivat dppaS $_{2}{ }^{\ominus}$ entsteht, beobachtet. In derartigen Reaktionen wird fast immer die fünfkernige Verbindung $\underline{23}$ als Hauptprodukt gebildet, während die Verbindungen $\underline{24}$ und $\underline{25}$ nur in geringen Mengen entstehen.

Die im folgenden besprochenen Verbindungen stellen weitere Beispiele für die Vielfalt der Koordinationsarten des Liganden $\operatorname{dmpaS}_{2}{ }^{\ominus}$ an Metallatome dar (vgl. Abb. 5 (c) bis (j)).

\section{Die Struktur von $\left[\mathrm{Cu}_{5}\left(\mathrm{dmpaS}_{2}\right)_{5}\right] \underline{23}$ :}

Das Grundgerüst der fünfkernigen Verbindung $\underline{23}$, die monoklin in der Raumgruppe $C 2 / c$ kristallisiert, besteht aus zwei $\mathrm{Cu}_{3} \mathrm{~S}_{3}$-Sechsringen, die durch $\mathrm{Cu}(1)$ zu einem Spirocyclus verknüpft sind (Abb. 39).

Vier $\mathrm{Cu}$-Atome sind annähernd trigonal-planar von je drei Schwefelatomen umgeben. $\mathrm{Cu}(1)$ besitzt dagegen eine verzerrt tetraedrische Koordinationssphäre. Jedes der CuIonen wird von einem Liganden chelatisiert, wobei zwei Koordinationsarten der Liganden unterschieden werden können: Vier der Liganden binden gemäß Abb. 5 (d) mit dem einen Schwefelatom endständig und mit dem anderen Schwefelatom $\mu_{2}$-verbrückend an die Metallatome. Zudem ist ein fünfter Ligand durch seine zweifach $\mu_{2}$-verbrückende Funktion an drei Cu-Ionen gebunden (Abb. 5 (f)).

Die Cu-S- und P-S-Bindungslängen sind auch in $\underline{23}$ von der Art der Verbrückung der Schwefelatome abhängig, denn Die Cu-S-Abstände der $\mu_{2}$-koordinierten S-Atome sind mit 225 pm um 3 pm länger als die Cu-S-Abstände mit terminal bindenden S-Atomen. Die P-N-Bindungslängen bleiben davon unbeeinflußt und betragen einheitlich 160 pm. Die 


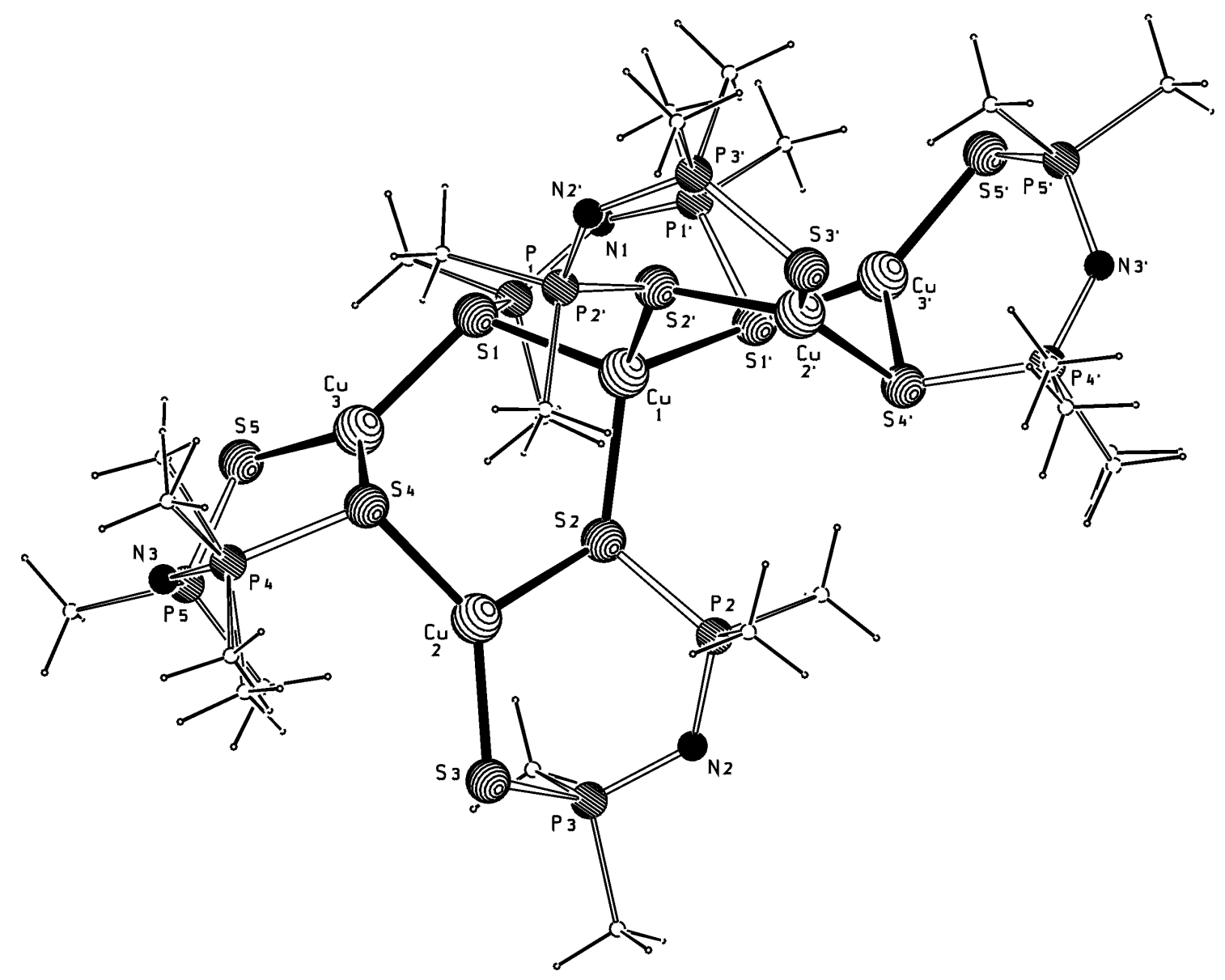

Abb. 39: Die Struktur von $\left[\mathrm{Cu}_{5}\left(\mathrm{dmpaS}_{2}\right)_{5}\right] \underline{23}$ im Kristall

gleiche Tendenz einer Bindungsverlängerung ist bei den P-S-Bindungen beobachtbar, denn die Länge der P- $\mu_{2}$-S-Bindungen beträgt 204 pm, während die P- $\mu_{1}$-S-Bindungen 202 pm lang sind. Da sich mit einer Erhöhung der Koordinationszahl des Metallions auch der Abstand zu den Liganddonoratomen vergrößert, sind die Abstände der $\mu_{2}$-verbrückenden S-Atome zum vierfach koordinierten $\mathrm{Cu}(1)$-Atom signifikant länger; sie betragen durchschnittlich 232 pm. Ein Vergleich der P-N- und P-S-Abstände von $\underline{23}$ mit denen, die im freien Liganden dppaS ${ }_{2} \mathrm{H}[70]$ bzw. seinem Methylester $\mathrm{MeS}-\mathrm{PPh}_{2}=\mathrm{NP}(\mathrm{S}) \mathrm{Ph}_{2}$ [105] gefunden werden, macht deutlich, daß es sich in $\underline{23}$ um Bindungen mit einer Bindungsordnung zwischen eins und zwei handelt, wobei die P-N-Bindungen mit einer Länge von 159 pm eher Doppelbindungscharakter und die P-S-Bindungen mit einer Länge von ca. 203 pm eher Einfachbindungscharakter besitzen. 
Die Struktur von $\left[\mathrm{Cu}_{8}\left(\operatorname{dmpaS}_{2}\right)_{6} \mathrm{Cl}_{2}\right] \underline{24}$ :

Nach Gl. 30 können nadelförmige Kristalle der Verbindung 24 isoliert werden, die monoklin in der Raumgruppe $P 2_{1} / n$ kristallisiert (Abb. 40). 24 ist aufgebaut aus acht $\mathrm{Cu}$-Ionen,

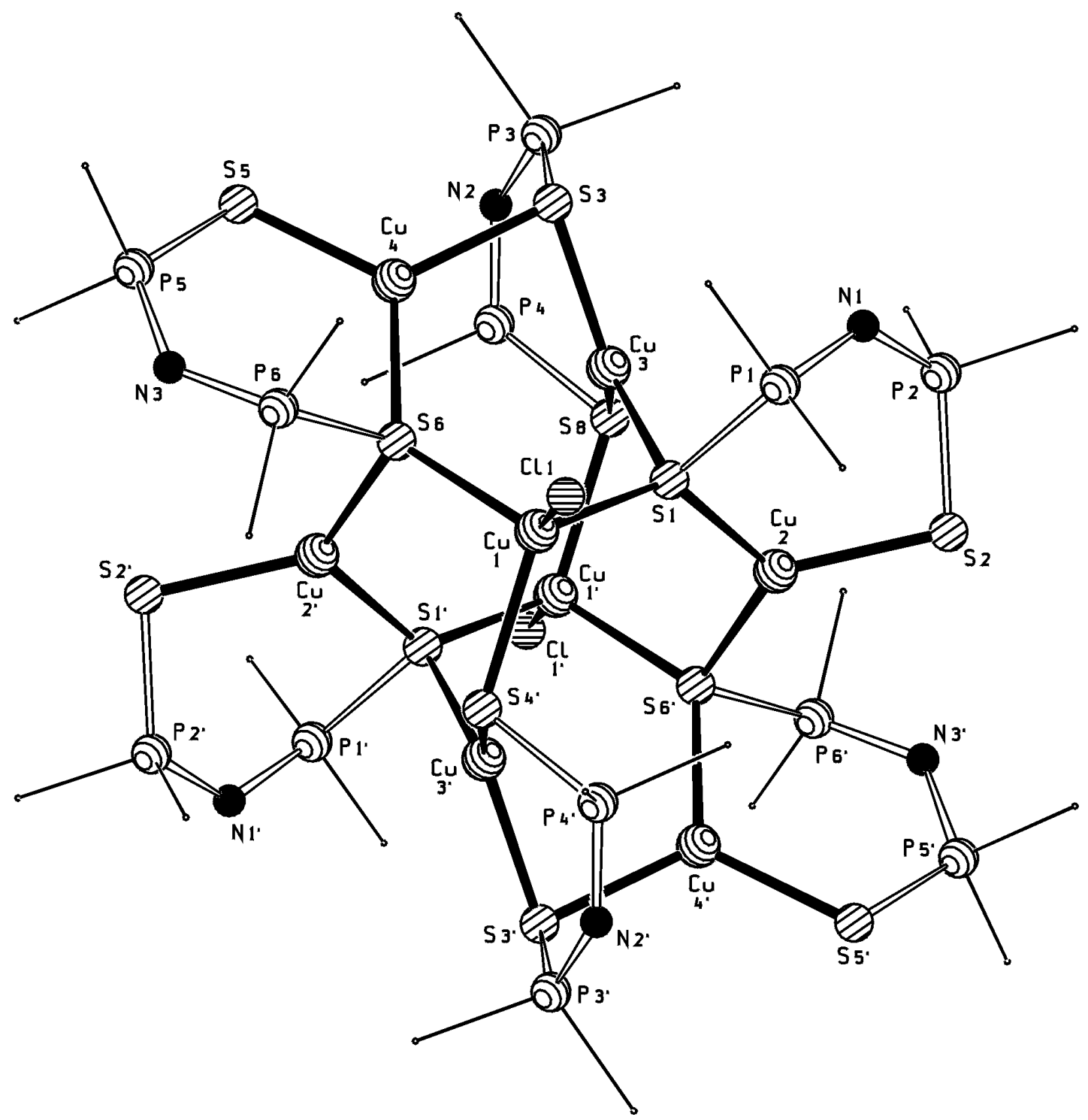

Abb. 40: Die Struktur von $\left[\mathrm{Cu}_{8}\left(\mathrm{dmpaS}_{2}\right)_{6} \mathrm{Cl}_{2}\right] \underline{24}$ im Kristall

an die sechs anionische $\operatorname{dmpaS}_{2}{ }^{\ominus}$-Liganden und zwei Chloroliganden gebunden sind. In Abb. 41 ist die Struktur von $\underline{24}$ gezeigt, in der das $\mathrm{Cu}_{8} \mathrm{~S}_{12} \mathrm{Cl}_{2}$-Gerüst verdeutlicht ist; die eingezeichneten $\mathrm{Cu}-\mathrm{Cu}$-Bindungen stellen keine echten Metall-Metall-Bindungen dar, sondern sollen nur die topologische Anordnung der $\mathrm{Cu}$-Atome hervorheben.

Wie in der Abbildung veranschaulicht, befinden sich sechs $\mathrm{Cu}$-Atome in den Ecken eines 
verzerrten Oktaeders; weiterhin sind zwei sich gegenüberliegende Oktaederflächen von je einem Cu-Ion überkappt, so daß die Anordnung der Cu-Atome als Oktaeder mit zwei flächenverknüpften Tetraedern beschrieben werden kann.

Die Schwefelatome S(1) und S(1') sind an zwei weitere sich gegenüberliegende Oktaederflächen $\mu_{3}$-artig koordiniert. Zudem befinden sich die zwei Atome $\mathrm{S}(4)$ und $\mathrm{S}\left(4^{\prime}\right)$ über zwei sich ebenfalls gegenüberliegenden Oktaederkanten. Eine Fläche und eine Kante jedes $\mathrm{Cu}$-Tetraeders ist von je einem $\mu_{3}$-bindenden $\mathrm{S}$-Atom überkappt $\left(\mathrm{S}(6), \mathrm{S}\left(6^{\prime}\right)\right)$. Die übrigen Schwefelatome und die Chloroliganden sind terminal an die Kupferatome gebunden, so daß sich für sechs $\mathrm{Cu}$-Ionen eine annähernd trigonal-planare und für die zwei Cu-Ionen $\mathrm{Cu}(1)$ und $\mathrm{Cu}\left(1^{\prime}\right)$ eine verzerrt tetraedrische Koordination ergibt.

Die achtkernige Verbindung zeigt einige Besonderheiten auf, denn einerseits ist die Anordnung der $\mathrm{Cu}$-Atome eher ungewöhnlich, da meist in achtkernigen $\mathrm{Cu}$-Komplexen die $\mathrm{Cu}$-Atome in den Ecken eines Würfels [120] oder eines quadratischen Antiprismas [121] positioniert sind und zum anderen sind in 24 zwei verschiedene Koordinationsmodi des Ligandensystems verwirklicht: die Schwefelatome zweier Liganden binden jeweils terminal und $\mu_{3}$-verbrückend (Abb. $5(\mathrm{~g})$ ) bzw. beide Schwefelatome eines dritten Liganden verbrücken $\mu_{2}$-artig, wie in Abb. 5 (f) dargestellt.

Wie es auch schon in den oben beschriebenen Verbindungen $\underline{22}$ und $\underline{23}$ der Fall ist, muß in 24 wiederum zwischen unterschiedlichen Bindungslängen der Cu-S-, P-S- und zudem der P-N-Bindungen differenziert werden. Je mehr Metallatome an ein Schwefelatom binden, desto länger werden die entsprechenden Cu-S- und P-S-Bindungen und umso kürzer werden die betreffenden P-N-Bindungen. Dieser Trend wird bei einem Vergleich der Cu-S- und P-S-Bindungslängen am deutlichsten.

Die Cu-S-Abstände für terminal, $\mu_{2}$ - und $\mu_{3}$-koordinierte Schwefelatome betragen $223 \mathrm{pm}$, 224 pm und 226 pm. Die entsprechenden P-S-Bindungen sind 201 pm, 205 pm und 207 pm lang. Eine analoge Verkürzung der betreffenden P-N-Bindungen, bei denen das Phophoratom an terminal, $\mu_{2^{-}}$und $\mu_{3}$-koordinierte Schwefelatome gebunden ist, zeigt sich weniger deutlich. Die P-N-Abstände betragen 160 pm, 159 pm und 158 pm. Auch sind in 24, wie bereits bei Verbindung 23 erwähnt, die Cu-S-Abstände bezüglich der tetraedrisch koordinierten $\mathrm{Cu}$-Atome mit durchschnittlich 236 pm signifikant länger, unabhängig davon, ob die Schwefelatome $\mu_{2}$ - oder $\mu_{3}$-artig koordiniert sind. 


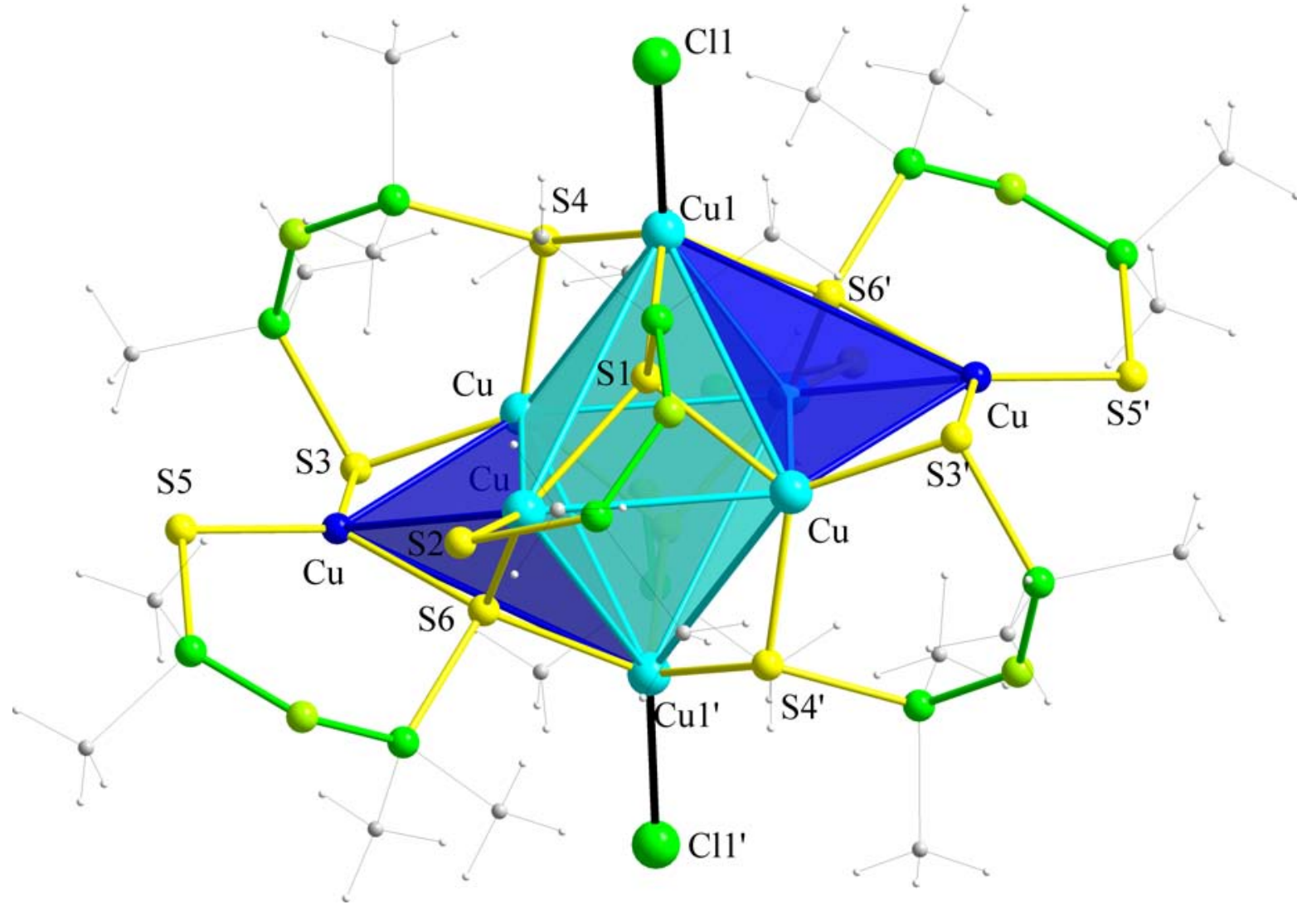

Abb. 41: Darstellung des molekularen Aufbaus von $\left[\mathrm{Cu}_{8}\left(\mathrm{dmpaS}_{2}\right)_{6} \mathrm{Cl}_{2}\right] \underline{24}$ 
Die Struktur von $\left[\mathrm{Cu}_{10}\left(\mathrm{dmpaS}_{2}\right)_{6} \mathrm{Cl}_{4}\right] \underline{25}$ :

Verbindung 25 kristallisiert triklin in der Raumgruppe $P \overline{1}$, deren Struktur in Abb. 42 gezeigt ist; die $\mathrm{Cu}-\mathrm{Cu}$-Bindungen sollen keine echten Bindungen darstellen, sondern nur die paarweise Anordnung der $\mathrm{Cu}$-Ionen verdeutlichen.

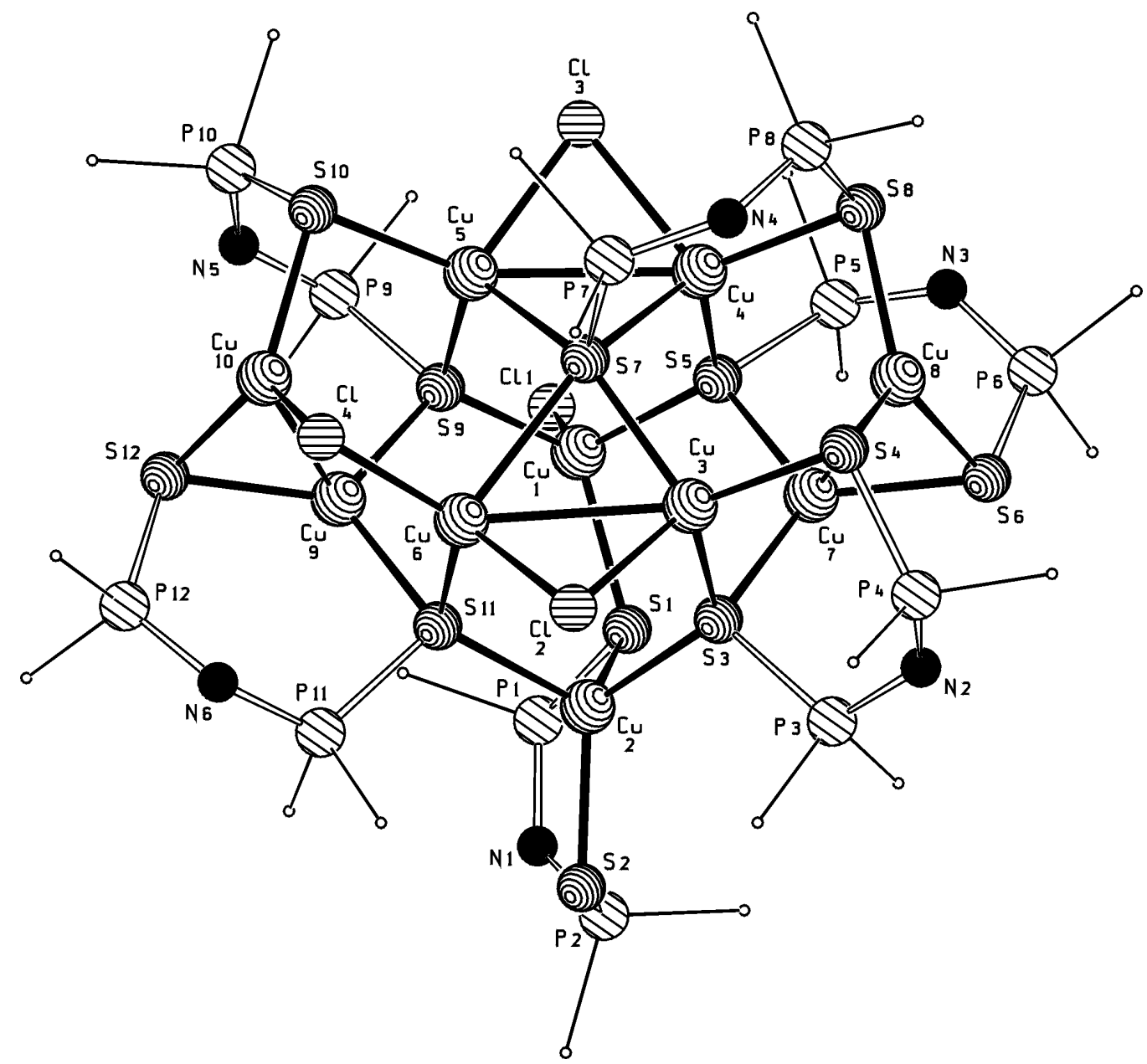

Abb. 42: Die Struktur von $\left[\mathrm{Cu}_{10}\left(\mathrm{dmpaS}_{2}\right)_{6} \mathrm{Cl}_{4}\right] \underline{25}$ im Kristall

Der Komplex besteht aus zehn $\mathrm{Cu}^{\mathrm{I}}$-Ionen, von denen acht paarweise mit $\mathrm{Cu}-\mathrm{Cu}$ Abständen von 262 bis $287 \mathrm{pm}$ zu einem $\mathrm{Cu}_{10}$-Polyeder angeordnet sind. Im Komplex sind weiterhin sechs anionische Ligandmoleküle und vier Chlorid-Ionen gebunden. Sechs $\mathrm{Cu}$-Atome sind verzerrt tetraedrisch und vier annähernd trigonal-planar koordiniert.

In Abb. 43 ist der $\mathrm{Cu}_{10}$-Polyeder mit den unterschiedlichen Koordinationsarten der Liganden dargestellt. Die Methylgruppen wurden aus Gründen der Übersichtlichkeit weggelassen. 


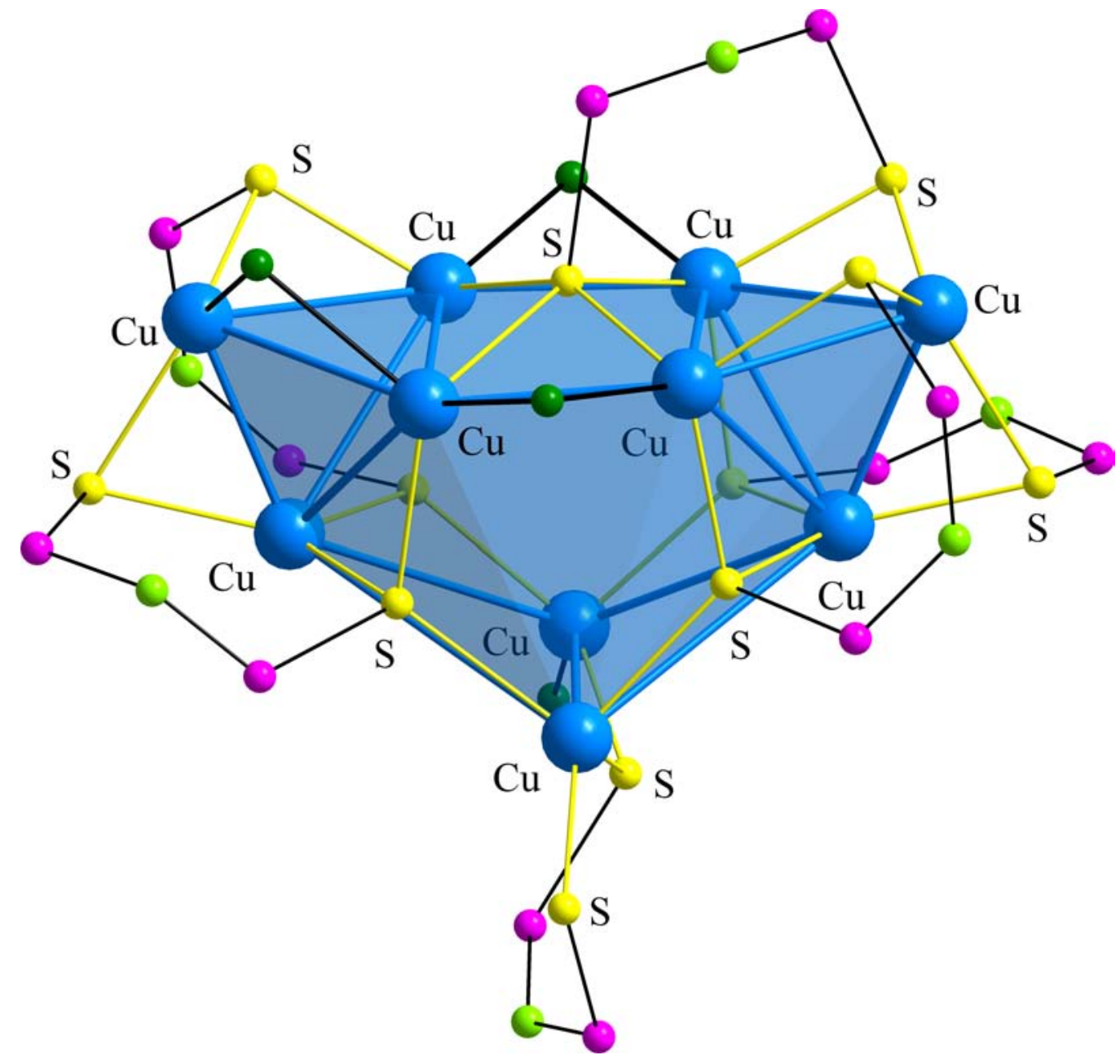

Abb. 43: Vereinfachte Darstellung der Molekülstruktur von $\left[\mathrm{Cu}_{10}\left(\mathrm{dmpaS}_{2}\right)_{6} \mathrm{Cl}_{4}\right] \underline{25}$

Unter den Chlorid-Ionen ist $\mathrm{Cl}(1)$ terminal gebunden, während $\mathrm{Cl}(2), \mathrm{Cl}(3)$ und $\mathrm{Cl}(4)$ $\mu_{2}$-verbrückend zwischen je zwei $\mathrm{Cu}$-Ionen wirken. Die Länge der $\mathrm{Cu}$-Cl-Bindungen ist abhängig von der Koordinationsart des Chloroliganden, denn das terminal gebundene Chloratom befindet sich in einem Abstand von 228 pm von $\mathrm{Cu}(1)$ entfernt, während die $\mu_{2}$-verbrückenden Chloroliganden Cu-Cl-Abstände von ca. $239 \mathrm{pm}$ aufweisen. Die in $\underline{25}$ vorliegenden $\mathrm{Cu}$-Cl-Abstände für die $\mu_{2}$-koordinierten Chloratome mit durchschnittlich 239 pm sind nicht ungewöhnlich, da sie ebenfalls den Abständen in Chlorokupraten wie $\left[\mathrm{Cu}_{4}(\mathrm{O})\left(\mu_{2}-\mathrm{Cl}\right)_{6} \mathrm{Cl}_{4}\right]^{4 \ominus}\left(\mathrm{Cu}-\mu_{2}-\mathrm{Cl}: 240 \mathrm{pm}\right)$ [87] ähneln.

In $\underline{25}$ sind die unterschiedlichen Varianten der Bindung des $\operatorname{dmpaS}_{2}{ }^{\ominus}$-Liganden auffällig. Im Komplex treten drei verschiedene Koordinationsmodi auf: entweder erfolgt die Bindung an zwei Metallatome gemäß Abb. 5 (d) oder der Ligand ist an insgesamt vier Metall- 
atome koodiniert, wie in Abb. 5 (i) dargestellt; weiterhin können fünf Metallionen an den Liganden gebunden sein (Abb.5 (j)).

Die Bindungslängen der Cu-S-, P-S- und P-N-Bindungen in $\underline{25}$ sind in Abhängigkeit von der Koordinationszahl (KoZa) des entsprechenden $\mathrm{Cu}$-Ions und der Koordinationsart der Schwefelatome in Tab. 1 gezeigt.

\begin{tabular}{|c|c|c|c|c|}
\hline $\begin{array}{c}\text { KoZa } \\
\text { von Cu }\end{array}$ & Verbrückung & $\begin{array}{c}\text { Cu-S-Abstand } \\
{[\mathrm{pm}]}\end{array}$ & $\begin{array}{c}\text { P-S-Abstand } \\
{[\mathrm{pm}]}\end{array}$ & $\begin{array}{c}\text { P-N-Abstand } \\
{[\mathrm{pm}]}\end{array}$ \\
\hline 3 & $\mu_{2}-\mathrm{S}$ & $224-228$ & $204-207$ & $158-160$ \\
3 & $\mu_{3}-\mathrm{S}$ & $223-224$ & $206-207$ & $158-159$ \\
4 & $\mu_{1}-\mathrm{S}$ & 226 & 201 & 160 \\
4 & $\mu_{2}-\mathrm{S}$ & $225-234$ & $203-205$ & $158-160$ \\
4 & $\mu_{3}-\mathrm{S}$ & $230-240$ & $206-207$ & $158-159$ \\
4 & $\mu_{4}-\mathrm{S}$ & $235-262$ & 208 & 159 \\
4 & $\mu_{1}-$ bis $\mu_{4}-\mathrm{S}$ & $225-262$ & $201-208$ & $158-160$ \\
\hline
\end{tabular}

Tab. 1: Bindungslängen in 25 in Abhängigkeit von der Verbrückung der Schwefelatome [pm]

Die Verlängerung der Cu-S-Bindungen bei einer Erhöhung der Koordinationszahl des Metalls ist deutlich erkennbar. Jedoch ist bei einem Vergleich der P-S-Bindungslängen die Unterscheidung der Bindungsart des Schwefelatoms nur bei einer Koordinationszahl von vier eindeutig. Hier kann eine sichtliche Abstufung bezüglich terminaler, $\mu_{2^{-}}, \mu_{3^{-}}$und $\mu_{4}$-Koordination festgestellt werden, die dagegen nicht beobachtet werden kann, wenn das betreffende $\mathrm{Cu}$-Atom eine Koordinationszahl von drei aufweist. Die P-N-Bindungslängen bleiben annähernd unbeeinflußt von der Änderung der Bindungsart des an das entsprechende Phosphoratom gebundenen Schwefelatoms. 


\subsubsection{Die Synthese und Struktur von $\left[\mathrm{KCu}\left(\operatorname{dmpaS}_{2}\right)_{2}(\operatorname{thf})\right]_{\infty} \underline{26}$}

Bei einer Variation des Deprotonierungsmittels und der Eduktverhältnisse können nicht nur fünf- bis zehnkernige Komplexverbindungen synthetisiert werden, es können sich auch polymere Strukturen ausbilden.

Wird der Ligand statt mit $\mathrm{NaN}\left(\mathrm{SiMe}_{3}\right)_{2}$ mit $\mathrm{KO}^{\mathrm{t}} \mathrm{Bu}$ deprotoniert und in einfachem Überschuß mit $\mathrm{CuCl}$ versetzt, so bildet sich die polymere Verbindung $\left[\mathrm{KCu}\left(\mathrm{dmpaS}_{2}\right)_{2}(\mathrm{thf})\right]_{\infty}$ $\underline{26}$ (Gl. 32).

$$
\mathrm{CuCl}+2\left[\mathrm{~K}\left(\mathrm{dmpaS}_{2}\right)\right] \stackrel{\mathrm{THF}}{\longrightarrow}\left[\mathrm{KCu}\left(\mathrm{dmpaS}_{2}\right)_{2}(\mathrm{thf})\right]_{\infty} \underline{26}
$$

Die Struktur von $\left[\mathrm{KCu}\left(\operatorname{dmpaS}_{2}\right)_{2}(\text { thf })\right]_{\infty} \underline{26}$ :

Die polymere Verbindung $\underline{26}$ kristallisiert monoklin in der Raumgruppe $P 2_{1} / c$ mit einer $\left[\mathrm{KCu}\left(\mathrm{dmpaS}_{2}\right)_{2}(\mathrm{thf})\right]$-Einheit in der asymmetrischen Einheit [55]. In Abb. 44 ist ein Ausschnitt aus der unendlichen Molekülkette dargestellt.

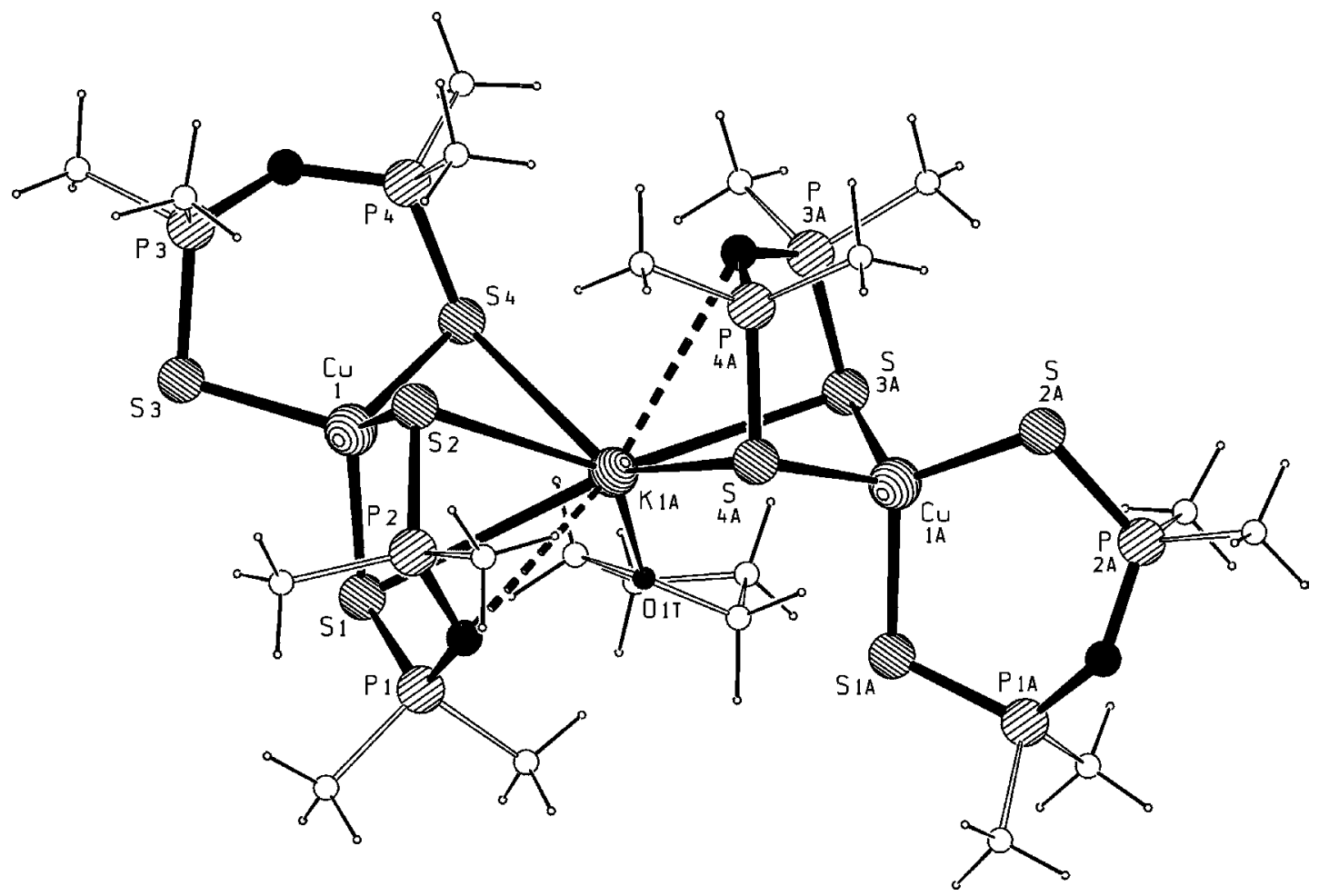

Abb. 44: Die Struktur von $\left[\mathrm{KCu}\left(\mathrm{dmpaS}_{2}\right)_{2}(\text { thf })\right]_{\infty} \underline{26}$ im Kristall (Ausschnitt) 
Der Komplex ist aus $\mathrm{Cu}(\mathrm{L})_{2}$-Einheiten aufgebaut, welche durch Kaliumionen zu einer unendlichen Molekülkette miteinander verknüpft werden. Die $\mathrm{Cu}$-Ionen besitzen eine verzerrt tetraedrische Koordinationsgeometrie, während die K-Atome von je fünf Schwefelatomen umgeben sind. Die verzerrt trigonal-prismatische Koordinationssphäre der K-Ionen wird durch ein weiteres, koordiniertes THF-Molekül ergänzt. Da zusätzlich zwei Amido-Stickstoffatome auf jedes K-Atom hin gerichtet sind, kann in diesem Fall von einer [6+2]-Koordination gesprochen werden. Wie die $\mathrm{Cu}(\mathrm{L})_{2}$-Einheiten durch die K-Ionen verbunden werden zeigt Abb. 45. Die $\mathrm{K}-\mathrm{Cu}(\mathrm{L})_{2}-\mathrm{K}-\mathrm{Cu}(\mathrm{L})_{2}$-Einheiten sind alternierend angeordnet, was zur Ausbildung der Kettenstruktur führt.

Auch in $\underline{26}$ werden zwei neue Koordinationsweisen des Liganden beobachtet. Zum einen kann der Ligand gemäß Abb. 5 (e) so an zwei Metallionen binden, daß beide Schwefelatome zwischen den beiden Metallionen $\mu_{2}$-artig verbrücken. Desweiteren wird eine Koordinationsmöglichkeit beobachtet, wie in Abb. 5 (h) dargestellt, bei der ein Schwefelatom $\mu_{3}$-artig an drei Metallatome und das andere S-Atom $\mu_{2}$-artig an zwei dieser Metallatome binden.

Entsprechend der unterschiedlichen Verbrückungen der Schwefelatome zwischen den $\mathrm{Cu}-$ und K-Ionen nehmen die Bindungslängen der Cu-S- und der P-S-Bindungen in der Reihenfolge $\mathrm{Cu}-\mu_{3}-\mathrm{S}(245 \mathrm{pm}) \succ \mathrm{Cu}-\mu_{2}-\mathrm{S}(234 \mathrm{pm})$ bzw. P- $\mu_{3}-\mathrm{S}(201 \mathrm{pm}) \succ \mathrm{P}-\mu_{2}-\mathrm{S}(200 \mathrm{pm})$ ab, wobei die Tendenz im Falle der P-S-Bindungen nur schwach ausgeprägt ist. 


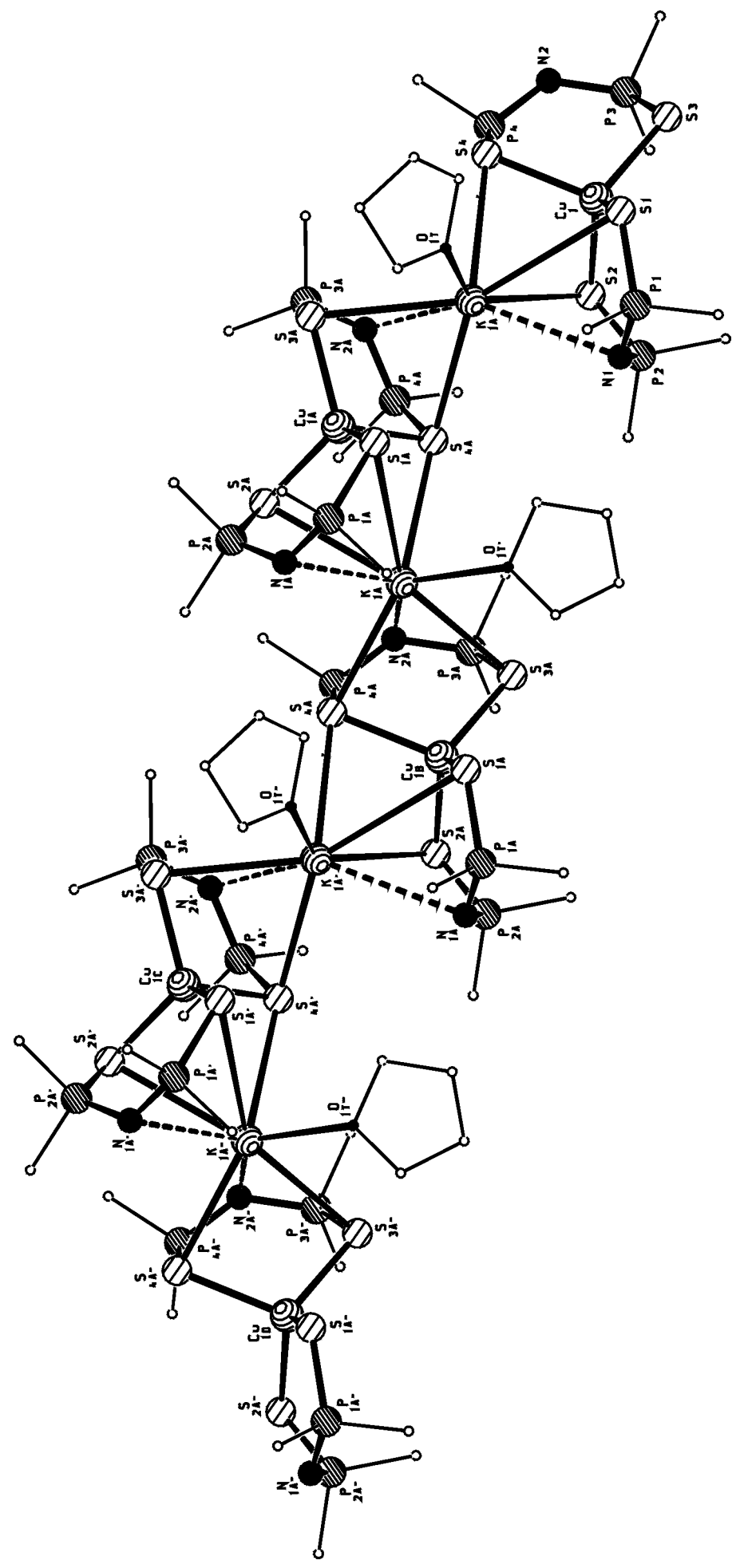

Abb. 45: Die Struktur von $\left[\mathrm{KCu}\left(\mathrm{dmpaS}_{2}\right)_{2}(\text { thf })\right]_{\infty} \underline{26}$ im Kristall (Ausschnitt) 


\subsubsection{NMR-spektroskopische Untersuchungen}

Zur weiteren Charakterisierung und zur Untersuchung des Lösungsverhaltens der isolierten Komplexe wurden ${ }^{31} \mathrm{P}-\mathrm{NMR}$-Spektren von Lösungen einiger Verbindungen aufgenommen.

Das Verhältnis $\mathrm{CuCl}$ zu Ligand hat einen Einfluß auf den Wert der chemischen Verschiebungen der Phosphoratome, denn ${ }^{31} \mathrm{P}-\mathrm{NMR}$-spektroskopische Untersuchungen ergeben, daß Verbindung 23 (Cu-Ligand-Verhältnis 1:1) in $\mathrm{CDCl}_{3}$ nur ein scharfes Signal bei $\delta=36,84$ ppm zeigt, während für Verbindung $\underline{24}$ (Cu-Ligand-Verhältnis 1,33) ein sehr breites Singulett mit einer chemischen Verschiebung von 34,20 ppm und für Verbindung 25 (Cu-Ligand-Verhälnis 1,66) ebenfalls ein breites Signal bei $+40{ }^{\circ} \mathrm{C}$ mit $\delta=33,73 \mathrm{ppm}$ beobachtet werden kann. Wird ein Cu-Ligand-Verhältnis von 0,5 gewählt, wie es z.B. in Verbindung 26 auftritt, so wird ein scharfes Singulett bei $\delta=38,41 \mathrm{ppm}\left(\mathrm{THF}, \mathrm{C}_{6} \mathrm{D}_{6}\right)$ beobachtet. Mit der Erhöhung des Cu-Ligand-Verhälnisses wird demnach im ${ }^{31} \mathrm{P}-\mathrm{NMR}$ Spektrum eine Verschiebung zu höherem Feld festgestellt.

Da breite ${ }^{31}$ P-NMR-Signale auf in der NMR-Zeit-Skala schnelle Austauschprozesse hinweisen, wurden von 25 mehrere Spektren in Abhängigkeit von der Temperatur aufgenommen, die in Abb. 47 dargestellt sind.

Bei $+40{ }^{\circ} \mathrm{C}$ zeigt das ${ }^{31} \mathrm{P}-\mathrm{NMR}-$ Spektrum der in THF und $\mathrm{CDCl}_{3}$ gelösten Verbindung $\underline{25}$ ein breites Singulett, das sich bei Verringerung der Temperatur zunächst in zwei ebenfalls breite Signale aufspaltet (bei $-30{ }^{\circ} \mathrm{C}: \delta=35,94 \mathrm{ppm}$ und 31,45 ppm). Schließlich können bei $-70{ }^{\circ} \mathrm{C}$ drei Signale bei $\delta=36,48 \mathrm{ppm}, 32,55 \mathrm{ppm}$ und 31,23 ppm unterschieden werden.

Diese Ergebnisse lassen vermuten, daß bei Raumtemperatur zwar mehrere nicht äquivalente Phosphoratome nebeneinander vorliegen, jedoch ihre Austauschgeschwindigkeit so groß ist, daß sie mit NMR-spektroskopischen Messungen nicht unterschieden werden können. Bei tieferen Temperaturen wird nun diese Austauschgeschwindigkeit soweit verringert, daß in den Spektren mehrere Signale von Phosphoratomen mit unterschiedlicher chemischer Umgebung zu erkennen sind. Weiterhin läßt die Beobachtung, daß bei einem Cu-Ligand-Verhältnis von 1:1 nur ein scharfes Signal auftritt, den Schluß zu, daß sich nur Phosphoratome mit gleicher chemischer Umgebung in Lösung befinden und die in Lösung befindliche Spezies ebenfalls in einem Cu-Ligand-Verhältnis von 1:1 vorliegt. Möglicherweise handelt es sich um die Spezies $\left[\mathrm{Cu}\left(\mathrm{dmpaS}_{2}\right)(\mathrm{thf})\right]$, in der der Ligand das $\mathrm{Cu}$-Ion chelatisiert und die für $\mathrm{Cu}^{\mathrm{I}}$ oft angetroffene Koordinationszahl von drei erreicht wird (vgl. Kapitel 3.4.5). Über das Lösungsverhalten von $\underline{24}$ und $\underline{25}$ und die Struktur 

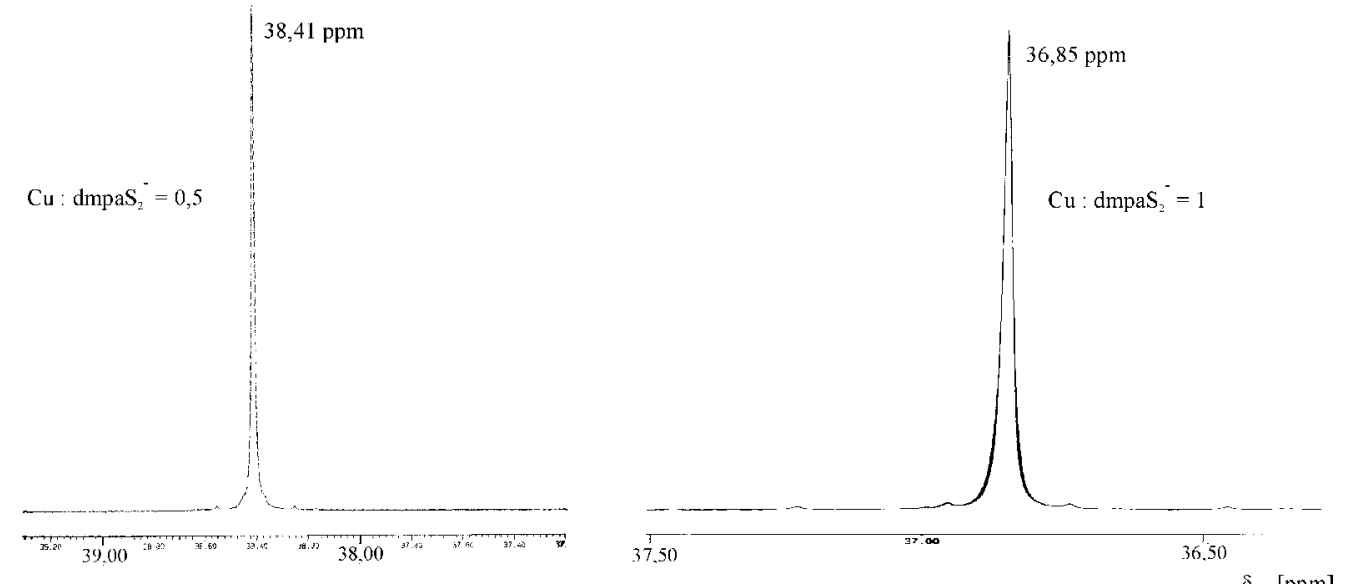

$\mathrm{Cu}: \mathrm{dmpaS}_{2}^{-}=1,33$
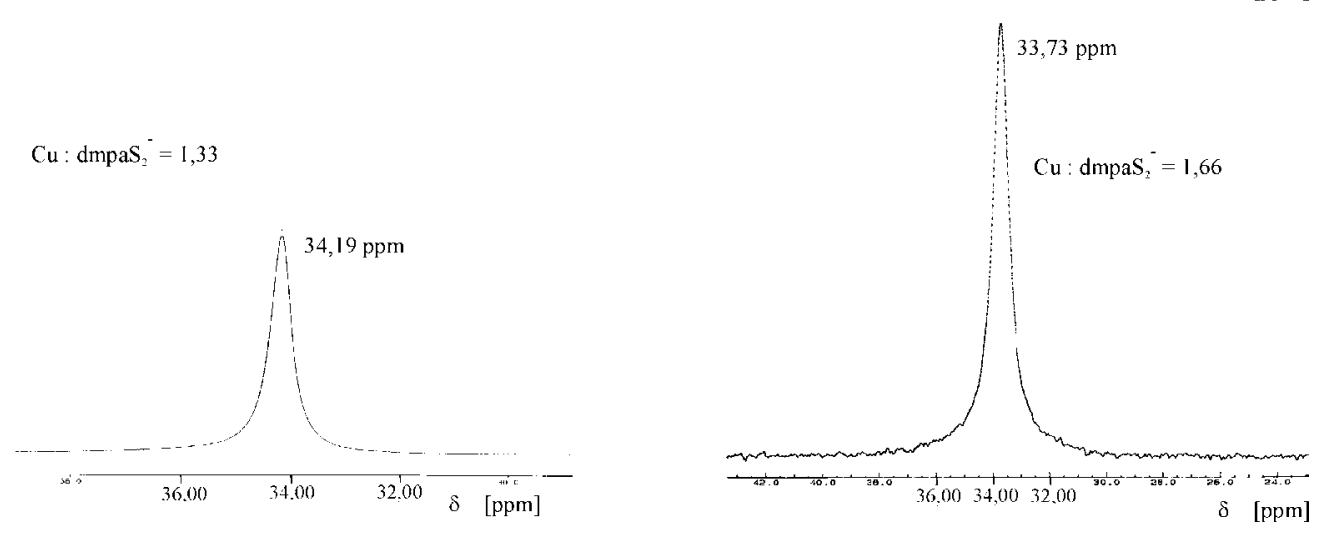

Abb. 46: ${ }^{31} \mathrm{P}-\mathrm{NMR}$-Spektren der Lösungen von $\mathrm{CuCl}$ und $\mathrm{dmpaS}_{2}{ }^{\ominus}$ in verschiedenen Molverhältnissen 


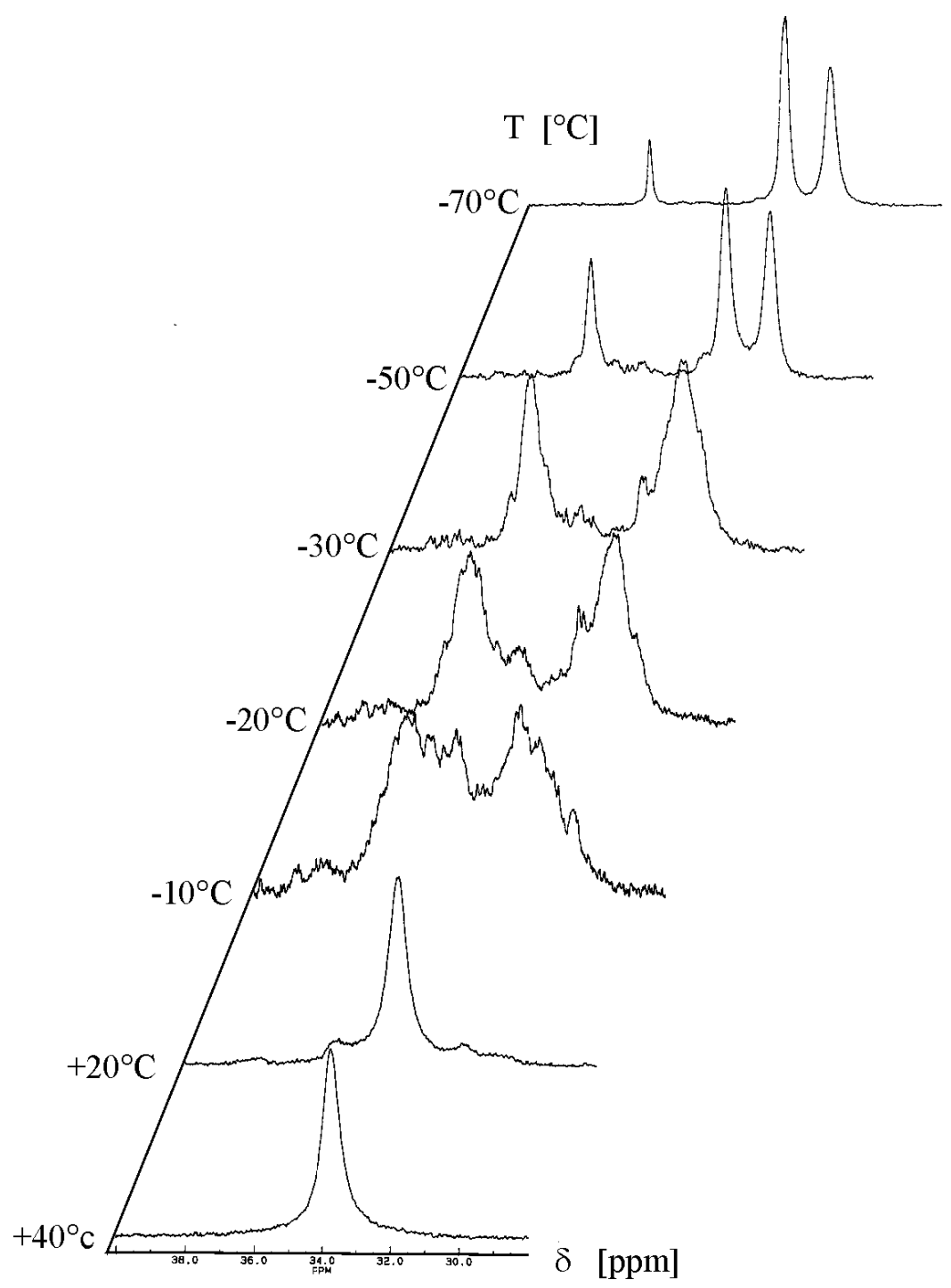

Abb. 47: Temperaturabhängige ${ }^{31} \mathrm{P}-\mathrm{NMR}-$ Spektren von $\left[\mathrm{Cu}_{10}\left(\mathrm{dmpaS}_{2}\right)_{6} \mathrm{Cl}_{4}\right] \underline{25}$ in $\mathrm{CDCl}_{3}$ 
der verschieden detektierten Spezies kann jedoch nur durch ${ }^{31} \mathrm{P}-\mathrm{NMR}$-spektroskopische Messungen keine sichere Aussage getroffen werden. Denkbar wären auch andere $\mathrm{Cu}_{\mathrm{m}} \mathrm{L}_{\mathrm{n}}$ Verbindungen mit unterschiedlicher Anzahl an Liganden ( $\mathrm{L}=\mathrm{LM}, \mathrm{Cl}$, dmpaS $\mathrm{S}_{2}$ ), wie z.B. $\left[\mathrm{Cu}\left(\mathrm{dmpaS}_{2}\right)(\text { thf })_{2}\right]$ oder $\left[\mathrm{Cu}\left(\mathrm{dmpaS}_{2}\right) \mathrm{Cl}\right]^{\ominus}$ oder sogar Komplexagglomerate mit mehreren $\mathrm{Cu}$-Ionen. Die beobachteten Hochfeldverschiebungen der beschriebenen Komplexverbindungen im Vergleich zum Natrium- oder Kaliumsalz des Liganden ( $\delta=43,8$ ppm [23] bzw. 44,0 ppm (MeOD) [26]) unterstützen zumindest die Annahme, daß die Kupferionen vom Liganden komplexiert werden. 


\subsection{Orientierende Versuche}

Um weitere komplexchemische Eigenschaften der Amido-Ligandensysteme gegenüber anderen Metallen zu untersuchen, wurden Reaktionen mit "späten" Übergangsmetallen wie $\mathrm{Hg}^{\mathrm{II}}$ durchgeführt.

\subsubsection{Die Synthese und Struktur von $\left[\mathrm{Hg}\left(\operatorname{dmpaS}_{2}\right)_{2}\right] \underline{27}$}

Da bisher nur ein Komplex von Quecksilber mit Diorganodiphosphorylamiden, die Verbindung $\left[\mathrm{Hg}\left(\mathrm{dppaSe}_{2}\right)_{2}\right]$ [31], strukturell charakterisiert ist, wurden Umsetzungen von $\mathrm{HgCl}_{2}$ mit $\operatorname{dmpaS}_{2}{ }^{\ominus}$ durchgeführt.

Durch die Reaktion von $\mathrm{HgCl}_{2}$ mit äquimolaren Mengen $\left[\mathrm{Na}\left(\mathrm{dmpaS}_{2}\right)\right]$ konnte die homoleptische Verbindung $\left[\mathrm{Hg}\left(\mathrm{dmpaS}_{2}\right)_{2}\right] \underline{27}$ isoliert werden (Gl. 33). Trotz des Mengenverhältnisses der Edukte von 1:1 bildet sich Verbindung 27, was möglicherweise auf die große Affinität des relativ weichen $\mathrm{Hg}^{\mathrm{II}}$-Ions gegenüber den weichen Schwefelatomen zurückzuführen ist.

$$
\mathrm{HgCl}_{2}+\left[\mathrm{Na}\left(\mathrm{dmpaS}_{2}\right)\right] \stackrel{\mathrm{THF}}{\longrightarrow}\left[\mathrm{Hg}\left(\mathrm{dmpaS}_{2}\right)_{2}\right] \underline{27}
$$

\section{Die Struktur von $\left[\mathrm{Hg}\left(\operatorname{dmpaS}_{2}\right)_{2}\right] \underline{\mathbf{2 7}}$ :}

In Abb. 48 ist Verbindung 27 dargestellt, die monoklin in der Raumgruppe $C 2 / c$ kristallisiert. Das Metallzentrum ist verzerrt tetraedrisch von vier terminal bindenden Schwefelatomen der Liganden umgeben, so daß sich zwei sechsgliedrige $\mathrm{HgP}_{2} \mathrm{~S}_{2}$-Ringe ausbilden (vgl. Abb. 5 (b)).

Die Hg-S-Bindungen sind 250 und 257 pm lang und liegen im Bereich der Hg-S-Abstände, die in der analog aufgebauten Verbindung $\left[\mathrm{Hg}\left(\mathrm{SP}\left(\mathrm{O}^{\mathrm{i}} \mathrm{Pr}\right)_{2} \mathrm{NC}(\mathrm{S}) \mathrm{Ph}\right]\right.$ [122] gefunden werden. Ein Vergleich der P-S- und P-N-Bindungslängen mit der isostrukturellen Verbindung $\left[\mathrm{Fe}\left(\mathrm{dmpaS}_{2}\right)_{2}\right]$ [34] zeigt, daß in beiden Fällen nahezu identische P-S- und P-NBindungslängen auftreten. In $\underline{27}$ betragen die P-S- bzw. die P-N-Bindungslängen 203 bzw. 159 pm, was eine $\pi$-Elektronen-Delokalisierung in $\underline{27}$ über die SPNPS-Systeme nahelegt. 


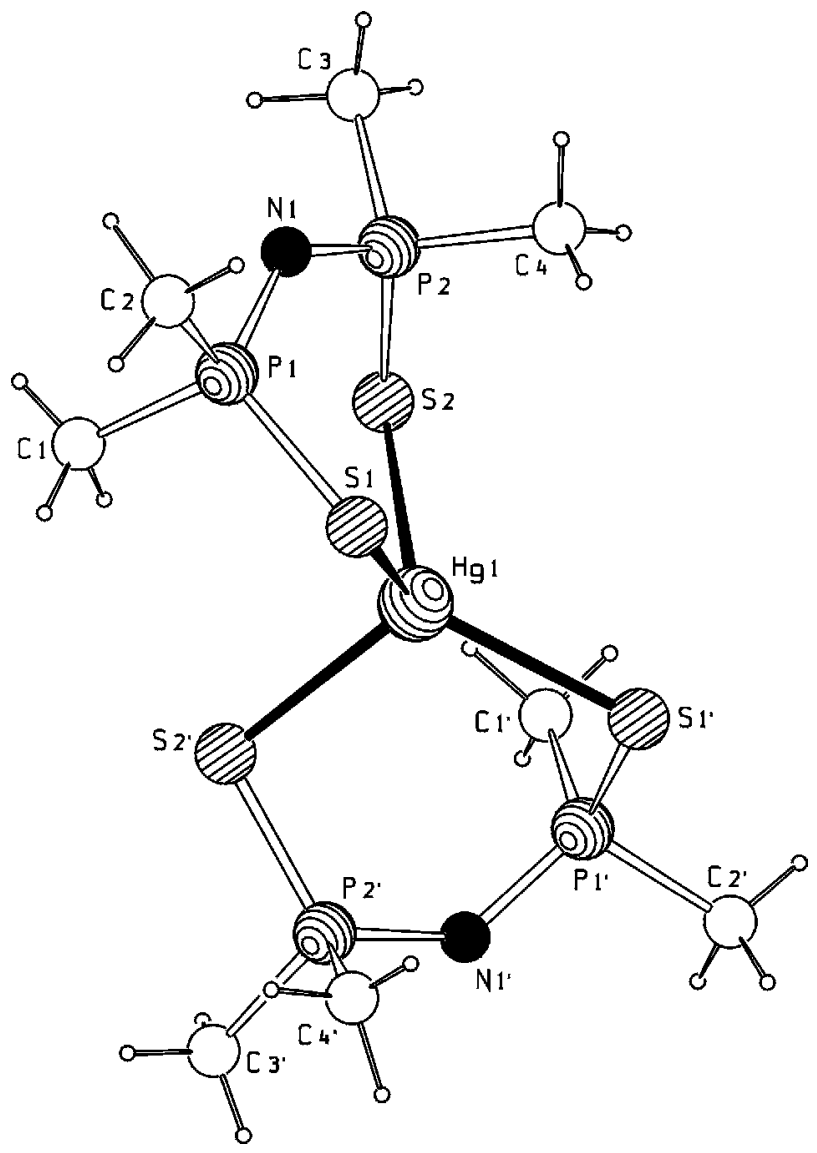

Abb. 48: Die Struktur von $\left[\mathrm{Hg}\left(\mathrm{dmpaS}_{2}\right)_{2}\right] \underline{27}$ im Kristall 


\section{Experimenteller Teil}

\subsection{Allgemeines}

Arbeitstechnik:

Aufgrund der Hydrolyseempfindlichkeit von einigen dargestellten und verwendeten Reagenzien werden alle Arbeitsschritte an einer Vakuumapparatur unter Stickstoffatmosphäre durchgeführt. Sämtliche Reaktionsgefäße werden mittels der Schlenk-Technik über Schlauchverbindungen an die Vakuumapparatur angeschlossen, mehrfach auf einen Druck von $10^{-3}$ torr evakuiert und danach mit Stickstoff (Reinheitsgrad 5.0) befüllt.

\section{Lösungsmittel:}

Alle verwendeten Lösungsmittel sind wasser- und sauerstofffrei und werden unter Stickstoffatmosphäre aufbewahrt. THF, Toluol, Heptan und Diethylether werden mit Natrium und Benzophenon bis zur Violettfärbung getrocknet (Ketyltrocknung) und anschließend abdestilliert. Methanol und Ethanol werden mit Natrium getrocknet und destilliert, Dichlormethan wird mehrere Stunden mit Phosphorpentoxid unter Rückfluß erhitzt und destilliert.

\section{Spektroskopische Untersuchungen:}

\section{Kernresonanzspektren:}

Die Kernresonanzspektren werden mit den Geräten AVANCE 200, AM 250, MSL 400 und AVANCE 500 der Firma Bruker bei Raumtemperatur aufgenommen. Die Meßfrequenz für die ${ }^{1}$ H-NMR-Spektren beträgt 200,13 MHz, für die ${ }^{31} \mathrm{P}-\mathrm{NMR}$-Spektren 161,96 MHz $\left({ }^{1} \mathrm{H}\right.$-entkoppelt) und für die ${ }^{77} \mathrm{Se}-\mathrm{NMR}-\mathrm{Spektren} 76,29 \mathrm{MHz}\left({ }^{1} \mathrm{H}\right.$-entkoppelt). Als interner Standard werden $\mathrm{CDCl}_{3}, \mathrm{C}_{6} \mathrm{D}_{6}$ oder THF- $\mathrm{d}_{8}$ verwendet. Die chemischen Verschiebungen sind in ppm angegeben und beziehen sich bei den ${ }^{1} \mathrm{H}-\mathrm{NMR}-\mathrm{Spektren}$ auf den externen Standard Tetramethylsilan, bei den ${ }^{31} \mathrm{P}-\mathrm{NMR}$-Spektren auf 85\%-ige Phosphorsäure und bei den ${ }^{77}$ Se-NMR-Spektren auf Dimethylselenid. Positive Werte relativ zum externen Standard entsprechen Tieffeldverschiebungen, negative entsprechen Hochfeldverschiebungen.

\section{IR-Spektren:}

Die IR-Spektren eines KBr-Presslings werden mit dem Gerät Bio-FTS-7 der Firma Biorad aufgenommen. Die angegebenen Wellenzahlen, bei denen eine charakteristische Absorbtion beobachtet wird, sind in $\mathrm{cm}^{-1}$ angegeben. 


\section{Massenspektrometrie:}

Die Erstellung der Massenspektren ist mit Hilfe eines Finnigan MAT System 8200 und Finnigan MAT System 95 Spektrometers bei einer Elektronenanregungsenergie von 70 eV erfolgt. Als Ionisierungsmethode wurde die Elektronenstoßionisation angewandt.

\subsection{Darstellung der Ausgangsverbindungen}

Die Verbindungen Bis(diphenylthiophosphoryl)amin $\left(\operatorname{dppaS}_{2} \mathrm{H}\right)$, Bis(diphenylselenophosphoryl)amin (dppaSe $\left.{ }_{2} \mathrm{H}\right)$, Bis(dimethylthiophosphoryl)amin $\left(\operatorname{dmpaS}_{2} \mathrm{H}\right)$, Bis(diphenylthiophosphoryl)methan $\left(\mathrm{dppmS}_{2} \mathrm{H}\right)$ und Bis(diphenylphosphino)amin (dppaH) wurde nach bekannten Literaturvorschriften synthetisiert [8,21-24,123].

\subsubsection{Darstellung von Bis(diphenylphosphoryl)amin $\left(\operatorname{dppaO}_{2} \mathbf{H}\right)[123]$}

Zu einer Lösung aus 10,0 g (25,9 mmol) Bis(diphenylphosphino)amin (dppaH) in $125 \mathrm{ml}$ THF werden bei Raumtemperatur langsam 5,67 ml (64,8 mmol) einer 35\%-igen wässrigen Wasserstoffperoxidlösung zugetropft und 15 min lang gerührt. Der gebildete weiße Niederschlag wird abfiltriert, mit insgesamt $80 \mathrm{ml}$ THF gewaschen und getrocknet.

Ausbeute: 10,8 g (25,9 mmol, 100\%)

${ }^{31} \mathrm{P}-\mathrm{NMR}\left(\mathrm{CDCl}_{3}\right): \delta=20,8 \mathrm{ppm}(\mathrm{s})$

IR-Spektrum (KBr) $\left[\mathrm{cm}^{-1}\right]: \nu(\mathrm{OH}) 3430 \mathrm{~m}, \mathrm{br}, \nu(\mathrm{PPh}) 1487 \mathrm{~s}, 1439 \mathrm{vs}, \nu(\mathrm{PO}) 1125 \mathrm{vs}$, $1109 \mathrm{~s}$.

\subsubsection{Darstellung von Diphenylphosphino(diphenylphosphoryl)amin (dppaOH) [124]}

Es werden 12,0 g (31,2 mmol) Bis(diphenylphosphino)amin (dppaH) in $225 \mathrm{ml}$ THF gelöst und anschließend bei $0{ }^{\circ} \mathrm{C} 2,73 \mathrm{ml}(31,2 \mathrm{mmol})$ einer 35\%-igen wässrigen Wasserstoffperoxidlösung zugetropft. Das Reaktionsgemisch wird für 10 min. bei dieser Temperatur gerührt. Innerhalb von $18 \mathrm{~h}$ fällt bei $-26{ }^{\circ} \mathrm{C}$ ein weißer Feststoff (dppaO $\mathrm{H}_{2}, 4 \mathrm{~g}$, 5,8 mmol, 19\%) aus, der durch Filtration von der Lösung abgetrennt wird. Das Filtrat wird bis auf ein Volumen von $40 \mathrm{ml}$ eingeengt. Durch Zugabe von $140 \mathrm{ml}$ Diethylether fällt das Produkt als weißer Feststoff aus, der in THF umkristallisiert wird.

Ausbeute: 8,7 g (21,8 mmol, 70\%)

${ }^{31} \mathrm{P}-\mathrm{NMR}\left(\mathrm{CDCl}_{3}\right): \delta=25,6 \mathrm{ppm}\left(\mathrm{d}, \mathrm{P}^{\mathrm{V}},{ }^{2} \mathrm{~J}(\mathrm{PP})=59,0 \mathrm{~Hz}\right), 28,1 \mathrm{ppm}\left(\mathrm{d}, \mathrm{P}^{\mathrm{III}}\right)$ 


\subsubsection{Darstellung von Diphenylphosphoryl(diphenylthiophosphoryl)amin (dppaOSH) und Diphenylphosphoryl(diphenylselenophosphoryl)amin (dppaOSeH) [20]}

5,9 g (14,7 mmol) Diphenylphosphino(diphenylphosphoryl)amin (dppaOH) werden in $200 \mathrm{ml}$ THF gelöst und mit 0,52 g (16,2 mmol, 1,1 Äq.) Schwefelblüte bzw. 1,7 g (21,2 mmol, 1,4 Äq.) grauem Selen versetzt und 1 h bzw. 3 h bei Raumtemperatur gerührt. Das ungelöste dppaO ${ }_{2} \mathrm{H}$ wird abfiltriert und das Filtrat auf $10 \mathrm{ml}$ eingeengt. Das Produkt wird vollständig durch Zugabe von $50 \mathrm{ml}$ Diethylether ausgefällt, abfiltriert, mit insgesamt $30 \mathrm{ml}$ Diethylether gewaschen und getrocknet.

Ausbeuten:

dppaOSH: 5,5 g (12,6 mmol, 86\%)

dppaOSeH: 6,4 g (13,2 mmol, 90\%)

${ }^{31} \mathrm{P}-\mathrm{NMR}\left(\mathrm{CDCl}_{3}\right)$ :

dppaOSH: $\delta=57,4 \mathrm{ppm}(\mathrm{PS}), 21,3 \mathrm{ppm}(\mathrm{PS})\left({ }^{2} \mathrm{~J}(\mathrm{PP})=19,8 \mathrm{~Hz}\right)$

dppaOSeH: $\delta=52,9 \mathrm{ppm}(\mathrm{d}$, PSe $), 21,5 \mathrm{ppm}(\mathrm{d}, \mathrm{PO})\left({ }^{2} \mathrm{~J}(\mathrm{PP})=23,5 \mathrm{~Hz}\right.$, $\left.{ }^{1} \mathrm{~J}(\mathrm{PSe})=791,9 \mathrm{~Hz}\right)$

IR-Spekren $(\mathrm{KBr})\left[\mathrm{cm}^{-1}\right]$ :

dppaOSH: $\nu(\mathrm{NH}) 2696 \mathrm{~s}$, br, $\nu(\mathrm{PPh}) 1479 \mathrm{~m}, 1438 \mathrm{vs}, \nu(\mathrm{PO}) 1205 \mathrm{vs}, 1190 \mathrm{~s}, \nu_{\mathrm{as}}(\mathrm{PNHP})$ $937 \mathrm{vs}, \nu(\mathrm{PS}) 628 \mathrm{~m}, 614 \mathrm{~m}$.

dppaOSeH: $\nu(\mathrm{NH}) 2716 \mathrm{~m}, 2660 \mathrm{~s}$, br, $\nu(\mathrm{PPh}) 1482 \mathrm{~m}, 1439 \mathrm{vs}, \nu(\mathrm{PO}) 1201 \mathrm{~s}, 1181 \mathrm{vs}$, $\nu_{\mathrm{as}}(\mathrm{PNHP}) 937 \mathrm{vs}, \nu(\mathrm{PSe}) 541 \mathrm{vs}$.

\subsubsection{Darstellung von Bis(diphenylphosphoryl)methan $\left(\operatorname{dppmO}_{2} \mathrm{H}\right)[125]$}

Eine Suspension aus 5,0 g (13,0 mmol) Bis(diphenylphosphino)methan (dppmH) und $60 \mathrm{ml}$ Toluol wird bei $0{ }^{\circ} \mathrm{C}$ mit 2,28 ml (26,0 mmol) 35\%-iger wässriger Wasserstoffperoxidlösung versetzt. Die Mischung wird eine Stunde bei $0{ }^{\circ} \mathrm{C}$ gerührt, auf ein Volumen von $10 \mathrm{ml}$ eingeengt und der auftretende Niederschlag abfiltriert. Das weiße Pulver wird mit insgesamt $300 \mathrm{ml}$ dest. Wasser gewaschen und getrocknet.

${ }^{31} \mathrm{P}-\mathrm{NMR}\left(\mathrm{CDCl}_{3}\right): \delta=27,3 \mathrm{ppm}(\mathrm{s})$

${ }^{1} \mathrm{H}-\mathrm{NMR}\left(\mathrm{CDCl}_{3}\right): \delta=3,6 \mathrm{ppm}\left(\mathrm{t}, \mathrm{CH}_{2},{ }^{2} \mathrm{~J}(\mathrm{PH})=14,0 \mathrm{~Hz}\right), 7,1-7,4 \mathrm{ppm}(\mathrm{m}, 12 \mathrm{H}, \mathrm{Ph})$, 7,6 - 7,7 ppm (m, $8 \mathrm{H}, \mathrm{Ph}), 10,0$ ppm (s, br, $1 \mathrm{H}, \mathrm{OH})$

IR-Spektrum $(\mathrm{KBr})\left[\mathrm{cm}^{-1}\right]: \nu(\mathrm{OH}) 3459 \mathrm{~s}, \mathrm{br}, \nu(\mathrm{PPh}) 1484 \mathrm{~m}, 1438 \mathrm{vs}, \nu(\mathrm{PO}) 1203 \mathrm{vs}$, 1190vs. 


\subsubsection{Darstellung von Bis(diphenylselenophosphoryl)methan (dppmSe $\left.\mathrm{d}_{2} \mathrm{H}\right)$}

[126]

Zu einer Lösung von 3,87 g (10,1 mmol) Bis(diphenylphosphino)methan (dppmH) in $25 \mathrm{ml}$ Toluol werden 1,56 g (19,7 mmol, 1,95 Äq.) graues Selen zugegeben. Das Reaktionsgemisch wird für $3 \mathrm{~h}$ zum Sieden erhitzt. Die schwach gelbe Lösung wird langsam abgekühlt, währenddessen ein weißer, kristalliner Niederschlag ausfällt, der vom Lösungsmittel abfiltriert, dreimal mit je $10 \mathrm{ml}$ Toluol gewaschen und unter Vakuum getrocknet wird.

Ausbeute: 4,72 g (8,7 mmol, 86\%)

${ }^{31} \mathrm{P}-\mathrm{NMR}\left(\mathrm{CDCl}_{3}\right): \delta=25,6 \mathrm{ppm}\left(\mathrm{s},{ }^{1} \mathrm{~J}(\mathrm{PSe})=745,7 \mathrm{~Hz},{ }^{2} \mathrm{~J}(\mathrm{PP})=17,4 \mathrm{~Hz}\right.$, $\left.{ }^{3} \mathrm{~J}(\mathrm{PSe})=2,1 \mathrm{~Hz}\right)$

${ }^{1} \mathrm{H}-\mathrm{NMR}\left(\mathrm{CDCl}_{3}\right): \delta=4,3 \mathrm{ppm}\left(\mathrm{t}, 2 \mathrm{H}, \mathrm{CH}_{2},{ }^{2} \mathrm{~J}(\mathrm{HP})=13,2 \mathrm{~Hz}\right), 7,2-7,4 \mathrm{ppm}(\mathrm{m}$, $12 \mathrm{H}, \mathrm{Ph}), 7,7$ - 7,9 ppm (m, $8 \mathrm{H}, \mathrm{Ph})$

MS (EI): 543,9 $\left(\mathrm{M}^{+}\right)$

IR-Spektrum (KBr) $\left[\mathrm{cm}^{-1}\right]: \nu(\mathrm{PPh}) 1482 \mathrm{~m}, 1435 \mathrm{vs}, \nu(\mathrm{PSe}) 531 \mathrm{~s}, 490 \mathrm{~m}, 471 \mathrm{~m}$.

\subsubsection{Darstellung von $\mathrm{CuO}^{\mathrm{t}} \mathrm{Bu}[127]$}

$\mathrm{Zu}$ einer Suspension aus 7,60 $\mathrm{g}(76,8 \mathrm{mmol}) \mathrm{CuCl}$ in $50 \mathrm{ml}$ THF werden bei $-78{ }^{\circ} \mathrm{C}$ 7,63 g (68,0 mmol) $\mathrm{KO}^{\mathrm{t}} \mathrm{Bu}$, gelöst in $90 \mathrm{ml} \mathrm{THF}$, zugetropft. Das Reaktionsgemisch wird langsam auf Raumtemperatur erwärmt und noch 12 h gerührt. Von der braunen Lösung mit gelbem Niederschlag wird das Lösungsmittel abkondensiert. Das grüne Produkt wird anschließend aus dem Rückstand bei $170{ }^{\circ} \mathrm{C}$ und einem Druck von $10^{-2}$ torr sublimiert. Ausbeute: $7,37 \mathrm{~g}(53,9 \mathrm{mmol}, 79 \%)$

\subsection{Umsetzungen von $\mathrm{CuCl}, \mathrm{CuCl}_{2}$ und $\mathrm{AuCl}\left(\mathrm{PPh}_{3}\right)$ mit $\operatorname{dppmE}_{2} \mathrm{H}(\mathrm{E}=\mathrm{O}, \mathrm{S}, \mathrm{Se})$}

\subsubsection{Darstellung von $\left[\mathrm{Cu}\left(\operatorname{dppmO}_{2} \mathrm{H}\right)_{3}\right]\left[\mathrm{CuCl}_{4}\right] \underline{1}$}

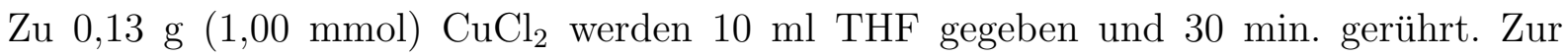
gelben Lösung mit orangefarbenem Niederschlag werden anschließend 0,67 g (1,50 mmol) dppmO $\mathrm{O}_{2} \mathrm{H}$ gegeben. Sofort wird die Lösung kräftig orangefarben. Der Niederschlag löst sich nach kurzem Erhitzen bis zum Siedepunkt des Lösungsmittels auf, und es entsteht eine dunkel orangefarbene Lösung mit schwarzem Niederschlag, der jedoch sofort wieder in Lösung geht. Beim Abkühlen auf Raumtemperatur bildet sich ein hellgelber Niederschlag. Nach zwei Tagen Rühren ist die Lösung gelb. Sie wird vom gelben Niederschlag 
abzentrifugiert und mit Heptan überschichtet. Es bilden sich an der Phasengrenze farblose, luftempfindliche Kristalle von 1.

Ausbeute: 0,44 g (2,25 mmol, 49\%)

\subsubsection{Darstellung von $\left[\mathrm{Cu}_{2}\left(\operatorname{dppmS}_{2} \mathrm{H}\right)_{2} \mathrm{Cl}\right]\left[\mathrm{CuCl}_{2}\right] \underline{2}$}

0,16 g (1,62 mmol) $\mathrm{CuCl}$ werden mit 0,72 $\mathrm{g}(1,62 \mathrm{mmol})$ dppmS $\mathrm{H}_{2} \mathrm{H}$ gut vermischt und bis zur Schmelztemperatur von $\mathrm{dppmS}_{2} \mathrm{H}\left(\mathrm{Smp} .178{ }^{\circ} \mathrm{C}\right) 5$ min. lang erhitzt. Die sehr viskose, gelbe Flüssigkeit wird auf Raumtemperatur abgekühlt und mit $8 \mathrm{ml}$ Dichlormethan versetzt. Die Suspension wird noch zwei Tage gerührt, die klare Lösung vom weißen Rückstand abpipettiert und mit Heptan überschichtet. An der Phasengrenze bilden sich farblose, kristalline Nadeln von 2 .

Ausbeute: 0,44 g ( 0,37 mmol, 69\%)

${ }^{31} \mathrm{P}-\mathrm{NMR}\left(\mathrm{CDCl}_{3}\right): 33,2 \mathrm{ppm}(\mathrm{s})$

${ }^{1} \mathrm{H}-\mathrm{NMR}\left(\mathrm{CDCl}_{3}\right): 4,1 \mathrm{ppm}\left(\mathrm{t}, 2 \mathrm{H}, \mathrm{CH}_{2},{ }^{2} \mathrm{~J}(\mathrm{HP})=12,8 \mathrm{~Hz}\right), 7,2-7,4 \mathrm{ppm}(\mathrm{m}, 12 \mathrm{H}$, $\mathrm{Ph}), 7,6-7,8 \mathrm{ppm}(\mathrm{m}, 8 \mathrm{H}, \mathrm{Ph})$

\subsubsection{Darstellung von $\left[\mathrm{Cu}_{2}\left(\mathrm{dppmSe}_{3}\right)_{2}\right] \underline{3}$}

In $4 \mathrm{ml}$ THF werden $0,20 \mathrm{~g}(0,37 \mathrm{mmol})$ dppmSe $\mathrm{H}_{2}$ gelöst und mit 0,37 $\mathrm{ml}(0,37 \mathrm{mmol})$ einer 1 M Lösung von $\mathrm{NaN}\left(\mathrm{SiMe}_{3}\right)_{2}$ in THF deprotoniert. Die gelbe Lösung wird anschließend zu einer Suspension aus 0,04 g (0,37 mmol) $\mathrm{CuCl}$ und $4 \mathrm{ml}$ THF getropft. Das gelbe Reaktionsgemisch wird einen Tag bei Raumtemperatur gerührt. Es entsteht eine dunkelviolette Lösung mit wenig grauem Niederschlag. Dieser wird abfiltriert und mit Heptan überschichtet. Nach drei Tagen bilden sich viel gelber, amorpher Niederschlag und wenige violette, quaderförmige Kristalle von $\underline{3}$.

Ausbeute: 0,06 g (0,08 mmol, 4,3\%)

\subsubsection{Darstellung von $\left[\mathrm{Au}_{2}\left(\mathrm{dppmS}_{2}\right)\left(\mathrm{PPh}_{3}\right)_{2}\right] \underline{4}$}

0,68 $\mathrm{g}(1,52 \mathrm{mmol}) \mathrm{dppmS}_{2} \mathrm{H}$ und $0,17 \mathrm{~g}(1,52 \mathrm{mmol}) \mathrm{KO}^{\mathrm{t}} \mathrm{Bu}$ werden in $4 \mathrm{ml} \mathrm{THF}$ und $4 \mathrm{ml}$ Heptan gelöst. Nach ca. 30 min. fällt aus der Lösung ein weißer Niederschlag von $\left[\mathrm{K}\left(\mathrm{dppmS}_{2}\right)\right]$ aus. Zu dieser Suspension werden sodann 0,75 g (1,52 mmol) $\mathrm{AuCl}\left(\mathrm{PPh}_{3}\right)$ gegeben. Nach 60 min. Rühren hat sich ein weißes Öl mit überstehender, farbloser Lösung gebildet. Es wird noch zwei Tage bei Raumtemperatur gerührt. Die überstehende Lösung wird abpipettiert und mit Heptan überschichtet. Innerhalb eines Tages entstehen als 
Hauptprodukt schwach gelbe, kristalline Nadeln von dppm $\mathrm{S}_{2} \mathrm{H}$, was mittels Vergleich der Gitterkonstanten überprüft wurde, und wenige farblose, plattenförmige Kristalle von $\underline{4}$. Die Ausbeute wurde nicht bestimmt.

\subsection{Umsetzungen von $\mathrm{CuCl}$ und $\mathrm{CuCl}_{2}$ mit $\operatorname{dppaO}_{2} \mathrm{H}$}

\subsubsection{Darstellung von $\left[\mathrm{Cu}\left(\operatorname{dppaO}_{2}\right)_{2}(\mathrm{MeOH})\right] \underline{5}$}

0, $24 \mathrm{~g}$ (1,79 mmol) $\mathrm{CuCl}_{2}$ werden in $10 \mathrm{ml}$ dest. Wasser gelöst und eine Lösung aus 1,50 g $(3,58 \mathrm{mmol}) \mathrm{dppaO}_{2} \mathrm{H}, 0,20 \mathrm{~g}(3,58 \mathrm{mmol})$ Kaliumhydroxid in $10 \mathrm{ml}$ Methanol zugegeben. Es fällt ein feiner, weißer Niederschlag aus der grünen Lösung aus. Das Lösungsmittel wird abkondensiert, und der getrocknete Rückstand wird in $10 \mathrm{ml}$ Methanol aufgenommen. Die grüne Lösung wird vom hellblauen Niederschlag abpipettiert. Bei $-26{ }^{\circ} \mathrm{C}$ bilden sich blaue Kristalle von $\underline{5}$.

Ausbeute: 0,64 g (0,70 mmol, $78 \%)$

\subsubsection{Darstellung von $\left[\mathrm{Cu}_{2}\left(\operatorname{dppaO}_{2}\right)_{2} \mathrm{Cl}_{2}(\operatorname{thf})_{2}\right] \underline{6}$}

$\mathrm{Zu}$ einer Suspension aus $0,15 \mathrm{~g}(1,10 \mathrm{mmol}) \mathrm{CuCl}_{2}$ und $5 \mathrm{ml}$ THF wird eine Suspension aus $0,46 \mathrm{~g}(1,10 \mathrm{mmol}) \mathrm{dppaO}_{2} \mathrm{H}$ in $15 \mathrm{ml}$ THF gegeben. $\mathrm{CuCl}_{2}$ löst sich sofort auf, gleichzeitig bildet sich ein leicht orangefarbener Niederschlag. Hierzu werden 0,12 g $(1,10 \mathrm{mmol}) \mathrm{KO}^{\mathrm{t}} \mathrm{Bu}$ gegeben. Die jetzt grüne Lösung wird für einen Tag bei Raumtemperatur gerührt. Nachdem der gelbe Niederschlag abfiltriert ist, wird die grüne Lösung mit Heptan überschichtet. Erst bilden sich an der Phasengrenze grüne Kristalle von $\underline{6}$. Nach einigen Wochen entstehen blaue, sehr verwachsene Kristalle von $\left[\mathrm{Cu}\left(\mathrm{dppaO}_{2}\right)_{2}\right]$.

Ausbeute an $\underline{6}$ : 0,18 g (0,09 mmol, 17\%)

\subsubsection{Darstellung von $\left[\mathrm{Cu}_{2}\left(\operatorname{dppaO}_{2}\right)_{2}(\mathrm{OMe})_{2}\right] \underline{7}$}

0,27 g (2,00 mmol) $\mathrm{CuCl}_{2}$ werden in $30 \mathrm{ml}$ Methanol gelöst. Anschließend werden in die grüne Lösung 0,83 g (2,00 mmol) dppaO ${ }_{2} \mathrm{H}$ hinzugegeben und 1,67 ml (1,21 g, 12,00 mmol) Triethylamin zugetropft. Es bildet sich ein dunkelblauer Niederschlag, während sich die Lösung entfärbt. Nach der Zugabe von 0,22 g (1,00 mmol) 3,5-Ditertiärbutylcatechol wird der Niederschlag grün. Dieser wird abfiltriert, gewaschen und in $20 \mathrm{ml}$ Methanol gelöst. Die dunkelgrüne Lösung wird mit Heptan überschichtet. Nach fünf Tagen entstehen an der Phasengrenze grüne Kristalle von $\underline{7}$.

Ausbeute: 0,49 g (0,48 mmol, 48\%) 


\subsubsection{Darstellung von $\left[\mathrm{Cu}_{3}\left(\operatorname{dppaO}_{2}\right)_{2}\left(\left(\mathrm{OSiMe}_{2}\right)_{2} \mathrm{O}\right)_{2}(\operatorname{thf})_{2}\right] \underline{8}$ und $\left[\mathrm{Cu}_{4}(\mathrm{O})\left(\operatorname{dppaO}_{2}\right)_{4} \mathrm{Cl}_{2}\right] \underline{9}$}

0,18 g (2,40 mmol) [OSiMe 2 (Schliffett) werden in $6 \mathrm{ml}$ THF mit 0,40 g (3,60 mmol) $\mathrm{KO}^{\mathrm{t}} \mathrm{Bu}$ gelöst und 1,00 g (2,40 mmol) $\mathrm{dppaO}_{2} \mathrm{H}$ hinzugegeben. Die farblose Lösung wird nun in eine Suspension von $0,24 \mathrm{~g}(1,80 \mathrm{mmol}) \mathrm{CuCl}_{2}$ in $8 \mathrm{ml}$ THF getropft. Es entsteht eine dunkelgrüne Lösung, die für eine Woche bei Raumtemperatur gerührt wird. Die Lösung wird vom grünen Niederschlag abfiltriert und mit Heptan überschichtet. Nach drei Tagen bilden sich erst blaue, sehr verwachsene Kristalle von $\underline{8}$ und dann wenige braune, rautenförmige Kristalle von $\underline{9}$. Nach einer Reaktionszeit von einem Tag entsteht die Verbindung $\underline{9}$ als Hauptprodukt und sehr wenig der Verbindung $\underline{8}$. Beträgt die Reaktionszeit jedoch eine Woche oder mehr, so verschiebt sich das Produktverhältnis zugunsten von Verbindung $\underline{8}$.

Ausbeute an $\underline{8}: 0,51 \mathrm{~g}(0,37 \mathrm{mmol}, 61 \%)$

Die Ausbeute an $\underline{9}$ wurde nicht bestimmt.

\subsection{Umsetzungen von $\mathrm{CuCl}$ und $\mathrm{CuCl}_{2}$ mit dppaOSH}

\subsubsection{Darstellung von $\left[\mathrm{Cu}_{3}(\operatorname{dppaOS})_{3}\right] \underline{10}$ und $\left[\mathrm{Cu}(\operatorname{dppaOS})_{2}\right]$}

In $10 \mathrm{ml}$ THF werden $0,07 \mathrm{~g}(0,52 \mathrm{mmol}) \mathrm{CuCl}_{2}$ und 0,05 g (0,52 mmol) $\mathrm{CuCl}$ suspendiert. Eine Lösung aus 0,45 g (1,04 mmol) dppaOSH, $5 \mathrm{ml}$ THF und 1,00 ml einer $1 \mathrm{M}$ Lösung von $\mathrm{NaN}\left(\mathrm{SiMe}_{3}\right)_{2}$ werden nun in diese Suspension getropft. $\mathrm{CuCl}_{2}$ löst sich sofort auf, und die Lösung wird dunkelbraun. Nach zwei Tagen Rühren wird die braune Lösung vom braunen Niederschlag abfiltriert und mit Heptan überschichtet. Nach einer Woche bilden sich an der Phasengrenze wenige farblose, quaderförmige Kristalle von 10 und stark verwachsene, dunkelgrüne Kristalle von $\left[\mathrm{Cu}(\mathrm{dppaOS})_{2}\right]$.

Die Ausbeute wurde nicht bestimmt.

${ }^{31} \mathrm{P}-\mathrm{NMR}$ von 10 (THF, $\left.\mathrm{CDCl}_{3}\right): 30,0$ ppm (s, PS), 16,9 ppm (s, PO)

\subsection{Umsetzungen von $\mathrm{CuCl}$ mit dppaOSeH}

\subsubsection{Darstellung von $\left[\mathrm{Cu}\left(\mathrm{dppaO}_{2}\right)_{2}\left(\mathrm{NH}_{3}\right)_{3}\right]_{\infty} \underline{11}$}

0,96 g (2,00 mmol) dppaOSeH werden in $6 \mathrm{ml}$ THF gelöst und mit 2,00 ml einer $1 \mathrm{M}$ Lösung von $\mathrm{NaN}\left(\mathrm{SiMe}_{3}\right)_{2}$ in THF deprotoniert. Diese gelbe Lösung wird anschließend in eine Suspension aus 0,20 g (2,00 mmol) $\mathrm{CuCl}$ in $5 \mathrm{ml}$ THF getropft. Die Lösung wird erst 
grün und nach vier Stunden Rühren bei Raumtemperatur gelb. Der entstandene Niederschlag wird von der Lösung abzentrifugiert und das Zentrifugat mit Heptan überschichtet. Es bilden sich erst farblose Kristalle, die sich jedoch nach zwei Wochen auflösen, während gleichzeitig ein Niederschlag von elementarem Selen und dunkelblaue, nadelförmige Kristalle von 11 entstehen.

Die Ausbeute wurde nicht bestimmt.

\subsubsection{Darstellung von $\left[\mathrm{H}\left((\mathrm{O}) \mathrm{PPh}_{2} \mathrm{NPPh}_{2}\right)_{2} \mathrm{O}\right]\left[\mathrm{CuCl}_{2}\right] \underline{12}$}

0,06 g (0,60 mmol) $\mathrm{CuCl}$ und 0,08 g (0,60 mmol) $\mathrm{CuCl}_{2}$ werden in $8 \mathrm{ml}$ THF suspendiert und eine Lösung aus 0,58 g (1,20 mmol) dppaOSeH und 0,13 g (1,2 mmol) $\mathrm{KO}^{\mathrm{t}} \mathrm{Bu}$ in $15 \mathrm{ml}$ THF zugetropft. Die rote Lösung wird für einen Tag bei Raumtemperatur gerührt. Die nun orange Lösung wird vom roten Niederschlag abfiltriert und mit Heptan überschichtet. Es bilden sich farblose Kristalle von 12 .

Die Ausbeute wurde nicht bestimmt.

\subsection{Umsetzungen von $\mathrm{CuCl}$ und $\mathrm{AgCl}$ mit $\operatorname{dppaE}_{2} \mathrm{H}(\mathrm{E}=\mathrm{S}, \mathrm{Se})$}

\subsubsection{Darstellung von $\left[\mathrm{NaCu}_{2}\left(\mathrm{dppaE}_{2}\right)_{3}(\mathrm{thf})_{2}\right](\mathrm{E}=\mathrm{S}: \underline{13} ; \mathrm{Se}: \underline{14})$}

3,96 g (8,80 mmol) dppaS ${ }_{2} \mathrm{H}$ bzw. 4,78 g $(8,80 \mathrm{mmol})$ dppaSe ${ }_{2} \mathrm{H}$ werden in $8 \mathrm{ml} \mathrm{THF}$ gelöst und mit 8,8 ml (8,80 mmol) einer $1 \mathrm{M}$ Lösung von $\mathrm{NaN}\left(\mathrm{SiMe}_{3}\right)_{2}$ in THF versetzt. Die farblose Lösung wird anschließend einer Suspension aus 0,58 g $(5,87 \mathrm{mmol}) \mathrm{CuCl}$ und $7 \mathrm{ml}$ THF zugegeben. Das Reaktionsgemisch wird einen Tag bei Raumtemperatur gerührt, die farblose bzw. gelbe Lösung vom weißen Niederschlag abfiltriert und mit Heptan überschichtet. Innerhalb von einigen Tagen bilden sich farblose Kristalle von $\underline{13}$ bzw. 14.

Ausbeute an 13: 0,87 g (0,53 mmol, 18\%)

${ }^{31} \mathrm{P}-\mathrm{NMR}$ (THF, $\mathrm{C}_{6} \mathrm{D}_{6}$ ): 34,1 ppm (s, br)

Ausbeute an 14: 0,62 g (0,32 mmol, 11\%)

${ }^{31} \mathrm{P}-\mathrm{NMR}\left(\mathrm{THF}, \mathrm{CDCl}_{3}\right): 19,8 \mathrm{ppm}\left(\mathrm{s}, \mathrm{br},{ }^{1} \mathrm{~J}(\mathrm{PSe})=556,2 \mathrm{~Hz}\right)$

\subsubsection{Darstellung von $\left[\mathrm{Cu}_{3}\left(\mathrm{dppaS}_{2}\right)_{3}\right] \underline{15}$}

Eine Lösung aus 0,49 g (1,10 mmol) dppaS $_{2} \mathrm{H}$ in $6 \mathrm{ml}$ THF und 0,69 $\mathrm{ml}(1,10 \mathrm{mmol})$ einer 1,6 M Lösung von ${ }^{\mathrm{n}}$ Butyllithium in Hexan werden zu einer Suspension von 0,11 g $(1,10 \mathrm{mmol}) \mathrm{CuCl}$ in $5 \mathrm{ml}$ THF getropft. Die gelbe Lösung mit gelbem Niederschlag wird 
einen Tag gerührt, dann vom Niederschlag abfiltriert und mit Heptan überschichtet. Es bilden sich quaderförmige Kristalle von 15.

Ausbeute: $0,27 \mathrm{~g}(0,17 \mathrm{mmol}, 46 \%)$

${ }^{31} \mathrm{P}-\mathrm{NMR}\left(\mathrm{THF}, \mathrm{C}_{6} \mathrm{D}_{6}\right): 31,8 \mathrm{ppm}(\mathrm{s})$

\subsubsection{Darstellung von $\left[\mathrm{Cu}_{3}\left(\mathrm{dppaSe}_{2}\right)_{3}\right] \underline{16}$}

1,10 g (2,02 mmol) dppaSe $\mathrm{H}_{2} \mathrm{H}$ werden in $10 \mathrm{ml}$ THF gelöst und mit 0,23 g (2,02 mmol) $\mathrm{KO}^{t} \mathrm{Bu}$ deprotoniert. Das Lösungsmittel wird abkondensiert und der zurückbleibende, weiße Feststoff von $\left[\mathrm{K}\left(\mathrm{dppaSe}_{2}\right)\right]$ wird anschließend zu einer Suspension aus $0,20 \mathrm{~g}$ $(2,02 \mathrm{mmol}) \mathrm{CuCl}$ und $10 \mathrm{ml}$ THF gegeben, wobei sich eine grüne Lösung bildet. Diese wird nun drei Tage gerührt, anschließend vom grün-gelben Niederschlag abfiltriert und mit Heptan überschichtet. Es bilden sich quaderförmige Kristalle von 16.

Ausbeute: $0,36 \mathrm{~g}(0,19 \mathrm{mmol}, 28 \%)$

${ }^{31} \mathrm{P}-\mathrm{NMR}\left(\mathrm{THF}, \mathrm{C}_{6} \mathrm{D}_{6}\right): 24,5 \mathrm{ppm}(\mathrm{s}, \mathrm{br}),{ }^{1} \mathrm{~J}(\mathrm{PSe})=609,6 \mathrm{~Hz}$

${ }^{77} \mathrm{Se}-\mathrm{NMR}\left(\mathrm{THF}, \mathrm{C}_{6} \mathrm{D}_{6}\right.$ ): $-140,0 \mathrm{ppm}(\mathrm{d})$

\subsubsection{Darstellung von $\left[\mathrm{Cu}_{4}\left(\mathrm{dppaS}_{2}\right)_{3}\right]_{2}\left[\mathrm{CuCl}_{2}\right][\mathrm{OH}] \underline{19}$}

0,18 g (1,82 mmol) $\mathrm{CuCl}$ und 0,82 g (1,82 mmol) dppa $\mathrm{S}_{2} \mathrm{H}$ werden vermischt und $30 \mathrm{~min}$. lang auf $220{ }^{\circ} \mathrm{C}$ erhitzt. In der gelben Schmelze entsteht ein schwarzer Niederschlag, der sich unter heftiger Gasentwicklung wieder auflöst. Das Reaktionsgemisch wird auf Raumtemperatur abgekühlt. Der nun entstandene graue und gelbe Feststoff wird in $8 \mathrm{ml}$ Dichlormethan suspendiert. Die gelbe Lösung, die stark nach Schwefelwasserstoff riecht, wird vom weißen Niederschlag abzentrifugiert und mit Heptan überschichtet. Es entsteht ein weißer, amorpher Niederschlag und orangefarbene Kristalle von $\underline{19}$.

Ausbeute: 0,45 g (0,24 mmol, 66\%)

${ }^{31} \mathrm{P}-\mathrm{NMR}\left(\mathrm{THF}, \mathrm{CDCl}_{3}\right): 27,4 \mathrm{ppm}(\mathrm{s}, \mathrm{br})$

\subsubsection{Darstellung von $\left[\mathrm{Cu}_{4}\left(\operatorname{dppaSe}_{2}\right)_{3}\right]\left[\mathrm{CuCl}_{2}\right] \underline{20}$}

0,68 g (1,25 mmol) dppaSe ${ }_{2} \mathrm{H}$ und 0,12 g (1,25 mmol) $\mathrm{CuCl}$ werden vermischt und $5 \mathrm{~min}$. lang auf die Schmelztemperatur von dppaSe $\mathrm{H}_{2} \mathrm{H}\left(\mathrm{Smp} .210{ }^{\circ} \mathrm{C}\right.$ ) erhitzt. In der gelben Schmelze bildet sich ein schwarzer Feststoff. Das Reaktionsgemisch wird auf Raumtemperatur abgekühlt. Das graue Gemisch wird nun in $8 \mathrm{ml}$ Dichlormethan aufgenommen. Die entstandene farblose Lösung wird vom grauen Niederschlag abzentrifugiert und mit Heptan überschichtet. Es bilden sich farblose Kristalle von 20. 
Die Ausbeute wurde nicht bestimmt.

${ }^{31} \mathrm{P}-\mathrm{NMR}\left(\mathrm{CDCl}_{3}\right): 18,8 \mathrm{ppm}(\mathrm{d})\left({ }^{2} \mathrm{~J}(\mathrm{PP})=11,7 \mathrm{~Hz},{ }^{1} \mathrm{~J}(\mathrm{PSe})=489,8 \mathrm{~Hz}\right), 18,9 \mathrm{ppm}(\mathrm{d})$

${ }^{77} \mathrm{Se}-\mathrm{NMR}\left(\mathrm{CDCl}_{3}\right):-128,2 \mathrm{ppm}(\mathrm{d}),-143,8 \mathrm{ppm}(\mathrm{d})$

\subsubsection{Darstellung von $\left[\mathrm{Cu}\left(\operatorname{dppaS}_{2}\right)(\right.$ th $\left.)\right] \underline{21}$}

0,80 g (8,08 mmol) $\mathrm{CuCl}$ werden in $5 \mathrm{ml}$ Ethanol suspendiert und mit 0,62 $\mathrm{g}(8,08 \mathrm{mmol})$ Thioharnstoff versetzt. Nach vier Stunden bildet sich eine farblose Lösung, aus der nach zwei Tagen Rühren ein weißer, voluminöser Niederschlag ausfällt. 0,44 g (8,08 mmol) $\mathrm{NaOMe}$ werden in $10 \mathrm{ml}$ Ethanol gelöst und anschließend 3,63 g (8,08 mmol) dppaS $\mathrm{S}_{2} \mathrm{H}$ zugegeben. Ein weißer Niederschlag von $\left[\mathrm{Na}\left(\mathrm{dppaS}_{2}\right)\right]$ fällt aus. Diese Suspension wird nun zur ersten Reaktionslösung hinzugegeben und einen Tag gerührt. Danach wird das Lösungsmittel abkondensiert und der verbleibende weiße Rückstand in $5 \mathrm{ml}$ THF aufgenommen. Diese Lösung wird noch 20 min. zum Sieden erhitzt. Aus der farblosen Lösung bilden sich nach einem Tag bei $-26{ }^{\circ} \mathrm{C}$ nadelförmige farblose Kristalle, die nicht zur Röntgenstrukturanalyse geeignet sind, neben farblosen plättchenförmigen Kristallen von 21. Wird der verbleibende Rückstand in Toluol gelöst und mit Heptan überschichtet, so entstehen rautenförmige Kristalle von $\left[\mathrm{Cu}_{3}\left(\mathrm{dppaS}_{2}\right)_{3}\right] \underline{15}$.

Die Ausbeute wurde nicht bestimmt.

${ }^{31} \mathrm{P}-\mathrm{NMR}\left(\mathrm{MeOH}, \mathrm{CDCl}_{3}\right): 33,5 \mathrm{ppm}(\mathrm{s})$

\subsubsection{Darstellung von $\left[\operatorname{Ag}_{3}\left(\operatorname{dppaS}_{2}\right)_{3}\right] \underline{17}$}

$\mathrm{Zu}$ einer Suspension von 0,19 $\mathrm{g}(1,30 \mathrm{mmol}) \mathrm{AgCl}$ in $5 \mathrm{ml}$ THF wird eine Lösung aus 0,58 g (1,30 mmol) dppaS ${ }_{2} \mathrm{H}, 0,82 \mathrm{ml}$ (1,40 mmol) einer 1,7 M Lösung von ${ }^{\mathrm{n}}$ Butyllithium in Hexan und $5 \mathrm{ml}$ THF zugetropft und einen Tag bei Raumtemperatur gerührt. Die gelbe Lösung wird bis auf ein Volumen von $5 \mathrm{ml}$ eingeengt. Nach einigen Tagen bilden sich bei $-26{ }^{\circ} \mathrm{C}$ farblose, nadelförmige Kristalle von $\underline{17}$.

Ausbeute: $0,44 \mathrm{~g}(0,50 \mathrm{mmol}, 39 \%)$

\subsubsection{Darstellung von $\left[\mathrm{Na}_{2} \mathrm{Ag}_{2}\left(\mathrm{dppaS}_{2}\right)_{4}(\text { thf })_{4}\right] \underline{18}$}

In $10 \mathrm{ml}$ THF werden 0,63 g (1,40 mmol) dppaS ${ }_{2} \mathrm{H}$ gelöst und mit 0,20 g (1,40 mmol $) \mathrm{AgCl}$ versetzt. Anschließend werden 1,40 ml (1,40 mmol) einer $1 \mathrm{M}$ Lösung von $\mathrm{NaN}\left(\mathrm{SiMe}_{3}\right)_{2}$ in THF hinzugetropft und einen Tag lang gerührt. Der nun grünlichen Lösung werden 0,10 $\mathrm{ml}$ (1,40 mmol) Ethylendiamin zugegeben, und es wird eine weitere Woche bei Raum- 
temperatur gerührt. Die farblose Lösung wird vom weißen Niederschlag abzentrifugiert und mit Heptan überschichtet. An der Phasengrenze bilden sich farblose Kristalle von 18 . Ausbeute: $0,13 \mathrm{~g}(0,11 \mathrm{mmol}, 31 \%)$

${ }^{31} \mathrm{P}-\mathrm{NMR}\left(\mathrm{THF}, \mathrm{C}_{6} \mathrm{D}_{6}\right.$ ): $36,8 \mathrm{ppm}(\mathrm{s})$

\subsection{Umsetzungen von $\mathrm{CuCl}$ und $\mathrm{CuCl}_{2}$ mit $\operatorname{dmpaS} \mathrm{S}_{2} \mathrm{H}$}

\subsubsection{Darstellung von $\left[\mathrm{Cu}_{2}\left(\operatorname{dmpaS}_{2} \mathrm{H}\right)_{2} \mathrm{Cl}_{2}\right] \underline{22}$}

0,30 g (2,23 mmol) $\mathrm{CuCl}_{2}$ und 0,45 g $(2,23 \mathrm{mmol})$ dmpaS ${ }_{2} \mathrm{H}$ werden in $10 \mathrm{ml}$ Ethanol gegeben, wobei sich eine gelbe Lösung mit braunem Niederschlag bildet. Zum Reaktionsgemisch werden sodann 0,12 g (2,23 mmol) NaOMe, gelöst in $6 \mathrm{ml}$ Ethanol, zugegeben und für $24 \mathrm{~h}$ bei Raumtemperatur gerührt. Die entstandene farblose Lösung wird vom weißen Niederschlag abfiltriert und mit Diethylether überschichtet. Nach zwei Tagen bilden sich farblose Kristalle von $\underline{22}$, die sich nach zwei Wochen unter Bildung einer orangefarbenen Lösung auflösen.

Ausbeute: $0,10 \mathrm{~g}(0,21 \mathrm{mmol}, 19 \%)$

\subsubsection{Darstellung von $\left[\mathrm{Cu}_{5}\left(\operatorname{dmpaS}_{2}\right)_{5}\right] \underline{23}$}

Eine Lösung aus 0,81 g (4,02 mmol) dmpaS ${ }_{2} \mathrm{H}, 4,02 \mathrm{ml}$ (4,02 mmol) einer $1 \mathrm{M}$ Lösung von $\mathrm{NaN}\left(\mathrm{SiMe}_{3}\right)_{2}$ in THF und $5 \mathrm{ml}$ THF wird zu einer Suspension aus 0,40 g (4,04 mmol) $\mathrm{CuCl}$ und $5 \mathrm{ml}$ THF gegeben. Nach $30 \mathrm{~min}$. färbt sich das trübe gewordene Gemisch grün und nach einem Tag leicht braun. Nach drei Tagen Rühren bei Raumtemperatur wird die gelbe Lösung vom grauen Niederschlag abzentrifugiert und mit Heptan überschichtet. Es bilden sich farblose, rautenförmige Kristalle von $\underline{23}$.

Ausbeute: $0,45 \mathrm{~g}(0,35 \mathrm{mmol}, 44 \%)$

${ }^{31} \mathrm{P}-\mathrm{NMR}\left(\mathrm{THF}, \mathrm{C}_{6} \mathrm{D}_{6}\right): 36,5 \mathrm{ppm}(\mathrm{s})$

Elementaranalyse: gef.: C 20,39\%, H 5,02\%, N 5,00\%, S 23,97\%, P 20,1\% Cu 20,69\%

ber.: C $18,22 \%$, H 4,59\%, N 5,31\%, S 24,31\%, P 23,48\%, Cu 24,09\%

\subsubsection{Darstellung von $\left[\mathrm{Cu}_{8}\left(\mathrm{dmpaS}_{2}\right)_{6} \mathrm{Cl}_{2}\right] \underline{24}$}

0,69 g (0,05 mmol) der Verbindung $\left[\mathrm{Cu}_{5}\left(\mathrm{dmpaS}_{2}\right)_{5}\right] \underline{23}$ werden in $5 \mathrm{ml}$ THF gelöst und 0,09 g (0,09 mmol) CuCl zugegeben. Nach 5 min. Rühren bei Raumtemperatur löst sich das $\mathrm{CuCl}$ auf und es bildet sich langsam eine leuchtend grüne Lösung, deren Farbe sich im Verlaufe von zwei Tagen hin zu braun ändert. Es bildet sich ein brauner Niederschlag, der 
von der Lösung abfiltriert wird. Das Filtrat wird mit Heptan überschichtet. Nach einer Woche können neben vielen farblosen Kristallen von $\underline{23}$ kleine, nadelförmige Kristalle von $\underline{24}$ isoliert werden.

Die Ausbeute wurde nicht bestimmt.

${ }^{31} \mathrm{P}-\mathrm{NMR}\left(\mathrm{CDCl}_{3}\right): 34,2 \mathrm{ppm}(\mathrm{s}, \mathrm{br})$

\subsubsection{Darstellung von $\left[\mathrm{Cu}_{10}\left(\mathrm{dmpaS}_{2}\right)_{6} \mathrm{Cl}_{4}\right] \underline{25}$}

Eine Lösung aus 0,22 g (1,10 mmol) dmpaS $\mathrm{d}_{2} \mathrm{H}, 1,10 \mathrm{ml}$ (1,10 mmol) einer $1 \mathrm{M}$ Lösung von $\mathrm{NaN}\left(\mathrm{SiMe}_{3}\right)_{2}$ in THF und $5 \mathrm{ml}$ THF werden in eine Suspension von 0,22 $\mathrm{g}$ (2,20 mmol) $\mathrm{CuCl}$ in $5 \mathrm{ml}$ THF getropft. Die gelbe, trübe Lösung wird zwei Tage bei Raumtemperatur gerührt. Anschließend wird die grün gewordene Lösung bis zur Trockene eingeengt, und der verleibende grüne Rückstand in $5 \mathrm{ml}$ Dichlormethan aufgenommen. Die grüne Lösung wird vom grünen und weißen Niederschlag abfiltriert und mit Heptan überschichtet. Nach einigen Tagen bilden sich ein gelartiger, grüner Niederschlag und farblose, rautenförmige Kristalle von 25 .

Die Ausbeute wurde nicht bestimmt.

${ }^{31} \mathrm{P}-\mathrm{NMR}\left(\mathrm{THF}, \mathrm{C}_{6} \mathrm{D}_{6}, 40{ }^{\circ} \mathrm{C}\right): 33,73 \mathrm{ppm}(\mathrm{s}, \mathrm{br})$

\subsubsection{Darstellung von $\left[\mathrm{KCu}\left(\operatorname{dmpaS}_{2}\right)_{2}(\text { thf })\right]_{\infty} \underline{26}$}

0,52 g (2,60 mmol) dmpaS $_{2} \mathrm{H}$ werden in $5 \mathrm{ml}$ THF gelöst und mit 0,29 g (2,60 mmol) $\mathrm{KO}^{\mathrm{t}} \mathrm{Bu}$ versetzt. Sofort fällt $\left[\mathrm{K}\left(\mathrm{dmpaS}_{2}\right)\right]$ als weißer Niederschlag aus. Diese Suspension wird nun einen Tag lang gerührt und anschließend zu einer Suspension von 0,13 g $(1,30 \mathrm{mmol}) \mathrm{CuCl}$ und $5 \mathrm{ml}$ THF gegeben. Nachdem das Reaktionsgemisch zwei Tage bei Raumtemperatur gerührt wurde, wird die farblose Lösung vom braunen Niederschlag abzentrifugiert und das Zentrifugat mit Heptan überschichtet. Es bilden sich zwei Kristallsorten: plattenförmige, farblose Kristalle von $\underline{26}$ und farblose Kristalle, die nicht geeignet sind für eine Röntgenstrukturanalyse.

Ausbeute: $0,16 \mathrm{~g}(0,27 \mathrm{mmol}, 21 \%)$

\subsection{Darstellung von $\left[\mathrm{Hg}\left(\operatorname{dmpaS}_{2}\right)_{2}\right] \underline{27}$}

0,40 g (2,00 mmol) dmpaS ${ }_{2} \mathrm{H}$ werden in $10 \mathrm{ml}$ THF gelöst und mit 2,00 $\mathrm{ml}(2,00 \mathrm{mmol})$ einer 1 M Lösung von $\mathrm{NaN}\left(\mathrm{SiMe}_{3}\right)_{2}$ in THF deprotoniert. Diese Lösung wird nun in eine Suspension von $0,27 \mathrm{~g}(1,00 \mathrm{mmol}) \mathrm{HgCl}_{2}$ in $10 \mathrm{ml}$ THF getropft. Das Reaktionsgemisch wird zwei Tage gerührt. Anschließend wird die farblose Lösung vom weißen Niederschlag 
abfiltriert und mit Heptan überschichtet. Nach einem Tag bilden sich farblose Kristalle von 27.

Ausbeute: 0,34 g (0,56 mmol, 56\%) 


\section{Kristallstrukturuntersuchungen}

Die im Rahmen dieser Arbeit durchgeführten röntgenographischen Kristallstrukturanalysen wurden mit dem Gerät Flächendetektor STOE Siemens-Huber Eigenbau vorgenommen. Als Strahlungsquelle diente eine Röntgenröhre mit Mo- $\mathrm{K}_{\alpha}$-Strahlung $(\lambda=71,073 \mathrm{pm})$ und nachgeschaltetem Graphitmonochromator.

Die Bestimmung der Gitterkonstanten erfolgte mit Hilfe der Orientierungsparameter von ca. 800 Reflexen aus mehreren Images bei verschiedenen Drehwinkeln $\phi$. Die Reflexintensitäten wurden durch Definition einer dem Reflexprofil entsprechenden Integrationsbox ermittelt.

Die Bestimmung der Kristallstrukturen erfolgte mit den Programmen SHELXS-97 [128] und SHELXL-97 [129].

- Datenreduktion: Skalierung der aus den Rohdaten berechneten F-Werte anhand der Referenzreflexe. Die Reflexintensitäten wurden durch Anwendung einer Lorentzfaktor- und Polarisationskorrektur umgerechnet. Eine Mittelung erfolgte über symmetrieäquivalente Reflexe.

- Lösung der Kristallstruktur mit Hilfe von Patterson- oder direkten Methoden und anschließenden Differenz-Fouriersynthesen.

- Optimierung der Atomparameter über Full Matrix-LSq-Verfeinerungen. Dabei wurde folgende Gewichtsfunktion verwendet:

$$
\frac{1}{w}=\sigma^{2}\left(F_{o}^{2}\right)+(a P)^{2}+b P \quad \text { mit } \quad P=\frac{\operatorname{Max}\left(F_{o}^{2}, 0\right)+2 F_{c}^{2}}{3}
$$

Die Werte für die Parameter $a$ und $b$ werden bei Anwendung des Programms SHELXL-97 [129] automatisch ermittelt und angepaßt. Für die so ermittelten Gütewerte gilt:

$$
R 1=\frac{\sum|| F_{o}|-| F_{c}||}{\sum\left|F_{o}\right|} ; \quad \mathrm{w} R 2=\sqrt{\frac{\sum\left[\mathrm{w}\left(F_{o}^{2}-F_{c}^{2}\right)^{2}\right]}{\sum\left[\mathrm{w}\left(F_{o}^{2}\right)^{2}\right]}}
$$

Nach der von Debye und Waller [130,131] abgeleiteten Theorie wird der Atomformfaktor $f_{0}$, der sich auf ruhende Atome bezieht, mit einem temperaturabhängigen Faktor korrigiert:

$$
f=f_{0} \exp \left(-8 \pi^{2} U \frac{\sin ^{2} \theta}{\lambda^{2}}\right)
$$


Der Koeffizient $U$ des Temperaturfaktors läßt sich mit der mittleren Schwingunsamplitude $\bar{u}$ des Atoms senkrecht zur reflektierenden Netzebene durch folgende Beziehung in Verbindung setzen:

$$
U=\bar{u}^{2}
$$

Multipliziert man den isotropen Auslenkungsparameter $U$ mit $8 \pi^{2}$, so erhält den sogenannten Debye-Waller-Faktor $B$.

Realistischer ist es, für die Atome im Kristall anisotrope Schwingungen zuzulassen. Dargestellt wird die anisotrope Schwingung eines Atoms durch ein Schwingungsellipsoid. Letzteres wird durch sechs Komponenten $\left(U_{\mathrm{ij}}\right)$ eines Tensors in seiner räumlichen Lage beschrieben. Für $B$ und die Komponenten $\left(U_{\mathrm{ij}}\right)$ des Schwingungstensors gelten mit $T=-\ln \left(\frac{f}{f_{0}}\right)$ folgende Zusammenhänge:

$$
T_{\text {iso }}=B \frac{\sin ^{2} \theta}{\lambda^{2}} \quad \text { bzw. } \quad T_{\text {aniso }}=2 \pi^{2}\left(\sum_{\mathrm{i}=1}^{3} \sum_{\mathrm{j}=1}^{3} U_{\mathrm{ij}} h_{\mathrm{i}} h_{\mathrm{j}} a_{\mathrm{i}}^{*} a_{\mathrm{j}}^{*}\right)
$$

$U_{\text {eq }}$ berechnet sich aus den Koeffizienten $U_{\mathrm{ij}}$ nach der Gl.:

$$
U_{\text {eq }}=\frac{1}{3}\left(\sum_{\mathrm{i}=1}^{3} \sum_{\mathrm{j}=1}^{3} U_{\mathrm{ij}} a_{\mathrm{i}}^{*} a_{\mathrm{j}}^{*} a_{\mathrm{i}} a_{\mathrm{j}}\right)
$$

Mit $\mathrm{i}=1$ bis 3 bezeichnen $h_{\mathrm{i}}$ die Millerschen Indizes $\left(h_{1}=h, h_{2}=k, h_{3}=l\right), a_{\mathrm{i}}$ die Gitterkonstanten und $a_{\mathrm{i}}^{*}$ die reziproken Gitterkostanten.

Die Lagen der Wasserstoffatome wurden, wenn nicht gesondert angegeben, mit idealisierter Geometrie als starre Gruppe berechnet. Die Abbildungen der Molekülstrukturen wurden mit Hilfe des Programms Schakal [132] und Diamond [133] angefertigt.

In den folgenden Tabellen sind die Bindungslängen in [pm], die Bindungswinkel in []

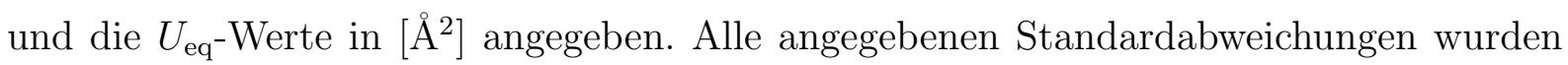
mittels des Programms SAINT [134] berechnet und sind unterschätzt. 


\subsection{Daten zur Kristallstrukturanalyse von $\left[\mathrm{Cu}\left(\mathrm{dppmO}_{2} \mathrm{H}\right)_{3}\right]\left[\mathrm{CuCl}_{4}\right] \underline{1}$}

\begin{tabular}{|ll|}
\hline Summenformel & $\mathrm{C}_{91} \mathrm{H}_{98} \mathrm{Cl}_{4} \mathrm{Cu}_{2} \mathrm{O}_{10} \mathrm{P}_{6}$ \\
Molekulargewicht $\left[\mathrm{g} \cdot \mathrm{mol}^{-1}\right]$ & 1806.39 \\
Temperatur & $133(2) \mathrm{K}$ \\
Wellenlänge & $71.073 \mathrm{pm}$ \\
Kristallsystem & monoklin \\
Raumgruppe & $P 2_{1} / n$ \\
Gitterkonstanten & $\mathrm{a}=16.1953(14) \AA, \alpha=90^{\circ}$ \\
& $\mathrm{b}=23.085(2) \AA, \beta=96.469(6)^{\circ} \circ$ \\
& $\mathrm{c}=23.382(2) \AA, \gamma=90^{\circ}{ }^{\circ}$ \\
Volumen & $8686.1(13) \AA^{3}$ \\
Formeleinheiten & 4 \\
Röntgenographische Dichte $\left[\mathrm{g} \cdot \mathrm{cm}^{-3}\right]$ & 1.381 \\
Absorptionskoeffizient $\left[\mathrm{mm}^{-1}\right]$ & 0.781 \\
$\mathrm{~F}(000)$ & 3760 \\
Meßbereich $\theta$ & $2.17-22.47^{\circ}$ \\
Indexbereiche & $-17 \leq \mathrm{h} \leq 12$ \\
& $-24 \leq \mathrm{k} \leq 24$ \\
Gemessene Reflexe & $-25 \leq 1 \leq 25$ \\
Unabhängige Reflexe & 80195 \\
Beobachtete Reflexe $[\mathrm{I}>2 \sigma]$ & 11310 \\
$\mathrm{R}_{\text {int }}$ & 5625 \\
Parameter & 0.1417 \\
R1; wR2[I>2 $\sigma(\mathrm{I})]$ & 872 \\
\hline
\end{tabular}


Tabelle 3: Atomkoordinaten und äquivalente isotrope Auslenkungsparameter $\left[\AA^{2}\right]$ für $\underline{1}$

\begin{tabular}{|c|c|c|c|c|}
\hline Atom & $\mathrm{x}$ & $\mathrm{y}$ & $\mathrm{z}$ & $\mathrm{U}_{e q}$ \\
\hline $\mathrm{Cu}(1)$ & $0.91055(8)$ & $0.20231(6)$ & $0.16819(6)$ & $0.0657(5)$ \\
\hline $\mathrm{Cu}(2)$ & $0.37472(8)$ & $0.17478(6)$ & $0.00434(6)$ & $0.0677(5)$ \\
\hline $\mathrm{P}(1)$ & $0.54474(16)$ & $0.13105(11)$ & $0.09265(11)$ & $0.0515(7)$ \\
\hline $\mathrm{P}(2)$ & $0.56165(18)$ & $0.24396(12)$ & $0.02844(12)$ & $0.0594(8)$ \\
\hline $\mathrm{P}(3)$ & $0.38656(17)$ & $0.14206(11)$ & $-0.13852(12)$ & $0.0550(8)$ \\
\hline $\mathrm{P}(4)$ & $0.29191(17)$ & $0.25226(12)$ & $-0.11461(14)$ & $0.0616(8)$ \\
\hline $\mathrm{P}(5)$ & $0.26026(18)$ & $0.24943(13)$ & $0.09538(12)$ & $0.0616(8)$ \\
\hline $\mathrm{P}(6)$ & $0.18957(17)$ & $0.13497(11)$ & $0.04664(11)$ & $0.0506(7)$ \\
\hline $\mathrm{Cl}(1)$ & $0.89752(18)$ & $0.10962(13)$ & $0.14141(15)$ & $0.0881(10)$ \\
\hline $\mathrm{Cl}(2)$ & $0.97729(17)$ & $0.25359(13)$ & $0.10406(14)$ & $0.0813(9)$ \\
\hline $\mathrm{Cl}(3)$ & $0.9919(2)$ & $0.20509(17)$ & $0.25460(15)$ & $0.1048(12)$ \\
\hline $\mathrm{Cl}(4)$ & $0.78394(17)$ & $0.24393(13)$ & $0.17831(13)$ & $0.0776(9)$ \\
\hline $\mathrm{O}(1)$ & $0.4561(4)$ & $0.1248(3)$ & $0.0684(3)$ & $0.0596(18)$ \\
\hline $\mathrm{O}(2)$ & $0.4847(4)$ & $0.2254(3)$ & $-0.0077(3)$ & $0.0587(18)$ \\
\hline $\mathrm{O}(3)$ & $0.4014(4)$ & $0.1280(3)$ & $-0.0756(3)$ & $0.0600(18)$ \\
\hline $\mathrm{O}(4)$ & $0.2977(4)$ & $0.2337(3)$ & $-0.0528(3)$ & $0.066(2)$ \\
\hline $\mathrm{O}(5)$ & $0.3405(4)$ & $0.2358(3)$ & $0.0733(3)$ & $0.071(2)$ \\
\hline $\mathrm{O}(6)$ & $0.2672(4)$ & $0.1232(3)$ & $0.0189(3)$ & $0.0552(17)$ \\
\hline $\mathrm{C}(1)$ & $0.5765(6)$ & $0.2065(4)$ & $0.0970(4)$ & $0.052(3)$ \\
\hline $\mathrm{C}(2)$ & $0.6114(6)$ & $0.0935(4)$ & $0.0493(4)$ & $0.044(2)$ \\
\hline $\mathrm{C}(3)$ & $0.5768(7)$ & $0.0577(5)$ & $0.0076(6)$ & $0.084(4)$ \\
\hline $\mathrm{C}(4)$ & $0.6243(8)$ & $0.0248(5)$ & $-0.0251(6)$ & $0.101(5)$ \\
\hline $\mathrm{C}(5)$ & $0.7095(8)$ & $0.0302(5)$ & $-0.0155(5)$ & $0.075(3)$ \\
\hline $\mathrm{C}(6)$ & $0.7466(6)$ & $0.0661(4)$ & $0.0261(4)$ & $0.054(3)$ \\
\hline $\mathrm{C}(7)$ & $0.6967(6)$ & $0.0979(4)$ & $0.0589(4)$ & $0.058(3)$ \\
\hline $\mathrm{C}(8 \mathrm{~A})$ & $0.5628(9)$ & $0.1085(7)$ & $0.1701(5)$ & $0.051(8)$ \\
\hline $\mathrm{C}(9 \mathrm{~A})$ & $0.6350(8)$ & $0.0798(8)$ & $0.1920(7)$ & $0.101(9)$ \\
\hline $\mathrm{C}(10 \mathrm{~A})$ & $0.6443(8)$ & $0.0605(7)$ & $0.2487(7)$ & $0.105(9)$ \\
\hline $\mathrm{C}(11 \mathrm{~A})$ & $0.5815(10)$ & $0.0699(7)$ & $0.2835(5)$ & $0.093(8)$ \\
\hline $\mathrm{C}(12 \mathrm{~A})$ & $0.5093(9)$ & $0.0985(7)$ & $0.2616(6)$ & $0.086(7)$ \\
\hline $\mathrm{C}(13 \mathrm{~A})$ & $0.4999(7)$ & $0.1178(6)$ & $0.2050(6)$ & $0.063(6)$ \\
\hline $\mathrm{C}(8 \mathrm{~B})$ & $0.5652(9)$ & $0.0976(6)$ & $0.1589(6)$ & $0.045(7)$ \\
\hline $\mathrm{C}(9 \mathrm{~B})$ & $0.6296(9)$ & $0.1175(6)$ & $0.1984(7)$ & $0.077(7)$ \\
\hline $\mathrm{C}(10 \mathrm{~B})$ & $0.6450(8)$ & $0.0911(7)$ & $0.2520(6)$ & $0.108(10)$ \\
\hline $\mathrm{C}(11 \mathrm{~B})$ & $0.5961(9)$ & $0.0449(7)$ & $0.2661(5)$ & $0.076(7)$ \\
\hline $\mathrm{C}(12 \mathrm{~B})$ & $0.5318(8)$ & $0.0250(6)$ & $0.2266(6)$ & $0.069(6)$ \\
\hline $\mathrm{C}(13 \mathrm{~B})$ & $0.5163(7)$ & $0.0514(6)$ & $0.1730(6)$ & $0.074(6)$ \\
\hline $\mathrm{C}(14)$ & $0.6545(7)$ & $0.2294(5)$ & $-0.0084(5)$ & $0.065(3)$ \\
\hline
\end{tabular}


Tabelle 3: Fortsetzung

\begin{tabular}{|r|r|r|r|r|}
\hline $\mathrm{C}(15)$ & $0.7327(8)$ & $0.2408(5)$ & $0.0187(5)$ & $0.073(3)$ \\
$\mathrm{C}(16)$ & $0.7983(8)$ & $0.2303(6)$ & $-0.0132(7)$ & $0.093(4)$ \\
$\mathrm{C}(17)$ & $0.7850(9)$ & $0.2077(7)$ & $-0.0691(6)$ & $0.099(5)$ \\
$\mathrm{C}(18)$ & $0.7051(9)$ & $0.1976(6)$ & $-0.0929(5)$ & $0.091(4)$ \\
$\mathrm{C}(19)$ & $0.6395(8)$ & $0.2081(5)$ & $-0.0638(5)$ & $0.074(3)$ \\
$\mathrm{C}(20)$ & $0.5556(8)$ & $0.3186(5)$ & $0.0444(5)$ & $0.063(3)$ \\
$\mathrm{C}(21)$ & $0.6097(11)$ & $0.3448(6)$ & $0.0839(6)$ & $0.122(6)$ \\
$\mathrm{C}(22)$ & $0.6007(14)$ & $0.4069(7)$ & $0.0934(7)$ & $0.164(8)$ \\
$\mathrm{C}(23)$ & $0.5424(12)$ & $0.4367(6)$ & $0.0643(7)$ & $0.116(5)$ \\
$\mathrm{C}(24)$ & $0.4865(8)$ & $0.4096(6)$ & $0.0227(7)$ & $0.098(4)$ \\
$\mathrm{C}(25)$ & $0.4939(8)$ & $0.3497(5)$ & $0.0137(6)$ & $0.084(4)$ \\
$\mathrm{C}(26)$ & $0.3703(6)$ & $0.2173(4)$ & $-0.1524(5)$ & $0.063(3)$ \\
$\mathrm{C}(27)$ & $0.2994(6)$ & $0.1044(4)$ & $-0.1722(5)$ & $0.058(3)$ \\
$\mathrm{C}(28)$ & $0.2720(7)$ & $0.0550(5)$ & $-0.1465(5)$ & $0.066(3)$ \\
$\mathrm{C}(29)$ & $0.2072(9)$ & $0.0228(5)$ & $-0.1715(6)$ & $0.086(4)$ \\
$\mathrm{C}(30)$ & $0.1667(9)$ & $0.0397(7)$ & $-0.2211(7)$ & $0.090(4)$ \\
$\mathrm{C}(31)$ & $0.1883(9)$ & $0.0877(8)$ & $-0.2478(6)$ & $0.098(5)$ \\
$\mathrm{C}(32)$ & $0.2572(8)$ & $0.1209(5)$ & $-0.2244(5)$ & $0.073(3)$ \\
$\mathrm{C}(33 \mathrm{~A})$ & $0.4693(9)$ & $0.1180(8)$ & $-0.1725(7)$ & $0.063(11)$ \\
$\mathrm{C}(34 \mathrm{~A})$ & $0.5091(11)$ & $0.1527(6)$ & $-0.2095(7)$ & $0.077(7)$ \\
$\mathrm{C}(35 \mathrm{~A})$ & $0.5751(11)$ & $0.1306(8)$ & $-0.2360(7)$ & $0.110(10)$ \\
$\mathrm{C}(36 \mathrm{~A})$ & $0.6012(10)$ & $0.0739(8)$ & $-0.2254(7)$ & $0.100(9)$ \\
$\mathrm{C}(37 \mathrm{~A})$ & $0.5614(11)$ & $0.0392(6)$ & $-0.1884(8)$ & $0.109(9)$ \\
$\mathrm{C}(38 \mathrm{~A})$ & $0.4954(10)$ & $0.0612(7)$ & $-0.1620(7)$ & $0.097(8)$ \\
$\mathrm{C}(33 \mathrm{~B})$ & $0.4787(7)$ & $0.1265(7)$ & $-0.1767(6)$ & $0.071(12)$ \\
$\mathrm{C}(34 \mathrm{~B})$ & $0.4832(8)$ & $0.1428(6)$ & $-0.2335(6)$ & $0.057(6)$ \\
$\mathrm{C}(35 \mathrm{~B})$ & $0.5542(9)$ & $0.1306(6)$ & $-0.2596(5)$ & $0.073(7)$ \\
$\mathrm{C}(36 \mathrm{~B})$ & $0.6207(7)$ & $0.1022(7)$ & $-0.2289(6)$ & $0.078(7)$ \\
$\mathrm{C}(37 \mathrm{~B})$ & $0.6163(7)$ & $0.0860(6)$ & $-0.1721(6)$ & $0.070(6)$ \\
$\mathrm{C}(38 \mathrm{~B})$ & $0.5453(9)$ & $0.0981(6)$ & $-0.1460(5)$ & $0.057(5)$ \\
$\mathrm{C}(39)$ & $0.1934(6)$ & $0.2349(4)$ & $-0.1501(4)$ & $0.049(3)$ \\
$\mathrm{C}(40)$ & $0.1620(8)$ & $0.2581(5)$ & $-0.2015(5)$ & $0.077(4)$ \\
$\mathrm{C}(41)$ & $0.0865(10)$ & $0.2432(6)$ & $-0.2281(5)$ & $0.090(4)$ \\
$\mathrm{C}(42)$ & $0.0367(7)$ & $0.2034(6)$ & $-0.2019(6)$ & $0.084(4)$ \\
$\mathrm{C}(43)$ & $0.0671(7)$ & $0.1795(5)$ & $-0.1485(5)$ & $0.067(3)$ \\
$\mathrm{C}(44)$ & $0.1435(6)$ & $0.1946(4)$ & $-0.1241(4)$ & $0.056(3)$ \\
$\mathrm{C}(45)$ & $0.3055(7)$ & $0.3286(5)$ & $-0.1244(7)$ & $0.088(4)$ \\
$\mathrm{C}(46)$ & $0.3344(10)$ & $0.3511(6)$ & $-0.1752(10)$ & $0.148(8)$ \\
\hline & $0.3428(15)$ & $0.4080(10)$ & $-0.1844(12)$ & $0.184(9)$ \\
$\mathrm{C}(15)$ & $0.4443(10)$ & $-0.1442(13)$ & $0.167(10)$ \\
\hline
\end{tabular}


Tabelle 3: Fortsetzung

\begin{tabular}{|r|r|r|r|r|}
\hline $\mathrm{C}(49)$ & $0.2911(11)$ & $0.4273(7)$ & $-0.0928(10)$ & $0.142(8)$ \\
$\mathrm{C}(50)$ & $0.2824(8)$ & $0.3646(6)$ & $-0.0836(8)$ & $0.113(6)$ \\
$\mathrm{C}(51)$ & $0.1738(6)$ & $0.2116(4)$ & $0.0577(4)$ & $0.048(2)$ \\
$\mathrm{C}(52)$ & $0.2676(8)$ & $0.2336(5)$ & $0.1708(4)$ & $0.066(3)$ \\
$\mathrm{C}(53)$ & $0.3435(8)$ & $0.2190(7)$ & $0.1978(5)$ & $0.099(5)$ \\
$\mathrm{C}(54)$ & $0.3525(8)$ & $0.2103(7)$ & $0.2570(6)$ & $0.109(5)$ \\
$\mathrm{C}(55)$ & $0.2851(9)$ & $0.2141(7)$ & $0.2875(5)$ & $0.101(5)$ \\
$\mathrm{C}(56)$ & $0.2109(9)$ & $0.2277(5)$ & $0.2608(5)$ & $0.085(4)$ \\
$\mathrm{C}(57)$ & $0.1982(8)$ & $0.2369(5)$ & $0.2007(4)$ & $0.072(3)$ \\
$\mathrm{C}(58)$ & $0.2320(9)$ & $0.3248(5)$ & $0.0879(5)$ & $0.071(3)$ \\
$\mathrm{C}(59)$ & $0.2910(10)$ & $0.3633(6)$ & $0.0756(6)$ & $0.111(5)$ \\
$\mathrm{C}(60)$ & $0.2776(12)$ & $0.4217(7)$ & $0.0732(8)$ & $0.140(7)$ \\
$\mathrm{C}(61)$ & $0.2004(11)$ & $0.4419(7)$ & $0.0837(7)$ & $0.115(5)$ \\
$\mathrm{C}(62)$ & $0.1389(9)$ & $0.4042(6)$ & $0.0966(5)$ & $0.095(4)$ \\
$\mathrm{C}(63)$ & $0.1572(9)$ & $0.3460(6)$ & $0.0973(5)$ & $0.084(4)$ \\
$\mathrm{C}(64)$ & $0.1957(7)$ & $0.1024(4)$ & $0.1162(4)$ & $0.061(3)$ \\
$\mathrm{C}(65)$ & $0.2699(7)$ & $0.0845(5)$ & $0.1435(5)$ & $0.068(3)$ \\
$\mathrm{C}(66)$ & $0.2742(9)$ & $0.0640(5)$ & $0.1977(6)$ & $0.090(4)$ \\
$\mathrm{C}(67)$ & $0.2045(12)$ & $0.0637(6)$ & $0.2281(6)$ & $0.106(5)$ \\
$\mathrm{C}(68)$ & $0.1296(9)$ & $0.0823(6)$ & $0.2012(6)$ & $0.092(4)$ \\
$\mathrm{C}(69)$ & $0.1236(8)$ & $0.1027(5)$ & $0.1457(5)$ & $0.074(3)$ \\
$\mathrm{C}(70)$ & $0.1009(6)$ & $0.1076(4)$ & $0.0029(4)$ & $0.047(2)$ \\
$\mathrm{C}(71)$ & $0.1122(6)$ & $0.0632(4)$ & $-0.0350(4)$ & $0.051(3)$ \\
$\mathrm{C}(72)$ & $0.0455(8)$ & $0.0379(5)$ & $-0.0670(4)$ & $0.064(3)$ \\
$\mathrm{C}(73)$ & $-0.0327(8)$ & $0.0552(4)$ & $-0.0609(4)$ & $0.063(3)$ \\
$\mathrm{C}(74)$ & $-0.0443(7)$ & $0.1008(5)$ & $-0.0244(5)$ & $0.068(3)$ \\
$\mathrm{C}(75)$ & $0.0203(6)$ & $0.1276(4)$ & $0.0077(4)$ & $0.055(3)$ \\
$\mathrm{C}(1 \mathrm{~L})$ & $0.4792(15)$ & $0.1903(10)$ & $0.4309(9)$ & $0.179(8)$ \\
$\mathrm{C}(2 \mathrm{~L})$ & $0.4226(11)$ & $0.1507(8)$ & $0.4044(7)$ & $0.129(5)$ \\
$\mathrm{C}(3 \mathrm{~L})$ & $0.4498(13)$ & $0.0969(10)$ & $0.4040(9)$ & $0.170(7)$ \\
$\mathrm{C}(4 \mathrm{LA})$ & $0.555(3)$ & $0.1248(18)$ & $0.3892(19)$ & $0.167(15)$ \\
$\mathrm{C}(4 \mathrm{LB})$ & $0.5466(18)$ & $0.1046(14)$ & $0.4366(14)$ & $0.113(10)$ \\
$\mathrm{C}(5 \mathrm{~L})$ & $0.5572(13)$ & $0.1679(10)$ & $0.4415(9)$ & $0.166(7)$ \\
$\mathrm{C}(6 \mathrm{~L})$ & $-0.0694(11)$ & $0.4614(8)$ & $0.1101(9)$ & $0.146(6)$ \\
$\mathrm{C}(7 \mathrm{~L})$ & $-0.1153(15)$ & $0.4544(13)$ & $0.0526(12)$ & $0.217(10)$ \\
$\mathrm{C}(8 \mathrm{~L})$ & $-0.1511(18)$ & $0.3955(14)$ & $0.0552(14)$ & $0.236(12)$ \\
$\mathrm{C}(9 \mathrm{~L})$ & $-0.162(2)$ & $0.3902(15)$ & $0.1100(17)$ & $0.261(14)$ \\
$\mathrm{C}(10 \mathrm{~L})$ & $-0.1162(17)$ & $0.4226(12)$ & $0.1492(11)$ & $0.214(10)$ \\
$\mathrm{C}(11 \mathrm{~L})$ & $-0.0598(14)$ & $0.0798(11)$ & $-0.2870(10)$ & $0.191(9)$ \\
$\mathrm{C}(12 \mathrm{~L})$ & $-0.1068(14)$ & $0.1394(10)$ & $-0.2940(9)$ & $0.176(8)$ \\
\hline & & & & \\
& & & \\
& & & \\
& &
\end{tabular}


Tabelle 3: Fortsetzung

\begin{tabular}{|r|r|r|r|r|}
\hline $\mathrm{C}(13 \mathrm{~L})$ & $-0.1806(14)$ & $0.1131(10)$ & $-0.2719(10)$ & $0.187(8)$ \\
$\mathrm{C}(14 \mathrm{~L})$ & $-0.1390(12)$ & $0.0936(9)$ & $-0.2038(9)$ & $0.161(7)$ \\
$\mathrm{C}(15 \mathrm{~L})$ & $-0.0586(12)$ & $0.0592(9)$ & $-0.2258(9)$ & $0.165(7)$ \\
$\mathrm{C}(16 \mathrm{~L})$ & $0.070(2)$ & $0.0752(17)$ & $0.3640(15)$ & $0.095(10)$ \\
$\mathrm{C}(17 \mathrm{~L})$ & $0.132(3)$ & $0.1349(16)$ & $0.3784(16)$ & $0.114(13)$ \\
$\mathrm{C}(18 \mathrm{~L})$ & $0.2120(19)$ & $0.0883(15)$ & $0.3950(14)$ & $0.112(10)$ \\
$\mathrm{C}(19 \mathrm{~L})$ & $0.1695(18)$ & $0.0716(13)$ & $0.4472(13)$ & $0.111(9)$ \\
$\mathrm{C}(20 \mathrm{~L})$ & $0.1086(12)$ & $0.0414(8)$ & $0.4237(9)$ & $0.051(5)$ \\
$\mathrm{C}(21 \mathrm{~L})$ & $0.112(3)$ & $0.0480(17)$ & $0.3794(18)$ & $0.118(11)$ \\
$\mathrm{C}(22 \mathrm{~L})$ & $0.163(2)$ & $0.0525(15)$ & $0.3673(15)$ & $0.120(11)$ \\
$\mathrm{C}(23 \mathrm{~L})$ & $0.1910(16)$ & $0.1279(11)$ & $0.4149(11)$ & $0.079(7)$ \\
$\mathrm{C}(24 \mathrm{~L})$ & $0.046(3)$ & $0.092(2)$ & $0.381(2)$ & $0.17(2)$ \\
$\mathrm{C}(25 \mathrm{~L})$ & $0.090(3)$ & $0.1413(16)$ & $0.3792(15)$ & $0.110(12)$ \\
\hline
\end{tabular}

Tabelle 4: Bindungslängen [pm] in 1

\begin{tabular}{|l|r||l|r|}
\hline $\mathrm{Cu}(1)-\mathrm{Cl}(1)$ & $223.3(3)$ & $\mathrm{Cu}(1)-\mathrm{Cl}(2)$ & $227.7(3)$ \\
$\mathrm{Cu}(1)-\mathrm{Cl}(3)$ & $228.5(4)$ & $\mathrm{Cu}(1)-\mathrm{Cl}(4)$ & $230.1(3)$ \\
$\mathrm{Cu}(2)-\mathrm{O}(6)$ & $216.7(6)$ & $\mathrm{Cu}(2)-\mathrm{O}(2)$ & $217.6(7)$ \\
$\mathrm{Cu}(2)-\mathrm{O}(4)$ & $219.5(7)$ & $\mathrm{Cu}(2)-\mathrm{O}(1)$ & $220.7(6)$ \\
$\mathrm{Cu}(2)-\mathrm{O}(3)$ & $224.3(6)$ & $\mathrm{Cu}(2)-\mathrm{O}(5)$ & $225.6(7)$ \\
$\mathrm{P}(1)-\mathrm{O}(1)$ & $149.0(6)$ & $\mathrm{P}(1)-\mathrm{C}(8 \mathrm{~B})$ & $173.0(11)$ \\
$\mathrm{P}(1)-\mathrm{C}(2)$ & $178.7(9)$ & $\mathrm{P}(1)-\mathrm{C}(1)$ & $181.6(9)$ \\
$\mathrm{P}(1)-\mathrm{C}(8 \mathrm{~A})$ & $187.6(12)$ & $\mathrm{P}(2)-\mathrm{O}(2)$ & $148.7(7)$ \\
$\mathrm{P}(2)-\mathrm{C}(20)$ & $176.9(12)$ & $\mathrm{P}(2)-\mathrm{C}(1)$ & $181.3(9)$ \\
$\mathrm{P}(2)-\mathrm{C}(14)$ & $184.6(11)$ & $\mathrm{P}(3)-\mathrm{O}(3)$ & $149.9(7)$ \\
$\mathrm{P}(3)-\mathrm{C}(33 \mathrm{~A})$ & $172.5(13)$ & $\mathrm{P}(3)-\mathrm{C}(27)$ & $176.6(11)$ \\
$\mathrm{P}(3)-\mathrm{C}(26)$ & $178.2(10)$ & $\mathrm{P}(3)-\mathrm{C}(33 \mathrm{~B})$ & $185.8(11)$ \\
$\mathrm{P}(4)-\mathrm{O}(4)$ & $150.1(8)$ & $\mathrm{P}(4)-\mathrm{C}(39)$ & $176.0(10)$ \\
$\mathrm{P}(4)-\mathrm{C}(45)$ & $179.3(13)$ & $\mathrm{P}(4)-\mathrm{C}(26)$ & $181.5(10)$ \\
$\mathrm{P}(5)-\mathrm{O}(5)$ & $148.6(7)$ & $\mathrm{P}(5)-\mathrm{C}(52)$ & $179.3(10)$ \\
$\mathrm{P}(5)-\mathrm{C}(51)$ & $179.6(9)$ & $\mathrm{P}(5)-\mathrm{C}(58)$ & $180.3(13)$ \\
$\mathrm{P}(6)-\mathrm{O}(6)$ & $150.4(6)$ & $\mathrm{P}(6)-\mathrm{C}(70)$ & $178.3(10)$ \\
$\mathrm{P}(6)-\mathrm{C}(64)$ & $178.5(10)$ & $\mathrm{P}(6)-\mathrm{C}(51)$ & $180.9(9)$ \\
\hline
\end{tabular}

Tabelle 5: Bindungswinkel $\left[^{\circ}\right]$ in $\underline{1}$

\begin{tabular}{|l|l||l|l|}
\hline $\mathrm{Cl}(1)-\mathrm{Cu}(1)-\mathrm{Cl}(2)$ & $110.52(12)$ & $\mathrm{Cl}(1)-\mathrm{Cu}(1)-\mathrm{Cl}(3)$ & $107.56(15)$ \\
$\mathrm{Cl}(2)-\mathrm{Cu}(1)-\mathrm{Cl}(3)$ & $107.37(14)$ & $\mathrm{Cl}(1)-\mathrm{Cu}(1)-\mathrm{Cl}(4)$ & $111.83(12)$ \\
\hline
\end{tabular}


Tabelle 5: Fortsetzung

\begin{tabular}{|l|r||l|r|}
\hline $\mathrm{Cl}(2)-\mathrm{Cu}(1)-\mathrm{Cl}(4)$ & $110.21(12)$ & $\mathrm{Cl}(3)-\mathrm{Cu}(1)-\mathrm{Cl}(4)$ & $109.21(12)$ \\
$\mathrm{O}(6)-\mathrm{Cu}(2)-\mathrm{O}(2)$ & $178.2(2)$ & $\mathrm{O}(6)-\mathrm{Cu}(2)-\mathrm{O}(4)$ & $91.4(2)$ \\
$\mathrm{O}(2)-\mathrm{Cu}(2)-\mathrm{O}(4)$ & $90.3(2)$ & $\mathrm{O}(6)-\mathrm{Cu}(2)-\mathrm{O}(1)$ & $92.0(2)$ \\
$\mathrm{O}(2)-\mathrm{Cu}(2)-\mathrm{O}(1)$ & $86.2(2)$ & $\mathrm{O}(4)-\mathrm{Cu}(2)-\mathrm{O}(1)$ & $173.0(3)$ \\
$\mathrm{O}(6)-\mathrm{Cu}(2)-\mathrm{O}(3)$ & $95.7(2)$ & $\mathrm{O}(2)-\mathrm{Cu}(2)-\mathrm{O}(3)$ & $85.0(2)$ \\
$\mathrm{O}(4)-\mathrm{Cu}(2)-\mathrm{O}(3)$ & $86.6(2)$ & $\mathrm{O}(1)-\mathrm{Cu}(2)-\mathrm{O}(3)$ & $99.1(2)$ \\
$\mathrm{O}(6)-\mathrm{Cu}(2)-\mathrm{O}(5)$ & $88.0(2)$ & $\mathrm{O}(2)-\mathrm{Cu}(2)-\mathrm{O}(5)$ & $91.6(2)$ \\
$\mathrm{O}(4)-\mathrm{Cu}(2)-\mathrm{O}(5)$ & $83.1(3)$ & $\mathrm{O}(1)-\mathrm{Cu}(2)-\mathrm{O}(5)$ & $91.0(3)$ \\
$\mathrm{O}(3)-\mathrm{Cu}(2)-\mathrm{O}(5)$ & $169.1(3)$ & $\mathrm{O}(1)-\mathrm{P}(1)-\mathrm{C}(8 \mathrm{~B})$ & $112.1(6)$ \\
$\mathrm{O}(1)-\mathrm{P}(1)-\mathrm{C}(2)$ & $110.8(4)$ & $\mathrm{O}(1)-\mathrm{P}(1)-\mathrm{C}(1)$ & $111.7(4)$ \\
$\mathrm{C}(2)-\mathrm{P}(1)-\mathrm{C}(1)$ & $108.1(4)$ & $\mathrm{O}(2)-\mathrm{P}(2)-\mathrm{C}(20)$ & $109.8(5)$ \\
$\mathrm{O}(2)-\mathrm{P}(2)-\mathrm{C}(1)$ & $112.6(4)$ & $\mathrm{O}(2)-\mathrm{P}(2)-\mathrm{C}(14)$ & $110.9(5)$ \\
$\mathrm{C}(1)-\mathrm{P}(2)-\mathrm{C}(14)$ & $106.9(5)$ & $\mathrm{O}(3)-\mathrm{P}(3)-\mathrm{C}(33 \mathrm{~A})$ & $109.6(6)$ \\
$\mathrm{O}(3)-\mathrm{P}(3)-\mathrm{C}(27)$ & $111.3(4)$ & $\mathrm{O}(3)-\mathrm{P}(3)-\mathrm{C}(26)$ & $113.2(5)$ \\
$\mathrm{O}(3)-\mathrm{P}(3)-\mathrm{C}(33 \mathrm{~B})$ & $112.3(5)$ & $\mathrm{O}(4)-\mathrm{P}(4)-\mathrm{C}(39)$ & $110.1(4)$ \\
$\mathrm{O}(4)-\mathrm{P}(4)-\mathrm{C}(45)$ & $114.1(6)$ & $\mathrm{O}(4)-\mathrm{P}(4)-\mathrm{C}(26)$ & $111.8(4)$ \\
$\mathrm{O}(5)-\mathrm{P}(5)-\mathrm{C}(52)$ & $109.6(5)$ & $\mathrm{O}(5)-\mathrm{P}(5)-\mathrm{C}(51)$ & $113.0(4)$ \\
$\mathrm{O}(5)-\mathrm{P}(5)-\mathrm{C}(58)$ & $113.1(5)$ & $\mathrm{O}(6)-\mathrm{P}(6)-\mathrm{C}(70)$ & $110.3(4)$ \\
$\mathrm{O}(6)-\mathrm{P}(6)-\mathrm{C}(64)$ & $110.8(5)$ & $\mathrm{O}(6)-\mathrm{P}(6)-\mathrm{C}(51)$ & $112.1(4)$ \\
$\mathrm{P}(1)-\mathrm{O}(1)-\mathrm{Cu}(2)$ & $133.2(4)$ & $\mathrm{P}(2)-\mathrm{O}(2)-\mathrm{Cu}(2)$ & $136.6(4)$ \\
$\mathrm{P}(3)-\mathrm{O}(3)-\mathrm{Cu}(2)$ & $133.6(4)$ & $\mathrm{P}(4)-\mathrm{O}(4)-\mathrm{Cu}(2)$ & $137.1(4)$ \\
$\mathrm{P}(5)-\mathrm{O}(5)-\mathrm{Cu}(2)$ & $132.5(4)$ & $\mathrm{P}(6)-\mathrm{O}(6)-\mathrm{Cu}(2)$ & $134.0(4)$ \\
$\mathrm{P}(2)-\mathrm{C}(1)-\mathrm{P}(1)$ & $113.6(5)$ & $\mathrm{P}(3)-\mathrm{C}(26)-\mathrm{P}(4)$ & $116.2(5)$ \\
$\mathrm{P}(5)-\mathrm{C}(51)-\mathrm{P}(6)$ & $115.5(5)$ & & \\
\hline & & & \\
\hline
\end{tabular}




\subsection{Daten zur Kristallstrukturanalyse von $\left[\mathrm{Cu}_{2}\left(\operatorname{dppmS}{ }_{2} \mathrm{H}\right)_{2} \mathrm{Cl}\right]\left[\mathrm{CuCl}_{2}\right] \underline{\mathbf{2}}$}

\begin{tabular}{|ll|}
\hline Summenformel & $\mathrm{C}_{50} \mathrm{H}_{44} \mathrm{Cl}_{3} \mathrm{Cu}_{3} \mathrm{P}_{4} \mathrm{~S}_{4}$ \\
Molekulargewicht $\left[\mathrm{g} \cdot \mathrm{mol}^{-1}\right]$ & 1193.94 \\
Temperatur & $293(2) \mathrm{K}$ \\
Wellenlänge & $71.073 \mathrm{pm}$ \\
Kristallsystem & orthorhombisch \\
Raumgruppe & $P c c n$ \\
Gitterkonstanten & $\mathrm{a}=11.280(7) \AA, \alpha=90^{\circ}$ \\
& $\mathrm{b}=16.251(14) \AA, \beta=90^{\circ}$ \\
& $\mathrm{c}=26.68(2) \AA, \gamma=90^{\circ}$ \\
Volumen & $4890(7) \AA^{3}$ \\
Formeleinheiten & 4 \\
Röntgenographische Dichte $\left[\mathrm{g} \cdot \mathrm{cm}^{-3}\right]$ & 1.622 \\
Absorptionskoeffizient $\left[\mathrm{mm}^{-1}\right]$ & 1.795 \\
F(000) & 2424 \\
Meßbereich $\theta$ & $2.20-22.51^{\circ}$ \\
Indexbereiche & $-5 \leq \mathrm{h} \leq 11$ \\
& $-15 \leq \mathrm{k} \leq 17$ \\
& $-27 \leq 1 \leq 28$ \\
Gemessene Reflexe & 13304 \\
Unabhängige Reflexe & 3191 \\
Beobachtete Reflexe $[\mathrm{I}>2 \sigma]$ & 2042 \\
$\mathrm{R}_{\text {int }}$ & 0.1240 \\
Parameter & 290 \\
R1; wR2[I $>2 \sigma(\mathrm{I})]$ & $0.0648 ; 0.1274$ \\
\hline
\end{tabular}


Tabelle 7: Atomkoordinaten und äquivalente isotrope Auslenkungsparameter $\left[\AA^{2}\right]$ für $\underline{2}$

\begin{tabular}{|c|c|c|c|c|}
\hline Atom & $\mathrm{x}$ & $\mathrm{y}$ & $\mathrm{z}$ & $\mathrm{U}_{e q}$ \\
\hline $\mathrm{Cu}(1)$ & $0.16325(9)$ & $0.67362(6)$ & $0.03002(4)$ & $0.0267(3)$ \\
\hline $\mathrm{Cu}(2)$ & 0.2500 & 0.7500 & $0.36188(6)$ & $0.0306(4)$ \\
\hline $\mathrm{P}(1)$ & $-0.06655(19)$ & $0.59439(13)$ & $0.08269(8)$ & $0.0195(6)$ \\
\hline $\mathrm{P}(2)$ & $0.1748(2)$ & $0.52650(13)$ & $0.11903(8)$ & $0.0213(6)$ \\
\hline $\mathrm{S}(1)$ & $-0.0295(2)$ & $0.65673(14)$ & $0.02130(8)$ & $0.0287(6)$ \\
\hline $\mathrm{S}(2)$ & $0.2642(2)$ & $0.62160(14)$ & $0.09288(8)$ & $0.0299(6)$ \\
\hline $\mathrm{Cl}(1)$ & 0.2500 & 0.7500 & $-0.02771(10)$ & $0.0303(8)$ \\
\hline $\mathrm{Cl}(2)$ & $0.1031(2)$ & $0.82801(13)$ & $0.35202(8)$ & $0.0322(6)$ \\
\hline $\mathrm{C}(1)$ & $0.0320(7)$ & $0.5082(4)$ & $0.0921(3)$ & $0.019(2)$ \\
\hline $\mathrm{C}(2)$ & $0.2598(7)$ & $0.4348(5)$ & $0.1080(3)$ & $0.023(2)$ \\
\hline $\mathrm{C}(3)$ & $0.3281(9)$ & $0.4030(6)$ & $0.1445(3)$ & $0.042(3)$ \\
\hline $\mathrm{C}(4)$ & $0.4015(9)$ & $0.3374(6)$ & $0.1347(4)$ & $0.051(3)$ \\
\hline $\mathrm{C}(5)$ & $0.4026(8)$ & $0.3031(5)$ & $0.0885(3)$ & $0.034(2)$ \\
\hline $\mathrm{C}(6)$ & $0.3356(7)$ & $0.3366(5)$ & $0.0513(3)$ & $0.028(2)$ \\
\hline $\mathrm{C}(7)$ & $0.2642(8)$ & $0.4024(5)$ & $0.0605(3)$ & $0.024(2)$ \\
\hline $\mathrm{C}(8)$ & $0.1524(7)$ & $0.5341(5)$ & $0.1852(3)$ & $0.022(2)$ \\
\hline $\mathrm{C}(9)$ & $0.1977(9)$ & $0.5995(6)$ & $0.2112(4)$ & $0.043(3)$ \\
\hline $\mathrm{C}(10)$ & $0.1850(10)$ & $0.6040(7)$ & $0.2616(4)$ & $0.057(3)$ \\
\hline $\mathrm{C}(11)$ & $0.1304(10)$ & $0.5433(7)$ & $0.2863(4)$ & $0.051(3)$ \\
\hline $\mathrm{C}(12)$ & $0.0806(10)$ & $0.4790(6)$ & $0.2617(4)$ & $0.058(3)$ \\
\hline $\mathrm{C}(13)$ & $0.0954(9)$ & $0.4731(6)$ & $0.2100(3)$ & $0.043(3)$ \\
\hline $\mathrm{C}(14)$ & $-0.0629(7)$ & $0.6539(4)$ & $0.1389(3)$ & $0.021(2)$ \\
\hline $\mathrm{C}(15)$ & $-0.0009(8)$ & $0.7272(5)$ & $0.1399(3)$ & $0.031(2)$ \\
\hline $\mathrm{C}(16)$ & $0.0041(8)$ & $0.7696(5)$ & $0.1841(4)$ & $0.039(3)$ \\
\hline $\mathrm{C}(17)$ & $-0.0552(10)$ & $0.7425(6)$ & $0.2254(4)$ & $0.048(3)$ \\
\hline $\mathrm{C}(18)$ & $-0.1191(10)$ & $0.6723(6)$ & $0.2235(3)$ & $0.046(3)$ \\
\hline $\mathrm{C}(19)$ & $-0.1223(9)$ & $0.6268(5)$ & $0.1800(3)$ & $0.033(2)$ \\
\hline $\mathrm{C}(20)$ & $-0.2124(7)$ & $0.5549(5)$ & $0.0783(3)$ & $0.018(2)$ \\
\hline $\mathrm{C}(21)$ & $-0.3053(8)$ & $0.6106(5)$ & $0.0819(3)$ & $0.034(2)$ \\
\hline $\mathrm{C}(22)$ & $-0.4179(8)$ & $0.5862(5)$ & $0.0742(3)$ & $0.038(3)$ \\
\hline $\mathrm{C}(23)$ & $-0.4440(8)$ & $0.5045(6)$ & $0.0631(3)$ & $0.033(2)$ \\
\hline $\mathrm{C}(24)$ & $-0.3533(8)$ & $0.4508(5)$ & $0.0590(3)$ & $0.030(2)$ \\
\hline $\mathrm{C}(25)$ & $-0.2393(8)$ & $0.4745(5)$ & $0.0662(3)$ & $0.022(2)$ \\
\hline
\end{tabular}

Tabelle 8: Bindungslängen $[\mathrm{pm}]$ in $\underline{2}$

\begin{tabular}{|l|l||l|l|}
\hline $\mathrm{Cu}(1)-\mathrm{S}(2)$ & $219.6(3)$ & $\mathrm{Cu}(1)-\mathrm{S}(1)$ & $220.4(3)$ \\
$\mathrm{Cu}(1)-\mathrm{Cl}(1)$ & $220.7(2)$ & $\mathrm{Cu}(2)-\mathrm{Cl}(2) \# 1$ & $210.3(2)$ \\
\hline
\end{tabular}


Tabelle 8: Fortsetzung

\begin{tabular}{|l|r||l|r|}
\hline $\mathrm{Cu}(2)-\mathrm{Cl}(2)$ & $210.3(2)$ & $\mathrm{P}(1)-\mathrm{C}(20)$ & $176.9(8)$ \\
$\mathrm{P}(1)-\mathrm{C}(14)$ & $178.5(8)$ & $\mathrm{P}(1)-\mathrm{C}(1)$ & $180.6(8)$ \\
$\mathrm{P}(1)-\mathrm{S}(1)$ & $197.0(3)$ & $\mathrm{P}(2)-\mathrm{C}(8)$ & $178.8(8)$ \\
$\mathrm{P}(2)-\mathrm{C}(1)$ & $178.9(8)$ & $\mathrm{P}(2)-\mathrm{C}(2)$ & $179.6(8)$ \\
$\mathrm{P}(2)-\mathrm{S}(2)$ & $197.3(3)$ & $\mathrm{Cl}(1)-\mathrm{Cu}(1) \# 1$ & $220.7(2)$ \\
$\mathrm{C}(2)-\mathrm{C}(3)$ & $134.4(11)$ & $\mathrm{C}(2)-\mathrm{C}(7)$ & $137.3(11)$ \\
$\mathrm{C}(3)-\mathrm{C}(4)$ & $137.6(12)$ & $\mathrm{C}(4)-\mathrm{C}(5)$ & $135.3(12)$ \\
$\mathrm{C}(5)-\mathrm{C}(6)$ & $136.1(11)$ & $\mathrm{C}(6)-\mathrm{C}(7)$ & $136.1(11)$ \\
$\mathrm{C}(8)-\mathrm{C}(13)$ & $135.4(11)$ & $\mathrm{C}(8)-\mathrm{C}(9)$ & $136.9(11)$ \\
$\mathrm{C}(9)-\mathrm{C}(10)$ & $135.4(12)$ & $\mathrm{C}(10)-\mathrm{C}(11)$ & $133.7(14)$ \\
$\mathrm{C}(11)-\mathrm{C}(12)$ & $135.5(14)$ & $\mathrm{C}(12)-\mathrm{C}(13)$ & $139.1(12)$ \\
$\mathrm{C}(14)-\mathrm{C}(19)$ & $136.0(11)$ & $\mathrm{C}(14)-\mathrm{C}(15)$ & $138.1(11)$ \\
$\mathrm{C}(15)-\mathrm{C}(16)$ & $136.7(11)$ & $\mathrm{C}(16)-\mathrm{C}(17)$ & $136.3(12)$ \\
$\mathrm{C}(17)-\mathrm{C}(18)$ & $135.1(12)$ & $\mathrm{C}(18)-\mathrm{C}(19)$ & $137.6(12)$ \\
$\mathrm{C}(20)-\mathrm{C}(25)$ & $138.0(10)$ & $\mathrm{C}(20)-\mathrm{C}(21)$ & $138.8(11)$ \\
$\mathrm{C}(21)-\mathrm{C}(22)$ & $134.6(11)$ & $\mathrm{C}(22)-\mathrm{C}(23)$ & $139.2(12)$ \\
$\mathrm{C}(23)-\mathrm{C}(24)$ & $134.9(11)$ & $\mathrm{C}(24)-\mathrm{C}(25)$ & $135.6(11)$ \\
\hline
\end{tabular}

Tabelle 9: Bindungswinkel $\left[^{\circ}\right]$ in $\underline{2}$

\begin{tabular}{|l|r||l|r|}
\hline $\mathrm{S}(2)-\mathrm{Cu}(1)-\mathrm{S}(1)$ & $122.96(9)$ & $\mathrm{S}(2)-\mathrm{Cu}(1)-\mathrm{Cl}(1)$ & $121.31(9)$ \\
$\mathrm{S}(1)-\mathrm{Cu}(1)-\mathrm{Cl}(1)$ & $115.71(8)$ & $\mathrm{Cl}(2) \# 1-\mathrm{Cu}(2)-\mathrm{Cl}(2)$ & $165.62(14)$ \\
$\mathrm{C}(20)-\mathrm{P}(1)-\mathrm{C}(14)$ & $105.9(4)$ & $\mathrm{C}(20)-\mathrm{P}(1)-\mathrm{C}(1)$ & $107.5(4)$ \\
$\mathrm{C}(14)-\mathrm{P}(1)-\mathrm{C}(1)$ & $106.9(4)$ & $\mathrm{C}(20)-\mathrm{P}(1)-\mathrm{S}(1)$ & $109.2(3)$ \\
$\mathrm{C}(14)-\mathrm{P}(1)-\mathrm{S}(1)$ & $114.5(3)$ & $\mathrm{C}(1)-\mathrm{P}(1)-\mathrm{S}(1)$ & $112.6(3)$ \\
$\mathrm{C}(8)-\mathrm{P}(2)-\mathrm{C}(1)$ & $106.3(4)$ & $\mathrm{C}(8)-\mathrm{P}(2)-\mathrm{C}(2)$ & $107.1(4)$ \\
$\mathrm{C}(1)-\mathrm{P}(2)-\mathrm{C}(2)$ & $106.1(4)$ & $\mathrm{C}(8)-\mathrm{P}(2)-\mathrm{S}(2)$ & $111.6(3)$ \\
$\mathrm{C}(1)-\mathrm{P}(2)-\mathrm{S}(2)$ & $116.6(3)$ & $\mathrm{C}(2)-\mathrm{P}(2)-\mathrm{S}(2)$ & $108.6(3)$ \\
$\mathrm{P}(1)-\mathrm{S}(1)-\mathrm{Cu}(1)$ & $100.68(11)$ & $\mathrm{P}(2)-\mathrm{S}(2)-\mathrm{Cu}(1)$ & $107.87(13)$ \\
$\mathrm{Cu}(1) \# 1-\mathrm{Cl}(1)-\mathrm{Cu}(1)$ & $91.49(13)$ & $\mathrm{P}(2)-\mathrm{C}(1)-\mathrm{P}(1)$ & $118.7(4)$ \\
$\mathrm{C}(3)-\mathrm{C}(2)-\mathrm{P}(2)$ & $120.4(7)$ & $\mathrm{C}(7)-\mathrm{C}(2)-\mathrm{P}(2)$ & $119.3(6)$ \\
$\mathrm{C}(13)-\mathrm{C}(8)-\mathrm{P}(2)$ & $120.0(7)$ & $\mathrm{C}(9)-\mathrm{C}(8)-\mathrm{P}(2)$ & $120.0(7)$ \\
$\mathrm{C}(19)-\mathrm{C}(14)-\mathrm{P}(1)$ & $119.4(6)$ & $\mathrm{C}(15)-\mathrm{C}(14)-\mathrm{P}(1)$ & $119.7(7)$ \\
$\mathrm{C}(25)-\mathrm{C}(20)-\mathrm{P}(1)$ & $124.3(6)$ & $\mathrm{C}(21)-\mathrm{C}(20)-\mathrm{P}(1)$ & $117.5(6)$ \\
\hline
\end{tabular}

$\# 1-\mathrm{x}+1 / 2,-\mathrm{y}+3 / 2, \mathrm{z}$ 


\subsection{Daten zur Kristallstrukturanalyse von $\left[\mathrm{Cu}_{2}(\mathrm{dppmSe})_{3}\right] 3$}

\begin{tabular}{|ll|}
\hline Summenformel & $\mathrm{C}_{27} \mathrm{H}_{24} \mathrm{CuO}_{0.5} \mathrm{P}_{2} \mathrm{Se}_{3}$ \\
Molekulargewicht $\left[\mathrm{g} \cdot \mathrm{mol}^{-1}\right]$ & 718.82 \\
Temperatur & $133(2) \mathrm{K}$ \\
Wellenlänge & $71.073 \mathrm{pm}$ \\
Kristallsystem & triklin \\
Raumgruppe & $P \overline{1}$ \\
Gitterkonstanten & $\mathrm{a}=10.2615(9) \AA, \alpha=110.558(5)^{\circ}$ \\
& $\mathrm{b}=10.5052(9) \AA, \beta=102.396(5)^{\circ}$ \\
& $\mathrm{c}=14.6480(12) \AA, \gamma=95.104(5)^{\circ}$ \\
& $\circ$ \\
Volumen & $1420.5(2) \AA^{3}$ \\
Formeleinheiten & 2 \\
Röntgenographische Dichte $\left[\mathrm{g} \cdot \mathrm{cm}^{-3}\right]$ & 1.681 \\
Absorptionskoeffizient $\left[\mathrm{mm}^{-1}\right]$ & 4.741 \\
F(000) & 702 \\
Meßbereich $\theta$ & $2.67-22.50^{\circ}$ \\
Indexbereiche & $-6 \leq \mathrm{h} \leq 11$ \\
& $-11 \leq \mathrm{k} \leq 11$ \\
& $-15 \leq 1 \leq 15$ \\
Gemessene Reflexe & 12911 \\
Unabhängige Reflexe & 3703 \\
Beobachtete Reflexe $[\mathrm{I}>2 \sigma]$ & 3036 \\
$\mathrm{R}_{\text {int }}$ & 0.0615 \\
Parameter & 308 \\
R1; wR2[I>2 $\sigma(\mathrm{I})]$ & $0.0499 ; 0.1112$ \\
\hline
\end{tabular}


Tabelle 11: Atomkoordinaten und äquivalente isotrope Auslenkungsparameter $\left[\AA^{2}\right]$ für $\underline{3}$

\begin{tabular}{|r|r|r|r|r|}
\hline Atom & \multicolumn{1}{|c|}{$\mathrm{x}$} & \multicolumn{1}{c|}{$\mathrm{y}$} & \multicolumn{1}{c|}{$\mathrm{z}$} & \multicolumn{1}{c|}{$\mathrm{U}_{e q}$} \\
\hline $\mathrm{Cu}(1)$ & $0.61373(11)$ & $0.17942(12)$ & $-0.36314(8)$ & $0.0271(3)$ \\
$\mathrm{Se}(1)$ & $0.56348(9)$ & $0.17864(9)$ & $-0.21547(7)$ & $0.0226(2)$ \\
$\mathrm{Se}(2)$ & $0.25412(9)$ & $-0.34787(9)$ & $-0.60893(7)$ & $0.0266(3)$ \\
$\mathrm{Se}(3)$ & $0.55847(8)$ & $-0.09427(8)$ & $-0.47399(6)$ & $0.0160(2)$ \\
$\mathrm{P}(1)$ & $0.5430(2)$ & $-0.0460(2)$ & $-0.26468(16)$ & $0.0164(5)$ \\
$\mathrm{P}(2)$ & $0.2973(2)$ & $-0.2408(2)$ & $-0.44671(16)$ & $0.0169(5)$ \\
$\mathrm{C}(1)$ & $0.4612(8)$ & $-0.1400(8)$ & $-0.3927(6)$ & $0.0158(19)$ \\
$\mathrm{C}(2)$ & $0.7104(9)$ & $-0.0897(9)$ & $-0.2406(6)$ & $0.019(2)$ \\
$\mathrm{C}(3)$ & $0.7329(9)$ & $-0.2221(9)$ & $-0.2939(7)$ & $0.021(2)$ \\
$\mathrm{C}(4)$ & $0.8565(9)$ & $-0.2585(9)$ & $-0.2706(7)$ & $0.027(2)$ \\
$\mathrm{C}(5)$ & $0.9609(9)$ & $-0.1648(10)$ & $-0.1953(7)$ & $0.032(2)$ \\
$\mathrm{C}(6)$ & $0.9431(10)$ & $-0.0336(11)$ & $-0.1423(7)$ & $0.037(3)$ \\
$\mathrm{C}(7)$ & $0.8183(9)$ & $0.0042(10)$ & $-0.1647(7)$ & $0.027(2)$ \\
$\mathrm{C}(8)$ & $0.4601(8)$ & $-0.0998(9)$ & $-0.1824(6)$ & $0.021(2)$ \\
$\mathrm{C}(9)$ & $0.3746(8)$ & $-0.0236(9)$ & $-0.1351(6)$ & $0.020(2)$ \\
$\mathrm{C}(10)$ & $0.3164(9)$ & $-0.0643(10)$ & $-0.0707(7)$ & $0.026(2)$ \\
$\mathrm{C}(11)$ & $0.3419(9)$ & $-0.1818(10)$ & $-0.0546(7)$ & $0.030(2)$ \\
$\mathrm{C}(12)$ & $0.4278(9)$ & $-0.2595(10)$ & $-0.1017(7)$ & $0.029(2)$ \\
$\mathrm{C}(13)$ & $0.4887(9)$ & $-0.2170(9)$ & $-0.1643(6)$ & $0.025(2)$ \\
$\mathrm{C}(14)$ & $0.1647(9)$ & $-0.1423(9)$ & $-0.4204(6)$ & $0.020(2)$ \\
$\mathrm{C}(15)$ & $0.0293(8)$ & $-0.2086(9)$ & $-0.4655(7)$ & $0.022(2)$ \\
$\mathrm{C}(16)$ & $-0.0747(9)$ & $-0.1374(10)$ & $-0.4470(7)$ & $0.029(2)$ \\
$\mathrm{C}(17)$ & $-0.0458(9)$ & $0.0014(9)$ & $-0.3835(7)$ & $0.026(2)$ \\
$\mathrm{C}(18)$ & $0.0885(9)$ & $0.0681(10)$ & $-0.3396(7)$ & $0.028(2)$ \\
$\mathrm{C}(19)$ & $0.1935(9)$ & $-0.0023(9)$ & $-0.3579(7)$ & $0.023(2)$ \\
$\mathrm{C}(20)$ & $0.2811(8)$ & $-0.3719(8)$ & $-0.3935(6)$ & $0.019(2)$ \\
$\mathrm{C}(21)$ & $0.1841(9)$ & $-0.3849(9)$ & $-0.3420(7)$ & $0.025(2)$ \\
$\mathrm{C}(22)$ & $0.1811(10)$ & $-0.4860(9)$ & $-0.3026(7)$ & $0.032(2)$ \\
$\mathrm{C}(23)$ & $0.2739(10)$ & $-0.5725(10)$ & $-0.3101(8)$ & $0.036(3)$ \\
$\mathrm{C}(24)$ & $0.3719(10)$ & $-0.5602(9)$ & $-0.3600(7)$ & $0.031(2)$ \\
$\mathrm{C}(25)$ & $0.3743(9)$ & $-0.4619(9)$ & $-0.4030(7)$ & $0.028(2)$ \\
$\mathrm{O}(1 \mathrm{~L})$ & $0.743(2)$ & $-0.448(2)$ & $-0.0958(15)$ & $0.080(6)$ \\
$\mathrm{C}(1 \mathrm{~L})$ & $0.697(3)$ & $-0.460(3)$ & $-0.004(2)$ & $0.092(10)$ \\
$\mathrm{C}(2 \mathrm{LA})$ & $0.746(4)$ & $-0.371(4)$ & $0.064(3)$ & $0.034(10)$ \\
$\mathrm{C}(3 \mathrm{LA})$ & $0.792(4)$ & $-0.255(3)$ & $0.047(3)$ & $0.048(12)$ \\
$\mathrm{C}(2 \mathrm{LB})$ & $0.842(6)$ & $-0.376(6)$ & $0.081(3)$ & $0.082(18)$ \\
$\mathrm{C}(3 \mathrm{LB})$ & $0.935(5)$ & $-0.390(8)$ & $0.003(5)$ & $0.13(3)$ \\
$\mathrm{C}(4 \mathrm{~L})$ & $0.816(3)$ & $-0.328(3)$ & $-0.0477(18)$ & $0.069(7)$ \\
\hline & & & & \\
\hline
\end{tabular}


Tabelle 12: Bindungslängen [pm] in $\underline{3}$

\begin{tabular}{|l|r||l|r|}
\hline $\mathrm{Cu}(1)-\mathrm{Se}(1)$ & $233.14(14)$ & $\mathrm{Cu}(1)-\mathrm{Se}(2) \# 1$ & $233.61(14)$ \\
$\mathrm{Cu}(1)-\mathrm{Se}(3) \# 1$ & $245.45(14)$ & $\mathrm{Cu}(1)-\mathrm{Se}(3)$ & $269.46(14)$ \\
$\mathrm{Se}(1)-\mathrm{P}(1)$ & $218.7(2)$ & $\mathrm{Se}(2)-\mathrm{P}(2)$ & $216.6(2)$ \\
$\mathrm{Se}(2)-\mathrm{Cu}(1) \# 1$ & $233.61(14)$ & $\mathrm{Se}(3)-\mathrm{C}(1)$ & $186.3(8)$ \\
$\mathrm{Se}(3)-\mathrm{Cu}(1) \# 1$ & $245.45(14)$ & $\mathrm{Se}(3)-\mathrm{Se}(3) \# 1$ & $265.22(16)$ \\
$\mathrm{P}(1)-\mathrm{C}(1)$ & $175.3(9)$ & $\mathrm{P}(1)-\mathrm{C}(2)$ & $181.1(9)$ \\
$\mathrm{P}(1)-\mathrm{C}(8)$ & $182.5(8)$ & $\mathrm{P}(2)-\mathrm{C}(1)$ & $176.1(8)$ \\
$\mathrm{P}(2)-\mathrm{C}(14)$ & $180.7(9)$ & $\mathrm{P}(2)-\mathrm{C}(20)$ & $181.6(8)$ \\
\hline
\end{tabular}

Tabelle 13: Bindungswinkel $\left[^{\circ}\right]$ in $\underline{3}$

\begin{tabular}{|l|r||l|r|}
\hline $\mathrm{Se}(1)-\mathrm{Cu}(1)-\mathrm{Se}(2) \# 1$ & $130.50(6)$ & $\mathrm{Se}(1)-\mathrm{Cu}(1)-\mathrm{Se}(3) \# 1$ & $122.27(5)$ \\
$\mathrm{Se}(2) \# 1-\mathrm{Cu}(1)-\mathrm{Se}(3) \# 1$ & $97.25(5)$ & $\mathrm{Se}(1)-\mathrm{Cu}(1)-\mathrm{Se}(3)$ & $99.77(5)$ \\
$\mathrm{Se}(2) \# 1-\mathrm{Cu}(1)-\mathrm{Se}(3)$ & $126.42(5)$ & $\mathrm{Se}(3) \# 1-\mathrm{Cu}(1)-\mathrm{Se}(3)$ & $61.80(4)$ \\
$\mathrm{P}(1)-\mathrm{Se}(1)-\mathrm{Cu}(1)$ & $93.02(7)$ & $\mathrm{P}(2)-\mathrm{Se}(2)-\mathrm{Cu}(1) \# 1$ & $96.30(7)$ \\
$\mathrm{C}(1)-\mathrm{Se}(3)-\mathrm{Cu}(1) \# 1$ & $101.2(3)$ & $\mathrm{C}(1)-\mathrm{Se}(3)-\mathrm{Se}(3) \# 1$ & $105.8(2)$ \\
$\mathrm{Cu}(1) \# 1-\mathrm{Se}(3)-\mathrm{Se}(3) \# 1$ & $63.56(4)$ & $\mathrm{C}(1)-\mathrm{Se}(3)-\mathrm{Cu}(1)$ & $95.3(2)$ \\
$\mathrm{Cu}(1) \# 1-\mathrm{Se}(3)-\mathrm{Cu}(1)$ & $118.20(4)$ & $\mathrm{Se}(3) \# 1-\mathrm{Se}(3)-\mathrm{Cu}(1)$ & $54.65(4)$ \\
$\mathrm{C}(1)-\mathrm{P}(1)-\mathrm{C}(2)$ & $107.5(4)$ & $\mathrm{C}(1)-\mathrm{P}(1)-\mathrm{C}(8)$ & $111.8(4)$ \\
$\mathrm{C}(2)-\mathrm{P}(1)-\mathrm{C}(8)$ & $104.8(4)$ & $\mathrm{C}(1)-\mathrm{P}(1)-\mathrm{Se}(1)$ & $115.4(3)$ \\
$\mathrm{C}(2)-\mathrm{P}(1)-\mathrm{Se}(1)$ & $108.8(3)$ & $\mathrm{C}(8)-\mathrm{P}(1)-\mathrm{Se}(1)$ & $108.0(3)$ \\
$\mathrm{C}(1)-\mathrm{P}(2)-\mathrm{C}(14)$ & $113.2(4)$ & $\mathrm{C}(1)-\mathrm{P}(2)-\mathrm{C}(20)$ & $109.4(4)$ \\
$\mathrm{C}(14)-\mathrm{P}(2)-\mathrm{C}(20)$ & $106.0(4)$ & $\mathrm{C}(1)-\mathrm{P}(2)-\mathrm{Se}(2)$ & $112.8(3)$ \\
$\mathrm{C}(14)-\mathrm{P}(2)-\mathrm{Se}(2)$ & $108.1(3)$ & $\mathrm{C}(20)-\mathrm{P}(2)-\mathrm{Se}(2)$ & $107.0(3)$ \\
$\mathrm{P}(1)-\mathrm{C}(1)-\mathrm{P}(2)$ & $128.5(5)$ & $\mathrm{P}(1)-\mathrm{C}(1)-\mathrm{Se}(3)$ & $110.3(4)$ \\
$\mathrm{P}(2)-\mathrm{C}(1)-\mathrm{Se}(3)$ & $120.2(5)$ & $\mathrm{C}(7)-\mathrm{C}(2)-\mathrm{P}(1)$ & $121.0(7)$ \\
$\mathrm{C}(3)-\mathrm{C}(2)-\mathrm{P}(1)$ & $120.7(7)$ & $\mathrm{C}(9)-\mathrm{C}(8)-\mathrm{P}(1)$ & $121.2(6)$ \\
$\mathrm{C}(13)-\mathrm{C}(8)-\mathrm{P}(1)$ & $119.1(7)$ & $\mathrm{C}(19)-\mathrm{C}(14)-\mathrm{P}(2)$ & $122.0(7)$ \\
$\mathrm{C}(15)-\mathrm{C}(14)-\mathrm{P}(2)$ & $118.7(7)$ & $\mathrm{C}(21)-\mathrm{C}(20)-\mathrm{P}(2)$ & $123.8(7)$ \\
$\mathrm{C}(25)-\mathrm{C}(20)-\mathrm{P}(2)$ & $117.4(6)$ & & \\
\hline
\end{tabular}

\#1 -x+1, -y, -z-1 


\subsection{Daten zur Kristallstrukturanalyse von}

$\left[\mathrm{Au}_{2}\left(\mathrm{dppmS}_{2}\right)\left(\mathrm{PPh}_{3}\right)_{2}\right] \underline{4}$

\begin{tabular}{|ll|}
\hline Summenformel & $\mathrm{C}_{71} \mathrm{H}_{70} \mathrm{Au}_{2} \mathrm{O}_{2.5} \mathrm{P}_{4} \mathrm{~S}_{2}$ \\
Molekulargewicht $\left[\mathrm{g} \cdot \mathrm{mol}^{-1}\right]$ & 1545.20 \\
Temperatur & $133(2) \mathrm{K}$ \\
Wellenlänge & $71.073 \mathrm{pm}$ \\
Kristallsystem & monoklin \\
Raumgruppe & $C 2 / c$ \\
Gitterkonstanten & $\mathrm{a}=42.994(5) \AA, \alpha=90^{\circ}$ \\
& $\mathrm{b}=13.463(2) \AA, \beta=103.44(3)^{\circ} \circ$ \\
& $\mathrm{c}=23.102(3) \AA, \gamma=90^{\circ}$ \\
Volumen & $13006(3) \AA^{3}$ \\
Formeleinheiten & 8 \\
Röntgenographische Dichte $\left[\mathrm{g} \cdot \mathrm{cm}^{-3}\right]$ & 1.578 \\
Absorptionskoeffizient $\left[\mathrm{mm}^{-1}\right]$ & 4.715 \\
F(000) & 6128 \\
Meßbereich $\theta$ & $2.10-25.14^{\circ}$ \\
Indexbereiche & $-51 \leq \mathrm{h} \leq 49$ \\
& $0 \leq \mathrm{k} \leq 15$ \\
Gemessene Reflexe & $0 \leq 1 \leq 27$ \\
Unabhängige Reflexe & 124438 \\
Beobachtete Reflexe $[\mathrm{I}>2 \sigma]$ & 11355 \\
$\mathrm{R}_{\text {int }}$ & 9551 \\
Parameter & 0.0631 \\
R1; wR2[I>2 $\sigma(\mathrm{I})]$ & 895 \\
\hline
\end{tabular}


Tabelle 15: Atomkoordinaten und äquivalente isotrope Auslenkungsparameter $\left[\AA^{2}\right]$ für $\underline{4}$

\begin{tabular}{|c|c|c|c|c|}
\hline Atom & $\mathrm{x}$ & $\mathrm{y}$ & $\mathrm{z}$ & $\mathrm{U}_{e q}$ \\
\hline $\mathrm{Au}(1)$ & $0.352685(4)$ & $0.385925(11)$ & $0.283716(7)$ & $0.02282(5)$ \\
\hline $\mathrm{Au}(2)$ & $0.384307(4)$ & $0.209965(12)$ & $0.255761(7)$ & $0.02425(5)$ \\
\hline $\mathrm{P}(1)$ & $0.41456(3)$ & $0.31367(8)$ & $0.37944(5)$ & $0.0244(2)$ \\
\hline $\mathrm{P}(2)$ & $0.35103(3)$ & $0.19861(8)$ & $0.37043(5)$ & $0.0236(2)$ \\
\hline $\mathrm{P}(3)$ & $0.33087(3)$ & $0.50995(8)$ & $0.22024(5)$ & $0.0246(2)$ \\
\hline $\mathrm{P}(4)$ & $0.38726(3)$ & $0.15296(8)$ & $0.16484(5)$ & $0.0266(3)$ \\
\hline $\mathrm{S}(2)$ & $0.33672(3)$ & $0.26114(9)$ & $0.43726(5)$ & $0.0344(3)$ \\
\hline $\mathrm{S}(1)$ & $0.44240(3)$ & $0.35295(9)$ & $0.32675(5)$ & $0.0344(3)$ \\
\hline $\mathrm{C}(1)$ & $0.37706(10)$ & $0.2690(3)$ & $0.33719(18)$ & $0.0232(9)$ \\
\hline $\mathrm{C}(11)$ & $0.43311(10)$ & $0.2186(3)$ & $0.4331(2)$ & $0.0325(11)$ \\
\hline $\mathrm{C}(12)$ & $0.42541(12)$ & $0.2098(4)$ & $0.4882(2)$ & $0.0483(14)$ \\
\hline $\mathrm{C}(13)$ & $0.43838(14)$ & $0.1346(5)$ & $0.5276(3)$ & $0.073(2)$ \\
\hline $\mathrm{C}(14)$ & $0.45818(16)$ & $0.0671(5)$ & $0.5119(4)$ & $0.083(2)$ \\
\hline $\mathrm{C}(15)$ & $0.46599(14)$ & $0.0721(4)$ & $0.4577(4)$ & $0.072(2)$ \\
\hline $\mathrm{C}(16)$ & $0.45367(12)$ & $0.1504(4)$ & $0.4173(3)$ & $0.0499(14)$ \\
\hline $\mathrm{C}(21)$ & $0.41266(10)$ & $0.4211(3)$ & $0.42691(17)$ & $0.0249(10)$ \\
\hline $\mathrm{C}(22)$ & $0.44101(12)$ & $0.4516(4)$ & $0.4658(2)$ & $0.0369(12)$ \\
\hline $\mathrm{C}(23)$ & $0.44245(13)$ & $0.5398(4)$ & $0.4981(2)$ & $0.0454(13)$ \\
\hline $\mathrm{C}(24)$ & $0.41570(14)$ & $0.5991(4)$ & $0.4910(2)$ & $0.0430(13)$ \\
\hline $\mathrm{C}(25)$ & $0.38749(13)$ & $0.5691(3)$ & $0.4530(2)$ & $0.0410(12)$ \\
\hline $\mathrm{C}(26)$ & $0.38567(12)$ & $0.4793(3)$ & $0.42192(19)$ & $0.0328(11)$ \\
\hline $\mathrm{C}(31)$ & $0.31678(10)$ & $0.1731(3)$ & $0.30771(18)$ & $0.0252(10)$ \\
\hline $\mathrm{C}(32)$ & $0.29086(11)$ & $0.2378(3)$ & $0.2966(2)$ & $0.0342(11)$ \\
\hline $\mathrm{C}(33)$ & $0.26497(12)$ & $0.2222(4)$ & $0.2484(2)$ & $0.0428(13)$ \\
\hline $\mathrm{C}(34)$ & $0.26467(12)$ & $0.1422(4)$ & $0.2111(2)$ & $0.0482(14)$ \\
\hline $\mathrm{C}(35)$ & $0.29016(12)$ & $0.0768(4)$ & $0.2217(2)$ & $0.0439(13)$ \\
\hline $\mathrm{C}(36)$ & $0.31599(11)$ & $0.0923(3)$ & $0.2696(2)$ & $0.0353(11)$ \\
\hline $\mathrm{C}(41)$ & $0.36651(10)$ & $0.0725(3)$ & $0.39316(18)$ & $0.0260(10)$ \\
\hline $\mathrm{C}(42)$ & $0.38793(11)$ & $0.0247(3)$ & $0.3672(2)$ & $0.0309(10)$ \\
\hline $\mathrm{C}(43)$ & $0.39778(12)$ & $-0.0726(3)$ & $0.3836(2)$ & $0.0399(12)$ \\
\hline $\mathrm{C}(44)$ & $0.38562(12)$ & $-0.1222(3)$ & $0.4250(2)$ & $0.0404(12)$ \\
\hline $\mathrm{C}(45)$ & $0.36423(12)$ & $-0.0758(3)$ & $0.4514(2)$ & $0.0375(12)$ \\
\hline $\mathrm{C}(46)$ & $0.35449(11)$ & $0.0216(3)$ & $0.43614(18)$ & $0.0307(10)$ \\
\hline $\mathrm{C}(51)$ & $0.38759(11)$ & $0.0175(3)$ & $0.15999(19)$ & $0.0304(10)$ \\
\hline $\mathrm{C}(52)$ & $0.40225(13)$ & $-0.0340(3)$ & $0.2109(2)$ & $0.0464(13)$ \\
\hline $\mathrm{C}(53)$ & $0.40487(16)$ & $-0.1379(4)$ & $0.2100(3)$ & $0.0612(17)$ \\
\hline $\mathrm{C}(54)$ & $0.39216(15)$ & $-0.1888(4)$ & $0.1579(3)$ & $0.0534(15)$ \\
\hline $\mathrm{C}(55)$ & $0.37727(14)$ & $-0.1392(4)$ & $0.1070(3)$ & $0.0499(14)$ \\
\hline
\end{tabular}


Tabelle 15: Fortsetzung

\begin{tabular}{|r|r|r|r|r|}
\hline $\mathrm{C}(56)$ & $0.37512(13)$ & $-0.0357(3)$ & $0.1073(2)$ & $0.0397(12)$ \\
$\mathrm{C}(61)$ & $0.42228(10)$ & $0.1950(3)$ & $0.14100(19)$ & $0.0272(10)$ \\
$\mathrm{C}(62)$ & $0.44152(12)$ & $0.2680(3)$ & $0.1736(2)$ & $0.0374(12)$ \\
$\mathrm{C}(63)$ & $0.46837(12)$ & $0.3018(4)$ & $0.1555(2)$ & $0.0449(13)$ \\
$\mathrm{C}(64)$ & $0.47552(12)$ & $0.2627(4)$ & $0.1053(2)$ & $0.0404(12)$ \\
$\mathrm{C}(65)$ & $0.45609(12)$ & $0.1908(4)$ & $0.0722(2)$ & $0.0383(12)$ \\
$\mathrm{C}(66)$ & $0.42978(11)$ & $0.1553(3)$ & $0.08934(19)$ & $0.0318(11)$ \\
$\mathrm{C}(71)$ & $0.35358(11)$ & $0.1959(3)$ & $0.10592(18)$ & $0.0288(10)$ \\
$\mathrm{C}(72)$ & $0.32442(12)$ & $0.1464(4)$ & $0.0940(2)$ & $0.0378(12)$ \\
$\mathrm{C}(73)$ & $0.29859(12)$ & $0.1823(4)$ & $0.0510(2)$ & $0.0446(13)$ \\
$\mathrm{C}(74)$ & $0.30124(13)$ & $0.2692(4)$ & $0.0206(2)$ & $0.0456(13)$ \\
$\mathrm{C}(75)$ & $0.32967(13)$ & $0.3201(4)$ & $0.0333(2)$ & $0.0413(12)$ \\
$\mathrm{C}(76)$ & $0.35589(12)$ & $0.2842(3)$ & $0.0754(2)$ & $0.0346(11)$ \\
$\mathrm{C}(81)$ & $0.29121(11)$ & $0.4846(3)$ & $0.17462(18)$ & $0.0275(10)$ \\
$\mathrm{C}(82)$ & $0.26722(11)$ & $0.5564(4)$ & $0.1610(2)$ & $0.0367(12)$ \\
$\mathrm{C}(83)$ & $0.23740(12)$ & $0.5337(4)$ & $0.1252(2)$ & $0.0440(13)$ \\
$\mathrm{C}(84)$ & $0.23109(12)$ & $0.4378(4)$ & $0.1028(2)$ & $0.0403(12)$ \\
$\mathrm{C}(85)$ & $0.25482(12)$ & $0.3660(4)$ & $0.1159(2)$ & $0.0414(12)$ \\
$\mathrm{C}(86)$ & $0.28454(12)$ & $0.3885(3)$ & $0.1516(2)$ & $0.0361(11)$ \\
$\mathrm{C}(91)$ & $0.35509(11)$ & $0.5312(3)$ & $0.16688(18)$ & $0.0289(10)$ \\
$\mathrm{C}(92)$ & $0.38748(12)$ & $0.5120(3)$ & $0.1845(2)$ & $0.0389(12)$ \\
$\mathrm{C}(93)$ & $0.40696(14)$ & $0.5287(4)$ & $0.1449(3)$ & $0.0484(14)$ \\
$\mathrm{C}(94)$ & $0.39351(18)$ & $0.5642(4)$ & $0.0883(3)$ & $0.0579(17)$ \\
$\mathrm{C}(95)$ & $0.36146(18)$ & $0.5824(4)$ & $0.0703(2)$ & $0.0546(17)$ \\
$\mathrm{C}(96)$ & $0.34195(13)$ & $0.5660(3)$ & $0.1091(2)$ & $0.0393(12)$ \\
$\mathrm{C}(101)$ & $0.32825(10)$ & $0.6305(3)$ & $0.25487(18)$ & $0.0257(10)$ \\
$\mathrm{C}(102)$ & $0.33134(12)$ & $0.7196(3)$ & $0.2268(2)$ & $0.0388(12)$ \\
$\mathrm{C}(103)$ & $0.32855(13)$ & $0.8101(3)$ & $0.2540(2)$ & $0.0432(13)$ \\
$\mathrm{C}(104)$ & $0.32254(11)$ & $0.8123(3)$ & $0.3099(2)$ & $0.0387(12)$ \\
$\mathrm{C}(105)$ & $0.31954(12)$ & $0.7240(4)$ & $0.3390(2)$ & $0.0381(12)$ \\
$\mathrm{C}(106)$ & $0.32247(11)$ & $0.6335(3)$ & $0.31197(19)$ & $0.0306(10)$ \\
$\mathrm{O}(1 \mathrm{~T})$ & $0.2620(3)$ & $-0.0199(7)$ & $0.0693(6)$ & $0.131(4)$ \\
$\mathrm{C}(1 \mathrm{~T})$ & $0.2801(5)$ & $-0.0810(10)$ & $0.0362(8)$ & $0.132(6)$ \\
$\mathrm{C}(2 \mathrm{~T})$ & $0.2819(5)$ & $-0.1783(12)$ & $0.0703(10)$ & $0.156(7)$ \\
$\mathrm{C}(3 \mathrm{~T})$ & $0.2565(4)$ & $-0.1707(11)$ & $0.1052(7)$ & $0.110(5)$ \\
$\mathrm{C}(4 \mathrm{~T})$ & $0.2365(3)$ & $-0.0777(10)$ & $0.0844(9)$ & $0.116(5)$ \\
$\mathrm{C}(1 \mathrm{U})$ & $0.4525(5)$ & $-0.2681(12)$ & $0.3804(10)$ & $0.168(6)$ \\
\hline & $0.4377(4)$ & $-0.3455(12)$ & $0.3378(8)$ & $0.118(5)$ \\
$\mathrm{C}(2)(6)$ & $-0.368(2)$ & $0.3119(11)$ & $0.157(8)$ \\
& & $-0.359(3)$ & $0.3650(14)$ & $0.208(9)$ \\
\hline
\end{tabular}


Tabelle 15: Fortsetzung

\begin{tabular}{|r|r|r|r|r|}
\hline $\mathrm{C}(4 \mathrm{U})$ & $0.4846(6)$ & $-0.298(2)$ & $0.4129(9)$ & $0.168(8)$ \\
$\mathrm{O}(1 \mathrm{~V})$ & $0.5110(16)$ & $0.008(5)$ & $0.290(2)$ & $0.130(11)$ \\
$\mathrm{C}(1 \mathrm{~V})$ & $0.5322(14)$ & $0.016(6)$ & $0.249(4)$ & $0.131(11)$ \\
$\mathrm{C}(2 \mathrm{~V})$ & $0.508(2)$ & $0.007(7)$ & $0.190(3)$ & $0.120(11)$ \\
$\mathrm{C}(3 \mathrm{~V})$ & $0.4796(17)$ & $-0.049(6)$ & $0.201(3)$ & $0.120(11)$ \\
$\mathrm{C}(4 \mathrm{~V})$ & $0.4846(18)$ & $-0.060(6)$ & $0.269(4)$ & $0.130(11)$ \\
$\mathrm{O}(1 \mathrm{~W})$ & $0.4517(4)$ & $-0.2545(11)$ & $0.3418(6)$ & $0.135(6)$ \\
$\mathrm{C}(1 \mathrm{~W})$ & $0.4501(5)$ & $-0.3589(16)$ & $0.3244(14)$ & $0.178(9)$ \\
$\mathrm{C}(2 \mathrm{~W})$ & $0.4853(5)$ & $-0.3907(15)$ & $0.3434(15)$ & $0.203(9)$ \\
$\mathrm{C}(3 \mathrm{~W})$ & $0.5019(4)$ & $-0.3156(16)$ & $0.3890(13)$ & $0.183(9)$ \\
$\mathrm{C}(4 \mathrm{~W})$ & $0.4766(5)$ & $-0.2396(14)$ & $0.3966(8)$ & $0.138(7)$ \\
$\mathrm{O}(1 \mathrm{X})$ & $0.2359(4)$ & $-0.0685(17)$ & $0.0171(6)$ & $0.183(7)$ \\
$\mathrm{C}(1 \mathrm{X})$ & $0.2645(7)$ & $-0.131(2)$ & $0.0262(9)$ & $0.191(9)$ \\
$\mathrm{C}(2 \mathrm{X})$ & $0.2798(5)$ & $-0.112(2)$ & $0.0921(10)$ & $0.174(8)$ \\
$\mathrm{C}(3 \mathrm{X})$ & $0.2511(6)$ & $-0.086(2)$ & $0.1172(7)$ & $0.161(7)$ \\
$\mathrm{C}(4 \mathrm{X})$ & $0.2317(5)$ & $-0.0158(17)$ & $0.0699(10)$ & $0.155(7)$ \\
$\mathrm{O}(1 \mathrm{Y})$ & $0.4964(9)$ & $0.0523(13)$ & $0.2775(13)$ & $0.134(7)$ \\
$\mathrm{C}(1 \mathrm{Y})$ & $0.5104(12)$ & $0.0826(14)$ & $0.2281(16)$ & $0.123(8)$ \\
$\mathrm{C}(2 \mathrm{Y})$ & $0.5122(9)$ & $-0.0149(16)$ & $0.1957(12)$ & $0.121(7)$ \\
$\mathrm{C}(3 \mathrm{Y})$ & $0.4998(12)$ & $-0.0959(10)$ & $0.2296(12)$ & $0.124(8)$ \\
$\mathrm{C}(4 \mathrm{Y})$ & $0.5000(12)$ & $-0.0535(16)$ & $0.2911(12)$ & $0.141(9)$ \\
\hline
\end{tabular}

Tabelle 16: Bindungslängen $[\mathrm{pm}]$ in $\underline{4}$

\begin{tabular}{|l|r||l|r|}
\hline $\mathrm{Au}(1)-\mathrm{C}(1)$ & $212.2(4)$ & $\mathrm{Au}(1)-\mathrm{P}(3)$ & $227.57(11)$ \\
$\mathrm{Au}(1)-\mathrm{Au}(2)$ & $287.85(4)$ & $\mathrm{Au}(2)-\mathrm{C}(1)$ & $213.1(4)$ \\
$\mathrm{Au}(2)-\mathrm{P}(4)$ & $226.74(11)$ & $\mathrm{C}(1)-\mathrm{P}(2)$ & $177.2(4)$ \\
$\mathrm{C}(1)-\mathrm{P}(1)$ & $178.4(4)$ & $\mathrm{P}(1)-\mathrm{C}(21)$ & $182.8(4)$ \\
$\mathrm{P}(1)-\mathrm{C}(11)$ & $183.1(4)$ & $\mathrm{P}(1)-\mathrm{S}(1)$ & $196.74(16)$ \\
$\mathrm{P}(2)-\mathrm{C}(31)$ & $184.2(4)$ & $\mathrm{P}(2)-\mathrm{C}(41)$ & $185.5(4)$ \\
$\mathrm{P}(2)-\mathrm{S}(2)$ & $197.81(16)$ & $\mathrm{P}(3)-\mathrm{C}(91)$ & $181.2(4)$ \\
$\mathrm{P}(3)-\mathrm{C}(81)$ & $181.6(5)$ & $\mathrm{P}(3)-\mathrm{C}(101)$ & $182.5(4)$ \\
$\mathrm{C}(51)-\mathrm{P}(4)$ & $182.7(4)$ & $\mathrm{C}(61)-\mathrm{P}(4)$ & $181.0(4)$ \\
$\mathrm{C}(71)-\mathrm{P}(4)$ & $183.5(5)$ & & \\
\hline
\end{tabular}


Tabelle 17: Bindungswinkel $\left[{ }^{\circ}\right.$ ] in $\underline{4}$

\begin{tabular}{|l|r||l|r|}
\hline $\mathrm{C}(1)-\mathrm{Au}(1)-\mathrm{P}(3)$ & $173.47(11)$ & $\mathrm{C}(1)-\mathrm{Au}(1)-\mathrm{Au}(2)$ & $47.53(11)$ \\
$\mathrm{P}(3)-\mathrm{Au}(1)-\mathrm{Au}(2)$ & $127.21(3)$ & $\mathrm{C}(1)-\mathrm{Au}(2)-\mathrm{P}(4)$ & $174.45(12)$ \\
$\mathrm{C}(1)-\mathrm{Au}(2)-\mathrm{Au}(1)$ & $47.27(11)$ & $\mathrm{P}(4)-\mathrm{Au}(2)-\mathrm{Au}(1)$ & $128.13(3)$ \\
$\mathrm{P}(2)-\mathrm{C}(1)-\mathrm{P}(1)$ & $121.7(2)$ & $\mathrm{P}(2)-\mathrm{C}(1)-\mathrm{Au}(1)$ & $111.9(2)$ \\
$\mathrm{P}(1)-\mathrm{C}(1)-\mathrm{Au}(1)$ & $109.24(19)$ & $\mathrm{P}(2)-\mathrm{C}(1)-\mathrm{Au}(2)$ & $114.8(2)$ \\
$\mathrm{P}(1)-\mathrm{C}(1)-\mathrm{Au}(2)$ & $108.1(2)$ & $\mathrm{Au}(1)-\mathrm{C}(1)-\mathrm{Au}(2)$ & $85.20(14)$ \\
$\mathrm{C}(1)-\mathrm{P}(1)-\mathrm{C}(21)$ & $115.7(2)$ & $\mathrm{C}(1)-\mathrm{P}(1)-\mathrm{C}(11)$ & $109.32(19)$ \\
$\mathrm{C}(21)-\mathrm{P}(1)-\mathrm{C}(11)$ & $102.7(2)$ & $\mathrm{C}(1)-\mathrm{P}(1)-\mathrm{S}(1)$ & $110.76(14)$ \\
$\mathrm{C}(21)-\mathrm{P}(1)-\mathrm{S}(1)$ & $106.08(14)$ & $\mathrm{C}(11)-\mathrm{P}(1)-\mathrm{S}(1)$ & $112.03(16)$ \\
$\mathrm{C}(1)-\mathrm{P}(2)-\mathrm{C}(31)$ & $103.24(19)$ & $\mathrm{C}(1)-\mathrm{P}(2)-\mathrm{C}(41)$ & $113.01(19)$ \\
$\mathrm{C}(31)-\mathrm{P}(2)-\mathrm{C}(41)$ & $102.74(19)$ & $\mathrm{C}(1)-\mathrm{P}(2)-\mathrm{S}(2)$ & $116.95(14)$ \\
$\mathrm{C}(31)-\mathrm{P}(2)-\mathrm{S}(2)$ & $110.88(15)$ & $\mathrm{C}(41)-\mathrm{P}(2)-\mathrm{S}(2)$ & $108.95(14)$ \\
$\mathrm{C}(91)-\mathrm{P}(3)-\mathrm{C}(81)$ & $104.2(2)$ & $\mathrm{C}(91)-\mathrm{P}(3)-\mathrm{C}(101)$ & $105.15(19)$ \\
$\mathrm{C}(81)-\mathrm{P}(3)-\mathrm{C}(101)$ & $106.0(2)$ & $\mathrm{C}(91)-\mathrm{P}(3)-\mathrm{Au}(1)$ & $109.77(15)$ \\
$\mathrm{C}(81)-\mathrm{P}(3)-\mathrm{Au}(1)$ & $115.28(14)$ & $\mathrm{C}(101)-\mathrm{P}(3)-\mathrm{Au}(1)$ & $115.37(14)$ \\
$\mathrm{C}(61)-\mathrm{P}(4)-\mathrm{C}(51)$ & $106.0(2)$ & $\mathrm{C}(61)-\mathrm{P}(4)-\mathrm{C}(71)$ & $104.2(2)$ \\
$\mathrm{C}(51)-\mathrm{P}(4)-\mathrm{C}(71)$ & $106.5(2)$ & $\mathrm{C}(61)-\mathrm{P}(4)-\mathrm{Au}(2)$ & $113.93(15)$ \\
$\mathrm{C}(51)-\mathrm{P}(4)-\mathrm{Au}(2)$ & $113.42(14)$ & $\mathrm{C}(71)-\mathrm{P}(4)-\mathrm{Au}(2)$ & $112.09(14)$ \\
\hline
\end{tabular}




\subsection{Daten zur Kristallstrukturanalyse von $\left[\mathrm{Cu}\left(\mathrm{dppaO}_{2}\right)_{2}(\mathrm{MeOH})\right] \underline{5}$}

\begin{tabular}{ll|}
\hline Summenformel & $\mathrm{C}_{49} \mathrm{H}_{44} \mathrm{CuN}_{2} \mathrm{O}_{5} \mathrm{P}_{4}$ \\
Molekulargewicht $\left[\mathrm{g} \cdot \mathrm{mol}^{-1}\right]$ & 928.28 \\
Temperatur & $133(2) \mathrm{K}$ \\
Wellenlänge & $71.073 \mathrm{pm}$ \\
Kristallsystem & triklin \\
Raumgruppe & $P \overline{1}$ \\
Gitterkonstanten & $\mathrm{a}=12.622(3) \AA, \alpha=74.42(3)^{\circ}$ \\
& $\mathrm{b}=12.659(3) \AA, \beta=77.66(3)^{\circ}$ \\
& $\mathrm{c}=14.556(3) \AA, \gamma=87.80(3)^{\circ}$ \\
Volumen & $2188.0(8) \AA^{3}$ \\
Formeleinheiten & 2 \\
Röntgenographische Dichte $\left[\mathrm{g} \cdot \mathrm{cm}^{-3}\right]$ & 1.409 \\
Absorptionskoeffizient $\left[\mathrm{mm}^{-1}\right]$ & 0.696 \\
F(000) & 962 \\
Meßbereich $\theta$ & $2.33-27.50^{\circ}$ \\
Indexbereiche & $-16 \leq \mathrm{h} \leq 16$ \\
& $-15 \leq \mathrm{k} \leq 16$ \\
Gemessene Reflexe & $0 \leq 1 \leq 18$ \\
Unabhängige Reflexe & 35172 \\
Beobachtete Reflexe $[\mathrm{I}>2 \sigma]$ & 10040 \\
$\mathrm{R}_{\text {int }}$ & 8596 \\
Parameter & 0.0264 \\
R1; wR2[I>2 $\sigma(\mathrm{I})]$ & 554 \\
\hline
\end{tabular}


Tabelle 19: Atomkoordinaten und äquivalente isotrope Auslenkungsparameter $\left[\AA^{2}\right]$ für $\underline{5}$

\begin{tabular}{|c|c|c|c|c|}
\hline Atom & $\mathrm{x}$ & $\mathrm{y}$ & Z & $\mathrm{U}_{e q}$ \\
\hline $\mathrm{Cu}(1)$ & $0.225008(17)$ & $0.003746(16)$ & $0.252888(14)$ & $0.01528(6)$ \\
\hline $\mathrm{P}(1)$ & $0.21871(4)$ & $0.20074(4)$ & $0.34424(3)$ & $0.01480(9)$ \\
\hline $\mathrm{P}(2)$ & $0.10911(4)$ & $0.22760(4)$ & $0.18967(3)$ & $0.01565(9)$ \\
\hline $\mathrm{P}(3)$ & $0.33570(4)$ & $-0.21091(4)$ & $0.33349(3)$ & $0.01625(9)$ \\
\hline $\mathrm{P}(4)$ & $0.27901(4)$ & $-0.18581(4)$ & $0.15395(3)$ & $0.01642(9)$ \\
\hline $\mathrm{O}(1)$ & $0.26849(10)$ & $0.09617(10)$ & $0.32319(9)$ & $0.0190(3)$ \\
\hline $\mathrm{O}(2)$ & $0.14024(11)$ & $0.11209(10)$ & $0.18208(9)$ & $0.0205(3)$ \\
\hline $\mathrm{O}(3)$ & $0.25527(10)$ & $-0.12210(10)$ & $0.35052(9)$ & $0.0192(3)$ \\
\hline $\mathrm{O}(4)$ & $0.23821(11)$ & $-0.07027(10)$ & $0.15167(9)$ & $0.0211(3)$ \\
\hline $\mathrm{N}(1)$ & $0.14177(12)$ & $0.26477(12)$ & $0.27597(11)$ & $0.0185(3)$ \\
\hline $\mathrm{N}(2)$ & $0.35025(13)$ & $-0.23961(13)$ & $0.23192(11)$ & $0.0208(3)$ \\
\hline $\mathrm{C}(11)$ & $0.14647(14)$ & $0.16518(15)$ & $0.46956(12)$ & $0.0177(3)$ \\
\hline $\mathrm{C}(12)$ & $0.12206(16)$ & $0.05591(16)$ & $0.51843(14)$ & $0.0252(4)$ \\
\hline $\mathrm{C}(13)$ & $0.06365(17)$ & $0.02782(18)$ & $0.61411(15)$ & $0.0308(4)$ \\
\hline $\mathrm{C}(14)$ & $0.03054(16)$ & $0.10787(19)$ & $0.66190(14)$ & $0.0305(5)$ \\
\hline $\mathrm{C}(15)$ & $0.05503(17)$ & $0.21680(19)$ & $0.61392(15)$ & $0.0302(4)$ \\
\hline $\mathrm{C}(16)$ & $0.11257(16)$ & $0.24579(17)$ & $0.51765(14)$ & $0.0250(4)$ \\
\hline $\mathrm{C}(21)$ & $0.32851(14)$ & $0.29133(14)$ & $0.33688(12)$ & $0.0174(3)$ \\
\hline $\mathrm{C}(22)$ & $0.34485(15)$ & $0.39274(15)$ & $0.26860(14)$ & $0.0232(4)$ \\
\hline $\mathrm{C}(23)$ & $0.43369(18)$ & $0.45869(18)$ & $0.26095(16)$ & $0.0315(5)$ \\
\hline $\mathrm{C}(24)$ & $0.50658(17)$ & $0.42245(18)$ & $0.32071(16)$ & $0.0312(5)$ \\
\hline $\mathrm{C}(25)$ & $0.49132(16)$ & $0.32119(19)$ & $0.38852(15)$ & $0.0297(4)$ \\
\hline $\mathrm{C}(26)$ & $0.40232(15)$ & $0.25583(17)$ & $0.39724(14)$ & $0.0240(4)$ \\
\hline $\mathrm{C}(31)$ & $0.16960(15)$ & $0.32088(15)$ & $0.07449(13)$ & $0.0201(4)$ \\
\hline $\mathrm{C}(32)$ & $0.26384(19)$ & $0.2922(2)$ & $0.01895(17)$ & $0.0394(5)$ \\
\hline $\mathrm{C}(33)$ & $0.3166(2)$ & $0.3677(3)$ & $-0.06555(19)$ & $0.0541(7)$ \\
\hline $\mathrm{C}(34)$ & $0.2755(2)$ & $0.4707(2)$ & $-0.09411(18)$ & $0.0468(6)$ \\
\hline $\mathrm{C}(35)$ & $0.1807(2)$ & $0.49929(19)$ & $-0.04036(17)$ & $0.0386(5)$ \\
\hline $\mathrm{C}(36)$ & $0.12770(18)$ & $0.42431(17)$ & $0.04396(15)$ & $0.0294(4)$ \\
\hline $\mathrm{C}(41)$ & $-0.03574(14)$ & $0.23782(14)$ & $0.20545(13)$ & $0.0180(3)$ \\
\hline $\mathrm{C}(42)$ & $-0.08996(16)$ & $0.19670(17)$ & $0.14789(14)$ & $0.0252(4)$ \\
\hline $\mathrm{C}(43)$ & $-0.20234(17)$ & $0.19556(18)$ & $0.16622(16)$ & $0.0306(4)$ \\
\hline $\mathrm{C}(44)$ & $-0.26144(17)$ & $0.23562(18)$ & $0.24067(16)$ & $0.0313(4)$ \\
\hline $\mathrm{C}(45)$ & $-0.20838(17)$ & $0.27767(19)$ & $0.29701(16)$ & $0.0317(5)$ \\
\hline $\mathrm{C}(46)$ & $-0.09552(16)$ & $0.27850(16)$ & $0.27967(15)$ & $0.0257(4)$ \\
\hline $\mathrm{C}(51)$ & $0.46723(15)$ & $-0.16940(16)$ & $0.34225(13)$ & $0.0222(4)$ \\
\hline $\mathrm{C}(52)$ & $0.47490(18)$ & $-0.08144(17)$ & $0.38125(15)$ & $0.0295(4)$ \\
\hline $\mathrm{C}(53)$ & $0.5766(2)$ & $-0.0412(2)$ & $0.37917(17)$ & $0.0413(6)$ \\
\hline
\end{tabular}


Tabelle 19: Fortsetzung

\begin{tabular}{|r|r|r|r|r|}
\hline $\mathrm{C}(54)$ & $0.6690(2)$ & $-0.0883(2)$ & $0.34041(18)$ & $0.0464(7)$ \\
$\mathrm{C}(55)$ & $0.66214(19)$ & $-0.1762(2)$ & $0.30288(19)$ & $0.0459(6)$ \\
$\mathrm{C}(56)$ & $0.56146(17)$ & $-0.21619(19)$ & $0.30255(16)$ & $0.0335(5)$ \\
$\mathrm{C}(61)$ & $0.29083(15)$ & $-0.33201(14)$ & $0.43127(12)$ & $0.0178(3)$ \\
$\mathrm{C}(62)$ & $0.18106(17)$ & $-0.34477(17)$ & $0.47392(15)$ & $0.0297(4)$ \\
$\mathrm{C}(63)$ & $0.14163(18)$ & $-0.44078(19)$ & $0.54354(16)$ & $0.0354(5)$ \\
$\mathrm{C}(64)$ & $0.21157(19)$ & $-0.52378(17)$ & $0.57197(15)$ & $0.0314(5)$ \\
$\mathrm{C}(65)$ & $0.32033(18)$ & $-0.51240(16)$ & $0.53010(15)$ & $0.0302(4)$ \\
$\mathrm{C}(66)$ & $0.36017(16)$ & $-0.41662(16)$ & $0.45948(14)$ & $0.0247(4)$ \\
$\mathrm{C}(71)$ & $0.36122(14)$ & $-0.18151(16)$ & $0.03602(12)$ & $0.0197(4)$ \\
$\mathrm{C}(72)$ & $0.40209(19)$ & $-0.08211(19)$ & $-0.02684(15)$ & $0.0328(5)$ \\
$\mathrm{C}(73)$ & $0.4695(2)$ & $-0.0788(2)$ & $-0.11659(16)$ & $0.0426(6)$ \\
$\mathrm{C}(74)$ & $0.49680(18)$ & $-0.1731(2)$ & $-0.14314(16)$ & $0.0408(6)$ \\
$\mathrm{C}(75)$ & $0.45702(19)$ & $-0.2731(2)$ & $-0.08086(17)$ & $0.0396(6)$ \\
$\mathrm{C}(76)$ & $0.38868(18)$ & $-0.27747(18)$ & $0.00858(15)$ & $0.0309(4)$ \\
$\mathrm{C}(81)$ & $0.16366(15)$ & $-0.27370(15)$ & $0.17048(13)$ & $0.0200(4)$ \\
$\mathrm{C}(82)$ & $0.15955(16)$ & $-0.38074(16)$ & $0.22967(14)$ & $0.0254(4)$ \\
$\mathrm{C}(83)$ & $0.07174(18)$ & $-0.44924(17)$ & $0.24133(16)$ & $0.0325(5)$ \\
$\mathrm{C}(84)$ & $-0.01265(18)$ & $-0.41098(19)$ & $0.19555(16)$ & $0.0336(5)$ \\
$\mathrm{C}(85)$ & $-0.01006(18)$ & $-0.3048(2)$ & $0.13694(16)$ & $0.0338(5)$ \\
$\mathrm{C}(86)$ & $0.07832(17)$ & $-0.23566(18)$ & $0.12405(14)$ & $0.0276(4)$ \\
$\mathrm{O}(1 \mathrm{~L})$ & $0.0875(2)$ & $0.0665(2)$ & $0.01167(18)$ & $0.0753(7)$ \\
$\mathrm{C}(1 \mathrm{~L})$ & $0.1572(3)$ & $0.0571(3)$ & $-0.0691(3)$ & $0.0744(10)$ \\
\hline & & & &
\end{tabular}

Tabelle 20: Bindungslängen [pm] in $\underline{5}$

\begin{tabular}{|l|r||l|r|}
\hline $\mathrm{Cu}(1)-\mathrm{O}(1)$ & $191.05(13)$ & $\mathrm{Cu}(1)-\mathrm{O}(3)$ & $191.64(14)$ \\
$\mathrm{Cu}(1)-\mathrm{O}(2)$ & $192.03(14)$ & $\mathrm{Cu}(1)-\mathrm{O}(4)$ & $192.37(13)$ \\
$\mathrm{P}(1)-\mathrm{O}(1)$ & $152.07(13)$ & $\mathrm{P}(1)-\mathrm{N}(1)$ & $158.33(16)$ \\
$\mathrm{P}(1)-\mathrm{C}(21)$ & $180.00(19)$ & $\mathrm{P}(1)-\mathrm{C}(11)$ & $180.02(19)$ \\
$\mathrm{P}(2)-\mathrm{O}(2)$ & $152.61(13)$ & $\mathrm{P}(2)-\mathrm{N}(1)$ & $158.84(15)$ \\
$\mathrm{P}(2)-\mathrm{C}(41)$ & $179.74(19)$ & $\mathrm{P}(2)-\mathrm{C}(31)$ & $180.2(2)$ \\
$\mathrm{P}(3)-\mathrm{O}(3)$ & $152.00(14)$ & $\mathrm{P}(3)-\mathrm{N}(2)$ & $158.71(16)$ \\
$\mathrm{P}(3)-\mathrm{C}(51)$ & $180.06(19)$ & $\mathrm{P}(3)-\mathrm{C}(61)$ & $180.17(19)$ \\
$\mathrm{P}(4)-\mathrm{O}(4)$ & $152.62(13)$ & $\mathrm{P}(4)-\mathrm{N}(2)$ & $159.20(16)$ \\
$\mathrm{P}(4)-\mathrm{C}(71)$ & $179.41(19)$ & $\mathrm{P}(4)-\mathrm{C}(81)$ & $180.2(2)$ \\
\hline
\end{tabular}


Tabelle 21: Bindungswinkel $\left[^{\circ}\right]$ in $\underline{5}$

\begin{tabular}{|l|r||l|r|}
\hline $\mathrm{O}(1)-\mathrm{Cu}(1)-\mathrm{O}(3)$ & $89.33(6)$ & $\mathrm{O}(1)-\mathrm{Cu}(1)-\mathrm{O}(2)$ & $95.61(6)$ \\
$\mathrm{O}(3)-\mathrm{Cu}(1)-\mathrm{O}(2)$ & $158.23(6)$ & $\mathrm{O}(1)-\mathrm{Cu}(1)-\mathrm{O}(4)$ & $156.94(6)$ \\
$\mathrm{O}(3)-\mathrm{Cu}(1)-\mathrm{O}(4)$ & $95.58(6)$ & $\mathrm{O}(2)-\mathrm{Cu}(1)-\mathrm{O}(4)$ & $88.15(6)$ \\
$\mathrm{O}(1)-\mathrm{P}(1)-\mathrm{N}(1)$ & $117.00(8)$ & $\mathrm{O}(1)-\mathrm{P}(1)-\mathrm{C}(21)$ & $107.40(8)$ \\
$\mathrm{N}(1)-\mathrm{P}(1)-\mathrm{C}(21)$ & $108.65(9)$ & $\mathrm{O}(1)-\mathrm{P}(1)-\mathrm{C}(11)$ & $107.15(8)$ \\
$\mathrm{N}(1)-\mathrm{P}(1)-\mathrm{C}(11)$ & $109.92(8)$ & $\mathrm{C}(21)-\mathrm{P}(1)-\mathrm{C}(11)$ & $106.18(8)$ \\
$\mathrm{O}(2)-\mathrm{P}(2)-\mathrm{N}(1)$ & $116.79(8)$ & $\mathrm{O}(2)-\mathrm{P}(2)-\mathrm{C}(41)$ & $108.27(8)$ \\
$\mathrm{N}(1)-\mathrm{P}(2)-\mathrm{C}(41)$ & $106.97(9)$ & $\mathrm{O}(2)-\mathrm{P}(2)-\mathrm{C}(31)$ & $106.95(9)$ \\
$\mathrm{N}(1)-\mathrm{P}(2)-\mathrm{C}(31)$ & $109.59(9)$ & $\mathrm{C}(41)-\mathrm{P}(2)-\mathrm{C}(31)$ & $107.98(9)$ \\
$\mathrm{O}(3)-\mathrm{P}(3)-\mathrm{N}(2)$ & $116.58(8)$ & $\mathrm{O}(3)-\mathrm{P}(3)-\mathrm{C}(51)$ & $108.71(9)$ \\
$\mathrm{N}(2)-\mathrm{P}(3)-\mathrm{C}(51)$ & $106.98(9)$ & $\mathrm{O}(3)-\mathrm{P}(3)-\mathrm{C}(61)$ & $106.36(8)$ \\
$\mathrm{N}(2)-\mathrm{P}(3)-\mathrm{C}(61)$ & $109.39(8)$ & $\mathrm{C}(51)-\mathrm{P}(3)-\mathrm{C}(61)$ & $108.62(9)$ \\
$\mathrm{O}(4)-\mathrm{P}(4)-\mathrm{N}(2)$ & $117.24(8)$ & $\mathrm{O}(4)-\mathrm{P}(4)-\mathrm{C}(71)$ & $107.73(9)$ \\
$\mathrm{N}(2)-\mathrm{P}(4)-\mathrm{C}(71)$ & $106.79(9)$ & $\mathrm{O}(4)-\mathrm{P}(4)-\mathrm{C}(81)$ & $108.16(8)$ \\
$\mathrm{N}(2)-\mathrm{P}(4)-\mathrm{C}(81)$ & $109.75(9)$ & $\mathrm{C}(71)-\mathrm{P}(4)-\mathrm{C}(81)$ & $106.67(9)$ \\
$\mathrm{P}(1)-\mathrm{O}(1)-\mathrm{Cu}(1)$ & $130.21(8)$ & $\mathrm{P}(2)-\mathrm{O}(2)-\mathrm{Cu}(1)$ & $131.15(8)$ \\
$\mathrm{P}(3)-\mathrm{O}(3)-\mathrm{Cu}(1)$ & $126.02(8)$ & $\mathrm{P}(4)-\mathrm{O}(4)-\mathrm{Cu}(1)$ & $127.54(8)$ \\
$\mathrm{P}(1)-\mathrm{N}(1)-\mathrm{P}(2)$ & $125.83(10)$ & $\mathrm{P}(3)-\mathrm{N}(2)-\mathrm{P}(4)$ & $122.63(10)$ \\
\hline
\end{tabular}

Tabelle 22: Wasserstoffbindungslängen [pm] in $\underline{5}$

\begin{tabular}{|l|l|l|l|l|}
\hline D-H $\cdots A$ & $\mathrm{~d}(\mathrm{D}-\mathrm{H})$ & $\mathrm{d}(\mathrm{H} \cdots \mathrm{A})$ & $\mathrm{d}(\mathrm{D} \cdots \mathrm{A})$ & $\left.\angle(D H A){ }^{\circ}\right]$ \\
\hline $\mathrm{O}(1 \mathrm{~L})-\mathrm{H}(1 \mathrm{~L}) \cdots \mathrm{O}(2)$ & $97.3(18)$ & $212(3)$ & $290.4(3)$ & $136(3)$ \\
$\mathrm{O}(1 \mathrm{~L})-\mathrm{H}(1 \mathrm{~L}) \cdots \mathrm{O}(4)$ & $97.3(18)$ & $233(2)$ & $320.6(3)$ & $150(3)$ \\
\hline
\end{tabular}




\subsection{Daten zur Kristallstrukturanalyse von \\ $\left.\left[\mathrm{Cu}_{2}(\operatorname{dppaO})_{2}\right)_{2} \mathrm{Cl}_{2}(\text { thf })_{2}\right] \underline{6}$}

\begin{tabular}{ll} 
Summenformel & $\mathrm{C}_{56} \mathrm{H}_{56} \mathrm{Cl}_{2} \mathrm{Cu}_{2} \mathrm{~N}_{2} \mathrm{O}_{6} \mathrm{P}_{4}$ \\
Molekulargewicht $\left[\mathrm{g} \cdot \mathrm{mol}^{-1}\right]$ & 1174.89 \\
Temperatur & $133(2) \mathrm{K}$ \\
Wellenlänge & $71.073 \mathrm{pm}$ \\
Kristallsystem & triklin \\
Raumgruppe & $P \overline{1}$ \\
Gitterkonstanten & $\mathrm{a}=9.7464(5) \AA, \alpha=95.092(3)^{\circ}$ \\
& $\mathrm{b}=11.1449(6) \AA, \beta=99.070(3)^{\circ}$ \\
& $\mathrm{c}=13.4118(7) \AA, \gamma=110.545(3) \circ$ \\
Volumen & $1330.66(12) \AA^{3}$ \\
Formeleinheiten & 1 \\
Röntgenographische Dichte $\left[\mathrm{g} \cdot \mathrm{cm}^{-3}\right]$ & 1.466 \\
Absorptionskoeffizient $\left[\mathrm{mm}^{-1}\right]$ & 1.072 \\
$\mathrm{~F}(000)$ & 606 \\
Meßbereich $\theta$ & $2.40-26.00^{\circ}$ \\
Indexbereiche & $-12 \leq \mathrm{h} \leq 8$ \\
& $-13 \leq \mathrm{k} \leq 13$ \\
& $-16 \leq 1 \leq 16$ \\
Gemessene Reflexe & 18586 \\
Unabhängige Reflexe & 5217 \\
Beobachtete Reflexe $[\mathrm{I}>2 \sigma]$ & 4191 \\
$\mathrm{R}_{\text {int }}$ & 0.0405 \\
Parameter & 323 \\
R1; wR2[I $>2 \sigma(\mathrm{I})]$ & $0.0377 ; 0.0905$ \\
\hline
\end{tabular}


Tabelle 24: Atomkoordinaten und äquivalente isotrope Auslenkungsparameter $\left[\AA^{2}\right]$ für $\underline{6}$

\begin{tabular}{|r|r|r|r|r|}
\hline Atom & \multicolumn{1}{|c|}{$\mathrm{x}$} & \multicolumn{1}{c|}{$\mathrm{y}$} & \multicolumn{1}{c|}{$\mathrm{z}$} & \multicolumn{1}{c|}{$\mathrm{U}_{e q}$} \\
\hline $\mathrm{Cu}(1)$ & $0.51969(3)$ & $0.12760(3)$ & $0.43726(2)$ & $0.02093(11)$ \\
$\mathrm{P}(1)$ & $0.42474(7)$ & $0.16247(6)$ & $0.21645(5)$ & $0.01846(15)$ \\
$\mathrm{P}(2)$ & $0.73197(7)$ & $0.25148(6)$ & $0.30260(5)$ & $0.01869(15)$ \\
$\mathrm{Cl}(1)$ & $0.33115(8)$ & $-0.06286(7)$ & $0.44688(6)$ & $0.03240(18)$ \\
$\mathrm{O}(1)$ & $0.3905(2)$ & $0.15412(18)$ & $0.32271(14)$ & $0.0230(4)$ \\
$\mathrm{O}(2)$ & $0.6962(2)$ & $0.26018(18)$ & $0.40909(13)$ & $0.0224(4)$ \\
$\mathrm{N}(1)$ & $0.5954(2)$ & $0.2062(2)$ & $0.20816(17)$ & $0.0215(5)$ \\
$\mathrm{C}(1)$ & $0.3453(3)$ & $0.2717(2)$ & $0.1624(2)$ & $0.0210(6)$ \\
$\mathrm{C}(2)$ & $0.2458(3)$ & $0.3096(3)$ & $0.2082(2)$ & $0.0285(6)$ \\
$\mathrm{C}(3)$ & $0.1871(4)$ & $0.3953(3)$ & $0.1670(2)$ & $0.0356(7)$ \\
$\mathrm{C}(4)$ & $0.2265(3)$ & $0.4424(3)$ & $0.0798(3)$ & $0.0388(8)$ \\
$\mathrm{C}(5)$ & $0.3223(4)$ & $0.4032(3)$ & $0.0325(3)$ & $0.0425(9)$ \\
$\mathrm{C}(6)$ & $0.3828(3)$ & $0.3190(3)$ & $0.0740(2)$ & $0.0318(7)$ \\
$\mathrm{C}(7)$ & $0.3248(3)$ & $0.0068(3)$ & $0.1356(2)$ & $0.0220(6)$ \\
$\mathrm{C}(8)$ & $0.3935(4)$ & $-0.0825(3)$ & $0.1275(3)$ & $0.0415(8)$ \\
$\mathrm{C}(9)$ & $0.3157(4)$ & $-0.2042(3)$ & $0.0707(3)$ & $0.0449(9)$ \\
$\mathrm{C}(10)$ & $0.1702(3)$ & $-0.2383(3)$ & $0.0213(2)$ & $0.0320(7)$ \\
$\mathrm{C}(11)$ & $0.1003(3)$ & $-0.1504(3)$ & $0.0293(2)$ & $0.0327(7)$ \\
$\mathrm{C}(12)$ & $0.1778(3)$ & $-0.0288(3)$ & $0.0859(2)$ & $0.0282(6)$ \\
$\mathrm{C}(13)$ & $0.8307(3)$ & $0.1421(3)$ & $0.2942(2)$ & $0.0260(6)$ \\
$\mathrm{C}(14)$ & $0.8088(3)$ & $0.0621(3)$ & $0.2027(3)$ & $0.0327(7)$ \\
$\mathrm{C}(15)$ & $0.8827(4)$ & $-0.0239(4)$ & $0.1981(3)$ & $0.0506(10)$ \\
$\mathrm{C}(16)$ & $0.9813(5)$ & $-0.0271(4)$ & $0.2833(4)$ & $0.0623(12)$ \\
$\mathrm{C}(17)$ & $1.0038(5)$ & $0.0511(4)$ & $0.3731(3)$ & $0.0572(12)$ \\
$\mathrm{C}(18)$ & $0.9282(4)$ & $0.1360(4)$ & $0.3794(3)$ & $0.0404(8)$ \\
$\mathrm{C}(19)$ & $0.8613(3)$ & $0.4085(3)$ & $0.29087(19)$ & $0.0201(5)$ \\
$\mathrm{C}(20)$ & $0.9902(3)$ & $0.4718(3)$ & $0.3667(2)$ & $0.0278(6)$ \\
$\mathrm{C}(21)$ & $1.0895(3)$ & $0.5929(3)$ & $0.3599(2)$ & $0.0314(7)$ \\
$\mathrm{C}(22)$ & $1.0644(3)$ & $0.6522(3)$ & $0.2761(2)$ & $0.0285(6)$ \\
$\mathrm{C}(23)$ & $0.9374(3)$ & $0.5909(3)$ & $0.2007(2)$ & $0.0284(6)$ \\
$\mathrm{C}(24)$ & $0.8363(3)$ & $0.4699(3)$ & $0.2083(2)$ & $0.0242(6)$ \\
$\mathrm{O}(3)$ & $0.4676(3)$ & $0.2551(3)$ & $0.55184(17)$ & $0.0476(6)$ \\
$\mathrm{C}(25)$ & $0.4522(7)$ & $0.2317(6)$ & $0.6528(3)$ & $0.0943(19)$ \\
$\mathrm{C}(27 \mathrm{~B})$ & $0.4013(6)$ & $0.3310(5)$ & $0.6982(4)$ & $0.0884(18)$ \\
$\mathrm{C}(28 \mathrm{~B})$ & $0.3739(14)$ & $0.4029(12)$ & $0.6106(9)$ & $0.086(3)$ \\
& $0.4010(11)$ & $0.3401(10)$ & $0.6148(7)$ & $0.069(3)$ \\
& $0.3441(12)$ & $0.3000(11)$ & $0.5197(8)$ & $0.079(3)$ \\
\hline
\end{tabular}


Tabelle 25: Bindungslängen [pm] in $\underline{6}$

\begin{tabular}{|l|r||l|r|}
\hline $\mathrm{Cu}(1)-\mathrm{O}(1)$ & $194.03(18)$ & $\mathrm{Cu}(1)-\mathrm{O}(2)$ & $195.10(18)$ \\
$\mathrm{Cu}(1)-\mathrm{O}(3)$ & $223.7(2)$ & $\mathrm{Cu}(1)-\mathrm{Cl}(1) \# 1$ & $229.60(7)$ \\
$\mathrm{Cu}(1)-\mathrm{Cl}(1)$ & $229.88(8)$ & $\mathrm{P}(1)-\mathrm{O}(1)$ & $151.74(19)$ \\
$\mathrm{P}(1)-\mathrm{N}(1)$ & $158.6(2)$ & $\mathrm{P}(1)-\mathrm{C}(1)$ & $180.3(3)$ \\
$\mathrm{P}(1)-\mathrm{C}(7)$ & $181.1(3)$ & $\mathrm{P}(2)-\mathrm{O}(2)$ & $152.52(19)$ \\
$\mathrm{P}(2)-\mathrm{N}(1)$ & $158.6(2)$ & $\mathrm{P}(2)-\mathrm{C}(19)$ & $180.0(3)$ \\
$\mathrm{P}(2)-\mathrm{C}(13)$ & $180.5(3)$ & $\mathrm{Cl}(1)-\mathrm{Cu}(1) \# 1$ & $229.60(7)$ \\
\hline
\end{tabular}

Tabelle 26: Bindungswinkel $\left[{ }^{\circ}\right]$ in $\underline{6}$

\begin{tabular}{|l|r||l|r|}
\hline $\mathrm{O}(1)-\mathrm{Cu}(1)-\mathrm{O}(2)$ & $91.84(8)$ & $\mathrm{O}(1)-\mathrm{Cu}(1)-\mathrm{O}(3)$ & $93.78(9)$ \\
$\mathrm{O}(2)-\mathrm{Cu}(1)-\mathrm{O}(3)$ & $97.23(9)$ & $\mathrm{O}(1)-\mathrm{Cu}(1)-\mathrm{Cl}(1) \# 1$ & $169.42(6)$ \\
$\mathrm{O}(2)-\mathrm{Cu}(1)-\mathrm{Cl}(1) \# 1$ & $90.42(6)$ & $\mathrm{O}(3)-\mathrm{Cu}(1)-\mathrm{Cl}(1) \# 1$ & $96.19(7)$ \\
$\mathrm{O}(1)-\mathrm{Cu}(1)-\mathrm{Cl}(1)$ & $89.63(6)$ & $\mathrm{O}(2)-\mathrm{Cu}(1)-\mathrm{Cl}(1)$ & $165.65(6)$ \\
$\mathrm{O}(3)-\mathrm{Cu}(1)-\mathrm{Cl}(1)$ & $96.91(7)$ & $\mathrm{Cl}(1) \# 1-\mathrm{Cu}(1)-\mathrm{Cl}(1)$ & $85.66(3)$ \\
$\mathrm{O}(1)-\mathrm{P}(1)-\mathrm{N}(1)$ & $116.91(11)$ & $\mathrm{O}(1)-\mathrm{P}(1)-\mathrm{C}(1)$ & $106.90(12)$ \\
$\mathrm{N}(1)-\mathrm{P}(1)-\mathrm{C}(1)$ & $109.90(12)$ & $\mathrm{O}(1)-\mathrm{P}(1)-\mathrm{C}(7)$ & $109.58(12)$ \\
$\mathrm{N}(1)-\mathrm{P}(1)-\mathrm{C}(7)$ & $107.61(12)$ & $\mathrm{C}(1)-\mathrm{P}(1)-\mathrm{C}(7)$ & $105.36(12)$ \\
$\mathrm{O}(2)-\mathrm{P}(2)-\mathrm{N}(1)$ & $117.20(11)$ & $\mathrm{O}(2)-\mathrm{P}(2)-\mathrm{C}(19)$ & $107.72(11)$ \\
$\mathrm{N}(1)-\mathrm{P}(2)-\mathrm{C}(19)$ & $108.65(12)$ & $\mathrm{O}(2)-\mathrm{P}(2)-\mathrm{C}(13)$ & $108.26(12)$ \\
$\mathrm{N}(1)-\mathrm{P}(2)-\mathrm{C}(13)$ & $108.25(13)$ & $\mathrm{C}(19)-\mathrm{P}(2)-\mathrm{C}(13)$ & $106.24(12)$ \\
$\mathrm{Cu}(1) \# 1-\mathrm{Cl}(1)-\mathrm{Cu}(1)$ & $94.34(3)$ & $\mathrm{P}(1)-\mathrm{O}(1)-\mathrm{Cu}(1)$ & $124.13(11)$ \\
$\mathrm{P}(2)-\mathrm{O}(2)-\mathrm{Cu}(1)$ & $119.51(11)$ & $\mathrm{P}(1)-\mathrm{N}(1)-\mathrm{P}(2)$ & $124.88(14)$ \\
\hline
\end{tabular}

$\# 1-\mathrm{x}+1,-\mathrm{y},-\mathrm{z}+1$ 


\subsection{Daten zur Kristallstrukturanalyse von $\left[\mathrm{Cu}_{2}\left(\mathrm{dppaO}_{2}\right)_{2}(\mathrm{OMe})_{2}\right] \underline{7}$}

\begin{tabular}{|ll|}
\hline Summenformel & $\mathrm{C}_{50} \mathrm{H}_{46} \mathrm{Cu}_{2} \mathrm{~N}_{2} \mathrm{O}_{6} \mathrm{P}_{4}$ \\
Molekulargewicht $\left[\mathrm{g} \cdot \mathrm{mol}^{-1}\right]$ & 1021.85 \\
Temperatur & $133(2) \mathrm{K}$ \\
Wellenlänge & $71.073 \mathrm{pm}$ \\
Kristallsystem & triklin \\
Raumgruppe & $P \overline{1}$ \\
Gitterkonstanten & $\mathrm{a}=8.6948(4) \AA, \alpha=94.391(3)^{\circ}$ \\
& $\mathrm{b}=9.9690(4) \AA, \beta=104.403(2)^{\circ}$ \\
& $\mathrm{c}=13.7878(6) \AA, \gamma=99.972(2)^{\circ}$
\end{tabular}


Tabelle 28: Atomkoordinaten und äquivalente isotrope Auslenkungsparameter $\left[\AA^{2}\right]$ für $\underline{7}$

\begin{tabular}{|r|r|r|r|r|}
\hline $\mathrm{Atom}$ & \multicolumn{1}{|c|}{$\mathrm{x}$} & \multicolumn{1}{c|}{$\mathrm{y}$} & \multicolumn{1}{c|}{$\mathrm{z}$} & \multicolumn{1}{c|}{$\mathrm{U}_{e q}$} \\
\hline $\mathrm{Cu}$ & $0.60818(3)$ & $0.61465(3)$ & $0.57610(2)$ & $0.01555(10)$ \\
$\mathrm{N}$ & $0.9126(2)$ & $0.86744(19)$ & $0.73486(14)$ & $0.0171(4)$ \\
$\mathrm{P}(1)$ & $0.89853(7)$ & $0.71698(6)$ & $0.76684(4)$ & $0.01560(14)$ \\
$\mathrm{P}(2)$ & $0.76864(7)$ & $0.92111(6)$ & $0.66241(4)$ & $0.01493(14)$ \\
$\mathrm{O}(1)$ & $0.7696(2)$ & $0.60416(16)$ & $0.69703(12)$ & $0.0201(4)$ \\
$\mathrm{O}(2)$ & $0.63674(19)$ & $0.81370(16)$ & $0.58848(12)$ & $0.0180(4)$ \\
$\mathrm{O}(3)$ & $0.4705(2)$ & $0.57978(16)$ & $0.44238(12)$ & $0.0233(4)$ \\
$\mathrm{C}(1)$ & $0.8637(3)$ & $0.7170(2)$ & $0.89096(18)$ & $0.0176(5)$ \\
$\mathrm{C}(2)$ & $0.8589(3)$ & $0.8365(3)$ & $0.94706(19)$ & $0.0230(5)$ \\
$\mathrm{C}(3)$ & $0.8265(3)$ & $0.8317(3)$ & $1.0404(2)$ & $0.0277(6)$ \\
$\mathrm{C}(4)$ & $0.7999(3)$ & $0.7087(3)$ & $1.0786(2)$ & $0.0276(6)$ \\
$\mathrm{C}(5)$ & $0.8057(4)$ & $0.5887(3)$ & $1.0231(2)$ & $0.0339(7)$ \\
$\mathrm{C}(6)$ & $0.8372(3)$ & $0.5925(3)$ & $0.9299(2)$ & $0.0284(6)$ \\
$\mathrm{C}(7)$ & $1.0921(3)$ & $0.6688(2)$ & $0.77867(17)$ & $0.0175(5)$ \\
$\mathrm{C}(8)$ & $1.2314(3)$ & $0.7485(3)$ & $0.84468(19)$ & $0.0232(5)$ \\
$\mathrm{C}(9)$ & $1.3817(3)$ & $0.7156(3)$ & $0.8497(2)$ & $0.0276(6)$ \\
$\mathrm{C}(10)$ & $1.3940(3)$ & $0.6041(3)$ & $0.7883(2)$ & $0.0292(6)$ \\
$\mathrm{C}(11)$ & $1.2566(3)$ & $0.5236(3)$ & $0.7236(2)$ & $0.0290(6)$ \\
$\mathrm{C}(12)$ & $1.1059(3)$ & $0.5548(3)$ & $0.71852(19)$ & $0.0238(6)$ \\
$\mathrm{C}(13)$ & $0.6754(3)$ & $1.0197(2)$ & $0.73854(17)$ & $0.0167(5)$ \\
$\mathrm{C}(14)$ & $0.5124(3)$ & $0.9879(3)$ & $0.73468(19)$ & $0.0250(6)$ \\
$\mathrm{C}(15)$ & $0.4506(3)$ & $1.0635(3)$ & $0.7992(2)$ & $0.0328(7)$ \\
$\mathrm{C}(16)$ & $0.5514(4)$ & $1.1694(3)$ & $0.8690(2)$ & $0.0317(6)$ \\
$\mathrm{C}(17)$ & $0.7141(3)$ & $1.2021(3)$ & $0.8735(2)$ & $0.0274(6)$ \\
$\mathrm{C}(18)$ & $0.7752(3)$ & $1.1286(2)$ & $0.80816(19)$ & $0.0226(5)$ \\
$\mathrm{C}(19)$ & $0.8610(3)$ & $1.0480(2)$ & $0.59710(17)$ & $0.0164(5)$ \\
$\mathrm{C}(20)$ & $1.0283(3)$ & $1.0868(2)$ & $0.61903(18)$ & $0.0204(5)$ \\
$\mathrm{C}(21)$ & $1.0979(3)$ & $1.1902(3)$ & $0.5722(2)$ & $0.0248(6)$ \\
$\mathrm{C}(22)$ & $1.0010(3)$ & $1.2564(3)$ & $0.5046(2)$ & $0.0261(6)$ \\
\hline & $0.8350(3)$ & $1.2182(3)$ & $0.48177(19)$ & $0.0255(6)$ \\
$\mathrm{C}(23)$ & $0.7640(3)$ & $1.1139(2)$ & $0.52712(18)$ & $0.0210(5)$ \\
& & & & $0.0214(5)$ \\
\hline
\end{tabular}


Tabelle 29: Bindungslängen [pm] in $\underline{7}$

\begin{tabular}{|l|r||l|r|}
\hline $\mathrm{Cu}-\mathrm{O}(3)$ & $190.07(16)$ & $\mathrm{Cu}-\mathrm{O}(3) \# 1$ & $191.52(16)$ \\
$\mathrm{Cu}-\mathrm{O}(1)$ & $191.70(16)$ & $\mathrm{Cu}-\mathrm{O}(2)$ & $194.54(15)$ \\
$\mathrm{Cu}-\mathrm{Cu} \# 1$ & $299.67(6)$ & $\mathrm{N}-\mathrm{P}(1)$ & $158.9(2)$ \\
$\mathrm{N}-\mathrm{P}(2)$ & $159.43(19)$ & $\mathrm{P}(1)-\mathrm{O}(1)$ & $151.94(17)$ \\
$\mathrm{P}(1)-\mathrm{C}(7)$ & $180.2(2)$ & $\mathrm{P}(1)-\mathrm{C}(1)$ & $181.1(2)$ \\
$\mathrm{P}(2)-\mathrm{O}(2)$ & $152.54(16)$ & $\mathrm{P}(2)-\mathrm{C}(19)$ & $179.9(2)$ \\
$\mathrm{P}(2)-\mathrm{C}(13)$ & $180.8(2)$ & $\mathrm{O}(3)-\mathrm{C}(25)$ & $141.1(3)$ \\
$\mathrm{O}(3)-\mathrm{Cu} \# 1$ & $191.52(16)$ & & \\
\hline
\end{tabular}

Tabelle 30: Bindungswinkel $\left[^{\circ}\right]$ in $\underline{7}$

\begin{tabular}{|l|r||l|r|}
\hline $\mathrm{O}(3)-\mathrm{Cu}-\mathrm{O}(3) \# 1$ & $76.50(8)$ & $\mathrm{O}(3)-\mathrm{Cu}-\mathrm{O}(1)$ & $163.06(7)$ \\
$\mathrm{O}(3) \# 1-\mathrm{Cu}-\mathrm{O}(1)$ & $91.65(7)$ & $\mathrm{O}(3)-\mathrm{Cu}-\mathrm{O}(2)$ & $96.62(7)$ \\
$\mathrm{O}(3) \# 1-\mathrm{Cu}-\mathrm{O}(2)$ & $167.12(7)$ & $\mathrm{O}(1)-\mathrm{Cu}-\mathrm{O}(2)$ & $97.19(7)$ \\
$\mathrm{O}(3)-\mathrm{Cu}-\mathrm{Cu} \# 1$ & $38.42(5)$ & $\mathrm{O}(3) \# 1-\mathrm{Cu}-\mathrm{Cu} \# 1$ & $38.08(5)$ \\
$\mathrm{O}(1)-\mathrm{Cu}-\mathrm{Cu} \# 1$ & $128.69(5)$ & $\mathrm{O}(2)-\mathrm{Cu}-\mathrm{Cu} \# 1$ & $134.12(5)$ \\
$\mathrm{P}(1)-\mathrm{N}-\mathrm{P}(2)$ & $124.37(13)$ & $\mathrm{O}(1)-\mathrm{P}(1)-\mathrm{N}$ & $117.26(10)$ \\
$\mathrm{O}(1)-\mathrm{P}(1)-\mathrm{C}(7)$ & $107.90(10)$ & N-P(1)-C(7) & $107.69(11)$ \\
$\mathrm{O}(1)-\mathrm{P}(1)-\mathrm{C}(1)$ & $107.23(11)$ & N-P(1)-C(1) & $110.20(11)$ \\
$\mathrm{C}(7)-\mathrm{P}(1)-\mathrm{C}(1)$ & $105.98(11)$ & $\mathrm{O}(2)-\mathrm{P}(2)-\mathrm{N}$ & $117.22(10)$ \\
$\mathrm{O}(2)-\mathrm{P}(2)-\mathrm{C}(19)$ & $111.04(10)$ & N-P $(2)-\mathrm{C}(19)$ & $106.69(11)$ \\
$\mathrm{O}(2)-\mathrm{P}(2)-\mathrm{C}(13)$ & $108.86(10)$ & $\mathrm{N}-\mathrm{P}(2)-\mathrm{C}(13)$ & $108.99(11)$ \\
$\mathrm{C}(19)-\mathrm{P}(2)-\mathrm{C}(13)$ & $103.09(10)$ & $\mathrm{P}(1)-\mathrm{O}(1)-\mathrm{Cu}$ & $129.96(10)$ \\
$\mathrm{P}(2)-\mathrm{O}(2)-\mathrm{Cu}$ & $128.88(9)$ & $\mathrm{C}(25)-\mathrm{O}(3)-\mathrm{Cu}$ & $130.19(15)$ \\
$\mathrm{C}(25)-\mathrm{O}(3)-\mathrm{Cu} \# 1$ & $126.29(14)$ & $\mathrm{Cu}-\mathrm{O}(3)-\mathrm{Cu} \# 1$ & $103.50(7)$ \\
\hline
\end{tabular}

$\#-x+1,-y+1,-z+1$ 


\subsection{Daten zur Kristallstrukturanalyse von $\left[\mathrm{Cu}_{3}\left(\operatorname{dppaO}_{2}\right)_{2}\left(\left(\mathrm{OSiMe}_{2}\right)_{2} \mathrm{O}\right)_{2}(\operatorname{thf})_{2}\right] \underline{8}$}

\begin{tabular}{|ll|}
\hline Summenformel & $\mathrm{C}_{64} \mathrm{H}_{80} \mathrm{Cu}_{3} \mathrm{~N}_{2} \mathrm{O}_{12} \mathrm{P}_{4} \mathrm{Si}_{4}$ \\
Molekulargewicht $\left[\mathrm{g} \cdot \mathrm{mol}^{-1}\right]$ & 1496.16 \\
Temperatur & $133(2) \mathrm{K}$ \\
Wellenlänge & $71.073 \mathrm{pm}$ \\
Kristallsystem & monoklin \\
Raumgruppe & $P 2_{1} / c$ \\
Gitterkonstanten & $\mathrm{a}=13.582(2) \AA, \alpha=90^{\circ}$ \\
& $\mathrm{b}=16.772(3) \AA, \beta=107.67(3)^{\circ} \circ$ \\
& $\mathrm{c}=15.734(3) \AA, \gamma=90^{\circ}$ \\
Volumen & $3415.1(10) \AA^{3}$ \\
Formeleinheiten & 2 \\
Röntgenographische Dichte $\left[\mathrm{g} \cdot \mathrm{cm}^{-3}\right]$ & 1.455 \\
Absorptionskoeffizient $\left[\mathrm{mm}^{-1}\right]$ & 1.150 \\
F(000) & 1554 \\
Meßbereich $\theta$ & $1.74-25.05^{\circ}$ \\
Indexbereiche & $-15 \leq \mathrm{h} \leq 15$ \\
& $-19 \leq \mathrm{k} \leq 19$ \\
Gemessene Reflexe & $-12 \leq 1 \leq 18$ \\
Unabhängige Reflexe & 52841 \\
Beobachtete Reflexe $[\mathrm{I}>2 \sigma]$ & 8209 \\
$\mathrm{R}_{\text {int }}$ & 5755 \\
Parameter & 0.0974 \\
R1; wR2[I>2 $\sigma(\mathrm{I})]$ & 408 \\
\hline
\end{tabular}


Tabelle 32: Atomkoordinaten und äquivalente isotrope Auslenkungsparameter $\left[\AA^{2}\right]$ für $\underline{8}$

\begin{tabular}{|c|c|c|c|c|}
\hline Atom & $\mathrm{x}$ & $\mathrm{y}$ & z & $\mathrm{U}_{e q}$ \\
\hline $\mathrm{Cu}(1)$ & $0.41623(4)$ & $0.38603(3)$ & $0.60240(3)$ & $0.01575(15)$ \\
\hline $\mathrm{Cu}(2)$ & 0.5000 & 0.5000 & 0.5000 & $0.0224(2)$ \\
\hline $\mathrm{P}(1)$ & $0.40128(8)$ & $0.21774(6)$ & $0.68545(7)$ & $0.0157(2)$ \\
\hline $\mathrm{P}(2)$ & $0.27985(8)$ & $0.33449(6)$ & $0.72255(7)$ & $0.0155(2)$ \\
\hline $\mathrm{Si}(1)$ & $0.55288(9)$ & $0.31021(6)$ & $0.48875(8)$ & $0.0203(3)$ \\
\hline $\operatorname{Si}(2)$ & $0.34772(9)$ & $0.56978(6)$ & $0.60557(7)$ & $0.0189(3)$ \\
\hline $\mathrm{O}(1)$ & $0.4443(2)$ & $0.27434(14)$ & $0.62944(19)$ & $0.0207(6)$ \\
\hline $\mathrm{O}(2)$ & $0.3193(2)$ & $0.39657(13)$ & $0.66933(18)$ & $0.0190(6)$ \\
\hline $\mathrm{O}(3)$ & $0.4941(2)$ & $0.38488(14)$ & $0.51922(19)$ & $0.0210(6)$ \\
\hline $\mathrm{O}(4)$ & $0.4211(2)$ & $0.49946(14)$ & $0.58456(19)$ & $0.0206(6)$ \\
\hline $\mathrm{O}(5)$ & $0.3930(2)$ & $0.65523(15)$ & $0.5836(2)$ & $0.0304(7)$ \\
\hline $\mathrm{N}(1)$ & $0.2929(2)$ & $0.24336(16)$ & $0.6979(2)$ & $0.0156(7)$ \\
\hline $\mathrm{C}(11)$ & $0.5004(3)$ & $0.2075(2)$ & $0.7928(3)$ & $0.0209(9)$ \\
\hline $\mathrm{C}(12)$ & $0.4807(3)$ & $0.1610(2)$ & $0.8587(3)$ & $0.0306(11)$ \\
\hline $\mathrm{C}(13)$ & $0.5503(4)$ & $0.1571(3)$ & $0.9434(3)$ & $0.0412(13)$ \\
\hline $\mathrm{C}(14)$ & $0.6414(4)$ & $0.2002(3)$ & $0.9613(3)$ & $0.0494(15)$ \\
\hline $\mathrm{C}(15)$ & $0.6626(4)$ & $0.2460(3)$ & $0.8972(3)$ & $0.0429(13)$ \\
\hline $\mathrm{C}(16)$ & $0.5916(3)$ & $0.2493(3)$ & $0.8121(3)$ & $0.0306(11)$ \\
\hline $\mathrm{C}(21)$ & $0.3843(3)$ & $0.1199(2)$ & $0.6360(2)$ & $0.0175(9)$ \\
\hline $\mathrm{C}(22)$ & $0.4716(3)$ & $0.0783(2)$ & $0.6307(3)$ & $0.0253(10)$ \\
\hline $\mathrm{C}(23)$ & $0.4611(3)$ & $0.0023(2)$ & $0.5944(3)$ & $0.0303(11)$ \\
\hline $\mathrm{C}(24)$ & $0.3638(3)$ & $-0.0318(2)$ & $0.5622(3)$ & $0.0300(11)$ \\
\hline $\mathrm{C}(25)$ & $0.2772(3)$ & $0.0090(2)$ & $0.5669(3)$ & $0.0278(10)$ \\
\hline $\mathrm{C}(26)$ & $0.2877(3)$ & $0.0853(2)$ & $0.6038(3)$ & $0.0212(9)$ \\
\hline $\mathrm{C}(31)$ & $0.1454(3)$ & $0.3521(2)$ & $0.7070(2)$ & $0.0164(9)$ \\
\hline $\mathrm{C}(32)$ & $0.0789(3)$ & $0.2895(2)$ & $0.7112(3)$ & $0.0266(10)$ \\
\hline $\mathrm{C}(33)$ & $-0.0238(3)$ & $0.3048(2)$ & $0.7036(3)$ & $0.0296(10)$ \\
\hline $\mathrm{C}(34)$ & $-0.0613(3)$ & $0.3818(2)$ & $0.6907(3)$ & $0.0284(10)$ \\
\hline $\mathrm{C}(35)$ & $0.0041(3)$ & $0.4438(2)$ & $0.6859(3)$ & $0.0291(11)$ \\
\hline $\mathrm{C}(36)$ & $0.1065(3)$ & $0.4293(2)$ & $0.6952(3)$ & $0.0247(10)$ \\
\hline $\mathrm{C}(41)$ & $0.3468(3)$ & $0.3503(2)$ & $0.8398(2)$ & $0.0171(9)$ \\
\hline $\mathrm{C}(42)$ & $0.4358(3)$ & $0.3962(2)$ & $0.8654(3)$ & $0.0286(10)$ \\
\hline $\mathrm{C}(43)$ & $0.4941(3)$ & $0.4010(3)$ & $0.9556(3)$ & $0.0350(12)$ \\
\hline $\mathrm{C}(44)$ & $0.4636(4)$ & $0.3603(3)$ & $1.0184(3)$ & $0.0386(12)$ \\
\hline $\mathrm{C}(45)$ & $0.3747(3)$ & $0.3140(3)$ & $0.9938(3)$ & $0.0354(12)$ \\
\hline $\mathrm{C}(46)$ & $0.3158(3)$ & $0.3095(2)$ & $0.9039(3)$ & $0.0270(10)$ \\
\hline $\mathrm{C}(3 \mathrm{~A})$ & $0.3546(3)$ & $0.5765(2)$ & $0.7243(3)$ & $0.0252(10)$ \\
\hline $\mathrm{C}(3 \mathrm{~B})$ & $0.2127(4)$ & $0.5569(3)$ & $0.5314(3)$ & $0.0334(12)$ \\
\hline
\end{tabular}


Tabelle 32: Fortsetzung

\begin{tabular}{|l|r|r|r|r|}
\hline $\mathrm{C}(4 \mathrm{~A})$ & $0.6571(3)$ & $0.2680(3)$ & $0.5837(3)$ & $0.0318(11)$ \\
$\mathrm{C}(4 \mathrm{~B})$ & $0.4617(3)$ & $0.2306(2)$ & $0.4309(3)$ & $0.0279(10)$ \\
$\mathrm{O}(1 \mathrm{~L})$ & $0.6869(3)$ & $0.4882(2)$ & $0.6556(3)$ & $0.0618(12)$ \\
$\mathrm{C}(1 \mathrm{~L})$ & $0.6727(5)$ & $0.4608(3)$ & $0.7366(4)$ & $0.0599(16)$ \\
$\mathrm{C}(2 \mathrm{~L})$ & $0.6921(6)$ & $0.5317(4)$ & $0.8004(4)$ & $0.078(2)$ \\
$\mathrm{C}(3 \mathrm{~L})$ & $0.6861(5)$ & $0.6011(3)$ & $0.7397(4)$ & $0.0572(16)$ \\
$\mathrm{C}(4 \mathrm{~L})$ & $0.7268(4)$ & $0.5663(3)$ & $0.6675(4)$ & $0.0481(14)$ \\
\hline
\end{tabular}

Tabelle 33: Bindungslängen $[\mathrm{pm}]$ in $\underline{8}$

\begin{tabular}{|l|r||l|r|}
\hline $\mathrm{N}(1)-\mathrm{P}(2)$ & $160.0(3)$ & $\mathrm{N}(1)-\mathrm{P}(1)$ & $160.2(3)$ \\
$\mathrm{Cu}(1)-\mathrm{O}(3)$ & $191.6(3)$ & $\mathrm{Cu}(1)-\mathrm{O}(4)$ & $192.7(2)$ \\
$\mathrm{Cu}(1)-\mathrm{O}(2)$ & $192.7(3)$ & $\mathrm{Cu}(1)-\mathrm{O}(1)$ & $193.3(2)$ \\
$\mathrm{Cu}(1)-\mathrm{Cu}(2)$ & $294.23(6)$ & $\mathrm{O}(1)-\mathrm{P}(1)$ & $152.7(3)$ \\
$\mathrm{O}(2)-\mathrm{P}(2)$ & $153.1(3)$ & $\mathrm{P}(2)-\mathrm{C}(31)$ & $179.2(4)$ \\
$\mathrm{P}(2)-\mathrm{C}(41)$ & $181.1(4)$ & $\mathrm{P}(1)-\mathrm{C}(21)$ & $180.1(3)$ \\
$\mathrm{P}(1)-\mathrm{C}(11)$ & $182.0(4)$ & $\mathrm{Cu}(2)-\mathrm{O}(4)$ & $194.5(3)$ \\
$\mathrm{Cu}(2)-\mathrm{O}(3)$ & $196.0(2)$ & $\mathrm{Cu}(2)-\mathrm{O}(1 \mathrm{~L})$ & $292.5(6)$ \\
$\mathrm{Si}(1)-\mathrm{O}(3)$ & $163.3(3)$ & $\mathrm{Si}(1)-\mathrm{C}(4 \mathrm{~A})$ & $185.8(5)$ \\
$\mathrm{Si}(1)-\mathrm{C}(4 \mathrm{~B})$ & $186.1(4)$ & $\mathrm{Si}(2)-\mathrm{O}(5)$ & $163.7(3)$ \\
$\mathrm{Si}(2)-\mathrm{O}(4)$ & $164.1(3)$ & $\mathrm{Si}(2)-\mathrm{C}(3 \mathrm{~A})$ & $184.5(4)$ \\
$\mathrm{Si}(2)-\mathrm{C}(3 \mathrm{~B})$ & $186.4(5)$ & & \\
\hline
\end{tabular}

Tabelle 34: Bindungswinkel $\left[{ }^{\circ}\right]$ in $\underline{8}$

\begin{tabular}{|l|r||l|r|}
\hline $\mathrm{P}(2)-\mathrm{N}(1)-\mathrm{P}(1)$ & $117.36(18)$ & $\mathrm{O}(3)-\mathrm{Cu}(1)-\mathrm{O}(4)$ & $81.95(11)$ \\
$\mathrm{O}(3)-\mathrm{Cu}(1)-\mathrm{O}(2)$ & $169.79(12)$ & $\mathrm{O}(4)-\mathrm{Cu}(1)-\mathrm{O}(2)$ & $92.78(10)$ \\
$\mathrm{O}(3)-\mathrm{Cu}(1)-\mathrm{O}(1)$ & $91.58(11)$ & $\mathrm{O}(4)-\mathrm{Cu}(1)-\mathrm{O}(1)$ & $167.27(12)$ \\
$\mathrm{O}(2)-\mathrm{Cu}(1)-\mathrm{O}(1)$ & $95.19(10)$ & $\mathrm{O}(3)-\mathrm{Cu}(1)-\mathrm{Cu}(2)$ & $41.17(7)$ \\
$\mathrm{O}(4)-\mathrm{Cu}(1)-\mathrm{Cu}(2)$ & $40.78(8)$ & $\mathrm{O}(2)-\mathrm{Cu}(1)-\mathrm{Cu}(2)$ & $132.95(7)$ \\
$\mathrm{O}(1)-\mathrm{Cu}(1)-\mathrm{Cu}(2)$ & $131.82(8)$ & $\mathrm{P}(1)-\mathrm{O}(1)-\mathrm{Cu}(1)$ & $130.16(16)$ \\
$\mathrm{P}(2)-\mathrm{O}(2)-\mathrm{Cu}(1)$ & $130.31(15)$ & $\mathrm{O}(2)-\mathrm{P}(2)-\mathrm{N}(1)$ & $115.73(15)$ \\
$\mathrm{O}(2)-\mathrm{P}(2)-\mathrm{C}(31)$ & $108.93(16)$ & $\mathrm{N}(1)-\mathrm{P}(2)-\mathrm{C}(31)$ & $107.95(17)$ \\
$\mathrm{O}(2)-\mathrm{P}(2)-\mathrm{C}(41)$ & $107.84(16)$ & $\mathrm{N}(1)-\mathrm{P}(2)-\mathrm{C}(41)$ & $109.17(16)$ \\
$\mathrm{O}(1)-\mathrm{P}(1)-\mathrm{N}(1)$ & $115.52(15)$ & $\mathrm{O}(1)-\mathrm{P}(1)-\mathrm{C}(21)$ & $109.77(17)$ \\
$\mathrm{N}(1)-\mathrm{P}(1)-\mathrm{C}(21)$ & $107.18(16)$ & $\mathrm{O}(1)-\mathrm{P}(1)-\mathrm{C}(11)$ & $106.81(18)$ \\
$\mathrm{N}(1)-\mathrm{P}(1)-\mathrm{C}(11)$ & $111.11(18)$ & $\mathrm{O}(4)-\mathrm{Cu}(2)-\mathrm{O}(3)$ & $80.39(10)$ \\
$\mathrm{O}(4) \# 1-\mathrm{Cu}(2)-\mathrm{O}(3)$ & $99.61(10)$ & $\mathrm{O}(4)-\mathrm{Cu}(2)-\mathrm{O}(3) \# 1$ & $99.61(10)$ \\
$\mathrm{O}(4)-\mathrm{Cu}(2)-\mathrm{Cu}(1)$ & $40.32(7)$ & $\mathrm{O}(4) \# 1-\mathrm{Cu}(2)-\mathrm{Cu}(1)$ & $139.68(7)$ \\
\hline
\end{tabular}


Tabelle 34: Fortsetzung

\begin{tabular}{|l|r||l|r|}
\hline $\mathrm{O}(3)-\mathrm{Cu}(2)-\mathrm{Cu}(1)$ & $40.06(7)$ & $\mathrm{O}(3) \# 1-\mathrm{Cu}(2)-\mathrm{Cu}(1)$ & $139.94(7)$ \\
$\mathrm{O}(4)-\mathrm{Cu}(2)-\mathrm{Cu}(1) \# 1$ & $139.67(7)$ & $\mathrm{Cu}(1)-\mathrm{Cu}(2)-\mathrm{O}(1 \mathrm{~L})$ & $83.20(8)$ \\
$\mathrm{O}(3)-\mathrm{Si}(1)-\mathrm{O}(5) \# 1$ & $107.18(14)$ & $\mathrm{O}(3)-\mathrm{Si}(1)-\mathrm{C}(4 \mathrm{~A})$ & $112.14(18)$ \\
$\mathrm{O}(5) \# 1-\mathrm{Si}(1)-\mathrm{C}(4 \mathrm{~A})$ & $107.49(19)$ & $\mathrm{O}(3)-\mathrm{Si}(1)-\mathrm{C}(4 \mathrm{~B})$ & $112.43(17)$ \\
$\mathrm{O}(5) \# 1-\mathrm{Si}(1)-\mathrm{C}(4 \mathrm{~B})$ & $106.65(18)$ & $\mathrm{C}(4 \mathrm{~A})-\mathrm{Si}(1)-\mathrm{C}(4 \mathrm{~B})$ & $110.6(2)$ \\
$\mathrm{O}(5)-\mathrm{Si}(2)-\mathrm{O}(4)$ & $107.24(14)$ & $\mathrm{O}(5)-\mathrm{Si}(2)-\mathrm{C}(3 \mathrm{~A})$ & $104.68(17)$ \\
$\mathrm{O}(4)-\mathrm{Si}(2)-\mathrm{C}(3 \mathrm{~A})$ & $113.62(17)$ & $\mathrm{O}(5)-\mathrm{Si}(2)-\mathrm{C}(3 \mathrm{~B})$ & $109.29(19)$ \\
$\mathrm{O}(4)-\mathrm{Si}(2)-\mathrm{C}(3 \mathrm{~B})$ & $109.44(18)$ & $\mathrm{C}(3 \mathrm{~A})-\mathrm{Si}(2)-\mathrm{C}(3 \mathrm{~B})$ & $112.3(2)$ \\
$\mathrm{Si}(1) \# 1-\mathrm{O}(5)-\mathrm{Si}(2)$ & $137.18(18)$ & $\mathrm{Si}(1)-\mathrm{O}(3)-\mathrm{Cu}(1)$ & $128.94(15)$ \\
$\mathrm{Si}(1)-\mathrm{O}(3)-\mathrm{Cu}(2)$ & $131.57(15)$ & $\mathrm{Cu}(1)-\mathrm{O}(3)-\mathrm{Cu}(2)$ & $98.77(11)$ \\
$\mathrm{Si}(2)-\mathrm{O}(4)-\mathrm{Cu}(1)$ & $129.05(15)$ & $\mathrm{Si}(2)-\mathrm{O}(4)-\mathrm{Cu}(2)$ & $128.96(15)$ \\
$\mathrm{Cu}(1)-\mathrm{O}(4)-\mathrm{Cu}(2)$ & $98.90(11)$ & & \\
\hline
\end{tabular}

$\# 1-\mathrm{x}+1,-\mathrm{y}+1,-\mathrm{z}+1$ 


\subsection{Daten zur Kristallstrukturanalyse von \\ $\left[\mathrm{Cu}_{4}(\mathrm{O})\left(\mathrm{dppaO}_{2}\right)_{4} \mathrm{Cl}_{2}\right] \underline{\mathbf{9}}$}

\begin{tabular}{|ll|}
\hline Summenformel & $\mathrm{C}_{104} \mathrm{H}_{96} \mathrm{Cl}_{2} \mathrm{Cu}_{4} \mathrm{~N}_{4} \mathrm{O}_{11} \mathrm{P}_{8}$ \\
Molekulargewicht $\left[\mathrm{g} \cdot \mathrm{mol}^{-1}\right]$ & 2150.67 \\
Temperatur & $133(2) \mathrm{K}$ \\
Wellenlänge & $71.073 \mathrm{pm}$ \\
Kristallsystem & monoklin \\
Raumgruppe & $C 2 / c$ \\
Gitterkonstanten & $\mathrm{a}=36.208(3) \AA, \alpha=90^{\circ}$ \\
& $\mathrm{b}=12.2132(8) \AA, \beta=125.282(4)^{\circ}$ \\
& $\mathrm{c}=26.721(2) \AA, \gamma=90^{\circ}$ \\
Volumen & $9646.0(12) \AA^{3}$ \\
Formeleinheiten & 4 \\
Röntgenographische Dichte $\left[\mathrm{g} \cdot \mathrm{cm}^{-3}\right]$ & 1.481 \\
Absorptionskoeffizient $\left[\mathrm{mm}^{-1}\right]$ & 1.122 \\
$\mathrm{~F}(000)$ & 4424 \\
Meßbereich $\theta$ & $2.21-24.00^{\circ}$ \\
Indexbereiche & $-41 \leq \mathrm{h} \leq 41$ \\
& $-13 \leq \mathrm{k} \leq 10$ \\
& $-30 \leq 1 \leq 30$ \\
Gemessene Reflexe & 55248 \\
Unabhängige Reflexe & 7564 \\
Beobachtete Reflexe $[\mathrm{I}>2 \sigma]$ & 6366 \\
$\mathrm{R}_{\text {int }}$ & 0.0453 \\
Parameter & 599 \\
R1; wR2[I $>2 \sigma(\mathrm{I})]$ & $0.0314 ; 0.0821$ \\
\hline
\end{tabular}


Tabelle 36: Atomkoordinaten und äquivalente isotrope Auslenkungsparameter $\left[\AA^{2}\right]$ für $\underline{9}$

\begin{tabular}{|c|c|c|c|c|}
\hline Atom & $\mathrm{x}$ & $\mathrm{y}$ & $\mathrm{z}$ & $\mathrm{U}_{e q}$ \\
\hline $\mathrm{Cu}(1)$ & $0.042485(11)$ & $0.58551(3)$ & $0.751287(15)$ & $0.01956(10)$ \\
\hline $\mathrm{Cu}(2)$ & $-0.029026(11)$ & $0.76333(3)$ & $0.679229(15)$ & $0.01859(10)$ \\
\hline $\mathrm{N}(1)$ & $0.03665(8)$ & $0.5026(2)$ & $0.62764(11)$ & $0.0246(5)$ \\
\hline $\mathrm{N}(2)$ & $-0.14310(8)$ & $0.8209(2)$ & $0.58156(11)$ & $0.0250(6)$ \\
\hline $\mathrm{P}(1)$ & $0.00980(2)$ & $0.61394(6)$ & $0.61036(3)$ & $0.01992(17)$ \\
\hline $\mathrm{P}(2)$ & $0.07658(3)$ & $0.45203(6)$ & $0.69055(3)$ & $0.02193(17)$ \\
\hline $\mathrm{P}(3)$ & $-0.13539(2)$ & $0.73332(6)$ & $0.63025(3)$ & $0.01872(16)$ \\
\hline $\mathrm{P}(4)$ & $-0.10964(2)$ & $0.88278(6)$ & $0.57138(3)$ & $0.01989(17)$ \\
\hline $\mathrm{Cl}(1)$ & 0.0000 & $0.43827(8)$ & 0.7500 & $0.0341(3)$ \\
\hline $\mathrm{Cl}(2)$ & 0.0000 & $0.91159(8)$ & 0.7500 & $0.0335(3)$ \\
\hline $\mathrm{O}(1)$ & $0.01356(6)$ & $0.67362(16)$ & $0.66352(8)$ & $0.0210(4)$ \\
\hline $\mathrm{O}(2)$ & $0.08475(6)$ & $0.50114(16)$ & $0.74845(9)$ & $0.0242(4)$ \\
\hline $\mathrm{O}(3)$ & $-0.08741(6)$ & $0.68815(15)$ & $0.67197(8)$ & $0.0201(4)$ \\
\hline $\mathrm{O}(4)$ & $-0.06153(6)$ & $0.83999(16)$ & $0.60350(8)$ & $0.0211(4)$ \\
\hline $\mathrm{O}(5)$ & 0.0000 & $0.6752(2)$ & 0.7500 & $0.0164(6)$ \\
\hline $\mathrm{C}(1)$ & $0.02896(10)$ & $0.7035(2)$ & $0.57597(13)$ & $0.0241(7)$ \\
\hline $\mathrm{C}(2)$ & $0.04942(12)$ & $0.6589(3)$ & $0.54999(16)$ & $0.0372(8)$ \\
\hline $\mathrm{C}(3)$ & $0.06574(14)$ & $0.7260(3)$ & $0.52508(18)$ & $0.0473(10)$ \\
\hline $\mathrm{C}(4)$ & $0.06243(13)$ & $0.8380(3)$ & $0.52728(17)$ & $0.0432(9)$ \\
\hline $\mathrm{C}(5)$ & $0.04176(12)$ & $0.8830(3)$ & $0.55231(16)$ & $0.0377(8)$ \\
\hline $\mathrm{C}(6)$ & $0.02494(10)$ & $0.8164(3)$ & $0.57671(14)$ & $0.0286(7)$ \\
\hline $\mathrm{C}(7)$ & $-0.04884(10)$ & $0.5850(2)$ & $0.55300(13)$ & $0.0225(6)$ \\
\hline $\mathrm{C}(8)$ & $-0.07262(11)$ & $0.5260(3)$ & $0.57020(15)$ & $0.0351(8)$ \\
\hline $\mathrm{C}(9)$ & $-0.11808(11)$ & $0.5034(3)$ & $0.52780(16)$ & $0.0402(8)$ \\
\hline $\mathrm{C}(10)$ & $-0.13990(12)$ & $0.5382(3)$ & $0.46823(16)$ & $0.0403(9)$ \\
\hline $\mathrm{C}(11)$ & $-0.11661(12)$ & $0.5957(3)$ & $0.45052(16)$ & $0.0428(9)$ \\
\hline $\mathrm{C}(12)$ & $-0.07099(11)$ & $0.6198(3)$ & $0.49273(14)$ & $0.0336(8)$ \\
\hline $\mathrm{C}(13)$ & $0.06588(10)$ & $0.3075(2)$ & $0.68742(14)$ & $0.0275(7)$ \\
\hline $\mathrm{C}(14)$ & $0.08158(16)$ & $0.2501(3)$ & $0.74047(18)$ & $0.0559(12)$ \\
\hline $\mathrm{C}(15)$ & $0.07326(18)$ & $0.1389(3)$ & $0.7383(2)$ & $0.0695(14)$ \\
\hline $\mathrm{C}(16)$ & $0.05010(14)$ & $0.0847(3)$ & $0.68348(18)$ & $0.0483(10)$ \\
\hline $\mathrm{C}(17)$ & $0.03465(12)$ & $0.1411(3)$ & $0.63045(16)$ & $0.0380(8)$ \\
\hline $\mathrm{C}(18)$ & $0.04201(11)$ & $0.2527(3)$ & $0.63185(15)$ & $0.0322(7)$ \\
\hline $\mathrm{C}(19)$ & $0.13009(10)$ & $0.4662(3)$ & $0.70057(14)$ & $0.0277(7)$ \\
\hline $\mathrm{C}(20)$ & $0.15763(11)$ & $0.3782(3)$ & $0.71054(16)$ & $0.0404(9)$ \\
\hline $\mathrm{C}(21)$ & $0.19898(13)$ & $0.3960(4)$ & $0.71968(19)$ & $0.0545(11)$ \\
\hline $\mathrm{C}(22)$ & $0.21288(13)$ & $0.5002(4)$ & $0.71957(19)$ & $0.0567(12)$ \\
\hline $\mathrm{C}(23)$ & $0.18645(13)$ & $0.5875(4)$ & $0.7116(2)$ & $0.0554(11)$ \\
\hline
\end{tabular}


Tabelle 36: Fortsetzung

\begin{tabular}{|r|r|r|r|r|}
\hline $\mathrm{C}(24)$ & $0.14505(12)$ & $0.5710(3)$ & $0.70214(19)$ & $0.0463(10)$ \\
$\mathrm{C}(25)$ & $-0.17521(9)$ & $0.6239(2)$ & $0.58916(13)$ & $0.0231(6)$ \\
$\mathrm{C}(26)$ & $-0.17598(11)$ & $0.5363(3)$ & $0.62149(15)$ & $0.0347(8)$ \\
$\mathrm{C}(27)$ & $-0.20549(12)$ & $0.4506(3)$ & $0.59061(18)$ & $0.0447(9)$ \\
$\mathrm{C}(28)$ & $-0.23467(12)$ & $0.4528(3)$ & $0.52774(18)$ & $0.0475(10)$ \\
$\mathrm{C}(29)$ & $-0.23473(12)$ & $0.5399(3)$ & $0.49544(17)$ & $0.0477(10)$ \\
$\mathrm{C}(30)$ & $-0.20502(11)$ & $0.6261(3)$ & $0.52586(15)$ & $0.0358(8)$ \\
$\mathrm{C}(31)$ & $-0.15148(10)$ & $0.7905(2)$ & $0.67746(13)$ & $0.0221(6)$ \\
$\mathrm{C}(32)$ & $-0.11966(11)$ & $0.8243(3)$ & $0.73657(15)$ & $0.0356(8)$ \\
$\mathrm{C}(33)$ & $-0.13280(12)$ & $0.8708(3)$ & $0.77140(17)$ & $0.0448(9)$ \\
$\mathrm{C}(34)$ & $-0.17793(12)$ & $0.8841(3)$ & $0.74693(17)$ & $0.0424(9)$ \\
$\mathrm{C}(35)$ & $-0.20994(12)$ & $0.8493(3)$ & $0.68834(17)$ & $0.0440(9)$ \\
$\mathrm{C}(36)$ & $-0.19712(11)$ & $0.8028(3)$ & $0.65358(15)$ & $0.0360(8)$ \\
$\mathrm{C}(37)$ & $-0.13503(10)$ & $0.8822(2)$ & $0.48999(13)$ & $0.0221(6)$ \\
$\mathrm{C}(38)$ & $-0.10837(11)$ & $0.8835(3)$ & $0.46804(14)$ & $0.0299(7)$ \\
$\mathrm{C}(39)$ & $-0.12803(12)$ & $0.8847(3)$ & $0.40566(15)$ & $0.0393(8)$ \\
$\mathrm{C}(40)$ & $-0.17442(12)$ & $0.8838(3)$ & $0.36494(15)$ & $0.0392(8)$ \\
$\mathrm{C}(41)$ & $-0.20126(12)$ & $0.8830(3)$ & $0.38593(15)$ & $0.0414(9)$ \\
$\mathrm{C}(42)$ & $-0.18171(11)$ & $0.8823(3)$ & $0.44834(14)$ & $0.0332(8)$ \\
$\mathrm{C}(43)$ & $-0.10750(10)$ & $1.0252(2)$ & $0.59071(13)$ & $0.0242(7)$ \\
$\mathrm{C}(44)$ & $-0.14337(12)$ & $1.0745(3)$ & $0.58672(17)$ & $0.0433(9)$ \\
$\mathrm{C}(45)$ & $-0.14246(14)$ & $1.1855(3)$ & $0.59811(19)$ & $0.0547(11)$ \\
$\mathrm{C}(46)$ & $-0.10556(16)$ & $1.2469(3)$ & $0.61493(18)$ & $0.0509(11)$ \\
$\mathrm{C}(47)$ & $-0.06904(16)$ & $1.1985(3)$ & $0.6209(2)$ & $0.0577(12)$ \\
$\mathrm{C}(48)$ & $-0.07001(13)$ & $1.0884(3)$ & $0.60856(19)$ & $0.0456(9)$ \\
$\mathrm{C}(1 \mathrm{LA})$ & $0.2187(6)$ & $0.8180(14)$ & $0.6401(8)$ & $0.111(5)$ \\
$\mathrm{C}(1 \mathrm{LB})$ & $0.1942(7)$ & $0.7821(17)$ & $0.6221(9)$ & $0.140(6)$ \\
$\mathrm{C}(2 \mathrm{~L})$ & $0.1904(3)$ & $0.7886(10)$ & $0.5664(3)$ & $0.160(4)$ \\
$\mathrm{C}(3 \mathrm{LA})$ & $0.2437(6)$ & $0.7095(13)$ & $0.5802(8)$ & $0.111(4)$ \\
$\mathrm{C}(3 \mathrm{LB})$ & $0.2159(3)$ & $0.7160(7)$ & $0.5643(4)$ & $0.0466(19)$ \\
$\mathrm{C}(4 \mathrm{~L})$ & $0.2522(4)$ & $0.6762(7)$ & $0.6312(5)$ & $0.172(4)$ \\
$\mathrm{C}(5 \mathrm{~L})$ & $0.2411(3)$ & $0.7297(6)$ & $0.6634(3)$ & $0.111(2)$ \\
\hline & & & & \\
\hline
\end{tabular}

Tabelle 37: Bindungslängen [pm] in $\underline{9}$

\begin{tabular}{|l|r||l|r|}
\hline $\mathrm{Cu}(1)-\mathrm{O}(5)$ & $187.23(15)$ & $\mathrm{Cu}(1)-\mathrm{O}(2)$ & $188.23(19)$ \\
$\mathrm{Cu}(1)-\mathrm{O}(3) \# 1$ & $213.76(19)$ & $\mathrm{Cu}(1)-\mathrm{O}(1)$ & $221.76(19)$ \\
$\mathrm{Cu}(1)-\mathrm{Cl}(1)$ & $235.37(9)$ & $\mathrm{Cu}(1)-\mathrm{Cu}(1) \# 1$ & $303.73(7)$ \\
$\mathrm{Cu}(2)-\mathrm{O}(5)$ & $188.26(15)$ & $\mathrm{Cu}(2)-\mathrm{O}(4)$ & $189.88(18)$ \\
$\mathrm{Cu}(2)-\mathrm{O}(1)$ & $212.19(19)$ & $\mathrm{Cu}(2)-\mathrm{O}(3)$ & $220.81(18)$ \\
\hline
\end{tabular}


Tabelle 37: Fortsetzung

\begin{tabular}{|l|r||l|r|}
\hline $\mathrm{Cu}(2)-\mathrm{Cl}(2)$ & $237.98(9)$ & $\mathrm{N}(1)-\mathrm{P}(1)$ & $157.7(2)$ \\
$\mathrm{N}(1)-\mathrm{P}(2)$ & $157.8(3)$ & $\mathrm{N}(2)-\mathrm{P}(4)$ & $157.9(2)$ \\
$\mathrm{N}(2)-\mathrm{P}(3)$ & $157.9(2)$ & $\mathrm{P}(1)-\mathrm{O}(1)$ & $153.10(19)$ \\
$\mathrm{P}(2)-\mathrm{O}(2)$ & $152.1(2)$ & $\mathrm{P}(3)-\mathrm{O}(3)$ & $152.57(19)$ \\
$\mathrm{P}(4)-\mathrm{O}(4)$ & $152.2(2)$ & $\mathrm{Cl}(1)-\mathrm{Cu}(1) \# 1$ & $235.36(9)$ \\
$\mathrm{O}(5)-\mathrm{Cu}(1) \# 1$ & $187.22(15)$ & $\mathrm{O}(5)-\mathrm{Cu}(2) \# 1$ & $188.25(15)$ \\
\hline
\end{tabular}

Tabelle 38: Bindungswinkel $\left[^{\circ}\right]$ in $\underline{9}$

\begin{tabular}{|l|r||l|r|}
\hline $\mathrm{O}(5)-\mathrm{Cu}(1)-\mathrm{O}(2)$ & $176.23(8)$ & $\mathrm{O}(5)-\mathrm{Cu}(1)-\mathrm{O}(3) \# 1$ & $80.59(6)$ \\
$\mathrm{O}(2)-\mathrm{Cu}(1)-\mathrm{O}(3) \# 1$ & $100.01(8)$ & $\mathrm{O}(5)-\mathrm{Cu}(1)-\mathrm{O}(1)$ & $79.38(6)$ \\
$\mathrm{O}(2)-\mathrm{Cu}(1)-\mathrm{O}(1)$ & $96.97(8)$ & $\mathrm{O}(3) \# 1-\mathrm{Cu}(1)-\mathrm{O}(1)$ & $111.27(7)$ \\
$\mathrm{O}(5)-\mathrm{Cu}(1)-\mathrm{Cl}(1)$ & $85.61(7)$ & $\mathrm{O}(2)-\mathrm{Cu}(1)-\mathrm{Cl}(1)$ & $96.94(7)$ \\
$\mathrm{O}(3) \# 1-\mathrm{Cu}(1)-\mathrm{Cl}(1)$ & $126.22(5)$ & $\mathrm{O}(1)-\mathrm{Cu}(1)-\mathrm{Cl}(1)$ & $116.74(5)$ \\
$\mathrm{O}(5)-\mathrm{Cu}(1)-\mathrm{Cu}(1) \# 1$ & $35.79(6)$ & $\mathrm{O}(2)-\mathrm{Cu}(1)-\mathrm{Cu}(1) \# 1$ & $146.66(6)$ \\
$\mathrm{O}(3) \# 1-\mathrm{Cu}(1)-\mathrm{Cu}(1) \# 1$ & $102.79(5)$ & $\mathrm{O}(1)-\mathrm{Cu}(1)-\mathrm{Cu}(1) \# 1$ & $97.04(5)$ \\
$\mathrm{Cl}(1)-\mathrm{Cu}(1)-\mathrm{Cu}(1) \# 1$ & $49.816(18)$ & $\mathrm{O}(5)-\mathrm{Cu}(2)-\mathrm{O}(4)$ & $174.44(9)$ \\
$\mathrm{O}(5)-\mathrm{Cu}(2)-\mathrm{O}(1)$ & $81.69(6)$ & $\mathrm{O}(4)-\mathrm{Cu}(2)-\mathrm{O}(1)$ & $96.76(8)$ \\
$\mathrm{O}(5)-\mathrm{Cu}(2)-\mathrm{O}(3)$ & $78.53(6)$ & $\mathrm{O}(4)-\mathrm{Cu}(2)-\mathrm{O}(3)$ & $97.97(8)$ \\
$\mathrm{O}(1)-\mathrm{Cu}(2)-\mathrm{O}(3)$ & $122.42(7)$ & $\mathrm{O}(5)-\mathrm{Cu}(2)-\mathrm{Cl}(2)$ & $84.43(7)$ \\
$\mathrm{O}(4)-\mathrm{Cu}(2)-\mathrm{Cl}(2)$ & $100.90(6)$ & $\mathrm{O}(1)-\mathrm{Cu}(2)-\mathrm{Cl}(2)$ & $120.81(5)$ \\
$\mathrm{O}(3)-\mathrm{Cu}(2)-\mathrm{Cl}(2)$ & $110.32(5)$ & $\mathrm{P}(1)-\mathrm{N}(1)-\mathrm{P}(2)$ & $132.84(16)$ \\
$\mathrm{P}(4)-\mathrm{N}(2)-\mathrm{P}(3)$ & $132.66(16)$ & $\mathrm{O}(1)-\mathrm{P}(1)-\mathrm{N}(1)$ & $115.42(12)$ \\
$\mathrm{O}(1)-\mathrm{P}(1)-\mathrm{C}(7)$ & $108.27(12)$ & $\mathrm{O}(1)-\mathrm{P}(1)-\mathrm{C}(1)$ & $109.23(13)$ \\
$\mathrm{O}(2)-\mathrm{P}(2)-\mathrm{N}(1)$ & $116.71(12)$ & $\mathrm{O}(2)-\mathrm{P}(2)-\mathrm{C}(13)$ & $110.15(13)$ \\
$\mathrm{O}(2)-\mathrm{P}(2)-\mathrm{C}(19)$ & $104.47(13)$ & $\mathrm{O}(3)-\mathrm{P}(3)-\mathrm{N}(2)$ & $115.58(12)$ \\
$\mathrm{O}(3)-\mathrm{P}(3)-\mathrm{C}(25)$ & $110.49(12)$ & $\mathrm{O}(3)-\mathrm{P}(3)-\mathrm{C}(31)$ & $108.65(12)$ \\
$\mathrm{O}(4)-\mathrm{P}(4)-\mathrm{N}(2)$ & $118.72(12)$ & $\mathrm{O}(4)-\mathrm{P}(4)-\mathrm{C}(43)$ & $108.89(13)$ \\
$\mathrm{O}(4)-\mathrm{P}(4)-\mathrm{C}(37)$ & $107.35(12)$ & $\mathrm{Cu}(1) \# 1-\mathrm{Cl}(1)-\mathrm{Cu}(1)$ & $80.36(4)$ \\
$\mathrm{Cu}(2)-\mathrm{Cl}(2)-\mathrm{Cu}(2) \# 1$ & $80.92(4)$ & $\mathrm{P}(1)-\mathrm{O}(1)-\mathrm{Cu}(2)$ & $138.07(12)$ \\
$\mathrm{P}(1)-\mathrm{O}(1)-\mathrm{Cu}(1)$ & $119.00(11)$ & $\mathrm{Cu}(2)-\mathrm{O}(1)-\mathrm{Cu}(1)$ & $89.69(7)$ \\
$\mathrm{P}(2)-\mathrm{O}(2)-\mathrm{Cu}(1)$ & $124.89(12)$ & $\mathrm{P}(3)-\mathrm{O}(3)-\mathrm{Cu}(1) \# 1$ & $145.83(11)$ \\
$\mathrm{P}(3)-\mathrm{O}(3)-\mathrm{Cu}(2)$ & $122.82(11)$ & $\mathrm{Cu}(1) \# 1-\mathrm{O}(3)-\mathrm{Cu}(2)$ & $90.01(7)$ \\
$\mathrm{P}(4)-\mathrm{O}(4)-\mathrm{Cu}(2)$ & $124.98(11)$ & $\mathrm{Cu}(1) \# 1-\mathrm{O}(5)-\mathrm{Cu}(1)$ & $108.41(13)$ \\
$\mathrm{Cu}(1) \# 1-\mathrm{O}(5)-\mathrm{Cu}(2) \# 1$ & $109.208(15)$ & $\mathrm{Cu}(1)-\mathrm{O}(5)-\mathrm{Cu}(2) \# 1$ & $109.884(16)$ \\
$\mathrm{Cu}(1) \# 1-\mathrm{O}(5)-\mathrm{Cu}(2)$ & $109.877(15)$ & $\mathrm{Cu}(1)-\mathrm{O}(5)-\mathrm{Cu}(2)$ & $109.209(15)$ \\
$\mathrm{Cu}(2) \# 1-\mathrm{O}(5)-\mathrm{Cu}(2)$ & $110.22(13)$ & & \\
& & & \\
\hline
\end{tabular}

$\# 1-\mathrm{x}, \mathrm{y},-\mathrm{z}+3 / 2$ 


\subsection{Daten zur Kristallstrukturanalyse von $\left[\mathrm{Cu}_{3}(\mathrm{dppaOS})_{3}\right] \underline{10}$}

\begin{tabular}{|ll|}
\hline Summenformel & $\mathrm{C}_{78} \mathrm{H}_{72} \mathrm{Cu}_{3} \mathrm{~N}_{3} \mathrm{O}_{4.5} \mathrm{P}_{6} \mathrm{~S}_{3}$ \\
Molekulargewicht $\left[\mathrm{g} \cdot \mathrm{mol}^{-1}\right]$ & 1596.01 \\
Temperatur & $203(2) \mathrm{K}$ \\
Wellenlänge & $71.073 \mathrm{pm}$ \\
Kristallsystem & monoklin \\
Raumgruppe & $C 2 / c$ \\
Gitterkonstanten & $\mathrm{a}=44.1400(18) \AA, \alpha=90^{\circ}$ \\
& $\mathrm{b}=15.1030(10) \AA, \beta=126.972(10)^{\circ} \circ$ \\
& $\mathrm{c}=27.1010(14) \AA, \gamma=90^{\circ}{ }^{\circ}, \AA^{3}$ \\
Volumen & $14434.1(13) \AA^{3}$ \\
Formeleinheiten & 8 \\
Röntgenographische Dichte $\left[\mathrm{g} \cdot \mathrm{cm}^{-3}\right]$ & 1.469 \\
Absorptionskoeffizient $\left[\mathrm{mm}^{-1}\right]$ & 1.150 \\
$\mathrm{~F}(000)$ & 6576 \\
Meßbereich $\theta$ & $2.09-25.00^{\circ}$ \\
Indexbereiche & $-38 \leq \mathrm{h} \leq 52$ \\
& $-17 \leq \mathrm{k} \leq 16$ \\
& $-31 \leq \mathrm{l} \leq 14$ \\
Gemessene Reflexe & 18849 \\
Unabhängige Reflexe & 10249 \\
Beobachtete Reflexe $[\mathrm{I}>2 \sigma]$ & 8341 \\
$\mathrm{R}_{\text {int }}$ & 0.0398 \\
Parameter & 874 \\
R1; wR2[I $>2 \sigma(\mathrm{I})]$ & $0.0450 ; 0.1126$ \\
\hline
\end{tabular}


Tabelle 40: Atomkoordinaten und äquivalente isotrope Auslenkungsparameter $\left[\AA^{2}\right]$ für $\underline{10}$

\begin{tabular}{|c|c|c|c|c|}
\hline Atom & $\mathrm{x}$ & $\mathrm{y}$ & z & $\mathrm{U}_{e q}$ \\
\hline $\mathrm{Cu}(1)$ & $0.122994(12)$ & $0.86281(3)$ & $0.07234(2)$ & $0.02043(13)$ \\
\hline $\mathrm{Cu}(2)$ & $0.155239(11)$ & $0.69815(3)$ & $0.09072(2)$ & $0.01951(13)$ \\
\hline $\mathrm{Cu}(3)$ & $0.102198(12)$ & $0.72879(3)$ & $0.10766(2)$ & $0.02038(13)$ \\
\hline $\mathrm{P}(1)$ & $0.07731(2)$ & $0.92302(5)$ & $0.13269(4)$ & $0.0151(2)$ \\
\hline $\mathrm{P}(2)$ & $0.12699(2)$ & $0.80089(5)$ & $0.23459(4)$ & $0.0161(2)$ \\
\hline $\mathrm{P}(3)$ & $0.21290(2)$ & $0.86656(5)$ & $0.11414(4)$ & $0.0156(2)$ \\
\hline $\mathrm{P}(4)$ & $0.14931(2)$ & $0.93697(5)$ & $-0.00550(4)$ & $0.0161(2)$ \\
\hline $\mathrm{P}(5)$ & $0.08695(2)$ & $0.56303(5)$ & $-0.00674(4)$ & $0.0157(2)$ \\
\hline $\mathrm{P}(6)$ & $0.15819(2)$ & $0.56469(5)$ & $0.01394(4)$ & $0.0159(2)$ \\
\hline $\mathrm{S}(1)$ & $0.06712(2)$ & $0.85055(5)$ & $0.05927(4)$ & $0.0183(2)$ \\
\hline $\mathrm{S}(2)$ & $0.18383(2)$ & $0.82056(5)$ & $0.14690(4)$ & $0.0178(2)$ \\
\hline $\mathrm{S}(3)$ & $0.11423(2)$ & $0.59647(5)$ & $0.08498(4)$ & $0.0178(2)$ \\
\hline $\mathrm{O}(1)$ & $0.12992(7)$ & $0.72588(16)$ & $0.20037(12)$ & $0.0246(7)$ \\
\hline $\mathrm{O}(2)$ & $0.11941(6)$ & $0.92859(16)$ & $0.00480(12)$ & $0.0216(6)$ \\
\hline $\mathrm{O}(3)$ & $0.17039(7)$ & $0.65949(15)$ & $0.03574(12)$ & $0.0210(6)$ \\
\hline $\mathrm{N}(1)$ & $0.11132(8)$ & $0.89465(18)$ & $0.20043(13)$ & $0.0182(7)$ \\
\hline $\mathrm{N}(2)$ & $0.18941(8)$ & $0.89102(19)$ & $0.04332(14)$ & $0.0192(7)$ \\
\hline $\mathrm{N}(3)$ & $0.11393(8)$ & $0.54201(19)$ & $-0.02548(14)$ & $0.0201(7)$ \\
\hline $\mathrm{C}(1)$ & $0.08724(10)$ & $1.0349(2)$ & $0.12245(16)$ & $0.0203(9)$ \\
\hline $\mathrm{C}(2)$ & $0.12210(11)$ & $1.0531(2)$ & $0.1356(2)$ & $0.0281(10)$ \\
\hline $\mathrm{C}(3)$ & $0.12973(12)$ & $1.1370(3)$ & $0.1240(2)$ & $0.0340(11)$ \\
\hline $\mathrm{C}(4)$ & $0.10318(14)$ & $1.2038(3)$ & $0.1009(2)$ & $0.0394(13)$ \\
\hline $\mathrm{C}(5)$ & $0.06864(13)$ & $1.1869(3)$ & $0.0890(2)$ & $0.0386(12)$ \\
\hline $\mathrm{C}(6)$ & $0.06061(11)$ & $1.1032(2)$ & $0.10000(19)$ & $0.0263(10)$ \\
\hline $\mathrm{C}(7)$ & $0.03251(9)$ & $0.9285(2)$ & $0.12205(17)$ & $0.0185(8)$ \\
\hline $\mathrm{C}(8)$ & $0.03295(11)$ & $0.9294(3)$ & $0.1733(2)$ & $0.0307(10)$ \\
\hline $\mathrm{C}(9)$ & $-0.00059(13)$ & $0.9386(3)$ & $0.1668(2)$ & $0.0442(13)$ \\
\hline $\mathrm{C}(10)$ & $-0.03491(12)$ & $0.9481(3)$ & $0.1075(2)$ & $0.0394(13)$ \\
\hline $\mathrm{C}(11)$ & $-0.03511(11)$ & $0.9480(3)$ & $0.0563(2)$ & $0.0333(11)$ \\
\hline $\mathrm{C}(12)$ & $-0.00175(10)$ & $0.9374(3)$ & $0.06303(19)$ & $0.0264(10)$ \\
\hline $\mathrm{C}(13)$ & $0.09855(10)$ & $0.7632(2)$ & $0.25803(17)$ & $0.0231(9)$ \\
\hline $\mathrm{C}(14)$ & $0.07004(11)$ & $0.7010(3)$ & $0.2220(2)$ & $0.0356(11)$ \\
\hline $\mathrm{C}(15)$ & $0.04700(14)$ & $0.6738(4)$ & $0.2385(3)$ & $0.0504(15)$ \\
\hline $\mathrm{C}(16)$ & $0.05158(14)$ & $0.7069(4)$ & $0.2890(3)$ & $0.0566(18)$ \\
\hline $\mathrm{C}(17)$ & $0.07942(13)$ & $0.7710(4)$ & $0.3248(2)$ & $0.0544(16)$ \\
\hline $\mathrm{C}(18)$ & $0.10277(12)$ & $0.7973(3)$ & $0.3093(2)$ & $0.0382(12)$ \\
\hline $\mathrm{C}(19)$ & $0.17256(9)$ & $0.8255(2)$ & $0.30587(17)$ & $0.0186(8)$ \\
\hline $\mathrm{C}(20)$ & $0.19876(10)$ & $0.7570(3)$ & $0.33761(19)$ & $0.0256(9)$ \\
\hline
\end{tabular}


Tabelle 40: Fortsetzung

\begin{tabular}{|c|c|c|c|c|}
\hline $\mathrm{C}(21)$ & $0.23337(11)$ & $0.7738(3)$ & $0.3937(2)$ & $0.0346(11)$ \\
\hline $\mathrm{C}(22)$ & $0.24197(10)$ & $0.8570(3)$ & $0.4194(2)$ & $0.0341(11)$ \\
\hline $\mathrm{C}(23)$ & $0.21642(11)$ & $0.9257(3)$ & $0.3888(2)$ & $0.0316(11)$ \\
\hline $\mathrm{C}(24)$ & $0.18171(10)$ & $0.9104(3)$ & $0.33174(19)$ & $0.0253(9)$ \\
\hline $\mathrm{C}(25)$ & $0.24816(9)$ & $0.7853(2)$ & $0.13121(18)$ & $0.0205(9)$ \\
\hline $\mathrm{C}(26)$ & $0.26947(11)$ & $0.7410(3)$ & $0.1879(2)$ & $0.0331(11)$ \\
\hline $\mathrm{C}(27)$ & $0.29870(11)$ & $0.6847(3)$ & $0.2020(2)$ & $0.0407(14)$ \\
\hline $\mathrm{C}(28)$ & $0.30609(11)$ & $0.6708(3)$ & $0.1603(3)$ & $0.0406(14)$ \\
\hline $\mathrm{C}(29)$ & $0.28435(12)$ & $0.7125(3)$ & $0.1026(3)$ & $0.0398(12)$ \\
\hline $\mathrm{C}(30)$ & $0.25526(11)$ & $0.7696(3)$ & $0.0890(2)$ & $0.0304(11)$ \\
\hline $\mathrm{C}(31)$ & $0.23923(9)$ & $0.9618(2)$ & $0.16278(16)$ & $0.0180(8)$ \\
\hline $\mathrm{C}(32)$ & $0.27841(10)$ & $0.9627(3)$ & $0.2082(2)$ & $0.0320(11)$ \\
\hline $\mathrm{C}(33)$ & $0.29619(11)$ & $1.0388(3)$ & $0.2426(2)$ & $0.0389(13)$ \\
\hline $\mathrm{C}(34)$ & $0.27615(12)$ & $1.1132(3)$ & $0.2342(2)$ & $0.0345(12)$ \\
\hline $\mathrm{C}(35)$ & $0.23720(13)$ & $1.1134(3)$ & $0.1897(2)$ & $0.0414(14)$ \\
\hline $\mathrm{C}(36)$ & $0.21896(12)$ & $1.0379(2)$ & $0.1544(2)$ & $0.0374(13)$ \\
\hline $\mathrm{C}(37)$ & $0.13175(9)$ & $0.8899(2)$ & $-0.07954(18)$ & $0.0211(9)$ \\
\hline $\mathrm{C}(38)$ & $0.13765(11)$ & $0.7999(2)$ & $-0.08291(19)$ & $0.0263(10)$ \\
\hline $\mathrm{C}(39)$ & $0.12033(13)$ & $0.7591(3)$ & $-0.1404(2)$ & $0.0358(11)$ \\
\hline $\mathrm{C}(40)$ & $0.09743(14)$ & $0.8071(3)$ & $-0.1940(2)$ & $0.0467(15)$ \\
\hline $\mathrm{C}(41)$ & $0.09167(14)$ & $0.8969(3)$ & $-0.1910(2)$ & $0.0470(15)$ \\
\hline$C(42)$ & $0.10879(12)$ & $0.9385(3)$ & $-0.13443(19)$ & $0.0338(11)$ \\
\hline $\mathrm{C}(43)$ & $0.15516(9)$ & $1.0540(2)$ & $-0.01139(16)$ & $0.0178(8)$ \\
\hline $\mathrm{C}(44)$ & $0.13542(10)$ & $1.1145(2)$ & $-0.00085(18)$ & $0.0236(9)$ \\
\hline$C(45)$ & $0.13977(11)$ & $1.2052(2)$ & $-0.0041(2)$ & $0.0320(11)$ \\
\hline $\mathrm{C}(46)$ & $0.16305(12)$ & $1.2357(3)$ & $-0.0180(2)$ & $0.0342(12)$ \\
\hline $\mathrm{C}(47)$ & $0.18270(11)$ & $1.1771(3)$ & $-0.0285(2)$ & $0.0314(10)$ \\
\hline $\mathrm{C}(48)$ & $0.17895(10)$ & $1.0865(2)$ & $-0.02474(19)$ & $0.0253(9)$ \\
\hline $\mathrm{C}(49)$ & $0.05888(9)$ & $0.4645(2)$ & $-0.02256(17)$ & $0.0178(8)$ \\
\hline $\mathrm{C}(50)$ & $0.05743(11)$ & $0.3998(2)$ & $-0.0613(2)$ & $0.0294(10)$ \\
\hline $\mathrm{C}(51)$ & $0.03521(13)$ & $0.3240(3)$ & $-0.0755(2)$ & $0.0398(13)$ \\
\hline $\mathrm{C}(52)$ & $0.01436(11)$ & $0.3137(3)$ & $-0.0535(2)$ & $0.0353(12)$ \\
\hline $\mathrm{C}(53)$ & $0.01596(12)$ & $0.3777(3)$ & $-0.0154(2)$ & $0.0339(11)$ \\
\hline $\mathrm{C}(54)$ & $0.03869(11)$ & $0.4524(3)$ & $0.0008(2)$ & $0.0290(10)$ \\
\hline $\mathrm{C}(55)$ & $0.05438(10)$ & $0.6510(2)$ & $-0.05482(17)$ & $0.0200(8)$ \\
\hline $\mathrm{C}(56)$ & $0.01510(10)$ & $0.6426(3)$ & $-0.08916(19)$ & $0.0261(10)$ \\
\hline $\mathrm{C}(57)$ & $-0.00815(11)$ & $0.7133(3)$ & $-0.1253(2)$ & $0.0363(12)$ \\
\hline $\mathrm{C}(58)$ & $0.00712(12)$ & $0.7922(3)$ & $-0.1266(2)$ & $0.0334(11)$ \\
\hline $\mathrm{C}(59)$ & $0.04630(11)$ & $0.8006(2)$ & $-0.0932(2)$ & $0.0317(11)$ \\
\hline $\mathrm{C}(60)$ & $0.06949(10)$ & $0.7305(2)$ & $-0.05789(19)$ & $0.0242(9)$ \\
\hline
\end{tabular}


Tabelle 40: Fortsetzung

\begin{tabular}{|r|r|r|r|r|}
\hline $\mathrm{C}(61)$ & $0.17208(10)$ & $0.5309(2)$ & $-0.03373(18)$ & $0.0218(9)$ \\
$\mathrm{C}(62)$ & $0.20554(11)$ & $0.5622(2)$ & $-0.0213(2)$ & $0.0272(10)$ \\
$\mathrm{C}(63)$ & $0.21889(13)$ & $0.5303(3)$ & $-0.0523(2)$ & $0.0364(11)$ \\
$\mathrm{C}(64)$ & $0.19904(15)$ & $0.4648(3)$ & $-0.0971(3)$ & $0.0446(13)$ \\
$\mathrm{C}(65)$ & $0.16523(15)$ & $0.4344(3)$ & $-0.1106(2)$ & $0.0434(13)$ \\
$\mathrm{C}(66)$ & $0.15161(12)$ & $0.4664(3)$ & $-0.0798(2)$ & $0.0310(10)$ \\
$\mathrm{C}(67)$ & $0.18529(9)$ & $0.4926(2)$ & $0.08071(17)$ & $0.0196(8)$ \\
$\mathrm{C}(68)$ & $0.17176(11)$ & $0.4108(2)$ & $0.0824(2)$ & $0.0302(11)$ \\
$\mathrm{C}(69)$ & $0.19407(13)$ & $0.3551(3)$ & $0.1327(2)$ & $0.0425(14)$ \\
$\mathrm{C}(70)$ & $0.22989(13)$ & $0.3809(3)$ & $0.1821(2)$ & $0.0369(12)$ \\
$\mathrm{C}(71)$ & $0.24344(12)$ & $0.4631(3)$ & $0.1821(2)$ & $0.0343(11)$ \\
$\mathrm{C}(72)$ & $0.22149(10)$ & $0.5192(3)$ & $0.13203(19)$ & $0.0261(9)$ \\
$\mathrm{C}(1 \mathrm{LA})$ & $0.0834(3)$ & $0.4648(6)$ & $0.2253(5)$ & $0.062(2)$ \\
$\mathrm{C}(2 \mathrm{LA})$ & $0.0916(3)$ & $0.4930(6)$ & $0.1827(5)$ & $0.065(3)$ \\
$\mathrm{C}(3 \mathrm{LA})$ & $0.1077(3)$ & $0.4119(5)$ & $0.1744(4)$ & $0.057(2)$ \\
$\mathrm{C}(4 \mathrm{LA})$ & $0.1215(3)$ & $0.3570(6)$ & $0.2311(6)$ & $0.059(3)$ \\
$\mathrm{O}(1 \mathrm{LA})$ & $0.09459(17)$ & $0.3761(4)$ & $0.2437(3)$ & $0.0612(15)$ \\
$\mathrm{C}(1 \mathrm{LB})$ & $0.1100(6)$ & $0.4959(12)$ & $0.2379(9)$ & $0.077(6)$ \\
$\mathrm{C}(2 \mathrm{LB})$ & $0.0748(6)$ & $0.4852(14)$ & $0.1759(10)$ & $0.082(7)$ \\
$\mathrm{C}(3 \mathrm{LB})$ & $0.0819(5)$ & $0.4039(11)$ & $0.1559(8)$ & $0.062(5)$ \\
$\mathrm{C}(4 \mathrm{LB})$ & $0.1199(5)$ & $0.3686(14)$ & $0.2072(11)$ & $0.068(6)$ \\
$\mathrm{O}(1 \mathrm{LB})$ & $0.1291(5)$ & $0.4117(12)$ & $0.2627(8)$ & $0.110(6)$ \\
$\mathrm{O}(1 \mathrm{~L})$ & 0.0000 & $0.5139(4)$ & 0.2500 & $0.074(2)$ \\
$\mathrm{C}(6 \mathrm{~L})$ & $0.02929(18)$ & $0.4616(4)$ & $0.2961(3)$ & $0.0624(18)$ \\
$\mathrm{C}(7 \mathrm{~L})$ & $0.02129(17)$ & $0.3681(4)$ & $0.2723(3)$ & $0.0573(16)$ \\
\hline & & & &
\end{tabular}

Tabelle 41: Bindungslängen [pm] in 10

\begin{tabular}{|l|r||l|r|}
\hline $\mathrm{Cu}(1)-\mathrm{O}(2)$ & $200.4(3)$ & $\mathrm{Cu}(1)-\mathrm{S}(2)$ & $227.48(9)$ \\
$\mathrm{Cu}(1)-\mathrm{S}(1)$ & $227.84(11)$ & $\mathrm{Cu}(1)-\mathrm{Cu}(3)$ & $262.93(7)$ \\
$\mathrm{Cu}(1)-\mathrm{Cu}(2)$ & $275.79(6)$ & $\mathrm{Cu}(2)-\mathrm{O}(3)$ & $205.1(3)$ \\
$\mathrm{Cu}(2)-\mathrm{S}(2)$ & $224.00(9)$ & $\mathrm{Cu}(2)-\mathrm{S}(3)$ & $230.64(10)$ \\
$\mathrm{Cu}(2)-\mathrm{Cu}(3)$ & $268.32(7)$ & $\mathrm{Cu}(3)-\mathrm{O}(1)$ & $202.8(3)$ \\
$\mathrm{Cu}(3)-\mathrm{S}(3)$ & $224.66(10)$ & $\mathrm{Cu}(3)-\mathrm{S}(1)$ & $224.86(9)$ \\
$\mathrm{P}(1)-\mathrm{N}(1)$ & $157.9(3)$ & $\mathrm{P}(1)-\mathrm{C}(1)$ & $180.9(4)$ \\
$\mathrm{P}(1)-\mathrm{C}(7)$ & $182.1(4)$ & $\mathrm{P}(1)-\mathrm{S}(1)$ & $206.94(14)$ \\
$\mathrm{P}(2)-\mathrm{O}(1)$ & $151.8(3)$ & $\mathrm{P}(2)-\mathrm{N}(1)$ & $160.3(3)$ \\
$\mathrm{P}(2)-\mathrm{C}(19)$ & $180.1(3)$ & $\mathrm{P}(2)-\mathrm{C}(13)$ & $180.6(5)$ \\
$\mathrm{P}(3)-\mathrm{N}(2)$ & $158.2(3)$ & $\mathrm{P}(3)-\mathrm{C}(25)$ & $181.1(4)$ \\
$\mathrm{P}(3)-\mathrm{C}(31)$ & $182.0(3)$ & $\mathrm{P}(3)-\mathrm{S}(2)$ & $207.05(15)$ \\
\hline
\end{tabular}


Tabelle 41: Fortsetzung

\begin{tabular}{|l|r||l|r|}
\hline $\mathrm{P}(4)-\mathrm{O}(2)$ & $151.0(3)$ & $\mathrm{P}(4)-\mathrm{N}(2)$ & $159.6(3)$ \\
$\mathrm{P}(4)-\mathrm{C}(37)$ & $180.6(4)$ & $\mathrm{P}(4)-\mathrm{C}(43)$ & $180.7(3)$ \\
$\mathrm{P}(5)-\mathrm{N}(3)$ & $158.3(4)$ & $\mathrm{P}(5)-\mathrm{C}(55)$ & $181.1(3)$ \\
$\mathrm{P}(5)-\mathrm{C}(49)$ & $181.5(3)$ & $\mathrm{P}(5)-\mathrm{S}(3)$ & $206.97(14)$ \\
$\mathrm{P}(6)-\mathrm{O}(3)$ & $151.9(2)$ & $\mathrm{P}(6)-\mathrm{N}(3)$ & $160.1(3)$ \\
$\mathrm{P}(6)-\mathrm{C}(61)$ & $180.5(5)$ & $\mathrm{P}(6)-\mathrm{C}(67)$ & $181.3(4)$ \\
\hline
\end{tabular}

Tabelle 42: Bindungswinkel $\left[^{\circ}\right]$ in $\underline{10}$

\begin{tabular}{|l|r||l|r|}
\hline $\mathrm{O}(2)-\mathrm{Cu}(1)-\mathrm{S}(2)$ & $110.67(8)$ & $\mathrm{O}(2)-\mathrm{Cu}(1)-\mathrm{S}(1)$ & $114.52(7)$ \\
$\mathrm{S}(2)-\mathrm{Cu}(1)-\mathrm{S}(1)$ & $134.78(4)$ & $\mathrm{O}(2)-\mathrm{Cu}(1)-\mathrm{Cu}(3)$ & $149.47(7)$ \\
$\mathrm{S}(2)-\mathrm{Cu}(1)-\mathrm{Cu}(3)$ & $86.83(3)$ & $\mathrm{S}(1)-\mathrm{Cu}(1)-\mathrm{Cu}(3)$ & $53.97(3)$ \\
$\mathrm{O}(2)-\mathrm{Cu}(1)-\mathrm{Cu}(2)$ & $111.15(8)$ & $\mathrm{S}(2)-\mathrm{Cu}(1)-\mathrm{Cu}(2)$ & $51.78(2)$ \\
$\mathrm{S}(1)-\mathrm{Cu}(1)-\mathrm{Cu}(2)$ & $110.34(3)$ & $\mathrm{Cu}(3)-\mathrm{Cu}(1)-\mathrm{Cu}(2)$ & $59.689(18)$ \\
$\mathrm{O}(3)-\mathrm{Cu}(2)-\mathrm{S}(2)$ & $115.60(7)$ & $\mathrm{O}(3)-\mathrm{Cu}(2)-\mathrm{S}(3)$ & $110.30(7)$ \\
$\mathrm{S}(2)-\mathrm{Cu}(2)-\mathrm{S}(3)$ & $133.94(4)$ & $\mathrm{O}(3)-\mathrm{Cu}(2)-\mathrm{Cu}(3)$ & $150.77(7)$ \\
$\mathrm{S}(2)-\mathrm{Cu}(2)-\mathrm{Cu}(3)$ & $86.23(3)$ & $\mathrm{S}(3)-\mathrm{Cu}(2)-\mathrm{Cu}(3)$ & $52.86(3)$ \\
$\mathrm{O}(3)-\mathrm{Cu}(2)-\mathrm{Cu}(1)$ & $118.93(7)$ & $\mathrm{S}(2)-\mathrm{Cu}(2)-\mathrm{Cu}(1)$ & $52.92(3)$ \\
$\mathrm{S}(3)-\mathrm{Cu}(2)-\mathrm{Cu}(1)$ & $106.82(3)$ & $\mathrm{Cu}(3)-\mathrm{Cu}(2)-\mathrm{Cu}(1)$ & $57.772(17)$ \\
$\mathrm{O}(1)-\mathrm{Cu}(3)-\mathrm{S}(3)$ & $104.70(7)$ & $\mathrm{O}(1)-\mathrm{Cu}(3)-\mathrm{S}(1)$ & $115.54(8)$ \\
$\mathrm{S}(3)-\mathrm{Cu}(3)-\mathrm{S}(1)$ & $139.56(4)$ & $\mathrm{O}(1)-\mathrm{Cu}(3)-\mathrm{Cu}(1)$ & $112.65(8)$ \\
$\mathrm{S}(3)-\mathrm{Cu}(3)-\mathrm{Cu}(1)$ & $113.16(4)$ & $\mathrm{S}(1)-\mathrm{Cu}(3)-\mathrm{Cu}(1)$ & $55.03(3)$ \\
$\mathrm{O}(1)-\mathrm{Cu}(3)-\mathrm{Cu}(2)$ & $105.65(9)$ & $\mathrm{S}(3)-\mathrm{Cu}(3)-\mathrm{Cu}(2)$ & $54.93(3)$ \\
$\mathrm{S}(1)-\mathrm{Cu}(3)-\mathrm{Cu}(2)$ & $114.01(4)$ & $\mathrm{Cu}(1)-\mathrm{Cu}(3)-\mathrm{Cu}(2)$ & $62.539(18)$ \\
$\mathrm{N}(1)-\mathrm{P}(1)-\mathrm{S}(1)$ & $118.64(13)$ & $\mathrm{O}(1)-\mathrm{P}(2)-\mathrm{N}(1)$ & $118.16(17)$ \\
$\mathrm{N}(2)-\mathrm{P}(3)-\mathrm{S}(2)$ & $118.40(13)$ & $\mathrm{O}(2)-\mathrm{P}(4)-\mathrm{N}(2)$ & $117.85(17)$ \\
$\mathrm{N}(3)-\mathrm{P}(5)-\mathrm{S}(3)$ & $115.32(11)$ & $\mathrm{O}(3)-\mathrm{P}(6)-\mathrm{N}(3)$ & $117.77(15)$ \\
$\mathrm{O}(3)-\mathrm{P}(6)-\mathrm{C}(61)$ & $110.60(18)$ & $\mathrm{O}(3)-\mathrm{P}(6)-\mathrm{C}(67)$ & $108.11(15)$ \\
$\mathrm{P}(1)-\mathrm{S}(1)-\mathrm{Cu}(3)$ & $101.33(5)$ & $\mathrm{P}(1)-\mathrm{S}(1)-\mathrm{Cu}(1)$ & $102.62(4)$ \\
$\mathrm{Cu}(3)-\mathrm{S}(1)-\mathrm{Cu}(1)$ & $71.01(3)$ & $\mathrm{P}(3)-\mathrm{S}(2)-\mathrm{Cu}(2)$ & $103.10(5)$ \\
$\mathrm{P}(3)-\mathrm{S}(2)-\mathrm{Cu}(1)$ & $103.29(4)$ & $\mathrm{Cu}(2)-\mathrm{S}(2)-\mathrm{Cu}(1)$ & $75.30(3)$ \\
$\mathrm{P}(5)-\mathrm{S}(3)-\mathrm{Cu}(3)$ & $118.89(4)$ & $\mathrm{P}(5)-\mathrm{S}(3)-\mathrm{Cu}(2)$ & $96.30(5)$ \\
$\mathrm{Cu}(3)-\mathrm{S}(3)-\mathrm{Cu}(2)$ & $72.21(3)$ & $\mathrm{P}(2)-\mathrm{O}(1)-\mathrm{Cu}(3)$ & $122.07(14)$ \\
$\mathrm{P}(4)-\mathrm{O}(2)-\mathrm{Cu}(1)$ & $127.81(14)$ & $\mathrm{P}(6)-\mathrm{O}(3)-\mathrm{Cu}(2)$ & $110.91(17)$ \\
$\mathrm{P}(1)-\mathrm{N}(1)-\mathrm{P}(2)$ & $133.59(19)$ & $\mathrm{P}(3)-\mathrm{N}(2)-\mathrm{P}(4)$ & $134.3(3)$ \\
$\mathrm{P}(5)-\mathrm{N}(3)-\mathrm{P}(6)$ & $126.5(2)$ & & \\
\hline & & & \\
\hline
\end{tabular}

\#1-x, y, -z+1/2 


\subsection{Daten zur Kristallstrukturanalyse von $\left[\mathrm{Cu}\left(\operatorname{dppaO}_{2}\right)_{2}\left(\mathrm{NH}_{3}\right)_{3}\right]_{\infty} \underline{11}$}

\begin{tabular}{|ll|}
\hline Summenformel & $\mathrm{C}_{56} \mathrm{H}_{65} \mathrm{CuN}_{5} \mathrm{O}_{6} \mathrm{P}_{4}$ \\
Molekulargewicht $\left[\mathrm{g} \cdot \mathrm{mol}^{-1}\right]$ & 1091.55 \\
Temperatur & $133(2) \mathrm{K}$ \\
Wellenlänge & $71.073 \mathrm{pm}$ \\
Kristallsystem & triklin \\
Raumgruppe & $P \overline{1}$ \\
Gitterkonstanten & $\mathrm{a}=11.8293(16) \AA, \alpha=76.057(9)^{\circ}$ \\
& $\mathrm{b}=15.341(2) \AA, \beta=69.624(8)^{\circ}$ \\
& $\mathrm{c}=16.655(2) \AA, \gamma=77.039(9)^{\circ}$ \\
& $\circ$ \\
Volumen & $2716.9(7) \AA^{3}$ \\
Formeleinheiten & 2 \\
Röntgenographische Dichte $\left[\mathrm{g} \cdot \mathrm{cm}^{-3}\right]$ & 1.334 \\
Absorptionskoeffizient $\left[\mathrm{mm}^{-1}\right]$ & 0.574 \\
$\mathrm{~F}(000)$ & 1146 \\
Meßbereich $\theta$ & $2.04-22.46^{\circ}$ \\
Indexbereiche & $-12 \leq \mathrm{h} \leq 9$ \\
& $-16 \leq \mathrm{k} \leq 16$ \\
& $-17 \leq \mathrm{l} \leq 17$ \\
Gemessene Reflexe & 24580 \\
Unabhängige Reflexe & 7058 \\
Beobachtete Reflexe $[\mathrm{I}>2 \sigma]$ & 4830 \\
$\mathrm{R}_{\text {int }}$ & 0.0961 \\
Parameter & 661 \\
R1; wR2[I>2 $\sigma(\mathrm{I})]$ & $0.0604 ; 0.1508$ \\
\hline
\end{tabular}


Tabelle 44: Atomkoordinaten und äquivalente isotrope Auslenkungsparameter $\left[\AA^{2}\right]$ für $\underline{11}$

\begin{tabular}{|c|c|c|c|c|}
\hline Atom & $\mathrm{x}$ & $\mathrm{y}$ & z & $\mathrm{U}_{e q}$ \\
\hline $\mathrm{Cu}(1)$ & $0.68286(5)$ & $1.02379(4)$ & $0.60695(4)$ & $0.0201(2)$ \\
\hline $\mathrm{P}(1)$ & $0.49430(12)$ & $1.24043(10)$ & $0.62917(9)$ & $0.0197(4)$ \\
\hline $\mathrm{P}(2)$ & $0.37646(12)$ & $1.21651(10)$ & $0.51370(10)$ & $0.0219(4)$ \\
\hline $\mathrm{P}(3)$ & $0.75831(12)$ & $0.92717(10)$ & $0.78627(9)$ & $0.0202(4)$ \\
\hline $\mathrm{P}(4)$ & $1.01100(12)$ & $0.85494(10)$ & $0.69705(9)$ & $0.0219(4)$ \\
\hline $\mathrm{O}(1)$ & $0.6037(3)$ & $1.1675(2)$ & $0.6257(2)$ & $0.0213(9)$ \\
\hline $\mathrm{O}(2)$ & $0.3991(3)$ & $1.1144(2)$ & $0.5346(3)$ & $0.0293(10)$ \\
\hline $\mathrm{O}(3)$ & $0.6809(3)$ & $0.9776(2)$ & $0.7283(2)$ & $0.0248(9)$ \\
\hline $\mathrm{O}(4)$ & $1.0501(3)$ & $0.9322(2)$ & $0.6245(2)$ & $0.0259(9)$ \\
\hline $\mathrm{N}(1)$ & $0.4459(4)$ & $1.2712(3)$ & $0.5463(3)$ & $0.0209(11)$ \\
\hline $\mathrm{C}(1)$ & $0.5284(4)$ & $1.3424(4)$ & $0.6465(4)$ & $0.0190(13)$ \\
\hline $\mathrm{C}(2)$ & $0.5971(5)$ & $1.3339(4)$ & $0.7016(4)$ & $0.0337(16)$ \\
\hline $\mathrm{C}(3)$ & $0.6261(6)$ & $1.4100(4)$ & $0.7157(4)$ & $0.0411(18)$ \\
\hline $\mathrm{C}(4)$ & $0.5907(5)$ & $1.4950(4)$ & $0.6746(4)$ & $0.0339(16)$ \\
\hline $\mathrm{C}(5)$ & $0.5221(6)$ & $1.5051(4)$ & $0.6197(4)$ & $0.0368(16)$ \\
\hline $\mathrm{C}(6)$ & $0.4916(5)$ & $1.4286(4)$ & $0.6057(4)$ & $0.0281(15)$ \\
\hline $\mathrm{C}(7)$ & $0.3737(4)$ & $1.2081(4)$ & $0.7285(4)$ & $0.0214(13)$ \\
\hline $\mathrm{C}(8)$ & $0.3966(5)$ & $1.1405(4)$ & $0.7949(4)$ & $0.0333(16)$ \\
\hline $\mathrm{C}(9)$ & $0.3063(5)$ & $1.1184(4)$ & $0.8718(4)$ & $0.0363(16)$ \\
\hline $\mathrm{C}(10)$ & $0.1883(5)$ & $1.1632(4)$ & $0.8827(4)$ & $0.0395(17)$ \\
\hline $\mathrm{C}(11)$ & $0.1631(5)$ & $1.2313(5)$ & $0.8179(4)$ & $0.0399(18)$ \\
\hline $\mathrm{C}(12)$ & $0.2542(5)$ & $1.2543(4)$ & $0.7413(4)$ & $0.0325(16)$ \\
\hline $\mathrm{C}(13)$ & $0.2137(5)$ & $1.2541(4)$ & $0.5494(4)$ & $0.0274(15)$ \\
\hline $\mathrm{C}(14)$ & $0.1336(6)$ & $1.1939(5)$ & $0.5878(4)$ & $0.049(2)$ \\
\hline $\mathrm{C}(15)$ & $0.0105(7)$ & $1.2216(7)$ & $0.6149(6)$ & $0.076(3)$ \\
\hline $\mathrm{C}(16)$ & $-0.0333(6)$ & $1.3134(9)$ & $0.6046(5)$ & $0.082(3)$ \\
\hline $\mathrm{C}(17)$ & $0.0435(6)$ & $1.3787(6)$ & $0.5662(5)$ & $0.060(2)$ \\
\hline $\mathrm{C}(18)$ & $0.1692(5)$ & $1.3469(5)$ & $0.5384(4)$ & $0.0421(18)$ \\
\hline $\mathrm{C}(19)$ & $0.4177(5)$ & $1.2503(4)$ & $0.3967(4)$ & $0.0224(14)$ \\
\hline $\mathrm{C}(20)$ & $0.3390(5)$ & $1.2482(4)$ & $0.3519(4)$ & $0.0245(14)$ \\
\hline $\mathrm{C}(21)$ & $0.3773(5)$ & $1.2609(4)$ & $0.2622(4)$ & $0.0314(15)$ \\
\hline $\mathrm{C}(22)$ & $0.4951(5)$ & $1.2744(4)$ & $0.2149(4)$ & $0.0337(16)$ \\
\hline $\mathrm{C}(23)$ & $0.5746(5)$ & $1.2777(4)$ & $0.2581(4)$ & $0.0326(16)$ \\
\hline $\mathrm{C}(24)$ & $0.5357(5)$ & $1.2653(4)$ & $0.3486(4)$ & $0.0267(14)$ \\
\hline $\mathrm{N}(2)$ & $0.8703(3)$ & $0.8537(3)$ & $0.7491(3)$ & $0.0215(11)$ \\
\hline $\mathrm{C}(25)$ & $0.6604(4)$ & $0.8680(4)$ & $0.8847(3)$ & $0.0201(13)$ \\
\hline $\mathrm{C}(26)$ & $0.5434(5)$ & $0.9131(4)$ & $0.9221(4)$ & $0.0348(16)$ \\
\hline $\mathrm{C}(27)$ & $0.4668(5)$ & $0.8713(4)$ & $0.9966(4)$ & $0.0349(16)$ \\
\hline
\end{tabular}


Tabelle 44: Fortsetzung

\begin{tabular}{|r|r|r|r|r|}
\hline $\mathrm{C}(28)$ & $0.5032(5)$ & $0.7842(4)$ & $1.0359(4)$ & $0.0353(16)$ \\
$\mathrm{C}(29)$ & $0.6183(5)$ & $0.7396(4)$ & $0.9996(4)$ & $0.0372(16)$ \\
$\mathrm{C}(30)$ & $0.6951(5)$ & $0.7816(4)$ & $0.9237(4)$ & $0.0276(14)$ \\
$\mathrm{C}(31)$ & $0.8024(4)$ & $1.0124(4)$ & $0.8229(3)$ & $0.0207(13)$ \\
$\mathrm{C}(32)$ & $0.8634(5)$ & $0.9878(4)$ & $0.8843(4)$ & $0.0295(15)$ \\
$\mathrm{C}(33)$ & $0.8966(5)$ & $1.0551(4)$ & $0.9126(4)$ & $0.0352(16)$ \\
$\mathrm{C}(34)$ & $0.8668(5)$ & $1.1447(4)$ & $0.8797(4)$ & $0.0297(15)$ \\
$\mathrm{C}(35)$ & $0.8050(5)$ & $1.1701(4)$ & $0.8188(4)$ & $0.0304(15)$ \\
$\mathrm{C}(36)$ & $0.7712(4)$ & $1.1042(4)$ & $0.7916(3)$ & $0.0217(14)$ \\
$\mathrm{C}(37)$ & $1.0887(5)$ & $0.8446(4)$ & $0.7757(4)$ & $0.0270(15)$ \\
$\mathrm{C}(38)$ & $1.0776(5)$ & $0.7754(5)$ & $0.8475(4)$ & $0.0376(17)$ \\
$\mathrm{C}(39)$ & $1.1331(6)$ & $0.7713(5)$ & $0.9101(4)$ & $0.050(2)$ \\
$\mathrm{C}(40)$ & $1.2014(6)$ & $0.8360(6)$ & $0.9004(5)$ & $0.054(2)$ \\
$\mathrm{C}(41)$ & $1.2142(5)$ & $0.9060(6)$ & $0.8301(5)$ & $0.054(2)$ \\
$\mathrm{C}(42)$ & $1.1595(5)$ & $0.9109(4)$ & $0.7676(4)$ & $0.0383(17)$ \\
$\mathrm{C}(43)$ & $1.0622(4)$ & $0.7492(4)$ & $0.6570(3)$ & $0.0207(13)$ \\
$\mathrm{C}(44)$ & $0.9914(5)$ & $0.6803(4)$ & $0.6833(4)$ & $0.0367(16)$ \\
$\mathrm{C}(45)$ & $1.0343(6)$ & $0.6002(5)$ & $0.6512(5)$ & $0.0487(19)$ \\
$\mathrm{C}(46)$ & $1.1503(5)$ & $0.5882(4)$ & $0.5909(4)$ & $0.0410(17)$ \\
$\mathrm{C}(47)$ & $1.2206(5)$ & $0.6561(4)$ & $0.5636(4)$ & $0.0339(16)$ \\
$\mathrm{C}(48)$ & $1.1763(5)$ & $0.7353(4)$ & $0.5953(4)$ & $0.0259(14)$ \\
$\mathrm{N}(3)$ & $0.8484(3)$ & $1.0595(3)$ & $0.5695(3)$ & $0.0234(12)$ \\
$\mathrm{N}(4)$ & $0.5225(4)$ & $0.9792(3)$ & $0.6430(3)$ & $0.0259(12)$ \\
$\mathrm{N}(5)$ & $0.6868(4)$ & $1.0401(3)$ & $0.4824(3)$ & $0.0261(12)$ \\
$\mathrm{O}(1 \mathrm{~L})$ & $0.7547(5)$ & $1.3639(3)$ & $0.8914(4)$ & $0.0686(15)$ \\
$\mathrm{C}(1 \mathrm{~L})$ & $0.6546(8)$ & $1.4092(5)$ & $0.9475(6)$ & $0.080(3)$ \\
$\mathrm{C}(2 \mathrm{~L})$ & $0.6461(6)$ & $1.5074(5)$ & $0.9081(5)$ & $0.055(2)$ \\
$\mathrm{C}(3 \mathrm{~L})$ & $0.7710(6)$ & $1.5181(5)$ & $0.8454(5)$ & $0.053(2)$ \\
$\mathrm{C}(4 \mathrm{~L})$ & $0.8410(6)$ & $1.4243(5)$ & $0.8559(6)$ & $0.068(2)$ \\
$\mathrm{O}(2 \mathrm{~L})$ & $0.2142(6)$ & $1.4673(4)$ & $0.9471(4)$ & $0.0870(19)$ \\
$\mathrm{C}(5 \mathrm{~L})$ & $0.1357(9)$ & $1.4254(7)$ & $0.9266(7)$ & $0.104(4)$ \\
$\mathrm{C}(6 \mathrm{~L})$ & $0.1235(10)$ & $1.4848(8)$ & $0.8431(8)$ & $0.118(4)$ \\
$\mathrm{C}(7 \mathrm{~L})$ & $0.2354(8)$ & $1.5215(8)$ & $0.7989(6)$ & $0.107(4)$ \\
$\mathrm{C}(8 \mathrm{~L})$ & $0.2893(10)$ & $1.5069(8)$ & $0.8671(8)$ & $0.137(5)$ \\
\hline & & & & \\
& & & \\
& & & \\
& & & \\
& & & \\
& &
\end{tabular}

Tabelle 45: Bindungslängen [pm] in 11

\begin{tabular}{|l|l||l|l|}
\hline $\mathrm{Cu}(1)-\mathrm{O}(3)$ & $196.9(4)$ & $\mathrm{Cu}(1)-\mathrm{N}(3)$ & $199.7(4)$ \\
$\mathrm{Cu}(1)-\mathrm{N}(4)$ & $200.4(4)$ & $\mathrm{Cu}(1)-\mathrm{N}(5)$ & $201.4(4)$ \\
$\mathrm{Cu}(1)-\mathrm{O}(1)$ & $224.5(3)$ & $\mathrm{P}(1)-\mathrm{O}(1)$ & $150.3(3)$ \\
\hline
\end{tabular}


Tabelle 45: Fortsetzung

\begin{tabular}{|l|l||l|l|}
\hline $\mathrm{P}(1)-\mathrm{N}(1)$ & $160.3(4)$ & $\mathrm{P}(1)-\mathrm{C}(1)$ & $180.9(5)$ \\
$\mathrm{P}(1)-\mathrm{C}(7)$ & $181.6(5)$ & $\mathrm{P}(2)-\mathrm{O}(2)$ & $150.3(4)$ \\
$\mathrm{P}(2)-\mathrm{N}(1)$ & $158.5(4)$ & $\mathrm{P}(2)-\mathrm{C}(13)$ & $180.7(5)$ \\
$\mathrm{P}(2)-\mathrm{C}(19)$ & $180.8(6)$ & $\mathrm{P}(3)-\mathrm{O}(3)$ & $151.9(4)$ \\
$\mathrm{P}(3)-\mathrm{N}(2)$ & $158.2(4)$ & $\mathrm{P}(3)-\mathrm{C}(31)$ & $180.7(6)$ \\
$\mathrm{P}(3)-\mathrm{C}(25)$ & $181.3(5)$ & $\mathrm{P}(4)-\mathrm{O}(4)$ & $149.9(4)$ \\
$\mathrm{P}(4)-\mathrm{N}(2)$ & $158.9(4)$ & $\mathrm{P}(4)-\mathrm{C}(43)$ & $180.2(6)$ \\
$\mathrm{P}(4)-\mathrm{C}(37)$ & $180.5(6)$ & & \\
\hline
\end{tabular}

Tabelle 46: Bindungswinkel $\left[^{\circ}\right]$ in $\underline{11}$

\begin{tabular}{|l|r||l|r|}
\hline $\mathrm{O}(3)-\mathrm{Cu}(1)-\mathrm{N}(3)$ & $93.66(17)$ & $\mathrm{O}(3)-\mathrm{Cu}(1)-\mathrm{N}(4)$ & $86.03(18)$ \\
$\mathrm{N}(3)-\mathrm{Cu}(1)-\mathrm{N}(4)$ & $175.99(19)$ & $\mathrm{O}(3)-\mathrm{Cu}(1)-\mathrm{N}(5)$ & $166.53(18)$ \\
$\mathrm{N}(3)-\mathrm{Cu}(1)-\mathrm{N}(5)$ & $91.42(19)$ & $\mathrm{N}(4)-\mathrm{Cu}(1)-\mathrm{N}(5)$ & $88.0(2)$ \\
$\mathrm{O}(3)-\mathrm{Cu}(1)-\mathrm{O}(1)$ & $95.36(14)$ & $\mathrm{N}(3)-\mathrm{Cu}(1)-\mathrm{O}(1)$ & $87.97(16)$ \\
$\mathrm{N}(4)-\mathrm{Cu}(1)-\mathrm{O}(1)$ & $96.04(16)$ & $\mathrm{N}(5)-\mathrm{Cu}(1)-\mathrm{O}(1)$ & $97.28(17)$ \\
$\mathrm{O}(1)-\mathrm{P}(1)-\mathrm{N}(1)$ & $118.6(2)$ & $\mathrm{O}(2)-\mathrm{P}(2)-\mathrm{N}(1)$ & $118.3(2)$ \\
$\mathrm{N}(1)-\mathrm{P}(2)-\mathrm{C}(13)$ & $111.1(3)$ & $\mathrm{N}(1)-\mathrm{P}(2)-\mathrm{C}(19)$ & $106.8(2)$ \\
$\mathrm{O}(3)-\mathrm{P}(3)-\mathrm{N}(2)$ & $118.0(2)$ & $\mathrm{N}(2)-\mathrm{P}(3)-\mathrm{C}(31)$ & $112.5(2)$ \\
$\mathrm{O}(3)-\mathrm{P}(3)-\mathrm{C}(25)$ & $108.1(2)$ & $\mathrm{N}(2)-\mathrm{P}(3)-\mathrm{C}(25)$ & $106.3(2)$ \\
$\mathrm{O}(4)-\mathrm{P}(4)-\mathrm{N}(2)$ & $120.0(2)$ & $\mathrm{N}(2)-\mathrm{P}(4)-\mathrm{C}(43)$ & $104.5(2)$ \\
$\mathrm{P}(1)-\mathrm{O}(1)-\mathrm{Cu}(1)$ & $145.4(2)$ & $\mathrm{P}(3)-\mathrm{O}(3)-\mathrm{Cu}(1)$ & $144.4(2)$ \\
$\mathrm{P}(2)-\mathrm{N}(1)-\mathrm{P}(1)$ & $128.0(3)$ & $\mathrm{P}(3)-\mathrm{N}(2)-\mathrm{P}(4)$ & $135.0(3)$ \\
\hline
\end{tabular}

Tabelle 47: Wasserstoffbindungslängen [pm] in $\underline{\mathbf{1 1}}$

\begin{tabular}{|l|l|l|l|l|}
\hline $\mathrm{D}-\mathrm{H} \cdots \mathrm{A}$ & $\mathrm{d}(\mathrm{D}-\mathrm{H})$ & $\mathrm{d}(\mathrm{H} \cdots \mathrm{A})$ & $\mathrm{d}(\mathrm{D} \cdots \mathrm{A})$ & $\angle(D H A)\left[^{\circ}\right]$ \\
\hline $\mathrm{N}(3)-\mathrm{H}(3) \cdots \mathrm{O}(4) \# 1$ & 91 & 210 & $301.2(6)$ & 176.0 \\
$\mathrm{~N}(3)-\mathrm{H}(3) \cdots \mathrm{O}(4)$ & 91 & 211 & $298.6(5)$ & 161.8 \\
$\mathrm{~N}(4)-\mathrm{H}(4) \cdots \mathrm{O}(2)$ & 91 & 207 & $289.3(6)$ & 150.5 \\
$\mathrm{~N}(5)-\mathrm{H}(5) \cdots \mathrm{O}(4) \# 1$ & 91 & 216 & $306.7(6)$ & 171.1 \\
$\mathrm{~N}(5)-\mathrm{H}(5) \cdots \mathrm{O}(2) \# 2$ & 91 & 199 & $288.6(6)$ & 166.8 \\
\hline
\end{tabular}

$\# 1-x+2,-y+2,-z+1 \quad \# 2-x+1,-y+2,-z+1$ 


\subsection{Daten zur Kristallstrukturanalyse von $\left[\mathrm{H}\left((\mathrm{O}) \mathrm{PPh}_{2} \mathrm{NPPh}_{2}\right)_{2} \mathrm{O}\right]\left[\mathrm{CuCl}_{2}\right] \underline{12}$}

\begin{tabular}{|ll|}
\hline Summenformel & $\mathrm{C}_{52} \mathrm{H}_{49} \mathrm{Cl}_{2} \mathrm{CuN}_{2} \mathrm{O}_{4} \mathrm{P}_{4}$ \\
Molekulargewicht $\left[\mathrm{g} \cdot \mathrm{mol}^{-1}\right]$ & 1024.25 \\
Temperatur & $293(2) \mathrm{K}$ \\
Wellenlänge & $71.073 \mathrm{pm}$ \\
Kristallsystem & monoklin \\
Raumgruppe & $P 2_{1} / n$ \\
Gitterkonstanten & $\mathrm{a}=16.3744(11) \AA, \alpha=90^{\circ}$ \\
& $\mathrm{b}=12.8328(8) \AA, \beta=102.837(4)^{\circ} \circ$ \\
& $\mathrm{c}=24.2758(16) \AA, \gamma=90^{\circ}{ }^{\circ}$ \\
Volumen & $4973.6(6) \AA^{3}$ \\
Formeleinheiten & 4 \\
Röntgenographische Dichte $\left[\mathrm{g} \cdot \mathrm{cm}^{-3}\right]$ & 1.368 \\
Absorptionskoeffizient $\left[\mathrm{mm}^{-1}\right]$ & 0.722 \\
$\mathrm{~F}(000)$ & 2120 \\
Meß3bereich $\theta$ & $2.04-22.46^{\circ}$ \\
Indexbereiche & $-17 \leq \mathrm{h} \leq 17$ \\
& $-13 \leq \mathrm{k} \leq 7$ \\
Gemessene Reflexe & $-26 \leq 1 \leq 26$ \\
Unabhängige Reflexe & 43664 \\
Beobachtete Reflexe $[\mathrm{I}>2 \sigma]$ & 6474 \\
$\mathrm{R}_{\text {int }}$ & 4562 \\
Parameter & 0.0963 \\
R1; wR2[I>2 $\sigma(\mathrm{I})]$ & 590 \\
\hline
\end{tabular}


Tabelle 49: Atomkoordinaten und äquivalente isotrope Auslenkungsparameter $\left[\AA^{2}\right]$ für $\underline{12}$

\begin{tabular}{|r|r|r|r|r|}
\hline Atom & \multicolumn{1}{|c|}{$\mathrm{x}$} & \multicolumn{1}{c|}{$\mathrm{y}$} & \multicolumn{1}{c|}{$\mathrm{z}$} & \multicolumn{1}{c|}{$\mathrm{U}_{e q}$} \\
\hline $\mathrm{Cu}$ & $1.25857(4)$ & $-0.54007(4)$ & $0.58543(3)$ & $0.03188(19)$ \\
$\mathrm{P}(1)$ & $0.73989(8)$ & $-0.16555(9)$ & $0.73186(5)$ & $0.0213(3)$ \\
$\mathrm{P}(2)$ & $0.88301(7)$ & $-0.27469(9)$ & $0.70781(5)$ & $0.0198(3)$ \\
$\mathrm{P}(3)$ & $0.91508(7)$ & $-0.14801(9)$ & $0.61526(5)$ & $0.0195(3)$ \\
$\mathrm{P}(4)$ & $0.73806(7)$ & $-0.10172(9)$ & $0.56539(5)$ & $0.0213(3)$ \\
$\mathrm{Cl}(1)$ & $1.33360(8)$ & $-0.41163(10)$ & $0.57644(6)$ & $0.0453(4)$ \\
$\mathrm{Cl}(2)$ & $1.18195(8)$ & $-0.66681(10)$ & $0.59395(6)$ & $0.0411(4)$ \\
$\mathrm{N}(1)$ & $0.7887(2)$ & $-0.2542(2)$ & $0.70630(15)$ & $0.0195(9)$ \\
$\mathrm{N}(2)$ & $0.8240(2)$ & $-0.1655(3)$ & $0.58185(15)$ & $0.0205(9)$ \\
$\mathrm{O}(1)$ & $0.74967(19)$ & $-0.0558(2)$ & $0.70974(14)$ & $0.0260(8)$ \\
$\mathrm{O}(2)$ & $0.9276(2)$ & $-0.1801(3)$ & $0.68152(14)$ & $0.0010(9)$ \\
$\mathrm{O}(3)$ & $0.71877(18)$ & $-0.0251(2)$ & $0.60833(12)$ & $0.0247(8)$ \\
$\mathrm{C}(1)$ & $0.6323(3)$ & $-0.2041(3)$ & $0.71551(18)$ & $0.0221(11)$ \\
$\mathrm{C}(2)$ & $0.5716(3)$ & $-0.1316(4)$ & $0.7204(2)$ & $0.0336(13)$ \\
$\mathrm{C}(3)$ & $0.4878(3)$ & $-0.1571(5)$ & $0.7068(2)$ & $0.0462(15)$ \\
$\mathrm{C}(4)$ & $0.4639(3)$ & $-0.2560(5)$ & $0.6882(2)$ & $0.0462(15)$ \\
$\mathrm{C}(5)$ & $0.5230(3)$ & $-0.3296(4)$ & $0.6836(2)$ & $0.0414(14)$ \\
$\mathrm{C}(6)$ & $0.6068(3)$ & $-0.3034(4)$ & $0.6972(2)$ & $0.0318(13)$ \\
$\mathrm{C}(7)$ & $0.7745(3)$ & $-0.1610(3)$ & $0.80728(19)$ & $0.0218(11)$ \\
$\mathrm{C}(8)$ & $0.8409(3)$ & $-0.0977(4)$ & $0.8327(2)$ & $0.0306(12)$ \\
$\mathrm{C}(9)$ & $0.8733(3)$ & $-0.1041(4)$ & $0.8900(2)$ & $0.0417(14)$ \\
$\mathrm{C}(10)$ & $0.8377(4)$ & $-0.1716(5)$ & $0.9222(2)$ & $0.0490(16)$ \\
$\mathrm{C}(11)$ & $0.7723(4)$ & $-0.2334(4)$ & $0.8976(2)$ & $0.0461(15)$ \\
$\mathrm{C}(12)$ & $0.7401(3)$ & $-0.2296(4)$ & $0.8399(2)$ & $0.0345(13)$ \\
$\mathrm{C}(13)$ & $0.9501(3)$ & $-0.2888(3)$ & $0.77590(19)$ & $0.0229(11)$ \\
$\mathrm{C}(14)$ & $1.0301(3)$ & $-0.2466(4)$ & $0.7903(2)$ & $0.0387(14)$ \\
$\mathrm{C}(15)$ & $1.0802(4)$ & $-0.2650(5)$ & $0.8439(2)$ & $0.0557(17)$ \\
$\mathrm{C}(16)$ & $1.0496(4)$ & $-0.3242(4)$ & $0.8822(2)$ & $0.0510(16)$ \\
$\mathrm{C}(17)$ & $0.9707(4)$ & $-0.3664(4)$ & $0.8682(2)$ & $0.0390(14)$ \\
$\mathrm{C}(18)$ & $0.9210(3)$ & $-0.3492(3)$ & $0.81538(19)$ & $0.0278(12)$ \\
$\mathrm{C}(19)$ & $0.8928(3)$ & $-0.3871(3)$ & $0.66674(18)$ & $0.0176(11)$ \\
$\mathrm{C}(20)$ & $0.9693(3)$ & $-0.4371(3)$ & $0.6727(2)$ & $0.0252(12)$ \\
$\mathrm{C}(21)$ & $0.9765(3)$ & $-0.5207(3)$ & $0.6385(2)$ & $0.0302(13)$ \\
$\mathrm{C}(22)$ & $0.9077(3)$ & $-0.5553(3)$ & $0.5989(2)$ & $0.0308(13)$ \\
$\mathrm{C}(23)$ & $0.8321(3)$ & $-0.5049(4)$ & $0.5923(2)$ & $0.0304(12)$ \\
$\mathrm{C}(24)$ & $0.8236(3)$ & $-0.4216(3)$ & $0.62638(19)$ & $0.0226(12)$ \\
$\mathrm{C}(25)$ & $0.9534(3)$ & $-0.0175(3)$ & $0.62090(19)$ & $0.0212(11)$ \\
$\mathrm{C}(26)$ & $0.9962(3)$ & $0.0179(4)$ & $0.5813(2)$ & $0.0423(15)$ \\
\hline & & & & \\
\hline
\end{tabular}


Tabelle 49: Fortsetzung

\begin{tabular}{|r|r|r|r|r|}
$\mathrm{C}(27)$ & $1.0231(4)$ & $0.1197(4)$ & $0.5827(2)$ & $0.0514(17)$ \\
$\mathrm{C}(28)$ & $1.0078(3)$ & $0.1861(4)$ & $0.6236(2)$ & $0.0390(14)$ \\
$\mathrm{C}(29)$ & $0.9663(3)$ & $0.1522(4)$ & $0.6629(2)$ & $0.0377(14)$ \\
$\mathrm{C}(30)$ & $0.9377(3)$ & $0.0501(4)$ & $0.6618(2)$ & $0.0310(13)$ \\
$\mathrm{C}(31)$ & $0.9850(3)$ & $-0.2290(3)$ & $0.58824(18)$ & $0.0199(11)$ \\
$\mathrm{C}(32)$ & $1.0702(3)$ & $-0.2307(3)$ & $0.61374(19)$ & $0.0265(12)$ \\
$\mathrm{C}(33)$ & $1.1234(3)$ & $-0.2959(4)$ & $0.5927(2)$ & $0.0343(13)$ \\
$\mathrm{C}(34)$ & $1.0917(3)$ & $-0.3588(4)$ & $0.5468(2)$ & $0.0352(13)$ \\
$\mathrm{C}(35)$ & $1.0072(3)$ & $-0.3568(4)$ & $0.5215(2)$ & $0.0317(13)$ \\
$\mathrm{C}(36)$ & $0.9536(3)$ & $-0.2922(3)$ & $0.54218(19)$ & $0.0231(11)$ \\
$\mathrm{C}(37)$ & $0.6575(3)$ & $-0.1988(3)$ & $0.54960(18)$ & $0.0200(11)$ \\
$\mathrm{C}(38)$ & $0.5800(3)$ & $-0.1808(4)$ & $0.5617(2)$ & $0.0331(13)$ \\
$\mathrm{C}(39)$ & $0.5178(3)$ & $-0.2557(4)$ & $0.5498(2)$ & $0.0411(14)$ \\
$\mathrm{C}(40)$ & $0.5330(3)$ & $-0.3484(4)$ & $0.5261(2)$ & $0.0373(14)$ \\
$\mathrm{C}(41)$ & $0.6084(3)$ & $-0.3667(4)$ & $0.5125(2)$ & $0.0342(13)$ \\
$\mathrm{C}(42)$ & $0.6708(3)$ & $-0.2923(4)$ & $0.52447(19)$ & $0.0274(12)$ \\
$\mathrm{C}(43)$ & $0.7360(3)$ & $-0.0308(3)$ & $0.50118(19)$ & $0.0229(11)$ \\
$\mathrm{C}(44)$ & $0.7706(3)$ & $-0.0740(4)$ & $0.4595(2)$ & $0.0410(14)$ \\
$\mathrm{C}(45)$ & $0.7682(3)$ & $-0.0203(4)$ & $0.4098(2)$ & $0.0480(16)$ \\
$\mathrm{C}(46)$ & $0.7310(4)$ & $0.0759(4)$ & $0.4015(2)$ & $0.0490(16)$ \\
$\mathrm{C}(47)$ & $0.6964(4)$ & $0.1185(4)$ & $0.4425(3)$ & $0.0578(18)$ \\
$\mathrm{C}(48)$ & $0.6972(3)$ & $0.0647(4)$ & $0.4920(2)$ & $0.0420(15)$ \\
$\mathrm{O}(1 \mathrm{~L})$ & $0.8256(4)$ & $0.0857(5)$ & $0.2758(2)$ & $0.1029(17)$ \\
$\mathrm{C}(1 \mathrm{~L})$ & $0.8468(5)$ & $-0.0064(7)$ & $0.2528(4)$ & $0.103(3)$ \\
$\mathrm{C}(2 \mathrm{~L})$ & $0.7780(5)$ & $-0.0771(6)$ & $0.2401(3)$ & $0.091(2)$ \\
$\mathrm{C}(3 \mathrm{~L})$ & $0.7008(4)$ & $-0.0046(8)$ & $0.2389(3)$ & $0.101(3)$ \\
$\mathrm{C}(4 \mathrm{~L})$ & $0.7414(6)$ & $0.0982(7)$ & $0.2464(3)$ & $0.102(3)$ \\
\hline & & & & \\
& & &
\end{tabular}

Tabelle 50: Bindungslängen [pm] in $\underline{12}$

\begin{tabular}{|l|r||l|r|}
\hline $\mathrm{Cu}-\mathrm{Cl}(2)$ & $209.22(14)$ & $\mathrm{Cu}-\mathrm{Cl}(1)$ & $209.60(14)$ \\
$\mathrm{P}(1)-\mathrm{O}(1)$ & $152.9(3)$ & $\mathrm{P}(1)-\mathrm{N}(1)$ & $159.3(3)$ \\
$\mathrm{P}(1)-\mathrm{C}(1)$ & $178.7(5)$ & $\mathrm{P}(1)-\mathrm{C}(7)$ & $179.3(5)$ \\
$\mathrm{P}(2)-\mathrm{N}(1)$ & $155.9(3)$ & $\mathrm{P}(2)-\mathrm{O}(2)$ & $161.9(3)$ \\
$\mathrm{P}(2)-\mathrm{C}(13)$ & $177.9(5)$ & $\mathrm{P}(2)-\mathrm{C}(19)$ & $178.1(4)$ \\
$\mathrm{P}(3)-\mathrm{N}(2)$ & $154.8(4)$ & $\mathrm{P}(3)-\mathrm{O}(2)$ & $162.9(4)$ \\
$\mathrm{P}(3)-\mathrm{C}(31)$ & $177.6(4)$ & $\mathrm{P}(3)-\mathrm{C}(25)$ & $178.3(4)$ \\
$\mathrm{P}(4)-\mathrm{O}(3)$ & $151.6(3)$ & $\mathrm{P}(4)-\mathrm{N}(2)$ & $159.9(4)$ \\
$\mathrm{P}(4)-\mathrm{C}(37)$ & $179.3(5)$ & $\mathrm{P}(4)-\mathrm{C}(43)$ & $179.9(5)$ \\
\hline
\end{tabular}


Tabelle 51: Bindungswinkel $\left[^{\circ}\right.$ ] in $\underline{12}$

\begin{tabular}{|l|r||l|r|}
\hline $\mathrm{Cl}(2)-\mathrm{Cu}-\mathrm{Cl}(1)$ & $179.08(7)$ & $\mathrm{O}(1)-\mathrm{P}(1)-\mathrm{N}(1)$ & $114.88(18)$ \\
$\mathrm{O}(1)-\mathrm{P}(1)-\mathrm{C}(1)$ & $110.61(19)$ & $\mathrm{N}(1)-\mathrm{P}(1)-\mathrm{C}(1)$ & $105.7(2)$ \\
$\mathrm{O}(1)-\mathrm{P}(1)-\mathrm{C}(7)$ & $107.14(19)$ & $\mathrm{N}(1)-\mathrm{P}(1)-\mathrm{C}(7)$ & $110.43(19)$ \\
$\mathrm{N}(1)-\mathrm{P}(2)-\mathrm{O}(2)$ & $113.30(19)$ & $\mathrm{N}(1)-\mathrm{P}(2)-\mathrm{C}(13)$ & $116.3(2)$ \\
$\mathrm{O}(2)-\mathrm{P}(2)-\mathrm{C}(13)$ & $101.7(2)$ & $\mathrm{N}(1)-\mathrm{P}(2)-\mathrm{C}(19)$ & $109.6(2)$ \\
$\mathrm{O}(2)-\mathrm{P}(2)-\mathrm{C}(19)$ & $106.40(19)$ & $\mathrm{N}(2)-\mathrm{P}(3)-\mathrm{O}(2)$ & $112.07(19)$ \\
$\mathrm{N}(2)-\mathrm{P}(3)-\mathrm{C}(31)$ & $110.3(2)$ & $\mathrm{O}(2)-\mathrm{P}(3)-\mathrm{C}(31)$ & $105.50(19)$ \\
$\mathrm{N}(2)-\mathrm{P}(3)-\mathrm{C}(25)$ & $117.2(2)$ & $\mathrm{O}(2)-\mathrm{P}(3)-\mathrm{C}(25)$ & $101.33(19)$ \\
$\mathrm{O}(3)-\mathrm{P}(4)-\mathrm{N}(2)$ & $117.77(18)$ & $\mathrm{O}(3)-\mathrm{P}(4)-\mathrm{C}(37)$ & $110.00(19)$ \\
$\mathrm{N}(2)-\mathrm{P}(4)-\mathrm{C}(37)$ & $105.2(2)$ & $\mathrm{O}(3)-\mathrm{P}(4)-\mathrm{C}(43)$ & $107.51(19)$ \\
$\mathrm{N}(2)-\mathrm{P}(4)-\mathrm{C}(43)$ & $108.78(19)$ & $\mathrm{P}(2)-\mathrm{N}(1)-\mathrm{P}(1)$ & $133.7(2)$ \\
$\mathrm{P}(3)-\mathrm{N}(2)-\mathrm{P}(4)$ & $138.5(2)$ & $\mathrm{P}(2)-\mathrm{O}(2)-\mathrm{P}(3)$ & $127.6(2)$ \\
\hline
\end{tabular}

Tabelle 52: Wasserstoffbindungslängen [pm] in 12

\begin{tabular}{|l|l|l|l|l|}
\hline $\mathrm{D}-\mathrm{H} \cdots \mathrm{A}$ & $\mathrm{d}(\mathrm{D}-\mathrm{H})$ & $\mathrm{d}(\mathrm{H} \cdots \mathrm{A})$ & $\mathrm{d}(\mathrm{D} \cdots \mathrm{A})$ & $\angle(D H A)\left[^{\circ}\right]$ \\
\hline $\mathrm{O}(1)-\mathrm{H}(49) \cdots \mathrm{O}(3)$ & $106(5)$ & $137(5)$ & $243.4(4)$ & $177(5)$ \\
\hline
\end{tabular}




\subsection{Daten zur Kristallstrukturanalyse von $\left[\mathrm{NaCu}_{2}\left(\operatorname{dppaS}_{2}\right)_{3}(\text { thf })_{2}\right] \underline{13}$}

\begin{tabular}{|ll|}
\hline Summenformel & $\mathrm{C}_{80} \mathrm{H}_{76} \mathrm{Cu}_{2} \mathrm{~N}_{3} \mathrm{NaO}_{2} \mathrm{P}_{6} \mathrm{~S}_{6}$ \\
Molekulargewicht $\left[\mathrm{g} \cdot \mathrm{mol}^{-1}\right]$ & 1639.69 \\
Temperatur & $133(2) \mathrm{K}$ \\
Wellenlänge & $71.073 \mathrm{pm}$ \\
Kristallsystem & triklin \\
Raumgruppe & $P 1$ \\
Gitterkonstanten & $\mathrm{a}=9.2570(10) \AA, \alpha=87.560(10)^{\circ}$ \\
& $\mathrm{b}=14.2390(10) \AA, \beta=74.640(10)^{\circ} \circ$ \\
& $\mathrm{c}=15.3280(10) \AA, \gamma=84.250(10)^{\circ}$
\end{tabular}


Tabelle 54: Atomkoordinaten und äquivalente isotrope Auslenkungsparameter $\left[\AA^{2}\right]$ für $\underline{13}$

\begin{tabular}{|c|c|c|c|c|}
\hline Atom & $\mathrm{x}$ & $\mathrm{y}$ & Z & $\mathrm{U}_{e q}$ \\
\hline $\mathrm{C}(1)$ & $1.1456(3)$ & $-0.8399(2)$ & $1.57627(19)$ & $0.0230(6)$ \\
\hline $\mathrm{C}(2)$ & $1.1848(5)$ & $-0.7651(2)$ & $1.5184(2)$ & $0.0405(9)$ \\
\hline $\mathrm{C}(3)$ & $1.2872(5)$ & $-0.7783(3)$ & $1.4351(3)$ & $0.0553(12)$ \\
\hline $\mathrm{C}(4)$ & $1.3527(5)$ & $-0.8673(3)$ & $1.4104(2)$ & $0.0534(12)$ \\
\hline $\mathrm{C}(5)$ & $1.3152(5)$ & $-0.9420(3)$ & $1.4678(3)$ & $0.0552(12)$ \\
\hline $\mathrm{C}(6)$ & $1.2128(5)$ & $-0.9289(2)$ & $1.5506(2)$ & $0.0423(9)$ \\
\hline $\mathrm{C}(7)$ & $0.8314(4)$ & $-0.8055(2)$ & $1.6466(2)$ & $0.0262(7)$ \\
\hline $\mathrm{C}(8)$ & $0.7757(5)$ & $-0.7158(3)$ & $1.6254(3)$ & $0.0471(10)$ \\
\hline $\mathrm{C}(9)$ & $0.6488(6)$ & $-0.7047(4)$ & $1.5927(3)$ & $0.0656(14)$ \\
\hline $\mathrm{C}(10)$ & $0.5794(5)$ & $-0.7806(4)$ & $1.5796(3)$ & $0.0606(13)$ \\
\hline $\mathrm{C}(11)$ & $0.6311(5)$ & $-0.8681(4)$ & $1.6016(3)$ & $0.0580(12)$ \\
\hline $\mathrm{C}(12)$ & $0.7566(5)$ & $-0.8817(3)$ & $1.6361(3)$ & $0.0454(9)$ \\
\hline $\mathrm{C}(13)$ & $0.9428(3)$ & $-0.5520(2)$ & $1.7954(2)$ & $0.0235(6)$ \\
\hline $\mathrm{C}(14)$ & $1.0057(4)$ & $-0.5089(2)$ & $1.7128(2)$ & $0.0286(7)$ \\
\hline $\mathrm{C}(15)$ & $0.9723(4)$ & $-0.4133(2)$ & $1.6998(3)$ & $0.0348(8)$ \\
\hline $\mathrm{C}(16)$ & $0.8783(5)$ & $-0.3603(2)$ & $1.7686(3)$ & $0.0421(9)$ \\
\hline $\mathrm{C}(17)$ & $0.8167(6)$ & $-0.4029(3)$ & $1.8502(3)$ & $0.0527(12)$ \\
\hline $\mathrm{C}(18)$ & $0.8482(5)$ & $-0.4979(2)$ & $1.8643(2)$ & $0.0400(9)$ \\
\hline $\mathrm{C}(19)$ & $1.1659(4)$ & $-0.6766(2)$ & $1.8473(2)$ & $0.0262(6)$ \\
\hline $\mathrm{C}(20)$ & $1.2962(4)$ & $-0.7271(3)$ & $1.8006(3)$ & $0.0379(8)$ \\
\hline $\mathrm{C}(21)$ & $1.4276(4)$ & $-0.7261(3)$ & $1.8292(3)$ & $0.0498(10)$ \\
\hline $\mathrm{C}(22)$ & $1.4278(5)$ & $-0.6756(4)$ & $1.9026(3)$ & $0.0552(11)$ \\
\hline $\mathrm{C}(23)$ & $1.2993(5)$ & $-0.6243(4)$ & $1.9480(3)$ & $0.0583(12)$ \\
\hline $\mathrm{C}(24)$ & $1.1679(4)$ & $-0.6249(3)$ & $1.9207(2)$ & $0.0422(9)$ \\
\hline $\mathrm{C}(25)$ & $0.5908(3)$ & $-1.0802(2)$ & $1.8887(2)$ & $0.0228(6)$ \\
\hline $\mathrm{C}(26)$ & $0.5753(4)$ & $-1.1668(2)$ & $1.8537(2)$ & $0.0299(7)$ \\
\hline $\mathrm{C}(27)$ & $0.4993(4)$ & $-1.1679(3)$ & $1.7877(2)$ & $0.0401(9)$ \\
\hline $\mathrm{C}(28)$ & $0.4367(4)$ & $-1.0852(3)$ & $1.7575(3)$ & $0.0468(10)$ \\
\hline $\mathrm{C}(29)$ & $0.4503(5)$ & $-1.0012(3)$ & $1.7924(3)$ & $0.0533(11)$ \\
\hline $\mathrm{C}(30)$ & $0.5303(4)$ & $-0.9977(3)$ & $1.8566(2)$ & $0.0372(8)$ \\
\hline $\mathrm{C}(31)$ & $0.8555(3)$ & $-1.1569(2)$ & $1.94411(19)$ & $0.0210(6)$ \\
\hline $\mathrm{C}(32)$ & $0.9030(4)$ & $-1.2191(3)$ & $2.0053(2)$ & $0.0345(8)$ \\
\hline $\mathrm{C}(33)$ & $1.0401(4)$ & $-1.2712(3)$ & $1.9788(2)$ & $0.0424(9)$ \\
\hline $\mathrm{C}(34)$ & $1.1313(4)$ & $-1.2626(3)$ & $1.8925(2)$ & $0.0378(8)$ \\
\hline $\mathrm{C}(35)$ & $1.0842(4)$ & $-1.2019(2)$ & $1.8314(2)$ & $0.0312(7)$ \\
\hline $\mathrm{C}(36)$ & $0.9467(4)$ & $-1.1495(2)$ & $1.8568(2)$ & $0.0245(6)$ \\
\hline $\mathrm{C}(37)$ & $0.3455(3)$ & $-1.2352(2)$ & $2.14172(19)$ & $0.0206(6)$ \\
\hline $\mathrm{C}(38)$ & $0.4232(4)$ & $-1.3099(2)$ & $2.0882(2)$ & $0.0284(7)$ \\
\hline
\end{tabular}


Tabelle 54: Fortsetzung

\begin{tabular}{|c|c|c|c|c|}
\hline $\mathrm{C}(39)$ & $0.3590(5)$ & $-1.3936(2)$ & $2.0919(3)$ & $0.0386(9)$ \\
\hline $\mathrm{C}(40)$ & $0.2180(5)$ & $-1.4035(2)$ & $2.1478(3)$ & $0.0394(9)$ \\
\hline $\mathrm{C}(41)$ & $0.1404(4)$ & $-1.3301(3)$ & $2.2013(2)$ & $0.0363(8)$ \\
\hline $\mathrm{C}(42)$ & $0.2047(4)$ & $-1.2467(2)$ & $2.1992(2)$ & $0.0283(7)$ \\
\hline $\mathrm{C}(43)$ & $0.3001(3)$ & $-1.0421(2)$ & $2.0883(2)$ & $0.0241(6)$ \\
\hline $\mathrm{C}(44)$ & $0.2294(4)$ & $-1.0710(2)$ & $2.0261(2)$ & $0.0316(7)$ \\
\hline $\mathrm{C}(45)$ & $0.1458(4)$ & $-1.0062(3)$ & $1.9854(3)$ & $0.0416(9)$ \\
\hline$C(46)$ & $0.1333(5)$ & $-0.9129(3)$ & $2.0040(3)$ & $0.0559(12)$ \\
\hline $\mathrm{C}(47)$ & $0.2050(6)$ & $-0.8834(3)$ & $2.0640(4)$ & $0.0753(18)$ \\
\hline $\mathrm{C}(48)$ & $0.2861(6)$ & $-0.9473(3)$ & $2.1073(3)$ & $0.0559(12)$ \\
\hline $\mathrm{C}(49)$ & $0.5170(3)$ & $-1.4484(2)$ & $2.37565(19)$ & $0.0206(6)$ \\
\hline $\mathrm{C}(50)$ & $0.4912(4)$ & $-1.4993(2)$ & $2.4570(2)$ & $0.0310(7)$ \\
\hline $\mathrm{C}(51)$ & $0.3535(4)$ & $-1.5337(2)$ & $2.4940(2)$ & $0.0343(8)$ \\
\hline $\mathrm{C}(52)$ & $0.2411(4)$ & $-1.5187(2)$ & $2.4508(2)$ & $0.0313(7)$ \\
\hline $\mathrm{C}(53)$ & $0.2656(4)$ & $-1.4702(2)$ & $2.3696(2)$ & $0.0310(7)$ \\
\hline $\mathrm{C}(54)$ & $0.4032(4)$ & $-1.4344(2)$ & $2.3320(2)$ & $0.0285(7)$ \\
\hline $\mathrm{C}(55)$ & $0.8300(4)$ & $-1.5049(2)$ & $2.2923(2)$ & $0.0339(8)$ \\
\hline $\mathrm{C}(56)$ & $0.8733(5)$ & $-1.5312(3)$ & $2.2033(3)$ & $0.0523(11)$ \\
\hline $\mathrm{C}(57)$ & $0.9709(6)$ & $-1.6136(4)$ & $2.1790(4)$ & $0.0753(18)$ \\
\hline $\mathrm{C}(58)$ & $1.0192(5)$ & $-1.6673(4)$ & $2.2414(5)$ & $0.0781(19)$ \\
\hline $\mathrm{C}(59)$ & $0.9784(6)$ & $-1.6415(4)$ & $2.3292(5)$ & $0.090(2)$ \\
\hline $\mathrm{C}(60)$ & $0.8849(5)$ & $-1.5613(3)$ & $2.3546(3)$ & $0.0606(14)$ \\
\hline $\mathrm{C}(61)$ & $0.8443(3)$ & $-1.2440(2)$ & $2.51227(19)$ & $0.0219(6)$ \\
\hline $\mathrm{C}(62)$ & $0.9897(4)$ & $-1.2752(2)$ & $2.4648(2)$ & $0.0306(7)$ \\
\hline $\mathrm{C}(63)$ & $1.1122(4)$ & $-1.2410(3)$ & $2.4850(2)$ & $0.0366(8)$ \\
\hline $\mathrm{C}(64)$ & $1.0896(4)$ & $-1.1770(2)$ & $2.5525(2)$ & $0.0360(8)$ \\
\hline $\mathrm{C}(65)$ & $0.9457(4)$ & $-1.1465(2)$ & $2.6005(2)$ & $0.0354(8)$ \\
\hline $\mathrm{C}(66)$ & $0.8229(4)$ & $-1.1797(2)$ & $2.5809(2)$ & $0.0296(7)$ \\
\hline $\mathrm{C}(67)$ & $0.5838(3)$ & $-1.3454(2)$ & $2.5861(2)$ & $0.0254(6)$ \\
\hline $\mathrm{C}(68)$ & $0.4291(4)$ & $-1.3306(2)$ & $2.6211(2)$ & $0.0332(8)$ \\
\hline $\mathrm{C}(69)$ & $0.3553(4)$ & $-1.3846(3)$ & $2.6934(3)$ & $0.0401(9)$ \\
\hline $\mathrm{C}(70)$ & $0.4345(5)$ & $-1.4538(3)$ & $2.7315(3)$ & $0.0454(9)$ \\
\hline $\mathrm{C}(71)$ & $0.5893(5)$ & $-1.4688(3)$ & $2.6982(3)$ & $0.0428(9)$ \\
\hline $\mathrm{C}(72)$ & $0.6626(4)$ & $-1.4155(3)$ & $2.6258(2)$ & $0.0335(8)$ \\
\hline $\mathrm{C}(73 \mathrm{~A})$ & $0.5011(18)$ & $-0.7594(10)$ & $2.1360(10)$ & $0.056(3)$ \\
\hline $\mathrm{C}(74 \mathrm{~A})$ & $0.494(2)$ & $-0.6570(11)$ & $2.1359(11)$ & $0.061(4)$ \\
\hline $\mathrm{C}(75 \mathrm{~A})$ & $0.5310(18)$ & $-0.6501(11)$ & $2.2329(10)$ & $0.064(4)$ \\
\hline $\mathrm{C}(76 \mathrm{~A})$ & $0.614(2)$ & $-0.7312(12)$ & $2.2542(13)$ & $0.082(5)$ \\
\hline $\mathrm{C}(77 \mathrm{~A})$ & $0.852(2)$ & $-0.8757(13)$ & $2.3291(13)$ & $0.080(5)$ \\
\hline $\mathrm{C}(78 \mathrm{~A})$ & $1.025(2)$ & $-0.9213(13)$ & $2.3239(13)$ & $0.083(5)$ \\
\hline
\end{tabular}


Tabelle 54: Fortsetzung

\begin{tabular}{r|r|r|r|r|}
$\mathrm{C}(79 \mathrm{~A})$ & $0.938(2)$ & $-0.9851(14)$ & $2.4021(12)$ & $0.071(4)$ \\
$\mathrm{C}(80 \mathrm{~A})$ & $0.820(3)$ & $-1.0123(16)$ & $2.3833(15)$ & $0.104(6)$ \\
$\mathrm{C}(73 \mathrm{~B})$ & $0.4378(8)$ & $-0.7599(5)$ & $2.1728(5)$ & $0.0487(15)$ \\
$\mathrm{C}(74 \mathrm{~B})$ & $0.4327(10)$ & $-0.6549(6)$ & $2.1795(6)$ & $0.066(2)$ \\
$\mathrm{C}(75 \mathrm{~B})$ & $0.5894(10)$ & $-0.6387(6)$ & $2.1538(6)$ & $0.072(2)$ \\
$\mathrm{C}(76 \mathrm{~B})$ & $0.6648(10)$ & $-0.7258(6)$ & $2.1889(6)$ & $0.076(2)$ \\
$\mathrm{C}(77 \mathrm{~B})$ & $0.7763(12)$ & $-0.9183(8)$ & $2.3659(7)$ & $0.095(3)$ \\
$\mathrm{C}(78 \mathrm{~B})$ & $0.9453(11)$ & $-0.9221(7)$ & $2.3814(7)$ & $0.078(2)$ \\
$\mathrm{C}(79 \mathrm{~B})$ & $0.9798(9)$ & $-1.0259(5)$ & $2.3587(5)$ & $0.0589(17)$ \\
$\mathrm{C}(80 \mathrm{~B})$ & $0.9119(8)$ & $-1.0389(5)$ & $2.2823(5)$ & $0.0560(16)$ \\
$\mathrm{N}(1)$ & $1.0330(3)$ & $-0.72240(17)$ & $1.71613(16)$ & $0.0263(6)$ \\
$\mathrm{N}(2)$ & $0.5883(3)$ & $-1.13934(18)$ & $2.06593(17)$ & $0.0281(6)$ \\
$\mathrm{N}(3)$ & $0.7574(3)$ & $-1.36539(18)$ & $2.41050(17)$ & $0.0253(6)$ \\
$\mathrm{O}(1)$ & $0.5703(3)$ & $-0.8020(2)$ & $2.19883(19)$ & $0.0498(7)$ \\
$\mathrm{O}(2)$ & $0.8031(4)$ & $-0.9637(3)$ & $2.2854(2)$ & $0.0654(9)$ \\
$\mathrm{Na}(1)$ & $0.65963(19)$ & $-0.96264(12)$ & $2.19129(10)$ & $0.0483(4)$ \\
$\mathrm{P}(1)$ & $1.00391(9)$ & $-0.82019(5)$ & $1.68195(5)$ & $0.02043(15)$ \\
$\mathrm{P}(2)$ & $0.99294(8)$ & $-0.67575(5)$ & $1.81248(5)$ & $0.01929(15)$ \\
$\mathrm{P}(3)$ & $0.68019(8)$ & $-1.08399(5)$ & $1.98032(5)$ & $0.01933(15)$ \\
$\mathrm{P}(4)$ & $0.42540(8)$ & $-1.12387(5)$ & $2.13291(5)$ & $0.01790(15)$ \\
$\mathrm{P}(5)$ & $0.69893(8)$ & $-1.40196(5)$ & $2.33115(5)$ & $0.02207(16)$ \\
$\mathrm{P}(6)$ & $0.68445(9)$ & $-1.28676(5)$ & $2.48432(5)$ & $0.02216(16)$ \\
$\mathrm{S}(1)$ & $1.00843(10)$ & $-0.93668(5)$ & $1.75937(5)$ & $0.02847(17)$ \\
$\mathrm{S}(2)$ & $0.83284(9)$ & $-0.72358(5)$ & $1.91555(5)$ & $0.02683(16)$ \\
$\mathrm{S}(3)$ & $0.71521(10)$ & $-0.95210(5)$ & $2.00558(5)$ & $0.02942(18)$ \\
$\mathrm{S}(4)$ & $0.41933(10)$ & $-1.07985(5)$ & $2.25701(5)$ & $0.02874(17)$ \\
$\mathrm{S}(5)$ & $0.69155(10)$ & $-1.31626(6)$ & $2.22595(5)$ & $0.03119(18)$ \\
$\mathrm{S}(6)$ & $0.54863(11)$ & $-1.17800(6)$ & $2.45532(6)$ & $0.0366(2)$ \\
$\mathrm{Cu}(1)$ & $0.85493(4)$ & $-0.87823(2)$ & $1.88610(2)$ & $0.02364(8)$ \\
$\mathrm{Cu}(2)$ & $0.55760(4)$ & $-1.19648(3)$ & $2.30964(3)$ & $0.03011(9)$ \\
\hline & & & & \\
& & & \\
& & & \\
& &
\end{tabular}

Tabelle 55: Bindungslängen [pm] in $\underline{13}$

\begin{tabular}{|l|l||l|l|}
\hline $\mathrm{C}(1)-\mathrm{P}(1)$ & $180.6(3)$ & $\mathrm{C}(7)-\mathrm{P}(1)$ & $180.8(3)$ \\
$\mathrm{C}(13)-\mathrm{P}(2)$ & $180.3(3)$ & $\mathrm{C}(19)-\mathrm{P}(2)$ & $181.5(3)$ \\
$\mathrm{C}(25)-\mathrm{P}(3)$ & $180.5(3)$ & $\mathrm{C}(31)-\mathrm{P}(3)$ & $180.4(3)$ \\
$\mathrm{C}(37)-\mathrm{P}(4)$ & $180.0(3)$ & $\mathrm{C}(43)-\mathrm{P}(4)$ & $180.3(3)$ \\
$\mathrm{C}(49)-\mathrm{P}(5)$ & $182.0(3)$ & $\mathrm{C}(55)-\mathrm{P}(5)$ & $181.9(3)$ \\
$\mathrm{C}(61)-\mathrm{P}(6)$ & $181.2(3)$ & $\mathrm{C}(67)-\mathrm{P}(6)$ & $180.9(3)$ \\
$\mathrm{N}(1)-\mathrm{P}(1)$ & $158.0(3)$ & $\mathrm{N}(1)-\mathrm{P}(2)$ & $158.2(2)$ \\
\hline
\end{tabular}


Tabelle 55: Fortsetzung

\begin{tabular}{|l|r||l|r|}
\hline $\mathrm{N}(2)-\mathrm{P}(4)$ & $158.4(3)$ & $\mathrm{N}(2)-\mathrm{P}(3)$ & $158.8(3)$ \\
$\mathrm{N}(3)-\mathrm{P}(5)$ & $158.2(3)$ & $\mathrm{N}(3)-\mathrm{P}(6)$ & $159.4(3)$ \\
$\mathrm{O}(1)-\mathrm{Na}(1)$ & $235.0(3)$ & $\mathrm{O}(2)-\mathrm{Na}(1)$ & $220.3(3)$ \\
$\mathrm{Na}(1)-\mathrm{S}(3)$ & $275.55(16)$ & $\mathrm{Na}(1)-\mathrm{S}(4)$ & $285.69(19)$ \\
$\mathrm{P}(1)-\mathrm{S}(1)$ & $200.16(10)$ & $\mathrm{P}(2)-\mathrm{S}(2)$ & $200.66(11)$ \\
$\mathrm{P}(3)-\mathrm{S}(3)$ & $200.81(11)$ & $\mathrm{P}(4)-\mathrm{S}(4)$ & $201.31(10)$ \\
$\mathrm{P}(5)-\mathrm{S}(5)$ & $199.19(11)$ & $\mathrm{P}(6)-\mathrm{S}(6)$ & $200.60(11)$ \\
$\mathrm{S}(1)-\mathrm{Cu}(1)$ & $221.89(9)$ & $\mathrm{S}(2)-\mathrm{Cu}(1)$ & $224.50(8)$ \\
$\mathrm{S}(3)-\mathrm{Cu}(1)$ & $223.32(9)$ & $\mathrm{S}(4)-\mathrm{Cu}(2)$ & $224.80(9)$ \\
$\mathrm{S}(5)-\mathrm{Cu}(2)$ & $223.87(10)$ & $\mathrm{S}(6)-\mathrm{Cu}(2)$ & $223.83(9)$ \\
\hline
\end{tabular}

Tabelle 56: Bindungswinkel $\left[^{\circ}\right]$ in $\underline{13}$

\begin{tabular}{|l|r||l|r|}
\hline $\mathrm{P}(1)-\mathrm{N}(1)-\mathrm{P}(2)$ & $133.49(17)$ & $\mathrm{P}(4)-\mathrm{N}(2)-\mathrm{P}(3)$ & $135.01(17)$ \\
$\mathrm{P}(5)-\mathrm{N}(3)-\mathrm{P}(6)$ & $130.79(17)$ & $\mathrm{O}(2)-\mathrm{Na}(1)-\mathrm{O}(1)$ & $98.85(13)$ \\
$\mathrm{O}(2)-\mathrm{Na}(1)-\mathrm{S}(3)$ & $133.77(11)$ & $\mathrm{O}(1)-\mathrm{Na}(1)-\mathrm{S}(3)$ & $89.49(9)$ \\
$\mathrm{O}(2)-\mathrm{Na}(1)-\mathrm{S}(4)$ & $111.37(11)$ & $\mathrm{O}(1)-\mathrm{Na}(1)-\mathrm{S}(4)$ & $111.23(10)$ \\
$\mathrm{S}(3)-\mathrm{Na}(1)-\mathrm{S}(4)$ & $107.44(6)$ & $\mathrm{N}(1)-\mathrm{P}(1)-\mathrm{C}(1)$ & $105.15(14)$ \\
$\mathrm{N}(1)-\mathrm{P}(1)-\mathrm{C}(7)$ & $108.96(15)$ & $\mathrm{N}(1)-\mathrm{P}(1)-\mathrm{S}(1)$ & $120.21(10)$ \\
$\mathrm{C}(1)-\mathrm{P}(1)-\mathrm{S}(1)$ & $108.17(10)$ & $\mathrm{C}(7)-\mathrm{P}(1)-\mathrm{S}(1)$ & $110.20(11)$ \\
$\mathrm{N}(1)-\mathrm{P}(2)-\mathrm{S}(2)$ & $120.76(11)$ & $\mathrm{C}(13)-\mathrm{P}(2)-\mathrm{S}(2)$ & $108.05(11)$ \\
$\mathrm{C}(19)-\mathrm{P}(2)-\mathrm{S}(2)$ & $108.98(11)$ & $\mathrm{N}(2)-\mathrm{P}(3)-\mathrm{S}(3)$ & $114.38(11)$ \\
$\mathrm{N}(2)-\mathrm{P}(4)-\mathrm{S}(4)$ & $115.05(11)$ & $\mathrm{C}(37)-\mathrm{P}(4)-\mathrm{S}(4)$ & $109.87(10)$ \\
$\mathrm{C}(43)-\mathrm{P}(4)-\mathrm{S}(4)$ & $107.37(10)$ & $\mathrm{N}(3)-\mathrm{P}(5)-\mathrm{S}(5)$ & $118.96(11)$ \\
$\mathrm{C}(55)-\mathrm{P}(5)-\mathrm{S}(5)$ & $109.20(13)$ & $\mathrm{C}(49)-\mathrm{P}(5)-\mathrm{S}(5)$ & $109.41(10)$ \\
$\mathrm{N}(3)-\mathrm{P}(6)-\mathrm{S}(6)$ & $119.81(10)$ & $\mathrm{C}(67)-\mathrm{P}(6)-\mathrm{S}(6)$ & $108.40(11)$ \\
$\mathrm{C}(61)-\mathrm{P}(6)-\mathrm{S}(6)$ & $110.18(10)$ & $\mathrm{P}(1)-\mathrm{S}(1)-\mathrm{Cu}(1)$ & $97.56(4)$ \\
$\mathrm{P}(2)-\mathrm{S}(2)-\mathrm{Cu}(1)$ & $101.84(4)$ & $\mathrm{P}(3)-\mathrm{S}(3)-\mathrm{Cu}(1)$ & $113.79(4)$ \\
$\mathrm{P}(3)-\mathrm{S}(3)-\mathrm{Na}(1)$ & $100.08(5)$ & $\mathrm{Cu}(1)-\mathrm{S}(3)-\mathrm{Na}(1)$ & $140.47(5)$ \\
$\mathrm{P}(4)-\mathrm{S}(4)-\mathrm{Cu}(2)$ & $103.18(4)$ & $\mathrm{P}(4)-\mathrm{S}(4)-\mathrm{Na}(1)$ & $93.78(5)$ \\
$\mathrm{Cu}(2)-\mathrm{S}(4)-\mathrm{Na}(1)$ & $95.64(5)$ & $\mathrm{P}(5)-\mathrm{S}(5)-\mathrm{Cu}(2)$ & $95.02(4)$ \\
$\mathrm{P}(6)-\mathrm{S}(6)-\mathrm{Cu}(2)$ & $104.43(4)$ & $\mathrm{S}(1)-\mathrm{Cu}(1)-\mathrm{S}(3)$ & $130.00(3)$ \\
$\mathrm{S}(1)-\mathrm{Cu}(1)-\mathrm{S}(2)$ & $121.36(3)$ & $\mathrm{S}(3)-\mathrm{Cu}(1)-\mathrm{S}(2)$ & $108.50(3)$ \\
$\mathrm{S}(6)-\mathrm{Cu}(2)-\mathrm{S}(5)$ & $123.75(3)$ & $\mathrm{S}(6)-\mathrm{Cu}(2)-\mathrm{S}(4)$ & $112.14(3)$ \\
$\mathrm{S}(5)-\mathrm{Cu}(2)-\mathrm{S}(4)$ & $124.10(3)$ & & \\
\hline
\end{tabular}




\subsection{Daten zur Kristallstrukturanalyse von $\left[\mathrm{NaCu}_{2}\left(\operatorname{dppaS}_{2}\right)_{3}(\text { thf })_{2}\right] \underline{13}$}

\begin{tabular}{|ll|}
\hline Summenformel & $\mathrm{C}_{80} \mathrm{H}_{76} \mathrm{Cu}_{2} \mathrm{~N}_{3} \mathrm{NaO}_{2} \mathrm{P}_{6} \mathrm{~S}_{6}$ \\
Molekulargewicht $\left[\mathrm{g} \cdot \mathrm{mol}^{-1}\right]$ & 1639.69 \\
Temperatur & $193(2) \mathrm{K}$ \\
Wellenlänge & $71.073 \mathrm{pm}$ \\
Kristallsystem & monoklin \\
Raumgruppe & $P 2_{1} / c$ \\
Gitterkonstanten & $\mathrm{a}=9.4830(10) \AA, \alpha=90^{\circ}$ \\
& $\mathrm{b}=27.999(3) \AA, \beta=97.690(10)^{\circ}$ \\
& $\mathrm{c}=29.697(3) \AA, \gamma=90^{\circ}$ \\
Volumen & $7814.1(14) \AA^{3}$ \\
Formeleinheiten & 4 \\
Röntgenographische Dichte $\left[\mathrm{g} \cdot \mathrm{cm}^{-3}\right]$ & 1.394 \\
Absorptionskoeffizient $\left[\mathrm{mm}^{-1}\right]$ & 0.881 \\
$\mathrm{~F}(000)$ & 3392 \\
Meßbereich $\theta$ & $2.17-24.00^{\circ}$ \\
Indexbereiche & $-10 \leq \mathrm{h} \leq 10$ \\
& $-28 \leq \mathrm{k} \leq 32$ \\
& $-33 \leq 1 \leq 33$ \\
Gemessene Reflexe & 72374 \\
Unabhängige Reflexe & 12255 \\
Beobachtete Reflexe $[\mathrm{I}>2 \sigma]$ & 10080 \\
$\mathrm{R}_{\text {int }}$ & 0.0463 \\
Parameter & 885 \\
R1; wR2[I>2 $\sigma(\mathrm{I})]$ & $0.0462 ; 0.0962$ \\
\hline
\end{tabular}


Tabelle 58: Atomkoordinaten und äquivalente isotrope Auslenkungsparameter $\left[\AA^{2}\right]$ für $\underline{13}$

\begin{tabular}{|c|c|c|c|c|}
\hline Atom & $\mathrm{x}$ & $\mathrm{y}$ & $\mathrm{Z}$ & $\mathrm{U}_{e q}$ \\
\hline $\mathrm{C}(1)$ & $0.2343(4)$ & $0.87603(13)$ & $0.46677(12)$ & $0.0350(8)$ \\
\hline $\mathrm{C}(2)$ & $0.2818(4)$ & $0.90721(15)$ & $0.50122(14)$ & $0.0489(10)$ \\
\hline $\mathrm{C}(3)$ & $0.3417(5)$ & $0.9507(2)$ & $0.4915(2)$ & $0.0592(12)$ \\
\hline $\mathrm{C}(4)$ & $0.3543(5)$ & $0.9630(2)$ & $0.4478(2)$ & $0.0552(11)$ \\
\hline $\mathrm{C}(5)$ & $0.3098(5)$ & $0.93223(15)$ & $0.4133(2)$ & $0.0549(11)$ \\
\hline $\mathrm{C}(6)$ & $0.2494(4)$ & $0.88884(14)$ & $0.42251(13)$ & $0.0463(10)$ \\
\hline $\mathrm{C}(7)$ & $-0.0353(4)$ & $0.82984(13)$ & $0.47450(12)$ & $0.0358(8)$ \\
\hline $\mathrm{C}(8)$ & $-0.0935(5)$ & $0.8471(2)$ & $0.51123(15)$ & $0.0594(12)$ \\
\hline $\mathrm{C}(9)$ & $-0.2397(6)$ & $0.8551(2)$ & $0.5078(2)$ & $0.083(2)$ \\
\hline $\mathrm{C}(10)$ & $-0.3248(6)$ & $0.8457(2)$ & $0.4683(3)$ & $0.083(2)$ \\
\hline $\mathrm{C}(11)$ & $-0.2688(5)$ & $0.8288(2)$ & $0.4319(2)$ & $0.075(2)$ \\
\hline $\mathrm{C}(12)$ & $-0.1254(5)$ & $0.8207(2)$ & $0.4347(2)$ & $0.0549(11)$ \\
\hline $\mathrm{C}(13)$ & $0.1289(4)$ & $0.78387(13)$ & $0.61177(12)$ & $0.0371(9)$ \\
\hline $\mathrm{C}(14)$ & $0.1695(7)$ & $0.8264(2)$ & $0.6309(2)$ & $0.084(2)$ \\
\hline $\mathrm{C}(15)$ & $0.1184(7)$ & $0.8431(2)$ & $0.6692(2)$ & $0.082(2)$ \\
\hline $\mathrm{C}(16)$ & $0.0236(5)$ & $0.8185(2)$ & $0.6879(2)$ & $0.073(2)$ \\
\hline $\mathrm{C}(17)$ & $-0.0195(8)$ & $0.7765(3)$ & $0.6694(3)$ & $0.140(4)$ \\
\hline $\mathrm{C}(18)$ & $0.0322(7)$ & $0.7592(2)$ & $0.6312(2)$ & $0.101(2)$ \\
\hline $\mathrm{C}(19)$ & $0.3815(4)$ & $0.74561(14)$ & $0.58385(12)$ & $0.0411(9)$ \\
\hline $\mathrm{C}(20)$ & $0.4953(5)$ & $0.7611(2)$ & $0.56372(15)$ & $0.0577(12)$ \\
\hline $\mathrm{C}(21)$ & $0.6320(5)$ & $0.7469(2)$ & $0.5802(2)$ & $0.0732(15)$ \\
\hline $\mathrm{C}(22)$ & $0.6551(6)$ & $0.7170(2)$ & $0.6169(2)$ & $0.080(2)$ \\
\hline $\mathrm{C}(23)$ & $0.5449(6)$ & $0.7019(3)$ & $0.6373(2)$ & $0.108(2)$ \\
\hline $\mathrm{C}(24)$ & $0.4081(5)$ & $0.7162(2)$ & $0.6209(2)$ & $0.085(2)$ \\
\hline $\mathrm{C}(25)$ & $-0.0251(4)$ & $0.68956(12)$ & $0.34147(12)$ & $0.0316(8)$ \\
\hline $\mathrm{C}(26)$ & $0.0049(5)$ & $0.7178(2)$ & $0.3061(2)$ & $0.0566(12)$ \\
\hline $\mathrm{C}(27)$ & $-0.0866(5)$ & $0.7541(2)$ & $0.2896(2)$ & $0.075(2)$ \\
\hline $\mathrm{C}(28)$ & $-0.2092(5)$ & $0.7620(2)$ & $0.3074(2)$ & $0.0580(12)$ \\
\hline $\mathrm{C}(29)$ & $-0.2416(5)$ & $0.7340(2)$ & $0.3417(2)$ & $0.0543(11)$ \\
\hline $\mathrm{C}(30)$ & $-0.1502(4)$ & $0.69814(15)$ & $0.35911(14)$ & $0.0482(10)$ \\
\hline $\mathrm{C}(31)$ & $0.0025(3)$ & $0.58688(12)$ & $0.33790(12)$ & $0.0309(8)$ \\
\hline $\mathrm{C}(32)$ & $0.0343(4)$ & $0.56995(14)$ & $0.29653(13)$ & $0.0401(9)$ \\
\hline $\mathrm{C}(33)$ & $-0.0340(4)$ & $0.5302(2)$ & $0.27652(15)$ & $0.0517(11)$ \\
\hline $\mathrm{C}(34)$ & $-0.1371(4)$ & $0.5077(2)$ & $0.2972(2)$ & $0.0554(12)$ \\
\hline $\mathrm{C}(35)$ & $-0.1693(4)$ & $0.52384(14)$ & $0.3382(2)$ & $0.0493(11)$ \\
\hline $\mathrm{C}(36)$ & $-0.0995(4)$ & $0.56309(13)$ & $0.35894(13)$ & $0.0384(9)$ \\
\hline $\mathrm{C}(37)$ & $0.4692(4)$ & $0.63878(12)$ & $0.29971(11)$ & $0.0295(8)$ \\
\hline $\mathrm{C}(38)$ & $0.4022(5)$ & $0.6673(2)$ & $0.26590(14)$ & $0.0522(11)$ \\
\hline
\end{tabular}


Tabelle 58: Fortsetzung

\begin{tabular}{|c|c|c|c|c|}
\hline $\mathrm{C}(39)$ & $0.4715(6)$ & $0.6797(2)$ & $0.2291(2)$ & $0.073(2)$ \\
\hline $\mathrm{C}(40)$ & $0.6054(5)$ & $0.6635(2)$ & $0.22617(15)$ & $0.0543(12)$ \\
\hline $\mathrm{C}(41)$ & $0.6709(4)$ & $0.6342(2)$ & $0.25907(15)$ & $0.0560(12)$ \\
\hline $\mathrm{C}(42)$ & $0.6034(4)$ & $0.6219(2)$ & $0.29555(14)$ & $0.0514(11)$ \\
\hline $\mathrm{C}(43)$ & $0.4947(4)$ & $0.64808(12)$ & $0.39730(11)$ & $0.0311(8)$ \\
\hline $\mathrm{C}(44)$ & $0.4655(4)$ & $0.69306(14)$ & $0.41214(13)$ & $0.0435(10)$ \\
\hline $\mathrm{C}(45)$ & $0.5527(5)$ & $0.7140(2)$ & $0.4479(2)$ & $0.0632(13)$ \\
\hline $\mathrm{C}(46)$ & $0.6705(5)$ & $0.6905(2)$ & $0.4680(2)$ & $0.0692(15)$ \\
\hline $\mathrm{C}(47)$ & $0.7005(4)$ & $0.6454(2)$ & $0.45400(14)$ & $0.0574(12)$ \\
\hline $\mathrm{C}(48)$ & $0.6118(4)$ & $0.6236(2)$ & $0.41877(12)$ & $0.0401(9)$ \\
\hline $\mathrm{C}(49)$ & $0.2465(4)$ & $0.49928(12)$ & $0.13006(12)$ & $0.0344(8)$ \\
\hline $\mathrm{C}(50)$ & $0.2713(5)$ & $0.4685(2)$ & $0.09587(13)$ & $0.0511(11)$ \\
\hline $\mathrm{C}(51)$ & $0.1679(5)$ & $0.4592(2)$ & $0.05981(15)$ & $0.0604(12)$ \\
\hline $\mathrm{C}(52)$ & $0.0385(5)$ & $0.4803(2)$ & $0.0579(2)$ & $0.0665(14)$ \\
\hline $\mathrm{C}(53)$ & $0.0125(5)$ & $0.5115(2)$ & $0.0907(2)$ & $0.093(2)$ \\
\hline $\mathrm{C}(54)$ & $0.1164(5)$ & $0.5206(2)$ & $0.1271(2)$ & $0.0703(15)$ \\
\hline $\mathrm{C}(55)$ & $0.5167(4)$ & $0.54293(12)$ & $0.15700(12)$ & $0.0327(8)$ \\
\hline $\mathrm{C}(56)$ & $0.4799(4)$ & $0.57902(14)$ & $0.12599(13)$ & $0.0455(10)$ \\
\hline $\mathrm{C}(57)$ & $0.5824(5)$ & $0.6085(2)$ & $0.1120(2)$ & $0.0597(12)$ \\
\hline $\mathrm{C}(58)$ & $0.7224(5)$ & $0.6024(2)$ & $0.1290(2)$ & $0.0631(13)$ \\
\hline $\mathrm{C}(59)$ & $0.7611(5)$ & $0.5668(2)$ & $0.1596(2)$ & $0.0629(13)$ \\
\hline $\mathrm{C}(60)$ & $0.6595(4)$ & $0.5370(2)$ & $0.17370(14)$ & $0.0495(10)$ \\
\hline $\mathrm{C}(61)$ & $0.5256(4)$ & $0.36832(12)$ & $0.21925(12)$ & $0.0353(8)$ \\
\hline $\mathrm{C}(62)$ & $0.5323(4)$ & $0.35657(14)$ & $0.17437(13)$ & $0.0443(10)$ \\
\hline $\mathrm{C}(63)$ & $0.5955(5)$ & $0.31521(14)$ & $0.1629(2)$ & $0.0519(11)$ \\
\hline $\mathrm{C}(64)$ & $0.6556(5)$ & $0.2853(2)$ & $0.1954(2)$ & $0.0657(13)$ \\
\hline $\mathrm{C}(65)$ & $0.6551(7)$ & $0.2965(2)$ & $0.2402(2)$ & $0.082(2)$ \\
\hline $\mathrm{C}(66)$ & $0.5892(5)$ & $0.33802(15)$ & $0.25243(15)$ & $0.0599(12)$ \\
\hline $\mathrm{C}(67)$ & $0.2467(4)$ & $0.40507(13)$ & $0.22922(14)$ & $0.0435(10)$ \\
\hline $\mathrm{C}(68)$ & $0.1782(6)$ & $0.4008(2)$ & $0.2663(2)$ & $0.096(2)$ \\
\hline $\mathrm{C}(69)$ & $0.0323(8)$ & $0.3900(3)$ & $0.2609(3)$ & $0.134(4)$ \\
\hline $\mathrm{C}(70)$ & $-0.0386(7)$ & $0.3837(2)$ & $0.2185(4)$ & $0.117(3)$ \\
\hline $\mathrm{C}(71)$ & $0.0314(7)$ & $0.3858(3)$ & $0.1817(3)$ & $0.119(3)$ \\
\hline $\mathrm{C}(72)$ & $0.1730(6)$ & $0.3970(2)$ & $0.1870(2)$ & $0.080(2)$ \\
\hline $\mathrm{C}(73)$ & $0.3975(9)$ & $0.6028(3)$ & $0.5198(3)$ & $0.122(2)$ \\
\hline $\mathrm{C}(74 \mathrm{~A})$ & $0.4862(18)$ & $0.5983(6)$ & $0.5552(6)$ & $0.123(5)$ \\
\hline $\mathrm{C}(75 \mathrm{~A})$ & $0.4519(16)$ & $0.5526(5)$ & $0.5775(5)$ & $0.096(4)$ \\
\hline$C(76 \mathrm{~A})$ & $0.4048(15)$ & $0.5250(4)$ & $0.5403(4)$ & $0.080(4)$ \\
\hline $\mathrm{C}(74 \mathrm{~B})$ & $0.3962(18)$ & $0.6014(6)$ & $0.5739(5)$ & $0.112(5)$ \\
\hline $\mathrm{C}(75 \mathrm{~B})$ & $0.3631(21)$ & $0.5564(7)$ & $0.5801(6)$ & $0.136(6)$ \\
\hline
\end{tabular}


Tabelle 58: Fortsetzung

\begin{tabular}{|r|r|r|r|r|}
\hline $\mathrm{C}(76 \mathrm{~B})$ & $0.3335(16)$ & $0.5270(5)$ & $0.5451(5)$ & $0.090(4)$ \\
$\mathrm{C}(77 \mathrm{~A})$ & $0.1392(19)$ & $0.4402(6)$ & $0.4602(6)$ & $0.119(5)$ \\
$\mathrm{C}(78 \mathrm{~A})$ & $0.1279(17)$ & $0.3926(5)$ & $0.4307(5)$ & $0.113(5)$ \\
$\mathrm{C}(79 \mathrm{~A})$ & $0.0963(18)$ & $0.4007(5)$ & $0.3880(5)$ & $0.108(5)$ \\
$\mathrm{C}(78 \mathrm{~B})$ & $0.0175(16)$ & $0.4158(5)$ & $0.4443(5)$ & $0.108(4)$ \\
$\mathrm{C}(77 \mathrm{~B})$ & $0.0674(17)$ & $0.4594(6)$ & $0.4575(5)$ & $0.106(5)$ \\
$\mathrm{C}(79 \mathrm{~B})$ & $0.0159(21)$ & $0.4137(7)$ & $0.3998(7)$ & $0.146(7)$ \\
$\mathrm{C}(80)$ & $0.1474(8)$ & $0.4478(3)$ & $0.3853(2)$ & $0.105(2)$ \\
$\mathrm{N}(1)$ & $0.2169(4)$ & $0.80854(11)$ & $0.53011(10)$ & $0.0448(8)$ \\
$\mathrm{N}(2)$ & $0.2339(3)$ & $0.64924(10)$ & $0.34118(9)$ & $0.0316(7)$ \\
$\mathrm{N}(3)$ & $0.4552(3)$ & $0.45724(10)$ & $0.19229(10)$ & $0.0392(7)$ \\
$\mathrm{Cu}(1)$ & $0.13005(5)$ & $0.70667(2)$ & $0.46335(2)$ & $0.03859(12)$ \\
$\mathrm{Cu}(2)$ & $0.39872(5)$ & $0.51388(2)$ & $0.290867(15)$ & $0.03806(12)$ \\
$\mathrm{Na}(1)$ & $0.2459(3)$ & $0.54895(6)$ & $0.43289(6)$ & $0.0763(6)$ \\
$\mathrm{S}(1)$ & $0.19224(12)$ & $0.77333(4)$ & $0.43020(4)$ & $0.0483(3)$ \\
$\mathrm{S}(2)$ & $0.09020(12)$ & $0.70630(4)$ & $0.53653(3)$ & $0.0468(3)$ \\
$\mathrm{S}(3)$ & $0.09516(10)$ & $0.63465(3)$ & $0.43013(3)$ & $0.0362(2)$ \\
$\mathrm{S}(4)$ & $0.39293(11)$ & $0.55141(3)$ & $0.35778(3)$ & $0.0400(2)$ \\
$\mathrm{S}(5)$ & $0.29820(12)$ & $0.54544(3)$ & $0.22569(3)$ & $0.0435(2)$ \\
$\mathrm{S}(6)$ & $0.50481(12)$ & $0.44194(4)$ & $0.29639(3)$ & $0.0446(2)$ \\
$\mathrm{P}(1)$ & $0.15499(10)$ & $0.81931(3)$ & $0.47875(3)$ & $0.0325(2)$ \\
$\mathrm{P}(2)$ & $0.20183(11)$ & $0.76333(3)$ & $0.56158(3)$ & $0.0363(2)$ \\
$\mathrm{P}(3)$ & $0.08969(9)$ & $0.64086(3)$ & $0.36201(3)$ & $0.0279(2)$ \\
$\mathrm{P}(4)$ & $0.38409(9)$ & $0.62287(3)$ & $0.34836(3)$ & $0.0274(2)$ \\
$\mathrm{P}(5)$ & $0.38211(10)$ & $0.50744(3)$ & $0.17858(3)$ & $0.0312(2)$ \\
$\mathrm{P}(6)$ & $0.43303(10)$ & $0.42208(3)$ & $0.23262(3)$ & $0.0309(2)$ \\
$\mathrm{O}(1)$ & $0.3478(6)$ & $0.5565(2)$ & $0.50518(12)$ & $0.114(2)$ \\
$\mathrm{O}(2)$ & $0.1617(5)$ & $0.47427(13)$ & $0.42677(13)$ & $0.0901(12)$ \\
\hline & & & &
\end{tabular}

Tabelle 59: Bindungslängen [pm] in $\underline{13}$

\begin{tabular}{|l|r||l|r|}
\hline $\mathrm{Cu}(1)-\mathrm{S}(1)$ & $222.62(11)$ & $\mathrm{Cu}(1)-\mathrm{S}(3)$ & $224.99(10)$ \\
$\mathrm{Cu}(1)-\mathrm{S}(2)$ & $225.51(11)$ & $\mathrm{Cu}(2)-\mathrm{S}(5)$ & $222.32(11)$ \\
$\mathrm{Cu}(2)-\mathrm{S}(6)$ & $224.76(11)$ & $\mathrm{Cu}(2)-\mathrm{S}(4)$ & $225.50(10)$ \\
$\mathrm{Na}(1)-\mathrm{O}(2)$ & $223.7(4)$ & $\mathrm{Na}(1)-\mathrm{O}(1)$ & $224.3(4)$ \\
$\mathrm{Na}(1)-\mathrm{S}(4)$ & $278.5(2)$ & $\mathrm{Na}(1)-\mathrm{S}(3)$ & $278.9(2)$ \\
$\mathrm{S}(1)-\mathrm{P}(1)$ & $199.96(13)$ & $\mathrm{S}(2)-\mathrm{P}(2)$ & $200.32(14)$ \\
$\mathrm{S}(3)-\mathrm{P}(3)$ & $202.43(13)$ & $\mathrm{S}(4)-\mathrm{P}(4)$ & $202.05(13)$ \\
$\mathrm{S}(5)-\mathrm{P}(5)$ & $200.52(13)$ & $\mathrm{S}(6)-\mathrm{P}(6)$ & $200.34(13)$ \\
$\mathrm{C}(1)-\mathrm{P}(1)$ & $181.3(4)$ & $\mathrm{C}(7)-\mathrm{P}(1)$ & $181.6(4)$ \\
\hline
\end{tabular}


Tabelle 59: Fortsetzung

\begin{tabular}{|l|l||l|l|}
\hline $\mathrm{C}(80)-\mathrm{O}(2)$ & $142.8(7)$ & $\mathrm{N}(1)-\mathrm{P}(1)$ & $158.8(3)$ \\
$\mathrm{N}(1)-\mathrm{P}(2)$ & $159.1(3)$ & $\mathrm{N}(2)-\mathrm{P}(3)$ & $159.2(3)$ \\
$\mathrm{N}(2)-\mathrm{P}(4)$ & $159.3(3)$ & $\mathrm{N}(3)-\mathrm{P}(6)$ & $158.6(3)$ \\
$\mathrm{N}(3)-\mathrm{P}(5)$ & $159.6(3)$ & & \\
\hline
\end{tabular}

Tabelle 60: Bindungswinkel $\left[^{\circ}\right]$ in $\underline{13}$

\begin{tabular}{|l|r||l|r|}
\hline $\mathrm{S}(1)-\mathrm{Cu}(1)-\mathrm{S}(3)$ & $126.13(4)$ & $\mathrm{S}(1)-\mathrm{Cu}(1)-\mathrm{S}(2)$ & $121.23(4)$ \\
$\mathrm{S}(3)-\mathrm{Cu}(1)-\mathrm{S}(2)$ & $112.62(4)$ & $\mathrm{S}(5)-\mathrm{Cu}(2)-\mathrm{S}(6)$ & $123.73(4)$ \\
$\mathrm{S}(5)-\mathrm{Cu}(2)-\mathrm{S}(4)$ & $121.59(4)$ & $\mathrm{S}(6)-\mathrm{Cu}(2)-\mathrm{S}(4)$ & $114.63(4)$ \\
$\mathrm{O}(2)-\mathrm{Na}(1)-\mathrm{O}(1)$ & $105.6(2)$ & $\mathrm{O}(2)-\mathrm{Na}(1)-\mathrm{S}(4)$ & $99.76(12)$ \\
$\mathrm{O}(1)-\mathrm{Na}(1)-\mathrm{S}(4)$ & $124.5(2)$ & $\mathrm{O}(2)-\mathrm{Na}(1)-\mathrm{S}(3)$ & $128.74(15)$ \\
$\mathrm{O}(1)-\mathrm{Na}(1)-\mathrm{S}(3)$ & $95.79(12)$ & $\mathrm{S}(4)-\mathrm{Na}(1)-\mathrm{S}(3)$ & $105.36(6)$ \\
$\mathrm{P}(1)-\mathrm{S}(1)-\mathrm{Cu}(1)$ & $97.94(5)$ & $\mathrm{P}(2)-\mathrm{S}(2)-\mathrm{Cu}(1)$ & $101.88(5)$ \\
$\mathrm{P}(3)-\mathrm{S}(3)-\mathrm{Cu}(1)$ & $110.10(5)$ & $\mathrm{P}(3)-\mathrm{S}(3)-\mathrm{Na}(1)$ & $92.74(5)$ \\
$\mathrm{Cu}(1)-\mathrm{S}(3)-\mathrm{Na}(1)$ & $135.53(7)$ & $\mathrm{P}(4)-\mathrm{S}(4)-\mathrm{Cu}(2)$ & $110.17(5)$ \\
$\mathrm{P}(4)-\mathrm{S}(4)-\mathrm{Na}(1)$ & $96.86(6)$ & $\mathrm{Cu}(2)-\mathrm{S}(4)-\mathrm{Na}(1)$ & $140.19(7)$ \\
$\mathrm{P}(5)-\mathrm{S}(5)-\mathrm{Cu}(2)$ & $103.45(5)$ & $\mathrm{P}(6)-\mathrm{S}(6)-\mathrm{Cu}(2)$ & $95.03(5)$ \\
$\mathrm{N}(1)-\mathrm{P}(1)-\mathrm{S}(1)$ & $119.47(14)$ & $\mathrm{C}(1)-\mathrm{P}(1)-\mathrm{S}(1)$ & $107.73(13)$ \\
$\mathrm{C}(7)-\mathrm{P}(1)-\mathrm{S}(1)$ & $108.91(13)$ & $\mathrm{N}(1)-\mathrm{P}(2)-\mathrm{S}(2)$ & $120.03(12)$ \\
$\mathrm{C}(19)-\mathrm{P}(2)-\mathrm{S}(2)$ & $109.98(14)$ & $\mathrm{C}(13)-\mathrm{P}(2)-\mathrm{S}(2)$ & $108.45(13)$ \\
$\mathrm{N}(2)-\mathrm{P}(3)-\mathrm{S}(3)$ & $119.51(11)$ & $\mathrm{C}(25)-\mathrm{P}(3)-\mathrm{S}(3)$ & $109.71(12)$ \\
$\mathrm{C}(31)-\mathrm{P}(3)-\mathrm{S}(3)$ & $105.72(12)$ & $\mathrm{N}(2)-\mathrm{P}(4)-\mathrm{S}(4)$ & $119.81(12)$ \\
$\mathrm{C}(37)-\mathrm{P}(4)-\mathrm{S}(4)$ & $109.94(12)$ & $\mathrm{C}(43)-\mathrm{P}(4)-\mathrm{S}(4)$ & $105.37(12)$ \\
$\mathrm{N}(3)-\mathrm{P}(5)-\mathrm{S}(5)$ & $119.26(12)$ & $\mathrm{C}(55)-\mathrm{P}(5)-\mathrm{S}(5)$ & $108.75(12)$ \\
$\mathrm{C}(49)-\mathrm{P}(5)-\mathrm{S}(5)$ & $108.42(13)$ & $\mathrm{N}(3)-\mathrm{P}(6)-\mathrm{C}(67)$ & $110.3(2)$ \\
$\mathrm{N}(3)-\mathrm{P}(6)-\mathrm{S}(6)$ & $118.61(13)$ & $\mathrm{C}(61)-\mathrm{P}(6)-\mathrm{S}(6)$ & $108.93(13)$ \\
$\mathrm{C}(67)-\mathrm{P}(6)-\mathrm{S}(6)$ & $109.25(14)$ & $\mathrm{C}(76 \mathrm{~A})-\mathrm{O}(1)-\mathrm{Na}(1)$ & $136.0(6)$ \\
$\mathrm{C}(73)-\mathrm{O}(1)-\mathrm{Na}(1)$ & $117.3(4)$ & $\mathrm{C}(76 \mathrm{~B})-\mathrm{O}(1)-\mathrm{Na}(1)$ & $130.3(7)$ \\
$\mathrm{C}(77 \mathrm{~A})-\mathrm{O}(2)-\mathrm{Na}(1)$ & $131.3(7)$ & $\mathrm{C}(77 \mathrm{~B})-\mathrm{O}(2)-\mathrm{Na}(1)$ & $118.0(7)$ \\
$\mathrm{C}(80)-\mathrm{O}(2)-\mathrm{Na}(1)$ & $123.2(4)$ & $\mathrm{P}(1)-\mathrm{N}(1)-\mathrm{P}(2)$ & $131.6(2)$ \\
$\mathrm{P}(3)-\mathrm{N}(2)-\mathrm{P}(4)$ & $132.8(2)$ & $\mathrm{P}(6)-\mathrm{N}(3)-\mathrm{P}(5)$ & $130.0(2)$ \\
\hline
\end{tabular}




\subsection{Daten zur Kristallstrukturanalyse von $\left[\mathrm{NaCu}_{2}\left(\mathrm{dppaSe}_{2}\right)_{3}(\text { thf })_{2}\right] \underline{14}$}

\begin{tabular}{|ll|}
\hline Summenformel & $\mathrm{C}_{80} \mathrm{H}_{76} \mathrm{Cu}_{2} \mathrm{~N}_{3} \mathrm{NaO}_{2} \mathrm{P}_{6} \mathrm{Se}_{6}$ \\
Molekulargewicht $\left[\mathrm{g} \cdot \mathrm{mol}^{-1}\right]$ & 1921.09 \\
Temperatur & $133(2) \mathrm{K}$ \\
Wellenlänge & $71.073 \mathrm{pm}$ \\
Kristallsystem & monoklin \\
Raumgruppe & $P 2_{1} / c$ \\
Gitterkonstanten & $\mathrm{a}=39.389(3) \AA, \alpha=90^{\circ}$ \\
& $\mathrm{b}=9.5029(7) \AA, \beta=97.355(4)^{\circ}$ \\
& $\mathrm{c}=21.3806(18) \AA, \gamma=90^{\circ}$ \\
& $7937.1(11) \AA^{3}$ \\
Volumen & 4 \\
Formeleinheiten & 1.608 \\
Röntgenographische Dichte $\left[\mathrm{g} \cdot \mathrm{cm}^{-3}\right]$ & 3.462 \\
Absorptionskoeffizient $\left[\mathrm{mm}{ }^{-1}\right]$ & 3824 \\
F(000) & $2.09-22.50^{\circ}$ \\
Meßbereich $\theta$ & $-42 \leq \mathrm{h} \leq 42$ \\
Indexbereiche & $-10 \leq \mathrm{k} \leq 8$ \\
& $-23 \leq 1 \leq 23$ \\
Gemessene Reflexe & 66669 \\
Unabhängige Reflexe & 10373 \\
Beobachtete Reflexe $[\mathrm{I}>2 \sigma]$ & 7379 \\
$\mathrm{R}_{\text {int }}$ & 0.1116 \\
Parameter & 849 \\
R1; wR2[I>2 $\sigma(\mathrm{I})]$ & $0.0620 ; 0.1151$ \\
\hline
\end{tabular}


Tabelle 62: Atomkoordinaten und äquivalente isotrope Auslenkungsparameter $\left[\AA^{2}\right]$ für $\underline{14}$

\begin{tabular}{|r|r|r|r|r|}
\hline Atom & \multicolumn{1}{|c|}{$\mathrm{x}$} & \multicolumn{1}{c|}{$\mathrm{y}$} & $\mathrm{z}$ & \multicolumn{1}{c|}{$\mathrm{U}_{e q}$} \\
\hline $\mathrm{Se}(1)$ & $0.11332(2)$ & $0.94573(10)$ & $0.17846(4)$ & $0.0268(2)$ \\
$\mathrm{Se}(2)$ & $0.14198(2)$ & $0.76182(11)$ & $0.01814(4)$ & $0.0305(2)$ \\
$\mathrm{Se}(3)$ & $0.21498(2)$ & $0.90195(11)$ & $0.14526(5)$ & $0.0341(3)$ \\
$\mathrm{Se}(4)$ & $0.30540(3)$ & $1.13270(13)$ & $0.14503(5)$ & $0.0456(3)$ \\
$\mathrm{Se}(5)$ & $0.40960(3)$ & $1.02672(15)$ & $0.18275(5)$ & $0.0541(4)$ \\
$\mathrm{Se}(6)$ & $0.34997(3)$ & $0.94619(12)$ & $0.01358(5)$ & $0.0441(3)$ \\
$\mathrm{Cu}(1)$ & $0.15534(3)$ & $0.87606(12)$ & $0.11670(5)$ & $0.0258(3)$ \\
$\mathrm{Cu}(2)$ & $0.35711(3)$ & $1.02808(14)$ & $0.11831(6)$ & $0.0427(3)$ \\
$\mathrm{P}(1)$ & $0.07496(6)$ & $0.7985(2)$ & $0.13646(10)$ & $0.0205(5)$ \\
$\mathrm{P}(2)$ & $0.08879(6)$ & $0.8271(2)$ & $0.00713(10)$ & $0.0213(5)$ \\
$\mathrm{P}(3)$ & $0.22162(6)$ & $1.0680(3)$ & $0.21589(11)$ & $0.0245(6)$ \\
$\mathrm{P}(4)$ & $0.29576(6)$ & $1.0460(3)$ & $0.23514(12)$ & $0.0281(6)$ \\
$\mathrm{P}(5)$ & $0.44126(7)$ & $0.9747(3)$ & $0.10998(12)$ & $0.0429(7)$ \\
$\mathrm{P}(6)$ & $0.39447(7)$ & $0.8069(3)$ & $0.02132(14)$ & $0.0443(7)$ \\
$\mathrm{Na}(1)$ & $0.26999(12)$ & $0.9242(7)$ & $0.0600(2)$ & $0.0793(17)$ \\
$\mathrm{N}(1)$ & $0.06623(17)$ & $0.7922(8)$ & $0.0619(3)$ & $0.0263(18)$ \\
$\mathrm{N}(2)$ & $0.25882(17)$ & $1.0823(8)$ & $0.2545(3)$ & $0.0272(18)$ \\
$\mathrm{N}(3)$ & $0.42906(19)$ & $0.8518(9)$ & $0.0620(4)$ & $0.042(2)$ \\
$\mathrm{C}(1)$ & $0.0347(2)$ & $0.8456(9)$ & $0.1635(4)$ & $0.022(2)$ \\
$\mathrm{C}(2)$ & $0.0142(2)$ & $0.7500(10)$ & $0.1905(4)$ & $0.026(2)$ \\
$\mathrm{C}(3)$ & $-0.0173(2)$ & $0.7924(10)$ & $0.2054(4)$ & $0.033(2)$ \\
$\mathrm{C}(4)$ & $-0.0285(2)$ & $0.9292(10)$ & $0.1946(4)$ & $0.033(2)$ \\
$\mathrm{C}(5)$ & $-0.0079(2)$ & $1.0259(10)$ & $0.1692(4)$ & $0.031(2)$ \\
$\mathrm{C}(6)$ & $0.0233(2)$ & $0.9827(10)$ & $0.1531(4)$ & $0.029(2)$ \\
$\mathrm{C}(7)$ & $0.0858(2)$ & $0.6220(9)$ & $0.1649(4)$ & $0.024(2)$ \\
$\mathrm{C}(8)$ & $0.0742(2)$ & $0.5074(10)$ & $0.1282(5)$ & $0.030(2)$ \\
$\mathrm{C}(9)$ & $0.0807(3)$ & $0.3715(11)$ & $0.1496(5)$ & $0.046(3)$ \\
$\mathrm{C}(10)$ & $0.0996(2)$ & $0.3492(11)$ & $0.2072(5)$ & $0.039(3)$ \\
$\mathrm{C}(11)$ & $0.1119(2)$ & $0.4607(11)$ & $0.2445(5)$ & $0.037(3)$ \\
$\mathrm{C}(12)$ & $0.1052(2)$ & $0.5959(9)$ & $0.2230(4)$ & $0.029(2)$ \\
$\mathrm{C}(13)$ & $0.0674(2)$ & $0.7374(9)$ & $-0.0619(4)$ & $0.022(2)$ \\
$\mathrm{C}(14)$ & $0.0368(2)$ & $0.6696(10)$ & $-0.0591(4)$ & $0.032(2)$ \\
$\mathrm{C}(15)$ & $0.0214(2)$ & $0.5938(10)$ & $-0.1107(4)$ & $0.032(2)$ \\
$\mathrm{C}(16)$ & $0.0370(2)$ & $0.5847(9)$ & $-0.1647(4)$ & $0.029(2)$ \\
$\mathrm{C}(17)$ & $0.0675(2)$ & $0.6537(9)$ & $-0.1676(4)$ & $0.028(2)$ \\
$\mathrm{C}(18)$ & $0.0825(2)$ & $0.7290(9)$ & $-0.1167(4)$ & $0.028(2)$ \\
$\mathrm{C}(19)$ & $0.0852(2)$ & $1.0156(9)$ & $-0.0102(4)$ & $0.024(2)$ \\
$\mathrm{C}(20)$ & $0.1134(2)$ & $1.1009(10)$ & $-0.0128(4)$ & $0.038(3)$ \\
\hline & & & & \\
\hline
\end{tabular}


Tabelle 62: Fortsetzung

\begin{tabular}{|r|r|r|r|r|}
\hline $\mathrm{C}(21)$ & $0.1093(3)$ & $1.2417(11)$ & $-0.0248(5)$ & $0.052(3)$ \\
$\mathrm{C}(22)$ & $0.0773(3)$ & $1.2997(12)$ & $-0.0349(5)$ & $0.053(3)$ \\
$\mathrm{C}(23)$ & $0.0492(3)$ & $1.2170(13)$ & $-0.0331(5)$ & $0.054(3)$ \\
$\mathrm{C}(24)$ & $0.0528(3)$ & $1.0747(10)$ & $-0.0206(4)$ & $0.037(3)$ \\
$\mathrm{C}(25)$ & $0.1934(2)$ & $1.0352(9)$ & $0.2750(4)$ & $0.026(2)$ \\
$\mathrm{C}(26)$ & $0.1955(3)$ & $0.9078(12)$ & $0.3063(5)$ & $0.054(3)$ \\
$\mathrm{C}(27)$ & $0.1754(3)$ & $0.8838(12)$ & $0.3533(6)$ & $0.060(3)$ \\
$\mathrm{C}(28)$ & $0.1538(2)$ & $0.9825(12)$ & $0.3717(5)$ & $0.043(3)$ \\
$\mathrm{C}(29)$ & $0.1509(2)$ & $1.1092(11)$ & $0.3395(4)$ & $0.037(3)$ \\
$\mathrm{C}(30)$ & $0.1706(2)$ & $1.1351(10)$ & $0.2915(4)$ & $0.029(2)$ \\
$\mathrm{C}(31)$ & $0.2082(2)$ & $1.2385(10)$ & $0.1823(4)$ & $0.028(2)$ \\
$\mathrm{C}(32)$ & $0.2253(3)$ & $1.3567(11)$ & $0.2070(5)$ & $0.041(3)$ \\
$\mathrm{C}(33)$ & $0.2129(3)$ & $1.4904(11)$ & $0.1898(5)$ & $0.051(3)$ \\
$\mathrm{C}(34)$ & $0.1851(3)$ & $1.5026(12)$ & $0.1452(6)$ & $0.055(3)$ \\
$\mathrm{C}(35)$ & $0.1681(2)$ & $1.3879(11)$ & $0.1201(5)$ & $0.041(3)$ \\
$\mathrm{C}(36)$ & $0.1797(2)$ & $1.2530(11)$ & $0.1380(4)$ & $0.034(2)$ \\
$\mathrm{C}(37)$ & $0.3255(2)$ & $1.1207(10)$ & $0.2969(5)$ & $0.035(2)$ \\
$\mathrm{C}(38)$ & $0.3155(3)$ & $1.2228(15)$ & $0.3352(7)$ & $0.097(6)$ \\
$\mathrm{C}(39)$ & $0.3399(4)$ & $1.2892(17)$ & $0.3786(10)$ & $0.145(9)$ \\
$\mathrm{C}(40)$ & $0.3723(3)$ & $1.2513(15)$ & $0.3873(8)$ & $0.088(5)$ \\
$\mathrm{C}(41)$ & $0.3831(3)$ & $1.1435(17)$ & $0.3507(6)$ & $0.072(4)$ \\
$\mathrm{C}(42)$ & $0.3599(3)$ & $1.0787(14)$ & $0.3053(5)$ & $0.057(3)$ \\
$\mathrm{C}(43)$ & $0.3024(2)$ & $0.8586(10)$ & $0.2395(5)$ & $0.030(2)$ \\
$\mathrm{C}(44)$ & $0.2949(3)$ & $0.7888(11)$ & $0.2925(5)$ & $0.047(3)$ \\
$\mathrm{C}(45)$ & $0.2979(3)$ & $0.6433(14)$ & $0.2972(7)$ & $0.073(4)$ \\
$\mathrm{C}(46)$ & $0.3073(3)$ & $0.5675(15)$ & $0.2474(10)$ & $0.090(6)$ \\
$\mathrm{C}(47)$ & $0.3147(3)$ & $0.6344(15)$ & $0.1954(9)$ & $0.086(5)$ \\
$\mathrm{C}(48)$ & $0.3130(2)$ & $0.7787(13)$ & $0.1909(6)$ & $0.052(3)$ \\
$\mathrm{C}(49)$ & $0.4509(2)$ & $1.1299(11)$ & $0.0660(5)$ & $0.037(3)$ \\
$\mathrm{C}(50)$ & $0.4593(2)$ & $1.1133(12)$ & $0.0055(5)$ & $0.045(3)$ \\
$\mathrm{C}(51)$ & $0.4670(3)$ & $1.2282(12)$ & $-0.0294(5)$ & $0.048(3)$ \\
$\mathrm{C}(52)$ & $0.4659(3)$ & $1.3614(14)$ & $-0.0047(6)$ & $0.062(4)$ \\
$\mathrm{C}(53)$ & $0.4578(3)$ & $1.3777(14)$ & $0.0559(7)$ & $0.066(4)$ \\
$\mathrm{C}(54)$ & $0.4502(3)$ & $1.2642(14)$ & $0.0910(6)$ & $0.060(3)$ \\
$\mathrm{C}(55)$ & $0.4818(3)$ & $0.9158(14)$ & $0.1511(5)$ & $0.052(3)$ \\
$\mathrm{C}(56)$ & $0.5107(3)$ & $1.0011(17)$ & $0.1601(7)$ & $0.083(5)$ \\
$\mathrm{C}(57)$ & $0.5407(4)$ & $0.950(2)$ & $0.1932(7)$ & $0.099(6)$ \\
$\mathrm{C}(59)$ & $0.5431(4)$ & $0.818(2)$ & $0.2154(6)$ & $0.082(5)$ \\
& $0.5158(4)$ & $0.7306(18)$ & $0.2073(6)$ & $0.076(4)$ \\
& & & & \\
$\mathrm{C}(581(3)$ & $0.7805(17)$ & $0.1741(5)$ & $0.073(4)$ \\
\hline
\end{tabular}


Tabelle 62: Fortsetzung

\begin{tabular}{|r|r|r|r|r|}
\hline $\mathrm{C}(61)$ & $0.4053(2)$ & $0.7731(13)$ & $-0.0567(5)$ & $0.052(3)$ \\
$\mathrm{C}(62)$ & $0.4376(3)$ & $0.7168(12)$ & $-0.0623(6)$ & $0.053(3)$ \\
$\mathrm{C}(63)$ & $0.4471(3)$ & $0.6844(15)$ & $-0.1207(7)$ & $0.078(4)$ \\
$\mathrm{C}(64)$ & $0.4239(4)$ & $0.7093(19)$ & $-0.1753(7)$ & $0.105(6)$ \\
$\mathrm{C}(65)$ & $0.3921(4)$ & $0.763(2)$ & $-0.1701(7)$ & $0.115(7)$ \\
$\mathrm{C}(66)$ & $0.3836(3)$ & $0.7933(16)$ & $-0.1101(7)$ & $0.083(5)$ \\
$\mathrm{C}(67)$ & $0.3844(2)$ & $0.6403(9)$ & $0.0581(4)$ & $0.069(4)$ \\
$\mathrm{C}(68)$ & $0.3703(3)$ & $0.5271(12)$ & $0.0227(3)$ & $0.114(6)$ \\
$\mathrm{C}(69)$ & $0.3640(2)$ & $0.4013(10)$ & $0.0523(5)$ & $0.111(6)$ \\
$\mathrm{C}(70)$ & $0.3717(3)$ & $0.3888(9)$ & $0.1173(5)$ & $0.117(6)$ \\
$\mathrm{C}(71)$ & $0.3858(3)$ & $0.5020(11)$ & $0.1528(3)$ & $0.109(6)$ \\
$\mathrm{C}(72)$ & $0.3922(2)$ & $0.6278(9)$ & $0.1231(4)$ & $0.075(4)$ \\
$\mathrm{O}(1 \mathrm{~A})$ & $0.2654(7)$ & $0.665(4)$ & $0.0320(14)$ & $0.059(9)$ \\
$\mathrm{C}(73 \mathrm{~A})$ & $0.2669(15)$ & $0.559(7)$ & $0.066(3)$ & $0.116(18)$ \\
$\mathrm{C}(74 \mathrm{~A})$ & $0.2772(16)$ & $0.446(7)$ & $0.012(3)$ & $0.13(2)$ \\
$\mathrm{C}(75 \mathrm{~A})$ & $0.2321(16)$ & $0.492(7)$ & $-0.010(3)$ & $0.12(2)$ \\
$\mathrm{C}(76 \mathrm{~A})$ & $0.232(2)$ & $0.645(9)$ & $-0.018(4)$ & $0.19(3)$ \\
$\mathrm{O}(1 \mathrm{~B})$ & $0.2609(4)$ & $0.7207(17)$ & $0.0189(7)$ & $0.063(5)$ \\
$\mathrm{C}(73 \mathrm{~B})$ & $0.2391(7)$ & $0.618(3)$ & $0.0463(12)$ & $0.094(7)$ \\
$\mathrm{C}(74 \mathrm{~B})$ & $0.2475(8)$ & $0.465(3)$ & $0.0293(14)$ & $0.109(9)$ \\
$\mathrm{C}(75 \mathrm{~B})$ & $0.2684(7)$ & $0.506(3)$ & $-0.0363(13)$ & $0.110(9)$ \\
$\mathrm{C}(76 \mathrm{~B})$ & $0.2780(6)$ & $0.647(3)$ & $-0.0298(12)$ & $0.105(8)$ \\
$\mathrm{O}(2 \mathrm{~A})$ & $0.2499(5)$ & $0.993(2)$ & $-0.0362(10)$ & $0.041(6)$ \\
$\mathrm{C}(77 \mathrm{~A})$ & $0.2433(7)$ & $1.148(3)$ & $-0.0285(14)$ & $0.039(8)$ \\
$\mathrm{C}(78 \mathrm{~A})$ & $0.2054(9)$ & $1.167(4)$ & $-0.049(2)$ & $0.091(15)$ \\
$\mathrm{C}(79 \mathrm{~A})$ & $0.2003(16)$ & $1.060(6)$ & $-0.100(3)$ & $0.17(5)$ \\
$\mathrm{C}(80 \mathrm{~A})$ & $0.2241(11)$ & $0.935(4)$ & $-0.082(2)$ & $0.118(18)$ \\
$\mathrm{O}(2 \mathrm{~B})$ & $0.2443(4)$ & $1.064(2)$ & $-0.0200(7)$ & $0.079(4)$ \\
$\mathrm{C}(77 \mathrm{~B})$ & $0.2060(5)$ & $1.086(2)$ & $-0.0254(10)$ & $0.080(7)$ \\
$\mathrm{C}(78 \mathrm{~B})$ & $0.1926(5)$ & $1.044(2)$ & $-0.0916(10)$ & $0.056(7)$ \\
$\mathrm{C}(79 \mathrm{~B})$ & $0.2235(8)$ & $1.045(3)$ & $-0.1242(12)$ & $0.122(10)$ \\
$\mathrm{C}(80 \mathrm{~B})$ & $0.2549(5)$ & $1.092(2)$ & $-0.0794(10)$ & $0.085(7)$ \\
\hline & & & & \\
& & & \\
& & & \\
& & & \\
& &
\end{tabular}

Tabelle 63: Bindungslängen [pm] in $\underline{14}$

\begin{tabular}{|l|r||l|r|}
\hline $\mathrm{Se}(1)-\mathrm{P}(1)$ & $216.8(2)$ & $\mathrm{Se}(1)-\mathrm{Cu}(1)$ & $234.12(14)$ \\
$\mathrm{Se}(2)-\mathrm{P}(2)$ & $216.9(2)$ & $\mathrm{Se}(2)-\mathrm{Cu}(1)$ & $236.89(14)$ \\
$\mathrm{Se}(3)-\mathrm{P}(3)$ & $217.7(3)$ & $\mathrm{Se}(3)-\mathrm{Cu}(1)$ & $236.34(14)$ \\
$\mathrm{Se}(3)-\mathrm{Na}(1)$ & $301.2(5)$ & $\mathrm{Se}(4)-\mathrm{P}(4)$ & $217.3(3)$ \\
$\mathrm{Se}(4)-\mathrm{Cu}(2)$ & $240.03(16)$ & $\mathrm{Se}(4)-\mathrm{Na}(1)$ & $292.0(5)$ \\
\hline
\end{tabular}


Tabelle 63: Fortsetzung

\begin{tabular}{|l|r||l|r|}
\hline $\mathrm{Se}(5)-\mathrm{P}(5)$ & $217.2(3)$ & $\mathrm{Se}(5)-\mathrm{Cu}(2)$ & $233.34(17)$ \\
$\mathrm{Se}(6)-\mathrm{P}(6)$ & $218.6(3)$ & $\mathrm{Se}(6)-\mathrm{Cu}(2)$ & $235.33(17)$ \\
$\mathrm{Se}(6)-\mathrm{Na}(1)$ & $342.8(5)$ & $\mathrm{Cu}(2)-\mathrm{Na}(1)$ & $363.4(5)$ \\
$\mathrm{P}(1)-\mathrm{N}(1)$ & $158.9(7)$ & $\mathrm{P}(1)-\mathrm{C}(1)$ & $181.4(8)$ \\
$\mathrm{P}(1)-\mathrm{C}(7)$ & $181.6(9)$ & $\mathrm{P}(2)-\mathrm{N}(1)$ & $159.3(7)$ \\
$\mathrm{P}(2)-\mathrm{C}(13)$ & $181.6(9)$ & $\mathrm{P}(2)-\mathrm{C}(19)$ & $183.1(9)$ \\
$\mathrm{P}(3)-\mathrm{N}(2)$ & $159.3(7)$ & $\mathrm{P}(3)-\mathrm{C}(25)$ & $181.3(9)$ \\
$\mathrm{P}(3)-\mathrm{C}(31)$ & $182.3(9)$ & $\mathrm{P}(4)-\mathrm{N}(2)$ & $160.0(7)$ \\
$\mathrm{P}(4)-\mathrm{C}(37)$ & $179.5(9)$ & $\mathrm{P}(4)-\mathrm{C}(43)$ & $180.0(10)$ \\
$\mathrm{P}(5)-\mathrm{N}(3)$ & $158.8(9)$ & $\mathrm{P}(5)-\mathrm{C}(55)$ & $180.9(11)$ \\
$\mathrm{P}(5)-\mathrm{C}(49)$ & $181.5(10)$ & $\mathrm{P}(6)-\mathrm{N}(3)$ & $157.8(8)$ \\
$\mathrm{P}(6)-\mathrm{C}(61)$ & $180.2(11)$ & $\mathrm{P}(6)-\mathrm{C}(67)$ & $183.4(8)$ \\
$\mathrm{Na}(1)-\mathrm{O}(1 \mathrm{~B})$ & $213.6(16)$ & $\mathrm{Na}(1)-\mathrm{O}(2 \mathrm{~A})$ & $221(2)$ \\
$\mathrm{Na}(1)-\mathrm{O}(2 \mathrm{~B})$ & $229.8(16)$ & $\mathrm{Na}(1)-\mathrm{O}(1 \mathrm{~A})$ & $253(3)$ \\
$\mathrm{Na}(1)-\mathrm{C}(77 \mathrm{~A})$ & $295(3)$ & & \\
\hline
\end{tabular}

Tabelle 64: Bindungswinkel $\left[^{\circ}\right]$ in $\underline{14}$

\begin{tabular}{|l|r||l|r|}
\hline $\mathrm{P}(1)-\mathrm{Se}(1)-\mathrm{Cu}(1)$ & $95.13(7)$ & $\mathrm{P}(2)-\mathrm{Se}(2)-\mathrm{Cu}(1)$ & $93.84(7)$ \\
$\mathrm{P}(3)-\mathrm{Se}(3)-\mathrm{Cu}(1)$ & $106.37(7)$ & $\mathrm{P}(3)-\mathrm{Se}(3)-\mathrm{Na}(1)$ & $109.71(13)$ \\
$\mathrm{Cu}(1)-\mathrm{Se}(3)-\mathrm{Na}(1)$ & $128.25(10)$ & $\mathrm{P}(4)-\mathrm{Se}(4)-\mathrm{Cu}(2)$ & $107.69(8)$ \\
$\mathrm{P}(4)-\mathrm{Se}(4)-\mathrm{Na}(1)$ & $99.84(12)$ & $\mathrm{Cu}(2)-\mathrm{Se}(4)-\mathrm{Na}(1)$ & $85.58(11)$ \\
$\mathrm{P}(5)-\mathrm{Se}(5)-\mathrm{Cu}(2)$ & $97.17(9)$ & $\mathrm{P}(6)-\mathrm{Se}(6)-\mathrm{Cu}(2)$ & $97.55(9)$ \\
$\mathrm{P}(6)-\mathrm{Se}(6)-\mathrm{Na}(1)$ & $134.33(13)$ & $\mathrm{Cu}(2)-\mathrm{Se}(6)-\mathrm{Na}(1)$ & $75.34(9)$ \\
$\mathrm{Se}(1)-\mathrm{Cu}(1)-\mathrm{Se}(3)$ & $125.16(6)$ & $\mathrm{Se}(1)-\mathrm{Cu}(1)-\mathrm{Se}(2)$ & $122.60(5)$ \\
$\mathrm{Se}(3)-\mathrm{Cu}(1)-\mathrm{Se}(2)$ & $112.20(5)$ & $\mathrm{Se}(5)-\mathrm{Cu}(2)-\mathrm{Se}(6)$ & $123.29(6)$ \\
$\mathrm{Se}(5)-\mathrm{Cu}(2)-\mathrm{Se}(4)$ & $125.21(7)$ & $\mathrm{Se}(6)-\mathrm{Cu}(2)-\mathrm{Se}(4)$ & $111.21(6)$ \\
$\mathrm{Se}(5)-\mathrm{Cu}(2)-\mathrm{Na}(1)$ & $158.04(10)$ & $\mathrm{Se}(6)-\mathrm{Cu}(2)-\mathrm{Na}(1)$ & $65.87(9)$ \\
$\mathrm{Se}(4)-\mathrm{Cu}(2)-\mathrm{Na}(1)$ & $53.23(10)$ & $\mathrm{N}(1)-\mathrm{P}(1)-\mathrm{C}(1)$ & $104.1(4)$ \\
$\mathrm{N}(1)-\mathrm{P}(1)-\mathrm{C}(7)$ & $108.2(4)$ & $\mathrm{N}(1)-\mathrm{P}(1)-\mathrm{Se}(1)$ & $119.3(3)$ \\
$\mathrm{C}(1)-\mathrm{P}(1)-\mathrm{Se}(1)$ & $107.5(3)$ & $\mathrm{C}(7)-\mathrm{P}(1)-\mathrm{Se}(1)$ & $109.6(3)$ \\
$\mathrm{N}(1)-\mathrm{P}(2)-\mathrm{C}(13)$ & $104.8(4)$ & $\mathrm{N}(1)-\mathrm{P}(2)-\mathrm{C}(19)$ & $108.5(4)$ \\
$\mathrm{N}(1)-\mathrm{P}(2)-\mathrm{Se}(2)$ & $118.9(3)$ & $\mathrm{C}(13)-\mathrm{P}(2)-\mathrm{Se}(2)$ & $107.1(3)$ \\
$\mathrm{C}(19)-\mathrm{P}(2)-\mathrm{Se}(2)$ & $110.5(3)$ & $\mathrm{N}(2)-\mathrm{P}(3)-\mathrm{C}(25)$ & $105.0(4)$ \\
$\mathrm{N}(2)-\mathrm{P}(3)-\mathrm{C}(31)$ & $109.2(4)$ & $\mathrm{N}(2)-\mathrm{P}(3)-\mathrm{Se}(3)$ & $116.2(3)$ \\
$\mathrm{C}(25)-\mathrm{P}(3)-\mathrm{Se}(3)$ & $109.2(3)$ & $\mathrm{C}(31)-\mathrm{P}(3)-\mathrm{Se}(3)$ & $111.6(3)$ \\
$\mathrm{N}(2)-\mathrm{P}(4)-\mathrm{C}(37)$ & $104.7(4)$ & $\mathrm{N}(2)-\mathrm{P}(4)-\mathrm{C}(43)$ & $109.3(4)$ \\
$\mathrm{N}(2)-\mathrm{P}(4)-\mathrm{Se}(4)$ & $114.5(3)$ & $\mathrm{C}(37)-\mathrm{P}(4)-\mathrm{Se}(4)$ & $109.3(3)$ \\
$\mathrm{C}(43)-\mathrm{P}(4)-\mathrm{Se}(4)$ & $112.3(3)$ & $\mathrm{N}(3)-\mathrm{P}(5)-\mathrm{C}(55)$ & $105.2(5)$ \\
$\mathrm{N}(3)-\mathrm{P}(5)-\mathrm{C}(49)$ & $109.2(5)$ & $\mathrm{N}(3)-\mathrm{P}(5)-\mathrm{Se}(5)$ & $118.4(3)$ \\
\hline
\end{tabular}


Tabelle 64: Fortsetzung

\begin{tabular}{|l|r||l|r|}
\hline $\mathrm{C}(55)-\mathrm{P}(5)-\mathrm{Se}(5)$ & $105.9(3)$ & C(49)-P(5)-Se(5) & $111.3(3)$ \\
$\mathrm{N}(3)-\mathrm{P}(6)-\mathrm{C}(61)$ & $105.6(4)$ & N(3)-P(6)-C(67) & $102.5(5)$ \\
$\mathrm{N}(3)-\mathrm{P}(6)-\mathrm{Se}(6)$ & $120.3(3)$ & $\mathrm{C}(61)-\mathrm{P}(6)-\mathrm{Se}(6)$ & $108.7(4)$ \\
$\mathrm{C}(67)-\mathrm{P}(6)-\mathrm{Se}(6)$ & $109.9(3)$ & $\mathrm{O}(1 \mathrm{~B})-\mathrm{Na}(1)-\mathrm{O}(2 \mathrm{~A})$ & $82.1(7)$ \\
$\mathrm{O}(1 \mathrm{~B})-\mathrm{Na}(1)-\mathrm{O}(2 \mathrm{~B})$ & $100.6(6)$ & $\mathrm{O}(2 \mathrm{~A})-\mathrm{Na}(1)-\mathrm{O}(2 \mathrm{~B})$ & $20.5(5)$ \\
$\mathrm{O}(1 \mathrm{~B})-\mathrm{Na}(1)-\mathrm{O}(1 \mathrm{~A})$ & $11.5(8)$ & $\mathrm{O}(2 \mathrm{~A})-\mathrm{Na}(1)-\mathrm{O}(1 \mathrm{~A})$ & $93.5(9)$ \\
$\mathrm{O}(2 \mathrm{~B})-\mathrm{Na}(1)-\mathrm{O}(1 \mathrm{~A})$ & $112.1(9)$ & $\mathrm{O}(1 \mathrm{~B})-\mathrm{Na}(1)-\mathrm{Se}(4)$ & $155.8(5)$ \\
$\mathrm{O}(2 \mathrm{~A})-\mathrm{Na}(1)-\mathrm{Se}(4)$ & $117.8(6)$ & $\mathrm{O}(2 \mathrm{~B})-\mathrm{Na}(1)-\mathrm{Se}(4)$ & $101.5(5)$ \\
$\mathrm{O}(1 \mathrm{~A})-\mathrm{Na}(1)-\mathrm{Se}(4)$ & $145.1(7)$ & $\mathrm{O}(1 \mathrm{~B})-\mathrm{Na}(1)-\mathrm{C}(77 \mathrm{~A})$ & $111.2(7)$ \\
$\mathrm{O}(2 \mathrm{~A})-\mathrm{Na}(1)-\mathrm{C}(77 \mathrm{~A})$ & $29.9(7)$ & $\mathrm{O}(2 \mathrm{~B})-\mathrm{Na}(1)-\mathrm{C}(77 \mathrm{~A})$ & $10.8(7)$ \\
$\mathrm{O}(1 \mathrm{~A})-\mathrm{Na}(1)-\mathrm{C}(77 \mathrm{~A})$ & $122.7(9)$ & $\mathrm{Se}(4)-\mathrm{Na}(1)-\mathrm{C}(77 \mathrm{~A})$ & $90.7(5)$ \\
$\mathrm{O}(1 \mathrm{~B})-\mathrm{Na}(1)-\mathrm{Se}(3)$ & $95.2(4)$ & $\mathrm{O}(2 \mathrm{~A})-\mathrm{Na}(1)-\mathrm{Se}(3)$ & $112.7(6)$ \\
$\mathrm{O}(2 \mathrm{~B})-\mathrm{Na}(1)-\mathrm{Se}(3)$ & $102.1(4)$ & $\mathrm{O}(1 \mathrm{~A})-\mathrm{Na}(1)-\mathrm{Se}(3)$ & $92.3(7)$ \\
$\mathrm{Se}(4)-\mathrm{Na}(1)-\mathrm{Se}(3)$ & $89.66(14)$ & $\mathrm{C}(77 \mathrm{~A})-\mathrm{Na}(1)-\mathrm{Se}(3)$ & $102.3(6)$ \\
$\mathrm{O}(1 \mathrm{~B})-\mathrm{Na}(1)-\mathrm{Se}(6)$ & $92.7(4)$ & $\mathrm{O}(2 \mathrm{~A})-\mathrm{Na}(1)-\mathrm{Se}(6)$ & $86.8(5)$ \\
$\mathrm{O}(2 \mathrm{~B})-\mathrm{Na}(1)-\mathrm{Se}(6)$ & $94.7(4)$ & $\mathrm{O}(1 \mathrm{~A})-\mathrm{Na}(1)-\mathrm{Se}(6)$ & $91.8(7)$ \\
$\mathrm{Se}(4)-\mathrm{Na}(1)-\mathrm{Se}(6)$ & $75.85(11)$ & $\mathrm{C}(77 \mathrm{~A})-\mathrm{Na}(1)-\mathrm{Se}(6)$ & $92.1(6)$ \\
$\mathrm{Se}(3)-\mathrm{Na}(1)-\mathrm{Se}(6)$ & $159.76(17)$ & $\mathrm{O}(1 \mathrm{~B})-\mathrm{Na}(1)-\mathrm{Cu}(2)$ & $119.2(4)$ \\
$\mathrm{O}(2 \mathrm{~A})-\mathrm{Na}(1)-\mathrm{Cu}(2)$ & $116.7(6)$ & $\mathrm{O}(2 \mathrm{~B})-\mathrm{Na}(1)-\mathrm{Cu}(2)$ & $113.8(4)$ \\
$\mathrm{O}(1 \mathrm{~A})-\mathrm{Na}(1)-\mathrm{Cu}(2)$ & $112.4(7)$ & $\mathrm{Se}(4)-\mathrm{Na}(1)-\mathrm{Cu}(2)$ & $41.19(7)$ \\
$\mathrm{C}(77 \mathrm{~A})-\mathrm{Na}(1)-\mathrm{Cu}(2)$ & $105.5(5)$ & $\mathrm{Se}(3)-\mathrm{Na}(1)-\mathrm{Cu}(2)$ & $122.15(15)$ \\
$\mathrm{Se}(6)-\mathrm{Na}(1)-\mathrm{Cu}(2)$ & $38.79(6)$ & $\mathrm{P}(1)-\mathrm{N}(1)-\mathrm{P}(2)$ & $131.3(5)$ \\
$\mathrm{P}(3)-\mathrm{N}(2)-\mathrm{P}(4)$ & $130.9(5)$ & $\mathrm{P}(6)-\mathrm{N}(3)-\mathrm{P}(5)$ & $136.3(5)$ \\
\hline
\end{tabular}




\subsection{Daten zur Kristallstrukturanalyse von $\left[\mathrm{Cu}_{3}\left(\mathrm{dppaS}_{2}\right)_{3}\right] \underline{15}$}

\begin{tabular}{|ll|}
\hline Summenformel & $\mathrm{C}_{76} \mathrm{H}_{68} \mathrm{Cu}_{3} \mathrm{~N}_{3} \mathrm{OP}_{6} \mathrm{~S}_{6}$ \\
Molekulargewicht $\left[\mathrm{g} \cdot \mathrm{mol}^{-1}\right]$ & 1608.13 \\
Temperatur & $133(2) \mathrm{K}$ \\
Wellenlänge & $71.073 \mathrm{pm}$ \\
Kristallsystem & monoklin \\
Raumgruppe & $P 2_{1} / n$ \\
Gitterkonstanten & $\mathrm{a}=15.4929(7) \AA, \alpha=90^{\circ}$ \\
& $\mathrm{b}=22.4882(9) \AA, \beta=94.7612(19)^{\circ} \circ$ \\
& $\mathrm{c}=20.5866(8) \AA, \gamma=90^{\circ} \AA$ \\
Volumen & $7147.8(5) \AA^{3}$ \\
Formeleinheiten & 4 \\
Röntgenographische Dichte $\left[\mathrm{g} \cdot \mathrm{cm}^{-3}\right]$ & 1.494 \\
Absorptionskoeffizient $\left[\mathrm{mm}^{-1}\right]$ & 1.242 \\
F(000) & 3304 \\
Meßbereich $\theta$ & $1.82-24.71^{\circ}$ \\
Indexbereiche & $-18 \leq \mathrm{h} \leq 18$ \\
& $-26 \leq \mathrm{k} \leq 26$ \\
Gemessene Reflexe & $-19 \leq 1 \leq 24$ \\
Unabhängige Reflexe & 89247 \\
Beobachtete Reflexe $[\mathrm{I}>2 \sigma]$ & 12174 \\
$\mathrm{R}_{\text {int }}$ & 9942 \\
Parameter & 0.0426 \\
R1; wR2[I $>2 \sigma(\mathrm{I})]$ & 839 \\
& $0.0321 ; 0.0717$ \\
\hline
\end{tabular}


Tabelle 66: Atomkoordinaten und äquivalente isotrope Auslenkungsparameter $\left[\AA^{2}\right]$ für $\underline{15}$

\begin{tabular}{|c|c|c|c|c|}
\hline Atom & $\mathrm{x}$ & $\mathrm{y}$ & $\mathrm{z}$ & $\mathrm{U}_{e q}$ \\
\hline $\mathrm{C}(1)$ & $0.40694(18)$ & $0.12148(12)$ & $0.53041(13)$ & $0.0230(6)$ \\
\hline $\mathrm{C}(2)$ & $0.3295(2)$ & $0.13397(15)$ & $0.49323(15)$ & $0.0338(7)$ \\
\hline $\mathrm{C}(3)$ & $0.3289(2)$ & $0.14106(16)$ & $0.42670(15)$ & $0.0398(8)$ \\
\hline $\mathrm{C}(4)$ & $0.4052(2)$ & $0.13661(15)$ & $0.39665(15)$ & $0.0354(7)$ \\
\hline $\mathrm{C}(5)$ & $0.4817(2)$ & $0.12498(16)$ & $0.43294(15)$ & $0.0376(8)$ \\
\hline $\mathrm{C}(6)$ & $0.48272(19)$ & $0.11683(14)$ & $0.49985(14)$ & $0.0311(7)$ \\
\hline $\mathrm{C}(7)$ & $0.34528(17)$ & $0.03352(12)$ & $0.61433(14)$ & $0.0230(6)$ \\
\hline $\mathrm{C}(8)$ & $0.30610(18)$ & $0.01540(13)$ & $0.66884(15)$ & $0.0280(7)$ \\
\hline $\mathrm{C}(9)$ & $0.26226(19)$ & $-0.03840(13)$ & $0.66982(16)$ & $0.0333(7)$ \\
\hline $\mathrm{C}(10)$ & $0.2573(2)$ & $-0.07451(14)$ & $0.61544(18)$ & $0.0409(8)$ \\
\hline $\mathrm{C}(11)$ & $0.2969(2)$ & $-0.05713(15)$ & $0.56130(18)$ & $0.0455(9)$ \\
\hline $\mathrm{C}(12)$ & $0.3411(2)$ & $-0.00334(14)$ & $0.55993(16)$ & $0.0346(7)$ \\
\hline $\mathrm{C}(13)$ & $0.50637(18)$ & $0.02306(12)$ & $0.75438(14)$ & $0.0237(6)$ \\
\hline $\mathrm{C}(14)$ & $0.47742(19)$ & $0.02615(13)$ & $0.81664(14)$ & $0.0291(7)$ \\
\hline $\mathrm{C}(15)$ & $0.4379(2)$ & $-0.02277(15)$ & $0.84247(16)$ & $0.0360(8)$ \\
\hline $\mathrm{C}(16)$ & $0.4275(2)$ & $-0.07493(14)$ & $0.80734(17)$ & $0.0379(8)$ \\
\hline $\mathrm{C}(17)$ & $0.4579(2)$ & $-0.07868(14)$ & $0.74621(17)$ & $0.0353(7)$ \\
\hline $\mathrm{C}(18)$ & $0.49705(19)$ & $-0.02993(13)$ & $0.71977(15)$ & $0.0291(7)$ \\
\hline $\mathrm{C}(19)$ & $0.66034(18)$ & $0.07068(13)$ & $0.70255(14)$ & $0.0251(6)$ \\
\hline $\mathrm{C}(20)$ & $0.72877(19)$ & $0.09149(14)$ & $0.74441(15)$ & $0.0326(7)$ \\
\hline $\mathrm{C}(21)$ & $0.8133(2)$ & $0.07468(16)$ & $0.73466(17)$ & $0.0408(8)$ \\
\hline $\mathrm{C}(22)$ & $0.8295(2)$ & $0.03727(16)$ & $0.68405(17)$ & $0.0415(8)$ \\
\hline $\mathrm{C}(23)$ & $0.7616(2)$ & $0.01702(15)$ & $0.64200(16)$ & $0.0385(8)$ \\
\hline $\mathrm{C}(24)$ & $0.6774(2)$ & $0.03379(14)$ & $0.65093(15)$ & $0.0320(7)$ \\
\hline $\mathrm{C}(25)$ & $0.39228(18)$ & $0.16589(12)$ & $0.94481(13)$ & $0.0230(6)$ \\
\hline $\mathrm{C}(26)$ & $0.32088(19)$ & $0.12843(13)$ & $0.94606(14)$ & $0.0272(6)$ \\
\hline $\mathrm{C}(27)$ & $0.3289(2)$ & $0.07357(13)$ & $0.97638(14)$ & $0.0304(7)$ \\
\hline $\mathrm{C}(28)$ & $0.4080(2)$ & $0.05593(14)$ & $1.00603(15)$ & $0.0353(7)$ \\
\hline $\mathrm{C}(29)$ & $0.4795(2)$ & $0.09249(15)$ & $1.00400(17)$ & $0.0395(8)$ \\
\hline $\mathrm{C}(30)$ & $0.4721(2)$ & $0.14758(14)$ & $0.97355(15)$ & $0.0322(7)$ \\
\hline $\mathrm{C}(31)$ & $0.30736(18)$ & $0.27604(12)$ & $0.96269(13)$ & $0.0227(6)$ \\
\hline $\mathrm{C}(32)$ & $0.3431(2)$ & $0.30629(14)$ & $1.01755(14)$ & $0.0315(7)$ \\
\hline $\mathrm{C}(33)$ & $0.2896(2)$ & $0.32816(15)$ & $1.06297(15)$ & $0.0378(8)$ \\
\hline $\mathrm{C}(34)$ & $0.2013(2)$ & $0.32009(14)$ & $1.05423(16)$ & $0.0363(8)$ \\
\hline $\mathrm{C}(35)$ & $0.1658(2)$ & $0.29109(13)$ & $0.99949(16)$ & $0.0332(7)$ \\
\hline $\mathrm{C}(36)$ & $0.21804(19)$ & $0.26924(13)$ & $0.95352(15)$ & $0.0274(6)$ \\
\hline $\mathrm{C}(37)$ & $0.56306(18)$ & $0.37059(14)$ & $0.95782(14)$ & $0.0266(6)$ \\
\hline $\mathrm{C}(38)$ & $0.5733(2)$ & $0.43113(15)$ & $0.94966(17)$ & $0.0390(8)$ \\
\hline
\end{tabular}


Tabelle 66: Fortsetzung

\begin{tabular}{|c|c|c|c|c|}
\hline $\mathrm{C}(39)$ & $0.6010(2)$ & $0.46709(17)$ & $1.00200(19)$ & $0.0494(9)$ \\
\hline $\mathrm{C}(40)$ & $0.6179(2)$ & $0.44258(18)$ & $1.06308(18)$ & $0.0480(10)$ \\
\hline $\mathrm{C}(41)$ & $0.6090(2)$ & $0.38270(19)$ & $1.07161(17)$ & $0.0492(10)$ \\
\hline $\mathrm{C}(42)$ & $0.5816(2)$ & $0.34594(17)$ & $1.01930(16)$ & $0.0404(8)$ \\
\hline $\mathrm{C}(43)$ & $0.62038(18)$ & $0.29141(12)$ & $0.85903(13)$ & $0.0237(6)$ \\
\hline $\mathrm{C}(44)$ & $0.6486(2)$ & $0.30790(14)$ & $0.79979(14)$ & $0.0315(7)$ \\
\hline $\mathrm{C}(45)$ & $0.7223(2)$ & $0.28246(15)$ & $0.77741(15)$ & $0.0359(8)$ \\
\hline $\mathrm{C}(46)$ & $0.76875(19)$ & $0.24087(14)$ & $0.81463(15)$ & $0.0327(7)$ \\
\hline $\mathrm{C}(47)$ & $0.7411(2)$ & $0.22367(14)$ & $0.87423(17)$ & $0.0362(8)$ \\
\hline $\mathrm{C}(48)$ & $0.6678(2)$ & $0.24849(14)$ & $0.89645(16)$ & $0.0335(7)$ \\
\hline $\mathrm{C}(49)$ & $0.36535(18)$ & $0.47000(12)$ & $0.67397(13)$ & $0.0215(6)$ \\
\hline $\mathrm{C}(50)$ & $0.43110(19)$ & $0.49365(13)$ & $0.71669(13)$ & $0.0254(6)$ \\
\hline $\mathrm{C}(51)$ & $0.4184(2)$ & $0.54774(13)$ & $0.74726(15)$ & $0.0312(7)$ \\
\hline $\mathrm{C}(52)$ & $0.3414(2)$ & $0.57854(13)$ & $0.73485(14)$ & $0.0308(7)$ \\
\hline $\mathrm{C}(53)$ & $0.2763(2)$ & $0.55517(13)$ & $0.69225(14)$ & $0.0306(7)$ \\
\hline $\mathrm{C}(54)$ & $0.28785(19)$ & $0.50103(13)$ & $0.66233(14)$ & $0.0253(6)$ \\
\hline $\mathrm{C}(55)$ & $0.33285(18)$ & $0.41531(12)$ & $0.54935(13)$ & $0.0224(6)$ \\
\hline $\mathrm{C}(56)$ & $0.3787(2)$ & $0.45111(13)$ & $0.50889(14)$ & $0.0278(7)$ \\
\hline $\mathrm{C}(57)$ & $0.3444(2)$ & $0.46521(14)$ & $0.44666(15)$ & $0.0348(7)$ \\
\hline $\mathrm{C}(58)$ & $0.2630(2)$ & $0.44473(14)$ & $0.42475(15)$ & $0.0359(8)$ \\
\hline $\mathrm{C}(59)$ & $0.2168(2)$ & $0.40937(14)$ & $0.46384(15)$ & $0.0352(7)$ \\
\hline $\mathrm{C}(60)$ & $0.25125(19)$ & $0.39425(13)$ & $0.52611(14)$ & $0.0284(7)$ \\
\hline $\mathrm{C}(61)$ & $0.65456(17)$ & $0.36757(12)$ & $0.62931(13)$ & $0.0220(6)$ \\
\hline $\mathrm{C}(62)$ & $0.67122(19)$ & $0.41255(13)$ & $0.67495(14)$ & $0.0273(6)$ \\
\hline $\mathrm{C}(63)$ & $0.7564(2)$ & $0.42907(14)$ & $0.69336(15)$ & $0.0337(7)$ \\
\hline $\mathrm{C}(64)$ & $0.82412(19)$ & $0.40155(14)$ & $0.66594(15)$ & $0.0337(7)$ \\
\hline $\mathrm{C}(65)$ & $0.80767(19)$ & $0.35681(14)$ & $0.62019(14)$ & $0.0304(7)$ \\
\hline $\mathrm{C}(66)$ & $0.72329(18)$ & $0.33961(13)$ & $0.60227(14)$ & $0.0259(6)$ \\
\hline $\mathrm{C}(67)$ & $0.53420(17)$ & $0.34438(12)$ & $0.51810(13)$ & $0.0215(6)$ \\
\hline $\mathrm{C}(68)$ & $0.57462(19)$ & $0.38922(13)$ & $0.48503(14)$ & $0.0272(6)$ \\
\hline $\mathrm{C}(69)$ & $0.5613(2)$ & $0.39430(15)$ & $0.41806(15)$ & $0.0353(7)$ \\
\hline $\mathrm{C}(70)$ & $0.5080(2)$ & $0.35435(16)$ & $0.38341(15)$ & $0.0387(8)$ \\
\hline $\mathrm{C}(71)$ & $0.4686(2)$ & $0.30906(16)$ & $0.41531(15)$ & $0.0376(8)$ \\
\hline $\mathrm{C}(72)$ & $0.48107(18)$ & $0.30407(13)$ & $0.48268(14)$ & $0.0277(6)$ \\
\hline $\mathrm{N}(1)$ & $0.50277(15)$ & $0.09572(10)$ & $0.64374(11)$ & $0.0233(5)$ \\
\hline $\mathrm{N}(2)$ & $0.47399(15)$ & $0.26919(11)$ & $0.91974(11)$ & $0.0264(5)$ \\
\hline $\mathrm{N}(3)$ & $0.48385(14)$ & $0.39344(10)$ & $0.63353(11)$ & $0.0215(5)$ \\
\hline $\mathrm{P}(1)$ & $0.40488(5)$ & $0.10325(3)$ & $0.61561(3)$ & $0.02033(15)$ \\
\hline $\mathrm{P}(2)$ & $0.54862(5)$ & $0.08902(3)$ & $0.71597(3)$ & $0.02199(16)$ \\
\hline $\mathrm{P}(3)$ & $0.38150(4)$ & $0.24038(3)$ & $0.91079(3)$ & $0.02025(15)$ \\
\hline
\end{tabular}


Tabelle 66: Fortsetzung

\begin{tabular}{|r|r|r|r|r|}
\hline $\mathrm{P}(4)$ & $0.52419(5)$ & $0.32340(3)$ & $0.88956(3)$ & $0.02234(16)$ \\
$\mathrm{P}(5)$ & $0.38212(4)$ & $0.40099(3)$ & $0.63117(3)$ & $0.01979(15)$ \\
$\mathrm{P}(6)$ & $0.54477(4)$ & $0.34280(3)$ & $0.60642(3)$ & $0.01937(15)$ \\
$\mathrm{S}(1)$ & $0.32806(4)$ & $0.16305(3)$ & $0.66029(3)$ & $0.02231(15)$ \\
$\mathrm{S}(2)$ & $0.54707(5)$ & $0.15720(3)$ & $0.77838(4)$ & $0.02852(17)$ \\
$\mathrm{S}(3)$ & $0.31846(4)$ & $0.23354(3)$ & $0.81933(3)$ & $0.02322(15)$ \\
$\mathrm{S}(4)$ & $0.46312(5)$ & $0.37645(3)$ & $0.82240(4)$ & $0.02821(17)$ \\
$\mathrm{S}(5)$ & $0.30889(4)$ & $0.33442(3)$ & $0.66784(3)$ & $0.02213(15)$ \\
$\mathrm{S}(6)$ & $0.53332(5)$ & $0.25906(3)$ & $0.63756(4)$ & $0.02607(16)$ \\
$\mathrm{Cu}(1)$ & $0.40840(2)$ & $0.182869(15)$ & $0.757622(17)$ & $0.02634(9)$ \\
$\mathrm{Cu}(2)$ & $0.37180(2)$ & $0.313633(16)$ & $0.768449(16)$ & $0.02487(9)$ \\
$\mathrm{Cu}(3)$ & $0.39242(2)$ & $0.253362(15)$ & $0.650193(17)$ & $0.02383(9)$ \\
$\mathrm{O}(1 \mathrm{~A})$ & $0.4882(3)$ & $0.2929(2)$ & $0.2470(2)$ & $0.0726(13)$ \\
$\mathrm{O}(1 \mathrm{~B})$ & $0.4957(7)$ & $0.2376(4)$ & $0.2681(5)$ & $0.075(3)$ \\
$\mathrm{C}(1 \mathrm{~L})$ & $0.5719(3)$ & $0.2571(2)$ & $0.2679(2)$ & $0.0777(14)$ \\
$\mathrm{C}(2 \mathrm{~L})$ & $0.6045(3)$ & $0.2436(2)$ & $0.2053(2)$ & $0.0641(11)$ \\
$\mathrm{C}(3 \mathrm{~L})$ & $0.5216(3)$ & $0.2361(2)$ & $0.1597(2)$ & $0.0739(13)$ \\
$\mathrm{C}(4 \mathrm{LA})$ & $0.4642(4)$ & $0.2790(3)$ & $0.1830(3)$ & $0.0613(17)$ \\
$\mathrm{C}(4 \mathrm{LB})$ & $0.4535(7)$ & $0.2459(5)$ & $0.2078(5)$ & $0.045(3)$ \\
\hline
\end{tabular}

Tabelle 67: Bindungslängen [pm] in $\underline{15}$

\begin{tabular}{|l|r||l|r|}
\hline $\mathrm{Cu}(1)-\mathrm{Cu}(3)$ & $271.56(5)$ & $\mathrm{Cu}(1)-\mathrm{Cu}(2)$ & $300.66(5)$ \\
$\mathrm{Cu}(2)-\mathrm{Cu}(3)$ & $282.76(5)$ & $\mathrm{S}(1)-\mathrm{Cu}(3)$ & $227.97(8)$ \\
$\mathrm{S}(1)-\mathrm{Cu}(1)$ & $231.27(8)$ & $\mathrm{S}(2)-\mathrm{Cu}(1)$ & $223.14(8)$ \\
$\mathrm{S}(3)-\mathrm{Cu}(1)$ & $226.88(8)$ & $\mathrm{S}(3)-\mathrm{Cu}(2)$ & $227.33(8)$ \\
$\mathrm{S}(4)-\mathrm{Cu}(2)$ & $222.94(8)$ & $\mathrm{S}(5)-\mathrm{Cu}(2)$ & $226.35(8)$ \\
$\mathrm{S}(5)-\mathrm{Cu}(3)$ & $228.19(8)$ & $\mathrm{S}(6)-\mathrm{Cu}(3)$ & $222.34(8)$ \\
$\mathrm{N}(1)-\mathrm{P}(1)$ & $158.7(2)$ & $\mathrm{N}(1)-\mathrm{P}(2)$ & $160.1(2)$ \\
$\mathrm{N}(2)-\mathrm{P}(3)$ & $156.9(2)$ & $\mathrm{N}(2)-\mathrm{P}(4)$ & $159.9(2)$ \\
$\mathrm{N}(3)-\mathrm{P}(5)$ & $158.2(2)$ & $\mathrm{N}(3)-\mathrm{P}(6)$ & $160.8(2)$ \\
$\mathrm{P}(1)-\mathrm{S}(1)$ & $206.20(10)$ & $\mathrm{P}(2)-\mathrm{S}(2)$ & $200.18(10)$ \\
$\mathrm{P}(3)-\mathrm{S}(3)$ & $205.36(10)$ & $\mathrm{P}(4)-\mathrm{S}(4)$ & $200.31(10)$ \\
$\mathrm{P}(5)-\mathrm{S}(5)$ & $205.92(10)$ & $\mathrm{P}(6)-\mathrm{S}(6)$ & $200.21(10)$ \\
$\mathrm{C}(7)-\mathrm{P}(1)$ & $181.9(3)$ & $\mathrm{C}(13)-\mathrm{P}(2)$ & $182.8(3)$ \\
$\mathrm{C}(19)-\mathrm{P}(2)$ & $182.2(3)$ & $\mathrm{C}(25)-\mathrm{P}(3)$ & $181.8(3)$ \\
$\mathrm{C}(31)-\mathrm{P}(3)$ & $181.9(3)$ & $\mathrm{C}(37)-\mathrm{P}(4)$ & $182.3(3)$ \\
$\mathrm{C}(43)-\mathrm{P}(4)$ & $181.4(3)$ & $\mathrm{C}(49)-\mathrm{P}(5)$ & $181.4(3)$ \\
$\mathrm{C}(55)-\mathrm{P}(5)$ & $181.9(3)$ & $\mathrm{C}(61)-\mathrm{P}(6)$ & $181.5(3)$ \\
$\mathrm{C}(67)-\mathrm{P}(6)$ & $181.2(3)$ & & \\
\hline
\end{tabular}


Tabelle 68: Bindungswinkel $\left[^{\circ}\right]$ in $\underline{15}$

\begin{tabular}{|c|c|c|c|}
\hline $\mathrm{P}(1)-\mathrm{S}(1)-\mathrm{Cu}(3)$ & $105.50(4)$ & $\mathrm{P}(1)-\mathrm{S}(1)-\mathrm{Cu}(1)$ & $102.89(4)$ \\
\hline $\mathrm{Cu}(3)-\mathrm{S}(1)-\mathrm{Cu}(1)$ & $72.50(2)$ & $\mathrm{P}(2)-\mathrm{S}(2)-\mathrm{Cu}(1)$ & $97.93(4)$ \\
\hline $\mathrm{P}(3)-\mathrm{S}(3)-\mathrm{Cu}(1)$ & $106.47(4)$ & $\mathrm{P}(3)-\mathrm{S}(3)-\mathrm{Cu}(2)$ & $101.52(4)$ \\
\hline $\mathrm{Cu}(1)-\mathrm{S}(3)-\mathrm{Cu}(2)$ & $82.89(3)$ & $\mathrm{P}(4)-\mathrm{S}(4)-\mathrm{Cu}(2)$ & $102.14(4)$ \\
\hline $\mathrm{P}(5)-\mathrm{S}(5)-\mathrm{Cu}(2)$ & $106.08(4)$ & $\mathrm{P}(5)-\mathrm{S}(5)-\mathrm{Cu}(3)$ & $100.69(3)$ \\
\hline $\mathrm{Cu}(2)-\mathrm{S}(5)-\mathrm{Cu}(3)$ & $76.93(2)$ & $\mathrm{P}(6)-\mathrm{S}(6)-\mathrm{Cu}(3)$ & $101.86(4)$ \\
\hline $\mathrm{S}(2)-\mathrm{Cu}(1)-\mathrm{S}(3)$ & $130.47(3)$ & $\mathrm{S}(2)-\mathrm{Cu}(1)-\mathrm{S}(1)$ & $123.81(3)$ \\
\hline $\mathrm{S}(3)-\mathrm{Cu}(1)-\mathrm{S}(1)$ & $105.70(3)$ & $\mathrm{S}(2)-\mathrm{Cu}(1)-\mathrm{Cu}(3)$ & $109.16(3)$ \\
\hline $\mathrm{S}(3)-\mathrm{Cu}(1)-\mathrm{Cu}(3)$ & $98.29(2)$ & $\mathrm{S}(1)-\mathrm{Cu}(1)-\mathrm{Cu}(3)$ & $53.19(2)$ \\
\hline $\mathrm{S}(2)-\mathrm{Cu}(1)-\mathrm{Cu}(2)$ & $115.05(2)$ & $\mathrm{S}(3)-\mathrm{Cu}(1)-\mathrm{Cu}(2)$ & $48.62(2)$ \\
\hline $\mathrm{S}(1)-\mathrm{Cu}(1)-\mathrm{Cu}(2)$ & $99.29(2)$ & $\mathrm{Cu}(3)-\mathrm{Cu}(1)-\mathrm{Cu}(2)$ & $58.964(12)$ \\
\hline $\mathrm{S}(4)-\mathrm{Cu}(2)-\mathrm{S}(5)$ & $122.16(3)$ & $\mathrm{S}(4)-\mathrm{Cu}(2)-\mathrm{S}(3)$ & $120.83(3)$ \\
\hline $\mathrm{S}(5)-\mathrm{Cu}(2)-\mathrm{S}(3)$ & $116.10(3)$ & $\mathrm{S}(4)-\mathrm{Cu}(2)-\mathrm{Cu}(3)$ & $128.33(3)$ \\
\hline $\mathrm{S}(5)-\mathrm{Cu}(2)-\mathrm{Cu}(3)$ & $51.82(2)$ & $\mathrm{S}(3)-\mathrm{Cu}(2)-\mathrm{Cu}(3)$ & $95.07(2)$ \\
\hline $\mathrm{S}(4)-\mathrm{Cu}(2)-\mathrm{Cu}(1)$ & $122.74(2)$ & $\mathrm{S}(5)-\mathrm{Cu}(2)-\mathrm{Cu}(1)$ & $101.75(2)$ \\
\hline $\mathrm{S}(3)-\mathrm{Cu}(2)-\mathrm{Cu}(1)$ & $48.49(2)$ & $\mathrm{Cu}(3)-\mathrm{Cu}(2)-\mathrm{Cu}(1)$ & $55.378(12)$ \\
\hline $\mathrm{S}(6)-\mathrm{Cu}(3)-\mathrm{S}(1)$ & $120.21(3)$ & $\mathrm{S}(6)-\mathrm{Cu}(3)-\mathrm{S}(5)$ & $123.22(3)$ \\
\hline $\mathrm{S}(1)-\mathrm{Cu}(3)-\mathrm{S}(5)$ & $116.04(3)$ & $\mathrm{S}(6)-\mathrm{Cu}(3)-\mathrm{Cu}(1)$ & $96.02(2)$ \\
\hline $\mathrm{S}(1)-\mathrm{Cu}(3)-\mathrm{Cu}(1)$ & $54.31(2)$ & $\mathrm{S}(5)-\mathrm{Cu}(3)-\mathrm{Cu}(1)$ & $110.56(2)$ \\
\hline $\mathrm{S}(6)-\mathrm{Cu}(3)-\mathrm{Cu}(2)$ & $104.79(2)$ & $\mathrm{S}(1)-\mathrm{Cu}(3)-\mathrm{Cu}(2)$ & $105.49(2)$ \\
\hline $\mathrm{S}(5)-\mathrm{Cu}(3)-\mathrm{Cu}(2)$ & $51.24(2)$ & $\mathrm{Cu}(1)-\mathrm{Cu}(3)-\mathrm{Cu}(2)$ & $65.657(13)$ \\
\hline $\mathrm{P}(1)-\mathrm{N}(1)-\mathrm{P}(2)$ & $133.31(15)$ & $\mathrm{P}(3)-\mathrm{N}(2)-\mathrm{P}(4)$ & $137.54(16)$ \\
\hline $\mathrm{P}(5)-\mathrm{N}(3)-\mathrm{P}(6)$ & $132.63(15)$ & $\mathrm{N}(1)-\mathrm{P}(1)-\mathrm{C}(1)$ & $106.59(13)$ \\
\hline $\mathrm{N}(1)-\mathrm{P}(1)-\mathrm{C}(7)$ & $112.45(12)$ & $\mathrm{C}(1)-\mathrm{P}(1)-\mathrm{C}(7)$ & $103.39(13)$ \\
\hline $\mathrm{N}(1)-\mathrm{P}(1)-\mathrm{S}(1)$ & $118.45(9)$ & $\mathrm{C}(1)-\mathrm{P}(1)-\mathrm{S}(1)$ & $110.06(10)$ \\
\hline $\mathrm{C}(7)-\mathrm{P}(1)-\mathrm{S}(1)$ & $104.93(9)$ & $\mathrm{N}(1)-\mathrm{P}(2)-\mathrm{C}(19)$ & $103.55(13)$ \\
\hline $\mathrm{N}(1)-\mathrm{P}(2)-\mathrm{C}(13)$ & $109.33(13)$ & $\mathrm{N}(1)-\mathrm{P}(2)-\mathrm{S}(2)$ & $119.74(9)$ \\
\hline $\mathrm{C}(19)-\mathrm{P}(2)-\mathrm{S}(2)$ & 109.44(10) & $\mathrm{C}(13)-\mathrm{P}(2)-\mathrm{S}(2)$ & $108.67(10)$ \\
\hline $\mathrm{N}(2)-\mathrm{P}(3)-\mathrm{C}(25)$ & $106.34(13)$ & $\mathrm{N}(2)-\mathrm{P}(3)-\mathrm{C}(31)$ & $111.35(13)$ \\
\hline $\mathrm{N}(2)-\mathrm{P}(3)-\mathrm{S}(3)$ & $119.92(9)$ & $\mathrm{C}(25)-\mathrm{P}(3)-\mathrm{S}(3)$ & $107.80(10)$ \\
\hline $\mathrm{C}(31)-\mathrm{P}(3)-\mathrm{S}(3)$ & $107.23(10)$ & $\mathrm{N}(2)-\mathrm{P}(4)-\mathrm{C}(43)$ & $105.80(13)$ \\
\hline $\mathrm{N}(2)-\mathrm{P}(4)-\mathrm{C}(37)$ & $106.53(13)$ & $\mathrm{N}(2)-\mathrm{P}(4)-\mathrm{S}(4)$ & $120.34(9)$ \\
\hline $\mathrm{C}(43)-\mathrm{P}(4)-\mathrm{S}(4)$ & $110.59(10)$ & $\mathrm{C}(37)-\mathrm{P}(4)-\mathrm{S}(4)$ & $107.00(10)$ \\
\hline $\mathrm{N}(3)-\mathrm{P}(5)-\mathrm{C}(49)$ & $105.04(13)$ & $\mathrm{N}(3)-\mathrm{P}(5)-\mathrm{C}(55)$ & $112.82(12)$ \\
\hline $\mathrm{N}(3)-\mathrm{P}(5)-\mathrm{S}(5)$ & $119.25(9)$ & $\mathrm{C}(49)-\mathrm{P}(5)-\mathrm{S}(5)$ & $109.78(9)$ \\
\hline $\mathrm{C}(55)-\mathrm{P}(5)-\mathrm{S}(5)$ & $105.45(10)$ & $\mathrm{N}(3)-\mathrm{P}(6)-\mathrm{C}(67)$ & $109.13(12)$ \\
\hline $\mathrm{N}(3)-\mathrm{P}(6)-\mathrm{C}(61)$ & $104.90(12)$ & $\mathrm{N}(3)-\mathrm{P}(6)-\mathrm{S}(6)$ & $118.99(9)$ \\
\hline $\mathrm{C}(67)-\mathrm{P}(6)-\mathrm{S}(6)$ & $109.67(10)$ & $\mathrm{C}(61)-\mathrm{P}(6)-\mathrm{S}(6)$ & $108.16(9)$ \\
\hline
\end{tabular}




\subsection{Daten zur Kristallstrukturanalyse von $\left[\mathrm{Cu}_{3}\left(\mathrm{dppaSe}_{2}\right)_{3}\right] \underline{16}$}

\begin{tabular}{|ll|}
\hline Summenformel & $\mathrm{C}_{76} \mathrm{H}_{68} \mathrm{Cu}_{3} \mathrm{~N}_{3} \mathrm{OP}_{6} \mathrm{Se}_{6}$ \\
Molekulargewicht $\left[\mathrm{g} \cdot \mathrm{mol}^{-1}\right]$ & 1889.53 \\
Temperatur & $133(2) \mathrm{K}$ \\
Wellenlänge & $71.073 \mathrm{pm}$ \\
Kristallsystem & monoklin \\
Raumgruppe & $P 2_{1} / n$ \\
Gitterkonstanten & $\mathrm{a}=15.5015(13) \AA, \alpha=90^{\circ}$ \\
& $\mathrm{b}=22.7731(16) \AA, \beta=95.303(4)^{\circ}$ \\
& $\mathrm{c}=20.7483(18) \AA, \gamma=90^{\circ}$ \\
Volumen & $7293.2(10) \AA^{3}$ \\
Formeleinheiten & 4 \\
Röntgenographische Dichte $\left[\mathrm{g} \cdot \mathrm{cm}^{-3}\right]$ & 1.721 \\
Absorptionskoeffizient $\left[\mathrm{mm}^{-1}\right]$ & 4.040 \\
$\mathrm{~F}(000)$ & 3736 \\
Meßbereich $\theta$ & $1.81-23.26^{\circ}$ \\
Indexbereiche & $-17 \leq \mathrm{h} \leq 17$ \\
& $-25 \leq \mathrm{k} \leq 20$ \\
& $-23 \leq 1 \leq 23$ \\
Gemessene Reflexe & 73192 \\
Unabhängige Reflexe & 10488 \\
Beobachtete Reflexe $[\mathrm{I}>2 \sigma]$ & 7633 \\
$\mathrm{R}_{\text {int }}$ & 0.1058 \\
Parameter & 839 \\
R1; wR2[I $>2 \sigma(\mathrm{I})]$ & $0.0389 ; 0.0681$ \\
&
\end{tabular}


Tabelle 70: Atomkoordinaten und äquivalente isotrope Auslenkungsparameter $\left[\AA^{2}\right]$ für $\underline{16}$

\begin{tabular}{|c|c|c|c|c|}
\hline Atom & $\mathrm{x}$ & $\mathrm{y}$ & $\mathrm{z}$ & $\mathrm{U}_{e q}$ \\
\hline $\mathrm{C}(1)$ & $0.4028(3)$ & $0.1161(2)$ & $0.5306(2)$ & $0.0157(13)$ \\
$\mathrm{C}(2)$ & $0.3247(4)$ & $0.1239(2)$ & $0.4917(3)$ & $0.0234(14)$ \\
$\mathrm{C}(3)$ & $0.3250(4)$ & $0.1336(3)$ & $0.4261(3)$ & $0.0279(15)$ \\
$\mathrm{C}(4)$ & $0.4030(4)$ & $0.1364(3)$ & $0.3984(3)$ & $0.0278(15)$ \\
$\mathrm{C}(5)$ & $0.4802(4)$ & $0.1292(3)$ & $0.4362(3)$ & $0.0309(16)$ \\
$\mathrm{C}(6)$ & $0.4803(4)$ & $0.1196(2)$ & $0.5018(3)$ & $0.0239(14)$ \\
$\mathrm{C}(7)$ & $0.3443(3)$ & $0.0291(2)$ & $0.6154(3)$ & $0.0155(13)$ \\
$\mathrm{C}(8)$ & $0.3038(3)$ & $0.0122(2)$ & $0.6694(3)$ & $0.0198(13)$ \\
$\mathrm{C}(9)$ & $0.2622(4)$ & $-0.0415(2)$ & $0.6714(3)$ & $0.0274(15)$ \\
$\mathrm{C}(10)$ & $0.2617(4)$ & $-0.0791(3)$ & $0.6190(3)$ & $0.0325(16)$ \\
$\mathrm{C}(11)$ & $0.3022(4)$ & $-0.0635(3)$ & $0.5652(3)$ & $0.0297(16)$ \\
$\mathrm{C}(12)$ & $0.3437(4)$ & $-0.0096(2)$ & $0.5627(3)$ & $0.0264(15)$ \\
$\mathrm{C}(13)$ & $0.5072(3)$ & $0.0193(2)$ & $0.7523(3)$ & $0.0188(13)$ \\
$\mathrm{C}(14)$ & $0.4791(3)$ & $0.0199(2)$ & $0.8144(3)$ & $0.0218(14)$ \\
$\mathrm{C}(15)$ & $0.4426(4)$ & $-0.0301(3)$ & $0.8386(3)$ & $0.0374(17)$ \\
$\mathrm{C}(16)$ & $0.4322(4)$ & $-0.0802(3)$ & $0.8008(4)$ & $0.0371(18)$ \\
$\mathrm{C}(17)$ & $0.4608(4)$ & $-0.0816(3)$ & $0.7398(3)$ & $0.0317(16)$ \\
$\mathrm{C}(18)$ & $0.4981(4)$ & $-0.0318(2)$ & $0.7156(3)$ & $0.0235(14)$ \\
$\mathrm{C}(19)$ & $0.6587(3)$ & $0.0690(2)$ & $0.7020(3)$ & $0.0175(13)$ \\
$\mathrm{C}(20)$ & $0.7275(4)$ & $0.0884(2)$ & $0.7441(3)$ & $0.0246(14)$ \\
$\mathrm{C}(21)$ & $0.8121(4)$ & $0.0728(3)$ & $0.7344(3)$ & $0.0316(16)$ \\
$\mathrm{C}(22)$ & $0.8279(4)$ & $0.0375(3)$ & $0.6829(3)$ & $0.0352(17)$ \\
$\mathrm{C}(23)$ & $0.7601(4)$ & $0.0184(3)$ & $0.6403(3)$ & $0.0296(15)$ \\
$\mathrm{C}(24)$ & $0.6758(4)$ & $0.0345(2)$ & $0.6494(3)$ & $0.0222(14)$ \\
$\mathrm{C}(25)$ & $0.3945(4)$ & $0.1711(2)$ & $0.9491(3)$ & $0.0184(13)$ \\
$\mathrm{C}(26)$ & $0.3244(4)$ & $0.1334(2)$ & $0.9505(3)$ & $0.0234(14)$ \\
$\mathrm{C}(27)$ & $0.3349(4)$ & $0.0783(2)$ & $0.9782(3)$ & $0.0251(14)$ \\
$\mathrm{C}(28)$ & $0.4163(4)$ & $0.0604(3)$ & $1.0042(3)$ & $0.0320(16)$ \\
$\mathrm{C}(29)$ & $0.4862(4)$ & $0.0965(3)$ & $1.0011(3)$ & $0.0381(17)$ \\
$\mathrm{C}(30)$ & $0.4764(4)$ & $0.1525(3)$ & $0.9740(3)$ & $0.0275(15)$ \\
$\mathrm{C}(31)$ & $0.3074(3)$ & $0.2784(2)$ & $0.9698(2)$ & $0.0164(13)$ \\
$\mathrm{C}(32)$ & $0.3443(4)$ & $0.3104(3)$ & $1.0232(3)$ & $0.0272(15)$ \\
$\mathrm{C}(33)$ & $0.2926(4)$ & $0.3318(3)$ & $1.0689(3)$ & $0.0333(16)$ \\
$\mathrm{C}(34)$ & $0.2053(4)$ & $0.3209(2)$ & $1.0629(3)$ & $0.0297(16)$ \\
$\mathrm{C}(35)$ & $0.1674(4)$ & $0.2902(2)$ & $1.0096(3)$ & $0.0265(15)$ \\
$\mathrm{C}(36)$ & $0.2173(4)$ & $0.2692(2)$ & $0.9634(3)$ & $0.0201(14)$ \\
$\mathrm{C}(37)$ & $0.5631(3)$ & $0.3746(2)$ & $0.9613(3)$ & $0.0177(13)$ \\
$\mathrm{C}(38)$ & $0.5788(4)$ & $0.4341(3)$ & $0.9513(3)$ & $0.0290(15)$ \\
\hline & & & & \\
\hline
\end{tabular}


Tabelle 70: Fortsetzung

\begin{tabular}{|c|c|c|c|c|}
\hline $\mathrm{C}(39)$ & $0.6044(4)$ & $0.4708(3)$ & $1.0037(4)$ & $0.0434(19)$ \\
$\mathrm{C}(40)$ & $0.6136(4)$ & $0.4485(3)$ & $1.0656(3)$ & $0.0391(18)$ \\
$\mathrm{C}(41)$ & $0.6004(4)$ & $0.3894(3)$ & $1.0751(3)$ & $0.0423(18)$ \\
$\mathrm{C}(42)$ & $0.5755(4)$ & $0.3524(3)$ & $1.0242(3)$ & $0.0288(15)$ \\
$\mathrm{C}(43)$ & $0.6212(3)$ & $0.2934(2)$ & $0.8666(3)$ & $0.0196(13)$ \\
$\mathrm{C}(44)$ & $0.6472(4)$ & $0.3053(2)$ & $0.8056(3)$ & $0.0236(14)$ \\
$\mathrm{C}(45)$ & $0.7202(4)$ & $0.2781(3)$ & $0.7852(3)$ & $0.0284(15)$ \\
$\mathrm{C}(46)$ & $0.7674(4)$ & $0.2391(3)$ & $0.8250(3)$ & $0.0285(15)$ \\
$\mathrm{C}(47)$ & $0.7420(4)$ & $0.2265(3)$ & $0.8859(3)$ & $0.0302(15)$ \\
$\mathrm{C}(48)$ & $0.6685(3)$ & $0.2537(2)$ & $0.9066(3)$ & $0.0243(14)$ \\
$\mathrm{C}(49)$ & $0.3629(3)$ & $0.4739(2)$ & $0.6683(3)$ & $0.0143(12)$ \\
$\mathrm{C}(50)$ & $0.4291(4)$ & $0.4976(2)$ & $0.7099(3)$ & $0.0201(14)$ \\
$\mathrm{C}(51)$ & $0.4167(4)$ & $0.5507(2)$ & $0.7410(3)$ & $0.0220(14)$ \\
$\mathrm{C}(52)$ & $0.3388(4)$ & $0.5800(3)$ & $0.7301(3)$ & $0.0268(15)$ \\
$\mathrm{C}(53)$ & $0.2722(4)$ & $0.5567(2)$ & $0.6887(3)$ & $0.0235(14)$ \\
$\mathrm{C}(54)$ & $0.2845(4)$ & $0.5035(2)$ & $0.6582(3)$ & $0.0210(14)$ \\
$\mathrm{C}(55)$ & $0.3336(4)$ & $0.4210(2)$ & $0.5430(3)$ & $0.0170(13)$ \\
$\mathrm{C}(56)$ & $0.3837(4)$ & $0.4555(2)$ & $0.5042(3)$ & $0.0223(14)$ \\
$\mathrm{C}(57)$ & $0.3525(4)$ & $0.4702(3)$ & $0.4417(3)$ & $0.0318(16)$ \\
$\mathrm{C}(58)$ & $0.2712(5)$ & $0.4521(3)$ & $0.4169(3)$ & $0.0341(17)$ \\
$\mathrm{C}(59)$ & $0.2219(4)$ & $0.4182(3)$ & $0.4549(3)$ & $0.0308(16)$ \\
$\mathrm{C}(60)$ & $0.2527(4)$ & $0.4023(3)$ & $0.5168(3)$ & $0.0266(15)$ \\
$\mathrm{C}(61)$ & $0.6520(3)$ & $0.3702(2)$ & $0.6330(3)$ & $0.0169(13)$ \\
$\mathrm{C}(62)$ & $0.6690(4)$ & $0.4112(2)$ & $0.6821(3)$ & $0.0210(14)$ \\
$\mathrm{C}(63)$ & $0.7524(4)$ & $0.4265(3)$ & $0.7024(3)$ & $0.0277(15)$ \\
$\mathrm{C}(64)$ & $0.8211(4)$ & $0.4024(3)$ & $0.6738(3)$ & $0.0300(16)$ \\
$\mathrm{C}(65)$ & $0.8058(4)$ & $0.3610(3)$ & $0.6252(3)$ & $0.0284(15)$ \\
$\mathrm{C}(66)$ & $0.7216(4)$ & $0.3443(2)$ & $0.6050(3)$ & $0.0222(14)$ \\
$\mathrm{C}(67)$ & $0.5348(3)$ & $0.3440(2)$ & $0.5201(2)$ & $0.0152(12)$ \\
$\mathrm{C}(68)$ & $0.5770(4)$ & $0.3871(2)$ & $0.4869(3)$ & $0.0244(14)$ \\
$\mathrm{C}(69)$ & $0.5657(4)$ & $0.3899(3)$ & $0.4201(3)$ & $0.0278(15)$ \\
$\mathrm{C}(70)$ & $0.5126(4)$ & $0.3495(3)$ & $0.3860(3)$ & $0.0335(16)$ \\
$\mathrm{C}(71)$ & $0.4713(4)$ & $0.3060(3)$ & $0.4184(3)$ & $0.0331(16)$ \\
$\mathrm{C}(72)$ & $0.4823(4)$ & $0.3036(2)$ & $0.4850(3)$ & $0.0262(15)$ \\
$\mathrm{N}(1)$ & $0.4998(3)$ & $0.09433(18)$ & $0.6444(2)$ & $0.0159(11)$ \\
$\mathrm{N}(2)$ & $0.4725(3)$ & $0.27514(19)$ & $0.9244(2)$ & $0.0186(11)$ \\
\hline & $0.4802(3)$ & $0.39637(18)$ & $0.6313(2)$ & $0.0150(10)$ \\
$\mathrm{P}(3)$ & $0.54656(9)$ & $0.08570(6)$ & $0.71588(7)$ & $0.0167(3)$ \\
& & & & $0.0157(3)$ \\
\hline
\end{tabular}


Tabelle 70: Fortsetzung

\begin{tabular}{|r|r|r|r|r|}
\hline $\mathrm{P}(4)$ & $0.52487(9)$ & $0.32707(6)$ & $0.89428(7)$ & $0.0178(3)$ \\
$\mathrm{P}(5)$ & $0.37881(9)$ & $0.40592(6)$ & $0.62541(7)$ & $0.0157(3)$ \\
$\mathrm{P}(6)$ & $0.54273(9)$ & $0.34561(6)$ & $0.60801(7)$ & $0.0153(3)$ \\
$\mathrm{Se}(1)$ & $0.31424(3)$ & $0.16098(2)$ & $0.65949(3)$ & $0.01749(14)$ \\
$\mathrm{Se}(2)$ & $0.54586(4)$ & $0.15655(2)$ & $0.78527(3)$ & $0.02349(15)$ \\
$\mathrm{Se}(3)$ & $0.30798(4)$ & $0.23727(2)$ & $0.82050(3)$ & $0.01819(14)$ \\
$\mathrm{Se}(4)$ & $0.46292(4)$ & $0.38347(2)$ & $0.81985(3)$ & $0.02300(15)$ \\
$\mathrm{Se}(5)$ & $0.29400(3)$ & $0.33774(2)$ & $0.66236(3)$ & $0.01717(14)$ \\
$\mathrm{Se}(6)$ & $0.53162(4)$ & $0.25797(2)$ & $0.64597(3)$ & $0.02074(14)$ \\
$\mathrm{Cu}(1)$ & $0.40204(5)$ & $0.18369(3)$ & $0.75786(3)$ & $0.02213(18)$ \\
$\mathrm{Cu}(2)$ & $0.36810(5)$ & $0.31658(3)$ & $0.76576(3)$ & $0.02223(18)$ \\
$\mathrm{Cu}(3)$ & $0.38268(4)$ & $0.25390(3)$ & $0.65071(3)$ & $0.01952(17)$ \\
$\mathrm{O}(1 \mathrm{~A})$ & $0.4791(7)$ & $0.2889(5)$ & $0.2529(5)$ & $0.065(3)$ \\
$\mathrm{O}(1 \mathrm{~B})$ & $0.4880(8)$ & $0.2375(5)$ & $0.2694(6)$ & $0.083(4)$ \\
$\mathrm{C}(1 \mathrm{~L})$ & $0.5714(6)$ & $0.2538(4)$ & $0.2661(5)$ & $0.088(3)$ \\
$\mathrm{C}(2 \mathrm{~L})$ & $0.5936(6)$ & $0.2500(4)$ & $0.2011(4)$ & $0.072(3)$ \\
$\mathrm{C}(3 \mathrm{~L})$ & $0.5098(6)$ & $0.2438(4)$ & $0.1605(5)$ & $0.093(3)$ \\
$\mathrm{C}(4 \mathrm{LA})$ & $0.4528(11)$ & $0.2807(8)$ & $0.1894(9)$ & $0.069(5)$ \\
$\mathrm{C}(4 \mathrm{LB})$ & $0.4428(9)$ & $0.2480(7)$ & $0.2098(7)$ & $0.043(4)$ \\
\hline
\end{tabular}

Tabelle 71: Bindungslängen [pm] in $\underline{16}$

\begin{tabular}{|l|r||l|r|}
\hline $\mathrm{Cu}(1)-\mathrm{Cu}(3)$ & $273.25(10)$ & $\mathrm{Cu}(2)-\mathrm{Cu}(3)$ & $280.83(10)$ \\
$\mathrm{Se}(1)-\mathrm{Cu}(3)$ & $238.17(9)$ & $\mathrm{Se}(1)-\mathrm{Cu}(1)$ & $240.26(9)$ \\
$\mathrm{Se}(2)-\mathrm{Cu}(1)$ & $233.27(9)$ & $\mathrm{Se}(3)-\mathrm{Cu}(2)$ & $236.99(9)$ \\
$\mathrm{Se}(3)-\mathrm{Cu}(1)$ & $237.78(9)$ & $\mathrm{Se}(4)-\mathrm{Cu}(2)$ & $233.15(9)$ \\
$\mathrm{Se}(5)-\mathrm{Cu}(3)$ & $237.79(9)$ & $\mathrm{Se}(5)-\mathrm{Cu}(2)$ & $238.73(9)$ \\
$\mathrm{Se}(6)-\mathrm{Cu}(3)$ & $232.19(9)$ & $\mathrm{Cu}(1)-\mathrm{Cu}(3)$ & $273.25(10)$ \\
$\mathrm{Cu}(2)-\mathrm{Cu}(3)$ & $280.83(10)$ & $\mathrm{P}(1)-\mathrm{Se}(1)$ & $221.84(15)$ \\
$\mathrm{P}(2)-\mathrm{Se}(2)$ & $216.31(15)$ & $\mathrm{P}(3)-\mathrm{Se}(3)$ & $220.97(15)$ \\
$\mathrm{P}(4)-\mathrm{Se}(4)$ & $216.40(16)$ & $\mathrm{P}(5)-\mathrm{Se}(5)$ & $221.66(15)$ \\
$\mathrm{P}(6)-\mathrm{Se}(6)$ & $215.82(15)$ & $\mathrm{N}(1)-\mathrm{P}(1)$ & $159.2(5)$ \\
$\mathrm{N}(1)-\mathrm{P}(2)$ & $160.1(5)$ & $\mathrm{N}(2)-\mathrm{P}(3)$ & $157.5(4)$ \\
$\mathrm{N}(2)-\mathrm{P}(4)$ & $159.4(4)$ & $\mathrm{N}(3)-\mathrm{P}(5)$ & $158.1(4)$ \\
$\mathrm{N}(3)-\mathrm{P}(6)$ & $161.1(4)$ & & \\
\hline
\end{tabular}


Tabelle 72: Bindungswinkel $\left[^{\circ}\right]$ in $\underline{16}$

\begin{tabular}{|c|c|c|c|}
\hline $\mathrm{P}(1)-\mathrm{Se}(1)-\mathrm{Cu}(3)$ & $104.11(4)$ & $\mathrm{P}(1)-\mathrm{Se}(1)-\mathrm{Cu}(1)$ & $99.89(5)$ \\
\hline $\mathrm{Cu}(3)-\mathrm{Se}(1)-\mathrm{Cu}(1)$ & $69.66(3)$ & $\mathrm{P}(2)-\mathrm{Se}(2)-\mathrm{Cu}(1)$ & $95.67(5)$ \\
\hline $\mathrm{P}(3)-\mathrm{Se}(3)-\mathrm{Cu}(2)$ & $100.51(4)$ & $\mathrm{P}(3)-\mathrm{Se}(3)-\mathrm{Cu}(1)$ & $104.35(5)$ \\
\hline $\mathrm{Cu}(2)-\mathrm{Se}(3)-\mathrm{Cu}(1)$ & $80.85(3)$ & $\mathrm{P}(4)-\mathrm{Se}(4)-\mathrm{Cu}(2)$ & $100.03(5)$ \\
\hline $\mathrm{P}(5)-\mathrm{Se}(5)-\mathrm{Cu}(3)$ & $99.23(4)$ & $\mathrm{P}(5)-\mathrm{Se}(5)-\mathrm{Cu}(2)$ & $101.58(5)$ \\
\hline $\mathrm{Cu}(3)-\mathrm{Se}(5)-\mathrm{Cu}(2)$ & $72.22(3)$ & $\mathrm{P}(6)-\mathrm{Se}(6)-\mathrm{Cu}(3)$ & $99.53(5)$ \\
\hline $\mathrm{Se}(2)-\mathrm{Cu}(1)-\mathrm{Se}(3)$ & $128.55(4)$ & $\mathrm{Se}(2)-\mathrm{Cu}(1)-\mathrm{Se}(1)$ & $127.14(3)$ \\
\hline $\mathrm{Se}(3)-\mathrm{Cu}(1)-\mathrm{Se}(1)$ & $104.30(3)$ & $\mathrm{Se}(2)-\mathrm{Cu}(1)-\mathrm{Cu}(3)$ & $112.59(3)$ \\
\hline $\mathrm{Se}(3)-\mathrm{Cu}(1)-\mathrm{Cu}(3)$ & $96.76(3)$ & $\mathrm{Se}(1)-\mathrm{Cu}(1)-\mathrm{Cu}(3)$ & $54.81(2)$ \\
\hline $\mathrm{Se}(4)-\mathrm{Cu}(2)-\mathrm{Se}(3)$ & $121.71(4)$ & $\mathrm{Se}(4)-\mathrm{Cu}(2)-\mathrm{Se}(5)$ & $122.03(3)$ \\
\hline $\mathrm{Se}(3)-\mathrm{Cu}(2)-\mathrm{Se}(5)$ & $114.00(3)$ & $\mathrm{Se}(4)-\mathrm{Cu}(2)-\mathrm{Cu}(3)$ & $130.14(3)$ \\
\hline $\mathrm{Se}(3)-\mathrm{Cu}(2)-\mathrm{Cu}(3)$ & $94.94(3)$ & $\mathrm{Se}(5)-\mathrm{Cu}(2)-\mathrm{Cu}(3)$ & $53.74(2)$ \\
\hline $\mathrm{Se}(6)-\mathrm{Cu}(3)-\mathrm{Se}(5)$ & $123.97(3)$ & $\mathrm{Se}(6)-\mathrm{Cu}(3)-\mathrm{Se}(1)$ & $119.37(3)$ \\
\hline $\mathrm{Se}(5)-\mathrm{Cu}(3)-\mathrm{Se}(1)$ & $116.09(3)$ & $\mathrm{Se}(6)-\mathrm{Cu}(3)-\mathrm{Cu}(1)$ & $91.31(3)$ \\
\hline $\mathrm{Se}(5)-\mathrm{Cu}(3)-\mathrm{Cu}(1)$ & $114.09(3)$ & $\mathrm{Se}(1)-\mathrm{Cu}(3)-\mathrm{Cu}(1)$ & $55.53(2)$ \\
\hline $\mathrm{Se}(6)-\mathrm{Cu}(3)-\mathrm{Cu}(2)$ & $100.03(3)$ & $\mathrm{Se}(5)-\mathrm{Cu}(3)-\mathrm{Cu}(2)$ & $54.04(2)$ \\
\hline $\mathrm{Se}(1)-\mathrm{Cu}(3)-\mathrm{Cu}(2)$ & $108.37(3)$ & $\mathrm{Cu}(1)-\mathrm{Cu}(3)-\mathrm{Cu}(2)$ & $67.49(3)$ \\
\hline $\mathrm{N}(1)-\mathrm{P}(1)-\mathrm{Se}(1)$ & $118.63(17)$ & $\mathrm{C}(1)-\mathrm{P}(1)-\mathrm{Se}(1)$ & $109.04(18)$ \\
\hline $\mathrm{C}(7)-\mathrm{P}(1)-\mathrm{Se}(1)$ & $103.96(17)$ & $\mathrm{N}(1)-\mathrm{P}(2)-\mathrm{C}(13)$ & $110.2(2)$ \\
\hline $\mathrm{N}(1)-\mathrm{P}(2)-\mathrm{C}(19)$ & $103.6(2)$ & $\mathrm{N}(1)-\mathrm{P}(2)-\mathrm{Se}(2)$ & $119.68(16)$ \\
\hline $\mathrm{C}(13)-\mathrm{P}(2)-\mathrm{Se}(2)$ & $108.74(19)$ & $\mathrm{C}(19)-\mathrm{P}(2)-\mathrm{Se}(2)$ & $108.84(19)$ \\
\hline $\mathrm{N}(2)-\mathrm{P}(3)-\mathrm{C}(25)$ & $107.1(2)$ & $\mathrm{N}(2)-\mathrm{P}(3)-\mathrm{C}(31)$ & $111.5(2)$ \\
\hline $\mathrm{N}(2)-\mathrm{P}(3)-\mathrm{Se}(3)$ & $120.36(17)$ & $\mathrm{C}(25)-\mathrm{P}(3)-\mathrm{Se}(3)$ & $106.98(19)$ \\
\hline $\mathrm{C}(31)-\mathrm{P}(3)-\mathrm{Se}(3)$ & $106.50(18)$ & $\mathrm{N}(2)-\mathrm{P}(4)-\mathrm{C}(37)$ & $106.5(2)$ \\
\hline $\mathrm{N}(2)-\mathrm{P}(4)-\mathrm{C}(43)$ & $105.8(2)$ & $\mathrm{N}(2)-\mathrm{P}(4)-\mathrm{Se}(4)$ & $120.77(17)$ \\
\hline $\mathrm{C}(37)-\mathrm{P}(4)-\mathrm{Se}(4)$ & $106.49(18)$ & $\mathrm{C}(43)-\mathrm{P}(4)-\mathrm{Se}(4)$ & $110.39(19)$ \\
\hline $\mathrm{N}(3)-\mathrm{P}(5)-\mathrm{C}(49)$ & $105.0(2)$ & $\mathrm{N}(3)-\mathrm{P}(5)-\mathrm{C}(55)$ & $113.1(2)$ \\
\hline $\mathrm{N}(3)-\mathrm{P}(5)-\mathrm{Se}(5)$ & $119.62(16)$ & $\mathrm{C}(49)-\mathrm{P}(5)-\mathrm{Se}(5)$ & $108.46(17)$ \\
\hline $\mathrm{C}(55)-\mathrm{P}(5)-\mathrm{Se}(5)$ & $105.58(18)$ & $\mathrm{N}(3)-\mathrm{P}(6)-\mathrm{C}(61)$ & $105.3(2)$ \\
\hline $\mathrm{N}(3)-\mathrm{P}(6)-\mathrm{C}(67)$ & 109.2(2) & $\mathrm{N}(3)-\mathrm{P}(6)-\mathrm{Se}(6)$ & $118.92(16)$ \\
\hline $\mathrm{C}(61)-\mathrm{P}(6)-\mathrm{Se}(6)$ & $106.55(18)$ & $\mathrm{C}(67)-\mathrm{P}(6)-\mathrm{Se}(6)$ & $110.35(18)$ \\
\hline $\mathrm{P}(1)-\mathrm{N}(1)-\mathrm{P}(2)$ & $134.3(3)$ & $\mathrm{P}(3)-\mathrm{N}(2)-\mathrm{P}(4)$ & $140.6(3)$ \\
\hline $\mathrm{P}(5)-\mathrm{N}(3)-\mathrm{P}(6)$ & $134.2(3)$ & $\mathrm{N}(1)-\mathrm{P}(1)-\mathrm{C}(1)$ & $106.8(2)$ \\
\hline $\mathrm{N}(1)-\mathrm{P}(1)-\mathrm{C}(7)$ & $113.2(2)$ & & \\
\hline
\end{tabular}




\subsection{Daten zur Kristallstrukturanalyse von $\left[\mathrm{Ag}_{3}\left(\mathrm{dppaS}_{2}\right)_{3}\right] 17$}

\begin{tabular}{|ll|}
\hline Summenformel & $\mathrm{C}_{39} \mathrm{H}_{35} \mathrm{Ag}_{1.5} \mathrm{~N}_{1.5} \mathrm{O}_{0.75} \mathrm{P}_{3} \mathrm{~S}_{3}$ \\
Molekulargewicht $\left[\mathrm{g} \cdot \mathrm{mol}^{-1}\right]$ & 887.58 \\
Temperatur & $133(2) \mathrm{K}$ \\
Wellenlänge & $71.073 \mathrm{pm}$ \\
Kristallsystem & triklin \\
Raumgruppe & $P \overline{1}$ \\
Gitterkonstanten & $\mathrm{a}=9.0834(7) \AA, \alpha=82.122(4)^{\circ}$ \\
& $\mathrm{b}=15.2161(12) \AA, \beta=84.731(5)^{\circ} \circ$ \\
& $\mathrm{c}=27.444(2) \AA, \gamma=84.830(5)^{\circ}$ \\
& $\circ$ \\
Volumen & $3729.9(5) \AA^{3}$ \\
Formeleinheiten & 4 \\
Röntgenographische Dichte $\left[\mathrm{g} \cdot \mathrm{cm}^{-3}\right]$ & 1.581 \\
Absorptionskoeffizient $\left[\mathrm{mm}^{-1}\right]$ & 1.125 \\
$\mathrm{~F}(000)$ & 1796 \\
Meßbereich $\theta$ & $2.32-21.50^{\circ}$ \\
Indexbereiche & $-7 \leq \mathrm{h} \leq 9$ \\
& $-7 \leq \mathrm{k} \leq 15$ \\
& $-28 \leq 1 \leq 28$ \\
Gemessene Reflexe & 31135 \\
Unabhängige Reflexe & 8574 \\
Beobachtete Reflexe $[\mathrm{I}>2 \sigma]$ & 7478 \\
$\mathrm{R}_{\text {int }}$ & 0.0756 \\
Parameter & 812 \\
R1; wR2[I>2 $\sigma(\mathrm{I})]$ & $0.0886 ; 0.1665$ \\
\hline
\end{tabular}


Tabelle 74: Atomkoordinaten und äquivalente isotrope Auslenkungsparameter $\left[\AA^{2}\right]$ für $\underline{17}$

\begin{tabular}{|c|c|c|c|c|}
\hline Atom & $\mathrm{x}$ & $\mathrm{y}$ & z & $\mathrm{U}_{e q}$ \\
\hline $\operatorname{Ag}(1)$ & $0.6388(2)$ & $0.84505(13)$ & $0.22314(7)$ & $0.0257(6)$ \\
\hline $\operatorname{Ag}(2)$ & $0.6000(2)$ & $0.59991(12)$ & $0.18004(7)$ & $0.0216(5)$ \\
\hline $\operatorname{Ag}(3)$ & $0.4471(2)$ & $0.63408(12)$ & $0.32616(7)$ & $0.0254(6)$ \\
\hline $\mathrm{S}(1)$ & $0.7496(10)$ & $0.9822(6)$ & $0.1890(3)$ & $0.055(2)$ \\
\hline $\mathrm{S}(2)$ & $0.5703(7)$ & $0.7606(4)$ & $0.1566(2)$ & $0.0211(15)$ \\
\hline $\mathrm{S}(3)$ & $0.7340(7)$ & $0.4573(4)$ & $0.1650(2)$ & $0.0240(16)$ \\
\hline $\mathrm{S}(4)$ & $0.3934(7)$ & $0.5785(4)$ & $0.2509(2)$ & $0.0171(14)$ \\
\hline $\mathrm{S}(5)$ & $0.3454(7)$ & $0.6235(4)$ & $0.4118(2)$ & $0.0270(16)$ \\
\hline $\mathrm{S}(6)$ & $0.5964(7)$ & $0.7722(4)$ & $0.3075(2)$ & $0.0177(14)$ \\
\hline $\mathrm{P}(1)$ & $0.6808(7)$ & $0.9871(4)$ & $0.1227(3)$ & $0.0265(18)$ \\
\hline $\mathrm{P}(2)$ & $0.7202(7)$ & $0.8031(4)$ & $0.1003(2)$ & $0.0252(18)$ \\
\hline $\mathrm{P}(3)$ & $0.7451(7)$ & $0.4252(4)$ & $0.2380(2)$ & $0.0155(15)$ \\
\hline $\mathrm{P}(4)$ & $0.4333(7)$ & $0.4439(4)$ & $0.2670(2)$ & $0.0152(15)$ \\
\hline $\mathrm{P}(5)$ & $0.4505(6)$ & $0.7178(4)$ & $0.4357(2)$ & $0.0153(15)$ \\
\hline $\mathrm{P}(6)$ & $0.4498(7)$ & $0.8466(4)$ & $0.3473(2)$ & $0.0152(15)$ \\
\hline $\mathrm{N}(1)$ & $0.738(3)$ & $0.9056(15)$ & $0.0925(9)$ & $0.048(7)$ \\
\hline $\mathrm{N}(2)$ & $0.601(2)$ & $0.4038(12)$ & $0.2719(7)$ & $0.022(5)$ \\
\hline $\mathrm{N}(3)$ & $0.450(2)$ & $0.8154(12)$ & $0.4049(7)$ & $0.017(5)$ \\
\hline $\mathrm{C}(1)$ & $0.479(3)$ & $1.0016(19)$ & $0.1259(10)$ & $0.031(7)$ \\
\hline $\mathrm{C}(2)$ & $0.407(4)$ & $0.981(2)$ & $0.0855(13)$ & $0.067(12)$ \\
\hline $\mathrm{C}(3)$ & $0.246(5)$ & $0.992(2)$ & $0.0899(18)$ & $0.075(14)$ \\
\hline $\mathrm{C}(4)$ & $0.178(4)$ & $1.023(2)$ & $0.1300(18)$ & $0.058(11)$ \\
\hline $\mathrm{C}(5)$ & $0.248(4)$ & $1.036(2)$ & $0.1686(18)$ & $0.076(15)$ \\
\hline $\mathrm{C}(6)$ & $0.400(3)$ & $1.0282(16)$ & $0.1663(13)$ & $0.049(10)$ \\
\hline $\mathrm{C}(7 \mathrm{~A})$ & $0.731(4)$ & $1.086(2)$ & $0.0808(13)$ & $0.008(13)$ \\
\hline $\mathrm{C}(8 \mathrm{~A})$ & $0.769(5)$ & $1.159(3)$ & $0.1007(10)$ & $0.05(2)$ \\
\hline $\mathrm{C}(9 \mathrm{~A})$ & $0.813(5)$ & $1.234(2)$ & $0.0698(13)$ & $0.031(16)$ \\
\hline $\mathrm{C}(10 \mathrm{~A})$ & $0.818(4)$ & $1.236(2)$ & $0.0189(12)$ & $0.029(13)$ \\
\hline $\mathrm{C}(11 \mathrm{~A})$ & $0.781(5)$ & $1.162(3)$ & $-0.0011(10)$ & $0.052(18)$ \\
\hline $\mathrm{C}(12 \mathrm{~A})$ & $0.737(5)$ & $1.087(2)$ & $0.0299(13)$ & $0.057(19)$ \\
\hline $\mathrm{C}(7 \mathrm{~B})$ & $0.760(4)$ & $1.0880(19)$ & $0.0918(11)$ & $0.022(17)$ \\
\hline $\mathrm{C}(8 \mathrm{~B})$ & $0.746(4)$ & $1.169(2)$ & $0.1106(11)$ & $0.030(17)$ \\
\hline $\mathrm{C}(9 \mathrm{~B})$ & $0.815(4)$ & $1.2414(18)$ & $0.0849(12)$ & $0.027(15)$ \\
\hline $\mathrm{C}(10 \mathrm{~B})$ & $0.897(4)$ & $1.2330(16)$ & $0.0403(11)$ & $0.021(12)$ \\
\hline $\mathrm{C}(11 \mathrm{~B})$ & $0.911(3)$ & $1.1520(19)$ & $0.0215(9)$ & $0.020(11)$ \\
\hline $\mathrm{C}(12 \mathrm{~B})$ & $0.843(4)$ & $1.0796(16)$ & $0.0473(11)$ & $0.011(10)$ \\
\hline $\mathrm{C}(13)$ & $0.901(2)$ & $0.7473(17)$ & $0.1091(9)$ & $0.020(6)$ \\
\hline $\mathrm{C}(14)$ & $0.938(4)$ & $0.6650(19)$ & $0.0990(10)$ & $0.041(8)$ \\
\hline
\end{tabular}


Tabelle 74: Fortsetzung

\begin{tabular}{|r|r|r|r|r|}
\hline $\mathrm{C}(15)$ & $1.084(4)$ & $0.625(2)$ & $0.1055(13)$ & $0.055(10)$ \\
$\mathrm{C}(16)$ & $1.185(3)$ & $0.673(3)$ & $0.1216(11)$ & $0.047(9)$ \\
$\mathrm{C}(17)$ & $1.150(3)$ & $0.754(3)$ & $0.1331(14)$ & $0.061(10)$ \\
$\mathrm{C}(18)$ & $1.007(3)$ & $0.7952(19)$ & $0.1252(11)$ & $0.040(8)$ \\
$\mathrm{C}(19 \mathrm{~A})$ & $0.668(5)$ & $0.783(3)$ & $0.0475(14)$ & $0.031(18)$ \\
$\mathrm{C}(20 \mathrm{~A})$ & $0.771(4)$ & $0.792(4)$ & $0.0069(19)$ & $0.10(3)$ \\
$\mathrm{C}(21 \mathrm{~A})$ & $0.733(5)$ & $0.777(4)$ & $-0.0390(15)$ & $0.09(3)$ \\
$\mathrm{C}(22 \mathrm{~A})$ & $0.591(6)$ & $0.755(4)$ & $-0.0444(14)$ & $0.057(19)$ \\
$\mathrm{C}(23 \mathrm{~A})$ & $0.488(4)$ & $0.746(4)$ & $-0.0039(19)$ & $0.08(3)$ \\
$\mathrm{C}(24 \mathrm{~A})$ & $0.526(5)$ & $0.761(3)$ & $0.0421(15)$ & $0.07(2)$ \\
$\mathrm{C}(19 \mathrm{~B})$ & $0.656(4)$ & $0.761(2)$ & $0.0430(9)$ & $0.006(13)$ \\
$\mathrm{C}(20 \mathrm{~B})$ & $0.678(4)$ & $0.8145(17)$ & $-0.0021(11)$ & $0.041(15)$ \\
$\mathrm{C}(21 \mathrm{~B})$ & $0.634(4)$ & $0.7877(18)$ & $-0.0447(8)$ & $0.007(10)$ \\
$\mathrm{C}(22 \mathrm{~B})$ & $0.569(4)$ & $0.708(2)$ & $-0.0422(9)$ & $0.051(18)$ \\
$\mathrm{C}(23 \mathrm{~B})$ & $0.548(4)$ & $0.6546(17)$ & $0.0029(11)$ & $0.021(12)$ \\
$\mathrm{C}(24 \mathrm{~B})$ & $0.591(4)$ & $0.681(2)$ & $0.0455(9)$ & $0.029(13)$ \\
$\mathrm{C}(25)$ & $0.825(3)$ & $0.5114(15)$ & $0.2631(8)$ & $0.015(6)$ \\
$\mathrm{C}(26)$ & $0.784(3)$ & $0.5232(17)$ & $0.3114(9)$ & $0.025(7)$ \\
$\mathrm{C}(27)$ & $0.842(3)$ & $0.5909(15)$ & $0.3324(10)$ & $0.021(6)$ \\
$\mathrm{C}(28)$ & $0.937(3)$ & $0.6443(19)$ & $0.3029(12)$ & $0.038(8)$ \\
$\mathrm{C}(29)$ & $0.971(3)$ & $0.6366(16)$ & $0.2556(10)$ & $0.024(6)$ \\
$\mathrm{C}(30)$ & $0.918(2)$ & $0.5722(17)$ & $0.2352(9)$ & $0.021(7)$ \\
$\mathrm{C}(31)$ & $0.864(2)$ & $0.3237(14)$ & $0.2503(10)$ & $0.020(6)$ \\
$\mathrm{C}(32)$ & $0.849(3)$ & $0.2520(17)$ & $0.2275(11)$ & $0.029(7)$ \\
$\mathrm{C}(33)$ & $0.929(3)$ & $0.1718(17)$ & $0.2378(13)$ & $0.046(9)$ \\
$\mathrm{C}(34)$ & $1.034(3)$ & $0.1635(18)$ & $0.2742(13)$ & $0.042(8)$ \\
$\mathrm{C}(35)$ & $1.052(3)$ & $0.236(2)$ & $0.2962(10)$ & $0.042(9)$ \\
$\mathrm{C}(36)$ & $0.971(3)$ & $0.3165(19)$ & $0.2832(9)$ & $0.031(7)$ \\
$\mathrm{C}(37)$ & $0.336(2)$ & $0.4092(15)$ & $0.3256(9)$ & $0.019(6)$ \\
$\mathrm{C}(38)$ & $0.200(3)$ & $0.4504(18)$ & $0.3399(9)$ & $0.032(7)$ \\
$\mathrm{C}(39)$ & $0.123(3)$ & $0.422(2)$ & $0.3850(11)$ & $0.046(9)$ \\
$\mathrm{C}(46)$ & $0.218(3)$ & $0.310(2)$ & $0.1573(11)$ & $0.037(8)$ \\
$\mathrm{C}(40)$ & $0.192(3)$ & $0.3535(19)$ & $0.4153(11)$ & $0.040(8)$ \\
$\mathrm{C}(41)$ & $0.332(4)$ & $0.3182(18)$ & $0.4029(11)$ & $0.045(9)$ \\
$\mathrm{C}(42)$ & $0.404(4)$ & $0.3395(18)$ & $0.3582(10)$ & $0.048(9)$ \\
$\mathrm{C}(43)$ & $0.351(2)$ & $0.3950(17)$ & $0.2227(8)$ & $0.018(6)$ \\
\hline & $0.403(3)$ & $0.3105(15)$ & $0.2104(11)$ & $0.030(7)$ \\
$\mathrm{C}(45)$ & $0.3914(17)$ & $0.1686(10)$ & $0.031(7)$ \\
$\mathrm{C}(3)$ & $0.429(2)$ & $0.1985(9)$ & $0.034(7)$ \\
\hline
\end{tabular}


Tabelle 74: Fortsetzung

\begin{tabular}{|r|r|r|r|r|}
\hline $\mathrm{C}(49)$ & $0.643(2)$ & $0.6810(16)$ & $0.4440(9)$ & $0.015(6)$ \\
$\mathrm{C}(50)$ & $0.752(3)$ & $0.7323(17)$ & $0.4319(10)$ & $0.035(8)$ \\
$\mathrm{C}(51)$ & $0.902(3)$ & $0.6978(16)$ & $0.4373(10)$ & $0.026(7)$ \\
$\mathrm{C}(52)$ & $0.932(3)$ & $0.611(2)$ & $0.4551(10)$ & $0.029(7)$ \\
$\mathrm{C}(53)$ & $0.835(5)$ & $0.560(2)$ & $0.4671(11)$ & $0.054(10)$ \\
$\mathrm{C}(54)$ & $0.675(3)$ & $0.5898(18)$ & $0.4617(10)$ & $0.033(7)$ \\
$\mathrm{C}(55)$ & $0.366(2)$ & $0.7328(16)$ & $0.4957(9)$ & $0.016(6)$ \\
$\mathrm{C}(56)$ & $0.327(3)$ & $0.6640(18)$ & $0.5298(12)$ & $0.042(8)$ \\
$\mathrm{C}(57)$ & $0.258(3)$ & $0.678(2)$ & $0.5755(11)$ & $0.040(8)$ \\
$\mathrm{C}(58)$ & $0.231(3)$ & $0.758(3)$ & $0.5888(11)$ & $0.051(10)$ \\
$\mathrm{C}(59)$ & $0.275(4)$ & $0.831(3)$ & $0.5567(12)$ & $0.075(12)$ \\
$\mathrm{C}(60)$ & $0.337(4)$ & $0.817(2)$ & $0.5094(10)$ & $0.058(10)$ \\
$\mathrm{C}(61)$ & $0.264(3)$ & $0.8525(15)$ & $0.3268(10)$ & $0.027(7)$ \\
$\mathrm{C}(62)$ & $0.239(3)$ & $0.8489(18)$ & $0.2786(11)$ & $0.037(8)$ \\
$\mathrm{C}(63)$ & $0.093(3)$ & $0.8671(17)$ & $0.2643(10)$ & $0.031(7)$ \\
$\mathrm{C}(64)$ & $-0.020(3)$ & $0.891(2)$ & $0.2983(12)$ & $0.042(8)$ \\
$\mathrm{C}(65)$ & $0.008(3)$ & $0.895(2)$ & $0.3464(13)$ & $0.054(10)$ \\
$\mathrm{C}(66)$ & $0.149(3)$ & $0.8764(18)$ & $0.3601(9)$ & $0.025(7)$ \\
$\mathrm{C}(67)$ & $0.499(2)$ & $0.9594(16)$ & $0.3368(10)$ & $0.023(7)$ \\
$\mathrm{C}(68)$ & $0.628(3)$ & $0.9768(17)$ & $0.3504(12)$ & $0.043(8)$ \\
$\mathrm{C}(69)$ & $0.675(3)$ & $1.0615(18)$ & $0.3387(11)$ & $0.037(8)$ \\
$\mathrm{C}(70)$ & $0.593(3)$ & $1.129(2)$ & $0.3161(14)$ & $0.059(12)$ \\
$\mathrm{C}(71)$ & $0.466(6)$ & $1.115(2)$ & $0.3005(14)$ & $0.091(17)$ \\
$\mathrm{C}(72)$ & $0.417(4)$ & $1.027(2)$ & $0.3118(14)$ & $0.073(12)$ \\
$\mathrm{O}(1 \mathrm{~L})$ & $0.168(3)$ & $0.0657(15)$ & $0.5261(10)$ & $0.072(7)$ \\
$\mathrm{C}(1 \mathrm{~L})$ & $0.061(5)$ & $0.118(3)$ & $0.4931(15)$ & $0.091(14)$ \\
$\mathrm{C}(2 \mathrm{~L})$ & $0.128(5)$ & $0.094(3)$ & $0.4416(16)$ & $0.099(15)$ \\
$\mathrm{C}(3 \mathrm{~L})$ & $0.289(5)$ & $0.066(3)$ & $0.4465(15)$ & $0.085(13)$ \\
$\mathrm{C}(4 \mathrm{~L})$ & $0.314(4)$ & $0.075(3)$ & $0.5010(15)$ & $0.091(15)$ \\
$\mathrm{C}(5 \mathrm{~L})$ & $0.922(13)$ & $1.441(7)$ & $0.050(3)$ & $0.16(3)$ \\
$\mathrm{C}(6 \mathrm{~L})$ & $0.798(10)$ & $1.463(8)$ & $0.017(5)$ & $0.16(3)$ \\
$\mathrm{C}(7 \mathrm{~L})$ & $0.870(12)$ & $1.530(8)$ & $-0.025(4)$ & $0.16(3)$ \\
$\mathrm{C}(8 \mathrm{~L})$ & $1.034(11)$ & $1.515(8)$ & $-0.023(4)$ & $0.16(3)$ \\
$\mathrm{C}(9 \mathrm{~L})$ & $1.056(11)$ & $1.432(8)$ & $0.017(5)$ & $0.16(3)$ \\
\hline & & & & \\
& & & \\
& &
\end{tabular}

Tabelle 75: Bindungslängen [pm] in $\underline{17}$

\begin{tabular}{|c|c||c|c|}
\hline $\mathrm{Ag}(1)-\mathrm{S}(1)$ & $243.1(8)$ & $\mathrm{Ag}(1)-\mathrm{S}(6)$ & $243.7(6)$ \\
$\mathrm{Ag}(1)-\mathrm{S}(2)$ & $252.3(7)$ & $\mathrm{Ag}(2)-\mathrm{S}(2)$ & $243.9(6)$ \\
$\mathrm{Ag}(2)-\mathrm{S}(3)$ & $245.7(6)$ & $\mathrm{Ag}(2)-\mathrm{S}(4)$ & $258.3(6)$ \\
\hline
\end{tabular}


Tabelle 75: Fortsetzung

\begin{tabular}{|l|r||l|r|}
\hline $\mathrm{Ag}(3)-\mathrm{S}(5)$ & $243.6(7)$ & $\mathrm{Ag}(3)-\mathrm{S}(4)$ & $244.2(7)$ \\
$\mathrm{Ag}(3)-\mathrm{S}(6)$ & $257.2(6)$ & $\mathrm{S}(1)-\mathrm{P}(1)$ & $196.8(11)$ \\
$\mathrm{S}(2)-\mathrm{P}(2)$ & $203.9(8)$ & $\mathrm{S}(3)-\mathrm{P}(3)$ & $200.6(9)$ \\
$\mathrm{S}(4)-\mathrm{P}(4)$ & $204.3(8)$ & $\mathrm{S}(5)-\mathrm{P}(5)$ & $200.4(9)$ \\
$\mathrm{S}(6)-\mathrm{P}(6)$ & $201.2(8)$ & $\mathrm{P}(1)-\mathrm{N}(1)$ & $161(3)$ \\
$\mathrm{P}(1)-\mathrm{C}(7 \mathrm{~A})$ & $183(3)$ & $\mathrm{P}(1)-\mathrm{C}(1)$ & $182(2)$ \\
$\mathrm{P}(1)-\mathrm{C}(7 \mathrm{~B})$ & $183(2)$ & $\mathrm{P}(2)-\mathrm{N}(1)$ & $157(2)$ \\
$\mathrm{P}(2)-\mathrm{C}(19 \mathrm{~A})$ & $164(4)$ & $\mathrm{P}(2)-\mathrm{C}(13)$ & $180(2)$ \\
$\mathrm{P}(2)-\mathrm{C}(19 \mathrm{~B})$ & $193(2)$ & $\mathrm{P}(3)-\mathrm{N}(2)$ & $157(2)$ \\
$\mathrm{P}(3)-\mathrm{C}(25)$ & $180(2)$ & $\mathrm{P}(3)-\mathrm{C}(31)$ & $181(2)$ \\
$\mathrm{P}(4)-\mathrm{N}(2)$ & $160(2)$ & $\mathrm{P}(4)-\mathrm{C}(43)$ & $176(2)$ \\
$\mathrm{P}(4)-\mathrm{C}(37)$ & $180(3)$ & $\mathrm{P}(5)-\mathrm{N}(3)$ & $160(2)$ \\
$\mathrm{P}(5)-\mathrm{C}(49)$ & $181(2)$ & $\mathrm{P}(5)-\mathrm{C}(55)$ & $179(2)$ \\
$\mathrm{P}(6)-\mathrm{N}(3)$ & $159(2)$ & $\mathrm{P}(6)-\mathrm{C}(67)$ & $179(2)$ \\
$\mathrm{P}(6)-\mathrm{C}(61)$ & $182(3)$ & & \\
\hline
\end{tabular}

Tabelle 76: Bindungswinkel $\left[^{\circ}\right]$ in $\underline{17}$

\begin{tabular}{|l|r||l|r|}
\hline $\mathrm{S}(1)-\mathrm{Ag}(1)-\mathrm{S}(6)$ & $132.3(3)$ & $\mathrm{S}(1)-\mathrm{Ag}(1)-\mathrm{S}(2)$ & $112.0(3)$ \\
$\mathrm{S}(6)-\mathrm{Ag}(1)-\mathrm{S}(2)$ & $115.6(2)$ & $\mathrm{S}(2)-\mathrm{Ag}(2)-\mathrm{S}(3)$ & $147.5(2)$ \\
$\mathrm{S}(2)-\mathrm{Ag}(2)-\mathrm{S}(4)$ & $101.0(2)$ & $\mathrm{S}(3)-\mathrm{Ag}(2)-\mathrm{S}(4)$ & $111.5(2)$ \\
$\mathrm{S}(5)-\mathrm{Ag}(3)-\mathrm{S}(4)$ & $137.0(2)$ & $\mathrm{S}(5)-\mathrm{Ag}(3)-\mathrm{S}(6)$ & $108.3(2)$ \\
$\mathrm{S}(4)-\mathrm{Ag}(3)-\mathrm{S}(6)$ & $111.9(2)$ & $\mathrm{P}(1)-\mathrm{S}(1)-\mathrm{Ag}(1)$ & $95.7(4)$ \\
$\mathrm{P}(2)-\mathrm{S}(2)-\mathrm{Ag}(2)$ & $112.2(3)$ & $\mathrm{P}(2)-\mathrm{S}(2)-\mathrm{Ag}(1)$ & $100.3(3)$ \\
$\mathrm{Ag}(2)-\mathrm{S}(2)-\mathrm{Ag}(1)$ & $112.1(2)$ & $\mathrm{P}(3)-\mathrm{S}(3)-\mathrm{Ag}(2)$ & $89.2(3)$ \\
$\mathrm{P}(4)-\mathrm{S}(4)-\mathrm{Ag}(3)$ & $103.3(3)$ & $\mathrm{P}(4)-\mathrm{S}(4)-\mathrm{Ag}(2)$ & $96.6(3)$ \\
$\mathrm{Ag}(3)-\mathrm{S}(4)-\mathrm{Ag}(2)$ & $113.7(2)$ & $\mathrm{P}(5)-\mathrm{S}(5)-\mathrm{Ag}(3)$ & $100.5(3)$ \\
$\mathrm{P}(6)-\mathrm{S}(6)-\mathrm{Ag}(1)$ & $111.0(3)$ & $\mathrm{P}(6)-\mathrm{S}(6)-\mathrm{Ag}(3)$ & $93.6(3)$ \\
$\mathrm{Ag}(1)-\mathrm{S}(6)-\mathrm{Ag}(3)$ & $120.7(3)$ & $\mathrm{N}(1)-\mathrm{P}(1)-\mathrm{S}(1)$ & $116.8(10)$ \\
$\mathrm{C}(7 \mathrm{~A})-\mathrm{P}(1)-\mathrm{S}(1)$ & $113.6(13)$ & $\mathrm{C}(1)-\mathrm{P}(1)-\mathrm{S}(1)$ & $110.0(9)$ \\
$\mathrm{C}(7 \mathrm{~B})-\mathrm{P}(1)-\mathrm{S}(1)$ & $100.6(12)$ & $\mathrm{N}(1)-\mathrm{P}(2)-\mathrm{S}(2)$ & $115.2(10)$ \\
$\mathrm{C}(19 \mathrm{~A})-\mathrm{P}(2)-\mathrm{S}(2)$ & $110.2(18)$ & $\mathrm{C}(13)-\mathrm{P}(2)-\mathrm{S}(2)$ & $110.8(8)$ \\
$\mathrm{C}(19 \mathrm{~B})-\mathrm{P}(2)-\mathrm{S}(2)$ & $104.7(10)$ & $\mathrm{N}(2)-\mathrm{P}(3)-\mathrm{S}(3)$ & $119.8(8)$ \\
$\mathrm{C}(25)-\mathrm{P}(3)-\mathrm{S}(3)$ & $110.2(8)$ & $\mathrm{C}(31)-\mathrm{P}(3)-\mathrm{S}(3)$ & $109.8(9)$ \\
$\mathrm{N}(2)-\mathrm{P}(4)-\mathrm{S}(4)$ & $118.4(8)$ & $\mathrm{C}(43)-\mathrm{P}(4)-\mathrm{S}(4)$ & $107.1(9)$ \\
$\mathrm{C}(37)-\mathrm{P}(4)-\mathrm{S}(4)$ & $108.3(8)$ & $\mathrm{N}(3)-\mathrm{P}(5)-\mathrm{S}(5)$ & $119.7(7)$ \\
$\mathrm{C}(49)-\mathrm{P}(5)-\mathrm{S}(5)$ & $111.1(8)$ & $\mathrm{C}(55)-\mathrm{P}(5)-\mathrm{S}(5)$ & $106.9(8)$ \\
$\mathrm{N}(3)-\mathrm{P}(6)-\mathrm{S}(6)$ & $112.6(7)$ & $\mathrm{C}(67)-\mathrm{P}(6)-\mathrm{S}(6)$ & $108.8(8)$ \\
$\mathrm{C}(61)-\mathrm{P}(6)-\mathrm{S}(6)$ & $112.2(9)$ & $\mathrm{P}(2)-\mathrm{N}(1)-\mathrm{P}(1)$ & $135.1(16)$ \\
$\mathrm{P}(4)-\mathrm{N}(2)-\mathrm{P}(3)$ & $130.0(11)$ & $\mathrm{P}(6)-\mathrm{N}(3)-\mathrm{P}(5)$ & $130.8(10)$ \\
\hline
\end{tabular}




\subsection{Daten zur Kristallstrukturanalyse von}

$\left[\mathrm{Na}_{2} \mathrm{Ag}_{2}\left(\mathrm{dppaS}_{2}\right)_{4}(\mathrm{thf})_{4}\right] \underline{18}$

\begin{tabular}{|ll|}
\hline Summenformel & $\mathrm{C}_{112} \mathrm{H}_{112} \mathrm{Ag}_{2} \mathrm{~N}_{4} \mathrm{Na}_{2} \mathrm{O}_{4} \mathrm{P}_{8} \mathrm{~S}_{8}$ \\
Molekulargewicht $\left[\mathrm{g} \cdot \mathrm{mol}^{-1}\right]$ & 2344.02 \\
Temperatur & $133(2) \mathrm{K}$ \\
Wellenlänge & $71.073 \mathrm{pm}$ \\
Kristallsystem & triklin \\
Raumgruppe & $P \overline{1}$ \\
Gitterkonstanten & $\mathrm{a}=13.0667(7) \AA, \alpha=113.873(3)^{\circ}$ \\
& $\mathrm{b}=15.8571(9) \AA, \beta=96.608(4)^{\circ}$ \\
& $\mathrm{c}=15.9908(8) \AA, \gamma=111.743(3)^{\circ}$ \\
Volumen & $2673.8(2) \AA^{3}$ \\
Formeleinheiten & 1 \\
Röntgenographische Dichte $\left[\mathrm{g} \cdot \mathrm{cm}^{-3}\right]$ & 1.456 \\
Absorptionskoeffizient $\left[\mathrm{mm}^{-1}\right]$ & 0.705 \\
F(000) & 1208 \\
Meßbereich $\theta$ & $2.14-22.50^{\circ}$ \\
Indexbereiche & $-13 \leq \mathrm{h} \leq 14$ \\
& $-17 \leq \mathrm{k} \leq 17$ \\
Gemessene Reflexe & $-17 \leq 1 \leq 13$ \\
Unabhängige Reflexe & 24231 \\
Beobachtete Reflexe $[\mathrm{I}>2 \sigma]$ & 6967 \\
$\mathrm{R}_{\text {int }}$ & 5240 \\
Parameter & 0.0480 \\
R1; wR2[I $>2 \sigma(\mathrm{I})]$ & 595 \\
& $0.0411 ; 0.0890$ \\
&
\end{tabular}


Tabelle 78: Atomkoordinaten und äquivalente isotrope Auslenkungsparameter $\left[\AA^{2}\right]$ für $\underline{18}$

\begin{tabular}{|r|r|r|r|r|}
\hline Atom & \multicolumn{1}{|c|}{$\mathrm{x}$} & \multicolumn{1}{c|}{$\mathrm{y}$} & \multicolumn{1}{c|}{$\mathrm{z}$} & \multicolumn{1}{c|}{$\mathrm{U}_{e q}$} \\
\hline $\mathrm{Ag}$ & $0.95928(4)$ & $0.74855(3)$ & $0.31912(3)$ & $0.03934(14)$ \\
$\mathrm{Na}$ & $1.13723(15)$ & $1.07171(13)$ & $0.35239(12)$ & $0.0329(4)$ \\
$\mathrm{S}(1)$ & $1.14857(11)$ & $0.89179(9)$ & $0.35321(9)$ & $0.0355(3)$ \\
$\mathrm{S}(2)$ & $1.15120(10)$ & $1.03150(8)$ & $0.16454(8)$ & $0.0280(3)$ \\
$\mathrm{S}(3)$ & $0.86913(16)$ & $0.78298(10)$ & $0.45540(9)$ & $0.0605(5)$ \\
$\mathrm{S}(4)$ & $0.84656(11)$ & $0.56525(9)$ & $0.19085(8)$ & $0.0330(3)$ \\
$\mathrm{P}(1)$ & $1.19201(11)$ & $0.83185(9)$ & $0.23357(8)$ & $0.0314(3)$ \\
$\mathrm{P}(2)$ & $1.09550(11)$ & $0.88001(9)$ & $0.08914(8)$ & $0.0287(3)$ \\
$\mathrm{P}(3)$ & $0.83056(13)$ & $0.64195(9)$ & $0.43999(8)$ & $0.0390(4)$ \\
$\mathrm{P}(4)$ & $0.71082(11)$ & $0.51893(9)$ & $0.23721(8)$ & $0.0259(3)$ \\
$\mathrm{N}(1)$ & $1.1277(4)$ & $0.8188(3)$ & $0.1370(3)$ & $0.0423(11)$ \\
$\mathrm{N}(2)$ & $0.7311(3)$ & $0.5424(3)$ & $0.3467(2)$ & $0.0288(9)$ \\
$\mathrm{O}(1)$ & $1.3323(3)$ & $1.1772(3)$ & $0.4164(2)$ & $0.0570(11)$ \\
$\mathrm{O}(2)$ & $0.9402(3)$ & $1.0161(3)$ & $0.3229(2)$ & $0.0500(10)$ \\
$\mathrm{C}(1)$ & $1.3477(4)$ & $0.9079(3)$ & $0.2662(3)$ & $0.0339(12)$ \\
$\mathrm{C}(2)$ & $1.4248(5)$ & $0.9277(5)$ & $0.3477(4)$ & $0.0527(15)$ \\
$\mathrm{C}(3)$ & $1.5436(5)$ & $0.9835(5)$ & $0.3693(4)$ & $0.0672(19)$ \\
$\mathrm{C}(4)$ & $1.5865(5)$ & $1.0193(5)$ & $0.3102(5)$ & $0.0609(17)$ \\
$\mathrm{C}(5)$ & $1.5144(5)$ & $1.0027(4)$ & $0.2313(4)$ & $0.0524(15)$ \\
$\mathrm{C}(6)$ & $1.3954(4)$ & $0.9470(4)$ & $0.2087(4)$ & $0.0405(13)$ \\
$\mathrm{C}(7)$ & $1.1721(4)$ & $0.7031(3)$ & $0.2049(3)$ & $0.0346(12)$ \\
$\mathrm{C}(8)$ & $1.1820(6)$ & $0.6729(4)$ & $0.2743(4)$ & $0.0614(19)$ \\
$\mathrm{C}(9)$ & $1.1705(7)$ & $0.5750(5)$ & $0.2488(4)$ & $0.084(3)$ \\
$\mathrm{C}(10)$ & $1.1474(6)$ & $0.5054(4)$ & $0.1544(4)$ & $0.0642(19)$ \\
$\mathrm{C}(11)$ & $1.1369(5)$ & $0.5341(4)$ & $0.0853(3)$ & $0.0430(14)$ \\
$\mathrm{C}(12)$ & $1.1488(4)$ & $0.6319(3)$ & $0.1096(3)$ & $0.0340(12)$ \\
$\mathrm{C}(13)$ & $0.9392(4)$ & $0.8125(3)$ & $0.0369(3)$ & $0.0249(10)$ \\
$\mathrm{C}(14)$ & $0.8839(4)$ & $0.8458(3)$ & $-0.0131(3)$ & $0.0299(11)$ \\
$\mathrm{C}(15)$ & $0.7660(4)$ & $0.7919(3)$ & $-0.0575(3)$ & $0.0302(11)$ \\
$\mathrm{C}(16)$ & $0.7014(4)$ & $0.7030(3)$ & $-0.0534(3)$ & $0.0346(12)$ \\
$\mathrm{C}(17)$ & $0.7543(4)$ & $0.6676(4)$ & $-0.0061(3)$ & $0.0369(13)$ \\
$\mathrm{C}(18)$ & $0.8728(4)$ & $0.7220(3)$ & $0.0396(3)$ & $0.0298(11)$ \\
$\mathrm{C}(19)$ & $1.1421(4)$ & $0.8492(3)$ & $-0.0175(3)$ & $0.0286(11)$ \\
$\mathrm{C}(20)$ & $1.1210(6)$ & $0.7478(4)$ & $-0.0740(4)$ & $0.0617(18)$ \\
$\mathrm{C}(21)$ & $1.1486(6)$ & $0.7178(5)$ & $-0.1575(4)$ & $0.0623(18)$ \\
$\mathrm{C}(22)$ & $1.2003(5)$ & $0.7884(5)$ & $-0.1859(4)$ & $0.0606(17)$ \\
$\mathrm{C}(24)$ & $1.2237(6)$ & $0.8885(5)$ & $-0.1297(5)$ & $0.084(2)$ \\
& & & & \\
\hline
\end{tabular}


Tabelle 78: Fortsetzung

\begin{tabular}{|c|c|c|c|c|}
\hline $\mathrm{C}(25 \mathrm{~A})$ & $0.8132(5)$ & $0.6399(4)$ & $0.5469(3)$ & $0.0154(19)$ \\
\hline $\mathrm{C}(26 \mathrm{~A})$ & $0.7147(4)$ & $0.5557(3)$ & $0.5346(3)$ & $0.022(2)$ \\
\hline $\mathrm{C}(27 \mathrm{~A})$ & $0.6897(4)$ & $0.5487(4)$ & $0.6144(4)$ & $0.030(2)$ \\
\hline $\mathrm{C}(28 \mathrm{~A})$ & $0.7632(5)$ & $0.6258(4)$ & $0.7065(3)$ & $0.028(2)$ \\
\hline $\mathrm{C}(29 \mathrm{~A})$ & $0.8616(5)$ & $0.7100(4)$ & $0.7188(3)$ & $0.030(2)$ \\
\hline $\mathrm{C}(30 \mathrm{~A})$ & $0.8866(4)$ & $0.7170(3)$ & $0.6389(3)$ & $0.021(2)$ \\
\hline $\mathrm{C}(25 \mathrm{~B})$ & $0.7589(4)$ & $0.6283(4)$ & $0.5354(3)$ & $0.0133(19)$ \\
\hline $\mathrm{C}(26 \mathrm{~B})$ & $0.6481(4)$ & $0.5529(4)$ & $0.5156(3)$ & $0.023(2)$ \\
\hline $\mathrm{C}(27 \mathrm{~B})$ & $0.6104(4)$ & $0.5423(4)$ & $0.5903(4)$ & $0.029(2)$ \\
\hline $\mathrm{C}(28 \mathrm{~B})$ & $0.6835(5)$ & $0.6071(4)$ & $0.6847(3)$ & $0.030(2)$ \\
\hline $\mathrm{C}(29 \mathrm{~B})$ & $0.7943(4)$ & $0.6825(4)$ & $0.7044(3)$ & $0.024(2)$ \\
\hline $\mathrm{C}(30 \mathrm{~B})$ & $0.8320(4)$ & $0.6931(4)$ & $0.6298(3)$ & $0.021(2)$ \\
\hline $\mathrm{C}(31)$ & $0.9612(4)$ & $0.6268(4)$ & $0.4514(3)$ & $0.0372(13)$ \\
\hline $\mathrm{C}(32)$ & $0.9538(4)$ & $0.5287(4)$ & $0.4015(3)$ & $0.0349(12)$ \\
\hline $\mathrm{C}(33)$ & $1.0499(4)$ & $0.5136(4)$ & $0.4126(4)$ & $0.0427(14)$ \\
\hline $\mathrm{C}(34)$ & $1.1559(5)$ & $0.5963(6)$ & $0.4747(4)$ & $0.066(2)$ \\
\hline $\mathrm{C}(35)$ & $1.1648(6)$ & $0.6935(6)$ & $0.5234(4)$ & $0.083(3)$ \\
\hline $\mathrm{C}(36)$ & $1.0686(6)$ & $0.7096(4)$ & $0.5120(4)$ & $0.066(2)$ \\
\hline $\mathrm{C}(37)$ & $0.6297(4)$ & $0.3790(3)$ & $0.1652(3)$ & $0.0282(11)$ \\
\hline $\mathrm{C}(38)$ & $0.5702(4)$ & $0.3187(3)$ & $0.2045(3)$ & $0.0324(12)$ \\
\hline $\mathrm{C}(39)$ & $0.5066(4)$ & $0.2126(4)$ & $0.1486(4)$ & $0.0407(13)$ \\
\hline $\mathrm{C}(40)$ & $0.5006(4)$ & $0.1645(4)$ & $0.0540(4)$ & $0.0478(14)$ \\
\hline $\mathrm{C}(41)$ & $0.5584(5)$ & $0.2229(4)$ & $0.0142(4)$ & $0.0509(15)$ \\
\hline $\mathrm{C}(42)$ & $0.6217(4)$ & $0.3290(4)$ & $0.0695(3)$ & $0.0420(13)$ \\
\hline $\mathrm{C}(43)$ & $0.6110(4)$ & $0.5656(3)$ & $0.2102(3)$ & $0.0272(11)$ \\
\hline $\mathrm{C}(44 \mathrm{~A})$ & $0.5704(8)$ & $0.6076(7)$ & $0.2737(6)$ & $0.024(2)$ \\
\hline $\mathrm{C}(45 \mathrm{~A})$ & $0.4926(9)$ & $0.6420(8)$ & $0.2488(7)$ & $0.034(2)$ \\
\hline $\mathrm{C}(44 \mathrm{~B})$ & $0.5093(8)$ & $0.5520(7)$ & $0.2488(6)$ & $0.027(2)$ \\
\hline $\mathrm{C}(45 \mathrm{~B})$ & $0.4327(10)$ & $0.5873(8)$ & $0.2312(7)$ & $0.042(3)$ \\
\hline $\mathrm{C}(46)$ & $0.4567(5)$ & $0.6356(4)$ & $0.1671(4)$ & $0.0531(16)$ \\
\hline $\mathrm{C}(47 \mathrm{~A})$ & $0.5153(8)$ & $0.5866(7)$ & $0.0930(7)$ & $0.028(2)$ \\
\hline $\mathrm{C}(48 \mathrm{~A})$ & $0.5907(8)$ & $0.5536(8)$ & $0.1183(7)$ & $0.029(2)$ \\
\hline $\mathrm{C}(47 \mathrm{~B})$ & $0.5345(10)$ & $0.6524(8)$ & $0.1399(7)$ & $0.039(3)$ \\
\hline $\mathrm{C}(48 \mathrm{~B})$ & $0.6136(8)$ & $0.6171(8)$ & $0.1573(7)$ & $0.029(2)$ \\
\hline $\mathrm{C}(49 \mathrm{~A})$ & $1.3946(8)$ & $1.2469(8)$ & $0.3804(7)$ & $0.028(2)$ \\
\hline $\mathrm{C}(49 \mathrm{~B})$ & $1.4087(9)$ & $1.2035(9)$ & $0.3640(7)$ & $0.036(3)$ \\
\hline $\mathrm{C}(50)$ & $1.5211(5)$ & $1.2535(4)$ & $0.4119(4)$ & $0.0518(15)$ \\
\hline $\mathrm{C}(51)$ & $1.5265(5)$ & $1.2408(4)$ & $0.5002(4)$ & $0.0588(17)$ \\
\hline $\mathrm{C}(52)$ & $1.4039(5)$ & $1.1889(5)$ & $0.4952(5)$ & $0.0670(18)$ \\
\hline $\mathrm{C}(53)$ & $0.8735(4)$ & $0.9798(4)$ & $0.3768(3)$ & $0.0398(13)$ \\
\hline
\end{tabular}


Tabelle 78: Fortsetzung

\begin{tabular}{|r|r|r|r|r|}
\hline $\mathrm{C}(54)$ & $0.7658(5)$ & $0.8811(4)$ & $0.3030(4)$ & $0.072(2)$ \\
$\mathrm{C}(55)$ & $0.7568(5)$ & $0.8911(6)$ & $0.2116(4)$ & $0.084(2)$ \\
$\mathrm{C}(56 \mathrm{~A})$ & $0.8700(7)$ & $1.0122(6)$ & $0.2477(6)$ & $0.0206(19)$ \\
$\mathrm{C}(56 \mathrm{~B})$ & $0.8659(11)$ & $0.9206(10)$ & $0.2222(9)$ & $0.071(4)$ \\
\hline
\end{tabular}

Tabelle 79: Bindungslängen [pm] in $\underline{18}$

\begin{tabular}{|l|r||l|r|}
\hline $\mathrm{Ag}-\mathrm{S}(1)$ & $246.97(13)$ & $\mathrm{Ag}-\mathrm{S}(4)$ & $247.63(12)$ \\
$\mathrm{Ag}-\mathrm{S}(3)$ & $255.71(14)$ & $\mathrm{Na}-\mathrm{O}(1)$ & $228.0(4)$ \\
$\mathrm{Na}-\mathrm{O}(2)$ & $230.9(4)$ & $\mathrm{Na}-\mathrm{S}(2)$ & $285.0(2)$ \\
$\mathrm{Na}-\mathrm{S}(1)$ & $291.6(2)$ & $\mathrm{Na}-\mathrm{S}(3) \# 1$ & $303.8(2)$ \\
$\mathrm{S}(1)-\mathrm{P}(1)$ & $201.59(17)$ & $\mathrm{S}(2)-\mathrm{P}(2)$ & $197.80(15)$ \\
$\mathrm{S}(3)-\mathrm{P}(3)$ & $200.16(17)$ & $\mathrm{S}(3)-\mathrm{Na} \# 1$ & $303.8(2)$ \\
$\mathrm{S}(4)-\mathrm{P}(4)$ & $200.33(16)$ & $\mathrm{P}(1)-\mathrm{N}(1)$ & $156.4(4)$ \\
$\mathrm{P}(1)-\mathrm{C}(7)$ & $180.8(4)$ & $\mathrm{P}(1)-\mathrm{C}(1)$ & $181.5(5)$ \\
$\mathrm{P}(2)-\mathrm{N}(1)$ & $159.2(4)$ & $\mathrm{P}(2)-\mathrm{C}(13)$ & $181.1(4)$ \\
$\mathrm{P}(2)-\mathrm{C}(19)$ & $181.7(5)$ & $\mathrm{P}(3)-\mathrm{N}(2)$ & $159.2(4)$ \\
$\mathrm{P}(3)-\mathrm{C}(25 \mathrm{~A})$ & $176.2(4)$ & $\mathrm{P}(3)-\mathrm{C}(31)$ & $180.9(5)$ \\
$\mathrm{P}(3)-\mathrm{C}(25 \mathrm{~B})$ & $192.3(4)$ & $\mathrm{P}(4)-\mathrm{N}(2)$ & $160.2(3)$ \\
$\mathrm{P}(4)-\mathrm{C}(43)$ & $181.1(5)$ & $\mathrm{P}(4)-\mathrm{C}(37)$ & $181.2(4)$ \\
$\mathrm{O}(1)-\mathrm{C}(52)$ & $138.2(7)$ & $\mathrm{O}(1)-\mathrm{C}(49 \mathrm{~B})$ & $142.8(10)$ \\
$\mathrm{O}(1)-\mathrm{C}(49 \mathrm{~A})$ & $147.3(9)$ & $\mathrm{O}(2)-\mathrm{C}(56 \mathrm{~A})$ & $139.1(8)$ \\
$\mathrm{O}(2)-\mathrm{C}(53)$ & $142.7(6)$ & $\mathrm{O}(2)-\mathrm{C}(56 \mathrm{~B})$ & $155.2(13)$ \\
\hline
\end{tabular}

Tabelle 80: Bindungswinkel $\left[^{\circ}\right.$ ] in 18

\begin{tabular}{|l|r||l|r|}
\hline S(1)-Ag-S(4) & $134.12(4)$ & S(1)-Ag-S(3) & $114.95(5)$ \\
S(4)-Ag-S(3) & $110.18(5)$ & O(1)-Na-O(2) & $160.64(16)$ \\
O(1)-Na-S(2) & $90.26(10)$ & O(2)-Na-S(2) & $99.74(10)$ \\
O(1)-Na-S(1) & $95.41(12)$ & O(2)-Na-S(1) & $99.18(11)$ \\
S(2)-Na-S(1) & $99.55(6)$ & O(1)-Na-S(3)\#1 & $83.90(11)$ \\
O(2)-Na-S(3)\#1 & $78.93(10)$ & S(2)-Na-S(3)\#1 & $148.59(7)$ \\
S(1)-Na-S(3)\#1 & $111.70(6)$ & P(1)-S(1)-Ag & $102.44(6)$ \\
P(1)-S(1)-Na & $102.28(7)$ & Ag-S(1)-Na & $114.73(6)$ \\
P(2)-S(2)-Na & $106.00(6)$ & P(3)-S(3)-Ag & $90.91(6)$ \\
P(3)-S(3)-Na\#1 & $112.42(7)$ & Ag-S(3)-Na\#1 & $151.11(7)$ \\
P(4)-S(4)-Ag & $100.28(6)$ & N(1)-P(1)-C(7) & $104.6(2)$ \\
N(1)-P(1)-C(1) & $111.1(2)$ & N(1)-P(1)-S(1) & $120.12(18)$ \\
C(7)-P(1)-S(1) & $109.59(16)$ & C (1)-P(1)-S(1) & $106.21(15)$ \\
\hline
\end{tabular}


Tabelle 80: Fortsetzung

\begin{tabular}{|l|r||l|r|}
\hline $\mathrm{N}(1)-\mathrm{P}(2)-\mathrm{C}(13)$ & $106.2(2)$ & $\mathrm{N}(1)-\mathrm{P}(2)-\mathrm{C}(19)$ & $105.4(2)$ \\
$\mathrm{N}(1)-\mathrm{P}(2)-\mathrm{S}(2)$ & $120.69(15)$ & $\mathrm{C}(13)-\mathrm{P}(2)-\mathrm{S}(2)$ & $110.79(15)$ \\
$\mathrm{C}(19)-\mathrm{P}(2)-\mathrm{S}(2)$ & $110.23(15)$ & $\mathrm{N}(2)-\mathrm{P}(3)-\mathrm{C}(25 \mathrm{~A})$ & $112.3(2)$ \\
$\mathrm{N}(2)-\mathrm{P}(3)-\mathrm{C}(31)$ & $109.0(2)$ & $\mathrm{N}(2)-\mathrm{P}(3)-\mathrm{C}(25 \mathrm{~B})$ & $98.6(2)$ \\
$\mathrm{N}(2)-\mathrm{P}(3)-\mathrm{S}(3)$ & $119.11(15)$ & $\mathrm{C}(25 \mathrm{~A})-\mathrm{P}(3)-\mathrm{S}(3)$ & $110.05(18)$ \\
$\mathrm{C}(31)-\mathrm{P}(3)-\mathrm{S}(3)$ & $109.36(17)$ & $\mathrm{C}(25 \mathrm{~B})-\mathrm{P}(3)-\mathrm{S}(3)$ & $107.93(17)$ \\
$\mathrm{N}(2)-\mathrm{P}(4)-\mathrm{C}(43)$ & $108.13(19)$ & $\mathrm{N}(2)-\mathrm{P}(4)-\mathrm{C}(37)$ & $105.25(19)$ \\
$\mathrm{N}(2)-\mathrm{P}(4)-\mathrm{S}(4)$ & $120.22(15)$ & $\mathrm{C}(43)-\mathrm{P}(4)-\mathrm{S}(4)$ & $110.21(15)$ \\
$\mathrm{C}(37)-\mathrm{P}(4)-\mathrm{S}(4)$ & $107.45(15)$ & $\mathrm{P}(1)-\mathrm{N}(1)-\mathrm{P}(2)$ & $143.4(3)$ \\
$\mathrm{P}(3)-\mathrm{N}(2)-\mathrm{P}(4)$ & $127.7(2)$ & $\mathrm{C}(52)-\mathrm{O}(1)-\mathrm{C}(49 \mathrm{~B})$ & $103.5(5)$ \\
$\mathrm{C}(52)-\mathrm{O}(1)-\mathrm{C}(49 \mathrm{~A})$ & $112.3(5)$ & $\mathrm{C}(49 \mathrm{~B})-\mathrm{O}(1)-\mathrm{C}(49 \mathrm{~A})$ & $29.2(5)$ \\
$\mathrm{C}(52)-\mathrm{O}(1)-\mathrm{Na}$ & $125.3(3)$ & $\mathrm{C}(49 \mathrm{~B})-\mathrm{O}(1)-\mathrm{Na}$ & $125.6(5)$ \\
$\mathrm{C}(49 \mathrm{~A})-\mathrm{O}(1)-\mathrm{Na}$ & $122.2(4)$ & $\mathrm{C}(56 \mathrm{~A})-\mathrm{O}(2)-\mathrm{C}(53)$ & $110.8(5)$ \\
$\mathrm{C}(56 \mathrm{~A})-\mathrm{O}(2)-\mathrm{C}(56 \mathrm{~B})$ & $52.9(6)$ & $\mathrm{C}(53)-\mathrm{O}(2)-\mathrm{C}(56 \mathrm{~B})$ & $97.2(6)$ \\
$\mathrm{C}(56 \mathrm{~A})-\mathrm{O}(2)-\mathrm{Na}$ & $123.6(4)$ & $\mathrm{C}(53)-\mathrm{O}(2)-\mathrm{Na}$ & $125.6(3)$ \\
$\mathrm{C}(56 \mathrm{~B})-\mathrm{O}(2)-\mathrm{Na}$ & $113.7(5)$ & & \\
\hline
\end{tabular}

$\# 1-\mathrm{x}+2,-\mathrm{y}+2,-\mathrm{z}+1$ 


\subsection{Daten zur Kristallstrukturanalyse von $\left[\mathrm{Cu}_{4}\left(\operatorname{dppaS}_{2}\right)_{3}\right]_{2}\left[\mathrm{CuCl}_{2}\right][\mathrm{OH}] \underline{19}$}

\begin{tabular}{|ll|}
\hline Summenformel & $\mathrm{C}_{82} \mathrm{H}_{80.5} \mathrm{ClCu}_{4.5} \mathrm{~N}_{3} \mathrm{O}_{3} \mathrm{P}_{6} \mathrm{~S}_{6}$ \\
Molekulargewicht $\left[\mathrm{g} \cdot \mathrm{mol}^{-1}\right]$ & 1855.55 \\
Temperatur & $133(2) \mathrm{K}$ \\
Wellenlänge & $71.073 \mathrm{pm}$ \\
Kristallsystem & rhomboedrisch \\
Raumgruppe & $R \overline{3} c$ \\
Gitterkonstanten & $\mathrm{a}=18.923(2) \AA, \alpha=90^{\circ}$ \\
& $\mathrm{b}=18.923(2) \AA, \beta=90^{\circ}$ \\
& $\mathrm{c}=76.755(11) \AA, \gamma=120^{\circ}$ \\
Volumen & $23802(5) \AA^{3}$ \\
Formeleinheiten & 12 \\
Röntgenographische Dichte $\left[\mathrm{g} \cdot \mathrm{cm}{ }^{-3}\right]$ & 1.553 \\
Absorptionskoeffizient $\left[\mathrm{mm}^{-1}\right]$ & 1.552 \\
$\mathrm{~F}(000)$ & 11412 \\
Meßbereich $\theta$ & $2.15-22.49^{\circ}$ \\
Indexbereiche & $-20 \leq \mathrm{h} \leq 20$ \\
& $-20 \leq \mathrm{k} \leq 13$ \\
Gemessene Reflexe & $-82 \leq 1 \leq 82$ \\
Unabhängige Reflexe & 60724 \\
Beobachtete Reflexe $[\mathrm{I}>2 \sigma]$ & 3474 \\
$\left.\mathrm{R}_{\text {int }}\right]$ & 2748 \\
Parameter & 0.1058 \\
R1; wR2[I $>2 \sigma(\mathrm{I})]$ & 321 \\
\hline
\end{tabular}


Tabelle 82: Atomkoordinaten und äquivalente isotrope Auslenkungsparameter $\left[\AA^{2}\right]$ für $\underline{19}$

\begin{tabular}{|c|c|c|c|c|}
\hline Atom & $\mathrm{x}$ & $\mathrm{y}$ & $\mathrm{z}$ & $\mathrm{U}_{e q}$ \\
\hline $\mathrm{C}(1)$ & $0.3320(3)$ & $0.4053(3)$ & $0.05281(7)$ & $0.0283(12)$ \\
\hline $\mathrm{C}(2)$ & $0.2849(3)$ & $0.3292(3)$ & $0.06062(7)$ & $0.0392(14)$ \\
\hline $\mathrm{C}(3)$ & $0.3123(4)$ & $0.2741(3)$ & $0.06094(8)$ & $0.0505(17)$ \\
\hline $\mathrm{C}(4)$ & $0.3861(4)$ & $0.2938(4)$ & $0.05336(9)$ & $0.0555(18)$ \\
\hline $\mathrm{C}(5)$ & $0.4326(4)$ & $0.3680(4)$ & $0.04521(9)$ & $0.0557(18)$ \\
\hline $\mathrm{C}(6)$ & $0.4055(3)$ & $0.4245(3)$ & $0.04517(8)$ & $0.0436(15)$ \\
\hline $\mathrm{C}(7)$ & $0.2622(3)$ & $0.4834(3)$ & $0.07385(6)$ & $0.0244(11)$ \\
\hline $\mathrm{C}(8)$ & $0.1813(3)$ & $0.4408(3)$ & $0.07876(6)$ & $0.0311(12)$ \\
\hline $\mathrm{C}(9)$ & $0.1580(3)$ & $0.4465(3)$ & $0.09546(7)$ & $0.0353(13)$ \\
\hline $\mathrm{C}(10)$ & $0.2163(4)$ & $0.4961(3)$ & $0.10743(7)$ & $0.0383(14)$ \\
\hline $\mathrm{C}(11)$ & $0.2978(4)$ & $0.5391(3)$ & $0.10258(7)$ & $0.0389(14)$ \\
\hline $\mathrm{C}(12)$ & $0.3209(3)$ & $0.5329(3)$ & $0.08594(7)$ & $0.0318(12)$ \\
\hline $\mathrm{C}(13)$ & $0.1856(3)$ & $0.3471(3)$ & $0.00991(7)$ & $0.0255(11)$ \\
\hline $\mathrm{C}(14)$ & $0.1815(3)$ & $0.2851(3)$ & $0.02015(7)$ & $0.0333(13)$ \\
\hline $\mathrm{C}(15)$ & $0.1766(3)$ & $0.2165(3)$ & $0.01237(8)$ & $0.0400(14)$ \\
\hline $\mathrm{C}(16)$ & $0.1770(3)$ & $0.2103(3)$ & $-0.00507(9)$ & $0.0451(16)$ \\
\hline $\mathrm{C}(17)$ & $0.1808(3)$ & $0.2720(3)$ & $-0.01555(8)$ & $0.0434(15)$ \\
\hline $\mathrm{C}(18)$ & $0.1853(3)$ & $0.3408(3)$ & $-0.00785(7)$ & $0.0340(13)$ \\
\hline $\mathrm{C}(19)$ & $0.0820(3)$ & $0.4123(3)$ & $0.02013(6)$ & $0.0256(11)$ \\
\hline $\mathrm{C}(20)$ & $0.0382(3)$ & $0.3978(3)$ & $0.03532(7)$ & $0.0382(14)$ \\
\hline $\mathrm{C}(21)$ & $-0.0412(3)$ & $0.3841(4)$ & $0.03467(9)$ & $0.0509(17)$ \\
\hline $\mathrm{C}(22)$ & $-0.0767(3)$ & $0.3833(3)$ & $0.01920(8)$ & $0.0436(15)$ \\
\hline $\mathrm{C}(23)$ & $-0.0341(3)$ & $0.3968(4)$ & $0.00395(8)$ & $0.0453(15)$ \\
\hline $\mathrm{C}(24)$ & $0.0449(3)$ & $0.4103(3)$ & $0.00445(7)$ & $0.0414(14)$ \\
\hline $\mathrm{N}(1)$ & $0.2124(2)$ & $0.4409(2)$ & $0.04020(5)$ & $0.0246(9)$ \\
\hline $\mathrm{P}(1)$ & $0.29279(7)$ & $0.47562(7)$ & $0.051872(16)$ & $0.0228(3)$ \\
\hline $\mathrm{P}(2)$ & $0.18629(7)$ & $0.43319(7)$ & $0.020508(16)$ & $0.0226(3)$ \\
\hline $\mathrm{S}(1)$ & $0.39159(7)$ & $0.58700(7)$ & $0.045975(16)$ & $0.0242(3)$ \\
\hline $\mathrm{S}(2)$ & $0.25043(7)$ & $0.53130(7)$ & $0.004124(16)$ & $0.0244(3)$ \\
\hline $\mathrm{Cu}(1)$ & $0.37081(3)$ & $0.60621(3)$ & $0.017845(8)$ & $0.0254(2)$ \\
\hline $\mathrm{Cu}(2)$ & 0.3333 & 0.6667 & $0.047304(13)$ & $0.0249(3)$ \\
\hline $\mathrm{Cu}(3)$ & 0.0000 & 0.0000 & 0.0000 & $0.0303(4)$ \\
\hline $\mathrm{Cl}(1)$ & 0.0000 & 0.0000 & $0.02720(3)$ & $0.0438(6)$ \\
\hline $\mathrm{O}(111)$ & $0.6099(7)$ & $0.2766(7)$ & 0.0833 & $0.034(4)$ \\
\hline $\mathrm{O}(1 \mathrm{~L})$ & $0.5071(3)$ & $0.1582(3)$ & $0.08373(12)$ & $0.0278(17)$ \\
\hline $\mathrm{C}(1 \mathrm{~L})$ & $0.4589(9)$ & $0.1974(9)$ & $0.0856(2)$ & $0.095(5)$ \\
\hline $\mathrm{C}(2 \mathrm{~L})$ & $0.3691(8)$ & $0.1279(8)$ & $0.0846(2)$ & $0.065(4)$ \\
\hline $\mathrm{C}(3 \mathrm{~L})$ & $0.3723(5)$ & $0.0569(5)$ & $0.08522(14)$ & $0.022(2)$ \\
\hline
\end{tabular}


Tabelle 82: Fortsetzung

\begin{tabular}{|c|r|r|r|r|}
\hline $\mathrm{C}(4 \mathrm{~L})$ & $0.4497(10)$ & $0.0683(9)$ & $0.0858(3)$ & $0.100(6)$ \\
$\mathrm{O}(2 \mathrm{~L})$ & $-0.2302(9)$ & $0.3781(8)$ & $0.0387(2)$ & $0.078(5)$ \\
$\mathrm{C}(5 \mathrm{~L})$ & $-0.2822(12)$ & $0.2898(11)$ & $0.0423(3)$ & $0.054(7)$ \\
$\mathrm{C}(6 \mathrm{~L})$ & $-0.3637(11)$ & $0.2758(11)$ & $0.0381(4)$ & $0.040(7)$ \\
$\mathrm{C}(7 \mathrm{~L})$ & $-0.3640(14)$ & $0.3490(15)$ & $0.0401(6)$ & $0.094(14)$ \\
$\mathrm{C}(8 \mathrm{~L})$ & $-0.2792(10)$ & $0.4126(12)$ & $0.0447(3)$ & $0.047(6)$ \\
\hline
\end{tabular}

Tabelle 83: Bindungslängen [pm] in $\underline{19}$

\begin{tabular}{|l|r||l|r|}
\hline $\mathrm{C}(1)-\mathrm{P}(1)$ & $182.0(5)$ & $\mathrm{C}(7)-\mathrm{P}(1)$ & $181.3(5)$ \\
$\mathrm{C}(13)-\mathrm{P}(2)$ & $181.5(5)$ & $\mathrm{C}(19)-\mathrm{P}(2)$ & $180.8(5)$ \\
$\mathrm{N}(1)-\mathrm{P}(2)$ & $157.4(4)$ & $\mathrm{N}(1)-\mathrm{P}(1)$ & $159.6(4)$ \\
$\mathrm{P}(1)-\mathrm{S}(1)$ & $204.99(16)$ & $\mathrm{P}(2)-\mathrm{S}(2)$ & $206.10(17)$ \\
$\mathrm{S}(1)-\mathrm{Cu}(1)$ & $225.64(14)$ & $\mathrm{S}(1)-\mathrm{Cu}(2)$ & $227.17(12)$ \\
$\mathrm{S}(2)-\mathrm{Cu}(1)$ & $225.36(14)$ & $\mathrm{S}(2)-\mathrm{Cu}(1) \# 1$ & $226.90(13)$ \\
$\mathrm{Cu}(1)-\mathrm{Cu}(2)$ & $278.13(10)$ & $\mathrm{Cu}(1)-\mathrm{Cu}(1) \# 2$ & $280.52(10)$ \\
$\mathrm{Cu}(2)-\mathrm{Cu}(1) \# 2$ & $278.12(10)$ & $\mathrm{Cu}(3)-\mathrm{Cl}(1) \# 3$ & $208.8(2)$ \\
\hline
\end{tabular}

Tabelle 84: Bindungswinkel $\left[^{\circ}\right]$ in $\underline{19}$

\begin{tabular}{|l|r||l|r|}
\hline $\mathrm{P}(2)-\mathrm{N}(1)-\mathrm{P}(1)$ & $140.1(3)$ & $\mathrm{N}(1)-\mathrm{P}(1)-\mathrm{S}(1)$ & $119.40(15)$ \\
$\mathrm{C}(7)-\mathrm{P}(1)-\mathrm{S}(1)$ & $106.63(16)$ & $\mathrm{C}(1)-\mathrm{P}(1)-\mathrm{S}(1)$ & $105.37(16)$ \\
$\mathrm{N}(1)-\mathrm{P}(2)-\mathrm{S}(2)$ & $119.10(16)$ & $\mathrm{C}(19)-\mathrm{P}(2)-\mathrm{S}(2)$ & $105.89(16)$ \\
$\mathrm{C}(13)-\mathrm{P}(2)-\mathrm{S}(2)$ & $105.79(17)$ & $\mathrm{P}(1)-\mathrm{S}(1)-\mathrm{Cu}(1)$ & $103.54(6)$ \\
$\mathrm{P}(1)-\mathrm{S}(1)-\mathrm{Cu}(2)$ & $100.18(6)$ & $\mathrm{Cu}(1)-\mathrm{S}(1)-\mathrm{Cu}(2)$ & $75.79(5)$ \\
$\mathrm{P}(2)-\mathrm{S}(2)-\mathrm{Cu}(1)$ & $103.67(6)$ & $\mathrm{P}(2)-\mathrm{S}(2)-\mathrm{Cu}(1) \# 1$ & $101.21(6)$ \\
$\mathrm{Cu}(1)-\mathrm{S}(2)-\mathrm{Cu}(1) \# 1$ & $76.67(5)$ & $\mathrm{S}(2)-\mathrm{Cu}(1)-\mathrm{S}(1)$ & $123.27(5)$ \\
$\mathrm{S}(2)-\mathrm{Cu}(1)-\mathrm{S}(2) \# 2$ & $117.90(7)$ & $\mathrm{S}(1)-\mathrm{Cu}(1)-\mathrm{S}(2) \# 2$ & $118.03(5)$ \\
$\mathrm{S}(2)-\mathrm{Cu}(1)-\mathrm{Cu}(2)$ & $104.67(4)$ & $\mathrm{S}(1)-\mathrm{Cu}(1)-\mathrm{Cu}(2)$ & $52.35(4)$ \\
$\mathrm{S}(2) \# 2-\mathrm{Cu}(1)-\mathrm{Cu}(2)$ & $104.24(4)$ & $\mathrm{S}(2)-\mathrm{Cu}(1)-\mathrm{Cu}(1) \# 2$ & $103.89(4)$ \\
$\mathrm{S}(1)-\mathrm{Cu}(1)-\mathrm{Cu}(1) \# 2$ & $103.05(3)$ & $\mathrm{S}(2) \# 2-\mathrm{Cu}(1)-\mathrm{Cu}(1) \# 2$ & $51.42(4)$ \\
$\mathrm{S}(2)-\mathrm{Cu}(1)-\mathrm{Cu}(1) \# 1$ & $51.91(4)$ & $\mathrm{S}(1)-\mathrm{Cu}(1)-\mathrm{Cu}(1) \# 1$ & $105.74(3)$ \\
$\mathrm{S}(2) \# 2-\mathrm{Cu}(1)-\mathrm{Cu}(1) \# 1$ & $103.47(4)$ & $\mathrm{Cu}(2)-\mathrm{Cu}(1)-\mathrm{Cu}(1) \# 1$ & $59.714(14)$ \\
$\mathrm{Cu}(1) \# 2-\mathrm{Cu}(1)-\mathrm{Cu}(1) \# 1$ & 60.0 & $\mathrm{~S}(1) \# 1-\mathrm{Cu}(2)-\mathrm{S}(1)$ & $119.801(7)$ \\
$\mathrm{S}(1)-\mathrm{Cu}(2)-\mathrm{Cu}(1) \# 2$ & $103.37(4)$ & $\mathrm{S}(1)-\mathrm{Cu}(2)-\mathrm{Cu}(1) \# 1$ & $106.07(4)$ \\
$\mathrm{S}(1) \# 2-\mathrm{Cu}(2)-\mathrm{Cu}(1)$ & $106.06(4)$ & $\mathrm{S}(1) \# 1-\mathrm{Cu}(2)-\mathrm{Cu}(1)$ & $103.37(4)$ \\
$\mathrm{S}(1)-\mathrm{Cu}(2)-\mathrm{Cu}(1)$ & $51.86(4)$ & $\mathrm{Cu}(1) \# 2-\mathrm{Cu}(2)-\mathrm{Cu}(1)$ & $60.57(3)$ \\
\hline
\end{tabular}

$\# 1-x+y,-x+1, z \quad \# 2-y+1, x-y+1, z \quad \# 3-x,-y,-z$ 


\subsection{Daten zur Kristallstrukturanalyse von $\left[\mathrm{Cu}_{4}\left(\mathrm{dppaSe}_{2}\right)_{3}\right]\left[\mathrm{CuCl}_{2}\right] \underline{20}$}

\begin{tabular}{|ll|}
\hline Summenformel & $\mathrm{C}_{74.5} \mathrm{H}_{65.5} \mathrm{Cl}_{7.5} \mathrm{Cu}_{5} \mathrm{~N}_{3} \mathrm{P}_{6} \mathrm{Se}_{6}$ \\
Molekulargewicht $\left[\mathrm{g} \cdot \mathrm{mol}^{-1}\right]$ & 2245.95 \\
Temperatur & $133(2) \mathrm{K}$ \\
Wellenlänge & $71.073 \mathrm{pm}$ \\
Kristallsystem & triklin \\
Raumgruppe & $P \overline{1}$ \\
Gitterkonstanten & $\mathrm{a}=13.8888(11) \AA, \alpha=105.784(5)^{\circ}$ \\
& $\mathrm{b}=17.2817(14) \AA, \beta=99.879(5)^{\circ}$ \\
& $\mathrm{c}=20.0708(16) \AA, \gamma=110.647(4)^{\circ}$
\end{tabular}


Tabelle 86: Atomkoordinaten und äquivalente isotrope Auslenkungsparameter $\left[\AA^{2}\right]$ für $\underline{20}$

\begin{tabular}{|c|r|r|r|r|}
\hline Atom & \multicolumn{1}{|c|}{$\mathrm{x}$} & \multicolumn{1}{c|}{$\mathrm{y}$} & \multicolumn{1}{c|}{$\mathrm{z}$} & $\mathrm{U}_{e q}$ \\
\hline $\mathrm{Cu}(1)$ & $0.73284(11)$ & $0.43158(10)$ & $0.76418(8)$ & $0.0217(4)$ \\
$\mathrm{Cu}(2)$ & $0.78085(11)$ & $0.47785(10)$ & $0.91028(8)$ & $0.0223(4)$ \\
$\mathrm{Cu}(3)$ & $0.92973(11)$ & $0.57481(9)$ & $0.84694(8)$ & $0.0210(4)$ \\
$\mathrm{Cu}(4)$ & $0.90415(11)$ & $0.40982(9)$ & $0.83873(8)$ & $0.0208(4)$ \\
$\mathrm{Cu}(5)$ & $0.77067(15)$ & $1.02196(12)$ & $1.24473(10)$ & $0.0499(5)$ \\
$\mathrm{Se}(1)$ & $0.60508(9)$ & $0.42255(8)$ & $0.83163(7)$ & $0.0220(3)$ \\
$\mathrm{Se}(2)$ & $0.89957(9)$ & $0.63116(8)$ & $0.96041(7)$ & $0.0203(3)$ \\
$\mathrm{Se}(3)$ & $0.81587(9)$ & $0.56508(8)$ & $0.73990(7)$ & $0.0208(3)$ \\
$\mathrm{Se}(4)$ & $1.06876(9)$ & $0.52584(8)$ & $0.84789(7)$ & $0.0199(3)$ \\
$\mathrm{Se}(5)$ & $0.78536(9)$ & $0.31478(8)$ & $0.72046(7)$ & $0.0199(3)$ \\
$\mathrm{Se}(6)$ & $0.87236(9)$ & $0.39675(8)$ & $0.94918(6)$ & $0.0198(3)$ \\
$\mathrm{P}(1)$ & $0.5941(2)$ & $0.5453(2)$ & $0.82522(18)$ & $0.0194(7)$ \\
$\mathrm{P}(2)$ & $0.7979(2)$ & $0.6929(2)$ & $0.92122(18)$ & $0.0203(7)$ \\
$\mathrm{P}(3)$ & $0.8730(2)$ & $0.5022(2)$ & $0.65379(17)$ & $0.0191(7)$ \\
$\mathrm{P}(4)$ & $1.0589(2)$ & $0.4834(2)$ & $0.73122(17)$ & $0.0199(7)$ \\
$\mathrm{P}(5)$ & $0.6632(2)$ & $0.2125(2)$ & $0.74452(18)$ & $0.0211(8)$ \\
$\mathrm{P}(6)$ & $0.7678(2)$ & $0.2530(2)$ & $0.89337(17)$ & $0.0179(7)$ \\
$\mathrm{Cl}(1)$ & $0.6241(4)$ & $1.0256(3)$ & $1.1992(3)$ & $0.0718(14)$ \\
$\mathrm{Cl}(2)$ & $0.9130(3)$ & $1.0150(3)$ & $1.2928(2)$ & $0.0632(13)$ \\
$\mathrm{N}(1)$ & $0.7011(7)$ & $0.6334(6)$ & $0.8479(5)$ & $0.020(2)$ \\
$\mathrm{N}(2)$ & $0.9474(7)$ & $0.4544(6)$ & $0.6724(5)$ & $0.017(2)$ \\
$\mathrm{N}(3)$ & $0.6679(7)$ & $0.2269(6)$ & $0.8263(5)$ & $0.024(2)$ \\
$\mathrm{C}(1)$ & $0.5285(8)$ & $0.5208(8)$ & $0.7327(7)$ & $0.021(3)$ \\
$\mathrm{C}(2)$ & $0.4386(11)$ & $0.4407(9)$ & $0.6921(7)$ & $0.038(4)$ \\
$\mathrm{C}(3)$ & $0.3841(11)$ & $0.4226(10)$ & $0.6218(8)$ & $0.047(4)$ \\
$\mathrm{C}(4)$ & $0.4122(12)$ & $0.4820(10)$ & $0.5913(8)$ & $0.043(4)$ \\
$\mathrm{C}(5)$ & $0.4999(12)$ & $0.5633(11)$ & $0.6295(8)$ & $0.050(4)$ \\
$\mathrm{C}(6)$ & $0.5568(10)$ & $0.5816(9)$ & $0.6996(8)$ & $0.038(4)$ \\
$\mathrm{C}(7)$ & $0.5016(9)$ & $0.5646(8)$ & $0.8732(6)$ & $0.020(3)$ \\
$\mathrm{C}(8)$ & $0.4723(13)$ & $0.6341(11)$ & $0.8678(9)$ & $0.057(5)$ \\
$\mathrm{C}(9)$ & $0.4081(12)$ & $0.6592(11)$ & $0.9078(9)$ & $0.057(5)$ \\
$\mathrm{C}(10)$ & $0.3656(10)$ & $0.6104(9)$ & $0.9485(7)$ & $0.035(4)$ \\
$\mathrm{C}(11)$ & $0.3911(13)$ & $0.5394(11)$ & $0.9516(9)$ & $0.058(5)$ \\
$\mathrm{C}(12)$ & $0.4595(12)$ & $0.5192(11)$ & $0.9136(9)$ & $0.053(5)$ \\
$\mathrm{C}(13)$ & $0.8875(9)$ & $0.7871(8)$ & $0.9057(7)$ & $0.023(3)$ \\
$\mathrm{C}(14)$ & $0.9790(10)$ & $0.8500(8)$ & $0.9631(7)$ & $0.031(3)$ \\
$\mathrm{C}(16)$ & $1.0512(10)$ & $0.9205(9)$ & $0.9532(8)$ & $0.038(4)$ \\
\hline & & & & $0.045(4)$ \\
\hline
\end{tabular}


Tabelle 86: Fortsetzung

\begin{tabular}{|c|c|c|c|c|}
\hline $\mathrm{C}(17)$ & $0.9500(10)$ & $0.8666(8)$ & $0.8298(7)$ & $0.030(3)$ \\
\hline $\mathrm{C}(18)$ & $0.8751(10)$ & $0.7956(8)$ & $0.8387(7)$ & $0.026(3)$ \\
\hline $\mathrm{C}(19)$ & $0.7512(8)$ & $0.7384(7)$ & $0.9942(6)$ & $0.019(3)$ \\
\hline$C(20)$ & $0.7406(11)$ & $0.8170(9)$ & $1.0018(8)$ & $0.041(4)$ \\
\hline $\mathrm{C}(21)$ & $0.6970(11)$ & $0.8489(10)$ & $1.0535(8)$ & $0.045(4)$ \\
\hline $\mathrm{C}(22)$ & $0.6648(11)$ & $0.8039(11)$ & $1.0984(8)$ & $0.047(4)$ \\
\hline $\mathrm{C}(23)$ & $0.6734(14)$ & $0.7271(12)$ & $1.0900(9)$ & $0.060(5)$ \\
\hline $\mathrm{C}(24)$ & $0.7192(12)$ & $0.6947(10)$ & $1.0382(8)$ & $0.049(4)$ \\
\hline $\mathrm{C}(25)$ & $0.9360(9)$ & $0.5851(8)$ & $0.6169(7)$ & $0.028(3)$ \\
\hline $\mathrm{C}(26)$ & $0.9962(11)$ & $0.6729(9)$ & $0.6614(8)$ & $0.044(4)$ \\
\hline $\mathrm{C}(27)$ & $1.0535(13)$ & $0.7343(10)$ & $0.6332(10)$ & $0.057(5)$ \\
\hline$C(28)$ & $1.0470(13)$ & $0.7087(12)$ & $0.5625(10)$ & $0.055(5)$ \\
\hline $\mathrm{C}(29)$ & $0.9884(13)$ & $0.6220(11)$ & $0.5162(9)$ & $0.058(5)$ \\
\hline $\mathrm{C}(30)$ & $0.9321(13)$ & $0.5594(10)$ & $0.5448(8)$ & $0.055(5)$ \\
\hline $\mathrm{C}(31)$ & $0.7560(9)$ & $0.4194(8)$ & $0.5832(6)$ & $0.021(3)$ \\
\hline $\mathrm{C}(32)$ & $0.6606(10)$ & $0.4313(9)$ & $0.5702(7)$ & $0.033(3)$ \\
\hline $\mathrm{C}(33)$ & $0.5675(10)$ & $0.3651(9)$ & $0.5166(7)$ & $0.035(3)$ \\
\hline $\mathrm{C}(34)$ & $0.5703(11)$ & $0.2887(10)$ & $0.4743(7)$ & $0.038(4)$ \\
\hline $\mathrm{C}(35)$ & $0.6634(11)$ & $0.2750(9)$ & $0.4885(7)$ & $0.037(4)$ \\
\hline $\mathrm{C}(36)$ & $0.7556(11)$ & $0.3398(8)$ & $0.5395(7)$ & $0.031(3)$ \\
\hline $\mathrm{C}(37)$ & $1.1679(9)$ & $0.5730(9)$ & $0.7219(7)$ & $0.024(3)$ \\
\hline $\mathrm{C}(38)$ & $1.1998(11)$ & $0.6599(9)$ & $0.7645(8)$ & $0.042(4)$ \\
\hline $\mathrm{C}(39)$ & $1.2724(12)$ & $0.7261(11)$ & $0.7502(8)$ & $0.057(5)$ \\
\hline $\mathrm{C}(40)$ & $1.3108(10)$ & $0.7077(10)$ & $0.6912(8)$ & $0.048(4)$ \\
\hline $\mathrm{C}(41)$ & $1.2785(11)$ & $0.6211(10)$ & $0.6478(9)$ & $0.051(4)$ \\
\hline$C(42)$ & $1.2053(11)$ & $0.5529(10)$ & $0.6645(8)$ & $0.041(4)$ \\
\hline $\mathrm{C}(43)$ & $1.0904(9)$ & $0.3890(8)$ & $0.7127(7)$ & $0.025(3)$ \\
\hline $\mathrm{C}(44)$ & $1.0149(11)$ & $0.3074(9)$ & $0.6621(7)$ & $0.032(3)$ \\
\hline $\mathrm{C}(45)$ & $1.0322(13)$ & $0.2320(10)$ & $0.6495(9)$ & $0.050(4)$ \\
\hline $\mathrm{C}(46)$ & $1.1284(12)$ & $0.2376(10)$ & $0.6936(9)$ & $0.045(4)$ \\
\hline $\mathrm{C}(47)$ & $1.2023(12)$ & $0.3180(10)$ & $0.7421(9)$ & $0.044(4)$ \\
\hline $\mathrm{C}(48)$ & $1.1850(10)$ & $0.3954(9)$ & $0.7534(8)$ & $0.035(4)$ \\
\hline $\mathrm{C}(49)$ & $0.5313(8)$ & $0.2019(7)$ & $0.6985(7)$ & $0.019(3)$ \\
\hline $\mathrm{C}(50)$ & $0.5134(9)$ & $0.2184(8)$ & $0.6360(6)$ & $0.025(3)$ \\
\hline $\mathrm{C}(51)$ & $0.4138(10)$ & $0.2083(10)$ & $0.6011(8)$ & $0.043(4)$ \\
\hline $\mathrm{C}(52)$ & $0.3313(11)$ & $0.1821(10)$ & $0.6314(8)$ & $0.045(4)$ \\
\hline $\mathrm{C}(53)$ & $0.3478(10)$ & $0.1671(10)$ & $0.6958(8)$ & $0.041(4)$ \\
\hline $\mathrm{C}(54)$ & $0.4496(9)$ & $0.1767(9)$ & $0.7306(8)$ & $0.035(3)$ \\
\hline$C(55)$ & $0.6677(7)$ & $0.1084(5)$ & $0.7017(5)$ & $0.023(3)$ \\
\hline $\mathrm{C}(56)$ & $0.5846(6)$ & $0.0322(6)$ & $0.6988(6)$ & $0.063(5)$ \\
\hline
\end{tabular}


Tabelle 86: Fortsetzung

\begin{tabular}{|r|r|r|r|r|}
\hline $\mathrm{C}(57)$ & $0.5867(7)$ & $-0.0499(5)$ & $0.6706(6)$ & $0.078(6)$ \\
$\mathrm{C}(58)$ & $0.6718(9)$ & $-0.0558(5)$ & $0.6452(6)$ & $0.073(5)$ \\
$\mathrm{C}(59)$ & $0.7549(7)$ & $0.0204(6)$ & $0.6481(5)$ & $0.043(4)$ \\
$\mathrm{C}(60)$ & $0.7528(6)$ & $0.1025(5)$ & $0.6764(5)$ & $0.035(3)$ \\
$\mathrm{C}(61)$ & $0.8553(8)$ & $0.1981(7)$ & $0.8700(6)$ & $0.018(3)$ \\
$\mathrm{C}(62)$ & $0.8148(11)$ & $0.1088(8)$ & $0.8611(7)$ & $0.034(3)$ \\
$\mathrm{C}(63)$ & $0.8706(11)$ & $0.0591(9)$ & $0.8366(7)$ & $0.037(4)$ \\
$\mathrm{C}(64)$ & $0.9677(11)$ & $0.1026(10)$ & $0.8244(7)$ & $0.039(4)$ \\
$\mathrm{C}(65)$ & $1.0067(11)$ & $0.1908(9)$ & $0.8347(7)$ & $0.038(4)$ \\
$\mathrm{C}(66)$ & $0.9488(9)$ & $0.2381(8)$ & $0.8559(7)$ & $0.027(3)$ \\
$\mathrm{C}(67)$ & $0.7155(9)$ & $0.2106(8)$ & $0.9579(6)$ & $0.021(3)$ \\
$\mathrm{C}(68)$ & $0.6062(10)$ & $0.1611(9)$ & $0.9428(8)$ & $0.036(4)$ \\
$\mathrm{C}(69)$ & $0.5688(10)$ & $0.1211(9)$ & $0.9908(8)$ & $0.042(4)$ \\
$\mathrm{C}(70)$ & $0.6373(11)$ & $0.1263(8)$ & $1.0495(7)$ & $0.034(3)$ \\
$\mathrm{C}(71)$ & $0.7493(11)$ & $0.1755(9)$ & $1.0658(7)$ & $0.033(3)$ \\
$\mathrm{C}(72)$ & $0.7858(9)$ & $0.2169(8)$ & $1.0200(7)$ & $0.024(3)$ \\
\hline
\end{tabular}

Tabelle 87: Bindungslängen [pm] in 20

\begin{tabular}{|l|r||l|r|}
\hline $\mathrm{Cu}(1)-\mathrm{Se}(5)$ & $238.13(18)$ & $\mathrm{Cu}(1)-\mathrm{Se}(1)$ & $239.79(18)$ \\
$\mathrm{Cu}(1)-\mathrm{Se}(3)$ & $241.21(19)$ & $\mathrm{Cu}(1)-\mathrm{Cu}(2)$ & $271.3(2)$ \\
$\mathrm{Cu}(1)-\mathrm{Cu}(4)$ & $278.5(2)$ & $\mathrm{Cu}(1)-\mathrm{Cu}(3)$ & $279.2(2)$ \\
$\mathrm{Cu}(2)-\mathrm{Se}(1)$ & $236.14(19)$ & $\mathrm{Cu}(2)-\mathrm{Se}(2)$ & $237.72(19)$ \\
$\mathrm{Cu}(2)-\mathrm{Se}(6)$ & $238.76(18)$ & $\mathrm{Cu}(2)-\mathrm{Cu}(4)$ & $277.89(19)$ \\
$\mathrm{Cu}(2)-\mathrm{Cu}(3)$ & $288.39(19)$ & $\mathrm{Cu}(3)-\mathrm{Se}(4)$ & $236.51(18)$ \\
$\mathrm{Cu}(3)-\mathrm{Se}(3)$ & $236.54(19)$ & $\mathrm{Cu}(3)-\mathrm{Se}(2)$ & $239.15(19)$ \\
$\mathrm{Cu}(3)-\mathrm{Cu}(4)$ & $270.1(2)$ & $\mathrm{Cu}(4)-\mathrm{Se}(5)$ & $238.30(19)$ \\
$\mathrm{Cu}(4)-\mathrm{Se}(6)$ & $238.70(18)$ & $\mathrm{Cu}(4)-\mathrm{Se}(4)$ & $238.83(18)$ \\
$\mathrm{Cu}(5)-\mathrm{Cl}(2)$ & $210.4(5)$ & $\mathrm{Cu}(5)-\mathrm{Cl}(1)$ & $211.4(5)$ \\
$\mathrm{Se}(1)-\mathrm{P}(1)$ & $221.7(3)$ & $\mathrm{Se}(2)-\mathrm{P}(2)$ & $221.0(3)$ \\
$\mathrm{Se}(3)-\mathrm{P}(3)$ & $220.3(3)$ & $\mathrm{Se}(4)-\mathrm{P}(4)$ & $221.9(3)$ \\
$\mathrm{Se}(5)-\mathrm{P}(5)$ & $220.6(3)$ & $\mathrm{Se}(6)-\mathrm{P}(6)$ & $221.7(3)$ \\
$\mathrm{P}(1)-\mathrm{N}(1)$ & $159.1(10)$ & $\mathrm{P}(1)-\mathrm{C}(1)$ & $178.2(13)$ \\
$\mathrm{P}(1)-\mathrm{C}(7)$ & $180.0(11)$ & $\mathrm{P}(2)-\mathrm{N}(1)$ & $159.8(10)$ \\
$\mathrm{P}(2)-\mathrm{C}(19)$ & $180.2(11)$ & $\mathrm{P}(2)-\mathrm{C}(13)$ & $181.0(12)$ \\
$\mathrm{P}(3)-\mathrm{N}(2)$ & $159.0(9)$ & $\mathrm{P}(3)-\mathrm{C}(31)$ & $177.8(12)$ \\
$\mathrm{P}(3)-\mathrm{C}(25)$ & $181.2(13)$ & $\mathrm{P}(4)-\mathrm{N}(2)$ & $159.5(9)$ \\
$\mathrm{P}(4)-\mathrm{C}(43)$ & $179.6(12)$ & $\mathrm{P}(4)-\mathrm{C}(37)$ & $182.4(12)$ \\
$\mathrm{P}(5)-\mathrm{N}(3)$ & $157.8(10)$ & $\mathrm{P}(5)-\mathrm{C}(55)$ & $179.8(7)$ \\
$\mathrm{P}(5)-\mathrm{C}(49)$ & $182.6(11)$ & $\mathrm{P}(6)-\mathrm{N}(3)$ & $159.0(10)$ \\
\hline
\end{tabular}


Tabelle 87: Fortsetzung

\begin{tabular}{|l|l||l|l|}
\hline $\mathrm{P}(6)-\mathrm{C}(67)$ & $179.1(12)$ & $\mathrm{P}(6)-\mathrm{C}(61)$ & $183.4(11)$ \\
\hline
\end{tabular}

Tabelle 88: Bindungswinkel $\left[^{\circ}\right]$ in $\underline{20}$

\begin{tabular}{|c|c|c|c|}
\hline $\mathrm{Se}(5)-\mathrm{Cu}(1)-\mathrm{Se}(1)$ & $123.12(7)$ & $\mathrm{Se}(5)-\mathrm{Cu}(1)-\mathrm{Se}(3)$ & $117.40(7)$ \\
\hline $\mathrm{Se}(1)-\mathrm{Cu}(1)-\mathrm{Se}(3)$ & $119.47(7)$ & $\mathrm{Se}(5)-\mathrm{Cu}(1)-\mathrm{Cu}(2)$ & $107.08(7)$ \\
\hline $\mathrm{Se}(1)-\mathrm{Cu}(1)-\mathrm{Cu}(2)$ & $54.62(5)$ & $\mathrm{Se}(3)-\mathrm{Cu}(1)-\mathrm{Cu}(2)$ & $107.26(7)$ \\
\hline $\mathrm{Se}(5)-\mathrm{Cu}(1)-\mathrm{Cu}(4)$ & $54.25(5)$ & $\mathrm{Se}(1)-\mathrm{Cu}(1)-\mathrm{Cu}(4)$ & $108.66(7)$ \\
\hline $\mathrm{Se}(3)-\mathrm{Cu}(1)-\mathrm{Cu}(4)$ & $104.85(6)$ & $\mathrm{Cu}(2)-\mathrm{Cu}(1)-\mathrm{Cu}(4)$ & $60.70(5)$ \\
\hline $\mathrm{Se}(5)-\mathrm{Cu}(1)-\mathrm{Cu}(3)$ & $102.53(6)$ & $\mathrm{Se}(1)-\mathrm{Cu}(1)-\mathrm{Cu}(3)$ & $109.74(7)$ \\
\hline $\mathrm{Se}(3)-\mathrm{Cu}(1)-\mathrm{Cu}(3)$ & $53.46(5)$ & $\mathrm{Cu}(2)-\mathrm{Cu}(1)-\mathrm{Cu}(3)$ & $63.16(5)$ \\
\hline $\mathrm{Cu}(4)-\mathrm{Cu}(1)-\mathrm{Cu}(3)$ & $57.92(5)$ & $\mathrm{Se}(1)-\mathrm{Cu}(2)-\mathrm{Se}(2)$ & $122.82(7)$ \\
\hline $\mathrm{Se}(1)-\mathrm{Cu}(2)-\mathrm{Se}(6)$ & $128.39(8)$ & $\mathrm{Se}(2)-\mathrm{Cu}(2)-\mathrm{Se}(6)$ & $108.75(7)$ \\
\hline $\mathrm{Se}(1)-\mathrm{Cu}(2)-\mathrm{Cu}(1)$ & $55.88(5)$ & $\mathrm{Se}(2)-\mathrm{Cu}(2)-\mathrm{Cu}(1)$ & $106.24(7)$ \\
\hline $\mathrm{Se}(6)-\mathrm{Cu}(2)-\mathrm{Cu}(1)$ & 109.92(7) & $\mathrm{Se}(1)-\mathrm{Cu}(2)-\mathrm{Cu}(4)$ & $109.97(7)$ \\
\hline $\mathrm{Se}(2)-\mathrm{Cu}(2)-\mathrm{Cu}(4)$ & $100.34(6)$ & $\mathrm{Se}(6)-\mathrm{Cu}(2)-\mathrm{Cu}(4)$ & $54.40(5)$ \\
\hline $\mathrm{Cu}(1)-\mathrm{Cu}(2)-\mathrm{Cu}(4)$ & $60.93(5)$ & $\mathrm{Se}(1)-\mathrm{Cu}(2)-\mathrm{Cu}(3)$ & $107.85(7)$ \\
\hline $\mathrm{Se}(2)-\mathrm{Cu}(2)-\mathrm{Cu}(3)$ & $53.02(5)$ & $\mathrm{Se}(6)-\mathrm{Cu}(2)-\mathrm{Cu}(3)$ & $100.73(6)$ \\
\hline $\mathrm{Cu}(1)-\mathrm{Cu}(2)-\mathrm{Cu}(3)$ & $59.75(5)$ & $\mathrm{Cu}(4)-\mathrm{Cu}(2)-\mathrm{Cu}(3)$ & $56.93(5)$ \\
\hline $\mathrm{Se}(4)-\mathrm{Cu}(3)-\mathrm{Se}(3)$ & $123.48(7)$ & $\mathrm{Se}(4)-\mathrm{Cu}(3)-\mathrm{Se}(2)$ & $117.50(7)$ \\
\hline $\mathrm{Se}(3)-\mathrm{Cu}(3)-\mathrm{Se}(2)$ & $118.96(7)$ & $\mathrm{Se}(4)-\mathrm{Cu}(3)-\mathrm{Cu}(4)$ & $55.78(5)$ \\
\hline $\mathrm{Se}(3)-\mathrm{Cu}(3)-\mathrm{Cu}(4)$ & $108.86(7)$ & $\mathrm{Se}(2)-\mathrm{Cu}(3)-\mathrm{Cu}(4)$ & $102.21(6)$ \\
\hline $\mathrm{Se}(4)-\mathrm{Cu}(3)-\mathrm{Cu}(1)$ & $109.08(7)$ & $\mathrm{Se}(3)-\mathrm{Cu}(3)-\mathrm{Cu}(1)$ & $55.02(5)$ \\
\hline $\mathrm{Se}(2)-\mathrm{Cu}(3)-\mathrm{Cu}(1)$ & $103.44(6)$ & $\mathrm{Cu}(4)-\mathrm{Cu}(3)-\mathrm{Cu}(1)$ & $60.91(5)$ \\
\hline $\mathrm{Se}(4)-\mathrm{Cu}(3)-\mathrm{Cu}(2)$ & $108.29(6)$ & $\mathrm{Se}(3)-\mathrm{Cu}(3)-\mathrm{Cu}(2)$ & $103.35(6)$ \\
\hline $\mathrm{Se}(2)-\mathrm{Cu}(3)-\mathrm{Cu}(2)$ & $52.56(5)$ & $\mathrm{Cu}(4)-\mathrm{Cu}(3)-\mathrm{Cu}(2)$ & $59.58(5)$ \\
\hline $\mathrm{Cu}(1)-\mathrm{Cu}(3)-\mathrm{Cu}(2)$ & $57.08(5)$ & $\mathrm{Se}(5)-\mathrm{Cu}(4)-\mathrm{Se}(6)$ & $124.94(7)$ \\
\hline $\mathrm{Se}(5)-\mathrm{Cu}(4)-\mathrm{Se}(4)$ & $117.76(7)$ & $\mathrm{Se}(6)-\mathrm{Cu}(4)-\mathrm{Se}(4)$ & $117.29(7)$ \\
\hline $\mathrm{Se}(5)-\mathrm{Cu}(4)-\mathrm{Cu}(3)$ & $105.22(7)$ & $\mathrm{Se}(6)-\mathrm{Cu}(4)-\mathrm{Cu}(3)$ & $106.17(7)$ \\
\hline $\mathrm{Se}(4)-\mathrm{Cu}(4)-\mathrm{Cu}(3)$ & $54.97(5)$ & $\mathrm{Se}(5)-\mathrm{Cu}(4)-\mathrm{Cu}(2)$ & $104.99(6)$ \\
\hline $\mathrm{Se}(6)-\mathrm{Cu}(4)-\mathrm{Cu}(2)$ & $54.42(5)$ & $\mathrm{Se}(4)-\mathrm{Cu}(4)-\mathrm{Cu}(2)$ & $111.04(7)$ \\
\hline $\mathrm{Cu}(3)-\mathrm{Cu}(4)-\mathrm{Cu}(2)$ & $63.49(5)$ & $\mathrm{Se}(5)-\mathrm{Cu}(4)-\mathrm{Cu}(1)$ & $54.20(5)$ \\
\hline $\mathrm{Se}(6)-\mathrm{Cu}(4)-\mathrm{Cu}(1)$ & $107.60(6)$ & $\mathrm{Se}(4)-\mathrm{Cu}(4)-\mathrm{Cu}(1)$ & $108.60(7)$ \\
\hline $\mathrm{Cu}(3)-\mathrm{Cu}(4)-\mathrm{Cu}(1)$ & $61.16(5)$ & $\mathrm{Cu}(2)-\mathrm{Cu}(4)-\mathrm{Cu}(1)$ & $58.36(5)$ \\
\hline $\mathrm{Cl}(2)-\mathrm{Cu}(5)-\mathrm{Cl}(1)$ & $177.3(2)$ & $\mathrm{P}(1)-\mathrm{Se}(1)-\mathrm{Cu}(2)$ & $102.20(10)$ \\
\hline $\mathrm{P}(1)-\mathrm{Se}(1)-\mathrm{Cu}(1)$ & $93.18(9)$ & $\mathrm{Cu}(2)-\mathrm{Se}(1)-\mathrm{Cu}(1)$ & $69.50(6)$ \\
\hline $\mathrm{P}(2)-\mathrm{Se}(2)-\mathrm{Cu}(2)$ & $102.60(10)$ & $\mathrm{P}(2)-\mathrm{Se}(2)-\mathrm{Cu}(3)$ & $95.68(10)$ \\
\hline $\mathrm{Cu}(2)-\mathrm{Se}(2)-\mathrm{Cu}(3)$ & $74.42(6)$ & $\mathrm{P}(3)-\mathrm{Se}(3)-\mathrm{Cu}(3)$ & $103.22(10)$ \\
\hline $\mathrm{P}(3)-\mathrm{Se}(3)-\mathrm{Cu}(1)$ & $94.27(10)$ & $\mathrm{Cu}(3)-\mathrm{Se}(3)-\mathrm{Cu}(1)$ & $71.52(6)$ \\
\hline $\mathrm{P}(4)-\mathrm{Se}(4)-\mathrm{Cu}(3)$ & $101.10(10)$ & $\mathrm{P}(4)-\mathrm{Se}(4)-\mathrm{Cu}(4)$ & $97.71(10)$ \\
\hline
\end{tabular}


Tabelle 88: Fortsetzung

\begin{tabular}{|l|r||l|r|}
\hline $\mathrm{Cu}(3)-\mathrm{Se}(4)-\mathrm{Cu}(4)$ & $69.24(6)$ & $\mathrm{P}(5)-\mathrm{Se}(5)-\mathrm{Cu}(1)$ & $97.46(10)$ \\
$\mathrm{P}(5)-\mathrm{Se}(5)-\mathrm{Cu}(4)$ & $101.70(10)$ & $\mathrm{Cu}(1)-\mathrm{Se}(5)-\mathrm{Cu}(4)$ & $71.55(6)$ \\
$\mathrm{P}(6)-\mathrm{Se}(6)-\mathrm{Cu}(4)$ & $91.22(10)$ & $\mathrm{P}(6)-\mathrm{Se}(6)-\mathrm{Cu}(2)$ & $108.68(10)$ \\
$\mathrm{Cu}(4)-\mathrm{Se}(6)-\mathrm{Cu}(2)$ & $71.18(6)$ & $\mathrm{N}(1)-\mathrm{P}(1)-\mathrm{C}(1)$ & $105.7(5)$ \\
$\mathrm{N}(1)-\mathrm{P}(1)-\mathrm{C}(7)$ & $111.2(5)$ & $\mathrm{N}(1)-\mathrm{P}(1)-\mathrm{Se}(1)$ & $119.7(4)$ \\
$\mathrm{C}(1)-\mathrm{P}(1)-\mathrm{Se}(1)$ & $106.5(4)$ & $\mathrm{C}(7)-\mathrm{P}(1)-\mathrm{Se}(1)$ & $107.7(4)$ \\
$\mathrm{N}(1)-\mathrm{P}(2)-\mathrm{C}(19)$ & $111.5(5)$ & $\mathrm{N}(1)-\mathrm{P}(2)-\mathrm{C}(13)$ & $107.2(5)$ \\
$\mathrm{N}(1)-\mathrm{P}(2)-\mathrm{Se}(2)$ & $117.4(4)$ & $\mathrm{C}(19)-\mathrm{P}(2)-\mathrm{Se}(2)$ & $108.3(4)$ \\
$\mathrm{C}(13)-\mathrm{P}(2)-\mathrm{Se}(2)$ & $105.9(4)$ & $\mathrm{N}(2)-\mathrm{P}(3)-\mathrm{C}(31)$ & $106.3(5)$ \\
$\mathrm{N}(2)-\mathrm{P}(3)-\mathrm{C}(25)$ & $111.6(5)$ & $\mathrm{N}(2)-\mathrm{P}(3)-\mathrm{Se}(3)$ & $118.6(4)$ \\
$\mathrm{C}(31)-\mathrm{P}(3)-\mathrm{Se}(3)$ & $106.5(4)$ & $\mathrm{C}(25)-\mathrm{P}(3)-\mathrm{Se}(3)$ & $107.0(4)$ \\
$\mathrm{N}(2)-\mathrm{P}(4)-\mathrm{C}(43)$ & $106.6(6)$ & $\mathrm{N}(2)-\mathrm{P}(4)-\mathrm{C}(37)$ & $111.3(5)$ \\
$\mathrm{N}(2)-\mathrm{P}(4)-\mathrm{Se}(4)$ & $119.0(3)$ & $\mathrm{C}(43)-\mathrm{P}(4)-\mathrm{Se}(4)$ & $106.0(4)$ \\
$\mathrm{C}(37)-\mathrm{P}(4)-\mathrm{Se}(4)$ & $106.0(4)$ & $\mathrm{N}(3)-\mathrm{P}(5)-\mathrm{C}(55)$ & $109.9(5)$ \\
$\mathrm{N}(3)-\mathrm{P}(5)-\mathrm{C}(49)$ & $107.1(5)$ & $\mathrm{N}(3)-\mathrm{P}(5)-\mathrm{Se}(5)$ & $118.5(4)$ \\
$\mathrm{C}(55)-\mathrm{P}(5)-\mathrm{Se}(5)$ & $107.5(3)$ & $\mathrm{C}(49)-\mathrm{P}(5)-\mathrm{Se}(5)$ & $107.3(4)$ \\
$\mathrm{N}(3)-\mathrm{P}(6)-\mathrm{C}(67)$ & $107.3(5)$ & $\mathrm{N}(3)-\mathrm{P}(6)-\mathrm{C}(61)$ & $112.9(5)$ \\
$\mathrm{N}(3)-\mathrm{P}(6)-\mathrm{Se}(6)$ & $116.1(4)$ & $\mathrm{C}(67)-\mathrm{P}(6)-\mathrm{Se}(6)$ & $108.3(4)$ \\
$\mathrm{C}(61)-\mathrm{P}(6)-\mathrm{Se}(6)$ & $107.3(4)$ & $\mathrm{P}(1)-\mathrm{N}(1)-\mathrm{P}(2)$ & $133.7(6)$ \\
$\mathrm{P}(3)-\mathrm{N}(2)-\mathrm{P}(4)$ & $135.2(6)$ & $\mathrm{P}(5)-\mathrm{N}(3)-\mathrm{P}(6)$ & $129.1(6)$ \\
\hline & \multicolumn{3}{|}{}
\end{tabular}

$\# 1-\mathrm{x}+1,-\mathrm{y},-\mathrm{z}+1 \quad \# 2-\mathrm{x}+2,-\mathrm{y},-\mathrm{z}+1$ 


\subsection{Daten zur Kristallstrukturanalyse von $\left[\mathrm{Cu}\left(\mathrm{dppaS}_{2}\right)(\right.$ th $\left.)\right] \underline{21}$}

\begin{tabular}{|ll|}
\hline Summenformel & $\mathrm{C}_{74} \mathrm{H}_{96} \mathrm{Cu}_{2} \mathrm{~N}_{6} \mathrm{O}_{6} \mathrm{P}_{4} \mathrm{~S}_{6}$ \\
Molekulargewicht $\left[\mathrm{g} \cdot \mathrm{mol}^{-1}\right]$ & 1608.89 \\
Temperatur & $133(2) \mathrm{K}$ \\
Wellenlänge & $71.073 \mathrm{pm}$ \\
Kristallsystem & monoklin \\
Raumgruppe & $P 2_{1} / n$ \\
Gitterkonstanten & $\mathrm{a}=12.699(2) \AA, \alpha=90^{\circ}$ \\
& $\mathrm{b}=18.618(3) \AA, \beta=99.585(2)^{\circ} \circ$ \\
& $\mathrm{c}=33.721(6) \AA, \gamma=90^{\circ}$ \\
Volumen & $7861(2) \AA^{3}$ \\
Formeleinheiten & 4 \\
Röntgenographische Dichte $\left[\mathrm{g} \cdot \mathrm{cm}^{-3}\right]$ & 1.359 \\
Absorptionskoeffizient $\left[\mathrm{mm}^{-1}\right]$ & 0.835 \\
$\mathrm{~F}(000)$ & 3376 \\
Meßbereich $\theta$ & $1.96-22.50^{\circ}$ \\
Indexbereiche & $-13 \leq \mathrm{h} \leq 13$ \\
& $0 \leq \mathrm{k} \leq 20$ \\
Gemessene Reflexe & $0 \leq 1 \leq 36$ \\
Unabhängige Reflexe & 18305 \\
Beobachtete Reflexe $[\mathrm{I}>2 \sigma]$ & 9592 \\
$\mathrm{R}_{\text {int }}$ & 3839 \\
Parameter & 0.1152 \\
R1; wR2[I $>2 \sigma(\mathrm{I})]$ & 394 \\
& $0.0829 ; 0.1560$ \\
&
\end{tabular}


Tabelle 90: Atomkoordinaten und äquivalente isotrope Auslenkungsparameter $\left[\AA^{2}\right]$ für $\underline{21}$

\begin{tabular}{|c|c|c|c|c|}
\hline Atom & $\mathrm{x}$ & $\mathrm{y}$ & Z & $\mathrm{U}_{e q}$ \\
\hline $\mathrm{Cu}(1)$ & $0.24349(9)$ & $0.54666(6)$ & $0.03829(3)$ & $0.0268(3)$ \\
\hline $\mathrm{Cu}(2)$ & $1.24558(9)$ & $0.04654(6)$ & $0.04359(3)$ & $0.0254(3)$ \\
\hline $\mathrm{S}(1)$ & $0.35432(19)$ & $0.50279(11)$ & $0.09308(7)$ & $0.0271(6)$ \\
\hline $\mathrm{S}(2)$ & $0.08710(19)$ & $0.59699(12)$ & $0.04585(7)$ & $0.0278(6)$ \\
\hline $\mathrm{S}(3)$ & $0.29143(18)$ & $0.54737(12)$ & $-0.02289(7)$ & $0.0280(6)$ \\
\hline $\mathrm{S}(4)$ & $1.35416(18)$ & $0.00436(11)$ & $0.09947(7)$ & $0.0245(6)$ \\
\hline $\mathrm{S}(5)$ & $1.08662(19)$ & $0.09771(11)$ & $0.04795(7)$ & $0.0263(6)$ \\
\hline $\mathrm{S}(6)$ & $1.29386(18)$ & $0.04579(12)$ & $-0.01728(6)$ & $0.0260(6)$ \\
\hline $\mathrm{P}(1)$ & $0.30654(18)$ & $0.57279(11)$ & $0.13212(7)$ & $0.0203(6)$ \\
\hline $\mathrm{P}(2)$ & $0.08329(19)$ & $0.55885(12)$ & $0.10125(7)$ & $0.0214(6)$ \\
\hline $\mathrm{P}(3)$ & $1.29870(19)$ & $0.07360(11)$ & $0.13713(7)$ & $0.0207(6)$ \\
\hline $\mathrm{P}(4)$ & $1.07851(19)$ & $0.05773(12)$ & $0.10259(7)$ & $0.0207(6)$ \\
\hline $\mathrm{N}(1)$ & $0.1819(5)$ & $0.5757(3)$ & $0.13626(18)$ & $0.0198(18)$ \\
\hline $\mathrm{N}(2)$ & $0.4617(5)$ & $0.6009(3)$ & $-0.0476(2)$ & $0.031(2)$ \\
\hline $\mathrm{N}(3)$ & $0.4822(5)$ & $0.5818(3)$ & $0.0200(2)$ & $0.029(2)$ \\
\hline $\mathrm{N}(4)$ & $1.1743(5)$ & $0.0757(3)$ & $0.13905(19)$ & $0.0219(18)$ \\
\hline $\mathrm{N}(5)$ & $1.4635(6)$ & $0.0995(3)$ & $-0.0422(2)$ & $0.035(2)$ \\
\hline $\mathrm{N}(6)$ & $1.4840(6)$ & $0.0797(3)$ & $0.0249(2)$ & $0.029(2)$ \\
\hline $\mathrm{C}(1)$ & $0.3781(4)$ & $0.5522(3)$ & $0.18208(13)$ & $0.025(2)$ \\
\hline $\mathrm{C}(2)$ & $0.3752(4)$ & $0.4823(2)$ & $0.19633(16)$ & $0.038(2)$ \\
\hline $\mathrm{C}(3)$ & $0.4246(5)$ & $0.4658(2)$ & $0.23519(17)$ & $0.048(3)$ \\
\hline $\mathrm{C}(4)$ & $0.4769(5)$ & $0.5191(3)$ & $0.25980(13)$ & $0.059(3)$ \\
\hline $\mathrm{C}(5)$ & $0.4798(5)$ & $0.5890(3)$ & $0.24555(16)$ & $0.058(3)$ \\
\hline $\mathrm{C}(6)$ & $0.4304(5)$ & $0.6056(2)$ & $0.20669(17)$ & $0.046(3)$ \\
\hline $\mathrm{C}(7)$ & $0.3504(4)$ & $0.6626(2)$ & $0.12064(15)$ & $0.021(2)$ \\
\hline $\mathrm{C}(8)$ & $0.4551(4)$ & $0.6744(2)$ & $0.11518(16)$ & $0.030(2)$ \\
\hline $\mathrm{C}(9)$ & $0.4897(3)$ & $0.7437(3)$ & $0.10894(16)$ & $0.034(2)$ \\
\hline $\mathrm{C}(10)$ & $0.4195(4)$ & $0.8012(2)$ & $0.10817(16)$ & $0.031(2)$ \\
\hline $\mathrm{C}(11)$ & $0.3148(4)$ & $0.7893(2)$ & $0.11364(16)$ & $0.025(2)$ \\
\hline $\mathrm{C}(12)$ & $0.2803(3)$ & $0.7200(3)$ & $0.11987(16)$ & $0.028(2)$ \\
\hline $\mathrm{C}(13)$ & $-0.0326(4)$ & $0.5992(3)$ & $0.11804(16)$ & $0.021(2)$ \\
\hline $\mathrm{C}(14)$ & $-0.0194(3)$ & $0.6487(3)$ & $0.14933(15)$ & $0.030(2)$ \\
\hline $\mathrm{C}(15)$ & $-0.1080(5)$ & $0.6820(3)$ & $0.16041(14)$ & $0.036(2)$ \\
\hline $\mathrm{C}(16)$ & $-0.2098(4)$ & $0.6659(3)$ & $0.14021(17)$ & $0.043(3)$ \\
\hline $\mathrm{C}(17)$ & $-0.2230(3)$ & $0.6164(3)$ & $0.10892(16)$ & $0.043(3)$ \\
\hline $\mathrm{C}(18)$ & $-0.1344(5)$ & $0.5830(3)$ & $0.09784(14)$ & $0.036(2)$ \\
\hline $\mathrm{C}(19)$ & $0.0556(3)$ & $0.46311(17)$ & $0.09892(13)$ & $0.023(2)$ \\
\hline $\mathrm{C}(20)$ & $0.0718(4)$ & $0.4240(2)$ & $0.13454(11)$ & $0.027(2)$ \\
\hline
\end{tabular}


Tabelle 90: Fortsetzung

\begin{tabular}{|c|c|c|c|c|}
\hline $\mathrm{C}(21)$ & $0.0477(3)$ & $0.3512(2)$ & $0.13406(13)$ & $0.031(2)$ \\
\hline $\mathrm{C}(22)$ & $0.0074(4)$ & $0.31739(18)$ & $0.09795(15)$ & $0.031(2)$ \\
\hline $\mathrm{C}(23)$ & $-0.0088(3)$ & $0.3565(2)$ & $0.06233(12)$ & $0.033(2)$ \\
\hline $\mathrm{C}(24)$ & $0.0153(2)$ & $0.4293(2)$ & $0.06281(12)$ & $0.026(2)$ \\
\hline $\mathrm{C}(25)$ & $0.4183(5)$ & $0.5776(4)$ & $-0.0154(2)$ & $0.024(2)$ \\
\hline $\mathrm{C}(26)$ & $1.3441(4)$ & $0.1644(2)$ & $0.12696(15)$ & $0.019(2)$ \\
\hline $\mathrm{C}(27)$ & $1.4481(4)$ & $0.1758(2)$ & $0.12060(16)$ & $0.026(2)$ \\
\hline $\mathrm{C}(28)$ & $1.4842(3)$ & $0.2452(3)$ & $0.11582(16)$ & $0.034(2)$ \\
\hline $\mathrm{C}(29)$ & $1.4162(4)$ & $0.3033(2)$ & $0.11741(16)$ & $0.033(2)$ \\
\hline $\mathrm{C}(30)$ & $1.3122(4)$ & $0.2919(2)$ & $0.12377(16)$ & $0.031(2)$ \\
\hline $\mathrm{C}(31)$ & $1.2761(3)$ & $0.2224(3)$ & $0.12855(16)$ & $0.026(2)$ \\
\hline $\mathrm{C}(32)$ & $1.3625(4)$ & $0.0542(3)$ & $0.18850(11)$ & $0.020(2)$ \\
\hline $\mathrm{C}(33)$ & $1.4690(4)$ & $0.0331(3)$ & $0.19611(13)$ & $0.030(2)$ \\
\hline $\mathrm{C}(34)$ & $1.5196(3)$ & $0.0219(3)$ & $0.23544(15)$ & $0.034(2)$ \\
\hline $\mathrm{C}(35)$ & $1.4638(4)$ & $0.0317(3)$ & $0.26716(11)$ & $0.032(2)$ \\
\hline $\mathrm{C}(36)$ & $1.3573(4)$ & $0.0528(3)$ & $0.25954(12)$ & $0.028(2)$ \\
\hline $\mathrm{C}(37)$ & $1.3067(3)$ & $0.0640(2)$ & $0.22022(14)$ & $0.027(2)$ \\
\hline $\mathrm{C}(38)$ & $0.9584(4)$ & $0.0925(3)$ & $0.11820(16)$ & $0.024(2)$ \\
\hline $\mathrm{C}(39)$ & $0.8591(4)$ & $0.0692(2)$ & $0.09869(13)$ & $0.027(2)$ \\
\hline $\mathrm{C}(40)$ & $0.7664(3)$ & $0.0972(3)$ & $0.10947(16)$ & $0.041(3)$ \\
\hline $\mathrm{C}(41)$ & $0.7730(4)$ & $0.1485(3)$ & $0.13976(17)$ & $0.042(3)$ \\
\hline $\mathrm{C}(42)$ & $0.8722(5)$ & $0.1717(2)$ & $0.15927(14)$ & $0.034(2)$ \\
\hline $\mathrm{C}(43)$ & $0.9649(3)$ & $0.1437(3)$ & $0.14849(15)$ & $0.027(2)$ \\
\hline $\mathrm{C}(44)$ & $1.0578(4)$ & $-0.03951(18)$ & $0.09875(15)$ & $0.022(2)$ \\
\hline $\mathrm{C}(45)$ & $1.0178(4)$ & $-0.0715(2)$ & $0.06209(12)$ & $0.026(2)$ \\
\hline $\mathrm{C}(46)$ & $0.9984(4)$ & $-0.1450(3)$ & $0.06012(13)$ & $0.031(2)$ \\
\hline $\mathrm{C}(47)$ & $1.0190(4)$ & $-0.18650(19)$ & $0.09481(16)$ & $0.031(2)$ \\
\hline $\mathrm{C}(48)$ & $1.0591(4)$ & $-0.1545(2)$ & $0.13147(13)$ & $0.031(2)$ \\
\hline $\mathrm{C}(49)$ & $1.0785(4)$ & $-0.0810(3)$ & $0.13344(12)$ & $0.023(2)$ \\
\hline $\mathrm{C}(50)$ & $1.4230(7)$ & $0.0768(4)$ & $-0.0107(3)$ & $0.024(2)$ \\
\hline $\mathrm{O}(1 \mathrm{~L})$ & $0.6532(5)$ & $0.3962(3)$ & $0.12957(19)$ & $0.0462(18)$ \\
\hline $\mathrm{C}(1 \mathrm{~L})$ & $0.7660(8)$ & $0.3838(5)$ & $0.1353(3)$ & $0.045(3)$ \\
\hline $\mathrm{C}(2 \mathrm{~L})$ & $0.8118(9)$ & $0.4165(6)$ & $0.1745(3)$ & $0.075(4)$ \\
\hline $\mathrm{C}(3 \mathrm{~L})$ & $0.7315(8)$ & $0.4710(5)$ & $0.1840(3)$ & $0.061(3)$ \\
\hline $\mathrm{C}(4 \mathrm{~L})$ & $0.6359(9)$ & $0.4593(5)$ & $0.1513(3)$ & $0.067(3)$ \\
\hline $\mathrm{O}(2 \mathrm{~L})$ & $0.3384(5)$ & $0.8425(3)$ & $0.00216(17)$ & $0.0370(17)$ \\
\hline $\mathrm{C}(5 \mathrm{~L})$ & $0.3490(8)$ & $0.7694(5)$ & $-0.0114(3)$ & $0.046(3)$ \\
\hline $\mathrm{C}(6 \mathrm{~L})$ & $0.2381(7)$ & $0.7492(5)$ & $-0.0326(3)$ & $0.044(3)$ \\
\hline $\mathrm{C}(7 \mathrm{~L})$ & $0.1703(7)$ & $0.7848(4)$ & $-0.0051(3)$ & $0.037(3)$ \\
\hline $\mathrm{C}(8 \mathrm{~L})$ & $0.2282(7)$ & $0.8543(4)$ & $0.0074(3)$ & $0.031(2)$ \\
\hline
\end{tabular}


Tabelle 90: Fortsetzung

\begin{tabular}{|r|r|r|r|r|}
\hline $\mathrm{O}(3 \mathrm{~L})$ & $0.3379(5)$ & $0.3420(3)$ & $0.00720(17)$ & $0.0379(17)$ \\
$\mathrm{C}(9 \mathrm{~L})$ & $0.3484(8)$ & $0.2680(5)$ & $-0.0035(3)$ & $0.045(3)$ \\
$\mathrm{C}(10 \mathrm{~L})$ & $0.2384(7)$ & $0.2453(5)$ & $-0.0237(3)$ & $0.041(3)$ \\
$\mathrm{C}(11 \mathrm{~L})$ & $0.1692(7)$ & $0.2855(4)$ & $0.0007(3)$ & $0.037(3)$ \\
$\mathrm{C}(12 \mathrm{~L})$ & $0.2293(7)$ & $0.3546(5)$ & $0.0135(3)$ & $0.040(3)$ \\
$\mathrm{O}(4 \mathrm{~L})$ & $0.6648(5)$ & $0.8801(3)$ & $0.12233(19)$ & $0.0457(18)$ \\
$\mathrm{C}(13 \mathrm{~L})$ & $0.6414(9)$ & $0.8942(6)$ & $0.1608(3)$ & $0.077(4)$ \\
$\mathrm{C}(14 \mathrm{~L})$ & $0.7286(8)$ & $0.9351(5)$ & $0.1826(3)$ & $0.063(3)$ \\
$\mathrm{C}(15 \mathrm{~L})$ & $0.8249(8)$ & $0.9095(5)$ & $0.1643(3)$ & $0.062(3)$ \\
$\mathrm{C}(16 \mathrm{~L})$ & $0.7781(7)$ & $0.8750(4)$ & $0.1254(3)$ & $0.034(2)$ \\
$\mathrm{O}(5 \mathrm{~L})$ & $0.0747(6)$ & $0.2662(4)$ & $0.2325(2)$ & $0.076(2)$ \\
$\mathrm{C}(17 \mathrm{~L})$ & $0.1838(10)$ & $0.2531(6)$ & $0.2344(4)$ & $0.084(4)$ \\
$\mathrm{C}(18 \mathrm{~L})$ & $0.2451(11)$ & $0.2926(7)$ & $0.2685(4)$ & $0.116(5)$ \\
$\mathrm{C}(19 \mathrm{~L})$ & $0.1678(10)$ & $0.3502(6)$ & $0.2752(4)$ & $0.090(4)$ \\
$\mathrm{C}(20 \mathrm{~L})$ & $0.0597(10)$ & $0.3196(7)$ & $0.2601(4)$ & $0.094(4)$ \\
$\mathrm{O}(6 \mathrm{~L})$ & $0.9792(7)$ & $0.7912(5)$ & $0.2477(3)$ & $0.099(3)$ \\
$\mathrm{C}(21 \mathrm{~L})$ & $0.9985(10)$ & $0.7188(6)$ & $0.2672(4)$ & $0.094(4)$ \\
$\mathrm{C}(22 \mathrm{~L})$ & $0.8887(9)$ & $0.6937(6)$ & $0.2727(4)$ & $0.079(4)$ \\
$\mathrm{C}(23 \mathrm{~L})$ & $0.8167(15)$ & $0.7640(9)$ & $0.2686(5)$ & $0.161(7)$ \\
$\mathrm{C}(24 \mathrm{~L})$ & $0.9020(13)$ & $0.8195(8)$ & $0.2705(5)$ & $0.136(6)$ \\
\hline
\end{tabular}

Tabelle 91: Bindungslängen [pm] in 21

\begin{tabular}{|l|l||l|r|}
\hline $\mathrm{Cu}(1)-\mathrm{S}(3)$ & $224.6(2)$ & $\mathrm{Cu}(1)-\mathrm{S}(2)$ & $224.9(3)$ \\
$\mathrm{Cu}(1)-\mathrm{S}(1)$ & $227.9(3)$ & $\mathrm{Cu}(2)-\mathrm{S}(6)$ & $223.8(2)$ \\
$\mathrm{Cu}(2)-\mathrm{S}(5)$ & $225.9(3)$ & $\mathrm{Cu}(2)-\mathrm{S}(4)$ & $228.1(3)$ \\
$\mathrm{S}(1)-\mathrm{P}(1)$ & $201.6(3)$ & $\mathrm{S}(2)-\mathrm{P}(2)$ & $200.6(3)$ \\
$\mathrm{S}(3)-\mathrm{C}(25)$ & $168.6(6)$ & $\mathrm{S}(4)-\mathrm{P}(3)$ & $201.7(3)$ \\
$\mathrm{S}(5)-\mathrm{P}(4)$ & $200.6(3)$ & $\mathrm{S}(6)-\mathrm{C}(50)$ & $171.8(9)$ \\
$\mathrm{P}(1)-\mathrm{N}(1)$ & $161.3(7)$ & $\mathrm{P}(1)-\mathrm{C}(1)$ & $181.7(5)$ \\
$\mathrm{P}(1)-\mathrm{C}(7)$ & $182.4(4)$ & $\mathrm{P}(2)-\mathrm{N}(1)$ & $160.2(7)$ \\
$\mathrm{P}(2)-\mathrm{C}(19)$ & $181.6(4)$ & $\mathrm{P}(2)-\mathrm{C}(13)$ & $182.4(4)$ \\
$\mathrm{P}(3)-\mathrm{N}(4)$ & $159.2(7)$ & $\mathrm{P}(3)-\mathrm{C}(32)$ & $182.2(4)$ \\
$\mathrm{P}(3)-\mathrm{C}(26)$ & $183.6(4)$ & $\mathrm{P}(4)-\mathrm{N}(4)$ & $161.4(7)$ \\
$\mathrm{P}(4)-\mathrm{C}(38)$ & $181.4(4)$ & $\mathrm{P}(4)-\mathrm{C}(44)$ & $183.1(4)$ \\
$\mathrm{N}(2)-\mathrm{C}(25)$ & $136.7(7)$ & $\mathrm{N}(3)-\mathrm{C}(25)$ & $133.1(10)$ \\
$\mathrm{N}(5)-\mathrm{C}(50)$ & $132.3(9)$ & $\mathrm{N}(6)-\mathrm{C}(50)$ & $131.8(10)$ \\
\hline
\end{tabular}


Tabelle 92: Bindungswinkel $\left[^{\circ}\right]$ in $\underline{21}$

\begin{tabular}{|l|r||l|r|}
\hline $\mathrm{S}(3)-\mathrm{Cu}(1)-\mathrm{S}(2)$ & $118.78(10)$ & $\mathrm{S}(3)-\mathrm{Cu}(1)-\mathrm{S}(1)$ & $121.42(10)$ \\
$\mathrm{S}(2)-\mathrm{Cu}(1)-\mathrm{S}(1)$ & $119.68(9)$ & $\mathrm{S}(6)-\mathrm{Cu}(2)-\mathrm{S}(5)$ & $116.53(10)$ \\
$\mathrm{S}(6)-\mathrm{Cu}(2)-\mathrm{S}(4)$ & $122.36(9)$ & $\mathrm{S}(5)-\mathrm{Cu}(2)-\mathrm{S}(4)$ & $121.01(9)$ \\
$\mathrm{P}(1)-\mathrm{S}(1)-\mathrm{Cu}(1)$ & $94.89(11)$ & $\mathrm{P}(2)-\mathrm{S}(2)-\mathrm{Cu}(1)$ & $96.71(12)$ \\
$\mathrm{C}(25)-\mathrm{S}(3)-\mathrm{Cu}(1)$ & $105.3(3)$ & $\mathrm{P}(3)-\mathrm{S}(4)-\mathrm{Cu}(2)$ & $94.22(11)$ \\
$\mathrm{P}(4)-\mathrm{S}(5)-\mathrm{Cu}(2)$ & $94.96(11)$ & $\mathrm{C}(50)-\mathrm{S}(6)-\mathrm{Cu}(2)$ & $106.4(3)$ \\
$\mathrm{N}(1)-\mathrm{P}(1)-\mathrm{C}(1)$ & $105.9(3)$ & $\mathrm{N}(1)-\mathrm{P}(1)-\mathrm{C}(7)$ & $109.0(3)$ \\
$\mathrm{N}(1)-\mathrm{P}(1)-\mathrm{S}(1)$ & $119.0(3)$ & $\mathrm{C}(1)-\mathrm{P}(1)-\mathrm{S}(1)$ & $108.1(2)$ \\
$\mathrm{C}(7)-\mathrm{P}(1)-\mathrm{S}(1)$ & $108.5(2)$ & $\mathrm{N}(1)-\mathrm{P}(2)-\mathrm{C}(19)$ & $110.3(3)$ \\
$\mathrm{N}(1)-\mathrm{P}(2)-\mathrm{C}(13)$ & $105.2(3)$ & $\mathrm{N}(1)-\mathrm{P}(2)-\mathrm{S}(2)$ & $118.7(3)$ \\
$\mathrm{C}(19)-\mathrm{P}(2)-\mathrm{S}(2)$ & $109.98(18)$ & $\mathrm{C}(13)-\mathrm{P}(2)-\mathrm{S}(2)$ & $106.8(2)$ \\
$\mathrm{N}(4)-\mathrm{P}(3)-\mathrm{C}(32)$ & $104.7(3)$ & $\mathrm{N}(4)-\mathrm{P}(3)-\mathrm{C}(26)$ & $109.2(3)$ \\
$\mathrm{N}(4)-\mathrm{P}(3)-\mathrm{S}(4)$ & $119.6(3)$ & $\mathrm{C}(32)-\mathrm{P}(3)-\mathrm{S}(4)$ & $109.0(2)$ \\
$\mathrm{C}(26)-\mathrm{P}(3)-\mathrm{S}(4)$ & $108.5(2)$ & $\mathrm{N}(4)-\mathrm{P}(4)-\mathrm{C}(38)$ & $105.6(3)$ \\
$\mathrm{N}(4)-\mathrm{P}(4)-\mathrm{C}(44)$ & $109.9(3)$ & $\mathrm{N}(4)-\mathrm{P}(4)-\mathrm{S}(5)$ & $118.4(3)$ \\
$\mathrm{C}(38)-\mathrm{P}(4)-\mathrm{S}(5)$ & $108.0(2)$ & $\mathrm{C}(44)-\mathrm{P}(4)-\mathrm{S}(5)$ & $109.3(2)$ \\
$\mathrm{P}(2)-\mathrm{N}(1)-\mathrm{P}(1)$ & $126.0(4)$ & $\mathrm{P}(3)-\mathrm{N}(4)-\mathrm{P}(4)$ & $126.2(4)$ \\
$\mathrm{N}(3)-\mathrm{C}(25)-\mathrm{N}(2)$ & $115.4(6)$ & $\mathrm{N}(3)-\mathrm{C}(25)-\mathrm{S}(3)$ & $125.3(4)$ \\
$\mathrm{N}(2)-\mathrm{C}(25)-\mathrm{S}(3)$ & $119.3(6)$ & $\mathrm{N}(6)-\mathrm{C}(50)-\mathrm{N}(5)$ & $118.1(8)$ \\
$\mathrm{N}(6)-\mathrm{C}(50)-\mathrm{S}(6)$ & $122.3(6)$ & $\mathrm{N}(5)-\mathrm{C}(50)-\mathrm{S}(6)$ & $119.6(8)$ \\
\hline
\end{tabular}




\subsection{Daten zur Kristallstrukturanalyse von $\left[\mathrm{Cu}_{2}\left(\operatorname{dmpaS}_{2} \mathrm{H}\right)_{2} \mathrm{Cl}_{2}\right] \underline{22}$}

\begin{tabular}{|ll|}
\hline Summenformel & $\mathrm{C}_{8} \mathrm{H}_{26} \mathrm{Cl}_{2} \mathrm{Cu}_{2} \mathrm{~N}_{2} \mathrm{P}_{4} \mathrm{~S}_{4}$ \\
Molekulargewicht $\left[\mathrm{g} \cdot \mathrm{mol}^{-1}\right]$ & 600.41 \\
Temperatur & $133(2) \mathrm{K}$ \\
Wellenlänge & $71.073 \mathrm{pm}$ \\
Kristallsystem & orthorhombisch \\
Raumgruppe & $P b c a$ \\
Gitterkonstanten & $\mathrm{a}=12.0560(10) \AA, \alpha=90^{\circ}$ \\
& $\mathrm{b}=11.9350(10) \AA, \beta=90^{\circ}$ \\
& $\mathrm{c}=15.6260(10) \AA, \gamma=90^{\circ}$ \\
Volumen & $2248.4(3) \AA^{3}$ \\
Formeleinheiten & 4 \\
Röntgenographische Dichte $\left[\mathrm{g} \cdot \mathrm{cm}^{-3}\right]$ & 1.774 \\
Absorptionskoeffizient $\left[\mathrm{mm}^{-1}\right]$ & 2.781 \\
$\mathrm{~F}(000)$ & 1216 \\
Meßbereich $\theta$ & $2.61-27.53^{\circ}$ \\
Indexbereiche & $-15 \leq \mathrm{h} \leq 10$ \\
& $-13 \leq \mathrm{k} \leq 15$ \\
Gemessene Reflexe & $-20 \leq 1 \leq 14$ \\
Unabhängige Reflexe & 14077 \\
Beobachtete Reflexe $[\mathrm{I}>2 \sigma]$ & 2574 \\
Rint & 2166 \\
Parameter & 0.0380 \\
R1; wR2[I>2 $\sigma(\mathrm{I})]$ & 152 \\
\hline
\end{tabular}

Anmerkung: alle H-Atome sind frei verfeinert 
Tabelle 94: Atomkoordinaten und äquivalente isotrope Auslenkungsparameter $\left[\AA^{2}\right]$ für $\underline{22}$

\begin{tabular}{|r|r|r|r|r|}
\hline Atom & \multicolumn{1}{|c|}{$\mathrm{x}$} & \multicolumn{1}{c|}{$\mathrm{y}$} & \multicolumn{1}{c|}{$\mathrm{z}$} & \multicolumn{1}{c|}{$\mathrm{U}_{e q}$} \\
\hline $\mathrm{C}(1)$ & $0.0358(2)$ & $-0.2293(2)$ & $0.6927(2)$ & $0.0169(5)$ \\
$\mathrm{C}(2)$ & $0.2537(2)$ & $-0.1660(2)$ & $0.7487(2)$ & $0.0185(5)$ \\
$\mathrm{C}(3)$ & $0.3455(2)$ & $0.0203(2)$ & $0.5764(2)$ & $0.0181(5)$ \\
$\mathrm{C}(4)$ & $0.3160(2)$ & $-0.1310(2)$ & $0.43473(14)$ & $0.0177(5)$ \\
$\mathrm{N}(1)$ & $0.18623(15)$ & $-0.1466(2)$ & $0.57869(11)$ & $0.0108(4)$ \\
$\mathrm{P}(1)$ & $0.14161(4)$ & $-0.12660(5)$ & $0.67994(3)$ & $0.01006(12)$ \\
$\mathrm{P}(2)$ & $0.24412(4)$ & $-0.05123(5)$ & $0.51361(3)$ & $0.01060(12)$ \\
$\mathrm{S}(1)$ & $0.09135(5)$ & $0.02722(5)$ & $0.70716(3)$ & $0.01586(13)$ \\
$\mathrm{S}(2)$ & $0.13481(4)$ & $0.05214(5)$ & $0.45972(3)$ & $0.01354(12)$ \\
$\mathrm{Cl}(1)$ & $-0.04464(4)$ & $0.26470(5)$ & $0.57146(3)$ & $0.01481(12)$ \\
$\mathrm{Cu}(1)$ & $0.01006(2)$ & $0.07979(3)$ & $0.57969(2)$ & $0.02020(9)$ \\
\hline
\end{tabular}

Tabelle 95: Bindungslängen [pm] in $\underline{22}$

\begin{tabular}{|l|r||l|r|}
\hline $\mathrm{N}(1)-\mathrm{P}(2)$ & $167.9(2)$ & $\mathrm{N}(1)-\mathrm{P}(1)$ & $168.8(2)$ \\
$\mathrm{P}(1)-\mathrm{S}(1)$ & $197.94(8)$ & $\mathrm{P}(2)-\mathrm{S}(2)$ & $199.20(8)$ \\
$\mathrm{S}(1)-\mathrm{Cu}(1)$ & $230.68(6)$ & $\mathrm{S}(2)-\mathrm{Cu}(1)$ & $242.60(6)$ \\
$\mathrm{S}(2)-\mathrm{Cu}(1) \# 1$ & $243.09(6)$ & $\mathrm{Cl}(1)-\mathrm{Cu}(1)$ & $230.70(6)$ \\
$\mathrm{Cu}(1)-\mathrm{S}(2) \# 1$ & $243.09(6)$ & & \\
\hline
\end{tabular}

Tabelle 96: Bindungswinkel $\left[^{\circ}\right]$ in $\underline{22}$

\begin{tabular}{|l|r||l|l|}
\hline $\mathrm{P}(2)-\mathrm{N}(1)-\mathrm{P}(1)$ & $127.16(12)$ & $\mathrm{N}(1)-\mathrm{P}(1)-\mathrm{S}(1)$ & $115.49(7)$ \\
$\mathrm{N}(1)-\mathrm{P}(2)-\mathrm{S}(2)$ & $113.65(7)$ & $\mathrm{P}(1)-\mathrm{S}(1)-\mathrm{Cu}(1)$ & $101.35(3)$ \\
$\mathrm{P}(2)-\mathrm{S}(2)-\mathrm{Cu}(1)$ & $99.65(3)$ & $\mathrm{P}(2)-\mathrm{S}(2)-\mathrm{Cu}(1) \# 1$ & $100.44(3)$ \\
$\mathrm{Cu}(1)-\mathrm{S}(2)-\mathrm{Cu}(1) \# 1$ & $80.70(2)$ & $\mathrm{S}(1)-\mathrm{Cu}(1)-\mathrm{Cl}(1)$ & $115.45(2)$ \\
$\mathrm{S}(1)-\mathrm{Cu}(1)-\mathrm{S}(2)$ & $111.52(2)$ & $\mathrm{Cl}(1)-\mathrm{Cu}(1)-\mathrm{S}(2)$ & $105.32(2)$ \\
$\mathrm{S}(1)-\mathrm{Cu}(1)-\mathrm{S}(2) \# 1$ & $110.36(2)$ & $\mathrm{Cl}(1)-\mathrm{Cu}(1)-\mathrm{S}(2) \# 1$ & $113.59(2)$ \\
$\mathrm{S}(2)-\mathrm{Cu}(1)-\mathrm{S}(2) \# 1$ & $99.30(2)$ & & \\
\hline
\end{tabular}

Tabelle 97: Wasserstoffbindungslängen [pm] in $\underline{22}$

\begin{tabular}{|l|l|l|l|l|}
\hline $\mathrm{D}-\mathrm{H} \cdots \mathrm{A}$ & $\mathrm{d}(\mathrm{D}-\mathrm{H})$ & $\mathrm{d}(\mathrm{H} \cdots \mathrm{A})$ & $\mathrm{d}(\mathrm{D} \cdots \mathrm{A})$ & $\angle(D H A)\left[{ }^{\circ}\right]$ \\
\hline $\mathrm{N}(1)-\mathrm{H}(5) \cdots \mathrm{Cl}(1) \# 1$ & $71.6(13)$ & $254.5(17)$ & $322.6(5)$ & $79.9(5)$ \\
\hline
\end{tabular}

$\# 1-\mathrm{x},-\mathrm{y},-\mathrm{z}+1$ 


\subsection{Daten zur Kristallstrukturanalyse von $\left[\mathrm{Cu}_{5}\left(\mathrm{dmpaS}_{2}\right)_{5}\right] 23$}

\begin{tabular}{|ll|}
\hline Summenformel & $\mathrm{C}_{20} \mathrm{H}_{60} \mathrm{Cu}_{5} \mathrm{~N}_{5} \mathrm{P}_{10} \mathrm{~S}_{10}$ \\
Molekulargewicht $\left[\mathrm{g} \cdot \mathrm{mol}^{-1}\right]$ & 1318.73 \\
Temperatur & $133(2) \mathrm{K}$ \\
Wellenlänge & $71.073 \mathrm{pm}$ \\
Kristallsystem & monoklin \\
Raumgruppe & $C 2 / c$ \\
Gitterkonstanten & $\mathrm{a}=22.660(3) \AA, \alpha=90^{\circ}$ \\
& $\mathrm{b}=9.9090(10) \AA, \beta=107.16(2)^{\circ} \circ$ \\
& $\mathrm{c}=24.747(3) \AA, \gamma=90^{\circ}{ }^{\circ}, \AA$ \\
Volumen & $5309.3(11) \AA^{3}$ \\
Formeleinheiten & 4 \\
Röntgenographische Dichte $\left[\mathrm{g} \cdot \mathrm{cm}^{-3}\right]$ & 1.650 \\
Absorptionskoeffizient $\left[\mathrm{mm}^{-1}\right]$ & 2.689 \\
$\mathrm{~F}(000)$ & 2680 \\
Meßbereich $\theta$ & $2.26-25.00^{\circ}$ \\
Indexbereiche & $-29 \leq \mathrm{h} \leq 28$ \\
& $0 \leq \mathrm{k} \leq 12$ \\
Gemessene Reflexe & $0 \leq 1 \leq 32$ \\
Unabhängige Reflexe & 29398 \\
Beobachtete Reflexe $[\mathrm{I}>2 \sigma]$ & 4676 \\
$\mathrm{R}_{\text {int }}$ & 4248 \\
Parameter & 0.0296 \\
R1; wR2[I>2 $\sigma(\mathrm{I})]$ & 229 \\
\hline
\end{tabular}


Tabelle 99: Atomkoordinaten und äquivalente isotrope Auslenkungsparameter $\left[\AA^{2}\right]$ für $\underline{23}$

\begin{tabular}{|r|r|r|r|r|}
\hline Atom & \multicolumn{1}{|c|}{$\mathrm{x}$} & \multicolumn{1}{c|}{$\mathrm{y}$} & \multicolumn{1}{c|}{$\mathrm{z}$} & \multicolumn{1}{c|}{$\mathrm{U}_{e q}$} \\
\hline $\mathrm{C}(1)$ & $-0.1218(2)$ & $1.0059(3)$ & $0.19526(15)$ & $0.0297(7)$ \\
$\mathrm{C}(2)$ & $-0.0504(2)$ & $0.8511(4)$ & $0.14067(13)$ & $0.0311(8)$ \\
$\mathrm{C}(3)$ & $0.10637(14)$ & $0.4580(4)$ & $0.14980(14)$ & $0.0303(8)$ \\
$\mathrm{C}(4)$ & $0.0556(2)$ & $0.2208(4)$ & $0.1827(2)$ & $0.0446(10)$ \\
$\mathrm{C}(5)$ & $-0.0864(2)$ & $0.4646(4)$ & $0.00404(14)$ & $0.0318(8)$ \\
$\mathrm{C}(6)$ & $-0.0475(2)$ & $0.2089(4)$ & $-0.02785(15)$ & $0.0391(9)$ \\
$\mathrm{C}(7 \mathrm{~A})$ & $-0.2701(4)$ & $0.1944(9)$ & $0.1299(4)$ & $0.050(2)$ \\
$\mathrm{C}(7 \mathrm{~B})$ & $-0.2639(3)$ & $0.2027(7)$ & $0.1041(3)$ & $0.0267(14)$ \\
$\mathrm{C}(8 \mathrm{~A})$ & $-0.2962(5)$ & $0.3820(12)$ & $0.2084(4)$ & $0.061(3)$ \\
$\mathrm{C}(8 \mathrm{~B})$ & $-0.2973(4)$ & $0.3124(8)$ & $0.1977(3)$ & $0.034(2)$ \\
$\mathrm{C}(9)$ & $-0.2483(2)$ & $0.5281(4)$ & $0.0206(2)$ & $0.0394(9)$ \\
$\mathrm{C}(10)$ & $-0.3620(2)$ & $0.6448(4)$ & $0.0239(2)$ & $0.0376(9)$ \\
$\mathrm{N}(1)$ & 0.0000 & $0.9561(4)$ & 0.2500 & $0.0227(8)$ \\
$\mathrm{N}(2)$ & $0.01374(12)$ & $0.3213(3)$ & $0.07273(11)$ & $0.0249(6)$ \\
$\mathrm{N}(3)$ & $-0.30386(12)$ & $0.4623(3)$ & $0.10337(13)$ & $0.0336(7)$ \\
$\mathrm{P}(1)$ & $-0.06003(4)$ & $0.88614(8)$ & $0.20890(3)$ & $0.0190(2)$ \\
$\mathrm{P}(2)$ & $0.03585(4)$ & $0.36488(8)$ & $0.13772(3)$ & $0.0199(2)$ \\
$\mathrm{P}(3)$ & $-0.05509(4)$ & $0.30330(8)$ & $0.03181(3)$ & $0.0222(2)$ \\
$\mathrm{P}(4)$ & $-0.26339(4)$ & $0.36128(9)$ & $0.14742(4)$ & $0.0289(2)$ \\
$\mathrm{P}(5)$ & $-0.28893(4)$ & $0.58531(9)$ & $0.06829(4)$ & $0.0260(2)$ \\
$\mathrm{S}(1)$ & $-0.08710(3)$ & $0.71539(7)$ & $0.24102(3)$ & $0.0185(2)$ \\
$\mathrm{S}(2)$ & $-0.02565(4)$ & $0.48338(10)$ & $0.16145(4)$ & $0.0335(2)$ \\
$\mathrm{S}(3)$ & $-0.11781(4)$ & $0.20917(8)$ & $0.06224(3)$ & $0.0280(2)$ \\
$\mathrm{S}(4)$ & $-0.17228(3)$ & $0.40454(8)$ & $0.18636(3)$ & $0.0226(2)$ \\
$\mathrm{S}(5)$ & $-0.24395(5)$ & $0.74394(9)$ & $0.11328(4)$ & $0.0432(3)$ \\
$\mathrm{Cu}(1)$ & 0.0000 & $0.58237(5)$ & 0.2500 & $0.02133(13)$ \\
$\mathrm{Cu}(2)$ & $-0.11204(2)$ & $0.35488(4)$ & $0.13221(2)$ & $0.02431(11)$ \\
$\mathrm{Cu}(3)$ & $-0.16945(2)$ & $0.63156(4)$ & $0.17532(2)$ & $0.02572(11)$ \\
\hline & & & & \\
\hline
\end{tabular}

Tabelle 100: Bindungslängen [pm] in $\underline{23}$

\begin{tabular}{|l|r||l|r|}
\hline $\mathrm{N}(1)-\mathrm{P}(1)$ & $159.6(2)$ & $\mathrm{N}(1)-\mathrm{P}(1) \# 1$ & $159.6(2)$ \\
$\mathrm{N}(2)-\mathrm{P}(2)$ & $159.6(3)$ & $\mathrm{N}(2)-\mathrm{P}(3)$ & $160.0(3)$ \\
$\mathrm{N}(3)-\mathrm{P}(4)$ & $156.3(3)$ & $\mathrm{N}(3)-\mathrm{P}(5)$ & $158.9(3)$ \\
$\mathrm{P}(1)-\mathrm{S}(1)$ & $203.93(11)$ & $\mathrm{P}(2)-\mathrm{S}(2)$ & $203.66(12)$ \\
$\mathrm{P}(3)-\mathrm{S}(3)$ & $202.20(12)$ & $\mathrm{P}(5)-\mathrm{S}(5)$ & $201.98(13)$ \\
$\mathrm{S}(1)-\mathrm{Cu}(3)$ & $224.18(9)$ & $\mathrm{S}(2)-\mathrm{Cu}(2)$ & $226.75(10)$ \\
$\mathrm{S}(3)-\mathrm{Cu}(2)$ & $222.86(9)$ & $\mathrm{S}(4)-\mathrm{Cu}(2)$ & $223.17(9)$ \\
\hline
\end{tabular}


Tabelle 100: Fortsetzung

\begin{tabular}{|l|r||l|l|}
\hline $\mathrm{S}(5)-\mathrm{Cu}(3)$ & $221.76(11)$ & $\mathrm{Cu}(1)-\mathrm{S}(2) \# 1$ & $231.31(9)$ \\
$\mathrm{Cu}(1)-\mathrm{S}(1) \# 1$ & $232.88(8)$ & & \\
\hline
\end{tabular}

Tabelle 101: Bindungswinkel $\left[{ }^{\circ}\right]$ in $\underline{23}$

\begin{tabular}{|l|r||l|r|}
\hline $\mathrm{P}(1)-\mathrm{N}(1)-\mathrm{P}(1) \# 1$ & $128.6(2)$ & $\mathrm{P}(2)-\mathrm{N}(2)-\mathrm{P}(3)$ & $128.7(2)$ \\
$\mathrm{P}(4)-\mathrm{N}(3)-\mathrm{P}(5)$ & $134.2(2)$ & $\mathrm{N}(1)-\mathrm{P}(1)-\mathrm{S}(1)$ & $114.17(10)$ \\
$\mathrm{N}(2)-\mathrm{P}(2)-\mathrm{S}(2)$ & $114.10(11)$ & $\mathrm{N}(2)-\mathrm{P}(3)-\mathrm{S}(3)$ & $118.49(11)$ \\
$\mathrm{N}(3)-\mathrm{P}(4)-\mathrm{S}(4)$ & $119.76(11)$ & $\mathrm{N}(3)-\mathrm{P}(5)-\mathrm{S}(5)$ & $116.72(12)$ \\
$\mathrm{P}(1)-\mathrm{S}(1)-\mathrm{Cu}(3)$ & $107.85(4)$ & $\mathrm{P}(1)-\mathrm{S}(1)-\mathrm{Cu}(1)$ & $99.37(4)$ \\
$\mathrm{Cu}(3)-\mathrm{S}(1)-\mathrm{Cu}(1)$ & $111.11(4)$ & $\mathrm{P}(2)-\mathrm{S}(2)-\mathrm{Cu}(2)$ & $100.61(4)$ \\
$\mathrm{P}(2)-\mathrm{S}(2)-\mathrm{Cu}(1)$ & $121.04(5)$ & $\mathrm{Cu}(2)-\mathrm{S}(2)-\mathrm{Cu}(1)$ & $119.49(4)$ \\
$\mathrm{P}(3)-\mathrm{S}(3)-\mathrm{Cu}(2)$ & $95.97(4)$ & $\mathrm{P}(4)-\mathrm{S}(4)-\mathrm{Cu}(2)$ & $112.41(5)$ \\
$\mathrm{P}(4)-\mathrm{S}(4)-\mathrm{Cu}(3)$ & $102.18(4)$ & $\mathrm{Cu}(2)-\mathrm{S}(4)-\mathrm{Cu}(3)$ & $95.87(3)$ \\
$\mathrm{P}(5)-\mathrm{S}(5)-\mathrm{Cu}(3)$ & $98.51(5)$ & $\mathrm{S}(2) \# 1-\mathrm{Cu}(1)-\mathrm{S}(2)$ & $129.82(6)$ \\
$\mathrm{S}(2) \# 1-\mathrm{Cu}(1)-\mathrm{S}(1)$ & $107.42(3)$ & $\mathrm{S}(2)-\mathrm{Cu}(1)-\mathrm{S}(1)$ & $100.41(3)$ \\
$\mathrm{S}(2) \# 1-\mathrm{Cu}(1)-\mathrm{S}(1) \# 1$ & $100.41(3)$ & $\mathrm{S}(2)-\mathrm{Cu}(1)-\mathrm{S}(1) \# 1$ & $107.42(3)$ \\
$\mathrm{S}(1)-\mathrm{Cu}(1)-\mathrm{S}(1) \# 1$ & $111.05(4)$ & $\mathrm{S}(3)-\mathrm{Cu}(2)-\mathrm{S}(4)$ & $134.70(3)$ \\
$\mathrm{S}(3)-\mathrm{Cu}(2)-\mathrm{S}(2)$ & $117.32(4)$ & $\mathrm{S}(4)-\mathrm{Cu}(2)-\mathrm{S}(2)$ & $107.94(3)$ \\
$\mathrm{S}(5)-\mathrm{Cu}(3)-\mathrm{S}(1)$ & $128.08(4)$ & $\mathrm{S}(5)-\mathrm{Cu}(3)-\mathrm{S}(4)$ & $122.57(4)$ \\
$\mathrm{S}(1)-\mathrm{Cu}(3)-\mathrm{S}(4)$ & $109.09(3)$ & & \\
\hline
\end{tabular}

\#1 1-x, y, -z+1/2 


\subsection{Daten zur Kristallstrukturanalyse von $\left[\mathrm{Cu}_{8}\left(\mathrm{dmpaS}_{2}\right)_{6} \mathrm{Cl}_{2}\right] \underline{24}$}

\begin{tabular}{|ll|}
\hline Summenformel & $\mathrm{C}_{24} \mathrm{H}_{72} \mathrm{Cl}_{2} \mathrm{Cu}_{8} \mathrm{~N}_{6} \mathrm{P}_{12} \mathrm{~S}_{12}$ \\
Molekulargewicht $\left[\mathrm{g} \cdot \mathrm{mol}^{-1}\right]$ & 1780.46 \\
Temperatur & $133(2) \mathrm{K}$ \\
Wellenlänge & $71.073 \mathrm{pm}$ \\
Kristallsystem & monoklin \\
Raumgruppe & $P 2_{1} / n$ \\
Gitterkonstanten & $\mathrm{a}=11.7523(10) \AA, \alpha=90^{\circ}$ \\
& $\mathrm{b}=22.664(2) \AA, \beta=90.881(10)^{\circ} \circ$ \\
& $\mathrm{c}=12.2635(10) \AA, \gamma=90^{\circ}{ }^{\circ}$ \\
Volumen & $3266.0(5) \AA^{3}$ \\
Formeleinheiten & 2 \\
Röntgenographische Dichte $\left[\mathrm{g} \cdot \mathrm{cm}^{-3}\right]$ & 1.810 \\
Absorptionskoeffizient $\left[\mathrm{mm}^{-1}\right]$ & 3.340 \\
$\mathrm{~F}(000)$ & 1792 \\
Meßbereich $\theta$ & $2.38-22.31^{\circ}$ \\
Indexbereiche & $-9 \leq \mathrm{h} \leq 12$ \\
& $-24 \leq \mathrm{k} \leq 24$ \\
Gemessene Reflexe & $-13 \leq \mathrm{l} \leq 13$ \\
Unabhängige Reflexe & 25290 \\
Beobachtete Reflexe $[\mathrm{I}>2 \sigma]$ & 4144 \\
$\mathrm{R}_{\text {int }}$ & 2869 \\
Parameter & 0.1375 \\
R1; wR2[I>2 $\sigma(\mathrm{I})]$ & 301 \\
\hline
\end{tabular}


Tabelle 103: Atomkoordinaten und äquivalente isotrope Auslenkungsparameter $\left[\AA^{2}\right]$ für $\underline{24}$

\begin{tabular}{|r|r|r|r|r|}
\hline $\mathrm{Atom}$ & $\mathrm{x}$ & $\mathrm{y}$ & $\mathrm{z}$ & \multicolumn{1}{c|}{$\mathrm{U}_{e q}$} \\
\hline $\mathrm{Cu}(1)$ & $0.85889(8)$ & $-0.08273(4)$ & $0.59499(7)$ & $0.0238(3)$ \\
$\mathrm{Cu}(2)$ & $1.01255(8)$ & $0.07963(4)$ & $0.68436(7)$ & $0.0240(3)$ \\
$\mathrm{Cu}(3)$ & $0.83141(8)$ & $0.07348(4)$ & $0.47501(7)$ & $0.0250(3)$ \\
$\mathrm{Cu}(4)$ & $0.71494(8)$ & $-0.00727(4)$ & $0.30814(7)$ & $0.0253(3)$ \\
$\mathrm{S}(1)$ & $0.87132(16)$ & $0.01943(8)$ & $0.62671(14)$ & $0.0199(4)$ \\
$\mathrm{S}(2)$ & $0.97335(17)$ & $0.14409(9)$ & $0.81649(17)$ & $0.0330(5)$ \\
$\mathrm{S}(3)$ & $0.65657(17)$ & $0.07324(8)$ & $0.39906(16)$ & $0.0258(5)$ \\
$\mathrm{S}(4)$ & $0.96513(16)$ & $0.13137(8)$ & $0.40375(15)$ & $0.0249(5)$ \\
$\mathrm{S}(5)$ & $0.6589(2)$ & $-0.03048(9)$ & $0.13955(17)$ & $0.0352(6)$ \\
$\mathrm{S}(6)$ & $0.82175(15)$ & $-0.07333(8)$ & $0.40368(15)$ & $0.0202(5)$ \\
$\mathrm{Cl}(1)$ & $0.73608(16)$ & $-0.13503(8)$ & $0.69883(15)$ & $0.0260(5)$ \\
$\mathrm{P}(1)$ & $0.74085(17)$ & $0.04555(8)$ & $0.72855(16)$ & $0.0218(5)$ \\
$\mathrm{P}(2)$ & $0.81323(18)$ & $0.16587(9)$ & $0.77210(17)$ & $0.0260(5)$ \\
$\mathrm{P}(3)$ & $0.66664(17)$ & $0.14293(8)$ & $0.29235(16)$ & $0.0228(5)$ \\
$\mathrm{P}(4)$ & $0.91105(17)$ & $0.13845(9)$ & $0.24547(16)$ & $0.0231(5)$ \\
$\mathrm{P}(5)$ & $0.66953(18)$ & $-0.11889(9)$ & $0.12675(16)$ & $0.0271(5)$ \\
$\mathrm{P}(6)$ & $0.73811(17)$ & $-0.14880(8)$ & $0.35134(15)$ & $0.0215(5)$ \\
$\mathrm{N}(1)$ & $0.7257(5)$ & $0.1150(3)$ & $0.7385(5)$ & $0.0262(16)$ \\
$\mathrm{N}(2)$ & $0.7802(5)$ & $0.1517(3)$ & $0.2257(5)$ & $0.0275(16)$ \\
$\mathrm{N}(3)$ & $0.7232(5)$ & $-0.1569(2)$ & $0.2251(5)$ & $0.0243(15)$ \\
$\mathrm{C}(1)$ & $0.7683(7)$ & $0.0109(3)$ & $0.8558(6)$ & $0.034(2)$ \\
$\mathrm{C}(2)$ & $0.6125(6)$ & $0.0152(3)$ & $0.6765(6)$ & $0.0280(19)$ \\
$\mathrm{C}(3)$ & $0.7550(7)$ & $0.2061(3)$ & $0.8827(6)$ & $0.034(2)$ \\
$\mathrm{C}(4)$ & $0.8167(7)$ & $0.2174(3)$ & $0.6612(6)$ & $0.041(2)$ \\
$\mathrm{C}(5)$ & $0.6351(7)$ & $0.2067(3)$ & $0.3734(6)$ & $0.039(2)$ \\
$\mathrm{C}(6)$ & $0.5529(6)$ & $0.1347(3)$ & $0.1942(6)$ & $0.033(2)$ \\
$\mathrm{C}(7)$ & $0.9914(7)$ & $0.1966(3)$ & $0.1868(6)$ & $0.036(2)$ \\
$\mathrm{C}(8)$ & $0.9503(7)$ & $0.0742(3)$ & $0.1723(6)$ & $0.038(2)$ \\
$\mathrm{C}(10)$ & $0.5276(7)$ & $-0.1461(4)$ & $0.1004(7)$ & $0.045(2)$ \\
\hline
\end{tabular}

Tabelle 104: Bindungslängen [pm] in $\underline{24}$

\begin{tabular}{|l|l||l|l|}
\hline $\mathrm{Cu}(1)-\mathrm{Cl}(1)$ & $227.3(2)$ & $\mathrm{Cu}(1)-\mathrm{S}(4) \# 1$ & $234.3(2)$ \\
$\mathrm{Cu}(1)-\mathrm{S}(1)$ & $235.2(2)$ & $\mathrm{Cu}(1)-\mathrm{S}(6)$ & $238.9(2)$ \\
$\mathrm{Cu}(2)-\mathrm{S}(2)$ & $223.5(2)$ & $\mathrm{Cu}(2)-\mathrm{S}(6) \# 1$ & $224.6(2)$ \\
\hline
\end{tabular}


Tabelle 104: Fortsetzung

\begin{tabular}{|l|r||l|l|}
\hline $\mathrm{Cu}(2)-\mathrm{S}(1)$ & $225.4(2)$ & $\mathrm{Cu}(3)-\mathrm{S}(4)$ & $223.5(2)$ \\
$\mathrm{Cu}(3)-\mathrm{S}(3)$ & $224.3(2)$ & $\mathrm{Cu}(3)-\mathrm{S}(1)$ & $227.0(2)$ \\
$\mathrm{Cu}(3)-\mathrm{Cu}(4)$ & $305.36(13)$ & $\mathrm{Cu}(4)-\mathrm{S}(5)$ & $222.3(2)$ \\
$\mathrm{Cu}(4)-\mathrm{S}(3)$ & $225.1(2)$ & $\mathrm{Cu}(4)-\mathrm{S}(6)$ & $226.8(2)$ \\
$\mathrm{S}(1)-\mathrm{P}(1)$ & $207.8(3)$ & $\mathrm{S}(2)-\mathrm{P}(2)$ & $201.2(3)$ \\
$\mathrm{S}(3)-\mathrm{P}(3)$ & $205.6(3)$ & $\mathrm{S}(4)-\mathrm{P}(4)$ & $204.0(3)$ \\
$\mathrm{S}(4)-\mathrm{Cu}(1) \# 1$ & $234.3(2)$ & $\mathrm{S}(5)-\mathrm{P}(5)$ & $201.4(3)$ \\
$\mathrm{S}(6)-\mathrm{P}(6)$ & $207.0(3)$ & $\mathrm{S}(6)-\mathrm{Cu}(2) \# 1$ & $224.6(2)$ \\
$\mathrm{P}(1)-\mathrm{N}(1)$ & $158.9(6)$ & $\mathrm{P}(1)-\mathrm{C}(2)$ & $176.8(7)$ \\
$\mathrm{P}(1)-\mathrm{C}(1)$ & $177.2(7)$ & $\mathrm{P}(2)-\mathrm{N}(1)$ & $159.4(6)$ \\
$\mathrm{P}(2)-\mathrm{C}(3)$ & $178.0(7)$ & $\mathrm{P}(2)-\mathrm{C}(4)$ & $179.3(8)$ \\
$\mathrm{P}(3)-\mathrm{N}(2)$ & $158.8(6)$ & $\mathrm{P}(3)-\mathrm{C}(6)$ & $179.5(7)$ \\
$\mathrm{P}(3)-\mathrm{C}(5)$ & $179.6(8)$ & $\mathrm{P}(4)-\mathrm{N}(2)$ & $158.2(6)$ \\
$\mathrm{P}(4)-\mathrm{C}(8)$ & $177.6(8)$ & $\mathrm{P}(4)-\mathrm{C}(7)$ & $177.9(8)$ \\
$\mathrm{P}(5)-\mathrm{N}(3)$ & $160.3(6)$ & $\mathrm{P}(5)-\mathrm{C}(10)$ & $179.9(8)$ \\
$\mathrm{P}(5)-\mathrm{C}(9)$ & $180.3(8)$ & $\mathrm{P}(6)-\mathrm{N}(3)$ & $156.7(6)$ \\
$\mathrm{P}(6)-\mathrm{C}(11)$ & $177.4(7)$ & $\mathrm{P}(6)-\mathrm{C}(12)$ & $179.6(7)$ \\
\hline
\end{tabular}

Tabelle 105: Bindungswinkel $\left[^{\circ}\right]$ in $\underline{24}$

\begin{tabular}{|l|r||l|r|}
\hline $\mathrm{Cl}(1)-\mathrm{Cu}(1)-\mathrm{S}(4) \# 1$ & $108.60(7)$ & $\mathrm{Cl}(1)-\mathrm{Cu}(1)-\mathrm{S}(1)$ & $117.33(8)$ \\
$\mathrm{S}(4) \# 1-\mathrm{Cu}(1)-\mathrm{S}(1)$ & $114.17(8)$ & $\mathrm{Cl}(1)-\mathrm{Cu}(1)-\mathrm{S}(6)$ & $119.25(8)$ \\
$\mathrm{S}(4) \# 1-\mathrm{Cu}(1)-\mathrm{S}(6)$ & $101.32(7)$ & $\mathrm{S}(1)-\mathrm{Cu}(1)-\mathrm{S}(6)$ & $94.85(7)$ \\
$\mathrm{S}(2)-\mathrm{Cu}(2)-\mathrm{S}(6) \# 1$ & $125.45(8)$ & $\mathrm{S}(2)-\mathrm{Cu}(2)-\mathrm{S}(1)$ & $117.65(8)$ \\
$\mathrm{S}(6) \# 1-\mathrm{Cu}(2)-\mathrm{S}(1)$ & $116.78(8)$ & $\mathrm{S}(4)-\mathrm{Cu}(3)-\mathrm{S}(3)$ & $118.95(8)$ \\
$\mathrm{S}(4)-\mathrm{Cu}(3)-\mathrm{S}(1)$ & $119.96(8)$ & $\mathrm{S}(3)-\mathrm{Cu}(3)-\mathrm{S}(1)$ & $121.02(8)$ \\
$\mathrm{S}(4)-\mathrm{Cu}(3)-\mathrm{Cu}(4)$ & $113.60(6)$ & $\mathrm{S}(3)-\mathrm{Cu}(3)-\mathrm{Cu}(4)$ & $47.30(6)$ \\
$\mathrm{S}(1)-\mathrm{Cu}(3)-\mathrm{Cu}(4)$ & $108.10(6)$ & $\mathrm{S}(5)-\mathrm{Cu}(4)-\mathrm{S}(3)$ & $124.36(8)$ \\
$\mathrm{S}(5)-\mathrm{Cu}(4)-\mathrm{S}(6)$ & $118.54(8)$ & $\mathrm{S}(3)-\mathrm{Cu}(4)-\mathrm{S}(6)$ & $116.72(8)$ \\
$\mathrm{S}(5)-\mathrm{Cu}(4)-\mathrm{Cu}(3)$ & $152.66(7)$ & $\mathrm{S}(3)-\mathrm{Cu}(4)-\mathrm{Cu}(3)$ & $47.09(6)$ \\
$\mathrm{S}(6)-\mathrm{Cu}(4)-\mathrm{Cu}(3)$ & $79.08(6)$ & $\mathrm{P}(1)-\mathrm{S}(1)-\mathrm{Cu}(2)$ & $100.70(9)$ \\
$\mathrm{P}(1)-\mathrm{S}(1)-\mathrm{Cu}(3)$ & $101.17(9)$ & $\mathrm{Cu}(2)-\mathrm{S}(1)-\mathrm{Cu}(3)$ & $94.15(8)$ \\
$\mathrm{P}(1)-\mathrm{S}(1)-\mathrm{Cu}(1)$ & $109.58(10)$ & $\mathrm{Cu}(2)-\mathrm{S}(1)-\mathrm{Cu}(1)$ & $133.74(9)$ \\
$\mathrm{Cu}(3)-\mathrm{S}(1)-\mathrm{Cu}(1)$ & $112.59(8)$ & $\mathrm{P}(2)-\mathrm{S}(2)-\mathrm{Cu}(2)$ & $99.58(10)$ \\
$\mathrm{P}(3)-\mathrm{S}(3)-\mathrm{Cu}(3)$ & $101.60(10)$ & $\mathrm{P}(3)-\mathrm{S}(3)-\mathrm{Cu}(4)$ & $106.64(10)$ \\
$\mathrm{Cu}(3)-\mathrm{S}(3)-\mathrm{Cu}(4)$ & $85.61(8)$ & $\mathrm{P}(4)-\mathrm{S}(4)-\mathrm{Cu}(3)$ & $101.98(10)$ \\
$\mathrm{P}(4)-\mathrm{S}(4)-\mathrm{Cu}(1) \# 1$ & $107.78(10)$ & $\mathrm{Cu}(3)-\mathrm{S}(4)-\mathrm{Cu}(1) \# 1$ & $110.30(8)$ \\
$\mathrm{P}(5)-\mathrm{S}(5)-\mathrm{Cu}(4)$ & $106.87(11)$ & $\mathrm{P}(6)-\mathrm{S}(6)-\mathrm{Cu}(2) \# 1$ & $102.10(9)$ \\
$\mathrm{P}(6)-\mathrm{S}(6)-\mathrm{Cu}(4)$ & $97.40(9)$ & $\mathrm{Cu}(2) \# 1-\mathrm{S}(6)-\mathrm{Cu}(4)$ & $105.71(8)$ \\
$\mathrm{P}(6)-\mathrm{S}(6)-\mathrm{Cu}(1)$ & $108.03(9)$ & $\mathrm{Cu}(2) \# 1-\mathrm{S}(6)-\mathrm{Cu}(1)$ & $108.65(8)$ \\
\hline
\end{tabular}


Tabelle 105: Fortsetzung

\begin{tabular}{|l|r||l|l|}
\hline $\mathrm{Cu}(4)-\mathrm{S}(6)-\mathrm{Cu}(1)$ & $131.10(9)$ & $\mathrm{N}(1)-\mathrm{P}(1)-\mathrm{C}(2)$ & $108.5(3)$ \\
$\mathrm{N}(1)-\mathrm{P}(1)-\mathrm{C}(1)$ & $113.0(3)$ & $\mathrm{N}(1)-\mathrm{P}(1)-\mathrm{S}(1)$ & $114.4(2)$ \\
$\mathrm{C}(2)-\mathrm{P}(1)-\mathrm{S}(1)$ & $107.8(3)$ & $\mathrm{C}(1)-\mathrm{P}(1)-\mathrm{S}(1)$ & $106.1(3)$ \\
$\mathrm{N}(1)-\mathrm{P}(2)-\mathrm{C}(3)$ & $108.2(3)$ & $\mathrm{N}(1)-\mathrm{P}(2)-\mathrm{C}(4)$ & $107.3(4)$ \\
$\mathrm{N}(1)-\mathrm{P}(2)-\mathrm{S}(2)$ & $119.3(2)$ & $\mathrm{C}(3)-\mathrm{P}(2)-\mathrm{S}(2)$ & $106.8(3)$ \\
$\mathrm{C}(4)-\mathrm{P}(2)-\mathrm{S}(2)$ & $109.4(3)$ & $\mathrm{N}(2)-\mathrm{P}(3)-\mathrm{C}(6)$ & $106.9(3)$ \\
$\mathrm{N}(2)-\mathrm{P}(3)-\mathrm{C}(5)$ & $111.5(4)$ & $\mathrm{N}(2)-\mathrm{P}(3)-\mathrm{S}(3)$ & $118.7(2)$ \\
$\mathrm{C}(6)-\mathrm{P}(3)-\mathrm{S}(3)$ & $107.3(3)$ & $\mathrm{C}(5)-\mathrm{P}(3)-\mathrm{S}(3)$ & $104.6(3)$ \\
$\mathrm{N}(2)-\mathrm{P}(4)-\mathrm{C}(8)$ & $109.7(4)$ & $\mathrm{N}(2)-\mathrm{P}(4)-\mathrm{C}(7)$ & $108.6(3)$ \\
$\mathrm{N}(2)-\mathrm{P}(4)-\mathrm{S}(4)$ & $116.7(2)$ & $\mathrm{C}(8)-\mathrm{P}(4)-\mathrm{S}(4)$ & $109.7(3)$ \\
$\mathrm{C}(7)-\mathrm{P}(4)-\mathrm{S}(4)$ & $106.5(3)$ & $\mathrm{N}(3)-\mathrm{P}(5)-\mathrm{C}(10)$ & $106.5(4)$ \\
$\mathrm{N}(3)-\mathrm{P}(5)-\mathrm{C}(9)$ & $107.6(4)$ & $\mathrm{N}(3)-\mathrm{P}(5)-\mathrm{S}(5)$ & $120.0(2)$ \\
$\mathrm{C}(10)-\mathrm{P}(5)-\mathrm{S}(5)$ & $109.4(3)$ & $\mathrm{C}(9)-\mathrm{P}(5)-\mathrm{S}(5)$ & $107.2(3)$ \\
$\mathrm{N}(3)-\mathrm{P}(6)-\mathrm{C}(11)$ & $107.3(3)$ & $\mathrm{N}(3)-\mathrm{P}(6)-\mathrm{C}(12)$ & $114.5(3)$ \\
$\mathrm{N}(3)-\mathrm{P}(6)-\mathrm{S}(6)$ & $116.7(2)$ & $\mathrm{C}(11)-\mathrm{P}(6)-\mathrm{S}(6)$ & $106.3(3)$ \\
$\mathrm{C}(12)-\mathrm{P}(6)-\mathrm{S}(6)$ & $104.6(3)$ & $\mathrm{P}(1)-\mathrm{N}(1)-\mathrm{P}(2)$ & $131.6(4)$ \\
$\mathrm{P}(4)-\mathrm{N}(2)-\mathrm{P}(3)$ & $136.0(4)$ & $\mathrm{P}(6)-\mathrm{N}(3)-\mathrm{P}(5)$ & $135.8(4)$ \\
\hline
\end{tabular}

$\# 1$-x+2, -y, -z+1 


\subsection{Daten zur Kristallstrukturanalyse von $\left[\mathrm{Cu}_{10}\left(\mathrm{dmpaS}_{2}\right)_{6} \mathrm{Cl}_{4}\right] \underline{25}^{*}$}

\begin{tabular}{|ll|}
\hline Summenformel & $\mathrm{C}_{28.5} \mathrm{H}_{81} \mathrm{Cl}_{13} \mathrm{Cu}_{10} \mathrm{~N}_{6} \mathrm{P}_{12} \mathrm{~S}_{12}$ \\
Molekulargewicht $\left[\mathrm{g} \cdot \mathrm{mol}^{-1}\right]$ & 2360.60 \\
Temperatur & $133(2) \mathrm{K}$ \\
Wellenlänge & $154.178 \mathrm{pm}$ \\
Kristallsystem & triklin \\
Raumgruppe & $P \overline{1}$ \\
Gitterkonstanten & $\mathrm{a}=13.854(4) \AA, \alpha=91.741(18)^{\circ}$ \\
& $\mathrm{b}=16.625(5) \AA, \beta=97.180(18)^{\circ}$ \\
& $\mathrm{c}=20.070(7) \AA, \gamma=112.331(15)^{\circ}$ \\
& $\circ$ \\
Volumen & $4227(2) \AA^{3}$ \\
Formeleinheiten & 2 \\
Röntgenographische Dichte $\left[\mathrm{g} \cdot \mathrm{cm}^{-3}\right]$ & 1.855 \\
Absorptionskoeffizient $\left[\mathrm{mm}^{-1}\right]$ & 11.624 \\
$\mathrm{~F}(000)$ & 2354 \\
Meßbereich $\theta$ & $2.88-56.61^{\circ}$ \\
Indexbereiche & $-14 \leq \mathrm{h} \leq 14$ \\
& $-17 \leq \mathrm{k} \leq 17$ \\
Gemessene Reflexe & $-21 \leq \mathrm{l} \leq 21$ \\
Unabhängige Reflexe & 51728 \\
Beobachtete Reflexe $[\mathrm{I}>2 \sigma]$ & 10648 \\
$\mathrm{R}_{\text {int }}$ & 9112 \\
Parameter & 0.0476 \\
R1; wR2[I>2 $\sigma(\mathrm{I})]$ & 758 \\
\hline
\end{tabular}

\footnotetext{
* Das Beugungsexperiment wurde an der rotating anode mit $\mathrm{Cu}-\mathrm{K}_{\alpha}$-Strahlung und einem Multi-Wire-Proportional-Counter durchgeführt.
} 
Tabelle 107: Atomkoordinaten und äquivalente isotrope Auslenkungsparameter $\left[\AA^{2}\right]$ für $\underline{25}$

\begin{tabular}{|c|c|c|c|c|}
\hline Atom & $\mathrm{x}$ & $\mathrm{y}$ & $\mathrm{z}$ & $\mathrm{U}_{e q}$ \\
\hline $\mathrm{Cu}(1)$ & $1.22307(8)$ & $0.82845(7)$ & $0.39192(5)$ & $0.0164(3)$ \\
\hline $\mathrm{Cu}(2)$ & $1.06285(8)$ & $0.92774(7)$ & $0.26182(5)$ & $0.0170(3)$ \\
\hline $\mathrm{Cu}(3)$ & $1.16250(9)$ & $0.82337(7)$ & $0.10589(6)$ & $0.0188(3)$ \\
\hline $\mathrm{Cu}(4)$ & $1.32552(9)$ & $0.74265(7)$ & $0.22394(6)$ & $0.0198(3)$ \\
\hline $\mathrm{Cu}(5)$ & $1.13396(9)$ & $0.61342(7)$ & $0.25467(6)$ & $0.0207(3)$ \\
\hline $\mathrm{Cu}(6)$ & $0.96631(10)$ & $0.70145(7)$ & $0.13779(6)$ & $0.0277(3)$ \\
\hline $\mathrm{Cu}(7)$ & $1.33761(8)$ & $0.93869(7)$ & $0.23260(5)$ & $0.0147(3)$ \\
\hline $\mathrm{Cu}(8)$ & $1.41228(8)$ & $0.88992(7)$ & $0.12745(5)$ & $0.0155(3)$ \\
\hline $\mathrm{Cu}(9)$ & $0.94000(8)$ & $0.66992(7)$ & $0.29347(6)$ & $0.0183(3)$ \\
\hline $\mathrm{Cu}(10)$ & $0.88381(9)$ & $0.52774(7)$ & $0.21206(6)$ & $0.0235(3)$ \\
\hline $\mathrm{P}(1)$ & $1.09457(15)$ & $0.96588(12)$ & $0.43001(9)$ & $0.0147(4)$ \\
\hline $\mathrm{P}(2)$ & $1.03299(16)$ & $1.08454(12)$ & $0.34813(10)$ & $0.0170(4)$ \\
\hline $\mathrm{P}(3)$ & $1.21153(15)$ & $1.03850(12)$ & $0.12799(9)$ & $0.0142(4)$ \\
\hline $\mathrm{P}(4)$ & $1.28366(14)$ & $0.97912(12)$ & $0.01310(9)$ & $0.0140(4)$ \\
\hline $\mathrm{P}(5)$ & $1.49600(14)$ & $0.89088(12)$ & $0.35092(9)$ & $0.0146(4)$ \\
\hline $\mathrm{P}(6)$ & $1.60067(14)$ & $1.01644(12)$ & $0.25851(9)$ & $0.0137(4)$ \\
\hline $\mathrm{P}(7)$ & $1.17743(15)$ & $0.62708(12)$ & $0.07650(10)$ & $0.0153(4)$ \\
\hline $\mathrm{P}(8)$ & $1.40713(16)$ & $0.68965(13)$ & $0.08705(11)$ & $0.0213(5)$ \\
\hline $\mathrm{P}(9)$ & $1.07873(16)$ & $0.59716(12)$ & $0.41178(10)$ & $0.0180(4)$ \\
\hline $\mathrm{P}(10)$ & $0.99664(16)$ & $0.43266(12)$ & $0.32646(10)$ & $0.0194(5)$ \\
\hline $\mathrm{P}(11)$ & $0.78776(15)$ & $0.75848(12)$ & $0.21255(11)$ & $0.0190(4)$ \\
\hline $\mathrm{P}(12)$ & $0.67509(16)$ & $0.57565(14)$ & $0.23554(12)$ & $0.0264(5)$ \\
\hline $\mathrm{Cl}(1)$ & $1.27265(15)$ & $0.82786(12)$ & $0.50433(9)$ & $0.0222(4)$ \\
\hline $\mathrm{Cl}(2)$ & $0.98439(14)$ & $0.78454(12)$ & $0.04263(9)$ & $0.0214(4)$ \\
\hline $\mathrm{Cl}(3)$ & $1.30537(15)$ & $0.60646(12)$ & $0.27596(10)$ & $0.0240(4)$ \\
\hline $\mathrm{Cl}(4)$ & $0.85764(15)$ & $0.55548(12)$ & $0.10288(10)$ & $0.0228(4)$ \\
\hline $\mathrm{S}(1)$ & $1.17834(14)$ & $0.94499(11)$ & $0.35994(9)$ & $0.0152(4)$ \\
\hline $\mathrm{S}(2)$ & $0.97920(15)$ & $1.02141(12)$ & $0.25546(10)$ & $0.0178(4)$ \\
\hline $\mathrm{S}(3)$ & $1.17937(13)$ & $0.93238(11)$ & $0.18497(9)$ & $0.0123(4)$ \\
\hline $\mathrm{S}(4)$ & $1.29022(14)$ & $0.86162(11)$ & $0.03491(9)$ & $0.0148(4)$ \\
\hline $\mathrm{S}(5)$ & $1.33899(13)$ & $0.84412(11)$ & $0.30943(9)$ & $0.0127(4)$ \\
\hline $\mathrm{S}(6)$ & $1.48120(14)$ & $1.02183(11)$ & $0.18901(9)$ & $0.0145(4)$ \\
\hline $\mathrm{S}(7)$ & $1.15398(14)$ & $0.69584(11)$ & $0.15693(9)$ & $0.0133(4)$ \\
\hline $\mathrm{S}(8)$ & $1.46825(14)$ & $0.78578(12)$ & $0.16464(10)$ & $0.0175(4)$ \\
\hline $\mathrm{S}(9)$ & $1.10227(14)$ & $0.68934(11)$ & $0.34260(9)$ & $0.0137(4)$ \\
\hline $\mathrm{S}(10)$ & $1.00712(15)$ & $0.47141(11)$ & $0.23133(10)$ & $0.0198(4)$ \\
\hline $\mathrm{S}(11)$ & $0.94557(14)$ & $0.77810(11)$ & $0.22883(9)$ & $0.0146(4)$ \\
\hline $\mathrm{S}(12)$ & $0.79334(15)$ & $0.54960(12)$ & $0.29277(10)$ & $0.0227(4)$ \\
\hline
\end{tabular}


Tabelle 107: Fortsetzung

\begin{tabular}{|c|c|c|c|c|}
\hline $\mathrm{N}(1)$ & $1.0324(5)$ & $1.0276(4)$ & $0.4108(3)$ & $0.0224(15)$ \\
\hline $\mathrm{N}(2)$ & $1.2878(5)$ & $1.0440(4)$ & $0.0741(3)$ & $0.0167(14)$ \\
\hline $\mathrm{N}(3)$ & $1.5782(5)$ & $0.9336(4)$ & $0.3012(3)$ & $0.0164(14)$ \\
\hline $\mathrm{N}(4)$ & $1.2896(5)$ & $0.6682(4)$ & $0.0526(3)$ & $0.0204(15)$ \\
\hline $\mathrm{N}(5)$ & $1.0001(5)$ & $0.5009(4)$ & $0.3844(3)$ & $0.0216(15)$ \\
\hline $\mathrm{N}(6)$ & $0.7076(5)$ & $0.6605(4)$ & $0.1944(4)$ & $0.0261(16)$ \\
\hline $\mathrm{C}(1)$ & $1.1845(6)$ & $1.0114(5)$ & $0.5064(4)$ & $0.0233(18)$ \\
\hline $\mathrm{C}(2)$ & $1.0014(6)$ & $0.8624(5)$ & $0.4484(4)$ & $0.0220(18)$ \\
\hline $\mathrm{C}(3)$ & $1.1615(7)$ & $1.1686(5)$ & $0.3484(5)$ & $0.029(2)$ \\
\hline $\mathrm{C}(4)$ & $0.9487(7)$ & $1.1418(6)$ & $0.3625(4)$ & $0.028(2)$ \\
\hline $\mathrm{C}(5)$ & $1.2735(7)$ & $1.1345(5)$ & $0.1853(4)$ & $0.0248(19)$ \\
\hline $\mathrm{C}(6)$ & $1.0846(6)$ & $1.0348(5)$ & $0.0909(4)$ & $0.0224(18)$ \\
\hline $\mathrm{C}(7)$ & $1.3947(6)$ & $1.0363(5)$ & $-0.0283(4)$ & $0.0221(18)$ \\
\hline $\mathrm{C}(8)$ & $1.1690(6)$ & $0.9544(5)$ & $-0.0496(4)$ & $0.0207(18)$ \\
\hline $\mathrm{C}(9)$ & $1.5077(7)$ & $0.9610(6)$ & $0.4233(4)$ & $0.029(2)$ \\
\hline $\mathrm{C}(10)$ & $1.5212(7)$ & $0.7995(6)$ & $0.3812(5)$ & $0.028(2)$ \\
\hline $\mathrm{C}(11)$ & $1.7053(6)$ & $1.0202(6)$ & $0.2133(4)$ & $0.0235(18)$ \\
\hline $\mathrm{C}(12)$ & $1.6463(7)$ & $1.1174(5)$ & $0.3110(4)$ & $0.0256(19)$ \\
\hline $\mathrm{C}(13)$ & $1.1487(7)$ & $0.5179(5)$ & $0.0990(4)$ & $0.0217(18)$ \\
\hline $\mathrm{C}(14)$ & $1.0825(6)$ & $0.6201(5)$ & $0.0057(4)$ & $0.0200(17)$ \\
\hline $\mathrm{C}(15)$ & $1.4852(7)$ & $0.7205(7)$ & $0.0202(5)$ & $0.038(2)$ \\
\hline $\mathrm{C}(16)$ & $1.4279(7)$ & $0.5975(5)$ & $0.1190(5)$ & $0.035(2)$ \\
\hline $\mathrm{C}(17)$ & $1.0236(7)$ & $0.6307(5)$ & $0.4778(4)$ & $0.028(2)$ \\
\hline $\mathrm{C}(18)$ & $1.2066(7)$ & $0.6041(5)$ & $0.4476(5)$ & $0.031(2)$ \\
\hline $\mathrm{C}(19)$ & $1.0990(7)$ & $0.3916(6)$ & $0.3444(5)$ & $0.031(2)$ \\
\hline $\mathrm{C}(20)$ & $0.8741(7)$ & $0.3413(5)$ & $0.3239(4)$ & $0.027(2)$ \\
\hline $\mathrm{C}(21)$ & $0.7717(7)$ & $0.8210(6)$ & $0.1441(4)$ & $0.030(2)$ \\
\hline $\mathrm{C}(22)$ & $0.7625(7)$ & $0.8089(6)$ & $0.2857(5)$ & $0.029(2)$ \\
\hline $\mathrm{C}(23)$ & $0.6052(7)$ & $0.4834(6)$ & $0.1756(5)$ & $0.040(2)$ \\
\hline $\mathrm{C}(24)$ & $0.5850(7)$ & $0.5753(7)$ & $0.2929(6)$ & $0.044(3)$ \\
\hline $\mathrm{Cl}(1 \mathrm{~L})$ & $0.8296(2)$ & $1.24954(15)$ & $0.14237(12)$ & $0.0489(7)$ \\
\hline $\mathrm{Cl}(2 \mathrm{~L})$ & $0.9503(2)$ & $0.78569(17)$ & $-0.18102(14)$ & $0.0549(7)$ \\
\hline $\mathrm{Cl}(3 \mathrm{~L})$ & $0.6260(3)$ & $1.2562(3)$ & $0.15429(19)$ & $0.0835(11)$ \\
\hline $\mathrm{Cl}(4 \mathrm{~A})$ & $0.7509(6)$ & $0.6460(5)$ & $-0.1454(5)$ & $0.084(2)$ \\
\hline $\mathrm{Cl}(4 \mathrm{~B})$ & $0.7641(4)$ & $0.6365(4)$ & $-0.1811(3)$ & $0.0491(13)$ \\
\hline $\mathrm{Cl}(5 \mathrm{~L})$ & $0.7175(5)$ & $0.7480(5)$ & $-0.0348(3)$ & $0.152(2)$ \\
\hline $\mathrm{Cl}(6 \mathrm{~L})$ & $0.6049(6)$ & $0.5725(4)$ & $0.0029(4)$ & $0.157(2)$ \\
\hline $\mathrm{Cl}(7 \mathrm{~L})$ & $1.5699(3)$ & $1.8320(3)$ & $-0.4002(2)$ & $0.0964(13)$ \\
\hline $\mathrm{Cl}(8 \mathrm{~A})$ & $1.4371(8)$ & $1.6899(7)$ & $-0.3410(5)$ & $0.095(2)$ \\
\hline $\mathrm{Cl}(8 \mathrm{~B})$ & $1.3968(8)$ & $1.6569(6)$ & $-0.3694(5)$ & $0.092(2)$ \\
\hline
\end{tabular}


Tabelle 107: Fortsetzung

\begin{tabular}{|r|r|r|r|r|}
\hline $\mathrm{C}(1 \mathrm{~L})$ & $1.4552(11)$ & $1.7383(9)$ & $-0.4211(8)$ & $0.075(4)$ \\
$\mathrm{C}(2 \mathrm{~L})$ & $0.7415(9)$ & $1.2993(6)$ & $0.1174(5)$ & $0.046(3)$ \\
$\mathrm{C}(3 \mathrm{~L})$ & $0.8922(11)$ & $0.6889(7)$ & $-0.1415(8)$ & $0.069(4)$ \\
$\mathrm{C}(4 \mathrm{~L})$ & $0.734(2)$ & $0.6514(18)$ & $0.0033(14)$ & $0.161(10)$ \\
$\mathrm{Cl}(9)$ & $1.2513(5)$ & $0.3169(4)$ & $0.5276(3)$ & $0.0843(15)$ \\
$\mathrm{C}(5 \mathrm{LA})$ & $1.3691(13)$ & $0.380(2)$ & $0.505(2)$ & $0.093(14)$ \\
$\mathrm{Cl}(10)$ & $1.3666(11)$ & $0.4573(9)$ & $0.4519(7)$ & $0.095(3)$ \\
$\mathrm{C}(5 \mathrm{LB})$ & $1.3250(15)$ & $0.4228(11)$ & $0.518(3)$ & $0.076(12)$ \\
$\mathrm{Cl}(11)$ & $1.4534(13)$ & $0.4367(12)$ & $0.5200(9)$ & $0.132(5)$ \\
\hline
\end{tabular}

Tabelle 108: Bindungslängen [pm] in $\underline{25}$

\begin{tabular}{|l|r||l|r|}
\hline $\mathrm{Cu}(1)-\mathrm{Cl}(1)$ & $227.6(2)$ & $\mathrm{Cu}(1)-\mathrm{S}(1)$ & $233.2(2)$ \\
$\mathrm{Cu}(1)-\mathrm{S}(9)$ & $237.4(2)$ & $\mathrm{Cu}(1)-\mathrm{S}(5)$ & $240.0(2)$ \\
$\mathrm{Cu}(2)-\mathrm{S}(2)$ & $226.5(2)$ & $\mathrm{Cu}(2)-\mathrm{S}(1)$ & $231.3(2)$ \\
$\mathrm{Cu}(2)-\mathrm{S}(3)$ & $235.1(2)$ & $\mathrm{Cu}(2)-\mathrm{S}(11)$ & $241.4(2)$ \\
$\mathrm{Cu}(3)-\mathrm{S}(3)$ & $229.5(2)$ & $\mathrm{Cu}(3)-\mathrm{S}(4)$ & $232.3(2)$ \\
$\mathrm{Cu}(3)-\mathrm{S}(7)$ & $235.2(2)$ & $\mathrm{Cu}(3)-\mathrm{Cl}(2)$ & $246.5(2)$ \\
$\mathrm{Cu}(3)-\mathrm{Cu}(6)$ & $286.94(18)$ & $\mathrm{Cu}(4)-\mathrm{S}(5)$ & $230.9(2)$ \\
$\mathrm{Cu}(4)-\mathrm{S}(8)$ & $232.5(2)$ & $\mathrm{Cu}(4)-\mathrm{S}(7)$ & $240.7(2)$ \\
$\mathrm{Cu}(4)-\mathrm{Cl}(3)$ & $245.6(2)$ & $\mathrm{Cu}(4)-\mathrm{Cu}(5)$ & $286.30(17)$ \\
$\mathrm{Cu}(5)-\mathrm{S}(9)$ & $231.9(2)$ & $\mathrm{Cu}(5)-\mathrm{S}(10)$ & $233.6(2)$ \\
$\mathrm{Cu}(5)-\mathrm{Cl}(3)$ & $240.7(2)$ & $\mathrm{Cu}(5)-\mathrm{S}(7)$ & $241.1(2)$ \\
$\mathrm{Cu}(6)-\mathrm{S}(11)$ & $230.5(2)$ & $\mathrm{Cu}(6)-\mathrm{Cl}(4)$ & $234.6(2)$ \\
$\mathrm{Cu}(6)-\mathrm{Cl}(2)$ & $237.4(2)$ & $\mathrm{Cu}(6)-\mathrm{S}(7)$ & $261.8(2)$ \\
$\mathrm{Cu}(7)-\mathrm{S}(5)$ & $223.9(2)$ & $\mathrm{Cu}(7)-\mathrm{S}(3)$ & $224.2(2)$ \\
$\mathrm{Cu}(7)-\mathrm{S}(6)$ & $224.8(2)$ & $\mathrm{Cu}(7)-\mathrm{Cu}(8)$ & $269.46(16)$ \\
$\mathrm{Cu}(8)-\mathrm{S}(4)$ & $225.6(2)$ & $\mathrm{Cu}(8)-\mathrm{S}(8)$ & $226.0(2)$ \\
$\mathrm{Cu}(8)-\mathrm{S}(6)$ & $228.0(2)$ & $\mathrm{Cu}(9)-\mathrm{S}(11)$ & $223.3(2)$ \\
$\mathrm{Cu}(9)-\mathrm{S}(9)$ & $223.7(2)$ & $\mathrm{Cu}(9)-\mathrm{S}(12)$ & $224.3(2)$ \\
$\mathrm{Cu}(9)-\mathrm{Cu}(10)$ & $262.90(18)$ & $\mathrm{Cu}(10)-\mathrm{S}(10)$ & $224.0(2)$ \\
$\mathrm{Cu}(10)-\mathrm{Cl}(4)$ & $226.6(2)$ & $\mathrm{Cu}(10)-\mathrm{S}(12)$ & $226.8(2)$ \\
$\mathrm{P}(1)-\mathrm{N}(1)$ & $159.8(7)$ & $\mathrm{P}(1)-\mathrm{C}(1)$ & $179.2(8)$ \\
$\mathrm{P}(1)-\mathrm{C}(2)$ & $179.8(8)$ & $\mathrm{P}(1)-\mathrm{S}(1)$ & $203.2(3)$ \\
$\mathrm{P}(2)-\mathrm{N}(1)$ & $159.6(7)$ & $\mathrm{P}(2)-\mathrm{C}(3)$ & $179.7(9)$ \\
$\mathrm{P}(2)-\mathrm{C}(4)$ & $180.7(8)$ & $\mathrm{P}(2)-\mathrm{S}(2)$ & $201.4(3)$ \\
$\mathrm{P}(3)-\mathrm{N}(2)$ & $158.6(7)$ & $\mathrm{P}(3)-\mathrm{C}(5)$ & $179.0(8)$ \\
$\mathrm{P}(3)-\mathrm{C}(6)$ & $179.9(8)$ & $\mathrm{P}(3)-\mathrm{S}(3)$ & $206.7(3)$ \\
$\mathrm{P}(4)-\mathrm{N}(2)$ & $158.8(7)$ & $\mathrm{P}(4)-\mathrm{C}(7)$ & $179.3(8)$ \\
$\mathrm{P}(4)-\mathrm{C}(8)$ & $180.2(8)$ & $\mathrm{P}(4)-\mathrm{S}(4)$ & $204.7(3)$ \\
\hline & & &
\end{tabular}


Tabelle 108: Fortsetzung

\begin{tabular}{|l|r||l|l|}
\hline $\mathrm{P}(5)-\mathrm{N}(3)$ & $158.3(7)$ & $\mathrm{P}(5)-\mathrm{C}(9)$ & $178.6(8)$ \\
$\mathrm{P}(5)-\mathrm{C}(10)$ & $179.1(8)$ & $\mathrm{P}(5)-\mathrm{S}(5)$ & $206.2(3)$ \\
$\mathrm{P}(6)-\mathrm{N}(3)$ & $159.4(7)$ & $\mathrm{P}(6)-\mathrm{C}(11)$ & $178.6(8)$ \\
$\mathrm{P}(6)-\mathrm{C}(12)$ & $180.2(8)$ & $\mathrm{P}(6)-\mathrm{S}(6)$ & $205.8(3)$ \\
$\mathrm{P}(7)-\mathrm{N}(4)$ & $158.5(7)$ & $\mathrm{P}(7)-\mathrm{C}(14)$ & $178.0(8)$ \\
$\mathrm{P}(7)-\mathrm{C}(13)$ & $178.9(7)$ & $\mathrm{P}(7)-\mathrm{S}(7)$ & $207.8(3)$ \\
$\mathrm{P}(8)-\mathrm{N}(4)$ & $158.7(7)$ & $\mathrm{P}(8)-\mathrm{C}(16)$ & $178.6(8)$ \\
$\mathrm{P}(8)-\mathrm{C}(15)$ & $179.4(9)$ & $\mathrm{P}(8)-\mathrm{S}(8)$ & $204.6(3)$ \\
$\mathrm{P}(9)-\mathrm{N}(5)$ & $158.8(7)$ & $\mathrm{P}(9)-\mathrm{C}(17)$ & $178.3(9)$ \\
$\mathrm{P}(9)-\mathrm{C}(18)$ & $178.6(9)$ & $\mathrm{P}(9)-\mathrm{S}(9)$ & $206.0(3)$ \\
$\mathrm{P}(10)-\mathrm{N}(5)$ & $158.3(7)$ & $\mathrm{P}(10)-\mathrm{C}(20)$ & $179.0(8)$ \\
$\mathrm{P}(10)-\mathrm{C}(19)$ & $179.8(9)$ & $\mathrm{P}(10)-\mathrm{S}(10)$ & $203.8(3)$ \\
$\mathrm{P}(11)-\mathrm{N}(6)$ & $158.4(7)$ & $\mathrm{P}(11)-\mathrm{C}(21)$ & $179.0(8)$ \\
$\mathrm{P}(11)-\mathrm{C}(22)$ & $180.5(9)$ & $\mathrm{P}(11)-\mathrm{S}(11)$ & $206.6(3)$ \\
$\mathrm{P}(12)-\mathrm{N}(6)$ & $159.9(7)$ & $\mathrm{P}(12)-\mathrm{C}(23)$ & $179.0(9)$ \\
$\mathrm{P}(12)-\mathrm{C}(24)$ & $179.9(10)$ & $\mathrm{P}(12)-\mathrm{S}(12)$ & $207.1(3)$ \\
\hline
\end{tabular}

Tabelle 109: Bindungswinkel $\left[^{\circ}\right]$ in $\underline{25}$

\begin{tabular}{|l|r||l|r|}
\hline $\mathrm{Cl}(1)-\mathrm{Cu}(1)-\mathrm{S}(1)$ & $115.72(8)$ & $\mathrm{Cl}(1)-\mathrm{Cu}(1)-\mathrm{S}(9)$ & $112.27(8)$ \\
$\mathrm{S}(1)-\mathrm{Cu}(1)-\mathrm{S}(9)$ & $114.76(8)$ & $\mathrm{Cl}(1)-\mathrm{Cu}(1)-\mathrm{S}(5)$ & $123.63(8)$ \\
$\mathrm{S}(1)-\mathrm{Cu}(1)-\mathrm{S}(5)$ & $94.57(7)$ & $\mathrm{S}(9)-\mathrm{Cu}(1)-\mathrm{S}(5)$ & $93.33(7)$ \\
$\mathrm{S}(2)-\mathrm{Cu}(2)-\mathrm{S}(1)$ & $115.56(8)$ & $\mathrm{S}(2)-\mathrm{Cu}(2)-\mathrm{S}(3)$ & $119.22(8)$ \\
$\mathrm{S}(1)-\mathrm{Cu}(2)-\mathrm{S}(3)$ & $97.77(8)$ & $\mathrm{S}(2)-\mathrm{Cu}(2)-\mathrm{S}(11)$ & $113.05(8)$ \\
$\mathrm{S}(1)-\mathrm{Cu}(2)-\mathrm{S}(11)$ & $113.66(8)$ & $\mathrm{S}(3)-\mathrm{Cu}(2)-\mathrm{S}(11)$ & $95.21(7)$ \\
$\mathrm{S}(3)-\mathrm{Cu}(3)-\mathrm{S}(4)$ & $114.74(8)$ & $\mathrm{S}(3)-\mathrm{Cu}(3)-\mathrm{S}(7)$ & $111.00(8)$ \\
$\mathrm{S}(4)-\mathrm{Cu}(3)-\mathrm{S}(7)$ & $110.60(8)$ & $\mathrm{S}(3)-\mathrm{Cu}(3)-\mathrm{Cl}(2)$ & $104.09(8)$ \\
$\mathrm{S}(4)-\mathrm{Cu}(3)-\mathrm{Cl}(2)$ & $111.52(8)$ & $\mathrm{S}(7)-\mathrm{Cu}(3)-\mathrm{Cl}(2)$ & $104.20(7)$ \\
$\mathrm{S}(3)-\mathrm{Cu}(3)-\mathrm{Cu}(6)$ & $95.73(6)$ & $\mathrm{S}(4)-\mathrm{Cu}(3)-\mathrm{Cu}(6)$ & $149.07(7)$ \\
$\mathrm{S}(7)-\mathrm{Cu}(3)-\mathrm{Cu}(6)$ & $59.20(6)$ & $\mathrm{Cl}(2)-\mathrm{Cu}(3)-\mathrm{Cu}(6)$ & $52.18(6)$ \\
$\mathrm{S}(5)-\mathrm{Cu}(4)-\mathrm{S}(8)$ & $113.67(8)$ & $\mathrm{S}(5)-\mathrm{Cu}(4)-\mathrm{S}(7)$ & $109.28(8)$ \\
$\mathrm{S}(8)-\mathrm{Cu}(4)-\mathrm{S}(7)$ & $115.91(8)$ & $\mathrm{S}(5)-\mathrm{Cu}(4)-\mathrm{Cl}(3)$ & $106.82(8)$ \\
$\mathrm{S}(8)-\mathrm{Cu}(4)-\mathrm{Cl}(3)$ & $110.99(8)$ & $\mathrm{S}(7)-\mathrm{Cu}(4)-\mathrm{Cl}(3)$ & $98.86(7)$ \\
$\mathrm{S}(5)-\mathrm{Cu}(4)-\mathrm{Cu}(5)$ & $94.97(7)$ & $\mathrm{S}(8)-\mathrm{Cu}(4)-\mathrm{Cu}(5)$ & $151.06(7)$ \\
$\mathrm{S}(7)-\mathrm{Cu}(4)-\mathrm{Cu}(5)$ & $53.61(6)$ & $\mathrm{Cl}(3)-\mathrm{Cu}(4)-\mathrm{Cu}(5)$ & $53.15(6)$ \\
$\mathrm{S}(9)-\mathrm{Cu}(5)-\mathrm{S}(10)$ & $112.58(8)$ & $\mathrm{S}(9)-\mathrm{Cu}(5)-\mathrm{Cl}(3)$ & $112.13(8)$ \\
$\mathrm{S}(10)-\mathrm{Cu}(5)-\mathrm{Cl}(3)$ & $108.52(8)$ & $\mathrm{S}(9)-\mathrm{Cu}(5)-\mathrm{S}(7)$ & $110.14(8)$ \\
$\mathrm{S}(10)-\mathrm{Cu}(5)-\mathrm{S}(7)$ & $112.73(8)$ & $\mathrm{Cl}(3)-\mathrm{Cu}(5)-\mathrm{S}(7)$ & $100.11(8)$ \\
$\mathrm{S}(9)-\mathrm{Cu}(5)-\mathrm{Cu}(4)$ & $100.12(6)$ & $\mathrm{S}(10)-\mathrm{Cu}(5)-\mathrm{Cu}(4)$ & $147.27(7)$ \\
$\mathrm{Cl}(3)-\mathrm{Cu}(5)-\mathrm{Cu}(4)$ & $54.72(6)$ & $\mathrm{S}(7)-\mathrm{Cu}(5)-\mathrm{Cu}(4)$ & $53.48(5)$ \\
\hline
\end{tabular}


Tabelle 109: Fortsetzung

\begin{tabular}{|c|c|c|c|}
\hline $\mathrm{S}(11)-\mathrm{Cu}(6)-\mathrm{Cl}(4)$ & $123.64(9)$ & $\mathrm{S}(11)-\mathrm{Cu}(6)-\mathrm{Cl}(2)$ & $110.32(8)$ \\
\hline $\mathrm{Cl}(4)-\mathrm{Cu}(6)-\mathrm{Cl}(2)$ & $109.07(8)$ & $\mathrm{S}(11)-\mathrm{Cu}(6)-\mathrm{S}(7)$ & $109.58(8)$ \\
\hline $\mathrm{Cl}(4)-\mathrm{Cu}(6)-\mathrm{S}(7)$ & $102.10(8)$ & $\mathrm{Cl}(2)-\mathrm{Cu}(6)-\mathrm{S}(7)$ & $99.07(7)$ \\
\hline $\mathrm{S}(11)-\mathrm{Cu}(6)-\mathrm{Cu}(3)$ & $100.40(7)$ & $\mathrm{Cl}(4)-\mathrm{Cu}(6)-\mathrm{Cu}(3)$ & $135.28(7)$ \\
\hline $\mathrm{Cl}(2)-\mathrm{Cu}(6)-\mathrm{Cu}(3)$ & $55.10(6)$ & $\mathrm{S}(7)-\mathrm{Cu}(6)-\mathrm{Cu}(3)$ & $50.50(5)$ \\
\hline $\mathrm{S}(5)-\mathrm{Cu}(7)-\mathrm{S}(3)$ & $116.96(8)$ & $\mathrm{S}(5)-\mathrm{Cu}(7)-\mathrm{S}(6)$ & $123.72(8)$ \\
\hline $\mathrm{S}(3)-\mathrm{Cu}(7)-\mathrm{S}(6)$ & $118.49(8)$ & $\mathrm{S}(5)-\mathrm{Cu}(7)-\mathrm{Cu}(8)$ & $105.31(7)$ \\
\hline $\mathrm{S}(3)-\mathrm{Cu}(7)-\mathrm{Cu}(8)$ & $102.27(7)$ & $\mathrm{S}(6)-\mathrm{Cu}(7)-\mathrm{Cu}(8)$ & $54.02(6)$ \\
\hline $\mathrm{S}(4)-\mathrm{Cu}(8)-\mathrm{S}(8)$ & $120.74(8)$ & $\mathrm{S}(4)-\mathrm{Cu}(8)-\mathrm{S}(6)$ & $122.16(8)$ \\
\hline $\mathrm{S}(8)-\mathrm{Cu}(8)-\mathrm{S}(6)$ & $117.07(8)$ & $\mathrm{S}(4)-\mathrm{Cu}(8)-\mathrm{Cu}(7)$ & $108.55(7)$ \\
\hline $\mathrm{S}(8)-\mathrm{Cu}(8)-\mathrm{Cu}(7)$ & $104.89(7)$ & $\mathrm{S}(6)-\mathrm{Cu}(8)-\mathrm{Cu}(7)$ & $52.93(6)$ \\
\hline $\mathrm{S}(11)-\mathrm{Cu}(9)-\mathrm{S}(9)$ & $110.59(8)$ & $\mathrm{S}(11)-\mathrm{Cu}(9)-\mathrm{S}(12)$ & $122.57(8)$ \\
\hline $\mathrm{S}(9)-\mathrm{Cu}(9)-\mathrm{S}(12)$ & $126.07(8)$ & $\mathrm{S}(11)-\mathrm{Cu}(9)-\mathrm{Cu}(10)$ & $106.88(7)$ \\
\hline $\mathrm{S}(9)-\mathrm{Cu}(9)-\mathrm{Cu}(10)$ & $103.45(7)$ & $\mathrm{S}(12)-\mathrm{Cu}(9)-\mathrm{Cu}(10)$ & $54.80(7)$ \\
\hline $\mathrm{S}(10)-\mathrm{Cu}(10)-\mathrm{Cl}(4)$ & $112.10(9)$ & $\mathrm{S}(10)-\mathrm{Cu}(10)-\mathrm{S}(12)$ & $123.18(9)$ \\
\hline $\mathrm{Cl}(4)-\mathrm{Cu}(10)-\mathrm{S}(12)$ & $124.70(9)$ & $\mathrm{S}(10)-\mathrm{Cu}(10)-\mathrm{Cu}(9)$ & $108.59(7)$ \\
\hline $\mathrm{Cl}(4)-\mathrm{Cu}(10)-\mathrm{Cu}(9)$ & $111.27(7)$ & $\mathrm{S}(12)-\mathrm{Cu}(10)-\mathrm{Cu}(9)$ & $53.92(6)$ \\
\hline $\mathrm{N}(1)-\mathrm{P}(1)-\mathrm{C}(1)$ & 109.2(4) & $\mathrm{N}(1)-\mathrm{P}(1)-\mathrm{C}(2)$ & $108.5(4)$ \\
\hline $\mathrm{N}(1)-\mathrm{P}(1)-\mathrm{S}(1)$ & $116.2(3)$ & $\mathrm{C}(1)-\mathrm{P}(1)-\mathrm{S}(1)$ & $107.5(3)$ \\
\hline $\mathrm{C}(2)-\mathrm{P}(1)-\mathrm{S}(1)$ & $108.8(3)$ & $\mathrm{N}(1)-\mathrm{P}(2)-\mathrm{C}(3)$ & $110.8(4)$ \\
\hline $\mathrm{N}(1)-\mathrm{P}(2)-\mathrm{C}(4)$ & $105.9(4)$ & $\mathrm{N}(1)-\mathrm{P}(2)-\mathrm{S}(2)$ & $118.0(3)$ \\
\hline $\mathrm{C}(3)-\mathrm{P}(2)-\mathrm{S}(2)$ & 109.4(3) & $\mathrm{C}(4)-\mathrm{P}(2)-\mathrm{S}(2)$ & $106.6(3)$ \\
\hline $\mathrm{N}(2)-\mathrm{P}(3)-\mathrm{C}(5)$ & $107.4(4)$ & $\mathrm{N}(2)-\mathrm{P}(3)-\mathrm{C}(6)$ & $112.9(4)$ \\
\hline $\mathrm{N}(2)-\mathrm{P}(3)-\mathrm{S}(3)$ & $115.6(2)$ & $\mathrm{C}(5)-\mathrm{P}(3)-\mathrm{S}(3)$ & 107.1(3) \\
\hline $\mathrm{C}(6)-\mathrm{P}(3)-\mathrm{S}(3)$ & $105.2(3)$ & $\mathrm{N}(2)-\mathrm{P}(4)-\mathrm{C}(7)$ & 106.1(4) \\
\hline $\mathrm{N}(2)-\mathrm{P}(4)-\mathrm{C}(8)$ & $112.7(4)$ & $\mathrm{N}(2)-\mathrm{P}(4)-\mathrm{S}(4)$ & $117.9(2)$ \\
\hline $\mathrm{C}(7)-\mathrm{P}(4)-\mathrm{S}(4)$ & $107.6(3)$ & $\mathrm{C}(8)-\mathrm{P}(4)-\mathrm{S}(4)$ & 106.1(3) \\
\hline $\mathrm{N}(3)-\mathrm{P}(5)-\mathrm{C}(9)$ & $114.3(4)$ & $\mathrm{N}(3)-\mathrm{P}(5)-\mathrm{C}(10)$ & $107.0(4)$ \\
\hline $\mathrm{N}(3)-\mathrm{P}(5)-\mathrm{S}(5)$ & $116.0(2)$ & $\mathrm{C}(9)-\mathrm{P}(5)-\mathrm{S}(5)$ & $105.5(3)$ \\
\hline $\mathrm{C}(10)-\mathrm{P}(5)-\mathrm{S}(5)$ & $106.7(3)$ & $\mathrm{N}(3)-\mathrm{P}(6)-\mathrm{C}(11)$ & $105.9(4)$ \\
\hline $\mathrm{N}(3)-\mathrm{P}(6)-\mathrm{C}(12)$ & $112.6(4)$ & $\mathrm{N}(3)-\mathrm{P}(6)-\mathrm{S}(6)$ & 119.1(2) \\
\hline $\mathrm{C}(11)-\mathrm{P}(6)-\mathrm{S}(6)$ & $106.9(3)$ & $\mathrm{C}(12)-\mathrm{P}(6)-\mathrm{S}(6)$ & 104.6(3) \\
\hline $\mathrm{N}(4)-\mathrm{P}(7)-\mathrm{C}(14)$ & $106.6(4)$ & $\mathrm{N}(4)-\mathrm{P}(7)-\mathrm{C}(13)$ & $111.7(4)$ \\
\hline $\mathrm{N}(4)-\mathrm{P}(7)-\mathrm{S}(7)$ & $115.9(2)$ & $\mathrm{C}(14)-\mathrm{P}(7)-\mathrm{S}(7)$ & $108.6(3)$ \\
\hline $\mathrm{C}(13)-\mathrm{P}(7)-\mathrm{S}(7)$ & 107.1(3) & $\mathrm{N}(4)-\mathrm{P}(8)-\mathrm{C}(16)$ & $113.8(4)$ \\
\hline $\mathrm{N}(4)-\mathrm{P}(8)-\mathrm{C}(15)$ & $105.0(4)$ & $\mathrm{N}(4)-\mathrm{P}(8)-\mathrm{S}(8)$ & 117.1(3) \\
\hline $\mathrm{C}(16)-\mathrm{P}(8)-\mathrm{S}(8)$ & $105.4(3)$ & $\mathrm{C}(15)-\mathrm{P}(8)-\mathrm{S}(8)$ & 109.2(3) \\
\hline $\mathrm{N}(5)-\mathrm{P}(9)-\mathrm{C}(17)$ & $106.8(4)$ & $\mathrm{N}(5)-\mathrm{P}(9)-\mathrm{C}(18)$ & $113.4(4)$ \\
\hline $\mathrm{N}(5)-\mathrm{P}(9)-\mathrm{S}(9)$ & $115.8(3)$ & $\mathrm{C}(17)-\mathrm{P}(9)-\mathrm{S}(9)$ & 107.1(3) \\
\hline $\mathrm{C}(18)-\mathrm{P}(9)-\mathrm{S}(9)$ & $106.5(3)$ & $\mathrm{N}(5)-\mathrm{P}(10)-\mathrm{C}(20)$ & $106.4(4)$ \\
\hline
\end{tabular}


Tabelle 109: Fortsetzung

\begin{tabular}{|c|c|c|c|}
\hline $\mathrm{N}(5)-\mathrm{P}(10)-\mathrm{C}(19)$ & $112.4(4)$ & $\mathrm{N}(5)-\mathrm{P}(10)-\mathrm{S}(10)$ & $118.2(2)$ \\
\hline $\mathrm{C}(20)-\mathrm{P}(10)-\mathrm{S}(10)$ & 107.9(3) & $\mathrm{C}(19)-\mathrm{P}(10)-\mathrm{S}(10)$ & $105.0(3)$ \\
\hline $\mathrm{N}(6)-\mathrm{P}(11)-\mathrm{C}(21)$ & 108.5(4) & $\mathrm{N}(6)-\mathrm{P}(11)-\mathrm{C}(22)$ & $112.8(4)$ \\
\hline $\mathrm{N}(6)-\mathrm{P}(11)-\mathrm{S}(11)$ & $115.8(3)$ & $\mathrm{C}(21)-\mathrm{P}(11)-\mathrm{S}(11)$ & $106.4(3)$ \\
\hline $\mathrm{C}(22)-\mathrm{P}(11)-\mathrm{S}(11)$ & $106.8(3)$ & $\mathrm{N}(6)-\mathrm{P}(12)-\mathrm{C}(23)$ & 107.1(4) \\
\hline $\mathrm{N}(6)-\mathrm{P}(12)-\mathrm{C}(24)$ & $112.4(4)$ & $\mathrm{N}(6)-\mathrm{P}(12)-\mathrm{S}(12)$ & $118.5(3)$ \\
\hline $\mathrm{C}(23)-\mathrm{P}(12)-\mathrm{S}(12)$ & 107.1(3) & $\mathrm{C}(24)-\mathrm{P}(12)-\mathrm{S}(12)$ & $105.0(4)$ \\
\hline $\mathrm{Cu}(6)-\mathrm{Cl}(2)-\mathrm{Cu}(3)$ & $72.72(7)$ & $\mathrm{Cu}(5)-\mathrm{Cl}(3)-\mathrm{Cu}(4)$ & $72.13(6)$ \\
\hline $\mathrm{Cu}(10)-\mathrm{Cl}(4)-\mathrm{Cu}(6)$ & $86.81(8)$ & $\mathrm{P}(1)-\mathrm{S}(1)-\mathrm{Cu}(2)$ & $101.02(10)$ \\
\hline $\mathrm{P}(1)-\mathrm{S}(1)-\mathrm{Cu}(1)$ & $106.74(10)$ & $\mathrm{Cu}(2)-\mathrm{S}(1)-\mathrm{Cu}(1)$ & $119.30(9)$ \\
\hline $\mathrm{P}(2)-\mathrm{S}(2)-\mathrm{Cu}(2)$ & $99.49(10)$ & $\mathrm{P}(3)-\mathrm{S}(3)-\mathrm{Cu}(7)$ & $105.09(9)$ \\
\hline $\mathrm{P}(3)-\mathrm{S}(3)-\mathrm{Cu}(3)$ & $101.50(10)$ & $\mathrm{Cu}(7)-\mathrm{S}(3)-\mathrm{Cu}(3)$ & $92.01(8)$ \\
\hline $\mathrm{P}(3)-\mathrm{S}(3)-\mathrm{Cu}(2)$ & $113.35(9)$ & $\mathrm{Cu}(7)-\mathrm{S}(3)-\mathrm{Cu}(2)$ & $114.39(8)$ \\
\hline $\mathrm{Cu}(3)-\mathrm{S}(3)-\mathrm{Cu}(2)$ & $126.95(9)$ & $\mathrm{P}(4)-\mathrm{S}(4)-\mathrm{Cu}(8)$ & $106.05(10)$ \\
\hline $\mathrm{P}(4)-\mathrm{S}(4)-\mathrm{Cu}(3)$ & $97.10(9)$ & $\mathrm{Cu}(8)-\mathrm{S}(4)-\mathrm{Cu}(3)$ & $87.77(8)$ \\
\hline $\mathrm{P}(5)-\mathrm{S}(5)-\mathrm{Cu}(7)$ & $102.24(9)$ & $5)-\mathrm{Cu}(4)$ & $103.70(9)$ \\
\hline $\mathrm{Cu}(7)-\mathrm{S}(5)-\mathrm{Cu}(4)$ & $89.34(8)$ & $\mathrm{P}(5)-\mathrm{S}$ & $113.12(10)$ \\
\hline $\mathrm{Cu}(7)-\mathrm{S}(5)-\mathrm{Cu}(1)$ & $116.18(8)$ & $\mathrm{Cu}(4)-\mathrm{S}(5)-\mathrm{Cu}(1)$ & $127.64(8)$ \\
\hline $\mathrm{P}(6)-\mathrm{S}(6)-\mathrm{Cu}(7)$ & $101.47(10)$ & $(6)-\mathrm{Cu}(8)$ & $105.44(10)$ \\
\hline $\mathrm{Cu}(7)-\mathrm{S}(6)-\mathrm{Cu}(8)$ & $73.05(7)$ & $\mathrm{P}(7)-\mathrm{S}(7)-\mathrm{Cu}(3)$ & 99.51(9) \\
\hline $\mathrm{P}(7)-\mathrm{S}(7)-\mathrm{Cu}(4)$ & $99.70(9)$ & $\mathrm{Cu}(3)-\mathrm{S}(7)-\mathrm{Cu}(4)$ & $101.98(8)$ \\
\hline $\mathrm{P}(7)-\mathrm{S}(7)-\mathrm{Cu}(5)$ & $110.77(9)$ & $\mathrm{Cu}(3)-\mathrm{S}(7)-\mathrm{Cu}(5)$ & $149.71(9)$ \\
\hline $\mathrm{Cu}(4)-\mathrm{S}(7)-\mathrm{Cu}(5)$ & $72.91(7)$ & $\mathrm{P}(7)-\mathrm{S}(7)-\mathrm{Cu}(6)$ & $111.45(9)$ \\
\hline $\mathrm{Cu}(3)-\mathrm{S}(7)-\mathrm{Cu}(6)$ & $70.30(6)$ & $\mathrm{Cu}(4)-\mathrm{S}(7)-\mathrm{Cu}(6)$ & $148.66(9)$ \\
\hline $\mathrm{Cu}(5)-\mathrm{S}(7)-\mathrm{Cu}(6)$ & $98.21(7)$ & $\mathrm{P}(8)-\mathrm{S}(8)-\mathrm{Cu}(8)$ & $103.06(10)$ \\
\hline $\mathrm{P}(8)-\mathrm{S}(8)-\mathrm{Cu}(4)$ & $98.45(10)$ & $\mathrm{Cu}(8)-\mathrm{S}(8)-\mathrm{Cu}(4)$ & $86.23(8)$ \\
\hline $\mathrm{P}(9)-\mathrm{S}(9)-\mathrm{Cu}(9)$ & $104.74(10)$ & $\mathrm{P}(9)-\mathrm{S}(9)-\mathrm{Cu}(5)$ & $98.87(9)$ \\
\hline $\mathrm{Cu}(9)-\mathrm{S}(9)-\mathrm{Cu}(5)$ & $93.98(8)$ & $\mathrm{P}(9)-\mathrm{S}(9)-\mathrm{Cu}(1)$ & $111.04(10)$ \\
\hline $\mathrm{Cu}(9)-\mathrm{S}(9)-\mathrm{Cu}(1)$ & $121.07(8)$ & $\mathrm{Cu}(5)-\mathrm{S}(9)-\mathrm{Cu}(1)$ & $123.49(9)$ \\
\hline $\mathrm{P}(10)-\mathrm{S}(10)-\mathrm{Cu}(10)$ & $104.22(11)$ & $\mathrm{P}(10)-\mathrm{S}(10)-\mathrm{Cu}(5)$ & $100.40(10)$ \\
\hline $\mathrm{Cu}(10)-\mathrm{S}(10)-\mathrm{Cu}(5)$ & $88.53(8)$ & $\mathrm{P}(11)-\mathrm{S}(11)-\mathrm{Cu}(9)$ & $100.32(9)$ \\
\hline $\mathrm{P}(11)-\mathrm{S}(11)-\mathrm{Cu}(6)$ & $104.06(10)$ & $\mathrm{Cu}(9)-\mathrm{S}(11)-\mathrm{Cu}(6)$ & $90.62(8)$ \\
\hline $\mathrm{P}(11)-\mathrm{S}(11)-\mathrm{Cu}(2)$ & $114.07(10)$ & $\mathrm{Cu}(9)-\mathrm{S}(11)-\mathrm{Cu}(2)$ & $122.25(9)$ \\
\hline $\mathrm{Cu}(6)-\mathrm{S}(11)-\mathrm{Cu}(2)$ & $121.26(9)$ & $\mathrm{P}(12)-\mathrm{S}(12)-\mathrm{Cu}(9)$ & $105.02(10)$ \\
\hline $\mathrm{P}(12)-\mathrm{S}(12)-\mathrm{Cu}(10)$ & $101.37(11)$ & $\mathrm{Cu}(9)-\mathrm{S}(12)-\mathrm{Cu}(10)$ & $71.29(7)$ \\
\hline $\mathrm{P}(2)-\mathrm{N}(1)-\mathrm{P}(1)$ & $131.0(4)$ & $\mathrm{P}(3)-\mathrm{N}(2)-\mathrm{P}(4)$ & 133.1(4) \\
\hline $\mathrm{P}(5)-\mathrm{N}(3)-\mathrm{P}(6)$ & $133.3(4)$ & $\mathrm{P}(7)-\mathrm{N}(4)-\mathrm{P}(8)$ & $134.6(4)$ \\
\hline $\mathrm{P}(10)-\mathrm{N}(5)-\mathrm{P}(9)$ & $134.8(5)$ & $\mathrm{P}(11)-\mathrm{N}(6)-\mathrm{P}(12)$ & $133.5(5)$ \\
\hline
\end{tabular}

$\# 1-x+3,-y+1,-z+1$ 


\subsection{Daten zur Kristallstrukturanalyse von $\left[\mathrm{KCu}\left(\operatorname{dmpaS}_{2}\right)_{2}(\text { thf })\right]_{\infty} \underline{\mathbf{2 6}}$}

\begin{tabular}{|ll|}
\hline Summenformel & $\mathrm{C}_{12} \mathrm{H}_{32} \mathrm{CuKN}_{2} \mathrm{OP}_{4} \mathrm{~S}_{4}$ \\
Molekulargewicht $\left[\mathrm{g} \cdot \mathrm{mol}^{-1}\right]$ & 575.16 \\
Temperatur & $133(2) \mathrm{K}$ \\
Wellenlänge & $71.073 \mathrm{pm}$ \\
Kristallsystem & monoklin \\
Raumgruppe & $P 2_{1} / c$ \\
Gitterkonstanten & $\mathrm{a}=11.945(4) \AA, \alpha=90^{\circ}$ \\
& $\mathrm{b}=20.345(4) \AA, \beta=119.700(10)^{\circ}$ \\
& $\mathrm{c}=11.940(5) \AA, \gamma=90^{\circ}$ \\
Volumen & $2520.5(14) \AA^{3}$ \\
Formeleinheiten & 4 \\
Röntgenographische Dichte $\left[\mathrm{g} \cdot \mathrm{cm}^{-3}\right]$ & 1.516 \\
Absorptionskoeffizient $\left[\mathrm{mm}^{-1}\right]$ & 1.623 \\
F(000) & 1192 \\
Meßbereich $\theta$ & $1.96-26.39^{\circ}$ \\
Indexbereiche & $-14 \leq \mathrm{h} \leq 12$ \\
& $0 \leq \mathrm{k} \leq 25$ \\
Gemessene Reflexe & $0 \leq 1 \leq 14$ \\
Unabhängige Reflexe & 32593 \\
Beobachtete Reflexe $[\mathrm{I}>2 \sigma]$ & 5140 \\
$\mathrm{R}_{\text {int }}$ & 4320 \\
Parameter & 0.0394 \\
R1; wR2[I>2 $\sigma(\mathrm{I})]$ & 259 \\
\hline
\end{tabular}


Tabelle 111: Atomkoordinaten und äquivalente isotrope Auslenkungsparameter $\left[\AA^{2}\right]$ für $\underline{26}$

\begin{tabular}{|c|c|c|c|c|}
\hline Atom & $\mathrm{x}$ & $\mathrm{y}$ & z & $\mathrm{U}_{e q}$ \\
\hline $\mathrm{Cu}(1)$ & $0.17226(2)$ & $0.349333(12)$ & $0.32600(2)$ & $0.02496(7)$ \\
\hline $\mathrm{K}(1)$ & $0.20264(4)$ & $0.25436(2)$ & $0.06968(4)$ & $0.02616(11)$ \\
\hline $\mathrm{S}(2)$ & $0.06479(5)$ & $0.38670(2)$ & $0.43274(5)$ & $0.02302(11)$ \\
\hline $\mathrm{S}(1)$ & $0.39576(5)$ & $0.34270(3)$ & $0.45071(5)$ & $0.03754(15)$ \\
\hline $\mathrm{S}(3)$ & $0.13619(5)$ & $0.40343(2)$ & $0.13736(5)$ & $0.02306(11)$ \\
\hline $\mathrm{S}(4)$ & $0.09207(5)$ & $0.23693(2)$ & $0.26198(5)$ & $0.02141(11)$ \\
\hline $\mathrm{P}(4)$ & $-0.08729(5)$ & $0.25942(2)$ & $0.12133(5)$ & $0.01986(11)$ \\
\hline $\mathrm{P}(3)$ & $-0.05086(5)$ & $0.38323(2)$ & $0.01888(5)$ & $0.01919(11)$ \\
\hline $\mathrm{P}(2)$ & $0.20939(5)$ & $0.43244(2)$ & $0.58436(5)$ & $0.02118(11)$ \\
\hline $\mathrm{P}(1)$ & $0.45066(5)$ & $0.37628(3)$ & $0.62768(5)$ & $0.02292(12)$ \\
\hline $\mathrm{N}(2)$ & $-0.09769(16)$ & $0.30860(8)$ & $0.01213(15)$ & $0.0231(4)$ \\
\hline $\mathrm{N}(1)$ & $0.34117(16)$ & $0.39190(8)$ & $0.66407(15)$ & $0.0241(4)$ \\
\hline $\mathrm{C}(32)$ & $-0.0882(2)$ & $0.40258(10)$ & $-0.14292(18)$ & $0.0251(4)$ \\
\hline $\mathrm{C}(22)$ & $0.1569(2)$ & $0.45115(12)$ & $0.6982(2)$ & $0.0330(5)$ \\
\hline $\mathrm{C}(12)$ & $0.5560(2)$ & $0.31767(11)$ & $0.7457(2)$ & $0.0302(5)$ \\
\hline $\mathrm{C}(31)$ & $-0.1479(2)$ & $0.43891(10)$ & $0.0533(2)$ & $0.0276(5)$ \\
\hline $\mathrm{C}(11)$ & $0.5536(2)$ & $0.44728(11)$ & $0.6577(2)$ & $0.0367(5)$ \\
\hline $\mathrm{C}(21)$ & $0.2336(2)$ & $0.51093(11)$ & $0.5288(2)$ & $0.0374(6)$ \\
\hline $\mathrm{C}(42)$ & $-0.18167(19)$ & $0.28619(11)$ & $0.1936(2)$ & $0.0278(5)$ \\
\hline $\mathrm{C}(41)$ & $-0.1670(2)$ & $0.18561(10)$ & $0.0366(2)$ & $0.0267(4)$ \\
\hline $\mathrm{O}(1 \mathrm{~T})$ & $0.40796(16)$ & $0.32682(8)$ & $0.10134(16)$ & $0.0380(4)$ \\
\hline $\mathrm{C}(1 \mathrm{~T})$ & $0.4235(4)$ & $0.3356(2)$ & $-0.0120(4)$ & $0.0387(10)$ \\
\hline $\mathrm{C}(2 \mathrm{~T})$ & $0.4975(6)$ & $0.3986(3)$ & $0.0118(5)$ & $0.0367(13)$ \\
\hline $\mathrm{C}(3 \mathrm{~T})$ & $0.4679(5)$ & $0.43505(16)$ & $0.1055(4)$ & $0.0402(11)$ \\
\hline $\mathrm{C}(4 \mathrm{~T})$ & $0.4684(3)$ & $0.37937(12)$ & $0.1890(2)$ & $0.0439(6)$ \\
\hline $\mathrm{C}\left(1 \mathrm{~T}^{\prime}\right)$ & $0.4074(3)$ & $0.34681(12)$ & $0.0018(2)$ & $0.080(7)$ \\
\hline $\mathrm{C}\left(2 \mathrm{~T}^{\prime}\right)$ & $0.466(2)$ & $0.4127(8)$ & $0.0045(14)$ & $0.050(6)$ \\
\hline $\mathrm{C}\left(3 \mathrm{~T}^{\prime}\right)$ & $0.5505(10)$ & $0.4192(5)$ & $0.1505(9)$ & $0.029(3)$ \\
\hline
\end{tabular}

Tabelle 112: Bindungslängen [pm] in $\underline{26}$

\begin{tabular}{|l|r||l|r|}
\hline $\mathrm{Cu}(1)-\mathrm{S}(1)$ & $232.89(10)$ & $\mathrm{Cu}(1)-\mathrm{S}(2)$ & $233.91(8)$ \\
$\mathrm{Cu}(1)-\mathrm{S}(3)$ & $234.69(10)$ & $\mathrm{Cu}(1)-\mathrm{S}(4)$ & $245.06(7)$ \\
$\mathrm{Cu}(1)-\mathrm{K}(1) \# 1$ & $346.44(11)$ & $\mathrm{Cu}(1)-\mathrm{K}(1)$ & $378.57(12)$ \\
$\mathrm{K}(1)-\mathrm{O}(1 \mathrm{~T})$ & $272.26(17)$ & $\mathrm{K}(1)-\mathrm{S}(4)$ & $318.55(12)$ \\
$\mathrm{K}(1)-\mathrm{S}(4) \# 2$ & $323.50(16)$ & $\mathrm{K}(1)-\mathrm{S}(2) \# 2$ & $330.96(9)$ \\
$\mathrm{K}(1)-\mathrm{N}(1) \# 2$ & $331.89(19)$ & $\mathrm{K}(1)-\mathrm{S}(3)$ & $333.41(9)$ \\
$\mathrm{S}(2)-\mathrm{P}(2)$ & $200.72(9)$ & $\mathrm{S}(1)-\mathrm{P}(1)$ & $199.72(11)$ \\
\hline
\end{tabular}


Tabelle 112: Fortsetzung

\begin{tabular}{|l|r||l|r|}
\hline $\mathrm{S}(3)-\mathrm{P}(3)$ & $200.75(9)$ & $\mathrm{S}(4)-\mathrm{P}(4)$ & $201.36(9)$ \\
$\mathrm{P}(4)-\mathrm{N}(2)$ & $159.88(17)$ & $\mathrm{P}(4)-\mathrm{C}(41)$ & $179.7(2)$ \\
$\mathrm{P}(4)-\mathrm{C}(42)$ & $181.1(2)$ & $\mathrm{P}(3)-\mathrm{N}(2)$ & $160.63(17)$ \\
$\mathrm{P}(3)-\mathrm{C}(32)$ & $179.8(2)$ & $\mathrm{P}(3)-\mathrm{C}(31)$ & $180.7(2)$ \\
$\mathrm{P}(2)-\mathrm{N}(1)$ & $160.60(17)$ & $\mathrm{P}(2)-\mathrm{C}(22)$ & $179.8(2)$ \\
$\mathrm{P}(2)-\mathrm{C}(21)$ & $180.6(2)$ & $\mathrm{P}(1)-\mathrm{N}(1)$ & $160.11(18)$ \\
$\mathrm{P}(1)-\mathrm{C}(12)$ & $179.8(2)$ & $\mathrm{P}(1)-\mathrm{C}(11)$ & $181.4(2)$ \\
\hline
\end{tabular}

Tabelle 113: Bindungswinkel $\left[{ }^{\circ}\right]$ in $\underline{26}$

\begin{tabular}{|l|r||l|r|}
\hline $\mathrm{S}(1)-\mathrm{Cu}(1)-\mathrm{S}(2)$ & $115.80(3)$ & $\mathrm{S}(1)-\mathrm{Cu}(1)-\mathrm{S}(3)$ & $104.36(3)$ \\
$\mathrm{S}(2)-\mathrm{Cu}(1)-\mathrm{S}(3)$ & $118.76(3)$ & $\mathrm{S}(1)-\mathrm{Cu}(1)-\mathrm{S}(4)$ & $107.01(2)$ \\
$\mathrm{S}(2)-\mathrm{Cu}(1)-\mathrm{S}(4)$ & $103.77(2)$ & $\mathrm{S}(3)-\mathrm{Cu}(1)-\mathrm{S}(4)$ & $106.30(3)$ \\
$\mathrm{S}(1)-\mathrm{Cu}(1)-\mathrm{K}(1) \# 1$ & $79.51(2)$ & $\mathrm{S}(2)-\mathrm{Cu}(1)-\mathrm{K}(1) \# 1$ & $66.28(2)$ \\
$\mathrm{S}(3)-\mathrm{Cu}(1)-\mathrm{K}(1) \# 1$ & $169.930(17)$ & $\mathrm{S}(4)-\mathrm{Cu}(1)-\mathrm{K}(1) \# 1$ & $63.63(2)$ \\
$\mathrm{S}(1)-\mathrm{Cu}(1)-\mathrm{K}(1)$ & $87.06(3)$ & $\mathrm{S}(2)-\mathrm{Cu}(1)-\mathrm{K}(1)$ & $154.775(18)$ \\
$\mathrm{S}(3)-\mathrm{Cu}(1)-\mathrm{K}(1)$ & $60.60(3)$ & $\mathrm{S}(4)-\mathrm{Cu}(1)-\mathrm{K}(1)$ & $56.689(16)$ \\
$\mathrm{K}(1) \# 1-\mathrm{Cu}(1)-\mathrm{K}(1)$ & $110.86(3)$ & $\mathrm{S}(4)-\mathrm{K}(1)-\mathrm{S}(4) \# 2$ & $137.98(3)$ \\
$\mathrm{S}(4)-\mathrm{K}(1)-\mathrm{S}(2) \# 2$ & $88.69(2)$ & $\mathrm{S}(4) \# 2-\mathrm{K}(1)-\mathrm{S}(2) \# 2$ & $70.314(18)$ \\
$\mathrm{S}(4)-\mathrm{K}(1)-\mathrm{N}(1) \# 2$ & $88.31(3)$ & $\mathrm{S}(4) \# 2-\mathrm{K}(1)-\mathrm{N}(1) \# 2$ & $106.80(3)$ \\
$\mathrm{S}(2) \# 2-\mathrm{K}(1)-\mathrm{N}(1) \# 2$ & $55.43(3)$ & $\mathrm{S}(4)-\mathrm{K}(1)-\mathrm{S}(3)$ & $72.124(18)$ \\
$\mathrm{S}(4) \# 2-\mathrm{K}(1)-\mathrm{S}(3)$ & $102.31(2)$ & $\mathrm{S}(2) \# 2-\mathrm{K}(1)-\mathrm{S}(3)$ & $142.05(2)$ \\
$\mathrm{N}(1) \# 2-\mathrm{K}(1)-\mathrm{S}(3)$ & $150.67(4)$ & $\mathrm{S}(4)-\mathrm{K}(1)-\mathrm{Cu}(1) \# 2$ & $129.00(2)$ \\
$\mathrm{S}(4) \# 2-\mathrm{K}(1)-\mathrm{Cu}(1) \# 2$ & $42.743(19)$ & $\mathrm{S}(2) \# 2-\mathrm{K}(1)-\mathrm{Cu}(1) \# 2$ & $40.319(13)$ \\
$\mathrm{N}(1) \# 2-\mathrm{K}(1)-\mathrm{Cu}(1) \# 2$ & $64.30(3)$ & $\mathrm{S}(3)-\mathrm{K}(1)-\mathrm{Cu}(1) \# 2$ & $144.989(18)$ \\
$\mathrm{S}(4)-\mathrm{K}(1)-\mathrm{Cu}(1)$ & $40.010(18)$ & $\mathrm{S}(4) \# 2-\mathrm{K}(1)-\mathrm{Cu}(1)$ & $137.85(2)$ \\
$\mathrm{N}(1) \# 2-\mathrm{K}(1)-\mathrm{Cu}(1)$ & $114.49(4)$ & $\mathrm{S}(3)-\mathrm{K}(1)-\mathrm{Cu}(1)$ & $37.826(12)$ \\
$\mathrm{P}(2)-\mathrm{S}(2)-\mathrm{Cu}(1)$ & $100.53(4)$ & $\mathrm{P}(2)-\mathrm{S}(2)-\mathrm{K}(1) \# 1$ & $87.78(3)$ \\
$\mathrm{Cu}(1)-\mathrm{S}(2)-\mathrm{K}(1) \# 1$ & $73.40(3)$ & $\mathrm{P}(1)-\mathrm{S}(1)-\mathrm{Cu}(1)$ & $109.15(3)$ \\
$\mathrm{P}(3)-\mathrm{S}(3)-\mathrm{Cu}(1)$ & $101.03(3)$ & $\mathrm{Cu}(1)-\mathrm{S}(3)-\mathrm{K}(1)$ & $81.57(3)$ \\
$\mathrm{P}(4)-\mathrm{S}(4)-\mathrm{Cu}(1)$ & $97.94(3)$ & $\mathrm{P}(4)-\mathrm{S}(4)-\mathrm{K}(1)$ & $91.34(4)$ \\
$\mathrm{Cu}(1)-\mathrm{S}(4)-\mathrm{K}(1)$ & $83.30(2)$ & $\mathrm{P}(4)-\mathrm{S}(4)-\mathrm{K}(1) \# 1$ & $127.00(3)$ \\
$\mathrm{Cu}(1)-\mathrm{S}(4)-\mathrm{K}(1) \# 1$ & $73.629(16)$ & $\mathrm{K}(1)-\mathrm{S}(4)-\mathrm{K}(1) \# 1$ & $136.95(3)$ \\
$\mathrm{P}(4)-\mathrm{N}(2)-\mathrm{P}(3)$ & $131.78(11)$ & $\mathrm{P}(1)-\mathrm{N}(1)-\mathrm{P}(2)$ & $129.32(11)$ \\
$\mathrm{P}(1)-\mathrm{N}(1)-\mathrm{K}(1) \# 1$ & $93.83(7)$ & $\mathrm{P}(2)-\mathrm{N}(1)-\mathrm{K}(1) \# 1$ & $94.70(7)$ \\
\hline
\end{tabular}

$\# 1 \mathrm{x},-\mathrm{y}+1, \mathrm{z}+1 / 2 \quad \# 2 \mathrm{x},-\mathrm{y}+1 / 2, \mathrm{z}-1 / 2$ 


\subsection{Daten zur Kristallstrukturanalyse von $\left[\mathrm{Hg}\left(\mathrm{dmpaS}_{2}\right)_{2}\right] \underline{27}$}

\begin{tabular}{|ll|}
\hline Summenformel & $\mathrm{C}_{8} \mathrm{H}_{24} \mathrm{HgN}_{2} \mathrm{P}_{4} \mathrm{~S}_{4}$ \\
Molekulargewicht $\left[\mathrm{g} \cdot \mathrm{mol}^{-1}\right]$ & 601.00 \\
Temperatur & $133(2) \mathrm{K}$ \\
Wellenlänge & $71.073 \mathrm{pm}$ \\
Kristallsystem & monoklin \\
Raumgruppe & $C 2 / c$ \\
Gitterkonstanten & $\mathrm{a}=14.7684(6) \AA, \alpha=90^{\circ}$ \\
& $\mathrm{b}=11.1267(5) \AA, \beta=109.060(2)^{\circ} \circ$ \\
& $\mathrm{c}=13.3366(5) \AA, \gamma=90^{\circ}$ \\
Volumen & $2071.37(15) \AA^{3}$ \\
Formeleinheiten & 4 \\
Röntgenographische Dichte $\left[\mathrm{g} \cdot \mathrm{cm}^{-3}\right]$ & 1.927 \\
Absorptionskoeffizient $\left[\mathrm{mm}^{-1}\right]$ & 8.133 \\
F(000) & 1160 \\
Meßbereich $\theta$ & $2.34-27.48^{\circ}$ \\
Indexbereiche & $-19 \leq \mathrm{h} \leq 19$ \\
& $-14 \leq \mathrm{k} \leq 14$ \\
Gemessene Reflexe & $-10 \leq 1 \leq 17$ \\
Unabhängige Reflexe & 14225 \\
Beobachtete Reflexe $[\mathrm{I}>2 \sigma]$ & 2384 \\
$\mathrm{R}_{\text {int }}$ & 2150 \\
Parameter & 0.0470 \\
R1; wR2[I>2 $\sigma(\mathrm{I})]$ & 135 \\
\hline
\end{tabular}


Tabelle 115: Atomkoordinaten und äquivalente isotrope Auslenkungsparameter $\left[\AA^{2}\right]$ für $\underline{27}$

\begin{tabular}{|r|r|r|r|r|}
\hline Atom & \multicolumn{1}{|c|}{$\mathrm{x}$} & \multicolumn{1}{c|}{$\mathrm{y}$} & \multicolumn{1}{c|}{$\mathrm{z}$} & \multicolumn{1}{c|}{$\mathrm{U}_{e q}$} \\
\hline $\mathrm{Hg}(1)$ & 0.5000 & $0.194814(19)$ & 0.2500 & $0.02482(8)$ \\
$\mathrm{N}(1)$ & $0.7132(2)$ & $0.2500(3)$ & $0.1538(2)$ & $0.0239(6)$ \\
$\mathrm{P}(1)$ & $0.73912(7)$ & $0.20950(8)$ & $0.27385(7)$ & $0.02035(19)$ \\
$\mathrm{P}(2)$ & $0.61328(7)$ & $0.27575(8)$ & $0.06346(7)$ & $0.01883(19)$ \\
$\mathrm{S}(1)$ & $0.65514(7)$ & $0.08108(8)$ & $0.30877(8)$ & $0.0264(2)$ \\
$\mathrm{S}(2)$ & $0.50678(7)$ & $0.34838(9)$ & $0.10816(8)$ & $0.0256(2)$ \\
$\mathrm{C}(1)$ & $0.7475(4)$ & $0.3380(4)$ & $0.3588(4)$ & $0.0332(9)$ \\
$\mathrm{C}(2)$ & $0.8577(3)$ & $0.1483(5)$ & $0.3112(3)$ & $0.0303(9)$ \\
$\mathrm{C}(3)$ & $0.6372(3)$ & $0.3777(4)$ & $-0.0283(3)$ & $0.0273(8)$ \\
$\mathrm{C}(4)$ & $0.5715(4)$ & $0.1394(4)$ & $-0.0092(4)$ & $0.0349(10)$ \\
\hline
\end{tabular}

Tabelle 116: Bindungslängen [pm] in $\underline{27}$

\begin{tabular}{|l|r||l|r|}
\hline $\mathrm{Hg}(1)-\mathrm{S}(1) \# 1$ & $250.84(9)$ & $\mathrm{Hg}(1)-\mathrm{S}(1)$ & $250.84(9)$ \\
$\mathrm{Hg}(1)-\mathrm{S}(2) \# 1$ & $257.53(9)$ & $\mathrm{Hg}(1)-\mathrm{S}(2)$ & $257.53(9)$ \\
$\mathrm{N}(1)-\mathrm{P}(1)$ & $158.5(3)$ & $\mathrm{N}(1)-\mathrm{P}(2)$ & $159.7(3)$ \\
$\mathrm{P}(1)-\mathrm{C}(2)$ & $179.2(4)$ & $\mathrm{P}(1)-\mathrm{C}(1)$ & $180.3(5)$ \\
$\mathrm{P}(1)-\mathrm{S}(1)$ & $204.30(13)$ & $\mathrm{P}(2)-\mathrm{C}(3)$ & $178.6(4)$ \\
$\mathrm{P}(2)-\mathrm{C}(4)$ & $179.7(4)$ & $\mathrm{P}(2)-\mathrm{S}(2)$ & $202.35(13)$ \\
\hline
\end{tabular}

Tabelle 117: Bindungswinkel $\left[^{\circ}\right]$ in $\underline{27}$

\begin{tabular}{|l|r||l|r|}
\hline $\mathrm{S}(1) \# 1-\mathrm{Hg}(1)-\mathrm{S}(1)$ & $119.41(4)$ & $\mathrm{S}(1)-\mathrm{Hg}(1)-\mathrm{S}(2) \# 1$ & $110.84(3)$ \\
$\mathrm{S}(1)-\mathrm{Hg}(1)-\mathrm{S}(2)$ & $108.28(3)$ & $\mathrm{S}(2) \# 1-\mathrm{Hg}(1)-\mathrm{S}(2)$ & $96.86(5)$ \\
$\mathrm{P}(1)-\mathrm{N}(1)-\mathrm{P}(2)$ & $132.2(2)$ & $\mathrm{N}(1)-\mathrm{P}(1)-\mathrm{C}(2)$ & $106.27(19)$ \\
$\mathrm{N}(1)-\mathrm{P}(1)-\mathrm{C}(1)$ & $110.7(2)$ & $\mathrm{N}(1)-\mathrm{P}(1)-\mathrm{S}(1)$ & $117.70(13)$ \\
$\mathrm{C}(2)-\mathrm{P}(1)-\mathrm{S}(1)$ & $106.37(16)$ & $\mathrm{C}(1)-\mathrm{P}(1)-\mathrm{S}(1)$ & $109.46(16)$ \\
$\mathrm{N}(1)-\mathrm{P}(2)-\mathrm{C}(3)$ & $106.85(18)$ & $\mathrm{N}(1)-\mathrm{P}(2)-\mathrm{C}(4)$ & $109.0(2)$ \\
$\mathrm{N}(1)-\mathrm{P}(2)-\mathrm{S}(2)$ & $117.73(13)$ & $\mathrm{C}(3)-\mathrm{P}(2)-\mathrm{S}(2)$ & $107.14(14)$ \\
$\mathrm{C}(4)-\mathrm{P}(2)-\mathrm{S}(2)$ & $109.45(17)$ & $\mathrm{P}(1)-\mathrm{S}(1)-\mathrm{Hg}(1)$ & $97.84(4)$ \\
$\mathrm{P}(2)-\mathrm{S}(2)-\mathrm{Hg}(1)$ & $99.89(4)$ & & \\
\hline
\end{tabular}

$\# 1-\mathrm{x}+1, \mathrm{y},-\mathrm{z}+1 / 2$ 


\section{Zusammenfassung}

Am Beispiel der Münzmetalle $\mathrm{Cu}$, $\mathrm{Ag}$ und $\mathrm{Au}$ konnten in Komplexierungsreaktionen gezielt ein- und mehrkernige Komplexverbindungen der Ligandensysteme Bis(diorganochalkogenophosphoryl)amine und -methane synthetisiert und deren molekularen Aufbau mittels Röntgenstrukturanalysen ermittelt werden. In allen synthetisierten Verbindungen stellt der neutrale, monoanionische oder dianionische Ligand einen strukturbildenden Baustein dar, der eine große Vielfalt an strukturellen Variationen erlaubt.

Unter den Komplexverbindungen des Liganden $\operatorname{dppmE}_{2} \mathrm{H}(\mathrm{E}=\mathrm{O}, \mathrm{S}$, Se) konnten sowohl Verbindungen von $\mathrm{Cu}^{\mathrm{I}}\left(\left[\mathrm{Cu}_{2}\left(\mathrm{dppmS}_{2} \mathrm{H}\right)_{2} \mathrm{Cl}\right]\left[\mathrm{CuCl}_{2}\right] \underline{2},\left[\mathrm{Cu}_{2}\left(\mathrm{dppmSe}_{3}\right)_{2}\right] \underline{3}\right)$ und $\mathrm{Cu}^{\mathrm{II}}$ $\left(\left[\mathrm{Cu}\left(\mathrm{dppmO}_{2} \mathrm{H}\right)_{3}\right]\left[\mathrm{CuCl}_{4}\right]\right.$ 1) als auch von $\mathrm{Au}^{\mathrm{I}}\left(\left[\mathrm{Au}_{2}\left(\mathrm{dppmS}_{2}\right)\left(\mathrm{PPh}_{3}\right)_{2}\right]\right.$ 出) isoliert und röntgenstrukturanalytisch untersucht werden.

Abb. 49 stellt eine Auswahl der mit dppmE ${ }_{2} \mathrm{H}(\mathrm{E}=\mathrm{O}, \mathrm{S}, \mathrm{Se})$ dargestellten Verbindungen dar.
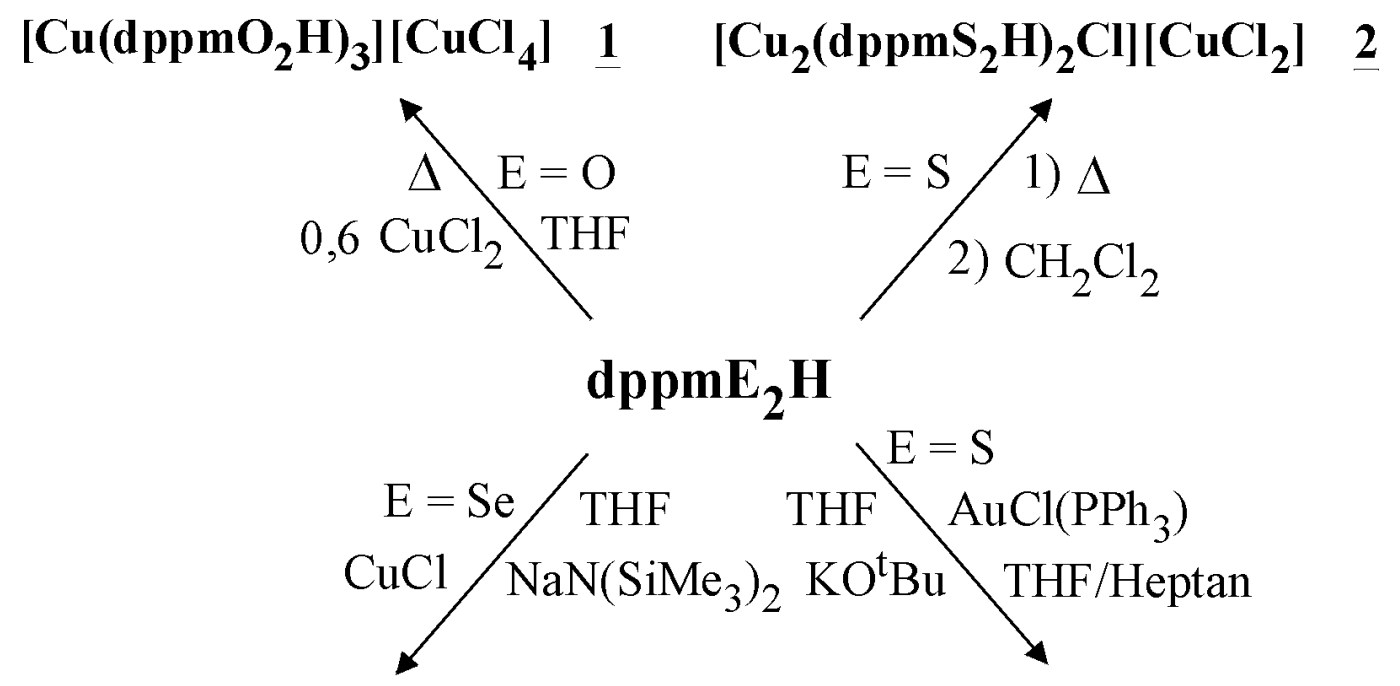

$\left[\mathrm{Cu}_{2}\left(\mathrm{dppmSe}_{3}\right)_{2}\right] \mathbf{3}$

$\left[\mathrm{Au}_{2}\left(\mathrm{dppmS}_{2}\right)\left(\mathrm{PPh}_{3}\right)_{2}\right] \underline{4}$

Abb. 49: Auswahl der Reaktionen mit dppmE $\mathrm{H}_{2} \mathrm{H}$

Verbindungen von $\mathrm{Cu}^{\mathrm{II}}$ konnten ebenfalls in Umsetzungen der Bis(diphenylphosphoryl)amide dppaOE ${ }^{\ominus}$ erhalten werden (Abb. 50). In diesen Fällen ergaben die Umsetzungen einige unerwartete Ergebnisse. Es konnte beispielsweise der Komplex $\left[\mathrm{Cu}_{3}\left(\mathrm{dppaO}_{2}\right)_{2}\left(\left(\mathrm{OSiMe}_{2}\right)_{2} \mathrm{O}\right)_{2}(\text { thf })_{2}\right] \quad \underline{8}$ isoliert werden, der das Schliffettfragment 
$\left[\mathrm{Cu}_{3}(\mathrm{dppaOS})_{3}\right] \underline{10}$

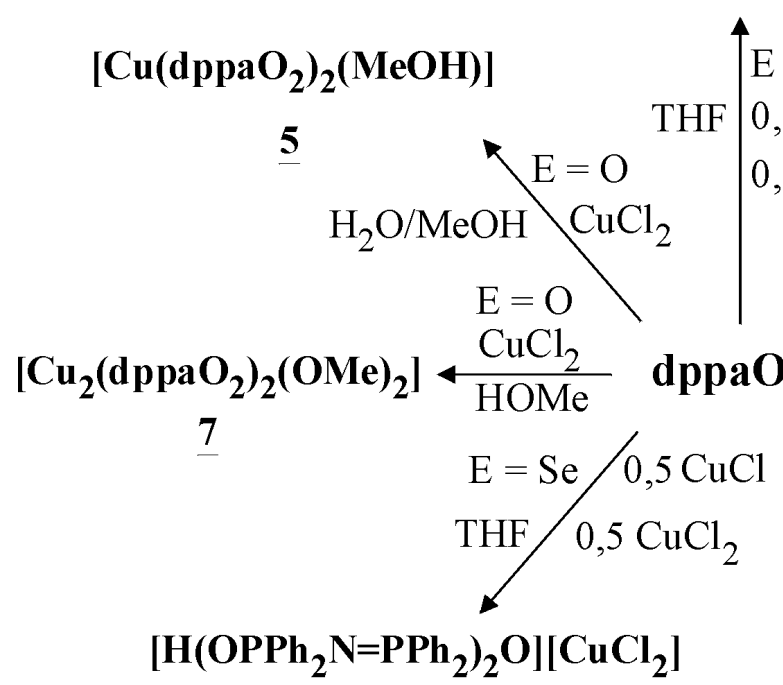

12

\section{$\left[\mathrm{Cu}_{4}(\mathrm{O})\left(\mathrm{dppaO}_{2}\right)_{4} \mathrm{Cl}_{2}\right] \underline{9}$}

Abb. 50: Reaktionen von dppaOE ${ }^{\ominus}$ mit $\mathrm{CuCl}$ und $\mathrm{CuCl}_{2}$

$\left[\left(\mathrm{OSiMe}_{2}\right)_{2} \mathrm{O}\right]^{2 \ominus}$ als Liganden enthält.

In Komplexierungsreaktionen von Bis(diphenylchalkogenophosphoryl)amiden $\operatorname{dppaE}_{2}{ }^{\ominus}$ $(\mathrm{E}=\mathrm{O}, \mathrm{S}, \mathrm{Se})$ mit $\mathrm{Cu}^{\mathrm{I}}$ und $\mathrm{Ag}^{\mathrm{I}}$ können sich, abhängig von den Mengenverhältnissen der Edukte und der Zugabe unterschiedlicher Basen, verschiedene mehrkernige $\mathrm{Cu}^{\mathrm{I}}$ - und $\mathrm{Ag}^{\mathrm{I}}$-Verbindungen bilden.

Mittels Röntgenstrukturanalyse konnten die Verbindungen $\left[\mathrm{NaCu}_{2}\left(\mathrm{dppaE}_{2}\right)_{3}(\mathrm{thf})_{2}\right]$ $(\mathrm{E}=\mathrm{S}: \underline{13} ; \mathrm{E}=\mathrm{Se}: \underline{14})$ sowie die trimeren Verbindungen $\left[\mathrm{Cu}_{3}\left(\mathrm{dppaE}_{2}\right)_{3}\right](\mathrm{E}=\mathrm{S}$ und $\mathrm{O}$ : $\underline{10} ; \mathrm{E}=\mathrm{S}: \underline{15} ; \mathrm{E}=\mathrm{Se}: \underline{16}),\left[\mathrm{Ag}_{3}\left(\mathrm{dppaS}_{2}\right)_{3}\right] \underline{17}$ und der Komplex $\left[\mathrm{Na}_{2} \mathrm{Ag}_{2}\left(\mathrm{dppaS}_{2}\right)_{4}(\mathrm{thf})_{4}\right]$ 18 charakterisiert werden.

Durch Reaktionen in der Schmelze gelang die Synthese der vierkernigen Verbindungen $\left[\mathrm{Cu}_{4}\left(\mathrm{dppaS}_{2}\right)_{3}\right]_{2}\left[\mathrm{CuCl}_{2}\right][\mathrm{OH}] \underline{19}$ und $\left[\mathrm{Cu}_{4}\left(\mathrm{dppaSe}_{2}\right)_{3}\right]\left[\mathrm{CuCl}_{2}\right] \underline{20}$. Werden zusätzlich andere Liganden wie Thioharnstoff eingesetzt, so bildet sich z.B. der Komplex $\left[\mathrm{Cu}\left(\mathrm{dppaS}_{2}\right)(\mathrm{th})\right]$ $\underline{21}$ (Abb. 51).

Geht man von den phenylsubstituierten Phosphorylamiden zu den Methylderivaten über, so bilden sich der fünfkernige Komplex $\left[\mathrm{Cu}_{5}\left(\mathrm{dmpaS}_{2}\right)_{5}\right] \underline{23}$ und die höherkernigen Verbindungen $\left[\mathrm{Cu}_{8}\left(\mathrm{dmpaS}_{2}\right)_{6} \mathrm{Cl}_{2}\right] \underline{24}$ und $\left[\mathrm{Cu}_{10}\left(\mathrm{dmpaS}_{2}\right)_{6} \mathrm{Cl}_{4}\right] \underline{25}$ sowie die polymere Verbindung $\left[\mathrm{KCu}\left(\mathrm{dmpaS}_{2}\right)_{2}(\mathrm{thf})\right]_{\infty} \underline{26}($ Abb. 52). 


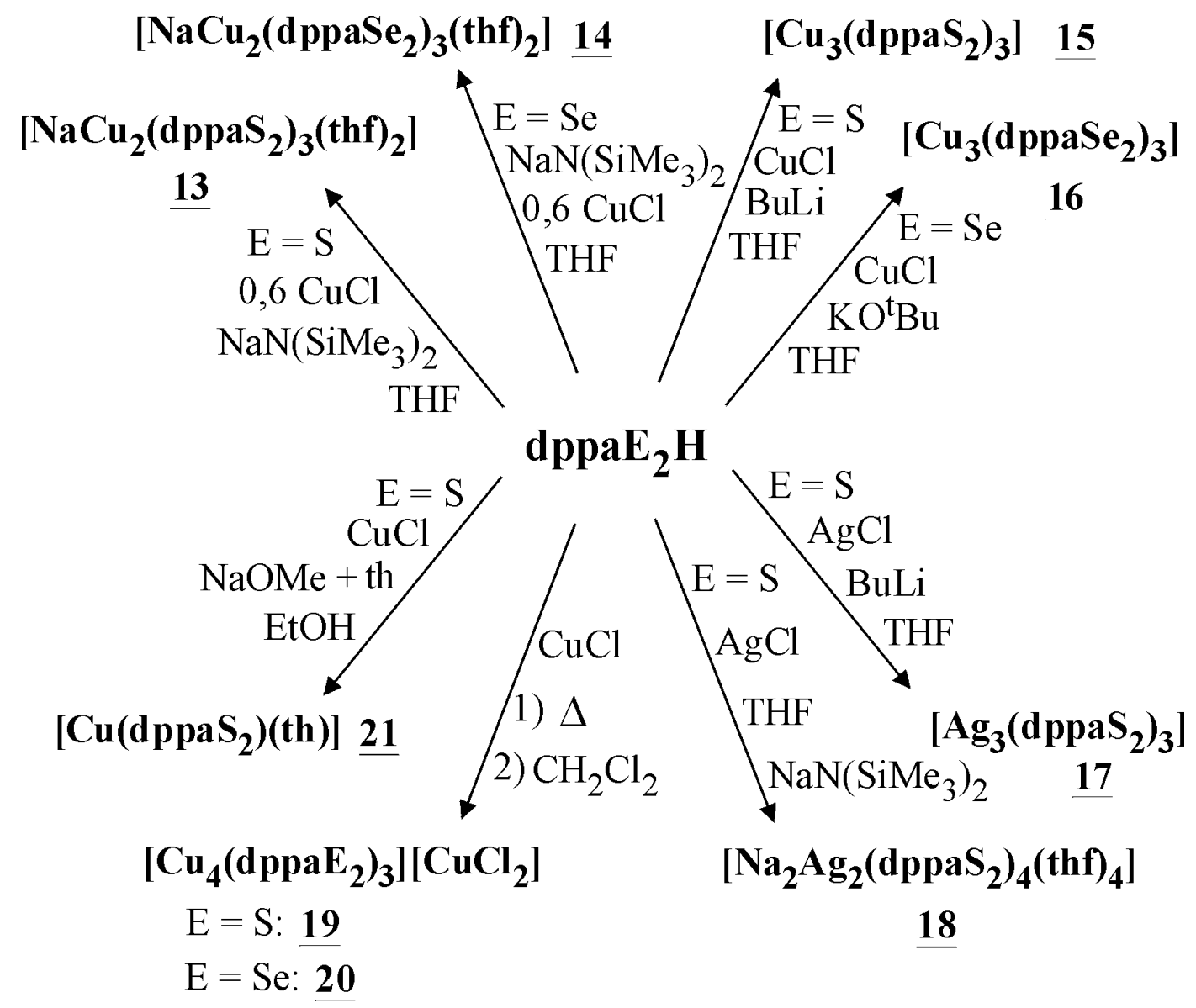

Abb. 51: Reaktionen von dppaE $\mathrm{E}_{2} \mathrm{H}$ mit $\mathrm{CuCl}$ und $\mathrm{AgCl}$

Es wurden außerdem ${ }^{31} \mathrm{P}-\mathrm{NMR}$-Untersuchungen zur weiteren Charakterisierung der Komplexe $\left[\mathrm{Cu}_{3}\left(\mathrm{dppaS}_{2}\right)_{3}\right] \underline{15},\left[\mathrm{NaCu}_{2}\left(\mathrm{dppaS}_{2}\right)_{3}(\mathrm{thf})_{2}\right] \underline{13}$ und $\left[\mathrm{Cu}_{10}\left(\mathrm{dmpaS}_{2}\right)_{6} \mathrm{Cl}_{4}\right] \underline{25}$ sowie deren Verhalten in Lösung bei unterschiedlichen Temperaturen durchgeführt. 
$\left[\mathrm{Cu}_{2}\left(\mathrm{dmpaS}_{2} \mathrm{H}\right)_{2} \mathrm{Cl}_{2}\right] \underline{21}$

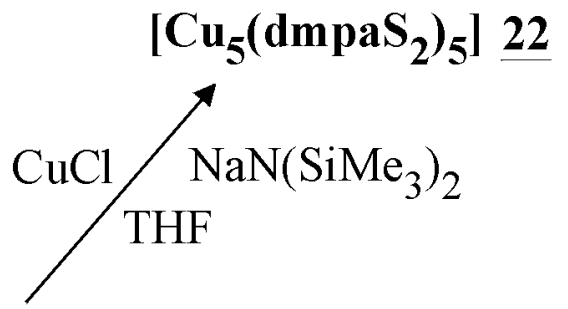

$4 / 3 \mathrm{CuCl}$

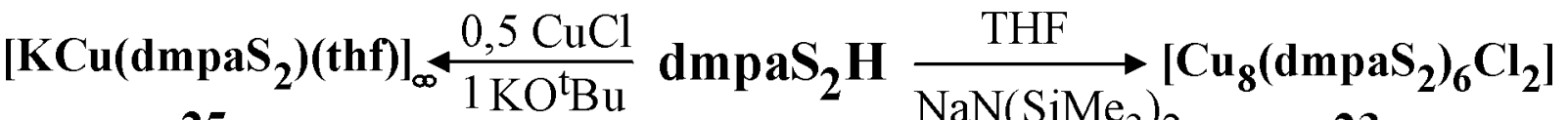
25

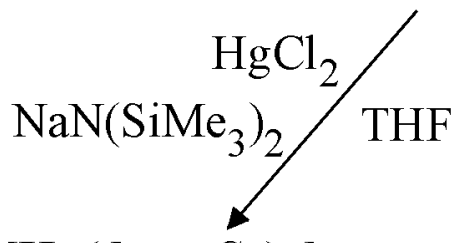

$\left[\mathrm{Hg}\left(\mathrm{dmpaS}_{2}\right)_{2}\right] \underline{26}$

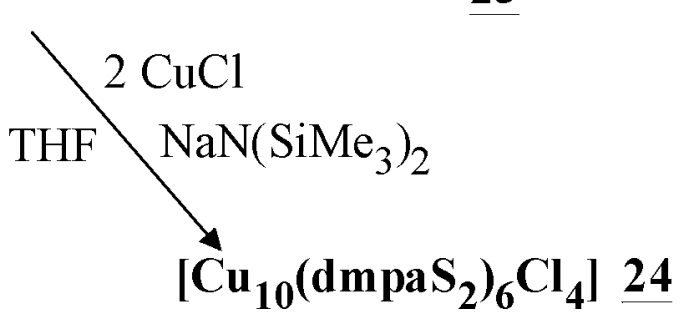

Abb. 52: Reaktionen von dmpaS $\mathrm{H}_{2} \mathrm{Hit} \mathrm{CuCl}, \mathrm{CuCl}_{2}$ und $\mathrm{HgCl}_{2}$ 


\section{Tabellarische Zusammenfassung der durchgeführ- ten Reaktionen}

\begin{tabular}{|c|c|c|c|}
\hline Edukt & Reaktanden & LM & Ergebnisse \\
\hline $\mathrm{CuCl}_{2}$ & $\mathrm{dppmO}{ }_{2} \mathrm{H}+\mathrm{KO}^{\mathrm{t}} \mathrm{Bu}$ & THF & {$\left[\mathrm{Cu}\left(\mathrm{dppmO}_{2} \mathrm{H}\right)_{3}\right]\left[\mathrm{CuCl}_{4}\right]$} \\
\hline $\mathrm{CuCl}_{2}$ & $1,5 \mathrm{dppmO}_{2} \mathrm{H}$ & THF & farblose Krist. \\
\hline $\mathrm{CuCl}$ & $\mathrm{dppmS}_{2} \mathrm{H}$ & Schmelze & {$\left[\mathrm{Cu}_{2}\left(\mathrm{dppmS} \mathrm{H}_{2} \mathrm{H}\right)_{2} \mathrm{Cl}\right]\left[\mathrm{CuCl}_{2}\right]$} \\
\hline $\mathrm{CuCl}$ & $\mathrm{dppmS}_{2} \mathrm{H}+\mathrm{NaN}\left(\mathrm{SiMe}_{3}\right)_{2}$ & THF & braunes Öl \\
\hline $\mathrm{CuCl}$ & $\mathrm{dppmS}_{2} \mathrm{H}+\mathrm{NaN}\left(\mathrm{SiMe}_{3}\right)_{2}$ & DME & amorpher, brauner Nd. \\
\hline $\mathrm{CuCl}$ & $\mathrm{dppmS}_{2} \mathrm{H}+\mathrm{KO}^{\mathrm{t}} \mathrm{Bu}$ & $\begin{array}{l}\text { THF/ } \\
\text { Heptan }\end{array}$ & $\begin{array}{l}{\left[\mathrm{K}_{2} \mathrm{Cu}_{2}\left(\mathrm{dppmS}_{2}\right)_{4}(\mathrm{thf})_{2}\right]} \\
\mathrm{dppmS}_{2} \mathrm{H}\end{array}$ \\
\hline $\mathrm{CuCl}$ & $\mathrm{dppmS}_{2} \mathrm{H}+\mathrm{BuLi}$ & THF, & $\mathrm{dppmS}_{2} \mathrm{H}$ \\
\hline $\mathrm{CuCl}$ & $\mathrm{dppmS}_{2} \mathrm{H}+2 \mathrm{BuLi}$ & THF & gelbe Krist. ${ }^{\star}$, gelbes Öl \\
\hline $\mathrm{CuCl}$ & $\mathrm{dppmS}_{2} \mathrm{H}+2 \mathrm{BuLi}$ & $\begin{array}{l}\text { THF/ } \\
\text { Heptan }\end{array}$ & orangefarbenes Öl \\
\hline $\mathrm{CuCl}$ & $2 \mathrm{dppmS}_{2} \mathrm{H}+2 \mathrm{BuLi}$ & $\begin{array}{l}\text { THF/ } \\
\text { Heptan }\end{array}$ & {$\left[\mathrm{Li}\left(\mathrm{dppmS}_{2}\right)(\mathrm{thf})_{2}\right]$} \\
\hline $\mathrm{CuCl}$ & $\mathrm{dppmS}_{2} \mathrm{H}+\mathrm{NaN}\left(\mathrm{SiMe}_{3}\right)_{2}$ & Toluol & {$\left[\mathrm{Na}\left(\mathrm{dppmS}_{2}\right)\right]$} \\
\hline $\mathrm{CuCl}$ & $\begin{array}{l}0,75 \mathrm{dppmS}_{2} \mathrm{H}+ \\
0,75 \mathrm{NaN}\left(\mathrm{SiMe}_{3}\right)_{2}+3 \mathrm{~S}\end{array}$ & THF & $\mathrm{dppmS}_{2} \mathrm{H}$ \\
\hline $\mathrm{CuCl}$ & $\begin{array}{l}\operatorname{dppmS} \mathrm{H}_{2} \mathrm{H}+ \\
\mathrm{NaN}\left(\mathrm{SiMe}_{3}\right)_{2}+2,5 \mathrm{~S}\end{array}$ & THF & {$\left[\mathrm{Na}\left(\mathrm{S}_{2} \mathrm{PPh}_{2}(\mathrm{thf})_{2}\right)\right]$} \\
\hline $\mathrm{CuCl}$ & $\mathrm{dppmS}_{2} \mathrm{H}+1,6 \mathrm{BuLi}+5 \mathrm{~S}$ & THF & dppmS $_{2} \mathrm{H}$, gelbe Krist. ${ }^{\star \star}$ \\
\hline $\mathrm{AgCl}$ & $2 \mathrm{dppmS}_{2} \mathrm{H}+2 \mathrm{KO}^{\mathrm{t}} \mathrm{Bu}$ & $\begin{array}{l}\text { THF/ } \\
\text { Heptan }\end{array}$ & $\mathrm{dppmS}_{2} \mathrm{H}$ \\
\hline $\mathrm{AuCl}\left(\mathrm{PPh}_{3}\right)$ & $\mathrm{dppmS}_{2} \mathrm{H}+\mathrm{KO}^{\mathrm{t}} \mathrm{Bu}$ & $\begin{array}{l}\text { THF/ } \\
\text { Heptan }\end{array}$ & $\begin{array}{l}{\left[\mathrm{Au}_{2}\left(\mathrm{dppmS}_{2}\right)\left(\mathrm{PPh}_{3}\right)_{2}\right],} \\
\mathrm{dppmS}_{2} \mathrm{H}\end{array}$ \\
\hline $\begin{array}{l}{\left[\mathrm{Au}_{2}\left(\mathrm{dppmS} \mathrm{S}_{2}\right)\right.} \\
\left.\left(\mathrm{PPh}_{3}\right)_{2}\right]\end{array}$ & $10 \mathrm{~S}$ & THF & $\mathrm{S}_{8}$ \\
\hline $\mathrm{CuCl}$ & $\mathrm{dppmSe}{ }_{2} \mathrm{H}+\mathrm{NaN}\left(\mathrm{SiMe}_{3}\right)_{2}$ & THF & {$\left[\mathrm{Cu}_{2}\left(\mathrm{dppmSe}_{3}\right)_{2}\right]$} \\
\hline $\mathrm{CuCl}$ & $\mathrm{dppmSe}_{2} \mathrm{H}+\mathrm{KO}^{\mathrm{t}} \mathrm{Bu}$ & $\begin{array}{l}\text { THF/ } \\
\text { Heptan }\end{array}$ & amorpher, brauner Nd. \\
\hline $\mathrm{AuCl}\left(\mathrm{PPh}_{3}\right)$ & $\mathrm{dppmSe}_{2} \mathrm{H}+2 \mathrm{BuLi}$ & $\begin{array}{l}\text { THF/ } \\
\text { Heptan }\end{array}$ & gelbes Öl \\
\hline $\mathrm{AuCl}\left(\mathrm{PPh}_{3}\right)$ & $\begin{array}{l}\mathrm{dppmSe}_{2} \mathrm{H}+2 \mathrm{BuLi}+ \\
5 \mathrm{~S}\end{array}$ & $\begin{array}{l}\text { THF/ } \\
\text { Heptan }\end{array}$ & dppmS $_{2} \mathrm{H}$, gelbe Krist. ${ }^{\star}$ \\
\hline $\mathrm{CuCl}$ & $\begin{array}{l}0,5 \mathrm{dppaO}_{2} \mathrm{H}+ \\
0,5 \mathrm{KO}^{\mathrm{t}} \mathrm{Bu}\end{array}$ & THF & $\begin{array}{l}{\left[\mathrm{Cu}_{3}\left(\mathrm{dppaO}_{2}\right)_{2}\left(\left(\mathrm{OSiMe}_{2}\right)_{2} \mathrm{O}\right)_{2}\right.} \\
\left.(\mathrm{thf})_{2}\right]\end{array}$ \\
\hline $\mathrm{CuCl}$ & $\begin{array}{l}0,5 \mathrm{dppaO}_{2} \mathrm{H}+ \\
0,5 \mathrm{NaN}\left(\mathrm{SiMe}_{3}\right)_{2}+\mathrm{THPh}_{2}\end{array}$ & THF & {$\left[\mathrm{CuCl}\left(\mathrm{thPh}_{2}\right)\left(\mathrm{thf}_{2}\right]\right.$} \\
\hline
\end{tabular}




\begin{tabular}{|c|c|c|c|}
\hline Edukt & Reaktanden & LM & Ergebnisse \\
\hline $\mathrm{CuCl}$ & $\begin{array}{l}0,67 \mathrm{dppaO}_{2} \mathrm{H}+ \\
0,67 \mathrm{KO}^{\mathrm{t}} \mathrm{Bu}+0,67 \mathrm{~K}_{4} \mathrm{P}_{2} \mathrm{O}_{7}\end{array}$ & THF & {$\left[\mathrm{Cu}\left(\mathrm{dppaO}_{2}\right)_{2}\right]$} \\
\hline $\mathrm{CuCl}_{2}$ & $\mathrm{dppaO}_{2} \mathrm{H}+\mathrm{KO}^{\mathrm{t}} \mathrm{Bu}$ & THF & $\begin{array}{l}\left.\left[\mathrm{Cu}_{2}(\mathrm{dppaO})_{2}\right)_{2} \mathrm{Cl}_{2}(\mathrm{thf})_{2}\right], \\
{\left[\mathrm{Cu}\left(\mathrm{dppaO}_{2}\right)_{2}\right]}\end{array}$ \\
\hline $\mathrm{CuCl}_{2}$ & $2 \mathrm{dppaO}_{2} \mathrm{H}+2 \mathrm{KO}^{t} \mathrm{Bu}$ & THF & {$\left[\mathrm{Cu}\left(\mathrm{dppaO}_{2}\right)_{2}\right]$} \\
\hline $\mathrm{CuCl}_{2}$ & $2 \mathrm{dppaO}_{2} \mathrm{H}+2 \mathrm{KOH}$ & $\begin{array}{l}\mathrm{H}_{2} \mathrm{O} / \\
\mathrm{MeOH}\end{array}$ & {$\left[\mathrm{Cu}\left(\mathrm{dppaO}_{2}\right)_{2}(\mathrm{MeOH})\right]$} \\
\hline $\mathrm{CuCl}_{2}$ & $\begin{array}{l}0,67 \mathrm{dppaO}_{2} \mathrm{H}+ \\
1,33\left[\mathrm{OSiMe}_{2}\right]_{\infty}+2 \mathrm{KO}^{\mathrm{t}} \mathrm{Bu}\end{array}$ & THF & $\begin{array}{l}{\left[\mathrm{Cu}_{3}\left(\mathrm{dppaO}_{2}\right)_{2}\left(\left(\mathrm{OSiMe}_{2}\right)_{2} \mathrm{O}\right)_{2}\right.} \\
\left.(\mathrm{thf})_{2}\right],\left[\mathrm{Cu}_{4}(\mathrm{O})\left(\mathrm{dppaO}_{2}\right)_{4} \mathrm{Cl}_{2}\right]\end{array}$ \\
\hline $\mathrm{CuCl}_{2}$ & $\begin{array}{l}0,5 \mathrm{dppaO}_{2} \mathrm{H}+3 \mathrm{NEt}_{3}+ \\
0,53,5-\mathrm{DTBC}\end{array}$ & $\mathrm{MeOH}$ & $\left.\left[\mathrm{Cu}_{2}(\mathrm{dppaO})_{2}\right)_{2}(\mathrm{OMe})_{2}\right]$ \\
\hline $\mathrm{Cu}\left(\mathrm{O}^{\mathrm{t}} \mathrm{Bu}\right)_{2}$ & $\mathrm{dppaO}_{2} \mathrm{H}$ & THF & {$\left[\mathrm{Cu}_{2}(\mathrm{dppaO})_{2}\left(\mathrm{O}^{\mathrm{t}} \mathrm{Bu}\right)_{2}\right]$} \\
\hline $\mathrm{CuCl}$ & $0,5 \mathrm{dppaOSH}+0,5 \mathrm{BuLi}$ & THF & amorpher, grüner Nd. \\
\hline $\mathrm{CuCl}$ & $\begin{array}{l}0,5 \text { dppaOSH }+ \\
\mathrm{NaN}_{\left(\mathrm{SiMe}_{3}\right)_{2}}\end{array}$ & THF & amorpher, brauner Nd. \\
\hline $\mathrm{CuCl}$ & dppaOSH & Schmelze & farblose und violette Krist.* \\
\hline $\mathrm{CuCl}$ & dppaOSH $+\mathrm{NaN}\left(\mathrm{SiMe}_{3}\right)_{2}$ & THF & gelbes Öl \\
\hline $\mathrm{CuCl}$ & $\begin{array}{l}\text { 1,5 dppaOSH }+ \\
1,5 \mathrm{NaN}\left(\mathrm{SiMe}_{3}\right)_{2}\end{array}$ & THF & gelbes Öl \\
\hline $\mathrm{CuCl}+\mathrm{CuCl}_{2}$ & $\begin{array}{l}2 \mathrm{dppaOSH}+ \\
2 \mathrm{NaN}\left(\mathrm{SiMe}_{3}\right)_{2} \\
\end{array}$ & THF & $\begin{array}{l}{\left[\mathrm{Cu}_{3}(\mathrm{dppaOS})_{3}\right]} \\
{\left[\mathrm{Cu}\left(\mathrm{dppaO}_{2}\right)_{2}\right]}\end{array}$ \\
\hline $\begin{array}{l}\mathrm{Cu}\left(\mathrm{O}^{\mathrm{t}} \mathrm{Bu}\right)_{2}+ \\
\mathrm{CuCl}_{2}\end{array}$ & dppaOSH & $\begin{array}{l}\text { THF } / \\
\mathrm{CH}_{2} \mathrm{Cl}_{2} \\
\end{array}$ & amorpher, brauner Nd. \\
\hline $\mathrm{CuCl}$ & dppaOSeH $+\mathrm{NaN}\left(\mathrm{SiMe}_{3}\right)_{2}$ & THF & braune und farblose Krist. \\
\hline $\mathrm{CuCl}$ & $\mathrm{dppaOSeH}+\mathrm{KO}^{t} \mathrm{Bu}$ & THF & $\left.\left[\mathrm{Cu}(\operatorname{dppaO})_{2}\right)_{2}\left(\mathrm{NH}_{3}\right)_{3}\right]_{\infty}$ \\
\hline $\mathrm{CuCl}+\mathrm{CuCl}_{2}$ & $2 \mathrm{dppaOSeH}+2 \mathrm{KO}^{\mathrm{t}} \mathrm{Bu}$ & THF & {$\left[\mathrm{H}\left((\mathrm{O}) \mathrm{PPh}_{2} \mathrm{NPPh}_{2}\right)_{2} \mathrm{O}\right]\left[\mathrm{CuCl}_{2}\right]$} \\
\hline $\mathrm{CuCl}$ & $\begin{array}{l}0,5 \mathrm{dppaS}_{2} \mathrm{H}+ \\
0,5 \mathrm{NaN}\left(\mathrm{SiMe}_{3}\right)_{2}\end{array}$ & THF & brauner, amorpher Nd. \\
\hline $\mathrm{CuCl}$ & $\mathrm{dppaS}_{2} \mathrm{H}$ & Schmelze & {$\left[\mathrm{Cu}_{4}\left(\mathrm{dppaS}_{2}\right)_{3}\right]_{2}\left[\mathrm{CuCl}_{2}\right][\mathrm{OH}]$} \\
\hline $\mathrm{CuCl}$ & $\mathrm{dppaS}_{2} \mathrm{H}+\mathrm{NaN}\left(\mathrm{SiMe}_{3}\right)_{2}$ & THF & $\begin{array}{l}{\left[\mathrm{Cu}_{3}\left(\mathrm{dppaS}_{2}\right)_{3}\right]} \\
{\left[\mathrm{NaCu}_{2}\left(\mathrm{dppaS}_{2}\right)_{3}(\mathrm{thf})_{2}\right]} \\
\end{array}$ \\
\hline $\mathrm{CuCl}$ & $\begin{array}{l}1,5 \mathrm{dppaS}_{2} \mathrm{H}+ \\
1,5 \mathrm{NaN}\left(\mathrm{SiMe}_{3}\right)_{2}\end{array}$ & THF & {$\left[\mathrm{NaCu}_{2}\left(\mathrm{dppaS}_{2}\right)_{3}(\mathrm{thf})_{2}\right]$} \\
\hline $\mathrm{CuCl}$ & $2 \mathrm{dppaS}_{2} \mathrm{H}+2 \mathrm{NaN}\left(\mathrm{SiMe}_{3}\right)_{2}$ & THF & {$\left[\mathrm{NaCu}_{2}\left(\mathrm{dppaS}_{2}\right)_{3}(\mathrm{thf})_{2}\right]$} \\
\hline $\mathrm{CuCl}$ & $\mathrm{dppaS}_{2} \mathrm{H}+\mathrm{BuLi}$ & THF & {$\left[\mathrm{Cu}_{3}\left(\mathrm{dppaS}_{2}\right)_{3}\right]$} \\
\hline $\mathrm{CuCl}$ & $\mathrm{dppaS}_{2} \mathrm{H}+\mathrm{KO}^{t} \mathrm{Bu}$ & THF & {$\left[\mathrm{Cu}_{3}\left(\mathrm{dppaS}_{2}\right)_{3}\right]$} \\
\hline $\mathrm{CuCl}$ & $\mathrm{dppaS}_{2} \mathrm{H}+\mathrm{KO}^{\mathrm{t}} \mathrm{Bu}$ & $\mathrm{CH}_{3} \mathrm{CN}$ & farblose und braune Krist. \\
\hline $\mathrm{CuCl}$ & $2 \mathrm{dppaS}_{2} \mathrm{H}+2 \mathrm{KO}^{t} \mathrm{Bu}$ & $\begin{array}{l}\mathrm{Et}_{2} \mathrm{O} / \\
\mathrm{CH}_{3} \mathrm{CN}\end{array}$ & farblose Krist. ${ }^{\star}$ \\
\hline
\end{tabular}




\begin{tabular}{|c|c|c|c|}
\hline Edukt & Reaktanden & LM & Ergebnisse \\
\hline $\mathrm{CuCl}$ & $\mathrm{dppaS}_{2} \mathrm{H}+\mathrm{NaOMe}+\mathrm{TH}$ & $\mathrm{EtOH}$ & $\begin{array}{l}{\left[\mathrm{Cu}\left(\mathrm{dppaS}_{2}\right)(\mathrm{th})\right]} \\
{\left[\mathrm{Cu}_{3}\left(\mathrm{dppaS}_{2}\right)_{3}\right]}\end{array}$ \\
\hline $\mathrm{CuCl}$ & $\mathrm{dppaS}_{2} \mathrm{H}+\mathrm{NaOMe}+\mathrm{TH}$ & $\mathrm{MeOH}$ & {$\left[\mathrm{Cu}\left(\mathrm{dppaS}_{2}\right)(\mathrm{th})\right]$} \\
\hline $\mathrm{CuCl}$ & $\begin{array}{l}\operatorname{dppaS}_{2} \mathrm{H}+2 \mathrm{NaN}\left(\mathrm{SiMe}_{3}\right)_{2}+ \\
\mathrm{TH}\end{array}$ & THF & farblose Krist. \\
\hline $\mathrm{CuCl}$ & $\begin{array}{l}0,5 \mathrm{dppaS}_{2} \mathrm{H}+0,5 \mathrm{THEt}_{2}+ \\
0,5 \mathrm{NaN}\left(\mathrm{SiMe}_{3}\right)_{2}\end{array}$ & THF & $\begin{array}{l}{\left[\mathrm{Cu}_{4}\left(\mathrm{dppaS}_{2}\right)_{3}\right]\left[\mathrm{Cu}_{2}\left(\mathrm{dppaS}_{2}\right)_{2}\right.} \\
\left.\left(\mathrm{thEt}_{2}\right) \mathrm{Cl}\right]\left[\mathrm{CuCl}_{2}\right]\end{array}$ \\
\hline $\mathrm{CuCl}$ & $\begin{array}{l}\operatorname{dppaS}_{2} \mathrm{H}+\mathrm{NaN}\left(\mathrm{SiMe}_{3}\right)_{2}+ \\
\mathrm{THPh}_{2}\end{array}$ & THF & {$\left[\mathrm{Cu}\left(\mathrm{dppaS}_{2}\right)\left(\mathrm{thPh}_{2}\right)\right]$} \\
\hline $\mathrm{CuCl}$ & $\begin{array}{l}0,5 \mathrm{dppaS}_{2} \mathrm{H}+ \\
0,5 \mathrm{NaN}\left(\mathrm{SiMe}_{3}\right)_{2}+\mathrm{TH}_{2}\end{array}$ & THF & {$\left[\mathrm{Na}\left(\mathrm{dppaS}_{2}\right)\right]$} \\
\hline $\mathrm{CuCl}$ & $\begin{array}{l}0,5 \text { dppaS }_{2} \mathrm{H}+\mathrm{NaOMe}+ \\
0,5 \mathrm{TH}_{2}\end{array}$ & $\begin{array}{l}\mathrm{MeOH} / \\
\mathrm{THF}\end{array}$ & weißer, amorpher Nd. \\
\hline $\mathrm{CuCl}$ & $\begin{array}{l}\mathrm{dppaS}_{2} \mathrm{H}+\mathrm{NaN}\left(\mathrm{SiMe}_{3}\right)_{2}+ \\
\mathrm{O}_{2(\mathrm{~g})}\end{array}$ & THF & farblose Krist. \\
\hline $\mathrm{CuCl}$ & $\begin{array}{l}\mathrm{dppaS}_{2} \mathrm{H}+\mathrm{NaN}\left(\mathrm{SiMe}_{3}\right)_{2}+ \\
3 \mathrm{~S}\end{array}$ & THF & $\begin{array}{l}{\left[\mathrm{Cu}_{3}\left(\mathrm{dppaS}_{2}\right)_{3}\right]} \\
{\left[\mathrm{Cu}_{4}\left(\mathrm{dppaS}_{2}\right)_{3}\right]_{2}\left[\mathrm{CuCl}_{2}\right][\mathrm{OH}]}\end{array}$ \\
\hline $\mathrm{CuCl}$ & $\begin{array}{l}\mathrm{dppaS}_{2} \mathrm{H}+\mathrm{NaN}\left(\mathrm{SiMe}_{3}\right)_{2}+ \\
10 \mathrm{Se}\end{array}$ & THF & {$\left[\mathrm{Cu}_{3}\left(\mathrm{dppaS}_{2}\right)_{3}\right]$} \\
\hline $\mathrm{CuCl}$ & $\begin{array}{l}0,5 \mathrm{dppaS}_{2} \mathrm{H}+0,5 \mathrm{KO}^{\mathrm{t}} \mathrm{Bu}+ \\
\mathrm{KI}\end{array}$ & $\mathrm{CH}_{3} \mathrm{CN}$ & amorpher, weißer Nd. \\
\hline $\mathrm{CuCl}$ & $\begin{array}{l}\operatorname{dppaS}_{2} \mathrm{H}+\mathrm{NaN}\left(\mathrm{SiMe}_{3}\right)_{2}+ \\
\mathrm{KI}\end{array}$ & $\begin{array}{l}\mathrm{THF} / \\
\mathrm{MeOH}\end{array}$ & rosafarbene Krist. \\
\hline $\mathrm{CuCl}$ & $\begin{array}{l}\mathrm{dppaS}_{2} \mathrm{H}+2 \mathrm{Na}+\mathrm{HS}^{\mathrm{t}} \mathrm{Bu} \\
+0,3\left(\mathrm{Me}_{2} \mathrm{SiS}\right)_{3}\end{array}$ & THF & weißes Gel \\
\hline $\mathrm{CuCl}+\mathrm{Cu}_{2} \mathrm{~S}$ & $\mathrm{dppaS}_{2} \mathrm{H}+\mathrm{NaN}\left(\mathrm{SiMe}_{3}\right)_{2}$ & THF & {$\left[\mathrm{Cu}_{3}\left(\mathrm{dppaS}_{2}\right)_{3}\right]$} \\
\hline $\mathrm{CuCl}+\mathrm{Fe}(\mathrm{CO})_{5}$ & $\mathrm{dppaS}_{2} \mathrm{H}+\mathrm{NaN}\left(\mathrm{SiMe}_{3}\right)_{2}$ & THF & $\begin{array}{l}{\left[\mathrm{NaCu}_{2}\left(\mathrm{dppaS}_{2}\right)_{3}(\mathrm{thf})_{2}\right],} \\
{\left[\mathrm{Cu}_{3}\left(\mathrm{dppaS}_{2}\right)_{3}\right], \mathrm{Fe}_{2}(\mathrm{CO})_{9}}\end{array}$ \\
\hline $\mathrm{CuO}^{\mathrm{t}} \mathrm{Bu}$ & $0,5 \mathrm{dppaS}_{2} \mathrm{H}$ & THF & amorpher, brauner Nd. \\
\hline $\mathrm{CuO}^{t} \mathrm{Bu}$ & $\mathrm{dppaS}_{2} \mathrm{H}$ & $\begin{array}{l}\text { Heptan/ } \\
\text { THF }\end{array}$ & gelbe Krist. $^{\star}$ \\
\hline $\mathrm{CuO}^{\mathrm{t}} \mathrm{Bu}+\mathrm{CuCl}_{2}$ & $\mathrm{dppaS}_{2} \mathrm{H}$ & $\mathrm{MeOH}$ & roter $\mathrm{Nd}$. \\
\hline $\mathrm{CuO}^{\mathrm{t}} \mathrm{Bu}+\mathrm{CuCl}_{2}$ & $\mathrm{dppaS}_{2} \mathrm{H}$ & $\mathrm{CH}_{2} \mathrm{Cl}_{2}$ & {$\left[\mathrm{Cu}_{4}\left(\mathrm{dppaS}_{2}\right)_{3}\right]_{2}\left[\mathrm{CuCl}_{2}\right][\mathrm{OH}]$} \\
\hline $\begin{array}{l}{\left[\mathrm{NaCu}_{2}\left(\mathrm{dppaS}_{2}\right)_{3}\right.} \\
\left.(\mathrm{thf})_{2}\right]\end{array}$ & $\mathrm{O}_{2(\mathrm{~g})}$ & THF & {$\left[\mathrm{NaCu}_{2}\left(\mathrm{dppaS}_{2}\right)_{3}(\mathrm{thf})_{2}\right]$} \\
\hline $\begin{array}{l}{\left[\mathrm{NaCu}_{2}\left(\mathrm{dppaS}_{2}\right)_{3}\right.} \\
\left.(\mathrm{thf})_{2}\right]\end{array}$ & $10 \mathrm{~S}$ & THF & {$\left[\mathrm{Cu}_{3}\left(\mathrm{dppaS}_{2}\right)_{3}\right]$} \\
\hline $\begin{array}{l}{\left[\mathrm{NaCu}_{2}\left(\mathrm{dppaS}_{2}\right)_{3}\right.} \\
\left.(\mathrm{thf})_{2}\right]\end{array}$ & $\mathrm{N}_{2} \mathrm{O}_{(\mathrm{g})}$ & THF & {$\left[\mathrm{NaCu}_{2}\left(\mathrm{dppaS}_{2}\right)_{3}(\mathrm{thf})_{2}\right]$} \\
\hline
\end{tabular}




\begin{tabular}{|c|c|c|c|}
\hline Edukt & Reaktanden & $\mathrm{LM}$ & Ergebnisse \\
\hline $\begin{array}{l}{\left[\mathrm{NaCu}_{2}\left(\mathrm{dppaS}_{2}\right)_{3}\right.} \\
\left.(\mathrm{thf})_{2}\right]\end{array}$ & 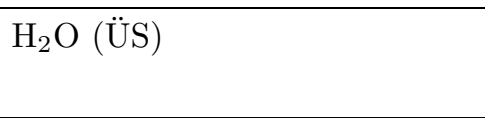 & $\mathrm{EtOH}$ & amorpher, weißer Nd. \\
\hline $\begin{array}{l}{\left[\mathrm{NaCu}_{2}\left(\mathrm{dppaS}_{2}\right)_{3}\right.} \\
\left.(\mathrm{thf})_{2}\right]\end{array}$ & $10 \mathrm{Na}_{2} \mathrm{O}_{2}$ & THF & amorpher, grüner Nd. \\
\hline $\begin{array}{l}{\left[\mathrm{NaCu}_{2}\left(\mathrm{dppaS}_{2}\right)_{3}\right.} \\
\left.(\mathrm{thf})_{2}\right]\end{array}$ & $\mathrm{H}_{2} \mathrm{O}_{2}$ (ÜS) & $\begin{array}{l}\text { THF/ } \\
\text { EtOH }\end{array}$ & amorpher, weißer Nd. \\
\hline $\begin{array}{l}{\left[\mathrm{NaCu}_{2}\left(\mathrm{dppaS}_{2}\right)_{3}\right.} \\
\left.(\mathrm{thf})_{2}\right]\end{array}$ & $\mathrm{H}_{2} \mathrm{O}_{2}$ (ÜS) & $\begin{array}{l}\text { THF / } \\
\mathrm{H}_{2} \mathrm{O}\end{array}$ & amorpher, weißer Nd. \\
\hline $\mathrm{CuCl}_{2}$ & $\mathrm{dppaS}_{2} \mathrm{H}$ & THF & amorpher, schwarzer Nd. \\
\hline $\mathrm{CuCl}_{2}$ & $\mathrm{dppaS}_{2} \mathrm{H}+\mathrm{NaN}\left(\mathrm{SiMe}_{3}\right)_{2}$ & THF & amorpher, weißer Nd. \\
\hline $\mathrm{CuCl}_{2}$ & $2 \mathrm{dppaS}_{2} \mathrm{H}+2 \mathrm{Na}$ & $\mathrm{H}_{2} \mathrm{O}$ & {$\left[\mathrm{Cu}_{3}\left(\mathrm{dppaS}_{2}\right)_{3}\right]$} \\
\hline $\mathrm{CuCl}_{2}+\mathrm{Cu}_{2} \mathrm{~S}$ & $\mathrm{dppaS}_{2} \mathrm{H}+\mathrm{NaN}\left(\mathrm{SiMe}_{3}\right)_{2}$ & THF & {$\left[\mathrm{Cu}_{4}\left(\mathrm{dppaS}_{2}\right)_{3}\right]_{2}\left[\mathrm{CuCl}_{2}\right][\mathrm{OH}]$} \\
\hline $\mathrm{AgCl}$ & $\begin{array}{l}0,5 \mathrm{dppaS}_{2} \mathrm{H}+ \\
0,5 \mathrm{NaN}\left(\mathrm{SiMe}_{3}\right)_{2}\end{array}$ & THF & farblose Krist. \\
\hline $\mathrm{AgCl}$ & $\mathrm{dppaS}_{2} \mathrm{H}+\mathrm{KO}^{\mathrm{t}} \mathrm{Bu}$ & THF & amorpher, weißer Nd. \\
\hline $\mathrm{AgCl}$ & $\mathrm{dppaS}_{2} \mathrm{H}+\mathrm{BuLi}$ & THF & {$\left[\mathrm{Ag}_{3}\left(\mathrm{dppaS}_{2}\right)_{3}\right]$} \\
\hline $\mathrm{AgCl}$ & $\begin{array}{l}\mathrm{dppaS}_{2} \mathrm{H}+\mathrm{NaN}\left(\mathrm{SiMe}_{3}\right)_{2}+ \\
\text { en }\end{array}$ & THF & {$\left[\mathrm{Na}_{2} \mathrm{Ag}_{2}\left(\mathrm{dppaS}_{2}\right)_{4}(\mathrm{thf})_{4}\right]$} \\
\hline $\mathrm{AuCl}\left(\mathrm{PPh}_{3}\right)$ & $\mathrm{dppaS}_{2} \mathrm{H}+\mathrm{KO}^{\mathrm{t}} \mathrm{Bu}+\mathrm{S}$ & THF & nadelförmige Krist. ${ }^{\star}$ \\
\hline $\mathrm{FeCl}_{2}$ & $\mathrm{dppaS}_{2} \mathrm{H}+\mathrm{NaN}\left(\mathrm{SiMe}_{3}\right)_{2}$ & THF & amorpher, brauner Nd. \\
\hline $\mathrm{FeCl}_{2}$ & $\mathrm{dppaS}_{2} \mathrm{H}+\mathrm{KO}^{t} \mathrm{Bu}$ & $\mathrm{CH}_{2} \mathrm{Cl}_{2}$ & {$\left[\mathrm{Fe}\left(\mathrm{dppaS}_{2} \mathrm{H}\right)_{3}\right]\left[\mathrm{FeCl}_{4}\right]$} \\
\hline $\mathrm{FeCl}_{3}$ & $\mathrm{dppaS}_{2} \mathrm{H}+\mathrm{NaN}\left(\mathrm{SiMe}_{3}\right)_{2}$ & THF & {$\left[\mathrm{Fe}_{2} \mathrm{Cl}_{3}(\mathrm{thf})_{6}\right]\left[\mathrm{FeCl}_{4}\right]$} \\
\hline $\mathrm{FeCl}_{3}$ & $\mathrm{dppaS}_{2} \mathrm{H}+3 \mathrm{NaN}\left(\mathrm{SiMe}_{3}\right)_{2}$ & THF & braune Krist. ${ }^{\star}$ \\
\hline $\mathrm{FeCl}_{3}$ & $\mathrm{dppaS}_{2} \mathrm{H}+\mathrm{BuLi}$ & $\mathrm{CH}_{2} \mathrm{Cl}_{2}$ & rote und gelbe Krist. \\
\hline $\mathrm{MnCl}_{2}$ & $\mathrm{dppaS}_{2} \mathrm{H}+\mathrm{NaOMe}$ & $\begin{array}{l}\text { THF/ } \\
\mathrm{MeOH}\end{array}$ & {$\left[\mathrm{Mn}\left(\mathrm{dppaS}_{2}\right)_{2}\right]$} \\
\hline$\left[\mathrm{Mn}\left(\mathrm{N}\left(\mathrm{SiMe}_{3}\right)_{2}\right)_{2}\right]$ & $\mathrm{dppaS}_{2} \mathrm{H}$ & Toluol & amorpher, brauner Nd. \\
\hline $\mathrm{CrCl}_{3}$ & $\mathrm{dppaS}_{2} \mathrm{H}+\mathrm{NaN}\left(\mathrm{SiMe}_{3}\right)_{2}$ & THF & {$\left[\mathrm{Cr}\left(\mathrm{dppaS}_{2}\right)_{3}\right]$} \\
\hline$\left[\mathrm{CrCl}_{3}(\mathrm{thf})_{3}\right]$ & $\mathrm{dppaS}_{2} \mathrm{H}+\mathrm{NaN}\left(\mathrm{SiMe}_{3}\right)_{2}$ & THF, & violette Krist. $^{\star}$ \\
\hline $\mathrm{CuCl}$ & $0,5 \mathrm{dppaSe}_{2} \mathrm{H}+0,5 \mathrm{BuLi}$ & THF & amorpher, weißer Nd. \\
\hline $\mathrm{CuCl}$ & $\begin{array}{l}0,67 \mathrm{dppaSe}_{2} \mathrm{H}+ \\
0,67 \mathrm{NaN}\left(\mathrm{SiMe}_{3}\right)_{2}\end{array}$ & THF & {$\left[\mathrm{NaCu}_{2}\left(\mathrm{dppaSe}_{2}\right)_{3}(\mathrm{thf})_{2}\right]$} \\
\hline $\mathrm{CuCl}$ & $0,75 \mathrm{dppaSe}_{2} \mathrm{H}+0,75 \mathrm{KO}^{\mathrm{t}} \mathrm{Bu}$ & THF & farblose, nadelförmige Krist. \\
\hline $\mathrm{CuCl}$ & $\mathrm{dppaSe}_{2} \mathrm{H}$ & Schmelze & {$\left[\mathrm{Cu}_{4}\left(\mathrm{dppaSe}_{2}\right)_{3}\right]\left[\mathrm{CuCl}_{2}\right]$} \\
\hline $\mathrm{CuCl}$ & $\mathrm{dppaSe}_{2} \mathrm{H}+\mathrm{NaN}\left(\mathrm{SiMe}_{3}\right)_{2}$ & THF & $\begin{array}{l}{\left[\mathrm{NaCu}_{2}\left(\mathrm{dppaSe}_{2}\right)_{3}(\mathrm{thf})_{2}\right],} \\
{\left[\mathrm{Cu}_{3}\left(\mathrm{dppaSe}_{2}\right)_{3}\right]}\end{array}$ \\
\hline $\mathrm{CuCl}$ & $\mathrm{dppaSe}_{2} \mathrm{H}+\mathrm{KN}\left(\mathrm{SiMe}_{3}\right)_{2}$ & THF & {$\left[\mathrm{Cu}_{3}\left(\mathrm{dppaSe}_{2}\right)_{3}\right]$} \\
\hline $\mathrm{CuCl}$ & $\mathrm{dppaSe} \mathrm{H}_{2} \mathrm{H}+\mathrm{KO}^{t} \mathrm{Bu}$ & THF & {$\left[\mathrm{Cu}_{3}\left(\mathrm{dppaSe}_{2}\right)_{3}\right]$} \\
\hline
\end{tabular}




\begin{tabular}{|c|c|c|c|}
\hline Edukt & Reaktanden & LM & Ergebnisse \\
\hline $\mathrm{CuCl}$ & $\mathrm{dppaSe}_{2} \mathrm{H}+\mathrm{KO}^{\mathrm{t}} \mathrm{Bu}$ & $\mathrm{CH}_{2} \mathrm{Cl}_{2}$ & {$\left[\mathrm{Cu}_{3}\left(\mathrm{dppaSe}_{2}\right)_{3}\right]$} \\
\hline $\mathrm{CuCl}$ & $\begin{array}{l}\text { 0,75 dppaSe } \mathrm{d}_{2} \mathrm{H}+ \\
0,75 \mathrm{NaN}\left(\mathrm{SiMe}_{3}\right)_{2}+\mathrm{S}\end{array}$ & THF & $\begin{array}{l}\text { amorpher, roter Nd., } \\
\text { gelbes Öl }\end{array}$ \\
\hline $\mathrm{CuO}^{\mathrm{t}} \mathrm{Bu}$ & $0,5 \mathrm{dppaSe}_{2} \mathrm{H}$ & THF & farblose Krist. ${ }^{\star}$ \\
\hline $\mathrm{CuCl}$ & $\begin{array}{l}\operatorname{dppaSe}_{2} \mathrm{H}+\mathrm{NaN}_{\left(\mathrm{SiMe}_{3}\right)_{2}} \\
+5 \mathrm{~S}\end{array}$ & THF & $\begin{array}{l}{\left[\mathrm{NaCu}_{2}\left(\mathrm{dppaSe}_{2}\right)_{3}(\mathrm{thf})_{2}\right],} \\
{\left[\mathrm{Cu}_{3}\left(\mathrm{dppaSe}_{2}\right)_{3}\right]}\end{array}$ \\
\hline $\mathrm{CuCl}$ & $\begin{array}{l}0,6 \mathrm{dppaSe}{ }_{2} \mathrm{H}+ \\
0,6 \mathrm{NaN}\left(\mathrm{SiMe}_{3}\right)_{2}+\mathrm{KI}\end{array}$ & $\begin{array}{l}\mathrm{THF} / \\
\mathrm{MeOH}\end{array}$ & farblose Krist. \\
\hline $\mathrm{CuCl}_{2}+\mathrm{Cu}_{2} \mathrm{~S}$ & $\mathrm{dppaSe}_{2} \mathrm{H}+\mathrm{NaN}\left(\mathrm{SiMe}_{3}\right)_{2}$ & THF, & amorpher, schwarzer Nd. \\
\hline $\mathrm{CuCl}_{2}+\mathrm{Cu}_{2} \mathrm{~S}$ & $\begin{array}{l}0,8 \mathrm{dppaSe}_{2} \mathrm{H}+ \\
0,8 \mathrm{NaN}\left(\mathrm{SiMe}_{3}\right)_{2}\end{array}$ & THF & $\begin{array}{l}{\left[\mathrm{Cu}\left(\mathrm{Ph}_{2} \mathrm{P}(\mathrm{Se}) \mathrm{NPPh}_{2}\right.\right.} \\
\left.\left(\mathrm{NHSiMe}_{3}\right)_{2} \mathrm{Cl}\right]\end{array}$ \\
\hline $\mathrm{CuCl}$ & $0,5 \mathrm{dmpaS}_{2} \mathrm{H}+0,5 \mathrm{BuLi}$ & THF & amorpher, brauner Nd. \\
\hline $\mathrm{CuCl}$ & $\begin{array}{l}0,5 \mathrm{dmpaS}_{2} \mathrm{H}+ \\
0,5 \mathrm{NaN}\left(\mathrm{SiMe}_{3}\right)_{2}\end{array}$ & $\begin{array}{l}\mathrm{THF} / \\
\mathrm{CH}_{2} \mathrm{Cl}_{2} \\
\end{array}$ & {$\left[\mathrm{Cu}_{10}\left(\mathrm{dmpaS}_{2}\right)_{6} \mathrm{Cl}_{4}\right]$} \\
\hline $\mathrm{CuCl}$ & $\mathrm{dmpaS}_{2} \mathrm{H}$ & THF & amorpher, weißer Nd. \\
\hline $\mathrm{CuCl}$ & $\mathrm{dmpaS}_{2} \mathrm{H}+\mathrm{NaN}\left(\mathrm{SiMe}_{3}\right)_{2}$ & THF & {$\left[\mathrm{Cu}_{5}\left(\mathrm{dmpaS}_{2}\right)_{5}\right]$} \\
\hline $\mathrm{CuCl}$ & $2 \mathrm{dmpaS}_{2} \mathrm{H}+2 \mathrm{NaN}\left(\mathrm{SiMe}_{3}\right)_{2}$ & THF & weißes Gel \\
\hline $\mathrm{CuCl}$ & $2 \mathrm{dmpaS}_{2} \mathrm{H}+2 \mathrm{KO}^{t} \mathrm{Bu}$ & THF & {$\left[\mathrm{KCu}\left(\mathrm{dmpaS}_{2}\right)_{2}(\mathrm{thf})\right]_{\infty}$} \\
\hline $\mathrm{CuCl}$ & $2 \mathrm{dmpaS}_{2} \mathrm{H}+2 \mathrm{BuLi}$ & THF & {$\left[\mathrm{Cu}_{5}\left(\mathrm{dmpaS}_{2}\right)_{5}\right]$} \\
\hline $\mathrm{CuCl}$ & $0,6\left[\mathrm{Cu}_{5}\left(\mathrm{dmpaS}_{2}\right)_{5}\right]$ & THF & {$\left[\mathrm{Cu}_{8}\left(\mathrm{dmpaS}_{2}\right)_{6} \mathrm{Cl}_{2}\right]$} \\
\hline $\mathrm{CuCl}$ & $\begin{array}{l}\mathrm{dmpaS}_{2} \mathrm{H}+\mathrm{NaN}\left(\mathrm{SiMe}_{3}\right)_{2}+ \\
5 \mathrm{~S}\end{array}$ & THF & {$\left[\mathrm{Cu}_{8}\left(\mathrm{dmpaS}_{2}\right)_{6} \mathrm{Cl}_{2}\right]$} \\
\hline $\mathrm{CuCl}$ & $\begin{array}{l}0,6 \mathrm{AgCl}+1,2 \mathrm{dmpaS}_{2} \mathrm{H}+ \\
1,2 \mathrm{NaN}\left(\mathrm{SiMe}_{3}\right)_{2}\end{array}$ & THF & {$\left[\mathrm{Ag}_{2} \mathrm{Cu}_{4}\left(\mathrm{dmpaS}_{2}\right)_{6}\right]$} \\
\hline$\left[\mathrm{Cu}_{5}\left(\mathrm{dmpaS}_{2}\right)_{5}\right]$ & $\mathrm{O}_{2(\mathrm{~g})}+\mathrm{S}(\ddot{\mathrm{USS}})$ & THF & {$\left[\mathrm{Cu}_{5}\left(\mathrm{dmpaS}_{2}\right)_{5}\right], \mathrm{S}_{8}$} \\
\hline$\left[\mathrm{Cu}_{5}\left(\mathrm{dmpaS}_{2}\right)_{5}\right]$ & $10 \mathrm{~S}$ & THF & $\mathrm{S}_{8}$ \\
\hline$\left[\mathrm{Cu}_{8}\left(\mathrm{dmpaS}_{2}\right)_{6} \mathrm{Cl}_{2}\right]$ & $\mathrm{CuCl}$ (ÜS) & THF & braunes Gel \\
\hline $\mathrm{CuCl}_{2}$ & $\mathrm{dmpaS}_{2} \mathrm{H}+\mathrm{NaOMe}$ & $\mathrm{EtOH}$ & {$\left[\mathrm{Cu}_{2}\left(\mathrm{dmpaS}_{2} \mathrm{H}\right)_{2} \mathrm{Cl}_{2}\right]$} \\
\hline $\mathrm{AgCl}$ & $\mathrm{dmpaS}_{2} \mathrm{H}+\mathrm{NaN}\left(\mathrm{SiMe}_{3}\right)_{2}$ & THF & farblose Krist. \\
\hline $\mathrm{FeCl}_{2}$ & $\mathrm{dmpaS}_{2} \mathrm{H}+\mathrm{NaOMe}$ & $\mathrm{EtOH}$ & amorpher, brauner Nd. \\
\hline $\mathrm{FeCl}_{3}$ & $\mathrm{dmpaS}_{2} \mathrm{H}+\mathrm{NaOMe}$ & $\mathrm{EtOH}$ & $\mathrm{dmpaS}_{2} \mathrm{H}$ \\
\hline
\end{tabular}

* für eine Röntgenstrukturanalyse nicht geeignet

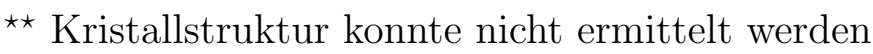




\section{Verzeichnis der verwendeten Abkürzungen}

\subsection{Moleküle und organische Gruppen}

\begin{tabular}{|c|c|}
\hline Ac & Acetat \\
\hline $\mathrm{BuH}$ & Butan \\
\hline${ }^{\mathrm{n}} \mathrm{Bu}$ & n-Butyl \\
\hline${ }^{t} \mathrm{Bu}$ & Tertiärbutyl \\
\hline $\mathrm{Cp}$ & Cyclopentadienid \\
\hline $\mathrm{Cp}^{\star}$ & Pentamethylcyclopentadienid \\
\hline dppaH & Bis(diphenylphosphino)amin \\
\hline dppaOH & Diphenylphosphino(diphenylphosphoryl)amin \\
\hline $\mathrm{dppaO}_{2} \mathrm{H}$ & Bis(diphenylphosphoryl)amin \\
\hline dppaOSH & Diphenylphosphoryl(diphenylthiophosphoryl)amin \\
\hline dppaOSeH & Diphenylphosphoryl(diphenylselenophosphoryl)amin \\
\hline $\mathrm{dppaS}_{2} \mathrm{H}$ & Bis(diphenylthiophosphoryl)amin \\
\hline dppaSe ${ }_{2} \mathrm{H}$ & Bis(diphenylselenophosphoryl)amin \\
\hline $\mathrm{dmpaS}_{2} \mathrm{H}$ & Bis(dimethylthiophosphoryl)amin \\
\hline $\mathrm{dppeS}_{2} \mathrm{H}$ & Bis(diphenylthiophosphoryl)ethan \\
\hline $\mathrm{dppmO}_{2} \mathrm{H}$ & Bis(diphenylphosphoryl)methan \\
\hline $\mathrm{dppmS}_{2} \mathrm{H}$ & Bis(diphenylthiophosphoryl)methan \\
\hline $\mathrm{dppmSe}_{2} \mathrm{H}$ & Bis(diphenylselenophosphoryl)methan \\
\hline $\mathrm{dppmSe}_{3}{ }^{\ominus}$ & {$\left[(\mathrm{Se}) \mathrm{PPh}_{2} \mathrm{CP}(\mathrm{Se}) \mathrm{Ph}_{2}\right]^{\ominus}$} \\
\hline 3,5 -DTBC & 3,5-Ditertiärbutylcatechol \\
\hline en & Ethylendiamin \\
\hline Et & Ethyl \\
\hline $\mathrm{Et}_{2} \mathrm{O}$ & Diethylether \\
\hline $\mathrm{EtOH}$ & Ethanol \\
\hline${ }^{i} \operatorname{Pr}$ & iso-Propyl \\
\hline$[18-\mathrm{K}-6]$ & [18-Krone-6] \\
\hline $\mathrm{K}_{4} \mathrm{P}_{2} \mathrm{O}_{7}$ & Kaliumdiphosphat \\
\hline $\mathrm{KO}^{\mathrm{t}} \mathrm{Bu}$ & Kaliumtertiärbutanolat \\
\hline $\mathrm{Me}$ & Methyl \\
\hline $\mathrm{MeOH}$ & Methanol \\
\hline $\mathrm{NaN}\left(\mathrm{SiMe}_{3}\right)_{2}$ & Natriumbis(trimethylsilyl)amid \\
\hline
\end{tabular}




$\begin{array}{ll}\mathrm{NaOMe} & \text { Natriummethanolat } \\ \mathrm{Naph} & \text { Naphthyl } \\ \mathrm{NEt}_{3} & \text { Triethylamin } \\ \mathrm{Ph} & \text { Phenyl } \\ \text { p-tol } & \text { para-Tolyl } \\ \text { py } & \text { Pyridin } \\ \mathrm{R} & \text { organischer Rest } \\ \text { th } & \text { Thioharnstoff } \\ \text { th }_{2} & \text { Dithiobiurea } \\ \text { thEt }_{2} & \text { N,N-Diethylthioharnstoff } \\ \mathrm{THF}_{\mathrm{H}} & \text { Tetrahydrofuran } \\ \text { thPh }_{2} & \text { N,N-Diphenylthioharnstoff } \\ \text { ÜS } & \text { Überschuß }\end{array}$

\subsection{Indizierung der IR-Spektren}

$\begin{array}{ll}\text { vs } & \text { sehr stark } \\ \text { s } & \text { stark } \\ \text { m } & \text { mittel }\end{array}$

\subsection{Sonstiges}

$\begin{array}{ll}\text { Äq. } & \text { Äquivalente } \\ \text { ber. } & \text { berechnet } \\ \text { B.O. } & \text { Bindungsordnung } \\ \text { br } & \text { breites Signal } \\ \Delta & \text { Differenz bzw. erhitzen } \\ \delta & \text { chemische Verschiebung in ppm } \\ \text { d } & \text { Duplett } \\ \text { E } & \text { Chalkogen } \\ \text { EI } & \text { Elektronenstoßionisation } \\ \text { eV } & \text { Elektronenvolt } \\ (\mathrm{g}) & \text { gasförmig }\end{array}$


gef. gefunden

h Stunden

HSAB hard and soft acids and bases

IR Infrarot

$\mathrm{J} \quad$ Kopplungskonstante in $\mathrm{Hz}$

KoZa Koordinationszahl

Krist. Kristalle

L Ligand

Ln Seltenerdmetall

LSq Least Square

m Multiplett

M Metall bzw. molar

$\mathrm{M}^{+} \quad$ Molekülpeak (Massespektrum)

min. Minuten

MS Massenspektrometrie

$\nu_{1 / 2} \quad$ Signalhalbwertsbreite in $\mathrm{Hz}$ (NMR-Spektren)

Nd. Niederschlag

NMR Kernmagnetische Resonanz

ppm parts per million

s Singulett

Smp. Schmelzpunkt

X Halogen 


\section{Verzeichnis der Abbildungen}

\section{Abbildungsverzeichnis}

1 Das Ligandensystem Bis(diorganochalkogenophosphoryl)amin (a) und

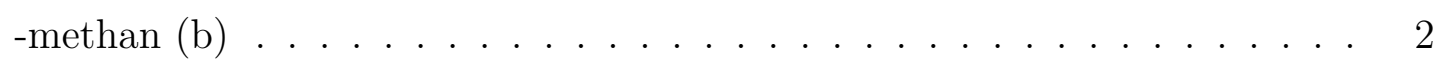

2 Koordinationsformen der Liganden $\operatorname{dppmE}_{2} \mathrm{H}, \mathrm{dppmE}_{2}{ }^{\ominus}$ und $\mathrm{dppmE}_{2}{ }^{2 \ominus}$. . 3

3 Grenzstrukturformeln der Bis(diorganochalkogenophosphoryl)amide . . . . 4

4 Koordinationsformen der Liganden $\mathrm{dppaE}_{2} \mathrm{H}$ und $\mathrm{dppaE}_{2}{ }^{\ominus} \ldots \ldots \ldots$

5 Koordinationsformen der Liganden $\mathrm{dmpaS}_{2} \mathrm{H}$ und $\mathrm{dmpaS}_{2}{ }^{\ominus} \ldots \ldots$. . . . . 6

6 Reaktion zur hypothetischen Spezies " $\mathrm{Cu}(\mathrm{L})$ " mit Koordinationszahl $=2 \quad{ }^{2} \quad 7$

7 Die verwendeten Ligandensysteme . . . . . . . . . . . . . . . 8

8 Die Struktur von $\left[\mathrm{Cu}\left(\mathrm{dppmO}_{2} \mathrm{H}\right)_{3}\right]\left[\mathrm{CuCl}_{4}\right] 1$ im Kristall . . . . . . . . . . . . 10

9 Die Struktur von $\left[\mathrm{Cu}_{2}\left(\mathrm{dppmS}_{2} \mathrm{H}\right)_{2} \mathrm{Cl}\right]\left[\mathrm{CuCl}_{2}\right] \underline{2}$ im Kristall . . . . . . . . . 12

10 Die Struktur von $\left[\mathrm{Cu}_{2}\left(\operatorname{dppeS}_{2} \mathrm{H}\right)_{2} \mathrm{Cl}_{2}\right]$ im Kristall . . . . . . . . . . . . . . 14

11 Die Struktur von $\left[\mathrm{K}_{2} \mathrm{Cu}_{2}\left(\mathrm{dppmS}_{2}\right)_{4}(\mathrm{thf})_{2}\right]$ im Kristall . . . . . . . . . . . 15

12 Die Struktur von $\left[\mathrm{Cu}_{2}\left(\mathrm{dppmSe}_{3}\right)_{2}\right]$ 3 im Kristall . . . . . . . . . . . . . 17

13 Die Struktur von $\left[\mathrm{Cu}\left(\mathrm{dppmSe}_{2}\right)(\mathrm{dppmSeH})\right]$ im Kristall . . . . . . . . . . . 18

14 Vorgeschlagener Reaktionsmechanismus der Reduktion von dppmSe $\mathrm{H}_{2} \mathrm{Hu}$ dppmSeH . . . . . . . . . . . . . . . . . . . . . . 19

15 Die Struktur von $\left[\mathrm{Au}_{2}\left(\mathrm{dppmS}_{2}\right)\left(\mathrm{PPh}_{3}\right)_{2}\right] \underline{4}$ im Kristall . . . . . . . . . . . . 21

16 Modell einer Drei-Zentren-zwei-Elektronen-Bindung . . . . . . . . . . . . . 21

17 Die Struktur von $\left[\mathrm{Ag}\left(\mathrm{dppmS}_{2}\right)\left(\mathrm{PPh}_{3}\right)\right]$ im Kristall _. . . . . . . . . . . . 23

18 Die Struktur von $\left[\mathrm{Cu}\left(\mathrm{dppaO}_{2}\right)_{2}(\mathrm{MeOH})\right] \underline{5}$ im Kristall . . . . . . . . . . . 25

19 Die Molekülstruktur von $\left[\mathrm{Cu}\left((\mathrm{PhO})_{2} \mathrm{P}(\mathrm{O}) \mathrm{NP}(\mathrm{O})(\mathrm{OPh})_{2}\right)_{2}\right] \ldots \ldots$. . . . . 26

20 Die Struktur von $\left[\mathrm{Cu}_{2}\left(\mathrm{dppaO}_{2}\right)_{2} \mathrm{Cl}_{2}(\text { thf })_{2}\right] \underline{6}$ im Kristall . . . . . . . . . . 28

21 Die Struktur von $\left[\mathrm{Cu}_{2}\left(\mathrm{dppaO}_{2}\right)_{2}\left(\mathrm{O}^{\mathrm{t}} \mathrm{Bu}\right)_{2}\right]$ im Kristall . . . . . . . . . . . 30

22 Die Struktur von $\left[\mathrm{Cu}_{2}\left(\mathrm{dppaO}_{2}\right)_{2}(\mathrm{OMe})_{2}\right] \underline{7}$ im Kristall . . . . . . . . . . . 31

23 Die Struktur von $\left[\mathrm{Cu}_{3}\left(\mathrm{dppaO}_{2}\right)_{2}\left(\left(\mathrm{OSiMe}_{2}\right)_{2} \mathrm{O}\right)_{2}(\text { thf })_{2}\right] \underline{8} \mathrm{im} \mathrm{Kristall} \ldots . . .33$

24 Der molekulare Aufbau von $\left[\mathrm{Cu}\left(\left(\mathrm{OSiPh}_{2}\right)_{2} \mathrm{O}\right)_{2}\left(\mathrm{Li}(\mathrm{py})_{2}\right)_{2}\right] \ldots \ldots$. . . . . . 34

25 Von S. Harvey et al. vorgeschlagener Mechanismus der Bildung von

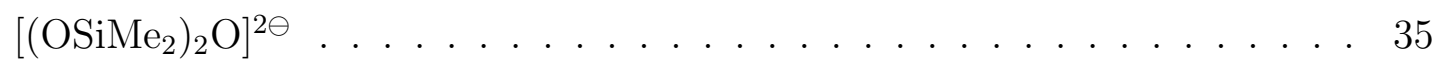

26 Die Struktur von $\left[\mathrm{Cu}_{4}(\mathrm{O})\left(\mathrm{dppaO}_{2}\right)_{4} \mathrm{Cl}_{2}\right] \underline{9}$ im Kristall . . . . . . . . . . . 36

27 Von R. Belfort et al. vorgeschlagener Mechanismus der Oxidioneneinlagerung 38

28 Ausschnitt aus der Struktur von $\left[\mathrm{Cu}\left(\mathrm{dppaO}_{2}\right)_{2}\left(\mathrm{NH}_{3}\right)_{3}\right]_{\infty} \underline{11}$ im Kristall . . 40 
29 Die Struktur von $\left[\mathrm{H}\left((\mathrm{O}) \mathrm{PPh}_{2} \mathrm{NPPh}_{2}\right)_{2} \mathrm{O}\right]\left[\mathrm{CuCl}_{2}\right] \underline{12} \mathrm{im} \mathrm{Kristall} \mathrm{.} \mathrm{.} \mathrm{.} \mathrm{.} \mathrm{.} \mathrm{.} \mathrm{.} 43$

30 Die Struktur von $\left[\mathrm{NaCu}_{2}\left(\mathrm{dppaSe}_{2}\right)_{3}(\mathrm{thf})_{2}\right] \underline{14} \mathrm{im} \mathrm{Kristall} \mathrm{\ldots .} \mathrm{.} \mathrm{.} \mathrm{.} \mathrm{.} \mathrm{.} \mathrm{.} \mathrm{.} 46$

31 Die Struktur von $\left[\mathrm{Cu}_{3}(\mathrm{dppaOS})_{3}\right] \underline{10}$ im Kristall . . . . . . . . . . . . . . 49

32 Vereinfachte Darstellung des molekularen Aufbaus von $\left[\mathrm{Cu}_{3}\left((\mathrm{PhO})_{2} \mathrm{P}(\mathrm{S}) \mathrm{NC}(\mathrm{S}) \mathrm{NEt}_{2}\right)_{3}\right] \ldots \ldots \ldots \ldots \ldots$

33 Die Struktur von $\left[\mathrm{Ag}_{3}\left(\mathrm{dppaS}_{2}\right)_{3}\right] 17$ im Kristall . . . . . . . . . . . . . 51

34 Die Struktur von $\left[\mathrm{Na}_{2} \mathrm{Ag}_{2}\left(\mathrm{dppaS}_{2}\right)_{4}(\mathrm{thf})_{4}\right] \underline{18}$ im Kristall . . . . . . . . 53

35 Die Struktur des Kations $\left[\mathrm{Cu}_{4}\left(\mathrm{dppaSe}_{2}\right)_{3}\right]^{\oplus}$ von $\underline{20} \mathrm{im} \mathrm{Kristall} \mathrm{.} \mathrm{.} \mathrm{.} \mathrm{.} \mathrm{.} \mathrm{.} \mathrm{.} 56$

36 Die Struktur von $\left[\mathrm{Cu}\left(\mathrm{dppaS}_{2}\right)(\mathrm{th})\right] \underline{21}$ im Kristall . . . . . . . . . . . . 58

$37{ }^{31} \mathrm{P}-\mathrm{NMR}-\mathrm{Spektrum}$ von $\left[\mathrm{NaCu}_{2}\left(\mathrm{dppaS}_{2}\right)_{3}(\text { thf })_{2}\right] \underline{13}$ in THF und $\mathrm{C}_{6} \mathrm{D}_{6}$ in Abhängigkeit von der Temperatur . . . . . . . . . . . . . . . . 61

38 Die Struktur von $\left[\mathrm{Cu}_{2}\left(\mathrm{dmpaS}_{2} \mathrm{H}\right)_{2} \mathrm{Cl}_{2}\right] \underline{22}$ im Kristall . . . . . . . . . . . 63

39 Die Struktur von $\left[\mathrm{Cu}_{5}\left(\mathrm{dmpaS}_{2}\right)_{5}\right] \underline{23}$ im Kristall . . . . . . . . . . . . . 65

40 Die Struktur von $\left[\mathrm{Cu}_{8}\left(\mathrm{dmpaS}_{2}\right)_{6} \mathrm{Cl}_{2}\right] 24$ im Kristall . . . . . . . . . . . . . 66

41 Darstellung des molekularen Aufbaus von $\left[\mathrm{Cu}_{8}\left(\mathrm{dmpaS}_{2}\right)_{6} \mathrm{Cl}_{2}\right]_{24}^{24} \ldots . .68$

42 Die Struktur von $\left[\mathrm{Cu}_{10}\left(\mathrm{dmpaS}_{2}\right)_{6} \mathrm{Cl}_{4}\right] 25$ im Kristall . . . . . . . . . . . . . 69

43 Vereinfachte Darstellung der Molekülstruktur von $\left[\mathrm{Cu}_{10}\left(\mathrm{dmpaS}_{2}\right)_{6} \mathrm{Cl}_{4}\right] \underline{25}$. 70

44 Die Struktur von $\left[\mathrm{KCu}\left(\mathrm{dmpaS}_{2}\right)_{2}(\text { thf })\right]_{\infty} \underline{26}$ im Kristall (Ausschnitt) . . . . 72

45 Die Struktur von $\left[\mathrm{KCu}\left(\mathrm{dmpaS}_{2}\right)_{2}(\mathrm{thf})\right]_{\infty} \underline{26} \mathrm{im}$ Kristall (Ausschnitt) . . . . 74

$46 \quad{ }^{31} \mathrm{P}-\mathrm{NMR}-\mathrm{Spektren}$ der Lösungen von $\mathrm{CuCl}$ und $\mathrm{dmpaS}_{2}{ }^{\ominus}$ in verschiedenen Molverhältnissen . . . . . . . . . . . . . . . 76

47 Temperaturabhängige ${ }^{31} \mathrm{P}-\mathrm{NMR}-$ Spektren von $\left[\mathrm{Cu}_{10}\left(\mathrm{dmpaS}_{2}\right)_{6} \mathrm{Cl}_{4}\right] \underline{25}$ in $\mathrm{CDCl}_{3} \ldots \ldots \ldots \ldots \ldots \ldots \ldots \ldots$

48 Die Struktur von $\left[\mathrm{Hg}\left(\mathrm{dmpaS}_{2}\right)_{2}\right] 27 \mathrm{im}$ Kristall . . . . . . . . . . . . . . . 80

49 Auswahl der Reaktionen mit $\mathrm{dppmE}_{2} \mathrm{H}$. . . . . . . . . . . . . . . . 215

50 Reaktionen von $\mathrm{dppaOE}^{\ominus}$ mit $\mathrm{CuCl}$ und $\mathrm{CuCl}_{2}$. . . . . . . . . . . . . 216

51 Reaktionen von $\mathrm{dppaE}_{2} \mathrm{H}$ mit $\mathrm{CuCl}$ und $\mathrm{AgCl}$. . . . . . . . . . . . . . 217

52 Reaktionen von $\mathrm{dmpaS}_{2} \mathrm{H}$ mit $\mathrm{CuCl}, \mathrm{CuCl}_{2}$ und $\mathrm{HgCl}_{2}$. . . . . . . . . . 218 


\section{Literaturverzeichnis}

[1] A. F. Holleman, E. Wiberg, N. Wiberg. "Lehrbuch der Anorganischen Chemie“. Walter de Gruyter, Berlin, 101. Aufl. (1995).

[2] N. N. Greenwood, A. Earnshaw. „Chemie der Elemente“. VCH Verlagsgesellschaft mbH, Weinheim, 1. Aufl. (1988).

[3] F. A. Cotton, G. Wilkinson. „Anorganische Chemie: eine zusammenfassende Darstellung für Fortgeschrittene“. Verlag Chemie GmbH, Weinheim, 4. Aufl. (1982).

[4] T. Greiser, E. Weiss. Chem. Ber. 109, 3142 (1976).

[5] I. G. Dance, J. C. Calabrese. Inorg. Chem. Acta 19, L41 (1976).

[6] S. L. Lawton, W. J. Rohrbaugh, G. T. Kokotailo. Inorg. Chem. 11, 621 (1972).

[7] S. O. Grim, L. C. Satek, J. D. Mitchell. Z. Naturforsch. 35 b, 832 (1980).

[8] C. J. Carmalt, A. H. Cowley, A. Decken, Y. G. Lawson, N. C. Norman. Acta Crystallogr. C52, 931 (1996).

[9] S. O. Grim, E. D. Walton. Inorg. Chem. 19, 1982 (1980).

[10] P. J. Carrol, D. D. Titus. J. Chem. Soc., Dalton Trans. 1977, 824.

[11] P. A. W. Dean. Can. J. Chem. 57, 754 (1979).

[12] M. Y. Antipin, Y. T. Struchkov, S. A. Pisareva, T. Y. Medved, M. I. Kabachnik. J. Struct. Chem. 1980, 644.

[13] E. W. Ainscough, H. A. Bergen, A. M. Brodie, K. A. Brown. J. Chem. Soc., Dalton Trans. 1976, 1649.

[14] G. Pelizzi, P. Tarasconi, F. Vitali, C. Pelizzi. Acta Crystallogr. C43, 1505 (1987).

[15] S. A. Sangokoya, B. Lee, M. F. Self, W. T. Pennington, G. H. Robinson. Polyhedron 8, 1497 (1989).

[16] E. W. Ainscough, A. M. Brodie, K. L. Brown. J. Chem. Soc., Dalton Trans. 1980, 1042.

[17] M. Valderrama, R. Contreras, M. Bascunan, D. Boys. Polyhedron 13, 1101 (1994). 
[18] D. H. Robinson, B. Lee, W. T. Pennington, S. A. Sangokoya. J. Am. Chem. Soc. 110, 6260 (1988).

[19] M. C. Gimeno, A. Laguna, M. Laguna, F. Sanmartin, P. G. Jones. Organometallics 12, 3984 (1993).

[20] A. M. Z. Slawin, M. B. Smith, J. D. Woollins. J. Chem. Soc., Dalton Trans. 1996, 3659.

[21] H. Nöth, L. Meinel. Z. anorg. allg. Chem. 349, 225 (1967).

[22] A. H. Cowley, H. Steinfink. Inorg. Chem. 4, 1827 (1965).

[23] A. Schmidpeter, J. Ebeling. Chem. Ber. 101, 815 (1968).

[24] H. Niebergall, B. Langenfeld. Chem. Ber. 95, 64 (1962).

[25] J. S. Casas, A. Castineiras, I. Haiduc, A. Sanchez, J. Sordo, E. M. Vazquez-Lopez. Polyhedron 13, 2873 (1994).

[26] C. Silvestru, R. Rösler, I. Haiduc, R. Cea-Olivares, G. Espinosa-Perez. Inorg. Chem. 34, 3352 (1995).

[27] M. R. Churchill, J. Cooke, J. P. Fennessey, J. Wormald. Inorg. Chem. 10, 1031 (1971).

[28] R. Cea-Olivares, J. Novosad, J. D. Woollins, A. M. Z. Slawin, V. Garcia-Montalvo, G. Espinosa-Perez, P. G. y Garcia. J. Chem. Soc., Chem. Commun. 1996, 519.

[29] M. Rietzel, H. W. Roesky, K. V. Katti, H. G. Schmidt, R. Herbst-Irmer, M. Noltemeyer, G. M. Sheldrick, M. C. R. Symons, A. Abu-Raqabah. J. Chem. Soc., Dalton Trans. 1990, 2387.

[30] E. N.-M. Ho, W.-T. Wong. J. Chem. Soc., Dalton Trans. 1997, 915.

[31] V. Garcia-Montalvo, J. Novosad, P. Kilian, J. D. Woollins, A. M. Slawin, P. G. y Garcia, M. Lopez-Cardoso, G. Espinosa-Perez, R. Cea-Olivares. J. Chem. Soc., Dalton Trans. 1997, 1025.

[32] C. P. Huber, M. L. Post, O. Siiman. Acta Crystallogr. B34, 2629 (1978). 
[33] H. Rudler, B. Denise, J. R. Gregorio, J. Vaissermann. J. Chem. Soc., Chem. Commun. 1997, 2299.

[34] M. R. Churchill, J. Wormald. Inorg. Chem. 10, 1778 (1971).

[35] M. Jansen. Angew. Chem. 99, 1136 (1987).

[36] F. A. Cotton, X. Feng, M. Matusz, R. Poli. J. Am. Chem. Soc. 110, 7077 (1988).

[37] F. A. Cotton, M. Matusz, R. Poli, X. Feng. J. Am. Chem. Soc. 110, 1144 (1988).

[38] F. A. Cotton, X. Feng, D. J. Timmons. Inorg. Chem. 37, 4066 (1998).

[39] G. R. Willey, M. D. Rudd, C. J. Samuel, M. G. B. Drew. J. Chem. Soc., Dalton Trans. 1995, 759 .

[40] E. J. Fernandez, P. G. Jones, A. Laguna, A. Mendia. Inorg. Chim. Acta 215, 229 (1994).

[41] J. E. Barkley, D. J. Evans, D. L. Hughes, G. J. Leigh. J. Chem. Soc., Dalton Trans. 1993, 69.

[42] Z. A. Sheka, K. B. Yatsimirskii, M. A. Ablova. Russ. J. Inorg. Chem. 15, 1527 (1970).

[43] K. B. Yatsimirskii, Y. T. Struchkov, A. S. Batsanov, E. I. Sinyavskaya. Koord. Khim. 11, 826 (1985).

[44] M. S. Abbassioun, P. A. Chaloner, C. Claver, P. B. Hitchcock, A. M. Masdeu, A. Ruiz, T. Saballs. J. Organomet. Chem. 403, 229 (1991).

[45] R. Contreras, M. Valderrama, S. Yanez. Transition Met. Chem. 18, 73 (1993).

[46] O. Crespo, M. C. Gimeno, P. G. Jones, A. Laguna. Inorg. Chem. 33, 6128 (1994).

[47] K. B. Yatsimirskii, G. G. Talanova, E. A. Nazarova, M. I. Kabachnik, T. A. Mastryukova, I. M. Aladzheva, E. V. Leonteva, M. Y. Antipin, Y. T. Struchkov. Koord. Khim. 19, 469 (1993).

[48] F. A. Cotton, B. A. Frenz, D. L. Hunter, Z. C. Mester. Inorg. Chim. Acta 11, 111 (1974).

[49] K. L. Brown. Acta Crystallogr. B35, 462 (1979). 
[50] F. H. Allen, O. Kennard, D. G. Watson, L. Brammer, A. G. Orpen, R. Taylor. J. Chem. Soc., Perkin Trans. II 1987, S1.

[51] D. Cauzzi, C. Graiff, M. Lanfranchi, G. Predieri, A. Tiripicchio. J. Chem. Soc., Chem. Commun. 1995, 2443.

[52] L. C. Göldner. „Synthese und Struktur von Chelatkomplexen der Übergangsmetalle. Hausarbeit im Rahmen der ersten Staatsprüfung für das Lehramt an Gymnasien“. Universität Göttingen (Januar 1999).

[53] P. Bhattacharyya, A. M. Z. Slawin, D. J. Williams, J. D. Woollins. J. Chem. Soc., Dalton Trans. 1995, 2489.

[54] P. Peringer, J. Schwald. J. Chem. Soc., Chem. Commun. 1986, 1625.

[55] C. Göbel. „Röntgenstrukturuntersuchungen an Chelatkomplexen. Hausarbeit im Rahmen der Ersten Staatsprüfung für das Lehramt an Gymnasien“. Universität Göttingen (Januar 1999).

[56] E. J. Fernandez, M. C. Gimeno, P. G. Jones, A. Laguna, J. M. L. de Luzuriaga. J. Chem. Soc., Dalton Trans. 1992, 3365.

[57] B. G. F. Johnson, J. Lewis, P. R. Raithby, A. Sanders. J. Organomet. Chem. 260, C29 (1984).

[58] J. Vincente, M. T. Chicote, J. A. Cayuelas, J. J. Fernandez-Baeza, P. G. Jones, G. M. Sheldrick, P. Espinet. J. Chem. Soc., Dalton Trans. 1985, 1163.

[59] M. C. Gimeno, P. G. Jones, A. Laguna, M. D. Villacampa. Chem. Ber. 129, 585 (1996).

[60] J. Vincente, M.-T. Chicote, M.-C. Lagunas, P. G. Jones. J. Chem. Soc., Dalton Trans. 1991, 2579.

[61] A. Laguna, M. Laguna. J. Organomet. Chem. 394, 743 (1990).

[62] R. O. Day, R. R. Holmes, A. Schmidpeter, K. Stoll, L. Howe. Chem. Ber. 124, 2443 (1991).

[63] C. S. Browning, D. H. Farrar, D. C. Frankel. Inorg. Chim. Acta 241, 111 (1996). 
[64] N. Platzer, H. Rudler, C. Alvarez, L. Barkaoui, B. Denise, N. Goasdoue, M.-N. Rager, J. Vaissermann, J.-C. Daran. Bull. Soc. Chim. Fr. 132, 95 (1995).

[65] C. Alvarez, L. Barkaoui, N. Goasdoue, J. C. Daran, N. Platzer, H. Rudler, J. Vaissermann. J. Chem. Soc., Chem. Commun. 1989, 1507.

[66] Die Strukturlösung und Verfeinerung wurden von K. Winkler und P. Müller durchgeführt.

[67] H. Richter, E. Fluck, H. Riffel, H. Hess. Z. anorg. allg. Chem. 496, 109 (1983).

[68] C. S. Browning, D. H. Farrar, D. C. Frankel. Inorg. Chim. Acta 241, 111 (1996).

[69] A. J. C. Wilson. „International Tables for Crystallography“, Bd. C. Kluwer Academic Publishers (1995).

[70] H. Nöth. Z. Naturforsch. 37 b, 1491 (1982).

[71] M. R. Churchill, Y.-J. Li, D. Nalewajek, P. M. Schaber, J. Dorfman. Inorg. Chem. 24, 2684 (1985).

[72] L.-P. Wu, T. Kuroda-Sova, M. Maekawa, Y. Suenaga, M. Munakata. J. Chem. Soc., Dalton Trans. 1996, 2179.

[73] M. B. Hursthouse, M. Motevalli, M. Sanganee, A. C. Sullivan. J. Chem. Soc., Chem. Commun. 1991, 1709.

[74] P. D. Lickiss, A. D. Redhouse, R. J. Thompson, W. A. Stanczyk, K. Rozga. J. Organomet. Chem. 453, 13 (1993).

[75] R. Sillanpaa, T. Nortia, L. Hiltunen. Inorg. Chim. Acta 83, 111 (1984).

[76] W. J. Evans, T. A. Ulibarri, J. W. Ziller. Organometallics 10, 134 (1991).

[77] S. Harvey, M. F. Lappert, C. L. Raston, B. W. Skelton, G. Srivastava, A. H. White. J. Chem. Soc., Chem. Commun. 1988, 1216.

[78] D. A. Edwards, R. M. Harker, M. F. Mahon, K. C. Molloy. J. Chem. Soc., Dalton Trans. 1997, 3509.

[79] X. Zhou, H. Ma, X. Huang, X. You. J. Chem. Soc., Chem. Commun. 1995, 2483. 
[80] M. R. Churchill, C. H. Lake, S.-H. L. Chao, J. O. T. Beachley. J. Chem. Soc., Chem. Commun. 1993, 1577.

[81] S. Kraut, J. Magull, U. Schaller, M. Karl, K. Harms, K. Dehnicke. Z. anorg. allg. Chem. 624, 1193 (1998).

[82] T. A. Bzhenova, R. M. Lobkovskaya, R. P. Shibaeva, A. E. Shilov. J. Organomet. Chem. 330, 9 (1987).

[83] A. Müller, B. Neumüller, K. Dehnicke, J. Magull, D. Fenske. Z. anor. allg. Chem. 623, 1306 (1997).

[84] A. J. Blake, C. M. Grant, I. C. Gregory, S. Parsons, J. M. Rawson, D. Reed, R. E. P. Winpenny. J. Chem. Soc., Dalton Trans. 1995, 163.

[85] R. Belford, D. E. Fenton, M. R. Truter. J. Chem. Soc., Dalton Trans. 1972, 2345.

[86] J. A. Bertrand. Inorg. Chem. 6, 495 (1967).

[87] J. A. Bertrand, J. A. Kelley. Inorg. Chem. 8, 1982 (1969).

[88] B. T. Kilbourn, J. D. Dunitz. Inorg. Chim. Acta 1, 209 (1967).

[89] H. Schumann, P. R. Lee, J. Loebel. Angew. Chem. 101, 1073 (1989).

[90] A. Schmidpeter, H. Groeger. Chem. Ber. 100, 3979 (1967).

[91] O. Siiman, J. Vetuskey. Inorg. Chem. 19, 1672 (1980).

[92] O. Siiman, C. P. Huber, M. L. Post. Inorg. Chim. Acta 25, L11 (1977).

[93] R. Rösler, J. E. Drake, C. Silvestru, J. Yang, I. Haiduc. J. Chem. Soc., Dalton Trans. 1996, 391.

[94] V. Gracia-Montalvo, R. Cea-Olivares, G. Espinosa-Perez. Polyhedron 15, 829 (1996).

[95] C. Silvestru, R. Rösler, I. Haiduc, R. A. Toscano, D. B. Sowerby. J. Organomet. Chem. 515, 131 (1996).

[96] P. Z. Zak, M. Fofana, J. Kamenicek, T. Glowiak. Acta Crystallogr. C45, 1686 (1989). 
[97] A. M. Z. Slawin, M. B. Smith, J. D. Woollins. J. Chem. Soc., Dalton Trans. 1998, 1537.

[98] J. Jang, J. E. Drake, S. Hernandez-Ortega, R. Rösler, C. Silvestru. Polyhedron 16, 4061 (1997).

[99] J. E. Drake, A. Silvestru, J. Yang, I. Haiduc. Inorg. Chim. Acta 271, 75 (1998).

[100] T. Q. Ly, F. E. Mabbs, E. J. L. McInnes, A. M. Z. Slawin, J. D. Woollins. Inorganic Chemistry Communications 1, 143 (1998).

[101] A. N. Chekhlov, A. I. Yurtanov, I. V. Martynov. Koord. Khim. 13, 1541 (1987).

[102] H. M. Colquhoun, J. F. Stoddart, D. J. Williams. J. Chem. Soc., Chem. Commun. 1981, 849.

[103] G. W. Svetich, C. N. Caughlan. Acta Crystallogr. 19, 645 (1965).

[104] J. D. Woollins. J. Chem. Soc., Dalton Trans. 1996, 2893.

[105] R. Rösler, C. Silvestru, G. Espinosa-Perez, I. Haiduc, R. Cea-Olivares. Inorg. Chim. Acta 241, 47 (1996).

[106] I. Haiduc, C. Silvestru, H. W. Roesky, H.-G. Schmidt, M. Noltemeyer. Polyhedron 12, 69 (1993).

[107] R. Rossi, A. Marchi, L. Marvelli, L. Magon, M. Peruzzini, U. Casellato, R. Graziani. J. Chem. Soc., Dalton Trans. 1993, 723.

[108] I. Haiduc, R. Cea-Olivares, R. A. Toscano, C. Silvestru. Polyhedron 14, 1067 (1995).

[109] M. J. Pilkington, A. M. Z. Slawin, D. J. Williams, J. D. Woollins. Polyhedron 10, 2641 (1991).

[110] D. J. Birsdall, D. Cupertino, J. D. Woollins, A. M. Z. Slawin. Phosphorus, Sulfur, and Silicon 124 \& 125, 465 (1997).

[111] E. Herrmann, R. Richter, N. Chau. Z. anorg. allg. Chem. 623, 403 (1997).

[112] C. W. Liu, J. T. Pitts, J. P. Fackler. Polyhedron 16, 3899 (1997).

[113] R. Hesse, L. Nilson. Acta Chem. Scand. 23, 825 (1969). 
[114] B. Krebs, G. Henkel. Z. anorg. allg. Chem. 475, 143 (1981).

[115] J. T. Shore, W. T. Pennington, M. C. Noble, A. W. Cordes. Phosphorus and Sulfur 39, 153 (1988).

[116] J. Cusick, M. L. Scudder, D. C. Craig, I. G. Dance. Polyhedron 8, 1139 (1989).

[117] W. A. Spofford, E. L. Amma. Acta Crystallogr. B26, 1474 (1970).

[118] A. Davison, E. S. Switkes. Inorg. Chem. 10, 837 (1971).

[119] D. Cupertino, R. Keyte, A. M. Z. Slawin, J. D. Woollins, D. J. Williams. Polyhedron 15, 4441 (1996).

[120] F. Hollander, D. Coucouvanis. J. Am. Chem. Soc. 99, 6268 (1977).

[121] T. H. Lemmen, K. Folting, J. C. Huffman, K. G. Caulton. J. Am. Chem. Soc. 107, $7774(1985)$.

[122] N. G. Zabirov, V. N. Solov'ev, F. M. Shamsevaleev, R. A. Cherkasov, I. V. Martynov. Zh. Obshch. Khim. 61, 1748 (1991).

[123] F. T. Wang, J. Najdzionek, K. L. Leneker, H. Wasserman, D. M. Braitsch. Synth. React. Inorg. Met.-Org. Chem. 8, 119 (1978).

[124] P. Bhattacharyya, A. M. Z. Slawin, M. B. Smith, J. D. Woollins. Inorg. Chem. 35, 3675 (1996).

[125] S. O. Grim, E. D. Walton. Inorg. Chem. 19, 1982 (1980).

[126] W. E. Slinkard, D. W. Meek. J. Chem. Soc., Dalton Trans. 1973, 1024.

[127] T. H. Lemmen, G. V. Goeden, J. C. Huffman, R. L. Geerts, K. G. Caulton. Inorg. Chem. 29, 3680 (1990).

[128] G. M. Sheldrick. „SHELXS-97. Program for Solution of Crystal Structures“. Göttingen (1997).

[129] G. M. Sheldrick. „SHELXL-97. Program for the Refinement of Crystal Structures“. Göttingen (1997).

[130] P. Debye. Ann. Phys. 43, 49 (1913). 
[131] I. Waller. Ann. Phys. 81, 154 (1927).

[132] E. Keller. „SCHAKAL. A Fortran Program for the Graphic Representation of Molecular and Crystallographic Models“. Universität Freiburg (1992).

[133] K. Brandenburg. „Diamont Version 2.1c“. Crystal Impact GbR (1999).

[134] Bruker Analytical X-ray Systems. „SAINT: SAX Area-detector Integration V5.00“ (1997). 


\section{Danksagung und Lebenslauf}

Danksagung

Die vorliegende Arbeit ist in der Zeit von August 1997 bis September 1999 unter der Leitung von Herrn Prof. Dr. J. Magull am Institut für Anorganische Chemie der Universität Göttingen angefertigt worden.

Ich danke allen herzlich, die durch ihre moralische und materielle Unterstützung zum Gelingen der vorliegenden Arbeit beigetragen haben.

Für die Durchführung von Kristallstrukturanalysen danke ich C. Göbel [55] (Verbindungen $\underline{4}$, $\underline{8}$ und $\underline{26}$ ), K. Winkler und P. Müller (Verbindung $\underline{5}$ ) und C. Lehmann (Verbindung 25).

Mein Dank gilt ebenfalls:

T. Albrecht, S. Dullnig, G. Elter, L. Friedrich, M. Geissinger, L.C. Göldner, T. Groh, U. Hasselhof, J. Haupt, M. Hesse, M. Hoffmann, J. Magull, U.H. Schaller, H.-J. Schlette, R. Schöne, G.M. Sheldrick, E. v. Steuber, A. Vielmäder, W. Zolke 